\title{
Transition Detection for Low Speed Wind Tunnel Testing Using Infrared Thermography
}

\author{
Liselle A. Joseph
}

Thesis submitted to the faculty of the Virginia Polytechnic Institute and State

University in partial fulfilment of the requirements for the degree of

Master of Science

In

Aerospace Engineering

\author{
Aurelien Borgoltz, Chair \\ William J. Devenport, Co-Chair \\ Kevin T. Lowe
}

$02 / 07 / 2014$

Blacksburg, Virginia

Keywords: Boundary Layer Transition, Infrared Thermography, Wind Turbine, Wind Tunnel, Stethoscope 


\title{
Transition Detection for Low Speed Wind Tunnel Testing Using Infrared Thermography
}

\author{
Liselle A. Joseph
}

\begin{abstract}
Transition is an important phenomenon in large scale, commercial, wind tunnel testing at low speeds because it is an excellent indicator of an airfoil performance. It is difficult to estimate transition through numerical techniques because of the complex nature of viscous flow. Therefore experimental techniques can be essential. Over the transition region the rate of heat transfer shows significant increases which can be detected using infrared thermography. This technique has been used predominantly at high speeds, on small models made of insulated materials, and for short test runs. Large scale testing has not been widely undertaken because the high sensitivity of transition to external factors makes it difficult to detect.

The present study records the process undertaken to develop, implement and validate a transition detection system for continual use in the Virginia Tech Stability Wind Tunnel: a low speed, commercial wind tunnel where large, aluminium models are tested. The final system developed comprises of two high resolution FLIR A655sc infrared cameras; four 63.5-mm diameter circular windows; aluminium models covered in $0.8-\mathrm{mm}$ silicone rubber insulation and a top layer of ConTact ${ }^{\odot}$ paper; and a series of 25.4-mm wide rubber silicone fiberglass insulated heaters mounted inside the model and controlled externally by experimenters. This system produces images or videos of the model and the associated transition location, which is later extracted through image processing methods to give a final transition location in percentage chord.

The system was validated using two DU96-W-180 airfoils of different chord lengths in the Virginia Tech Stability Wind Tunnel, each tested two months apart. The system proved to be robust and efficient, while not affecting the airfoil performance or any other system in use in the wind tunnel. Transition results produced by the system were compared to measurements obtained from pressure data and stethoscope tests as well as the numerical predictions of XFOIL. The transition results from all four methods showed excellent agreement with each other for the two models, for at least two Reynolds numbers and for several angles of attack on both suction and pressure side of the model. The agreement of data obtained under such different conditions and at different times suggests that the infrared thermography system efficiently and accurately detects transition for large aluminium models at low speeds.
\end{abstract}




\section{Acknowledgments}

First, and above all, I thank the Lord for His continual provision and guidance. He has blessed me with this research opportunity and with several wonderful people who have helped me to be successful.

I must express my sincere gratitude to General Electric Power and Water, particularly the Wind Blade Aerodynamics \& Acoustics Team, for funding this project and providing their technical expertise throughout.

A great debt of gratitude is owed to my academic advisor, Dr Aurelien Borgoltz, whose immense knowledge and unfailing support has proved invaluable to the success of this study. I especially want to thank $\mathrm{Dr}$ Borgoltz for going above and beyond to ensure that I gained a full spectrum of experiences and skills during my tenure as his graduate student, an effort which resulted in my growth as a researcher and as a young professional. I also thank $D r$. William J. Devenport, my committee co-chairman, for his guidance, motivation and unmatched interest in my wellbeing and success. In addition, I thank Dr Kevin T. Lowe for his critical analysis and insightful advice throughout this project, and for serving on my thesis committee.

I would be remiss if I did not acknowledge the outstanding contributions of technicians of the AOE Machine Shop: Mr James A. Lambert, Mr Cameron J. Hollandsworth, $\mathrm{Mr}$ J. Scott Patrick and Mr Matthew Del Mills. Your ideas, expertise and commitment went well past your daily duties and I greatly appreciate your work ethic. Very special thanks is also due to $\mathrm{Mr}$ Bill P. Oetjens, the Stability Wind Tunnel Engineer, and Mr Michael Morton, the Stability Wind Tunnel Test Engineer for willingly devoting several long hours during the testing phase of this project. I also thank Mr Mark R. Montgomery, the AOE Electrical Engineer, who assisted me with development of the electrical circuit for the new system.

I thank my colleagues of CREATe and Lab 7 for your assistance and the productive work environment you foster. In particular, I would like to thank Dan Cadel, Tim Meyers and Ken Brown who assisted me during testing in the Stability Wind Tunnel. I must also recognize the superb work of Stephen Young, an outstanding undergraduate researcher whose commitment to excellent research is reflected in his many long hours of work in this study.

Finally I express heartfelt gratitude to my parents and siblings in Grenada for their unconditional love, support and prayers. Likewise I acknowledge the encouragement of my Harvest Baptist Church family and my friends, and the unparalleled counsel of my most trusted personal advisors: Dr H. Pat Artis and Dr Rita F. Klein.

-Liselle

All photographs presented in this thesis were taken by the author, 2014 . 


\section{Table of Contents}

Chapter 1: INTRODUCTION ............................................................................................... 1

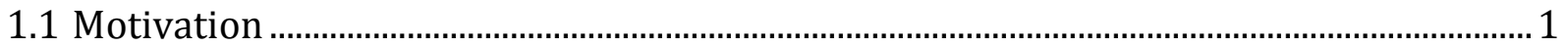

1.2 Literature Review................................................................................................................. 1

1.3 Scope and Aim of this Research ............................................................................................. 8

Chapter 2: APPARATUS AND INSTRUMENTATION _........................................................... 10

2.1 Open Jet Wind Tunnel............................................................................................................. 10

2.1.1 Open Jet Wind Tunnel Circuit .......................................................................................... 10

2.1.2 Model Mounting in Open Jet Wind Tunnel ................................................................... 12

2.2 Stability Wind Tunnel ........................................................................................................ 14

2.2.1 Stability Wind Tunnel Circuit .................................................................................... 15

2.2.2 Aerodynamic Test Section......................................................................................... 17

2.2.3 Flow Control System ................................................................................................... 18

2.2.4 Pressure Measurement Systems............................................................................. 21

2.2.4.1 Reference Pressure Measurements .................................................................... 21

2.2.4.2 Airfoil Surface Pressure Measurements .............................................................. 22

2.2.4.3 Test Section Wall Pressure Measurements .......................................................... 23

2.2.4.4 Wake Rake Pressure Measurement System ...................................................... 25

2.2.4.5 Measurement Corrections for Wall Interference ……………………………..... 27

2.2.4.6 Uncertainties in Measurements ........................................................................ 27

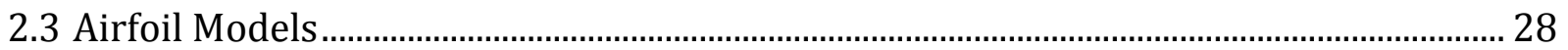

2.3 .1 0.8-m, 18\% Proprietary Model.................................................................................. 28

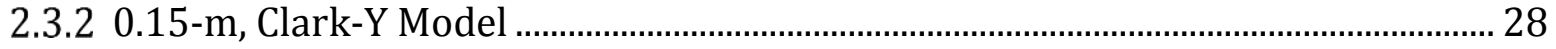

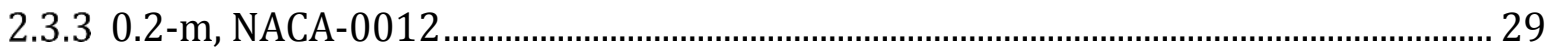

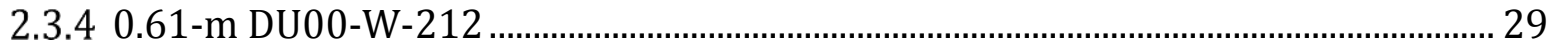

2.3.5 0.8-m DU96-W-180 ........................................................................................... 30

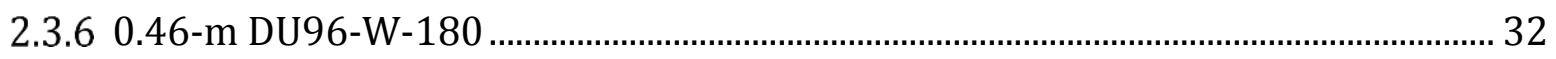

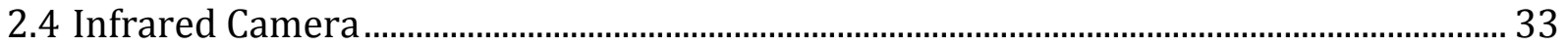

Chapter 3: TRANSITION DETECTION SYSTEM DEVELOPMENT …...................................... 35

3.1 Infrared Thermography System Requirements ................................................................ 35

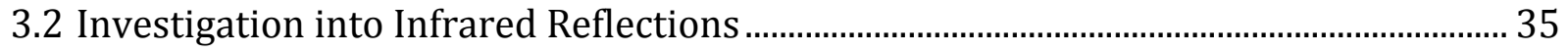


3.3 Development of Temperature Control System ………..................................................... 37

3.4 Investigation into Model Materials......................................................................................... 40

3.5 Aluminium Model Insulation ................................................................................................. 47

3.6 Additional Transition Detection Measurement Systems .................................................. 52

3.6.1 Mean Pressure Analysis ............................................................................................... 53

3.6.2 Stethoscope Measurements....................................................................................... 56

3.6.3 Preliminary Validation of Infrared Thermography Measurements.......................... 57

3.7 System Implementation in Stability Wind Tunnel .............................................................. 59

Chapter 4: TRANSITION DETECTION SYSTEM VALIDATION............................................67

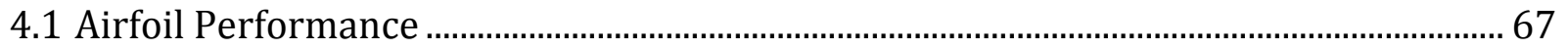

4.2 Effect of Infrared System on Airfoil Performance.................................................................. 70

4.2.1 Effect of Heated Model.................................................................................................... 70

4.2.2 Effect of Silicone Rubber Insulator ................................................................................ 73

4.2.3 Combined Effect of Heated Model and Silicone Rubber Insulator ............................ 77

4.3 Transition Detection Results................................................................................................... 79

4.3.1 Mean Pressure Results ............................................................................................ 79

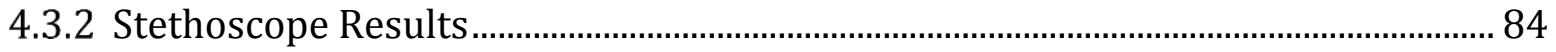

4.3.3 Infrared Thermography Results ................................................................................ 89

4.3.4 Validation of Infrared Results.................................................................................... 96

4.4 Boundary Layer Flow Diagnostics ………………………………………………….... 102

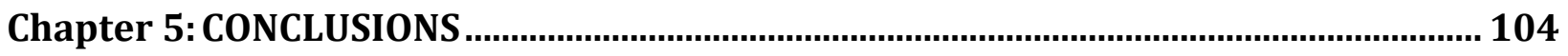

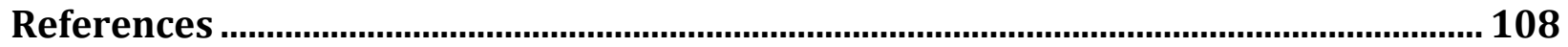

Appendix A: Additional Instrumentation \& Equipment............................................ 110

Appendix B: Airfoil Pressure Port Locations ……........................................................... 112

Appendix C: Infrared Videos of Flow .............................................................................. 114

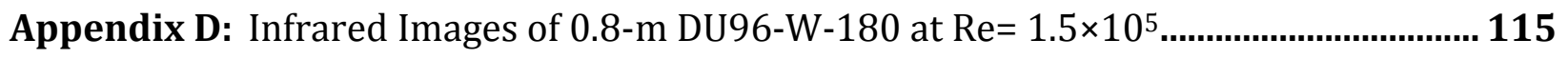

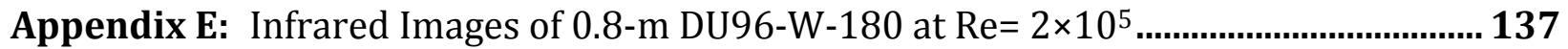

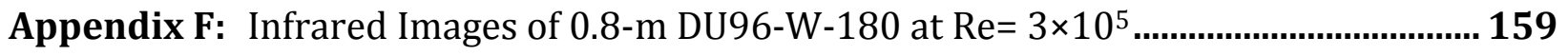

Appendix G: Infrared Images of 0.46-m DU96-W-180 at Re= $1.5 \times 10^{5} \ldots \ldots \ldots \ldots \ldots \ldots \ldots . . . . . . . . . . . . . . .175$

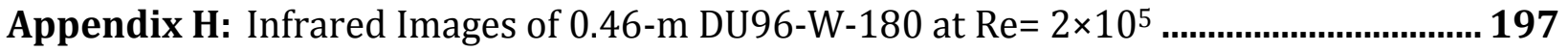




\section{Nomenclature}

\section{$\underline{\text { Roman }}$}

\begin{tabular}{|c|c|}
\hline$c$ & Airfoil Chord \\
\hline$C_{d}$ & Coefficient of Drag \\
\hline$C_{d w c}$ & Wake Corrected Coefficient of Drag \\
\hline$C_{l}$ & Coefficient of Lift \\
\hline$C_{l c}$ & Corrected Coefficient of Lift \\
\hline$C_{l w}$ & $\begin{array}{l}\text { Lift Coefficient from Integration of } \\
\text { the Wall Pressures }\end{array}$ \\
\hline$C_{m}$ & Coefficient of Moment \\
\hline$C_{m c}$ & Corrected Coefficient of Moment \\
\hline$C_{p}$ & Coefficient of Pressure \\
\hline $\mathrm{C}_{\mathrm{pc}}$ & Corrected Coefficient of Pressure \\
\hline$d$ & $\begin{array}{l}\text { Total Drag Force per Unit Span on } \\
\text { the Airfoil }\end{array}$ \\
\hline$h$ & $\begin{array}{l}\text { Stability Wind Tunnel Test Section } \\
\text { Width }\end{array}$ \\
\hline$H$ & $\begin{array}{l}\text { Vertical Height of the Open Jet } \\
\text { Wind Tunnel }\end{array}$ \\
\hline l & $\begin{array}{l}\text { Total Lift Force per Unit Span on } \\
\text { the Airfoil }\end{array}$ \\
\hline$p$ & Local Static Pressure \\
\hline$p_{\infty}$ & Free Stream Static Pressure \\
\hline$p_{o}$ & Local Stagnation Pressure \\
\hline$p_{o \infty}$ & Free Stream Stagnation Pressures \\
\hline$q$ & Dynamic Pressure \\
\hline$u$ & Local Mean Velocity, X-Direction \\
\hline$U_{\infty}$ & Free Stream Velocity \\
\hline$v$ & Local Mean Velocity, Y-Direction \\
\hline$w$ & Local Mean Velocity, Z-Direction \\
\hline$X$ & $\begin{array}{l}\text { Stream-Wise Tunnel Position, } \\
\text { Measured From Centre of Rotation } \\
\text { of Model }\end{array}$ \\
\hline$y$ & $\begin{array}{l}\text { Vertical Tunnel (Span-Wise) } \\
\text { Position, Measured From Centre of } \\
\text { Rotation of Model }\end{array}$ \\
\hline$z$ & Horizontal \\
\hline & $\begin{array}{l}\text { Measured Down From Tunnel } \\
\text { Centreline }\end{array}$ \\
\hline
\end{tabular}

\section{$\underline{\text { Greek }}$}

$\alpha_{c} \quad$ Blockage Corrected Angle of Attack

$\alpha_{e} \quad$ Effective Angle of Attack

$\alpha_{g} \quad$ Geometric Angle of Attack

$\rho \quad$ Density

$\zeta$ Open Jet Angle of Attack Correction Factor 


\section{List of Figures}

Figure 2.1 Full Circuit of the Virginia Tech Open Jet Wind Tunnel (adapted figure, used with permission of VT AOE Department, 2014) 11

Figure 2.2 Esterline 9816/98RK Scanners used in Open Jet Wind Tunnel.. 11

Figure 2.3 (a) Cart and Bearing System Attached to Lower End of Model (b) Downstream View of 1.82-m Span Model Mounted in Open Jet Wind Tunnel Test Section. 12

Figure 2.4 Setup of 0.61-m DU00-W-212 in Open Jet Wind Tunnel. 13

Figure 2.5 (a) Photograph and (b) Schematic showing Full Circuit of the Virginia Tech Stability Wind Tunnel (adapted figure, used with permission of VT AOE Department, 2014)

Figure 2.6 Downstream View of the Contraction and Turbulence Screens in the Settling Chamber 15

Figure 2.7 View Looking Downstream toward the Diffuser Entrance showing Vortex Generators and Turning Vanes..... 16

Figure 2.8 Downstream View of Empty, Aerodynamic Test Section 17

Figure 2.9 Upstream View of Aluminium, 0.08-mm Thick, All Weather Foil Tape Covering the Steps between the Contraction and Test Section. 18

Figure 2.10 Blowers for Flow Control System Installed in Compressor Room. 19

Figure 2.11 (a) Ducting for Flow Control System inside the Wind Tunnel Control Room (b) External Plenum Mounted Atop Test Section 20

Figure 2.12 Exploded View of the Lower Interior Plenum of the Boundary Layer Control System for the DU00-W212 20

Figure 2.13 View of the Upper End of the 0.8-m Chord DU96-W180 Airfoil with 5-mm Suction Slot 21

Figure 2.14 Side View Diagram Detailing the Location of the Pressure Taps Installed in the Test Section 23 
Figure 2.15 Wall Pressure Port View from (a) Isometric Drawing, (b) Outside the Test Section showing Tygon Tube Connection and (c) Inside the Test Section showing Smooth Surface with Side Wall 24

Figure 2.16 Control Volume used to Estimate the Lift from Side Wall Pressure Taps.......... 24

Figure 2.17 Upstream View of the Traversing Drag Rake Mounted in the Test Section ...... 25

Figure 2.18 Diagram of Traversing Wake Rake Showing Distribution of Pitot and Pitot-Static Probes. 26

Figure 2.19 Photographs of the (a) Full Span and (b) Profile of 0.15-m Wooden Clark-Y Model 28

Figure 2.20 (a) Full Span View and (b) Cross Section showing Thin Walls of the Aluminium, 0.2-m NACA-0012. 29

Figure 2.21 0.61-m DU00-W-212 Showing the Inner Cavities, the Leading Edge module and Main Element. 29

Figure 2.22 Lower Internal Suction System Assembly for 0.61-m DU00-W-212 30

Figure 2.23 0.8-m chord, DU96-W-180 Airfoil showing (a) Full Span and (b) Pressure Port arrangement

Figure 2.24 Single Laminate of the 0.8-m chord DU96-W-180 Airfoil showing the Wall Thicknesses and Inner Cavities 31

Figure 2.25 Lower Internal Suction System Assembly for 0.8-m DU96-W-180 ….................. 32

Figure 2.26 (a) Full span and (b) Pressure Port Configuration of 0.46-m DU96-W-180 model 32

Figure 2.27 Single Laminate of the 0.46-m chord DU96-W-180 Airfoil showing the Wall Thicknesses and Inner Cavities. 33

Figure 2.28 FLIR A655sc Infrared Camera used for Infrared Thermography in Transition Detection Study. 34

Figure 3.1 (a) Tear Drop Model at Room Temperature Set up Next to Heated Block (b) Resulting Coloured IR Image showing Clear Reflection of Heated Block on Tear Drop Model (Coldest Regions are Blue and Warmest Regions are White). 36 
Figure 3.2 (a) Tear Drop Model Covered in ConTact ${ }^{\circledR}$ Paper at Room Temperature Set up Next to Heated Block (b) Resulting Coloured IR Image showing No Environmental Reflections (Coldest Regions are Blue and Warmest Regions are White). 36

Figure 3.3 Lexan Flat Plate Mounted Vertically in Open Jet Wind Tunnel. 37

Figure 3.4 Coloured Infrared Image showing the Uneven Cooling Pattern Associated with the Dry Ice Cooling Method (Coldest Regions are Blue and Warmest Regions are White) .. 38

Figure 3.5 AC Cooling System Design 38

Figure 3.6 Kapton ${ }^{\circledR}$, Polyimide Film, Flexible, Insulated Heaters with Etched Foil Design. 39

Figure 3.7 Composite of Cross section through DU00-W-212 showing Internal Heater Arrangement. 39

Figure 3.8 Flexible Silicone Rubber Fiberglass Insulated Heaters. 40

Figure 3.9 Coloured Infrared Image of Suction Side of $0.8 \mathrm{~m} \mathrm{18 \%} \mathrm{Propriety} \mathrm{Model} \mathrm{Section} \mathrm{at}$ 30-ms ${ }^{-1}, 0^{\circ}$ Angle of Attack after (a) 1 minute and (b) 20 minutes (Coldest Regions are Blue and Warmest Regions are White). 40

Figure 3.10 Clark-Y Model Mounted in Open Jet Wind Tunnel, Suction Side Facing Camera 41

Figure 3.11 Coloured Infrared Image of Suction Side of Wooden Clark-Y Model at 30-ms-1, and (a) $0^{\circ}$ (b) $4^{\circ}$ Angle of Attack (Coldest Regions are Blue and Warmest Regions are White) 41

Figure 3.12 Trip Strip Made of 0.8-mm Dimple Tape Attached to Clark-Y Model. 42

Figure 3.13 Coloured Infrared Image of Suction Side of Clark-Y Model at 30-ms ${ }^{-1}, 4^{\circ}$ Angle of Attack (a) without Trip (b) with Trip (Coldest Regions are Blue and Warmest Regions are White)... 42

Figure 3.14 Coloured Infrared Image of Suction Side of NACA-0012 Model at 30-ms ${ }^{-1}$ and Angle of Attacks of (a) $0^{\circ}$ (b) $4^{\circ}$ (c) $8^{\circ}$ (Coldest Regions are Blue and Warmest Regions are White) 43

Figure 3.15 Setup of DU00-W-212 in Open Jet Wind Tunnel, Suction Side Facing Camera 44 
Figure 3.16 Coloured Infrared Image of Suction Side of DU00-W-212 Model at 30-ms ${ }^{-1}$ and Angle of Attacks of (a) $0^{\circ}$ (b) $2^{\circ}$ (c) $4^{\circ}$ and (d) $0^{\circ}$ with a Trip Strip (Coldest Regions are Blue and Warmest Regions are White). 45

Figure 3.17 Suction Side of DU00-W-212 covered in (a) ConTact ${ }^{\odot}$ paper and Thin Layer of Foam (b) ConTact ${ }^{\odot}$ paper, Thin Layer of Foam and Final Layer of ConTact ${ }^{\odot}$ Paper. 46

Figure 3.18 Coloured Infrared Image of Suction Side of DU00-W-212 Model Insulated with Foam at $30-\mathrm{ms}^{-1}$ and Angle of Attacks of (a) $-4^{\circ}$ (b) $0^{\circ}$ (c) $4^{\circ}$ and (d) $-6^{\circ}$ with a Trip Strip (Coldest Regions are Blue and Warmest Regions are White). 47

Figure 3.19 Coloured Infrared Image of Suction Side of DU00-W-212 Model, Insulted with Mylar, at 30- $\mathrm{ms}^{-1}$ and Angle of Attack of $0^{\circ}$ (Coldest Regions are Blue and Warmest Regions are White) 48

Figure 3.20 Coloured Infrared Image of Suction Side of DU00-W-212 Model, Insulted with Three Sheets of ConTact ${ }^{\odot}$ Paper, at 30-ms ${ }^{-1}$ and Angle of Attack of $4^{\circ}$ (Coldest Regions are Blue and Warmest Regions are White) 48

Figure 3.21 (a) Sample of 3-mm Cotton Sheet (b) Surface Quality of Suction Side of DU00W-212 Model After Attaching Cotton Sheet and ConTact ${ }^{\odot}$ Paper (c) Coloured Infrared Image of Suction Side of DU00-W-212 Model, Insulted with Cotton, at 30-ms ${ }^{-1}$ and Angle of Attack of $0^{\circ}$ (Coldest Regions are Blue and Warmest Regions are White) 49

Figure 3.22 0.46-m DU96-W-180 covered in (a) FDA Compliant Silicone Rubber Sheet (b) Final layer of ConTact ${ }^{\odot}$ Paper over Silicone Rubber Insulator 50

Figure 3.23 Coloured Infrared Image of Suction Side of DU96-W-180 Model, Insulated with Silicone Rubber, at 30-ms ${ }^{-1}$ and Angle of Attacks of (a) $-4^{\circ}$ (b) $-1^{\circ}$ (c) $2.5^{\circ}$ and (d) $5^{\circ}$ (Coldest Regions are Blue and Warmest Regions are White) 50

Figure 3.24 Photograph of TemperKote Insulating Coating (a) Immediately After Application to Airfoil (b) Sanded to Improve Surface Quality. 51

Figure 3.25 Coloured Infrared Image of Suction Side of 0.46-m DU96-W-180 Model, covered in TemperKote Insulating Paint, at 30- $\mathrm{ms}^{-1}$ and Angle of Attacks of (a) $0^{\circ}$ (b) $4^{\circ}$ (Coldest Regions are Blue and Warmest Regions are White) 52

Figure 3.26 Coefficient of Pressure Distribution for the $0.46-\mathrm{m}$ DU96-W- 180 at $\operatorname{Re}=1.5 \times 10^{6}$ and $\alpha=3^{\circ}$ 53 
Figure 3.27 Coefficient of Pressure Distribution with Transition Region Characterized by the Second Derivative of the Negative Coefficient of Pressure for the (a) Suction Side and (b) Pressure Side of the $0.46-\mathrm{m}$ DU96-W-180 at $\mathrm{Re}=1.5 \times 10^{6}$ and $\alpha=3^{\circ}$. 53

Figure 3.28 Coefficient of Pressure Distribution with Transition Region Characterized by the Maxima in Second Derivative of the -Cp for the Suction Side of the 0.46-m DU96-W-180 (a) $\alpha=-2^{\circ}$ (b) $\alpha=-0^{\circ}$ (c) $\alpha=0.5^{\circ}$ (d) $\alpha=1^{\circ}$ (e) $\alpha=2^{\circ}$. 55

Figure 3.29 Comparison of Infrared Thermography and Mean Pressure Results for Transition Location on the Suction Side of the 0.46-m DU96-W-180 ……………………....... 56

Figure 3.30 Stethoscope Outfitted with Pitot Probe for Transition Detection......................... 56

Figure 3.31 Comparison of Infrared Thermography and Stethoscope Results for Transition Location on the Suction Side of the 0.46-m DU96-W-180.. 57

Figure 3.32 Comparison of Transition Results from Mean Pressure, Stethoscope and Infrared Thermography for the Suction Side of the 0.46-m DU96-W-180 (a) $\alpha=-2^{\circ}$ (b) $\alpha=-0^{\circ}$ (c) $\alpha=0.5^{\circ}$ (d) $\alpha=1^{\circ}$ (e) $\alpha=2^{\circ}$ 58

Figure 3.33 (a) Diagram (b) Picture Showing Location of IR Windows in Starboard Glass and Lexan Panels 60

Figure 3.34 Infrared Window Sealed with Rapid Prototype Insert and Foil Tape 61

Figure 3.35 (a) Aluminium Framework for Camera Mounting on Suction Side of Test Section (b) View of Mounted Camera from Outside Suction Side of Test Section (c) View of Mounted Camera from Inside Test Section 62

Figure 3.36 Diagram of Heater Arrangement inside the Model for the (a) 0.8-m and (b) 0.46m DU96-W-180 (Locations are approximate and Drawings not to scale). 63

Figure 3.37 0.8-m DU96-W-180, Pressure Side Up, with Flexible Silicone Rubber Heaters Installed Inside. 63

Figure 3.38 (a) Suction Side of the 0.8-m DU96-W-180 covered in 0.8-mm Black Silicone Rubber Insulator (b) Thickness of Insulator.. 64

Figure 3.39 Downstream View of 0.8-m DU96-W-180 Mounted in Wind Tunnel with Infrared Thermography System 65

Figure 3.40 Downstream View of 0.46-m DU96-W-180 Mounted in Wind Tunnel with Infrared Thermography System. 65 
Figure 3.41 (a) Stethoscope Adapted for Use in the Stability Wind Tunnel (b) Slot in Rapid Prototype Insert for Inserting the Pitot Probe into Test Section 66

Figure 3.42 Experimenter Taking Stethoscope Measurements on the Pressure Side of the Insulated 0.8-m DU96-W-180 in the Stability Wind Tunnel (use of likeness with permission of experimenter) 66

Figure 4.1 Corrected Coefficient of (a) Lift (from integrated surface pressure) and (b) Drag Distribution over the 0.8-m DU96-W-180 at Three Reynolds Numbers. 67

Figure 4.2 Comparison of the (a) Corrected Coefficient of Lift (from integrated surface pressure) and (b) Corrected Coefficient of Drag at $\mathrm{Re}=2 \times 10^{6}$ for the $0.8 \mathrm{-m}$ and $0.46-\mathrm{m}$ DU96W-180 68

Figure 4.3 Corrected Coefficient of (a) Lift from integrated surface pressure) and (b) Drag at $\mathrm{Re}=1.5 \times 10^{6}$ for two Runs of the $0.8-\mathrm{m}$ DU96-W-180. 69

Figure 4.4 Effect of Heating on (a) Corrected Coefficient of Lift at $R e=1.5 \times 10^{6}$ (b) Corrected Coefficient of Drag at $\mathrm{Re}=1.5 \times 10^{6}$ (c) Corrected Coefficient of Lift and Drag at $\mathrm{Re}=2 \times 10^{6}$ (d) Corrected Coefficient of Drag at $\mathrm{Re}=2 \times 10^{6}$ (e) Corrected Coefficient of Lift $\mathrm{Re}=3 \times 10^{6}$ (f) Corrected Coefficient of Drag at $\mathrm{Re}=3 \times 10^{6}$ for the $0.8-\mathrm{m}$ DU96-W- 180 . 71

Figure 4.5 Comparisons of the Wake Cross Sections for the Clean, Unheated 0.8-m DU96-W180 (left) and the Clean, Heated Model (right) at $R e=1.5 \times 10^{6}$ and (a) $\alpha=-5^{\circ}$ and (b) $\alpha=0^{\circ} . .72$

Figure 4.6 Normalized Profile of DU96-W-180 showing the Insulator Thickness Relative to the Model Thickness. 73

Figure 4.7 Effect of Insulator on (a) Corrected Coefficient of Lift at $\mathrm{Re}=1.5 \times 10^{6}$ (b) Corrected Coefficient of Drag at $R e=1.5 \times 10^{6}$ (c) Corrected Coefficient of Lift at $R e=2 \times 10^{6}$ (d) Corrected Coefficient of Drag at $\mathrm{Re}=2 \times 10^{6}$ (e) Corrected Coefficient of Lift $\mathrm{Re}=3 \times 10^{6}$ (f) Corrected Coefficient of Drag at $\mathrm{Re}=3 \times 10^{6}$ for the $0.8-\mathrm{m}$ DU96-W-180. 75

Figure 4.8 Comparisons of the Wake Cross Sections for the Clean, Unheated 0.8-m DU96-W180 (left) and the Insulated, Unheated Model (right) at $\operatorname{Re}=1.5 \times 10^{6}$ and (a) $\alpha=-5^{\circ}$ (b) $\alpha=0^{\circ}$ and (c) $\alpha=5^{\circ}$. 76

Figure 4.9 Combined Effects of Insulation and Heat on (a) Corrected Coefficient of Lift at $\mathrm{Re}=1.5 \times 10^{6}$ (b) Corrected Coefficient of Drag at $\mathrm{Re}=1.5 \times 10^{6}$ (c) Corrected Coefficient of Lift at $\mathrm{Re}=2 \times 10^{6}$ (d) Corrected Coefficient of Drag at $\mathrm{Re}=2 \times 10^{6}$ (e) Corrected Coefficient of Lift $\mathrm{Re}=3 \times 10^{6}$ (f) Corrected Coefficient of Drag at $\mathrm{Re}=3 \times 10^{6}$ for the $0.8-\mathrm{m}$ DU96-W-180 ........... 78 
Figure 4.10 Coefficient of Pressure for Suction Side of 0.8-m DU96-W-180, at $\operatorname{Re}=2 \times 10^{6}$ and (a) $\alpha=0^{\circ}$ and (b) $\alpha=3^{\circ}$ 79

Figure 4.11 Coefficient of Pressure for Pressure Side of 0.8-m DU96-W-180, at $\mathrm{Re}=2 \times 10^{6}$ and (a) $\alpha=3^{\circ}$ and (b) $\alpha=-2^{\circ}$. 80

Figure 4.12 Coefficient of Pressure Prediction of Transition Range for 0.8-m DU96-W-180: (a) Suction Side at $R e=2 \times 10^{6}$ (b) Pressure Side at $R e=2 \times 10^{6}$ (c) Suction Side at $R e=1.5 \times 10^{6}$ (d) Suction Side at $\operatorname{Re}=3 \times 10^{6}$ (b) Pressure Side at $R e=3 \times 10^{6}$ 82

Figure 4.13 Coefficient of Pressure Prediction of Transition Range for 0.46-m DU96-W-180 (a) Suction Side at $R e=1.5 \times 10^{6}$ (b) Pressure Side at $R e=1.5 \times 10^{6}$ (c) Suction Side at $R e=2 \times 10^{6}$ and (d) Pressure Side at $\mathrm{Re}=2 \times 10^{6}$ 84

Figure 4.14 Infrared Images showing the (a) Before and (b) After Interference Effect of Inserting a Pitot Tube into the Boundary Layer of the 0.48-m DU96-W180 (at $\alpha=8^{\circ}$, $\left.\operatorname{Re}=2 \times 10^{6}\right)$ 85

Figure 4.15 Stethoscope Results for Transition Range for (a) Suction Side (b) Pressure Side of $0.8-\mathrm{m}$ DU96-W-180 at $\mathrm{Re}=1.5 \times 10^{6}$. 86

Figure 4.16 Stethoscope Results for Transition Range for (a) Suction Side (b) Pressure Side of $0.8-\mathrm{m}$ DU96-W-180 at $\mathrm{Re}=2 \times 10^{6}$ 87

Figure 4.17 Stethoscope Results for Transition Range for (a) Suction Side (b) Pressure Side of $0.46-\mathrm{m}$ DU96-W-180 at $\mathrm{Re}=1.5 \times 10^{6}$ 87

Figure 4.18 Comparison of Stethoscope Results on Insulated versus Clean (a) Suction Side (b) Pressure Side of 0.8-m DU96-W-180 at $\mathrm{Re}=2 \times 10^{6}$ 88

Figure 4.19 Infrared Images for Suction Side of the $0.8-\mathrm{m}$ DU96-W-180 at $\mathrm{Re}=1.5 \times 10^{6}$ and (a) $\alpha=-10^{\circ}$ (b) $\alpha=-5^{\circ}$ (c) $\alpha=-3^{\circ}$ (d) $\alpha=0^{\circ}$ (e) $\alpha=5^{\circ}$ (f) $\alpha=10^{\circ}$ 90

Figure 4.20 Infrared Images for Pressure Side of the $0.8-\mathrm{m}$ DU96-W-180 at $\mathrm{Re}=1.5 \times 10^{6}$ and (a) $\alpha=-5^{\circ}$ (b) $\alpha=-3^{\circ}$ (c) $\alpha=0^{\circ}$ (d) $\alpha=3^{\circ}$ (e) $\alpha=5^{\circ}$ (f) $\alpha=8^{\circ}$. 92

Figure 4.21 Infrared Images for Suction Side of the $0.46-\mathrm{m}$ DU96-W-180 at $\mathrm{Re}=1.5 \times 10^{6}$ and (a) $\alpha=-10^{\circ}$ (b) $\alpha=-5^{\circ}$ (c) $\alpha=0^{\circ}$ with Plot of Local Mean Chord-wise Temperature (d) $\alpha=5^{\circ}$ (e) $\alpha=10^{\circ}$ (f) $\alpha=-7^{\circ}$ with Highest Temperature Gradient as Identified by the Image Processing Algorithm 94 
Figure 4.22 Infrared Images for Pressure Side of the $0.46-\mathrm{m}$ DU96-W-180 at $\mathrm{Re}=1.5 \times 10^{6}$ and (a) $\alpha=-5^{\circ}$ (b) $\alpha=-3^{\circ}$ (c) $\alpha=0^{\circ}$ (d) $\alpha=5^{\circ}$ (e) $\alpha=8^{\circ}$ (f) $\alpha=-4^{\circ}$ with Highest Temperature Gradient as Identified by the Image Processing Algorithm 95

Figure 4.23 Comparison of Transition Results Obtained from Four Methods for 0.8-m DU96W-180 (a) Suction Side at $\operatorname{Re}=1.5 \times 10^{6}$ (b) Pressure Side at $R e=1.5 \times 10^{6}$ (c) Suction Side at $\mathrm{Re}=2 \times 10^{6}$ (d) Pressure Side at $\mathrm{Re}=2 \times 10^{6}$ (e) Suction Side at $\mathrm{Re}=3 \times 10^{6}$ (f) Pressure Side at $\mathrm{Re}=3 \times 10^{6}$. 97

Figure 4.24 Infrared Images showing Three Dimensionality of Transition for Pressure Side of the $0.8-\mathrm{m}$ DU96-W-180 at $\operatorname{Re}=3 \times 10^{6}$ and (a) $\alpha=-3^{\circ}$ (b) $\alpha=-1^{\circ}$ 98

Figure 4.25 Comparison of Transition Results Obtained from Four Methods for 0.46-m DU96-W-180 (a) Suction Side at $R e=1.5 \times 10^{6}$ (b) Pressure Side at $R e=1.5 \times 10^{6}$ (c) Suction Side at $\mathrm{Re}=2 \times 10^{6}(\mathrm{~d})$ Pressure Side at $\mathrm{Re}=2 \times 10^{6}$ 98

Figure 4.26 Infrared Images showing the Stethoscope Location when Detecting the (a) First Sound at $\alpha=-2^{\circ}$ (b) Second Sound at $\alpha=-2^{\circ}$ (c) First Sound at $\alpha=-4^{\circ}$ (d) Second Sound at $\alpha=-$ $4^{\circ}$ on the Suction Side of the 0.8-m DU96-W180 99

Figure 4.27 Effect of Chord Length of DU96-W-180 on the Infrared Thermography Transition Results, Investigated on (a) Suction Side at $\mathrm{Re}=1.5 \times 10^{6}$ (b) Pressure Side at $\mathrm{Re}=1.5 \times 10^{6}$ (c) Suction Side at $\mathrm{Re}=2 \times 10^{6}$ (d) Pressure Side at $\mathrm{Re}=2 \times 10^{6}$. 101

Figure 4.28 Variation of Transition Location with Increasing Turbulence Levels, as Predicted by XFOIL, for the DU96-W-180 at $\mathrm{Re}=2 \times 10^{6}$ 101

Figure 4.29 (a) 5-mm Air Bubble Formed in ConTact ${ }^{\odot}$ Paper on Model Surface and (b) Infrared Image showing the Turbulent Wedge Produced by the Air Bubble 102

Figure 4.30 (a) 2-mm Tear in ConTact ${ }^{\circledR}$ Paper on Model Surface (b) Infrared Image showing the Turbulent Wedge Produced by the Tear (c) Infrared Image after Tear is removed......103

\section{List of Tables}

Table 2.1 Freestream Turbulence Levels for the Virginia Tech Stability Wind Tunnel. 17 


\section{List of Appendix Items}

Figure A1: Close up of Turning Vanes between Diffuser and Fan of Virginia Tech Stability Wind Tunnel 110

Figure A2: Instrumentation to Control the (a) Tunnel Turntable and (b) Wind Tunnel Speed 110

Figure A3: Samples of Infrared Plexiglass of Different Thicknesses

Figure A4: General Radio Type W5MT Variac used to Control Heaters during Tests 111

Figure A5: Probe Microphone used to Record Sounds Heard by Stethoscope

Table B1: Pressure Port Locations on 0.8-m chord, DU96-W-180 Airfoil 112

Table B2: Pressure Port Locations on 0.46-m chord, DU96-W-180 Airfoil 113

Video C1: Flow over (a) Pressure and (b) Suction Sides of 0.8-m DU96-W-180 at $\mathrm{Re}=2 \times 10^{5}$, Rotating from $\alpha=-20^{\circ}$ to $20^{\circ}$, as seen through Infrared Camera 114

Video C2: Flow over (a) Pressure and (b) Suction Sides of 0.46-m DU96-W-180 at $\operatorname{Re}=2 \times 10^{5}$, Rotating from $\alpha=-20^{\circ}$ to $20^{\circ}$, as seen through Infrared Camera 114 


\section{Chapter 1:INTRODUCTION}

\subsection{Motivation}

The region over which laminar flow becomes turbulent is of utmost importance in wind tunnel testing at low speeds. This phenomenon, known as transition, is important in experimental design and testing because it is an important factor in airfoil performance. The transition point drives the frictional drag of the airfoil and is therefore essential in understanding lift and drag behaviour. In fact, the transition location influences separation and total lift because it changes the pressure distribution. Moreover, there are significant issues when estimating transition through numerical and simulated techniques. Hence, experimental data can be used to give accurate results which can validate these techniques.

The Virginia Tech Stability Wind Tunnel is a low speed, commercial testing facility which offers variable Reynolds numbers, very low free stream turbulence levels, low interference effects and interchangeable aerodynamic and aeroacoustic test sections. However, this facility currently has no system in place to report the transition location despite it being an integral component of airfoil performance. This thesis records the process undertaken to develop, implement and validate a transition detection system for continual use in this low speed, commercial wind tunnel where several large, aluminium models are tested and where accuracy and efficiency are of paramount importance.

\subsection{Literature Review}

Transition is the change from laminar to turbulent flow. It occurs over a region of flow, with the development of two dimensional instabilities called Tollmien Schlichting waves which later grow within the boundary layer causing the development of secondary instabilities all of which eventually coalesce into a fully turbulent nature. In this region there are marked increases in boundary layer thickness, velocity fluctuations, skin friction and consequently, in the rate of heat transfer. The transition is greatly influenced by surface roughness, adverse pressure gradients and the free-stream turbulence levels. Reynolds number, Mach number, acoustic radiation, surface temperature and surface curvature also affect the transition location. Therefore transition can occur naturally, based on the development of the boundary layer over a surface, or it can be intentionally induced in the flow using different kinds of 'trip' strips.

Several methods have been developed to detect the region over which transition occurs. Most of these involve the use of intrusive probes or flow visualization techniques. However, traditional flow visualization techniques (such as particle image velocimetry, smoke-wire, tuft filaments and oil-flow visualization) create flow issues stemming from the 
influence of the apparatus itself. One of the few non-intrusive methods for transition detection is infrared thermography (IRT), developed in the early 1980s at ONERA. It involves the use of a high resolution infrared camera to detect the model surface temperature distribution. Due to the increased mixing and shear stresses in flow undergoing transition, the temperature difference between model and flow in this regime rises. This leads to a higher rate of heat transfer in transition flow than in laminar flow. Consequently, a large surface temperature difference is created between the section of the model over which there is laminar flow and the section of the model over which there is turbulent flow. This temperature gradient can be clearly sensed and visualized by the infrared camera. In fact, it was stated that, when compared to other methods, IRT is extremely valuable because the IR camera is non-intrusive, has a high sensitivity and a low response time (Giovanni, 2010; Kuklova, 2012).

Giovanni (2010), who did a study of the main contributors to thermofluid-dynamics IRT research in the last two decades, concludes that this optical tool is very versatile and powerful. If coupled with the correct conditions, it can produce useful results for complex fluid flows, from hypersonic to natural convection. Astartita (2000), who also did a survey of infrared thermography, found that it is useful for observing the development of the boundary layer; complex flow development (over a delta wings or between jet and free stream); the aerodynamic heating over ellipsoidal bodies in a hypersonic tunnel; the location of reattachment points over a circular cylinder, and vortex rings. For several years infrared thermography has been used at large facilities in ONERA for boundary layer visualization. According to Le Sant et al. (2002), both transonic and hypersonic tests are done extensively in this facility, but in short runs. Similarly, IRT tests were done in the Ohio State's transonic wind tunnel (Gompertz, 2012) producing results which established that infrared thermography is a useful tool for transonic flow visualizations, in particular transition detection. A study of boundary layer transition using infrared thermography in supersonic flows on swept wings also showed that the transition front can be clearly identified (Zuccher, 2008). The success of these and other tests have proven the fidelity of IRT at high speeds, and thus this technique is usually attempted at these speeds.

However, as early as 1985, Schmitt and Chanetz (1985) proved that infrared thermography could be used to study low speed flows. Success was also achieved by Quast (1987) who conducted low speed IR thermography experiments. The low speed infrared systems developed by Ricci (2009) and Yokokawa (2005) have also showed promising results but focused on small scale testing of single models at few angles of attack in order to validate the concept of infrared thermography for transition detection. There has not been large scale implementation of this technique in such a way that transition results can be obtained efficiently and instantaneously, as would be needed in commercial wind tunnel testing. Considering the limited development in this area, it would appear that the 
development of several components would be needed to implement a transition detection system which utilizes infrared thermography in the Virginia Tech Stability Wind Tunnel.

\section{$\underline{\text { Model Material }}$}

One of the most important considerations for infrared thermography is the model material. Materials used in successful studies range from metal to plastics to composites. Most studies report that the most successful model candidates are those made of low thermal conductivity materials (Schmitt et al., 1985), and which have high insulating properties. These are usually made of insulating materials, which would eliminate the need for insulation. Possible materials include epoxy resin (Kuklova, 2012) or the use white ABS plastic fabricated from a rapid-prototyping machine (Freels, 2012). The research of Zuccher (2008) used a Bakelite model because it was both insulating and unaffected by reflections. Further insulating properties were introduced by adding a 76- $\mu \mathrm{m}$ thick coating made of DuPont ${ }^{\circledR}$ thermosetting epoxy, which worked well. However, these insulating methods must be pursued with caution as the enhanced temperature levels created by heating or cooling low thermal conductivity materials can change the behaviour of the boundary layer (Le Sant et al., 2002). There has also been documented success (Ricci, 2009) in the use of models made of insulated materials which are coated with thin metallic film to improve the surface heat transfer. In this case a $25-\mu \mathrm{m}$ thick aluminium coating was applied to a model (for Joule heating) and it was reported that no insulation was needed to obtain useful results.

The less popular option for models is the use of metals. Several researchers have stated that this option is not the ideal; aluminium and steel models should be avoided since they have high thermal conductivity which limits the time that the model can hold temperature differences (Zuccher, 2008; Freels, 2012). Furthermore, in the case of large metal models the increased model mass causes high material heat transfer rates which have the tendency to overshadow any heat transfer occurring due to flow effects. Models made of metals also create problems with reflections (Zuccher, 2008; Gompertz 2012; Ehrmann, 2014). According to Gompertz (2012) and (Ehrmann, 2014) metal models create "smearing effects" which impede the quality of the thermographs produced.

Tests on metallic models have been successfully carried out at hypersonic speeds at ONERA (Le Sant et al., 2002) using a top coat of insulating paint, an idea which is supported by other researchers in the high speed testing field (Zuccher, 2008). However, these paints are usually time consuming to apply and hard to remove, in most cases permanently altering the surface condition of the model. This is undesirable for the Virginia Tech Stability Wind Tunnel where models are frequently reused and preserving excellent surface quality is of utmost importance. If this method is to be used, it will be extremely beneficial if the paint 
can be replaced by another form of insulation (Le Sant et al., 2002), which is less permanent and easy to apply and remove.

The investigation of Yokokawa (2005) has also reported success in using metal models for infrared thermography, and achieving this at low speeds in the JAXA 6.5-m $\times$ 5.5$\mathrm{m}$ wind tunnel. In this case, a $0.4-\mathrm{m}$ section of the duralumin wing was cooled and no insulation was used. This method worked well for transition detection, with no reflections and useful results. However, only a few angles and speeds could be completed because of rapid equalization of the flow and model temperature. Zuccher (2008) conferred with Yokokawa that for short runs IRT may not require extra insulation to work.

On the other hand, Freels (2012) reported lack of success in using aluminium models at low speeds at Texas A\&M. He tested and compared ABS plastic (RP models) and aluminium airfoils, both $0.3-\mathrm{m}$ in chord and $0.61-\mathrm{m}$ in span, with and without coatings such as black paint and surface wraps. The models were both cooled and heated and it was found that aluminium did not produce good results; even with an insulator the results were only slightly improved from that of the uninsulated model. However, he did find that the aluminium configurations responded much quicker than the RP models, a very desirable outcome for commercial testing. Ehrmann (2014) also reported success with aluminium models at Reynolds numbers of $0.8-4.4 \times 10^{6}$. The transition was said to be difficult to view but painting the model with $225 \mu \mathrm{m}$ of Sherwin-Williams ${ }^{\circledR}$ Lusterless High Solids Polyurethane topcoat (unsanded) provided sufficient insulation and removed smearing. This variation in the success of using aluminium models, whether insulated or not, indicates that it is possible to use aluminium models but much research and testing is needed to fully understand the thermal behaviour of the aluminium models and to ensure that all other system components are designed to enhance the performance of aluminium.

\section{Infrared Reflections}

The issue of infrared reflections, on metal models or any other, has been circumvented in past research studies by increasing the model emissivity. It is found that this value should be as close to 1 (close to black body behaviour) as possible in order to limit the infrared radiation and reflections which occur during infrared thermography tests. This requires either the use of model materials which intrinsically offer this feature or, additional surface preparations. Surface treatments which have been shown to work well include: a thin layer of high emissivity, black paint as advocated by Le Sant et al. (2002) and used by Ricci (2009), Schmitt et al., (1985) and Kuklova (2012); a "thin black seat" glued to model surface as was used by Yokokawa (2005); covering metal models with thin plastic coatings (Quast, 1987; Freels, 2012). Ehrmann (2014) painted the metal model with 225- $\mu$ m of Sherwin-Williams ${ }^{\circledR}$ Lustreless High Solids polyurethane topcoat which acted as an insulator 
but also reduced surface reflections. If the option of adding some surface treatment to improve emissivity is undertaken, it is important to note that the surface condition must be particularly well treated since it has been proven that infrared thermography is very sensitive to even the smallest surface imperfections (Zuccher, 2008).

\section{Temperature Control}

Another important parameter to consider for a versatile infrared thermography system is the development of a reliable and sustainable temperature control mechanism. For infrared thermography to work, there must be a temperature difference between the model and the flow. When testing on large scales where angle of attack sweeps can range over fortyone angles and sweeps last for over an hour, a very efficient system is needed to create the temperature difference. The study of Quast (1987) investigated low speed IRT for transition and it was found that surface temperature differences as small as 1-K are enough to produce clear transition fronts. Moreover, in wind tunnels without flow temperature control systems and where the model and tunnel walls are made of materials of significantly different thermal properties, the naturally occurring temperature imbalances have proved to be sufficient to allow transition detection (Gartenberg et al., 1991; Quast, 1987).

The work of Zuccher (2008) compared three temperature states for infrared thermography on a Bakelite model: model at ambient temperature, heated model and a cooled model. The model at room temperature (which is at least $21^{\circ} \mathrm{C}$ ) was significantly warmer than the oncoming flow temperature by about $5-\mathrm{K}$. This method did not produce good results because the small temperature differences resulted in poor image resolution. This strongly suggests that a temperature difference should be introduced to obtain best results. Cooling the model showed more promising results and better resolution than at room temperature. The model was cooled to between $-233.15-\mathrm{K}$ and $-253.15-\mathrm{K}\left(\Delta \mathrm{T} \approx 50^{\circ} \mathrm{C}\right.$ with the flow) by spreading liquid nitrogen on its surface while it was mounted in tunnel. This method resulted in non-uniform surface cooling due to the manual operation and the variation in model thicknesses. Nevertheless, the non-uniform temperature distribution did not compromise transition detection. The model was then heated by the use of electric elements connected to the model surface, to approximately $343.15-\mathrm{K}-363.15-\mathrm{K}\left(\Delta \mathrm{T} \approx 60^{\circ} \mathrm{C}\right.$ with the flow). This method again resulted in non-uniform temperature distribution, but as with the cooling method it did not affect the final infrared results. However, it was stated that the cooling method worked better than the heating method because higher temperature differences could be created, resulting in better resolution in the final thermographs. Considering the high temperature differences created in this study and the fact that there were still issues with resolution it seems that the model material could have played a role in the heat transfer occurring. It should be noted that neither cooling nor heating the model, to 
the level described in this study, appeared to change the stability characteristics of the boundary layer in any way.

Other researchers who have recorded success with IRT without introducing a temperature difference have, like Zuccher (2008), been predominantly in the high speed field (Gompertz, 2012) where the temperature differences are inherently high due to the high speed. Yokokawa (2005) reported good results for a metal model at low speeds with no heating or cooling, but his tests were only done at a few angles of attack and speeds (and may not have been consecutive). Furthermore, the infrared images showed poor resolution in some cases because of the lack of a larger temperature difference. Hence, it appears that for commercial infrared thermography testing in a large wind tunnel to be successful a means of creating and sustaining a suitable temperature difference is necessary, but this hypothesis will need to be investigated fully through testing.

Techniques which have been used to create a temperature difference between the model and flow include pre-treating the model with cooling blankets (Yokokawa, 2005) or liquid nitrogen (Zuccher, 2008) or using continuous mechanisms for creating and maintaining the temperature difference during the test. While proven to work, the pretreatment methods are not well suited for large scale wind tunnel tests because there is no active control of the temperature difference - the temperature cannot be re-introduced as necessary during the test. This will reduce the efficiency of the system and increase tunnel testing time. The active, continuous methods used include: installing electric elements in the walls of the model so that heating occurs by the Joule effect (De Luca, 1990; Patorski, 2000; Ricci, 2009; Zuccher, 2008; De Luca, 1995); the use of internal heating sheets (Crawford, 2014); and the use of heat lamps (Baek, 2009; Freels 2012; Gaidos, 1990) during the test. When using heat lamps, a special window may be needed to maximize the heat transfer process, especially in large facilities with large models. In the case of Baek (2009), a quartz glass window was used to maximize the heat transfer from the heat lamp to model. It should be noted that in the study of Freels (2012) where both an external heat lamp and the circulation of cold fluid were used for the temperature difference, it was found that internal circulation of cold fluid was far more successful than the heat lamp. This was concluded because the heat lamp produced marked reflections in the infrared region which negatively affected the final results. Similarly, Gaidos (1990) found laser heating to be a very effective method for a sustainable temperature rise, more so than heat lamps.

\section{Camera Set up}

The last aspect of the infrared system which is of interest is the ideal system set up to ensure that the best infrared images are obtained. This involves finding the best camera angles and the installation of infrared windows for the cameras. 
Several studies reported that an infrared camera window is necessary because most materials are reflective and not transparent to infrared radiation. The system developed by Baek (2009), for the low speed wind tunnel located at LM Glasfiber, used a barium fluoride glass window for the infrared camera. The window was mounted on the ceiling of the test section. Other materials used for the camera windows include zinc selenide (Le Sant et al., 2002; Ricci, 2009), crystal sapphire (Gompertz, 2012) and an open cut hole in the test section walls (Yokokawa, 2005; Quast, 1987; Ehrmann, 2014). Quast also suggested that the camera should be positioned so that as little of the background is in the view of the camera to prevent automatic mean temperature adjustment of the IR system. Additionally, Baek (2009) used gold leaf markers (which are visible in infrared) on the model to help identify chord locations, a technique which may be useful at Virginia Tech.

\section{$\underline{\text { Validation Methods }}$}

In most studies researchers have used additional transition detection techniques to validate the results of IRT. Schmitt and Chanetz (1985) conducted tests over an ellipsoid configuration at speeds up to $70-\mathrm{ms}^{-1}$, and angles of attack to $40^{\circ}$ degrees. These results were compared to that of acenaphtene ablation and showed good agreement. Further validation techniques included liquid crystal film used by Yokokawa (2005) and hot film anemometry employed by Ehrmann (2014), both of whom concluded that IRT techniques surpassed that of the liquid crystal film. Kuklova (2012) also conducted a verification study of infrared thermography on a 250-mm chord NACA 63A-421 at an angle of attack of $\alpha=0^{\circ}$ and Reynolds number was $\operatorname{Re}=2 \times 10^{5}$. The model was covered with a high emissivity acrylic paint and uniformly heated before the test. The test proved that the IRT system produced results which agreed with both XFOIL and oil flow visualizations. Gompertz (2012) and Baek (2009) also used XFOIL for verification of the transition results from IRT and, within uncertainties, the results agreed. In addition to XFOIL, Baek (2009) utilized microphone data to produce transition results and these agreed closer to the infrared results than XFOIL did. The success of these verification studies for different models and conditions strongly suggests that IRT is a good transition detection technique while also endorses all of these techniques for IRT validation.

Independently of IRT, transition detection on an airfoil from the surface pressure distribution as was undertaken by Popov (2008). Two interpolation methods, the piecewise cubic Hermite interpolating polynomial and Spline, were used to calculate the second derivative of the pressure distribution, which is then used to find the maximum curvature of the pressure distribution. This pressure analysis was conducted on NACA 4415, WTEA-TE1 and, a 17 modified WTEA-TE1 airfoils and was validated using the XFOIL prediction code. The results had an uncertainty of $0.23 \%$ chord for the PCPH interpolation scheme and $0.33 \%$ 
chord for the spline. While affording the advantage of real time applicability, the method was found to be very limited in the range in which the transition point can be located. This is the case because the method is erroneous in the leading edge region (which must eliminated during analysis) and is highly dependent on the density of pressure measurements which were collected. The study did also stress that this method does not replace the theoretical $\mathrm{e}^{\mathrm{N}}$ method or other experimental methods but can only, at best, act as a validation of these schemes and should not be the sole transition detection method. Ehrmann (2014) also determined transition location using - $\mathrm{Cp}$ data in addition to the infrared thermography measurements. In his study, he stated outright that the infrared transition data was the truest measure of transition since it is non-invasive and boundary layer stability variations due to small temperature differentials is minimal. In his work, the data from -Cp and hot film anemometry had large uncertainties when compared with IRT and showed large variations at some angles of attack.

A more traditional and straightforward method of transition detection is the use of stethoscope. This is based on the unsteady nature of turbulent flow which leads to increased noise levels in the stethoscope and changes in the frequency of sound of the flow in the region of transition. These sound changes are distinct but results are subject to human error biases. This method is described by Meuller (2001) and has been used by Maughmer (2010) and Lutz (2001).

\subsection{Scope and Aim of this Research}

In order to implement a reliable and efficient transition detection system which utilizes infrared thermography in the Virginia Tech Stability Wind Tunnel, extensive development was required. This was undertaken in two major phases: a developmental phase and a validation phase.

Phase One, development of the system, involved in depth planning and execution of small scale experiments in the Virginia Tech Open Jet Wind Tunnel. The overarching aim of this phase was to gain an understanding of the concept of infrared thermography as it applies to transition detection, and to use this knowledge to develop the best system for large scale, low speed wind tunnel testing. Major goals of testing in this phase included:

1. Developing a method for creating a sustainable and controllable temperature difference between the model and the flow, and to ascertain the optimum temperature difference required to produce useful results.

2. Determining the best model material which can be used for transition detection without affecting the quality of flow or altering airfoil performance. 
3. The development of a software tool to quickly extract the transition location through image processing techniques.

4. Preliminary validation of the results produced by the infrared system and methods for extending validation schemes to the Virginia Tech Stability Wind Tunnel.

5. Installing infrared cameras appropriately to capture flow effects occurring on both sides of airfoils mounted in the Virginia Tech Stability Wind Tunnel.

6. Implementing all components of the system in the Virginia Tech Stability Wind Tunnel such that the flow remains unaffected and all other measurement systems are simultaneously functional.

The validation phase, Phase Two, was conducted to prove that the system was not only functional but was also able to reliably and consistently produce accurate results for transition. This phase was carried out in the Virginia Tech Stability Wind Tunnel and aimed to:

1. Prove that the infrared thermography system does not affect the flow or airfoil performance, and thus produces results which are solely due to flow effects on the model.

2. Gauge the efficiency and versatility of the system in an actual wind tunnel test with two models of different chord lengths at various angles of attack and Reynolds numbers.

3. Evaluate the sensitivity of the system and the uncertainty associated with its results.

4. Compare the transition results from infrared thermography to that of other transition detection methods as well as numerical predictions, in order to validate the results of the infrared system.

This report documents the ideas and procedures undertaken to achieve the stated goals. The major apparatus and facilities used will be described, thereafter details of Phase One will be given, followed by an in depth account of the Phase Two work. 


\section{Chapter 2: APPARATUS AND INSTRUMENTATION}

The current study was conducted in two phases. Phase One focused on the development of a transition detection system using infrared thermography. This phase was conducted in the Virginia Tech Open Jet Wind Tunnel. Phase Two was carried out the in Virginia Tech Stability Wind Tunnel and aimed to validate the transition detection system developed in Phase One. The following is a description of the major apparatus and instruments used in both phases of the present work.

\subsection{Open Jet Wind Tunnel}

The Virginia Tech Open Jet Wind Tunnel is an open test section, open circuit, subsonic wind tunnel. The wind tunnel comprises of a 30-hp BC-SW Size 365 Twin City centrifugal fan, a diffuser, settling chamber, contraction, open test section and a jet catcher.

\subsubsection{Open Jet Wind Tunnel Circuit}

The fan is able to produce a volumetric flow rate of up to $15-\mathrm{m}^{3} \mathrm{~s}^{-1}$ and a maximum speed of 1180-rpm. From the fan, flow passes through the diffuser which is $6^{\circ}$ and 4-m long thereby slowing down the flow. Flow then enters the settling chamber which is 1.78 -m wide and $1.47-\mathrm{m}$ high, and is finally released into the test section via a 5.5:1 contraction nozzle. The open test section is essentially a framework of $80 / 20$ aluminum beams with a variety of slots which allows copious options for mounting and positioning models. The test section is $1.07-\mathrm{m}$ wide, $1.07-\mathrm{m}$ high and $1.4-\mathrm{m}$ long and is fitted with a series of brackets to keep it perfectly square. The flow is finally directed into the $1.2-\mathrm{m}$ jet catcher which drastically reduces the velocity of the flow before it reaches the surrounding environment. Figure 2.1 shows the Open Jet Wind Tunnel and all its components.

The Open Jet Wind Tunnel is able to produce flow in the test section of up to $30-\mathrm{ms}^{-1}$ at maximum fan speed. The flow speed is controlled by an AF-600 General Electric variable frequency drive and is measured using static pressure taps located at the exit of the settling chamber. In order to increase the uniformity of flow and reduce turbulence, a $0.09-\mathrm{m}$ honeycomb with cell size of $0.01-\mathrm{m}$ and three, $0.3-\mathrm{mm}$ diameter fiberglass screens with 55\% open area ratio are erected at the exit of the settling chamber (the entrance of the contraction). 


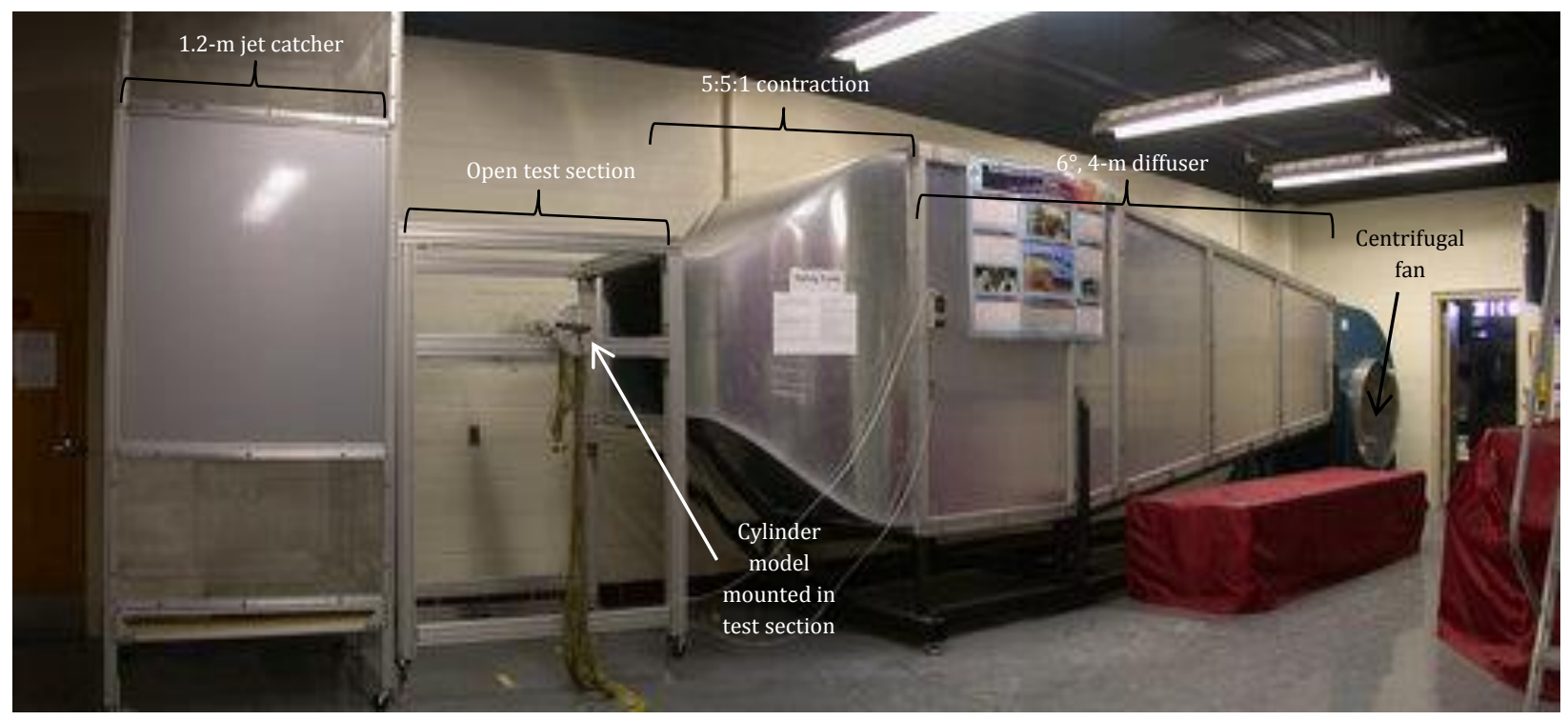

Figure 2.1 Full Circuit of the Virginia Tech Open Jet Wind Tunnel (adapted figure, used with permission of VT AOE Department, 2014)

For the transition detection tests, pressure measurements were made on airfoil models with pre-fabricated pressure ports along mid-span. In the Open Jet Wind Tunnel, pressure measurements are usually taken using three Esterline 9816/98RK pressure scanners each with 16 channels, a range of \pm 10 -inWC, resolution of \pm 0.003 and an accuracy of $0.05 \%$. The scanner is shown in Figure 2.2. In order to measure pressure along the full chord of these models, an additional three scanners were added to the system creating a total of 96 channels. Therefore all the airfoil surface pressure ports and the static pressure taps in the Open Jet tunnel settling chamber could be connected to these scanners. As is done in the Stability Wind Tunnel, the reliability of the pressure connections and the functionality of the pressure scanner were tested using a Fluke model 718-1G Pressure Calibrator. The Open Jet Wind Tunnel system normally uses a LABVIEW based program for pressure acquisition. However, for the current work, the Esterline scanners were connected via IP to a computer running the pressure acquisition software used in the Virginia Tech Stability Wind Tunnel (developed inhouse using MATLAB). This was done because the Stability Wind Tunnel acquisition code allows both data acquisition and post processing to be done in one step. Furthermore, it is programmed to take pressure along the chord of airfoils and thus is designed to control acquisition from a large number of channels.

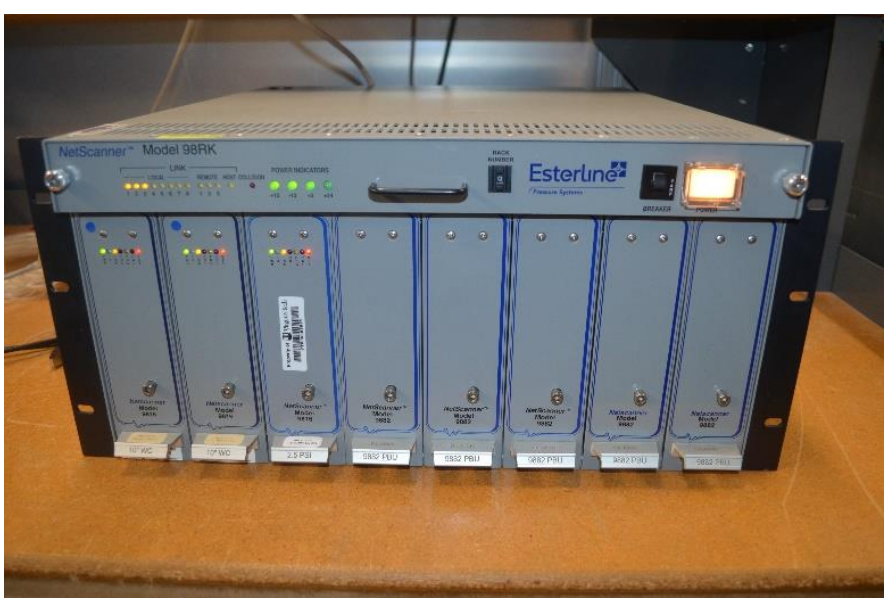

Figure 2.2 Esterline 9816/98RK Scanners used in Open Jet Wind Tunnel 


\subsubsection{Model Mounting in Open Jet Wind Tunnel}

The Virginia Tech Open Jet Wind Tunnel was intended for testing models with small chords lengths and wing spans. Small models are easily mounted using additional 80/20 aluminium bars and brackets in the test section, the construction of which lends itself readily to this. However, testing of models with larger chords and spans (spans $\approx 1.82-\mathrm{m}$, chords $\approx$ $0.46-\mathrm{m}$ or $0.61-\mathrm{m}$ ) such as those designed to be tested in the Stability Wind Tunnel, becomes problematic. Several issues arise with model mounting and in flow corrections. Nevertheless, this was necessary because there is pressure and transition data already available for these models, so comparisons can be done. Furthermore, it was beneficial to test these models which are similar to the types of models for which the final system will be used.

In order to mount these large models in the Open Jet Wind Tunnel a bearing system from the Stability Wind Tunnel was used. This bearing system allows models to rotate $360^{\circ}$ by means of a bearing, atop which a plate is secured. The airfoil centre support shaft fits into a slot in the bearing plate allowing the whole model to be rotated. The bearing plate was secured on a steel, rolling cart 38.1-mm tall. The bearing plate system supporting a mounted airfoil is shown in Figure 2.3. The model can then be hoisted (using a forklift) into the bearing plate slot and the entire cart wheeled into the Open Jet test section. This set up ensures that neither the cart nor the bearing plate is in the flow, or close enough to the test section to affect the flow. It also allows the model to be mounted precisely at the contraction exit, which ensures that the best quality flow is 'seen' by the model.

(a)

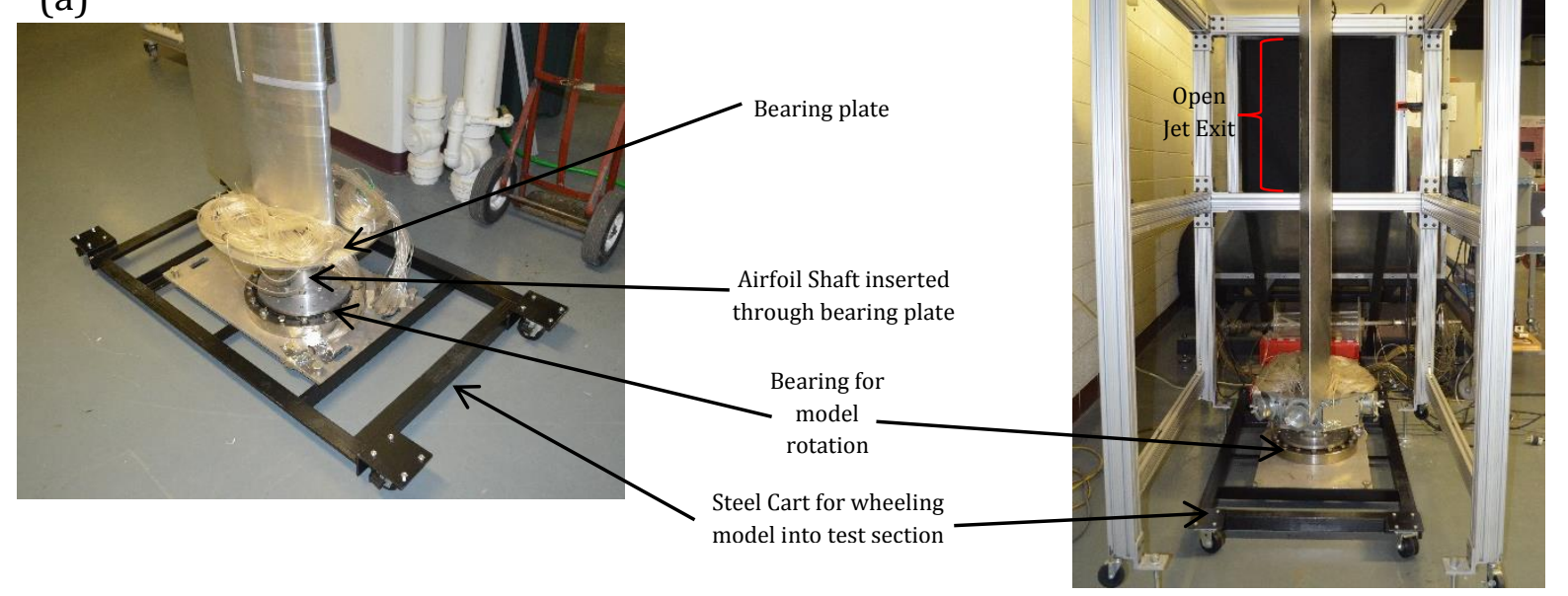

Figure 2.3 (a) Cart and Bearing System Attached to Lower End of Model (b) Downstream View of 1.82-m Span Model Mounted in Open Jet Wind Tunnel Test Section

To secure the large models during testing, a steel collar was fabricated to fasten the top of the airfoil shaft to the test section. The collar is welded to steel bars which is bolted to 
the $80 / 20$ beams at the top of the test section. While providing stability for the model, the collar also allowed angles of attack to be fixed during testing. The angle of attack of the model was manually set during the tests. The angles were calculated by geometric relations of the desired angle of attack and the chord length which provided precise leading edge and trailing edge locations. Figure 2.4 depicts the 0.61-m DU00-W-212 mounted in the Open Jet Wind Tunnel.

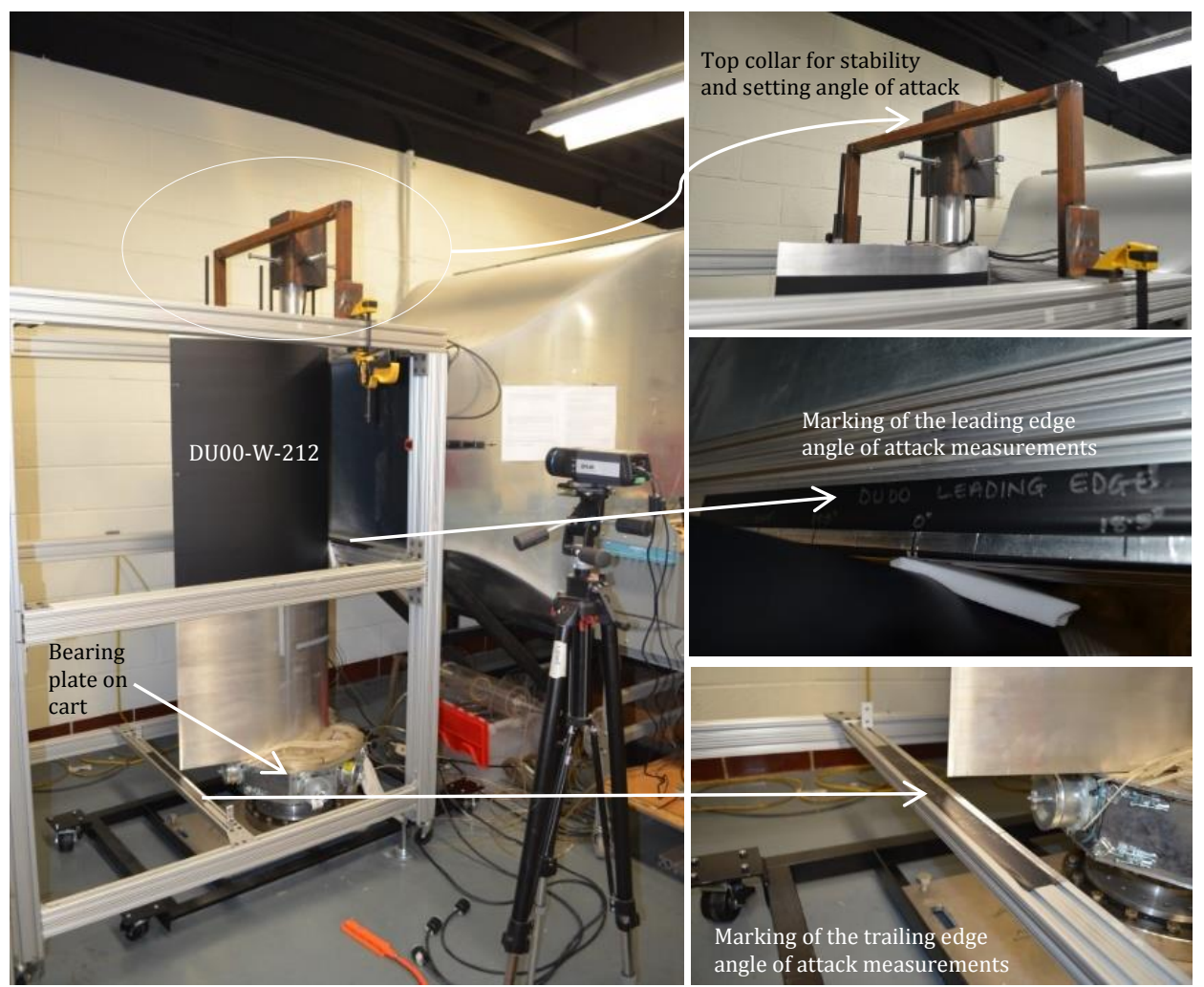

Figure 2.4 Setup of 0.61-m DU00-W-212 in Open Jet Wind Tunnel

The correction schemes for blockage effects in an open test section are less defined than those in a closed test section. They are sensitive to the jet dimensions and the dimensions of the test model. This created significant issues with correcting the data once obtained. Furthermore, angle of attack corrections were needed to compensate for the reductions in effective angle of attack caused by downwash and flow curvature. This is especially important for models with large chord length where the deflection of the flow is very significant. The angle of attack correction used is described by Brooks et al., (1989) and is based on the lifting surface theory and ignores small camber effects. The corrected (effective) angle of attack is given by equation (2-1),

$$
\alpha_{e}=\frac{\alpha}{\zeta}
$$


where $\alpha_{e}$ is the effective angle of attack, $\alpha$ is the geometric angle of attack and $\zeta$ is a correction factor. The effective angle is the angle of the flow which would produce the same (lift under ideal conditions) as is obtained from the geometric angle set in the tunnel. The correction factor is further described in equations (2-2) and (2-3), where $c$ is the airfoil chord and $H$ is the vertical height of the Open Jet Wind Tunnel.

$$
\begin{gathered}
\zeta=(1+2 \sigma)^{2}+\sqrt{12 \sigma} \\
\sigma=\frac{\pi^{2}}{48}\left(\frac{c}{H}\right)^{2}
\end{gathered}
$$

For the large models used in the Open Jet Wind Tunnel tests, the angle of attack ratios were $\frac{\alpha_{e}}{\alpha} \approx$ 0.32 (DU00-W-212) and $\frac{\alpha_{e}}{\alpha} \approx 0.42$ (DU96-W-180) which means that the effective of angle of attack was at least twice as large as the geometric angle of attack. This suggests that even after applying the correction, the true angle of attack determined is uncertain because the chord lengths are quite large compared to the test section.

\subsection{Stability Wind Tunnel}

The data which will be the prime focus of Phase Two of this study was collected in the Virginia Tech Stability Wind Tunnel. It is a closed circuit, subsonic wind tunnel. The continuous, single return system comprises a motor driving a fan, an air exchange tower and, a removable test section. Figure 2.5 shows the general schematic of the facility.
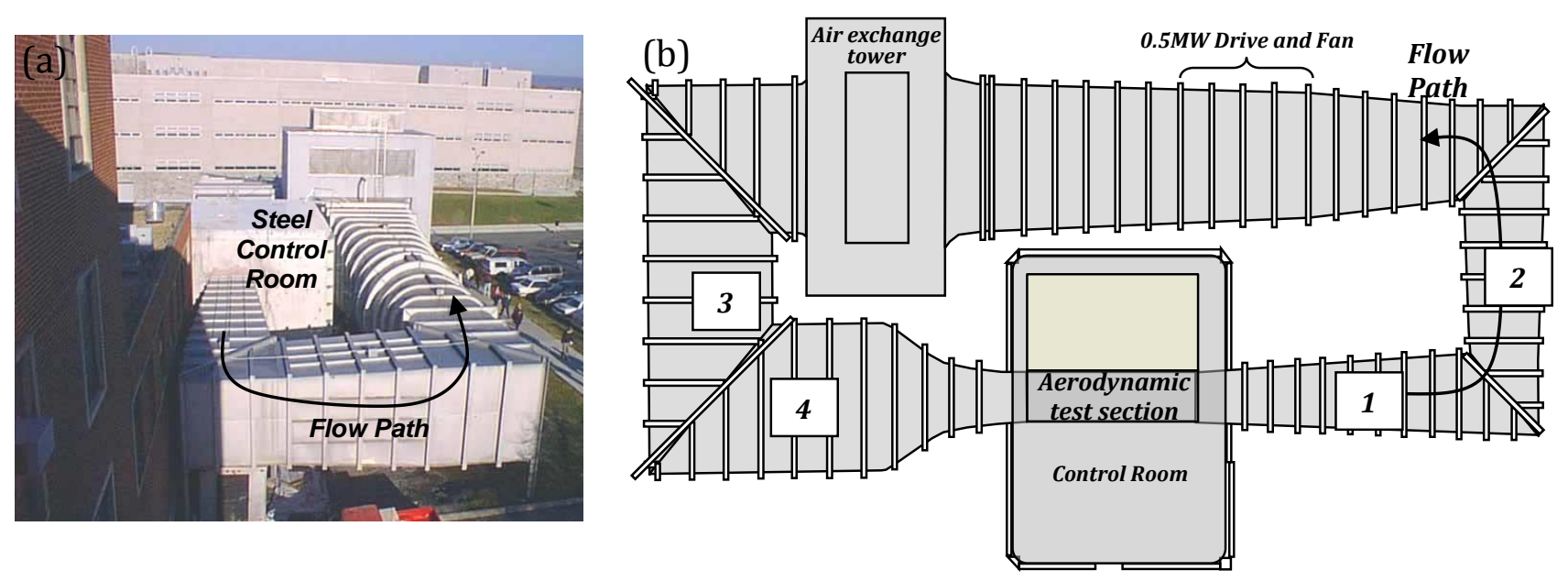

Figure 2.5 (a) Photograph and (b) Schematic showing Full Circuit of the Virginia Tech Stability Wind Tunnel (adapted figure, used with permission of VT AOE Department, 2014) 


\subsubsection{Stability Wind Tunnel Circuit}

The Virginia Tech Stability Wind Tunnel is powered by a 0.45-MW variable speed DC motor driving a $4.3-\mathrm{m}$ propeller. The maximum propeller rotation is $600-\mathrm{RPM}$ which produces flow speeds of up to $80-\mathrm{ms}^{-1}$ and Reynolds numbers per meter of approximately $5,000,000$, not accounting for blockage effects in the test section.

A custom designed Emerson VIP ES-6600 SCR Drive is used to regulate the speed of the wind tunnel. This is achieved by interfacing with the tunnel's computer-controlled data acquisition system. This state of the art drive system has markedly improved both the efficiency and accuracy of speed control when compared to previous drive models. In fact, this precision regulated drive removes both the cyclic unsteadiness in tunnel velocities and the turbulence inducing vibrations normally associated with older systems.

Downstream of the fan and motor is an air exchange tower which is open to the atmosphere, allowing for temperature stabilization. Following this, flow enters a 5.5- $\mathrm{m} \times 5.5$ $\mathrm{m}$ settling chamber housing seven turbulence-reducing screens. Turbulence levels are further reduced when the flow enters a 9:1 contraction nozzle depicted in Figure 2.6. In addition to this, the contraction nozzle also accelerates the flow to the test speed.

Directly downstream of the contraction nozzle is the test section of the Stability Wind Tunnel which is 7.3-m long and has a $1.83-\mathrm{m}$, square cross section. This component of the wind tunnel circuit is enclosed in a sealed steel building,

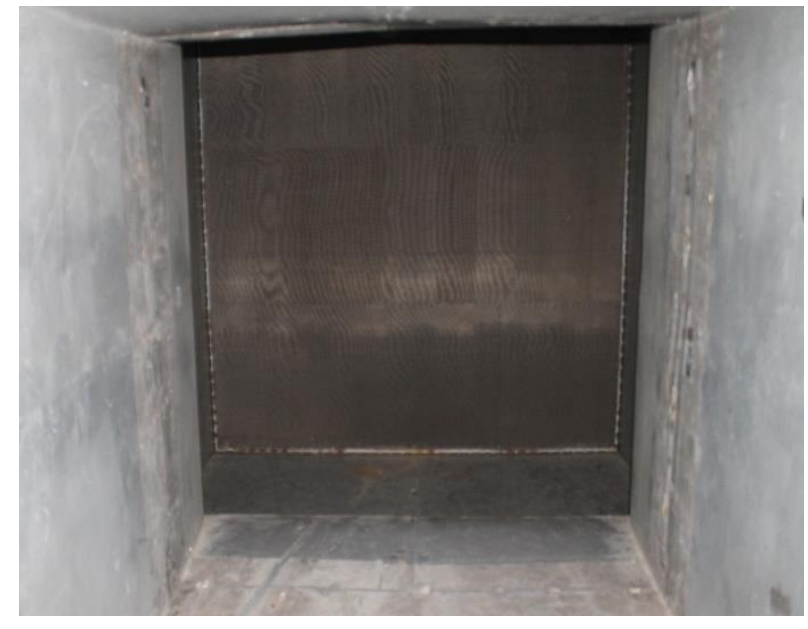

Figure 2.6 Downstream View of the Contraction and Turbulence Screens in the Settling Chamber impervious to air leakage, so that the pressure inside the control room can be equalized with the static pressure of the test section flow. This is achieved via sidewall slots located at the downstream end of the test section. There are two removable test sections available for use in the Stability Wind Tunnel: an anechoic test section and an aerodynamic, hard-wall test section. The data presented in this study was collected in the aerodynamic, hard wall test section which is discussed in detail in Section 2.2.2.

From the test section, flow is directed through a $3^{\circ}$ diffuser. The entrance to the diffuser is equipped with eight vortex generators, each $0.16-\mathrm{m}$ in height. Each of these is mounted $0.4-\mathrm{m}$ from the corners of the duct in order to minimize the possibility of separation 
and the instability and inefficiencies which result. A view looking downstream toward the diffuser, showing the vortex generators, is shown in Figure 2.7.

Additionally, turning vanes are installed in the four corners of the wind tunnel circuit to help direct flow and prevent corner stall and separation. One such turning vane is also shown in Figure 2.7 and in Figure A1. Each of these structures comprises of a series of shaped diagonal vanes spaced $0.3-\mathrm{m}$ apart, except for the turning vane erected immediately upstream of the settling chamber in which the vanes are spaced 0.056-m apart.

The wind tunnel circuit is acoustically treated in several areas to reduce noise reflection. Referring to the tunnel sections depicted in Figure 2.5, the four walls of the diffuser (labelled 1) are lined with 25-mm thick melamine; the walls and ceiling of the section between the diffuser and the drive and fan (labelled 2) are lined with 50-mm thick melamine and urethane foam; the

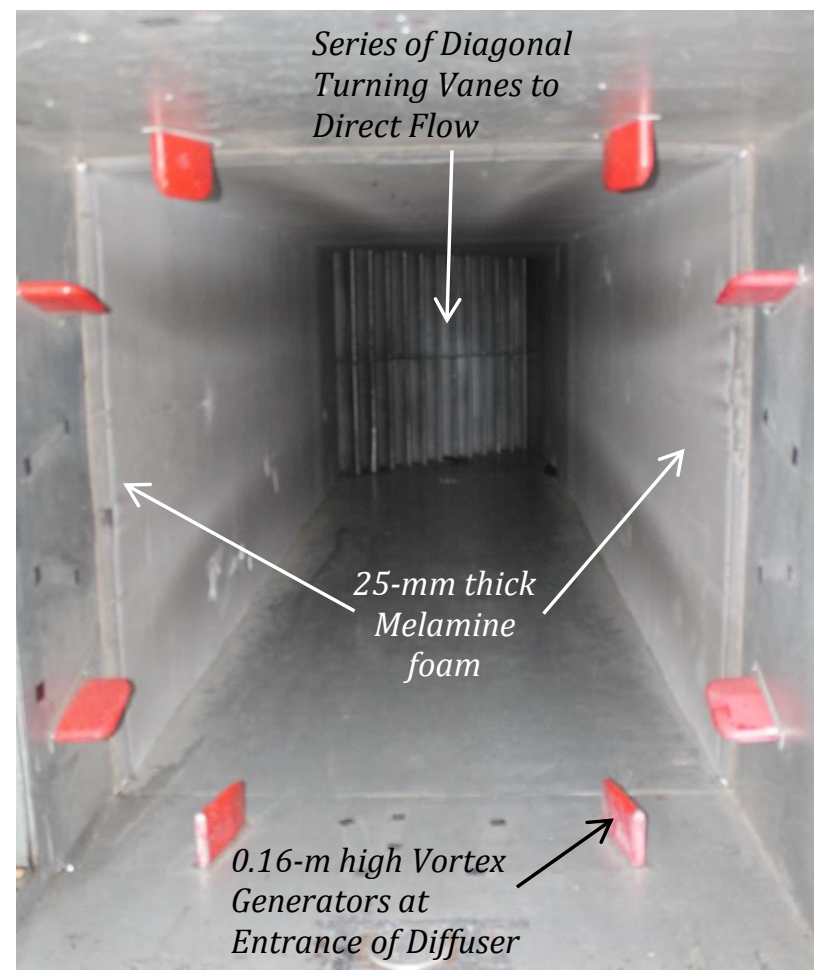

Figure 2.7 View Looking Downstream toward the Diffuser Entrance showing Vortex Generators and Turning Vanes walls and floor of the section between the air exchange tower and the settling chamber are lined with 50-mm thick urethane foam (labelled 3) as are the walls for section of the settling chamber upstream of the screens (labelled 4). The lower half of the fan blades are lined with Delrin ${ }^{\circledR}$ to reduce the tip gap from about $15 \pm 4-\mathrm{mm}$ to about $7 \pm 3-\mathrm{mm}$.

The flow generated in the Virginia Tech Stability Wind Tunnel has good uniformity (Aerospace Engineering Department, 2013) with turbulence levels are on the order of 0.03\% or less - an outstanding feature among wind tunnels of its size. The extremely low turbulence levels of the facility can be attributed to the turbulence screens and the other flow smoothing features previously discussed. The most recent hot-wire anemometry measurements of free stream turbulence as a function of flow speed was taken in 2006 and are showed in Table 2.1. From these results it is seen that turbulence levels are as low as $0.016 \%$ at $12-\mathrm{ms}^{-1}$ and increase with speed, rising to $0.031 \%$ at $57-\mathrm{ms}^{-1}$. 


\begin{tabular}{|c|c|}
\hline $\begin{array}{c}\text { Free-stream Velocity, } \\
U_{\infty}\left(m s^{-1}\right)\end{array}$ & $\begin{array}{c}\text { RMS Stream-wise Fluctuations, } \\
u^{\prime} / U_{\infty}\end{array}$ \\
\hline 12 & $0.016 \%$ \\
\hline 21 & $0.021 \%$ \\
\hline 30 & $0.024 \%$ \\
\hline 48 & $0.029 \%$ \\
\hline 57 & $0.031 \%$ \\
\hline
\end{tabular}

Table 2.1 Freestream Turbulence Levels for the Virginia Tech Stability Wind Tunnel

\subsubsection{Aerodynamic Test Section}

The Virginia Tech Stability Wind Tunnel is equipped with two removable test sections, each 7.3-m long with a $1.83-\mathrm{m}$, square cross section. The data which is the subject of this current work was collected in the aerodynamic, hard wall test section which is shown in Figure 2.8.

The side walls of the test section are essentially separate Lexan windows assembled individually to create a continuous hard surface. These Lexan panels are approximately $1.7-\mathrm{m}$ tall, $12.7-\mathrm{mm}$ thick and either $0.61-\mathrm{m}$ or $0.76-\mathrm{m}$ wide, depending on their location in the test section. They are fastened into a steel frame which extends the structure to the full height of the tunnel, 1.83-m. The Lexan windows are secured into the steel frame, using bolts, to create a smooth flow surface inside of the test section. Lexan panels (on each side of the test section) which are closest to the airfoil have been recently replaced with Starphire ${ }^{\circledR}$ glass panels of the same size. This development is part of the wind tunnel's flow visualization system.

The floor and ceiling of the test section consists of a collection of removable, interchangeable $0.61-\mathrm{m} \times 0.61-\mathrm{m}$ aluminium

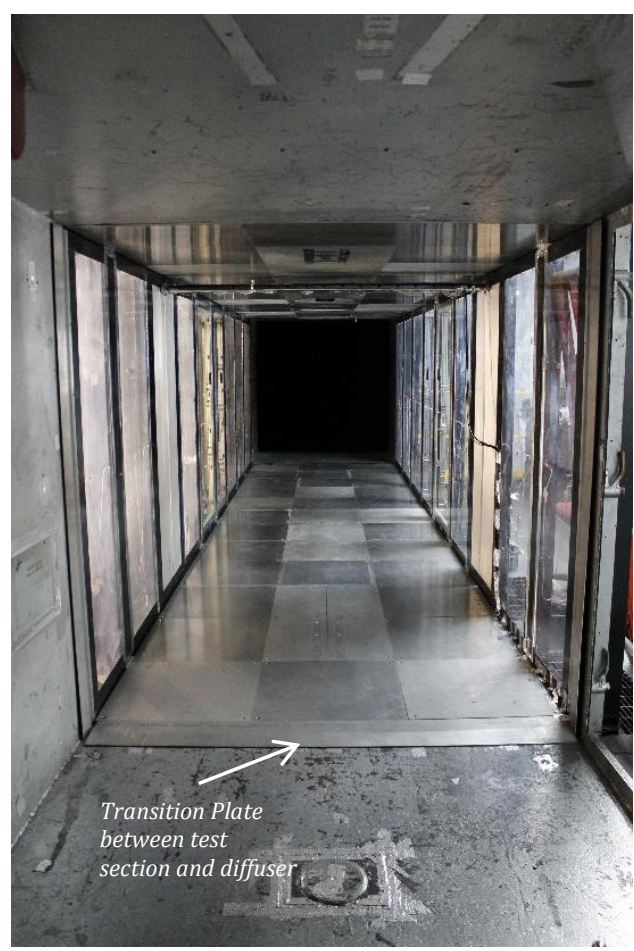

Figure 2.8 Downstream View of Empty, Aerodynamic Test Section panels. These panels are precisely aligned and carefully screwed into the steel structure supporting the test section so as to create continuous, smooth surfaces for flow.

Full span, vertically-mounted airfoils are held in the centre of the test section through supports installed in the floor and ceiling. Two panels, adjacent in the flow direction, are 
removed from the each of the ceiling and floor and replaced with $1.22-\mathrm{m} \times 0.61-\mathrm{m}$ rectangular plates containing a circular turntable system. The turntable system is used to support the model but also to change angle of attack of the model during testing. Collars are affixed in the centre of the turntables so that $88.9-\mathrm{mm}$ diameter shafts extended from the ends of an airfoil model can be inserted through the turntable, into the ceiling or floor. The design of the turntable system places the axis of the shaft, which coincides with the quarter chord of the model, midway between the two side walls, and 3.56-m from the upstream end of the test section.

A Kinematics model ZE14C slew drive powers the turntable system and provides up to $1000-\mathrm{Nm}$ of torque and a rotation rate of $0.1-\mathrm{RPM}$. The position of the turntable is recognized by a Renishaw LM10 linear magnetic encoder system. The system is also equipped with a Renishaw MS10B encoder strip which allows for angle of attack measurements from $-250^{\circ}$ to $100^{\circ}$. The instrumentation used to control the wind tunnel is shown in Figure A2.

The uncertainty in setting the absolute angle of attack is estimated based on first hand experiences completing this task. A conservative estimate of the uncertainty is $\pm 0.2^{\circ}$ but is highly dependent on the accuracy of the initial alignment of the airfoil. Hence the uncertainty can be as small as $25 \%$ of this estimate from run to run. The uncertainty associated with angle of attack readings is $0.01^{\circ}$ as this is the accuracy to which the measurements were read on this device.

The junction of the test section with the contraction exit and the diffuser are covered with 0.08-mm NASHUA All Weather aluminium foil tape and aluminium transition plates, respectively. The transition plates downstream the airfoil are shown in Figure 2.8. Figure 2.9 shows the foil tape covering the steps between the contraction and the test section. These ensure that there is a smooth transition between the test section and the rest of the wind tunnel circuit by reducing the steps and gaps at these junctions.

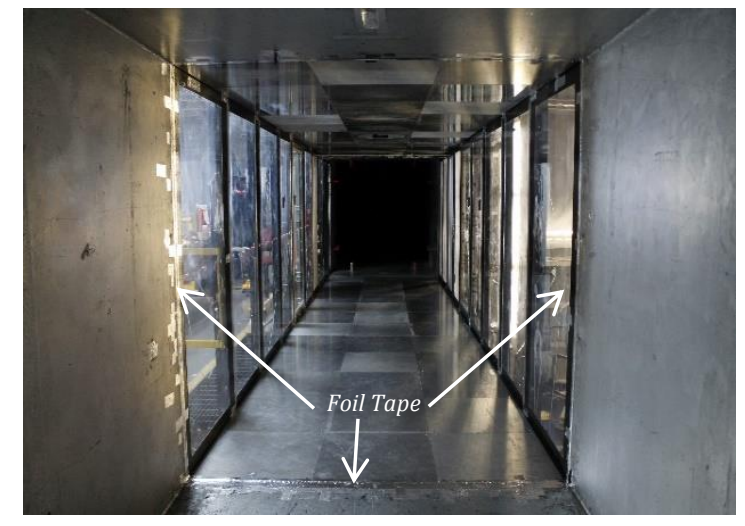

Figure 2.9 Upstream View of Aluminium, 0.08- $\mathrm{mm}$ Thick, All Weather Foil Tape Covering the Steps between the Contraction and Test Section

\subsubsection{Flow Control System}

The Virginia Tech Stability Wind Tunnel is also equipped with a boundary layer control system which ensures flow in the test section is two-dimensional at all test angles. The system draws air from the endwall junctions between the model and the test section, thus preventing or delaying separation over the model. 
The flow control system is powered by two Cincinnati Fan Model HP12F26 blowers, matching 60-hp motors, and General Electric variable frequency drives which are all housed in a separate part of the building (called the compressor room) from the wind tunnel control room. The blower set up is shown in Figure 2.10. This model blower is very powerful and can generate pressure differences of as much as 50 $\mathrm{inH}_{2} \mathrm{O}$ with no flow rate and $46-\mathrm{inH}_{2} \mathrm{O}$ with a flow rate of $4800 \mathrm{CFM}$.

Each blower is connected to a $0.254-\mathrm{m}$ inner diameter PVC pipe which then connects to two 0.254-m inner diameter steel ducts. The steel ducts penetrate the walls of the compressor room and run along the outside of the building before entering the wind tunnel control room.

Inside the control room, the exteriors steel

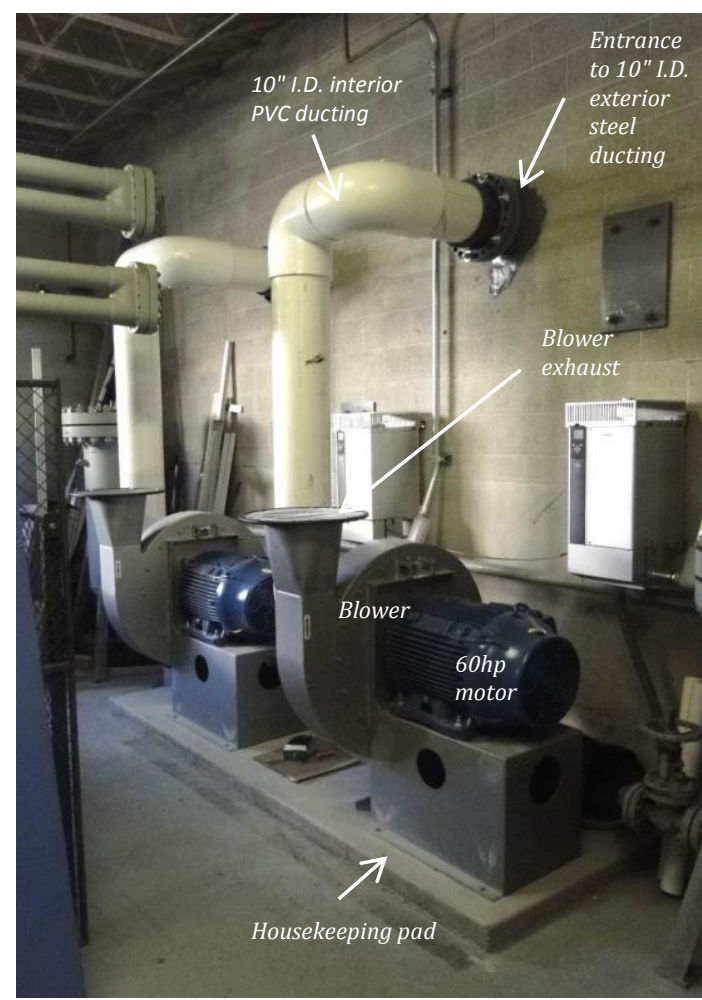

Figure 2.10 Blowers for Flow Control System Installed in Compressor Room ducts reconnect to two $0.254-\mathrm{m}$ inner diameter PVC pipes, one of which runs from the walls to the top of the test section and the other to the bottom of the test section. Two Dwyer PAFS-1005 averaging flow sensors, each with 0.251$\mathrm{mm}$ insertion length, are implanted into each PVC pipe to read the flow rate. Figure 2.11a shows the duct work inside the control room. The PVC pipes directly attach to 0.254-m inner diameter, flexible vacuum ducts which then attach to distribution boxes. Each distribution box is finally connected to an octagonal exterior plenum, shown in Figure 2.11b, by two flexible ducts each $0.1-\mathrm{m}$ in diameter.

The external plenum fits around the collar embedded in the turntable which supports the 88.9-mm airfoil shaft. The plenum is attached to the interior suction assembly which comprises of a shallow plenum and a series of end pieces, each tailored according to the airfoil profile. Figure 2.12 gives details of a typical interior suction assembly for the bottom of an airfoil. 


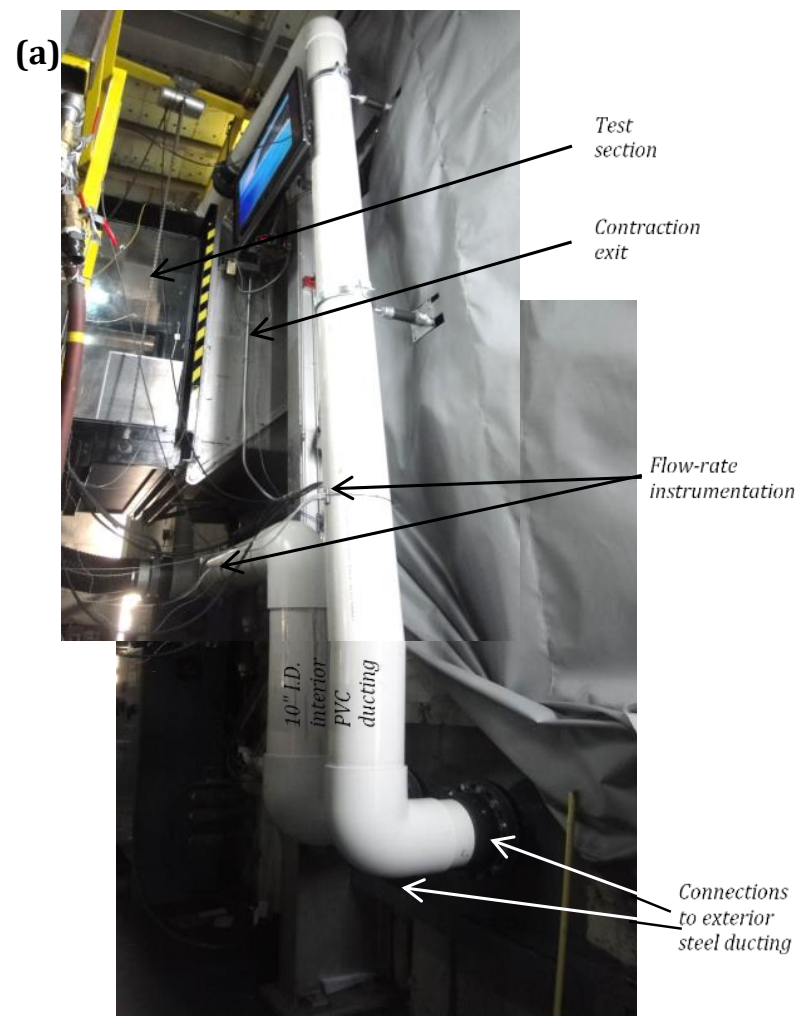

(b)

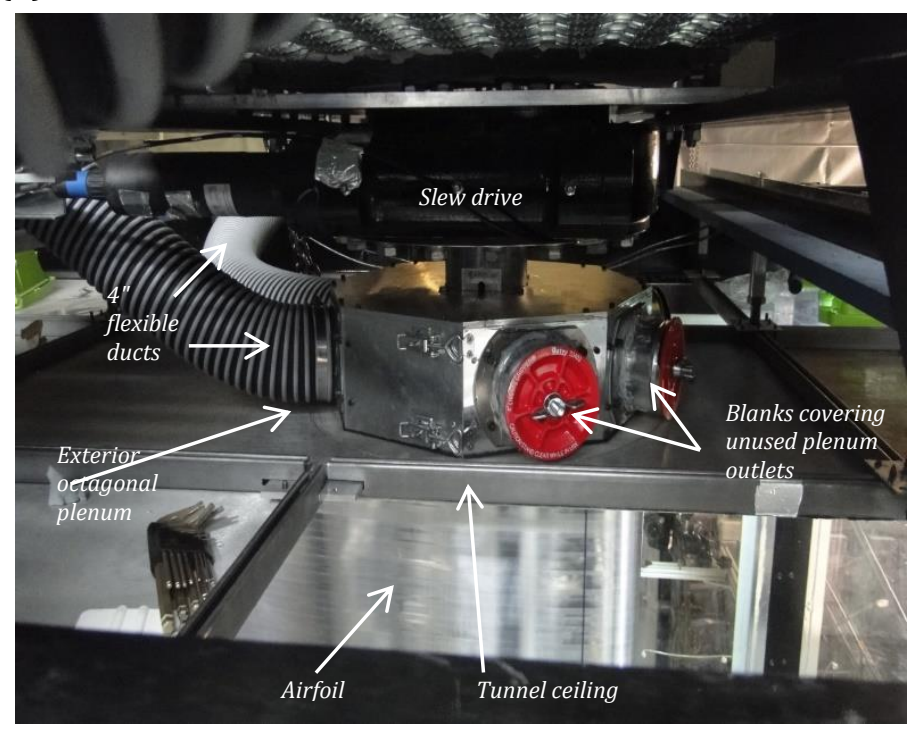

Figure 2.11 (a) Ducting for Flow Control System inside the Wind Tunnel Control Room (b) External Plenum Mounted Atop Test Section

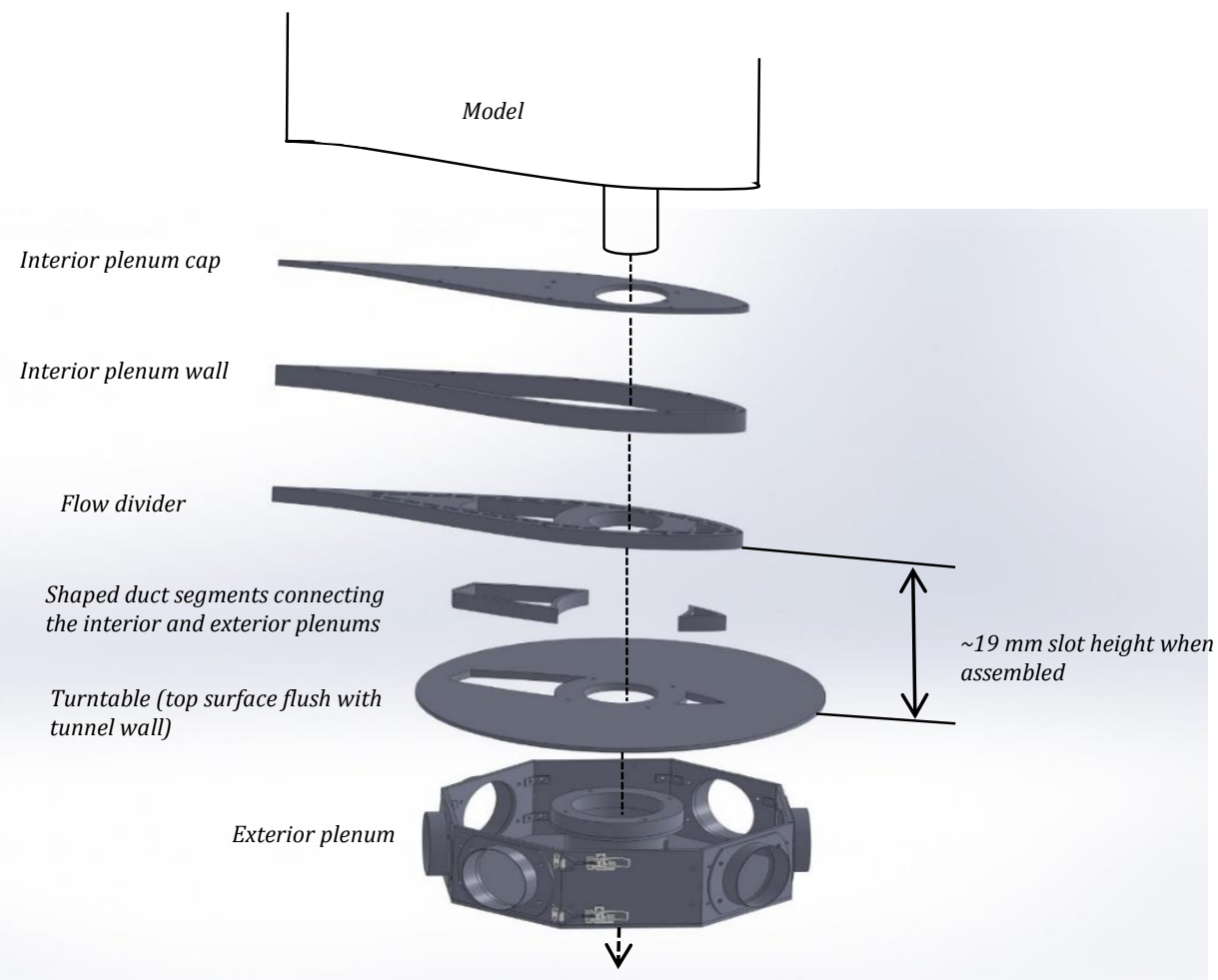

Figure 2.12 Exploded View of the Lower Interior Plenum of the Boundary Layer Control System for the DU00-W212 
When fully assembled, the end plates create a maximum suction slot height of 19-mm, but this height can be varied or completely covered using aluminium shim. Figure 2.13 shows the suction assembly attached to the top of an airfoil, with a 5-mm slot. The suction assembly is covered in foil tape and 0.25 $\mathrm{mm}$ Mylar to ensure no steps exist between the end pieces.

Air is pulled inward through the suction slot, into the internal plenum

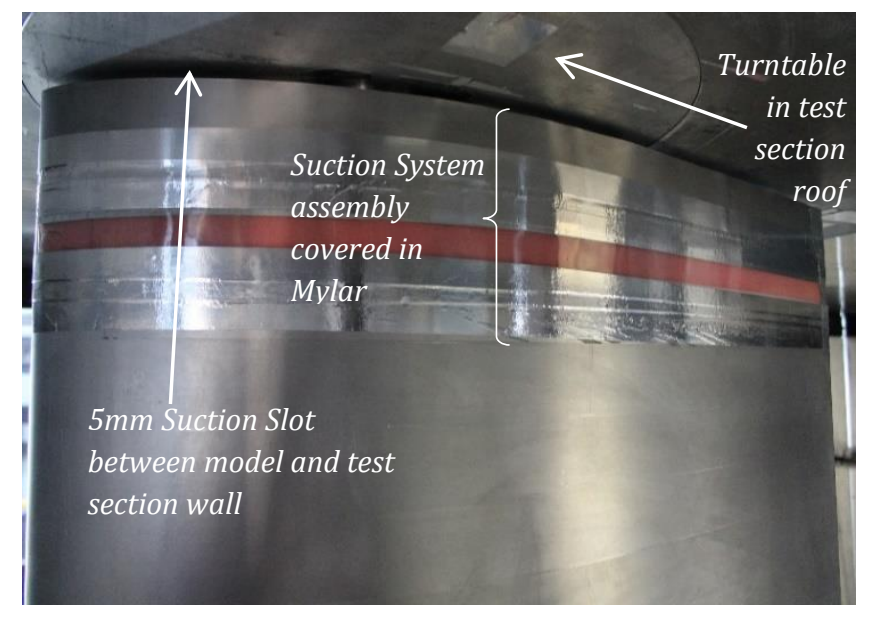

Figure 2.13 View of the Upper End of the 0.8-m Chord DU96-W180 Airfoil with 5-mm Suction Slot where it is then directed through the end pieces and into the external plenum. The air is removed to the compressor room through the PVC and steel pipes. Exhaust is finally expelled from the compressor room through small louvers installed in the walls.

\subsubsection{Pressure Measurement Systems}

The Virginia Tech Stability Wind Tunnel measures the flow speed, airfoil lift, airfoil drag, and airfoil pitching moment from pressure measurements. These pressure data are collected from four different systems, each using different computational methods, in the wind tunnel test section.

\subsubsection{Reference Pressure Measurements}

Flow speed is calculated using the pressure difference between static pressure taps embedded in the walls of the settling chamber and the contraction. Either a Datametrics Barocel Pressure Sensor Type 590D with traceable calibration or an Esterline 9816/98RK pressure scanner system is used to take these pressure measurements. These pressure taps are calibrated using a Pitot static probe mounted at the model position in the empty test section. The calibration is then verified using pressure taps installed in the wind tunnel floor. The absolute ambient pressure is measured by a Validyne DB-99 Digital Barometer with a resolution of 0.01-inHg. Temperature measurements, needed to produce useful flow results from the pressure measurements, are measured using an Omega Thermistor type 44004 with an accuracy of $\pm 0.2-{ }^{\circ} \mathrm{C}$. 


\subsubsection{Airfoil Surface Pressure Measurements}

Pressure measurements are taken along the chord of airfoils mounted in the Stability Wind Tunnel through pressure taps fabricated on the model surface. These pressure taps are precision cut holes, on the order of $0.5-\mathrm{mm}$ in diameter, which connect to pressure scanners using 1.8-mm diameter Tygon tubing. An Esterline 9816/98RK pressure scanner system, with 288 channels, is used to measure these airfoil surface pressures and allows data acquisition of up to three complete rows of pressure taps. The Tygon tubes then connect directly to the Esterline scanner system. The reliability of these connections and the functionality of the pressure scanner are tested using a Fluke model 718-1G Pressure Calibrator. The pressure calibrator is used to apply a known pressure to the pressure taps on the model surface which is then read from the pressure scanners, and should be the same as that applied to the taps. This pressure check gives a clear indication of faulty connections, leaking tubes or faulty pressure ports so that these can be removed from the final data set.

The Esterline scanner is connected via IP to the computer running the pressure acquisition software which was written in MATLAB by Virginia Tech Stability Wind Tunnel personnel. The acquisition program is designed so that any number of measurements (usually twenty five, each with average of 96 samples) can be collected from each scanner and then averaged to produce a final pressure distribution over the model.

As an extension of the acquisition code, the lift and moment coefficients are estimated. This is done by transforming the final pressure distribution measurements from functions of chord-wise position, $x$, to edge-length positions, $s$, using the specific airfoil shape. Thereafter, the pressures are integrated to approximate the lift per unit span and the moment per unit span using the trapezium rule. These are then used to evaluate the lift coefficient, $C_{l}$, and moment coefficients, $C_{m}$, (measured positive pitch up) using equations (2-4).

$$
\begin{aligned}
C_{l} & \equiv \frac{l}{\frac{1}{2} \rho U_{\infty}^{2} c} \\
C_{m} & \equiv \frac{m}{\frac{1}{2} \rho U_{\infty}^{2} c^{2}}
\end{aligned}
$$

The variables $\rho$ and $U_{\infty}$ refer to the free stream density and velocity, respectively, and $c$ refers to the airfoil chord length. In this study, the lift presented were predominantly obtained using this method. 


\subsubsection{Test Section Wall Pressure Measurements}

The walls of the aerodynamic test section are outfitted with a sequence of pressure taps which provide secondary measurements of lift. These measurements are instrumental in validating the airfoil surface pressure measurements as they are typically less sensitive to local flow features because they are based on span-wise averaging. Therefore, lift measurements can be made independently of model surface condition. Esterline 9816/98RK pressure scanners each with a range of \pm 10 -inWC, resolution of \pm 0.003 and an accuracy of $0.05 \%$ were used to measure pressures from an array of ports installed in the test section side walls.

The Lexan panels which make up the test section walls are equipped with a forty one pressure ports in both the span-wise and stream-wise directions. However, only the twenty four stream-wise ports located at mid-span are used for lift measurements. The locations of the ports are identical on both side walls and are depicted in Figure 2.14.

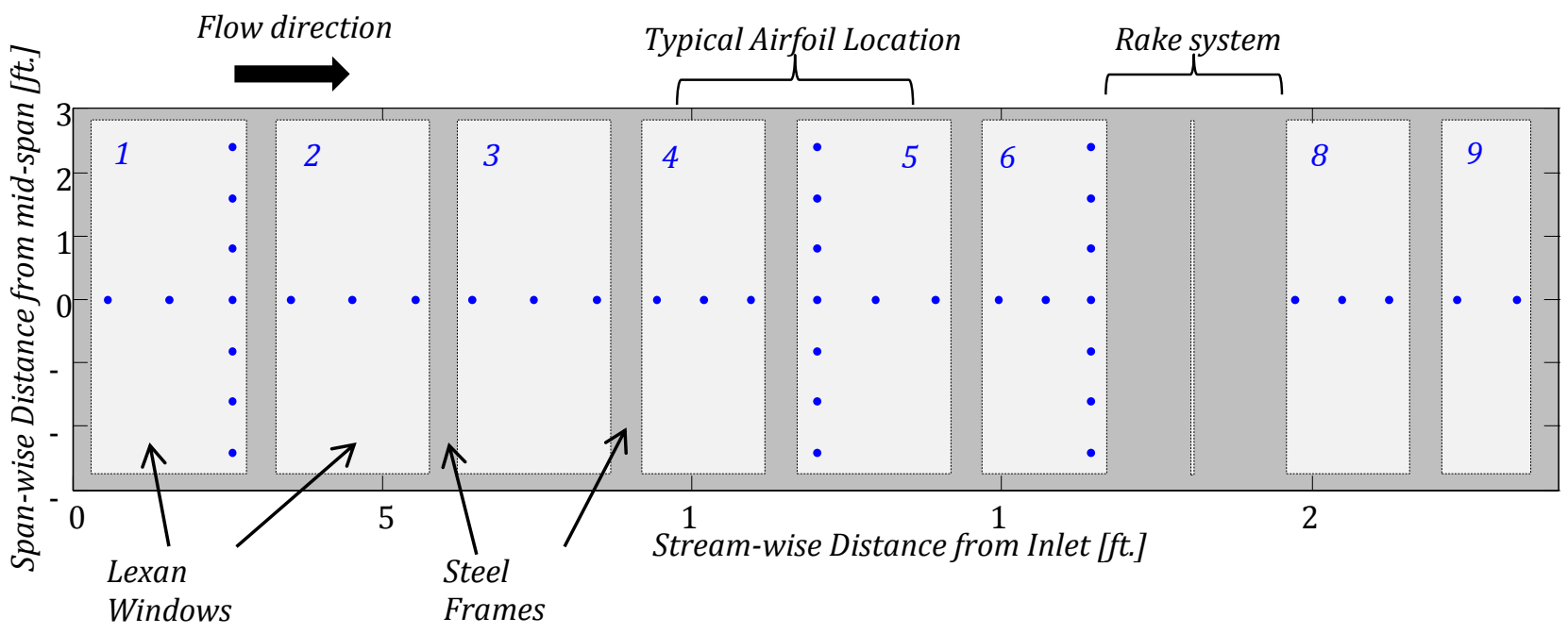

Figure 2.14 Side View Diagram Detailing the Location of the Pressure Taps Installed in the Test Section

The pressure ports are made from rapid-prototyped inserts which are either 4.76$\mathrm{mm}$ or $6.35-\mathrm{mm}$ in diameter and which contain a $0.64-\mathrm{mm}$ diameter through-hole. These taps are inserted into the test section side walls so that they are flush with the inner wall surface. They are connected to the pressure scanner using 1.8-mm Tygon tubes. Figure 2.15 shows the structure of these wall pressure taps. 

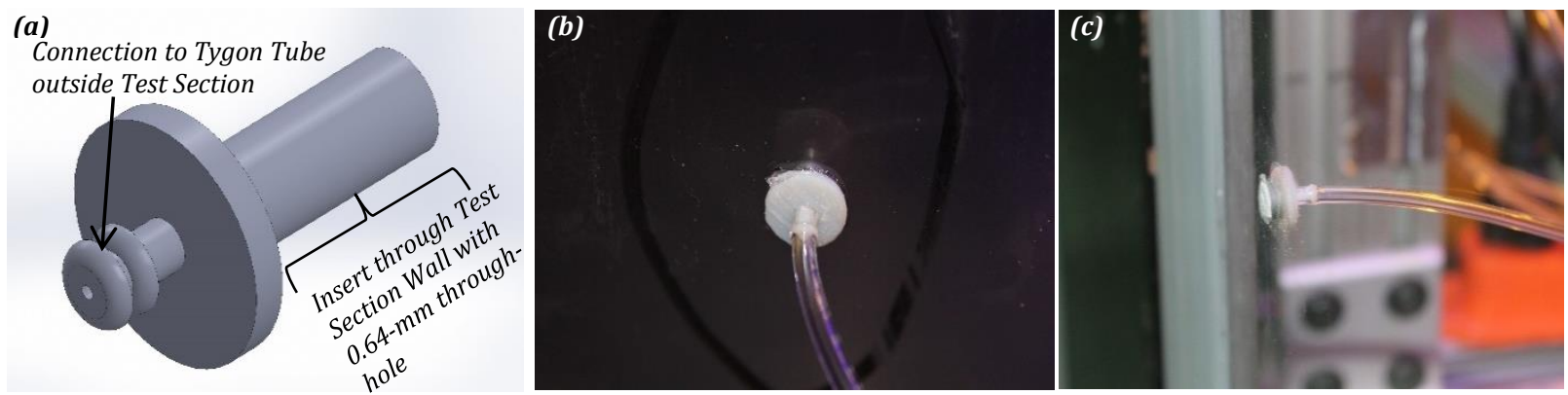

Figure 2.15 Wall Pressure Port View from (a) Isometric Drawing, (b) Outside the Test Section showing Tygon Tube Connection and (c) Inside the Test Section showing Smooth Surface with Side Wall

Lift on an airfoil is calculated by applying the vertical momentum equation and integrating the pressure measurements obtained from the side wall pressure over the control volume shown in Figure 2.16. The lift force on the airfoil is given by the difference between the pressure forces on face 3 and face 4 minus the difference in vertical momentum flux through faces 1 and 2, which is small because it is assumed that faces 1 and 2 are in aerodynamic far-field of the airfoil (W. Devenport, personal communication, December, 2012).

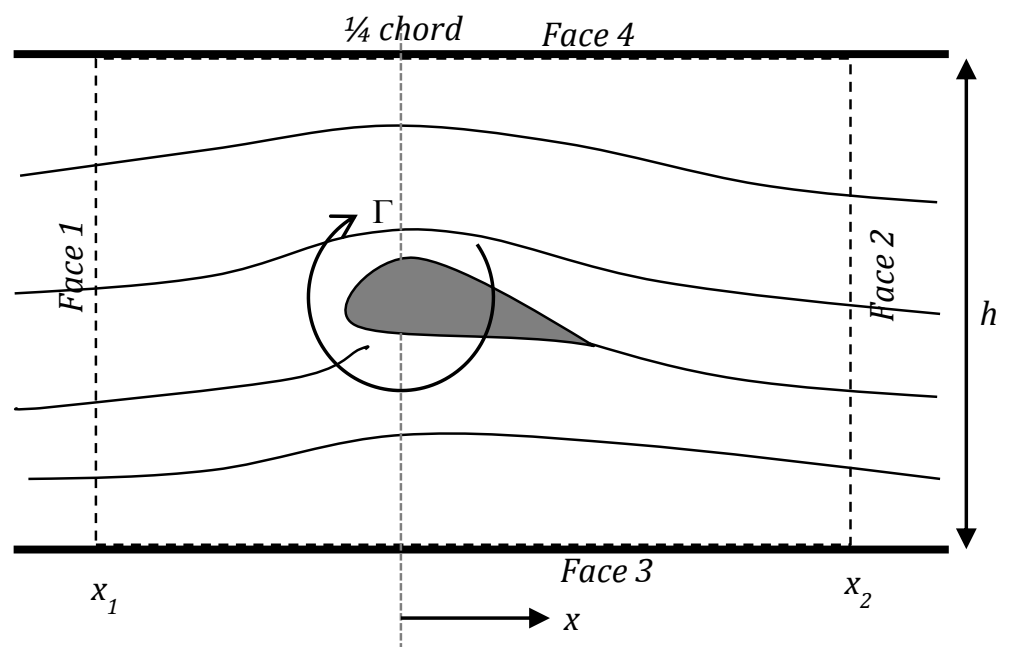

Figure 2.16 Control Volume used to Estimate the Lift from Side Wall Pressure Taps

The final lift coefficient estimated by this method is given by equation (2-5) where the $C_{l}$ is the true lift coefficient, $C_{l w}$ is the lift coefficient determined from integration of the wall pressures, and $\varepsilon$ is the error term given by equation (2-5) and (2-6).

$$
C_{l}=\frac{C_{l w}}{1-\varepsilon}
$$




$$
\varepsilon=\frac{1}{\pi}\left[\arctan \left(\operatorname{coth} \frac{\pi x}{2 h}\right)-\arctan \left(\tanh \frac{\pi x}{2 h}\right)\right]_{x_{1}}^{x_{2}}
$$

Here $h$ is the test section width, $x_{1}$ and $x_{2}$ are the stream-wise limits of the pressure integration on the test section walls measured relative to the quarter chord position (W. Devenport, personal communication, December, 2012).

\subsubsection{Wake Rake Pressure Measurement System}

A traversing drag rake comprising of Pitot and static probes is used to measure pressure data in the airfoil wake, from which drag is later determined by the momentum balance approach.

The traversing drag rake encompasses almost the full width of the test section, with only about a $3-\mathrm{cm}$ space between the mounted rake and the test section side walls. The front tip of the probe is approximately 1.7-m downstream of the axis of rotation of the airfoils due to the locations of the traversing slots in the test section side walls. The rake is essentially a combination of 112 Pitot probes and 7 Pitot static probes attached to a box shaped aluminium strut which traverses the height of the test section, thus allowing cross sections through the wake to be taken. Figure 2.17 shows the traversing wake rake mounted in the test section.

All pressure probes are supported by an oval clamp positioned 105-mm downstream of the probe tips. Seventeen 6.4-mm diameter steel rods, mounted downstream of the probes every $102-\mathrm{mm}$ along the width of the rake, support the clamp. These rods then attach the airfoil shaped aluminium strut, 29-mm thick and $89-\mathrm{mm}$ in chord. Four DTC Initium ESP32HD 32-channel pressure scanners, with a range of \pm 2.5 -psi, accuracy $0.05 \%$ full scale, are secured atop the aluminium strut and measure the pressure from the Pitot probes. These scanners are fairly small and therefore can be

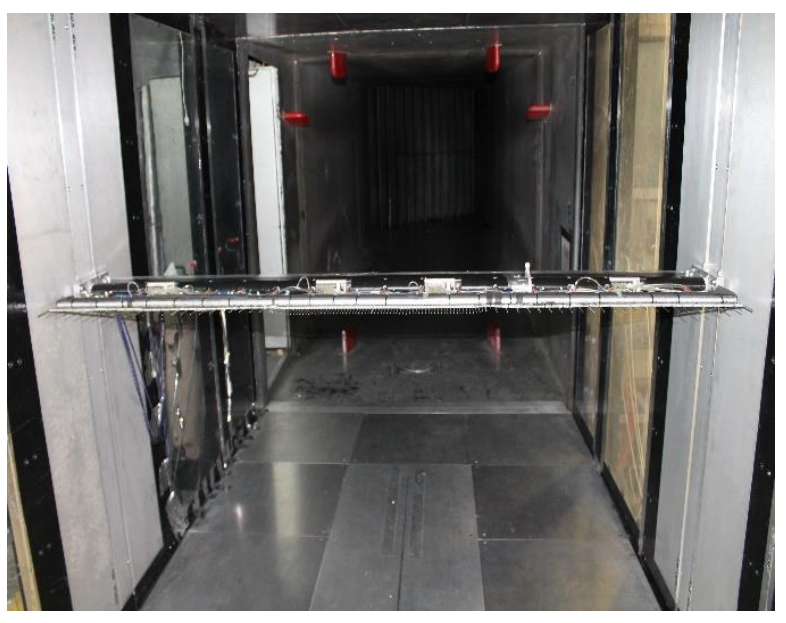

Figure 2.17 Upstream View of the Traversing Drag Rake Mounted in the Test Section placed in the flow without risk of extensive flow interference, which in turn produces more accurate results since scanners are connected to the probes using shorter tubes.

The Pitot probes themselves are simple tubes fabricated from 1.6-mm diameter stainless steel. The Pitot static probes however, are Dwyer, model 167, 3-mm in diameter. 
The Pitot probes are 102-mm long and arranged such that there is a higher concentration in the region close to the rake mid-span because this region is directly behind the airfoil at zero angle of attack. This central area is approximately 528-mm wide and the probes are spaced every 7.6-mm here, while the probes outside this section are spaced every 25.4-mm. Two of the seven Pitot static probes are positioned at the extreme ends of the rake, two are mounted on the outer limits of the centre region of concentrated probes, and the remaining three probes are each placed in the middle of one of the three regions created by the first four probes. A schematic of the traversing drake rake is shown in Figure 2.18.

The rake is traversed vertically using NEMA 34 High Torque Step Motors (model HT34-497DYAA) and an AC Advanced Microstep Drive with Encoder Input (model STAC5-SE120) both manufactured by Applied Motion Products. This motion control system is attached to the aluminium strut and is computer controlled using the same data acquisition software which controls the airfoil motion, surface pressure and wall pressure. Using this system, the rake position can be set within an uncertainty of 1-mm.

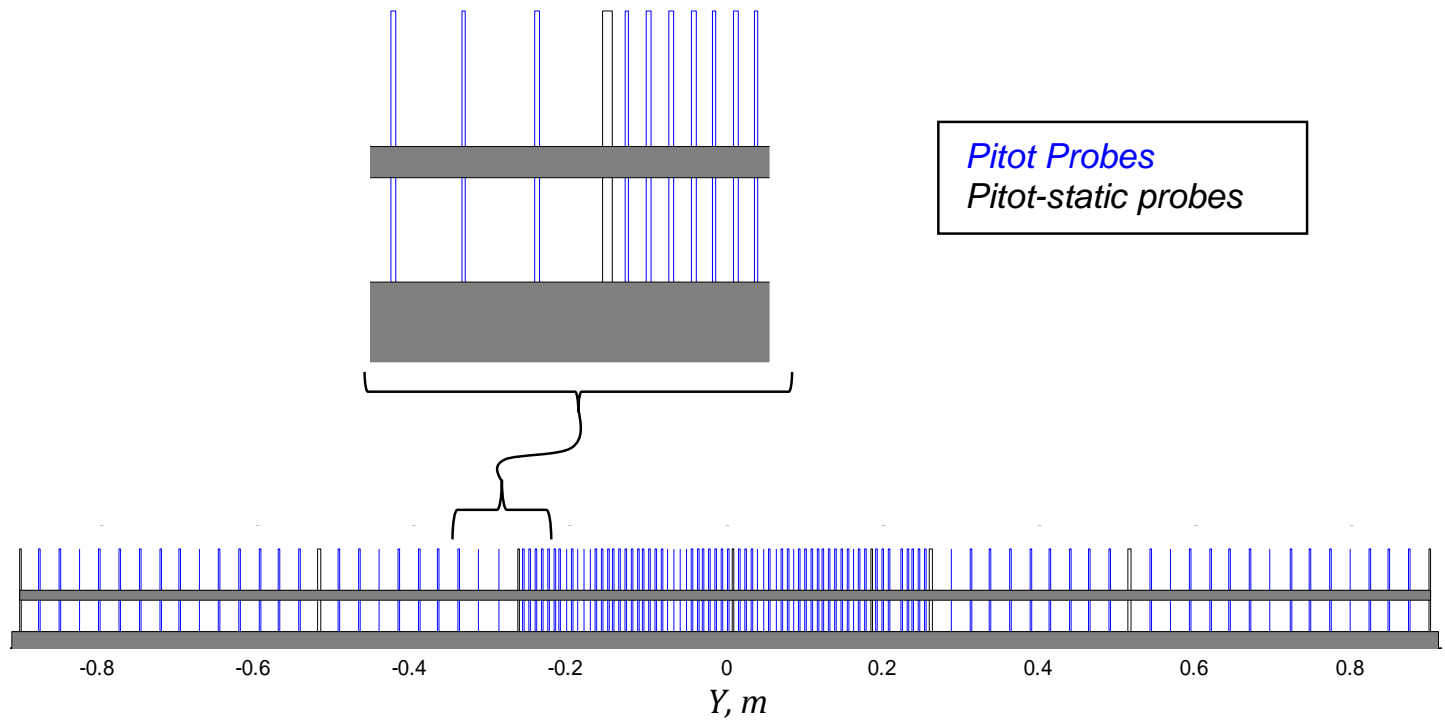

Figure 2.18 Diagram of Traversing Wake Rake Showing Distribution of Pitot and Pitot-Static Probes.

This system gives an estimate of drag coefficient by first evaluating the total drag per unit span on the model. Integrating the stagnation and static pressure profile and applying a momentum balance gives the airfoil drag. The stagnation and static pressure coefficient profiles are given in equations (2-7) and (2-8) where $p_{\infty}$ and $p_{0 \infty}$ are the free stream static and stagnation pressures respectively, and $p$ and $p_{o}$ are the local values measured from the rake (W. Devenport, personal communication, December, 2012).

$$
C_{p}=\frac{p-p_{\infty}}{p_{o \infty}-p_{\infty}}
$$




$$
C_{p o}=\frac{p_{o}-p_{\infty}}{p_{o \infty}-p_{\infty}}
$$

The total drag force per unit span on the airfoil, for the chosen control volume, is given in equation (2-9).

$$
d=\rho \int U_{\infty}^{2}-U^{2}-\frac{1}{2}\left(U_{\infty}+U_{e}\right)\left(U_{\infty}-U\right)-\frac{p-p_{\infty}}{\rho} d Y
$$

This produces a drag coefficient shown in equation (2-10) (W. Devenport, personal communication, December, 2012).

$$
C_{d} \equiv \frac{d}{\frac{1}{2} \rho U_{\infty}^{2} c}=\int\left(2-2 \frac{U^{2}}{U_{\infty}^{2}}-\left(1+\frac{U_{e}}{U_{\infty}}\right)\left(1-\frac{U}{U_{\infty}}\right)-C_{p}\right) d(Y / c)
$$

\subsubsection{Measurement Corrections for Wall Interference}

Corrections for the wall interference in the two-dimensional hard wall test section were done according to the work of Allen and Vicenti (1947). In wind tunnel testing, the free stream velocity and the angle of attack experienced by the model is slightly different from that encountered in free flight at the same conditions. This variation occurs due to the effect of the confining test section wall. The corrections suggested by Allen and Vicenti (1947) focused on the analysis of an approximate inviscid model and has proven to be robust and appropriate to modern wind tunnel tests.

The proposed correction scheme corrects for the effect of the solid wall blockage on the lift, moment, pressure coefficient and angle of attack. The blockage corrections for angle of attack vary as the square of the ratio of chord-length to test section width and the largest blockage corrections to angle of attack were typically 0.3 degrees. The drag was also corrected by accounting for wake blockage effects (Allen and Vicenti, 1947). The wake corrected drag is subscripted by 'wc'.

All results presented for the Stability Wind Tunnel in this study (Chapter 4:) will be corrected according to this correction scheme and be denoted by a subscript ' $c$ '.

\subsubsection{Uncertainties in Measurements}

Uncertainties associated with the pressure, lift and moment coefficients were estimated as $\pm 0.007, \pm 0.012$, and \pm 0.0014 respectively (Devenport et al., Personal Communication, 2010). The drag coefficient was predicted to have a $5 \%$ uncertainty for cases when the drag rake is able to capture the entire wake. This is applicable to measurements made when the flow is attached and in the locality of stall. The increased uncertainty for fully or partially stalled flow over the airfoil was also addressed by Devenport et al. (Personal Communication, 2010). 


\subsection{Airfoil Models}

A series of airfoil models were tested during the course of this study, during both Phase One and Phase Two. The following is a description of the models used.

\subsubsection{8-m, 18\% Proprietary Model}

An 18\% proprietary model of 0.8 -m chord was fabricated by the Aerospace and Ocean Engineering Department machine shop for a study of spoiler performance (A. Borgoltz, personal communication, December 2011). The model is built from a series of stacked 25$\mathrm{mm}$ thick aluminium laminates to create a full span of 1.82-m. Each laminate has four open regions to allow equipment to be run through model and connect to external instruments. The thickness of the laminate walls varies around the profile, ranging from 9.5-mm to over 25-mm in some regions. The model is instrumented with pressure taps for measuring the pressure along its chord.

This model was used during the early stages of the system development in Phase One. For this testing only six of the $25-\mathrm{mm}$ laminates were stacked to create a $0.15-\mathrm{m}$ span, $0.8-\mathrm{m}$ chord model.

\subsection{5-m, Clark-Y Model}

A close replica of a Clark-Y airfoil, 0.9-m in span, 0.15-m in chord with a maximum thickness of 19.1-mm (12.5\%), was used. This is not an exact Clark-Y model as the perfect Clark-Y profile has a maximum thickness of $11.7 \%$. This model was constructed from solid wood and painted black. It has an interior, circular metal disc at mid-span for attachment to a mounting strut. This disc is not visible on the surface but has two screw holes for attachment. This model has two rows of internal pressure taps located approximately 13$\mathrm{mm}$ from each end of the model and arranged diagonally. The taps were painted over and are not instrumented, hence it is not possible to measure pressure using the pressure taps. There were also several scratches and small dents on surface of the model. The model and its profile are shown in Figure 2.19.
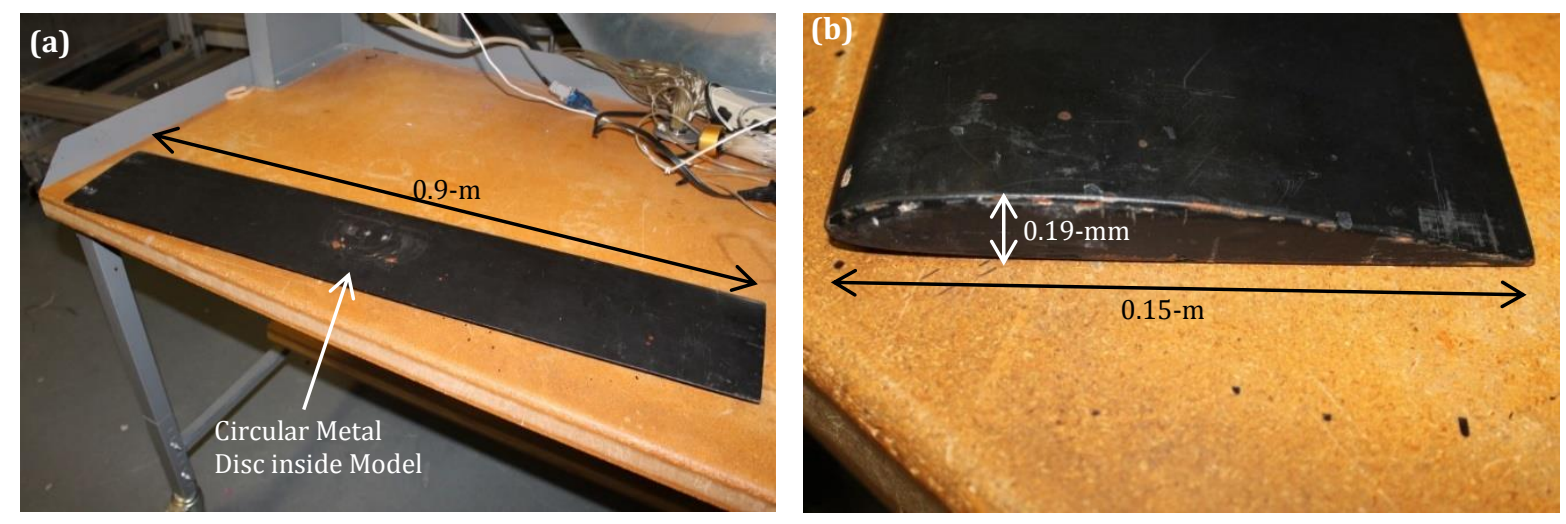

Figure 2.19 Photographs of the (a) Full Span and (b) Profile of 0.15-m Wooden Clark-Y Model 


\subsubsection{2-m, NACA-0012}

An aluminium, NACA-0012 model with 1.016-m span, 0.2-m chord and maximum thickness of 25.4-mm was tested in Phase One, in the Open Jet Wind Tunnel. This model is hollow with four inner extrusions and a solid leading edge. However, the model is not instrumented with pressure taps or sensors for measuring surface pressure. The model is symmetric with a maximum thickness of $12 \%$ occurring at $30 \%$ chord. This aluminium model has very thin walls, with the thickness region being approximately 5-mm. Figure 2.20 shows the model and its cross section.
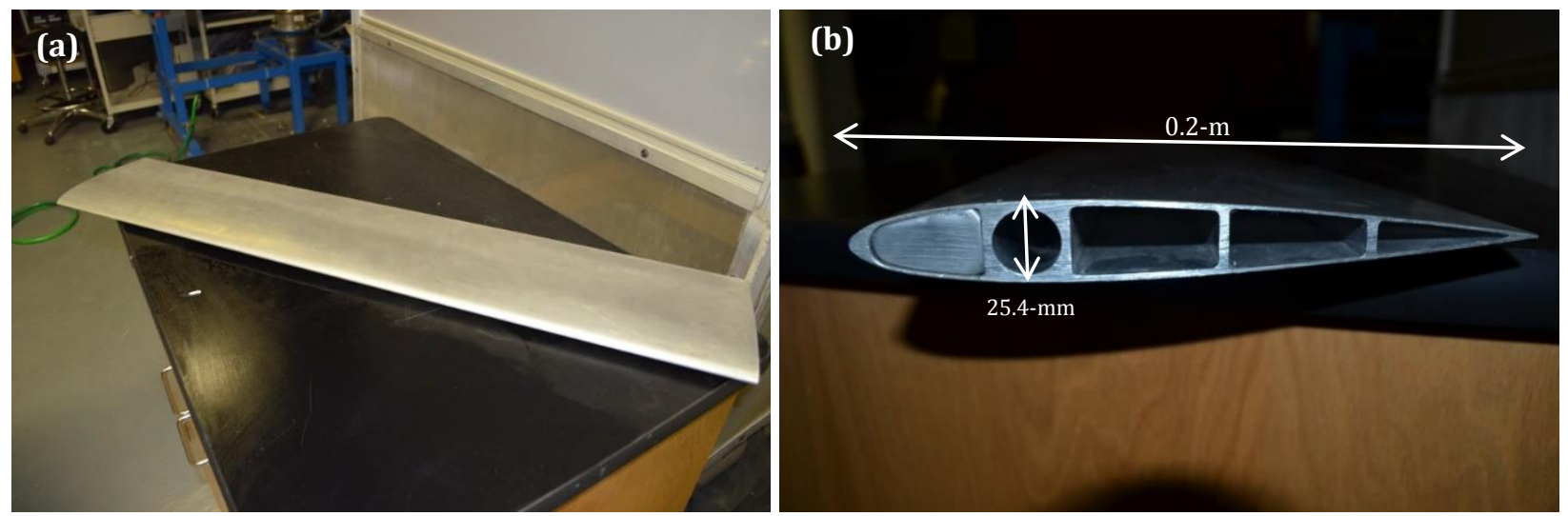

Figure 2.20 (a) Full Span View and (b) Cross Section showing Thin Walls of the Aluminium, 0.2-m NACA-0012

\subsection{1-m DU00-W-212}

This model was fabricated by the Aerospace and Ocean Engineering Department machine shop for a study of spoiler performance (A. Borgoltz, personal communication, December 2011). As a result the model comprises of two sections: a main element and a removable leading edge module. Together, the total airfoil chord is $0.61-\mathrm{m}$ and has a span of $1.82-\mathrm{m}$. The leading edge module is $1.0-\mathrm{m}$ in span and extends from the leading edge to the $40 \%$ chord location. The model is shown in Figure 2.21.

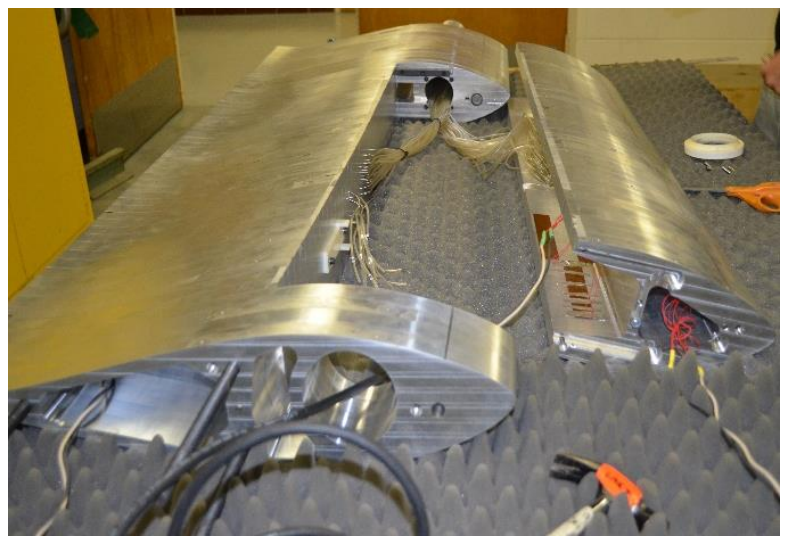

Figure 2.21 0.61-m DU00-W-212 Showing the Inner Cavities, the Leading Edge module and Main Element

The main element is made of 72

vertically-stacked, 25.4-mm thick, aluminium laminates with open interior cavities for instrumentation. A main circular shaft, 88.9- $\mathrm{mm}$ in diameter, is affixed to the ends of the main element thereby allowing the model to be mounted vertically. The centre of the shaft is at the quarter chord of the model allowing it to be mounted and rotated about this point. 
The leading edge module is designed and installed to minimize the step and gap between this element and the main element. The chord-wise gap between the leading edge module and the main element is estimated to be $0.13-\mathrm{mm}$ while the span-wise gap is approximately $1-\mathrm{mm}$. To minimize the effect of the step created (approximately $0.05-\mathrm{mm}$ high), 0.05-mm thick, clear Scotch tape was used to seal the junctions between the leading edge module and the main element of the airfoil.

Pressure taps are embedded in the model surface by drilling $0.5-\mathrm{mm}$ holes through the laminate walls which then attach first to stainless steel tubes and then Tygon tubes with inner diameter of $1.8-\mathrm{mm}$. The Tygon tubes are then routed to external pressure measurement instruments through the internal cavities in the model, specifically the cavity in the main airfoil shaft. A total of 73 pressure ports are installed on the model, 36 on each of the suction and pressure sides and 1 at the leading edge. Of the 73 pressure taps, 45 are in the leading edge module - 22 per side and 1 at the leading edge. The taps are arranged such that each side of the airfoil has three diagonal segments of taps as opposed to a single, chordwise row of taps. Each segment was approximately $15^{\circ}$ to the flow thus ensuring that no tap is in the wake of another upstream tap.

The internal suction assembly specifically tailored for this model is shown in Figure 2.22. Aluminium shim was used to reduce the 19-mm slot to a 5$\mathrm{mm}$ slot for extraction into the inner plenum.

\subsubsection{8-m DU96-W-180}

The 0.8-m chord DU96-W-180 model was designed, fabricated and instrumented by the machine shop of the Aerospace and Ocean Engineering Department. Aluminium laminates, each 50.8-mm in height, are stacked to create the full 1.82-m span model. The full scale
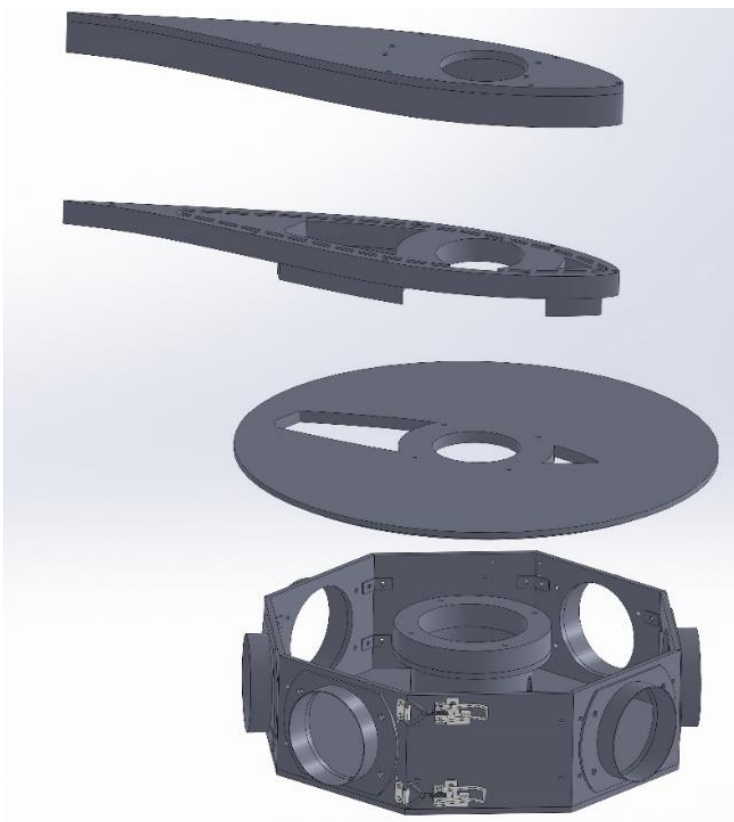

Figure 2.22 Lower Internal Suction System Assembly for 0.61-m DU00-W-212 model is shown in Figure 2.23a and a single laminate (from mid-span) is shown in Figure 2.24. Each laminate has inner cavities for instrumentation and the centre shaft (seen in Figure 2.24). The wall thickness of the laminates, and therefore the model, is small compared to the chord. The maximum wall 
(a)

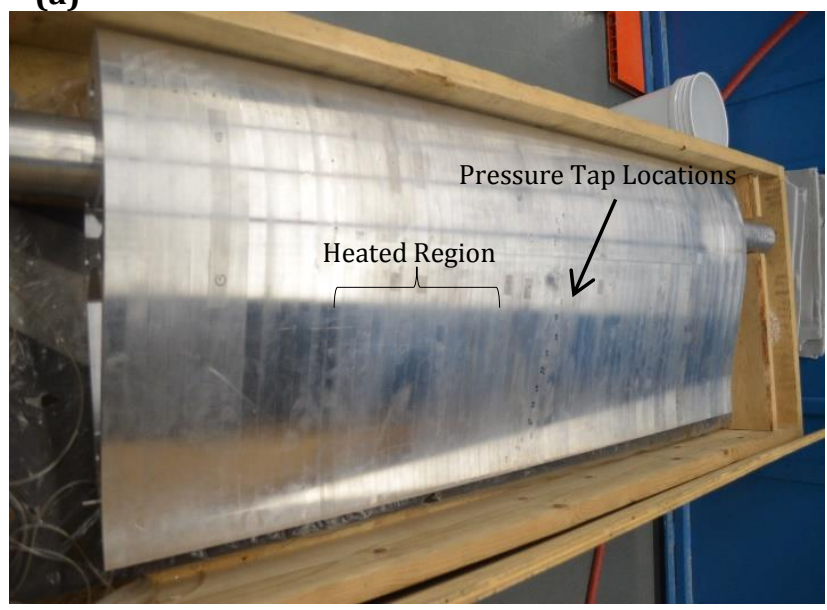

(b)

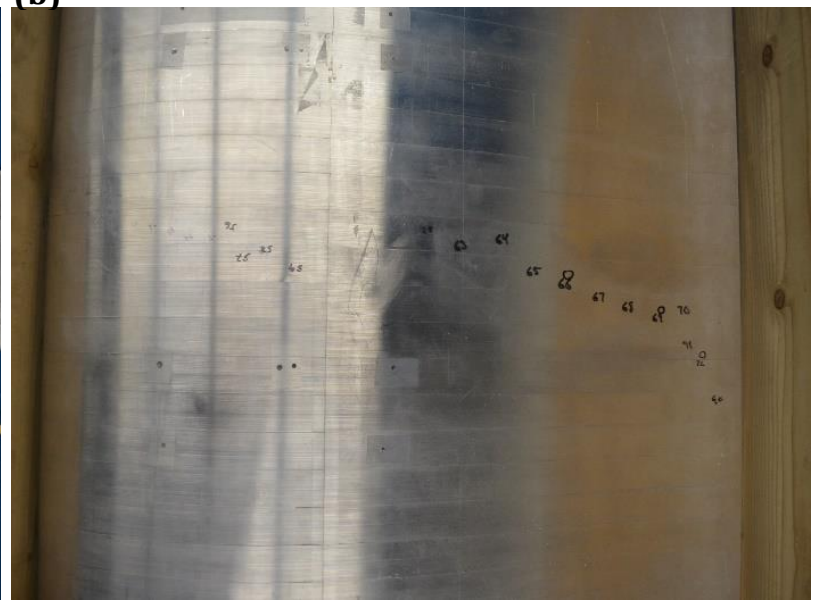

Figure 2.23 0.8-m chord, DU96-W-180 Airfoil showing (a) Full Span and (b) Pressure Port arrangement

thickness occurs at the trailing edge where it is about $0.203-\mathrm{m}$ but the average thickness in other regions is $13-\mathrm{mm}$.

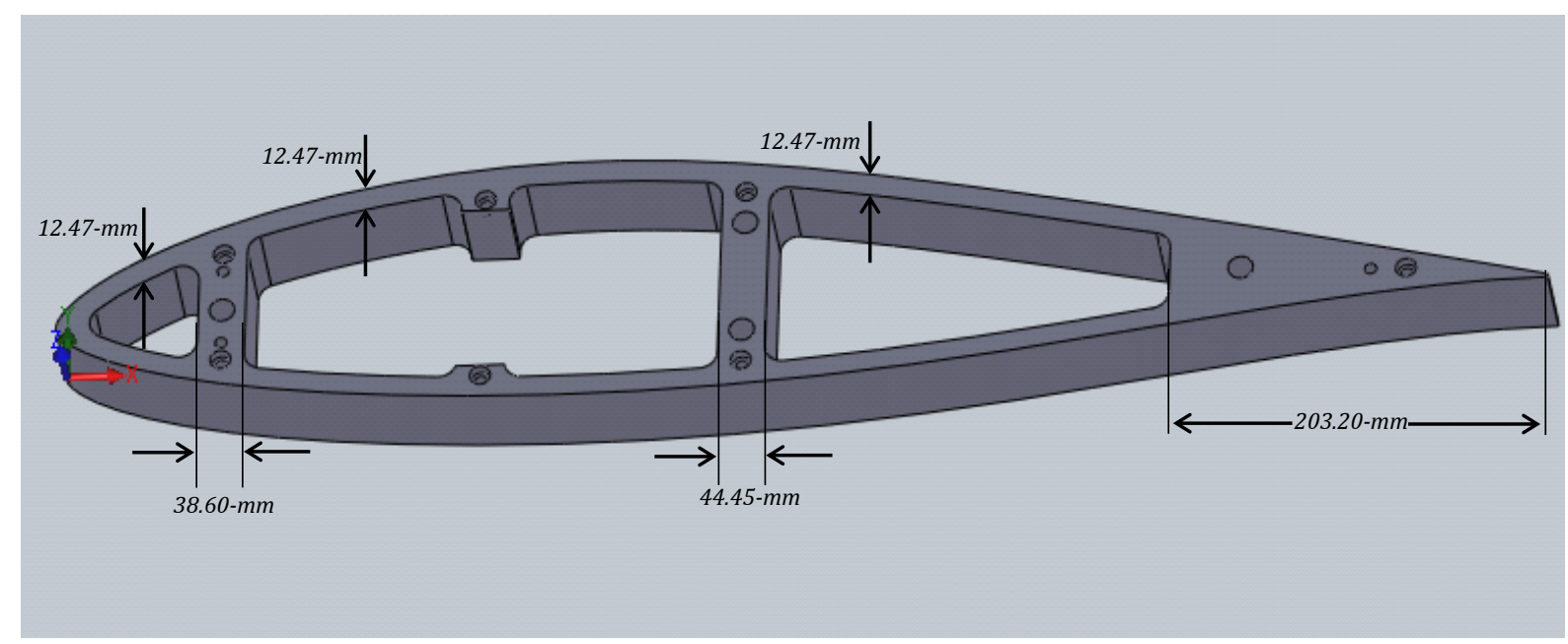

Figure 2.24 Single Laminate of the 0.8-m chord DU96-W-180 Airfoil showing the Wall Thicknesses and Inner Cavities

Surface pressure taps are inserted in the model surface by drilling 0.5-mm holes in the laminates, centred around mid-span. There is one row of pressure taps, arranged in two diagonal segments along the chord as shown in Figure 2.23b. The first segment of taps starts in the mid-span laminate at $0 \%$ chord and runs, at an $18^{\circ}$ angle, to $60 \%$ chord. The second segment of taps also begins in the mid-span laminate but at $68 \%$ chord and runs at a $20^{\circ}$ angle to $100 \%$ chord. There are a total of 79 pressure taps on the model; 39 on the pressure side, 39 on the suction side and one at the leading edge, the exact locations of which are listed in Table B1. The pressure taps are attached to stainless steel tubing embedded in the inner walls of the laminates. Tygon tubes, with inner diameter of $1.6-\mathrm{mm}$, are connected to these 
stainless steel tubes which then connect to the external pressure scanners through the main airfoil mounting shaft.

The end pieces designed to create the internal plenum for the boundary layer control system is shown in Figure 2.25. Aluminium shim stock was used to reduce the $19-\mathrm{mm}$ slot between the model and the test section to $5-\mathrm{mm}$, at both ends of the model.

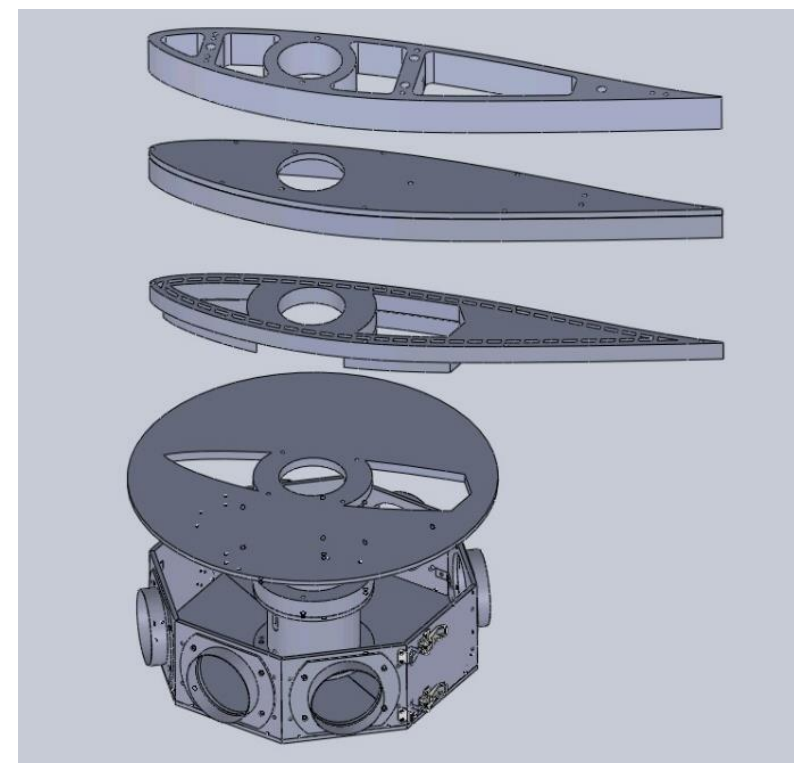

Figure 2.25 Lower Internal Suction System Assembly for 0.8-m DU96-W-180

\subsection{6-m DU96-W-180}

The 0.46-m chord DU96-W-180 model is fabricated from a series of aluminium laminates, each 50.8-mm thick. These laminates are stacked vertically to create the full 1.82m span model shown in Figure 2.26a. Each of the laminates has inner open areas to allow instrumentation to be passed through the full span and for the centre shaft to be inserted. A single laminate, from the mid-span region, is shown in Figure 2.27. The laminate has relatively small wall thickness compared to the chord - average wall thicknesses are 7-mm as seen in Figure 2.27. However, the leading and trailing edge have larger material thicknesses. The model fabrication and instrumentation was done by the machine shop of the Aerospace and Ocean Engineering Department.
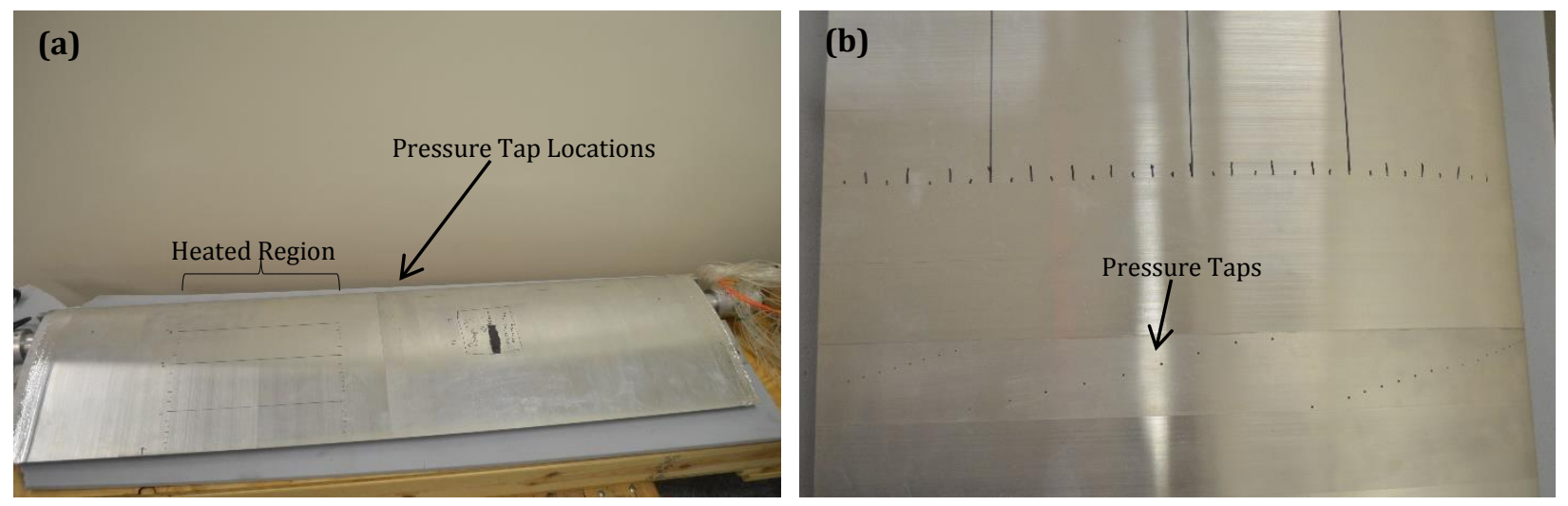

Figure 2.26 (a) Full span and (b) Pressure Port Configuration of 0.46-m DU96-W-180 model 
This model has a total of 79 pressure taps: 39 on the pressure side, 39 on the suction side and one at the leading edge. The pressure taps are attached to stainless steel tubing embedded in the inner walls of the laminates. Tygon tubes, with inner diameter of 1.6-mm, are connected to these stainless steel tubes which then connect to the external pressure scanners. As with the models discussed previously, the surface pressure taps are embedded in the model surface by drilling $0.5-\mathrm{mm}$ holes in the laminates. These taps are arranged around mid-span in three diagonal segments on each side of the model, as shown in Figure $2.26 b$. The first segment of taps begins at the leading edge at mid-span and extends upward at an $18^{\circ}$ angle. The second segment ranges from $0.37-\mathrm{m}$ along the model edge and extends to $0.55-\mathrm{m}$ along the edge, at a $15^{\circ}$ upward angle. The last segment of taps is arranged at a $17^{\circ}$ upward angle, starting at $0.08-\mathrm{m}$ from the trailing edge. The precise location of these pressure taps are tabulated in Table B2.

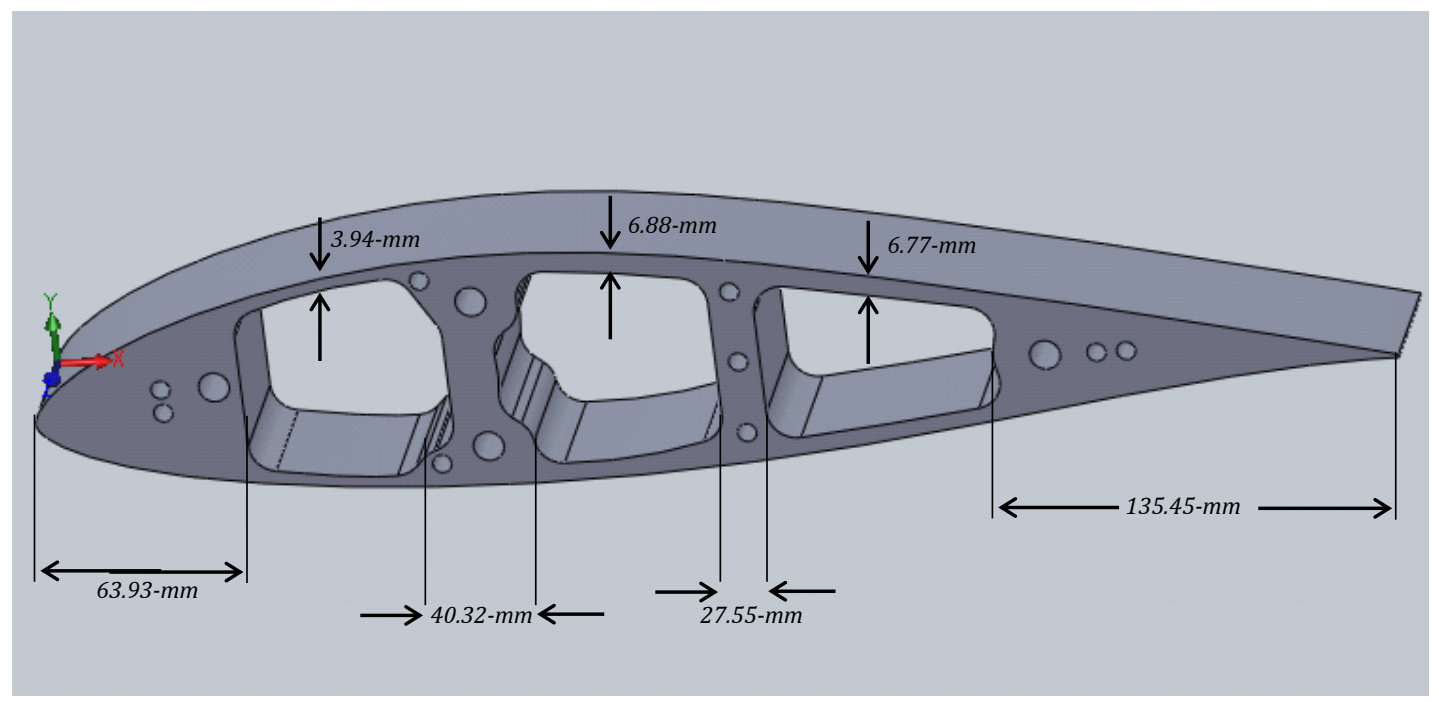

Figure 2.27 Single Laminate of the 0.46-m chord DU96-W-180 Airfoil showing the Wall Thicknesses and Inner Cavities

The end pieces designed to create the internal plenum for the boundary layer control system are the same as that of the $0.8-\mathrm{m}$ model. Aluminium shim stock was used to reduce the 19-mm slot between the model and the test section to 5-mm, at both ends of the model.

\subsection{Infrared Camera}

Thermal imaging utilized two identical high resolution, FLIR A655sc infrared cameras, one of which is shown in Figure 2.28. This device uses an uncooled microbolometer, 17 micron pixel detector with spectral range between $7.5-\mu \mathrm{m}$ and $14-\mu \mathrm{m}$ and an image resolution of $640 \times 480$. The standard temperature range is $233.15-\mathrm{K}$ to $423.15-\mathrm{K}\left(-40-{ }^{\circ} \mathrm{C}\right.$ to $\left.150-^{\circ} \mathrm{C}\right)$ and the camera has a reported accuracy of $\pm 2-\mathrm{K}\left(2^{\circ} \mathrm{C}\right)$ or $\pm 2 \%$ of the reading, whichever is larger. 
The dimensions of the camera are $216 \times 73 \times 75-\mathrm{mm}$ and was used with a $45^{\circ}$ angle lens which is 30.76 $\mathrm{mm}$ in diameter. The outer diameter of the lens is $67.39-\mathrm{mm}$. The camera aperture is fixed at 13.1-mm and both automatic and manual focus features are available. Both USB and Gigabit Ethernet connections are available for interfacing with a computer and various software.

Image acquisition was

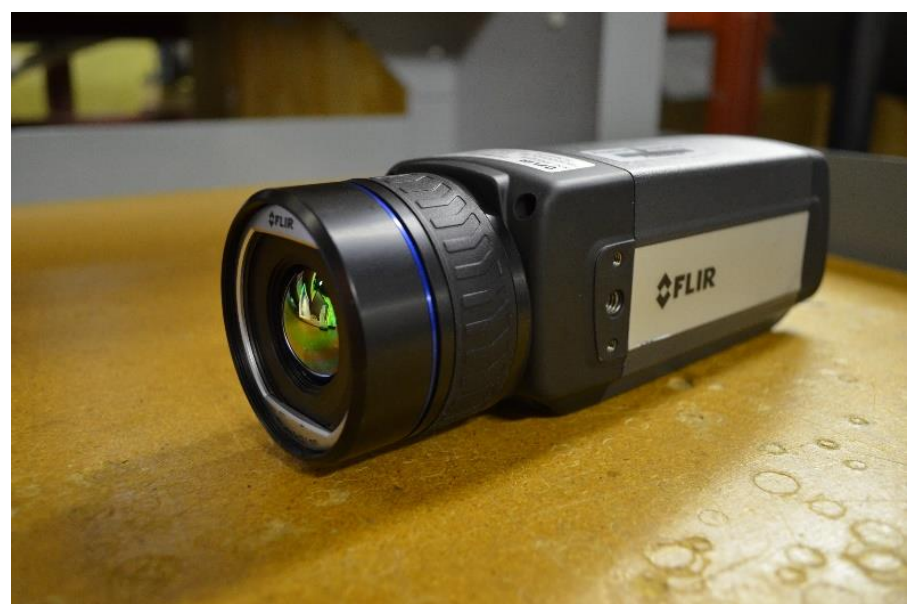

Figure 2.28 FLIR A655sc Infrared Camera used for Infrared Thermography in Transition Detection Study accomplished by use of the FLIR Tools software. This software allows users to stream live video via USB cable from A655sc infrared camera; import, search, filter, and view FLIR JPEG images from A655sc infrared camera; adjust parameters such as temperature range, emissivity, and reflective temperature; add measurement tools - spots, area boxes, circles, lines. Images can be taken in many colour schemes: artic, grey, iron, lava, rainbow, and rainbow HC. In this investigation the iron and grey colour pallets were most commonly used. 


\section{Chapter 3: TRANSITION DETECTION SYSTEM DEVELOPMENT}

\subsection{Infrared Thermography System Requirements}

In an effort to obtain clear, consistent, and accurate transition results for a range of models and conditions, it was necessary to develop a versatile and efficient infrared thermography system for implementation in the Virginia Tech Stability Wind Tunnel.

The main requirement of such a system is that it should be able to measure temperature differences as small as $0.1-\mathrm{K}$ on a surface. This would ensure that the changes in temperature occurring as a result of laminar to turbulent transition could be accurately measured.

Moreover, it is necessary that the system allow for the creation and maintenance of an adequate temperature difference between the model and the oncoming flow. This will ensure a versatile system by allowing the experimenter to choose a desired temperature difference at any point during the experiment.

Additionally, all system components should be quick and easy to implement without causing permanent alterations to the model or requiring extensive modifications to any other measurement system currently in use in the Stability Wind Tunnel.

Prior to testing in the Stability Wind Tunnel, several small scale tests were conducted in the Virginia Tech Open Jet Wind Tunnel. The overarching goal of these tests was to ascertain the best components which would create the ideal infrared thermography system that could be used efficiently in the Stability Wind Tunnel. One of the FLIR A655sc infrared cameras described Section 2.4 was used during these tests.

\subsection{Investigation into Infrared Reflections}

In the earliest stages of this transition detection work, the camera capabilities and the method of infrared thermography were explored. It was fundamental to understand the behaviour of infrared waves with materials in the lab and wind tunnel.

From the first use of the camera it was clear that neither Lexan, glass nor Plexiglass were transparent in the infrared range. These materials, which make up some of the major components of the Stability Wind Tunnel test section, appear completely opaque on the infrared camera. Therefore, the infrared camera cannot be mounted outside walls made of these materials. 
Based on previous research, environmental reflections were found to be a major issue in infrared thermography. To investigate this, a heated, triangular, aluminium block was set up next to a $0.3-\mathrm{m}$ tear-drop aluminium model. The resulting infrared image proved that a model made of aluminium, being very shiny and having a very low emissivity, was very reflective in the infrared region. This conclusion was made because there was a clear reflection of the heated block on the tear drop model image. Figure 3.1 demonstrates the test set up and the resulting infrared image with environmental reflections.
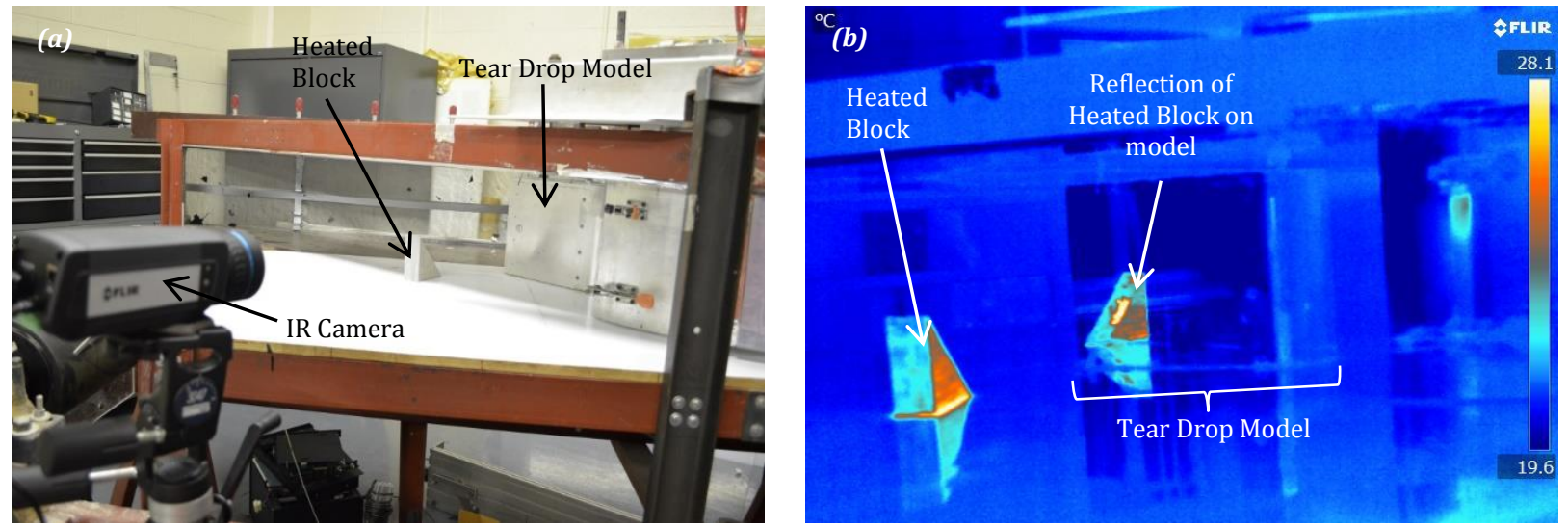

Figure 3.1 (a) Tear Drop Model at Room Temperature Set up Next to Heated Block (b) Resulting Coloured IR Image showing Clear Reflection of Heated Block on Tear Drop Model (Coldest Regions are Blue and Warmest Regions are White)

It is known that environmental reflections are significantly reduced as emissivity is increased. This can easily be done by creating a duller surface colour, ideally black. As a result, the tear drop model was covered in black ConTact ${ }^{\circledR}$ paper. The covered model was again set up next to a heated model and the infrared image examined for environmental reflections. As is seen in Figure 3.2, the addition of the ConTact ${ }^{\circledR}$ paper removed most of the environmental reflections - the heated block is not seen on the model surface.
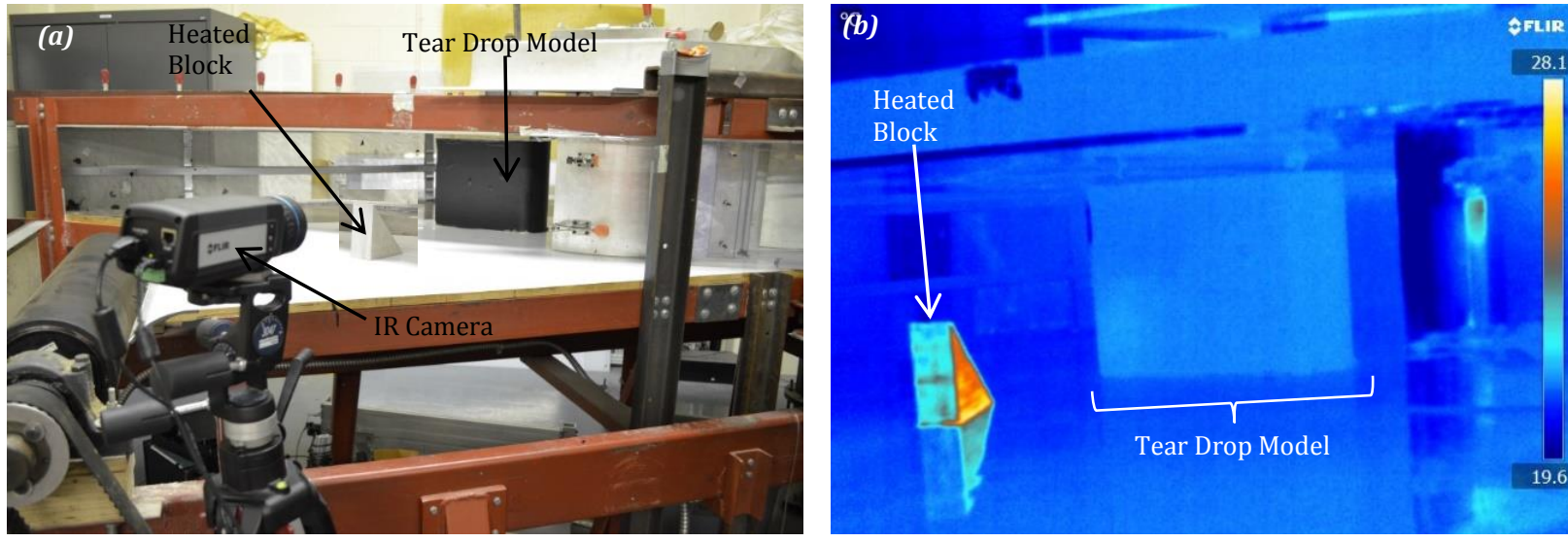

Figure 3.2 (a) Tear Drop Model Covered in ConTact ${ }^{\odot}$ Paper at Room Temperature Set up Next to Heated Block (b) Resulting Coloured IR Image showing No Environmental Reflections (Coldest Regions are Blue and Warmest Regions are White) 
These results prove that any model used in the infrared thermography transition detection system must be have a dull surface colour, ideally black, in order for the system to give accurate results. Otherwise, temperatures and gradients shown in the results may simply be as a result of environmental reflections.

\subsection{Development of Temperature Control System}

In order to detect transition using infrared thermography, it is crucial that there exists a large temperature difference between the model and the oncoming flow. Such a temperature difference can be created by either increasing or decreasing the temperature of the model in comparison to the flow. However, the method used to change the temperature of the model needs to be non-invasive such that it does not have any effect on the flow. Furthermore, it should be easy to implement and provide a means to sustain the temperature difference for at least 30 minutes in flow.

The first method investigated involved the use of dry ice to wrap the model before testing. This resembled the pre-treating methods used by Zuccher (2008) and Yokokawa (2005). This was done using a 1.2$\mathrm{m} \times 0.56-\mathrm{m} \times 12.7-\mathrm{mm}$ Lexan flat plate with rounded leading edge, mounted vertically in the Open Jet Wind Tunnel. The plate was covered in black ConTact ${ }^{\circledR}$ paper to increase the emissivity which in turn reduces the infrared reflections on the plate from the surrounding. Figure 3.3 shows the setup of the plate in the Open Jet Wind Tunnel.

Dry ice was packed in cloth which was then laid on the model

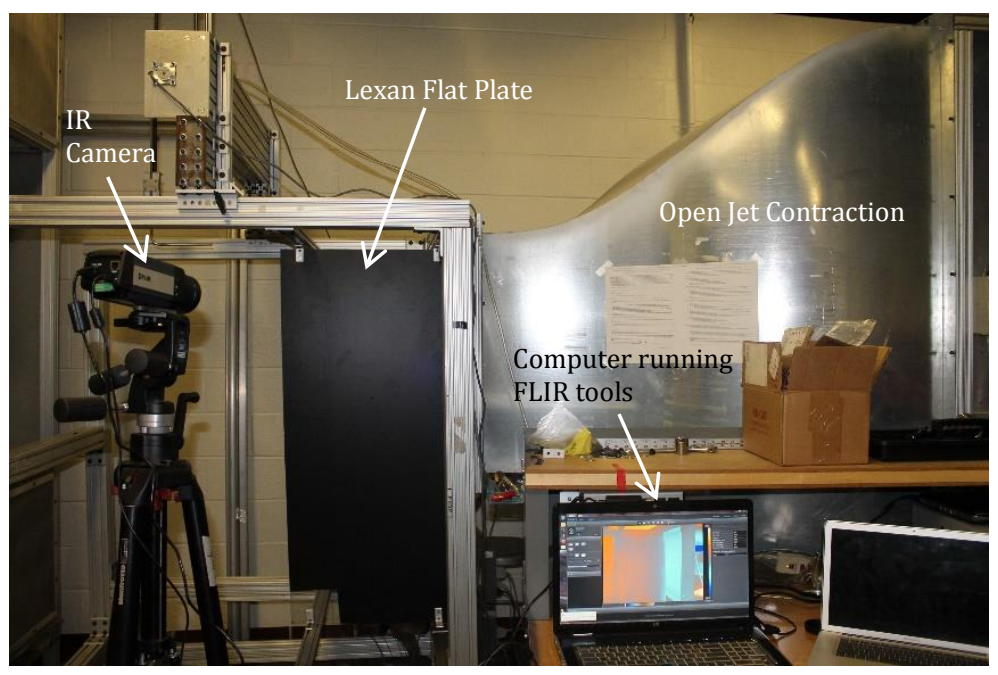

Figure 3.3 Lexan Flat Plate Mounted Vertically in Open Jet Wind Tunnel so that heat transfer could take place via conduction. This method proved to be very unreliable and tedious as the temperature could not be accurately controlled nor was it possible to cool the model evenly while it was mounted in the test section. This unevenness is shown in Figure 3.4. Most importantly, it was found that the model temperature increased back to that of the flow fairly quickly after flow was turned on - it took approximately five minutes for the plate to rise from $253.15-\mathrm{K}\left(-20^{\circ} \mathrm{C}\right)$ to $295.15-\mathrm{K}\left(22^{\circ} \mathrm{C}\right)$. While the thickness of the flat plate played a part in this rapid heat transfer, the result clearly indicated the need to actively control the temperature system during the test or risk having the pause the test 
to re-introduce the initial temperature difference. As a result, this method was reserved as an option for testing small models at few angles of attack. It would not be implemented in the Stability Wind Tunnel.

Again using the Lexan flat plate mounted vertically in the Open Jet test section, a heating blanket was used to wrap the model in order to increase the temperature to above that of the flow. While this method was relatively easy to implement, it required several hours to increase the model temperature. Furthermore, as was the case with the dry ice, once the flow was turned on the model was cooled to the temperature of the flow within ten minutes. Thus, this method was rejected.

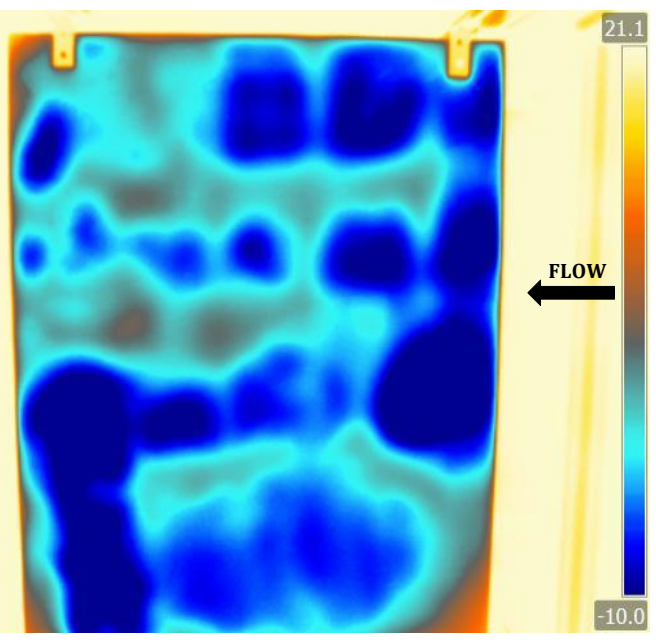

Figure 3.4 Coloured Infrared Image showing the Uneven Cooling Pattern Associated with the Dry Ice Cooling Method (Coldest Regions are Blue and Warmest Regions are White)

With the failure of simpler methods, effort was placed on developing a system that could provide active heating or cooling during the test, without affecting the flow. The first active system conceived involved using a MovinAir Office Pro 12, 13,500-BTU, 115-V airconditioning system to recirculate air through a 1.82-m DU00-W-212, with 0.61-m chord (similar to the work of Freels, 2012). The air was directed from the AC unit through short uninsulated plastic tubes into the upper end of the airfoil. The cold air then enters the inside of the model cooling it as it moves from the top to the bottom. The air discharged from the lower end of the model is then guided back into the AC system through short uninsulated plastic tubes. Figure 3.5 shows the general design for the AC cooling system.

From this test, it was found that the present AC system was not powerful enough to provide a sufficient temperature difference between the flow and a model of this size. Within fifty minutes of turning the AC system on, the model was only cooled by $5-\mathrm{K}\left(5^{\circ} \mathrm{C}\right)$. Additionally, the top $50 \%$ of the airfoil span was cooled more than the lower $50 \%$ because the flow through the model was heated along the flow path. These results suggested that the system would most likely be effective only if a more powerful cooling unit and insulated tubing were used.

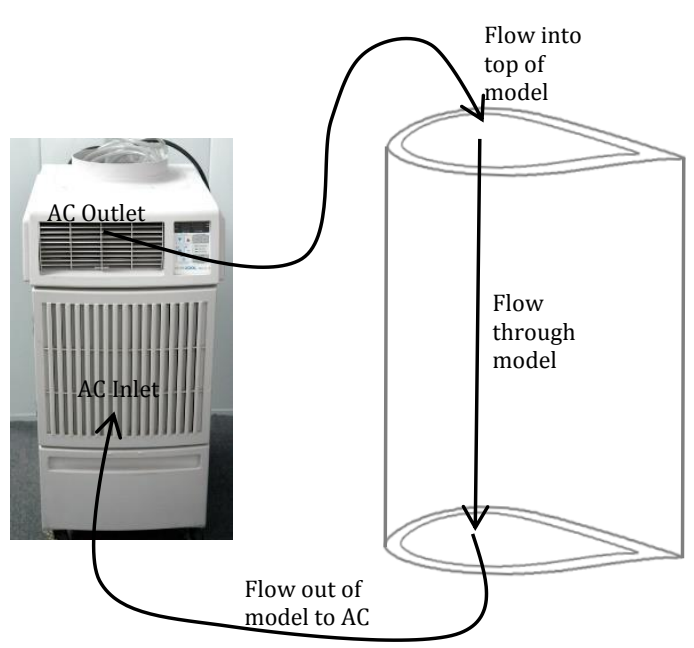

Figure 3.5 AC Cooling System Design 
The final method investigated was the use of flexible heaters adhered to the inner surface of models. This arrangement ensured that the system did not affect the flow or airfoil performance while still enabling the user to control the heaters and thus the temperature difference between the model and the flow. The heaters used were Kapton ${ }^{\circledR}$, polyimide film, flexible, insulated heaters with etched foil design from Omega. The selected model, KH-110/10-P, were $25-\mathrm{mm}$ wide, $152.4-\mathrm{mm}$ long and $0.25-\mathrm{mm}$ thick with pressure sensitive adhesive for easy adhesion to the airfoil. These heaters were rated at 10 -Win ${ }^{-2}$. Figure 3.6 shows one of these heaters

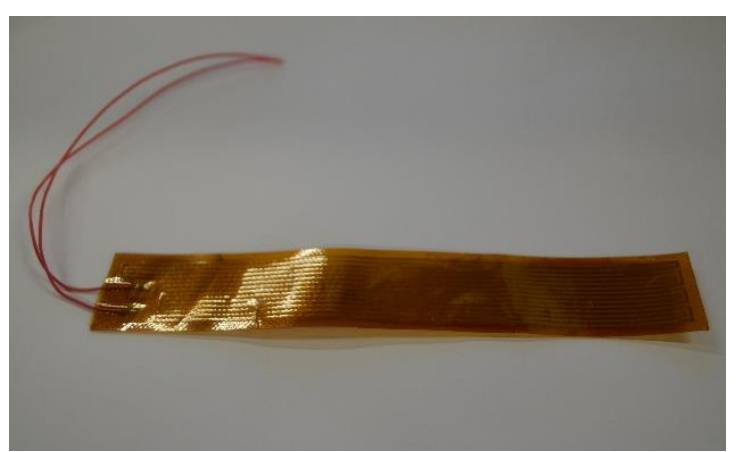

Figure 3.6 Kapton ${ }^{\circledR}$, Polyimide Film, Flexible, Insulated Heaters with Etched Foil Design while the arrangement of heaters inside the DU00-W-212 (1.82-m span, 0.61-m chord) model is depicted by Figure 3.7.

The Kapton etched foil heaters were fairly easy to apply though there were issues with flexibility which led to permanent distortion. The distortion caused to issues in applying the heaters smoothly to the surface which in turn led to burning up of heaters. Moreover, the attachment of wires to the heater was not very sturdy and wires were easily detached rendering the heater useless.

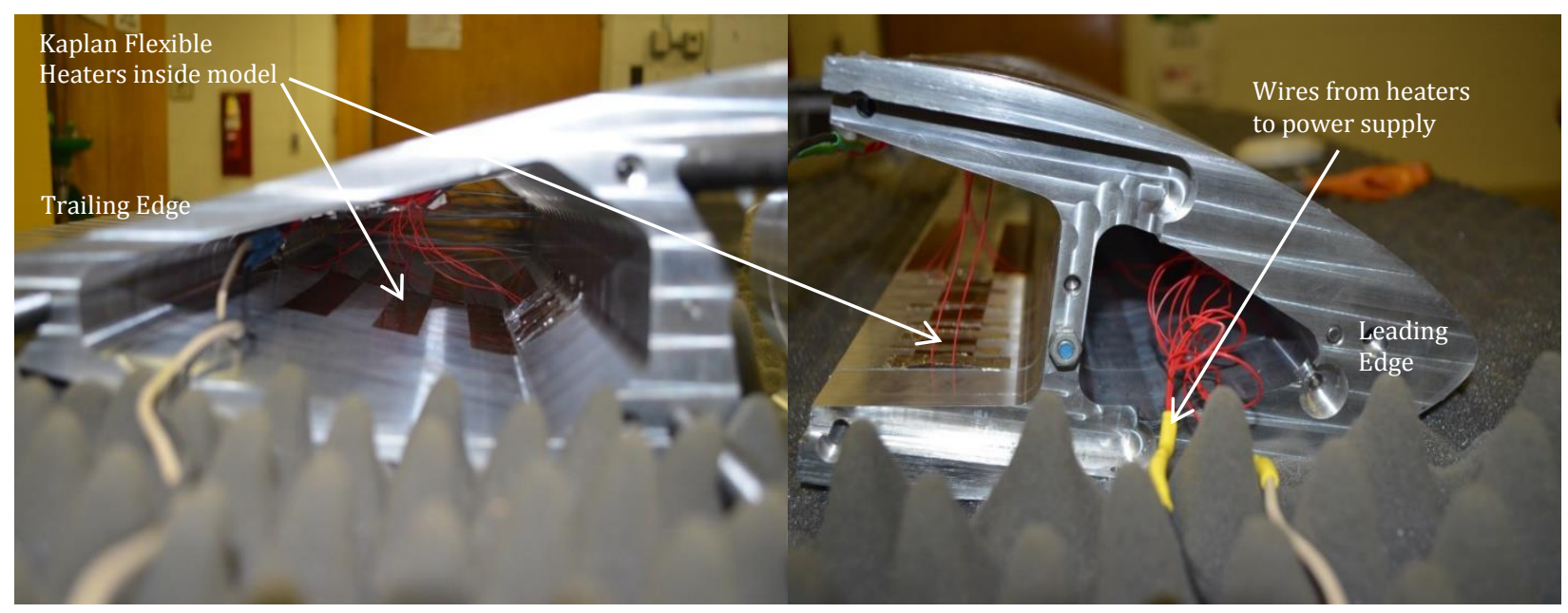

Figure 3.7 Composite of Cross section through DU00-W-212 showing Internal Heater Arrangement

Tests done in the Open Jet Wind Tunnel using these heaters to create a temperature a difference between the flow and the model showed that this method can provide a large temperature difference in a short period - a difference of $10-\mathrm{K}\left(10^{\circ} \mathrm{C}\right)$ could be created within 30-s. Furthermore, several angles could be tested as the heaters could be turned on and off as necessary to maintain the desired temperature difference. 
Due to the positive results of the flexible heater method, finding heaters which are easier to apply would increase the efficiency. The Kapton etched foil design heaters were replaced with lightweight, flexible, silicone rubber fiberglass insulated heaters. The selected model, SRFG-110/10-P, was 25$\mathrm{mm}$ wide and 152.4-mm long with pressure sensitive adhesive, rated at $10-\mathrm{Win}^{-2}$. These Omega heaters offered better attachment to the surface and were much more flexible than the previous heaters. In addition the wires were much more secure than in the

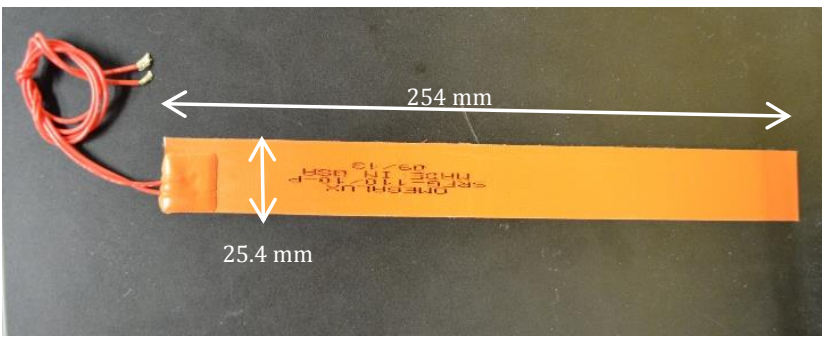

Figure 3.8 Flexible Silicone Rubber Fiberglass Insulated Heaters etched foil design. Figure 3.8 shows the silicone rubber heater.

\subsection{Investigation into Model Materials}

The $18 \%$ proprietary model of 0.8 -m chord, discussed in Section 2.3.1, was covered in black ConTact ${ }^{\odot}$ paper, cooled using the dry ice system, and mounted in the Open Jet Wind Tunnel in an effort to see transition. The resulting infrared images did not show any transition patterns. Figure 3.9 shows two of the infrared images for this model at $0^{\circ}$ angle of attack, one after one minute and another after 20 minutes.
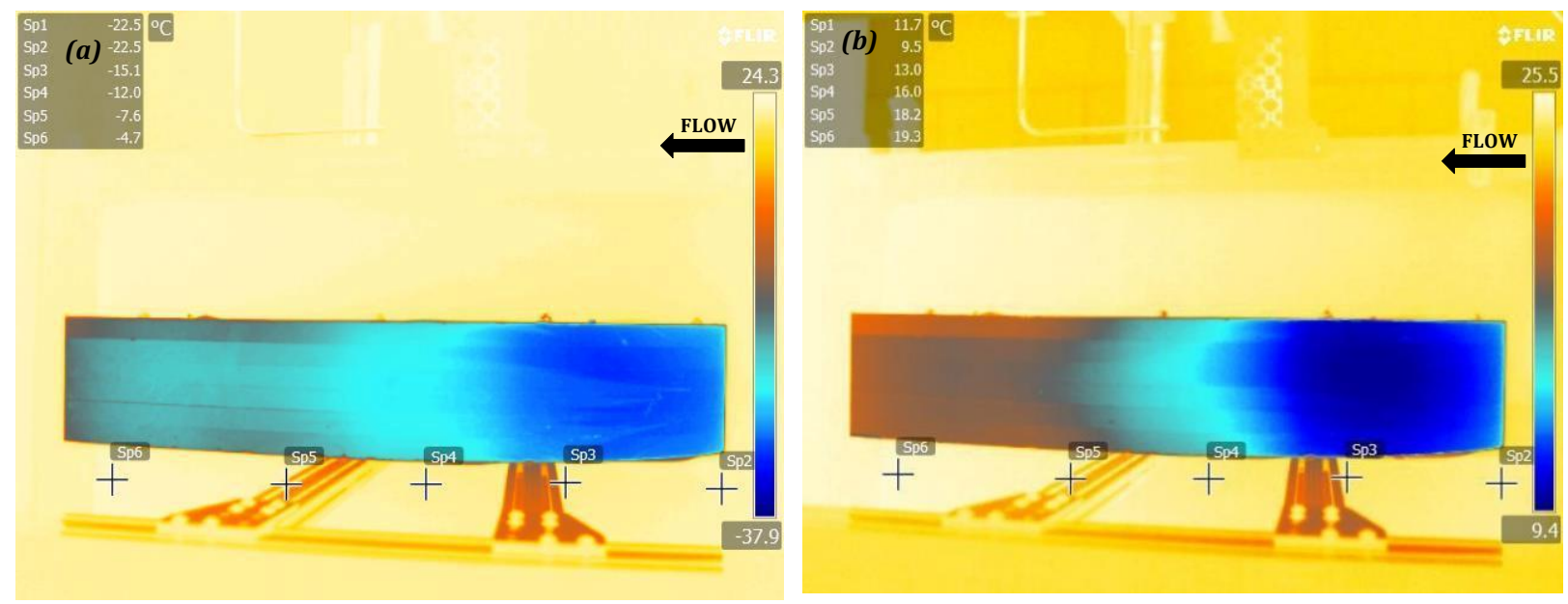

Figure 3.9 Coloured Infrared Image of Suction Side of $0.8 \mathrm{~m} 18 \%$ Propriety Model Section at 30-ms ${ }^{-1}$, $0^{\circ}$ Angle of Attack after (a) 1 minute and (b) 20 minutes (Coldest Regions are Blue and Warmest Regions are White)

These results show a temperature gradient which is most likely a result of material cooling along the chord of the model because the coolest regions coincide with areas of greatest material thickness - the leading edge. These results also suggest that for an aluminium model with large material thickness, transition cannot be detected by simply 
covering the model with ConTact $^{\complement}$ paper - an insulator is needed, as was suggested by Zuccher (2008) and Freels (2012).

The second model used in preliminary tests was the close replica of a Clark-Y airfoil, discussed in Section 2.3.2. The scratches on surface of the model which were sanded or covered in ConTact ${ }^{\odot}$ paper to reduce the effect on the flow. However, because the model itself was already painted black the entire model was not covered in ConTact ${ }^{\circledR}$ paper. Figure 3.10 illustrates the Clark-Y airfoil set up in the Open Jet Wind Tunnel.

The infrared results obtained from the Clark-Y model test showed that transition-like patterns (sharp gradient in temperature distribution) were detected at several angles of attack despite the metal inlays, rough edges and surface scratches. The location of this transition-like pattern changed with angle of attack further suggesting that the results show the natural transition for this model. Figure 3.11 shows the results for the Clark-Y model at two angles of attack.

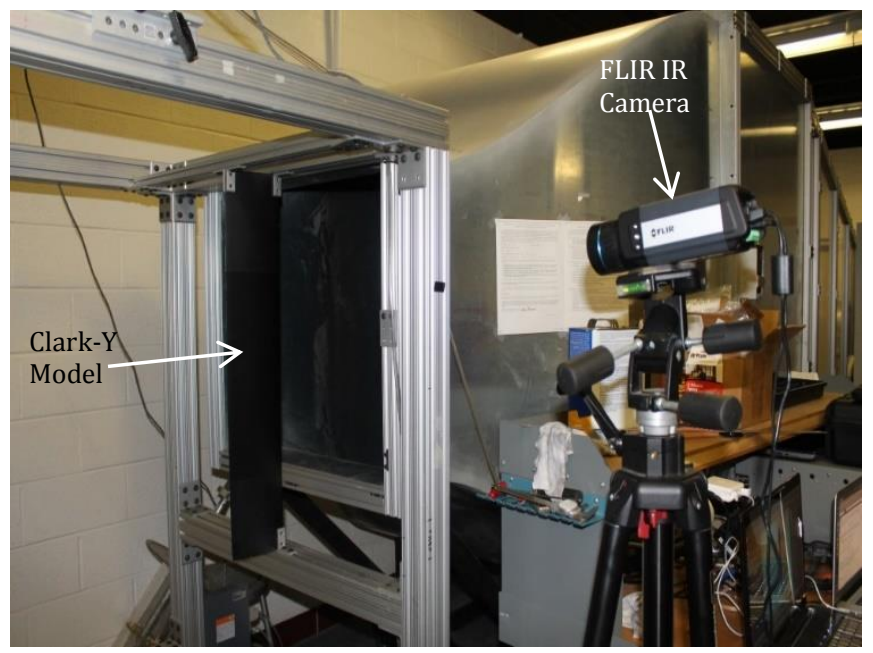

Figure 3.10 Clark-Y Model Mounted in Open Jet Wind Tunnel, Suction Side Facing Camera
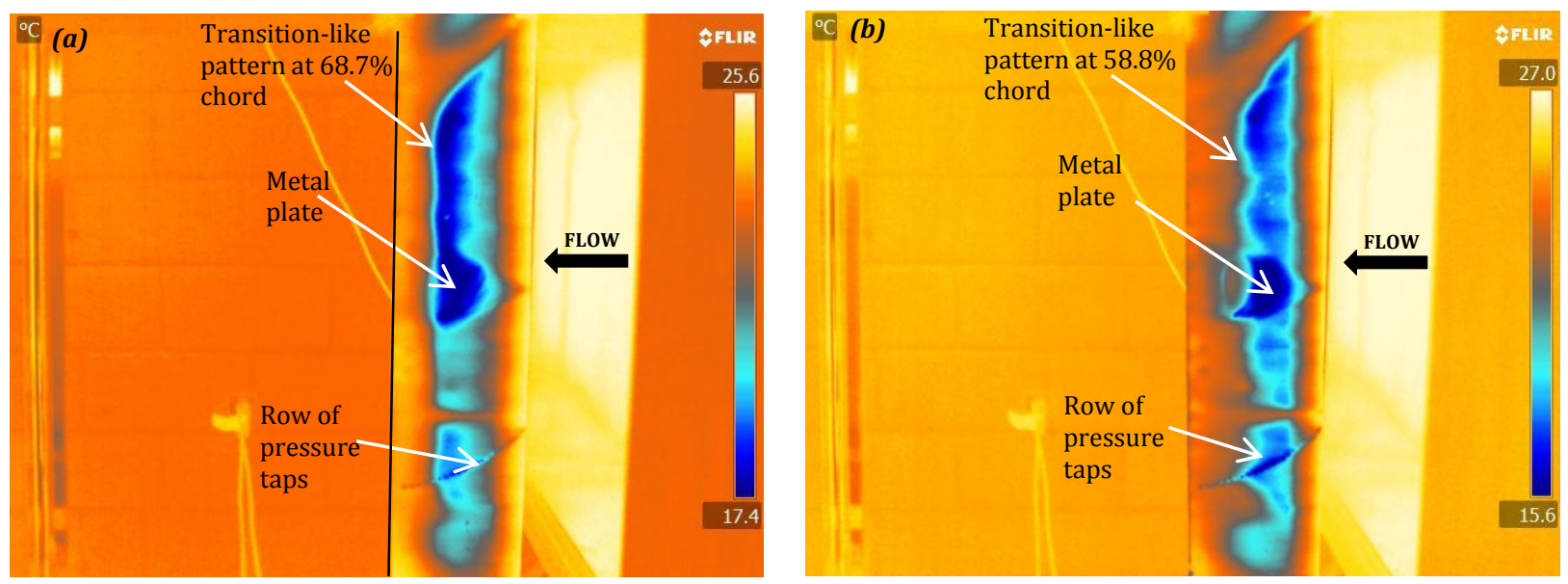

Figure 3.11 Coloured Infrared Image of Suction Side of Wooden Clark-Y Model at 30-ms $\mathrm{s}^{-1}$, and (a) $0^{\circ}$ (b) $4^{\circ}$ Angle of Attack (Coldest Regions are Blue and Warmest Regions are White)

These infrared patterns show that the leading edge heats up faster than other regions of the model. The most probably reason for this is that there is less material at this location and therefore the temperature gradient here is not characteristic of flow features. 
In order to definitively ascertain whether this model in the current set up showed transition, a trip strip was attached to the model with the premise that if the highest temperature gradient coincides with the chord-wise location of the trip strip, then the current set up is valid. The trip strip, comprising of a strip of $0.8-\mathrm{mm}$ dimples, was attached to the model as is shown in Figure 3.12.

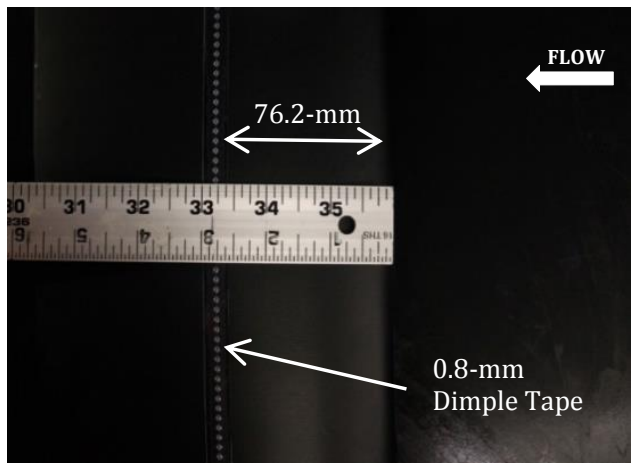

Figure 3.12 Trip Strip Made of 0.8- $\mathrm{mm}$ Dimple Tape Attached to Clark-Y Model the flow.
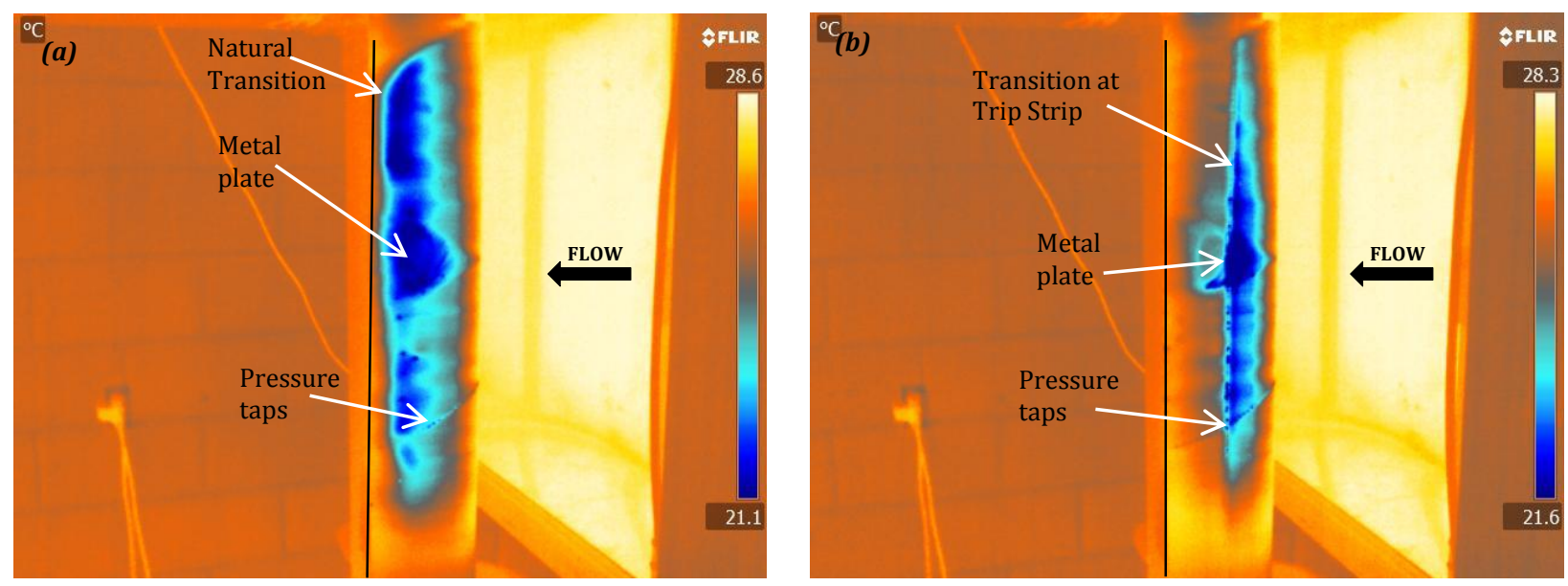

Figure 3.13 Coloured Infrared Image of Suction Side of Clark-Y Model at 30-ms ${ }^{-1} 4^{\circ}$ Angle of Attack (a) without Trip (b) with Trip (Coldest Regions are Blue and Warmest Regions are White)

From these results, it is clear that the trip strip initiated transition at this angle of attack because the highest temperature gradient moved from downstream to the exact location of the trip strip. This strongly suggests that the highest temperature gradient observed in the infrared images does in fact coincide with transition.

Conclusively, it can be said that the investigation into the Clark-Y model showed that wood is a good material candidate for transition detection and that even very small temperature differences, as small as $5-\mathrm{K}$, are sufficient to show transition. 
The aluminium, NACA-0012 model described in Section 2.3.3 was mounted in the Open Jet Wind Tunnel for testing. This model was unlike the $18 \%$ proprietary aluminium model in that the chord and the wall thicknesses were significantly smaller. The model was completely covered in ConTact ${ }^{\circledR}$ paper and cooled using the dry ice method. Figure 2.20 shows the cross section of the NACA-0012 model used. The setup of the NACA-0012 in the Open Jet Wind Tunnel is identical to that of the Clark-Y model shown Figure 3.10. The infrared results for the NACA-0012 are presented in Figure 3.14. These images show that as angle of attack increases, the location of highest temperature gradient moves further upstream - closer to the leading edge. This trend suggests that this location is indicative of the natural transition for the NACA-0012.
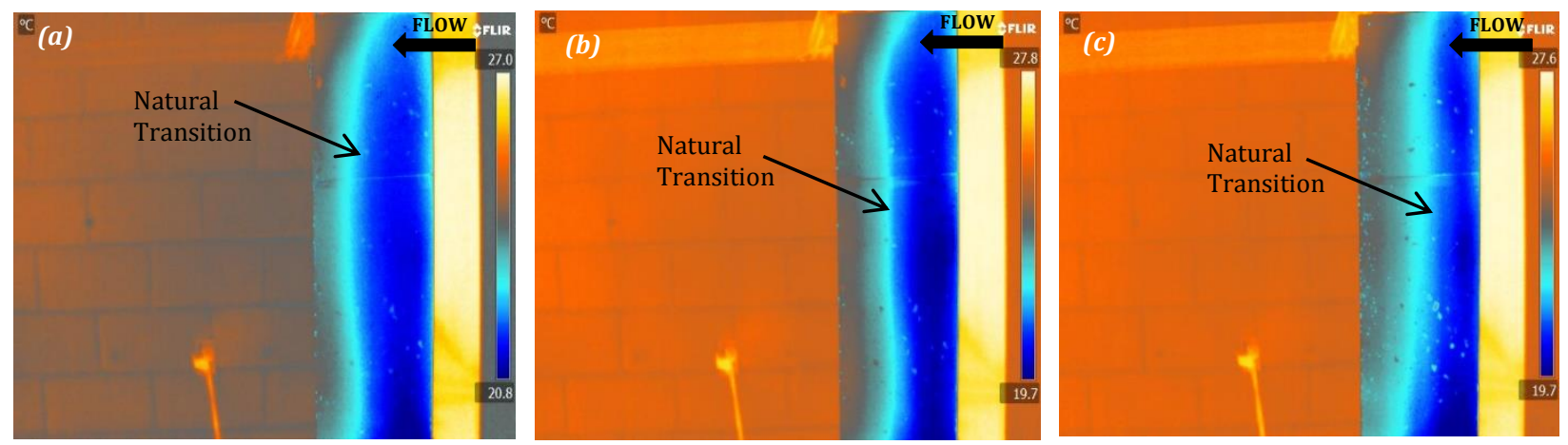

Figure 3.14 Coloured Infrared Image of Suction Side of NACA-0012 Model at 30-ms-1 and Angle of Attacks of (a) $0^{\circ}$ (b) $4^{\circ}$ (c) $8^{\circ}$ (Coldest Regions are Blue and Warmest Regions are White)

Considering the fact that the section of the $0.8-\mathrm{m}$ chord, $18 \%$ proprietary aluminium model did not produce any useful transition results but the aluminium NACA-0012 shows clear transition, it can be conceived that the differences in material thickness play a significant role in heat transfer between the flow and the model. Since the walls of the 18\% proprietary model are very much thicker than that of the NACA-0012 (approximately $5 \times$ thicker) and the proprietary model has regions of very large material thickness at the trailing edge and around quarter chord, this model most likely generates considerably higher heat transfer between flow and cooled/heated model than does the NACA-0012. Therefore, it is possible that the high heat transfer rates from model to flow in the case of the $18 \%$ proprietary model, overshadows the heat transfer occurring from of transition. Essentially, any heat transfer and temperature gradients which are produces from the transition phenomenon is smeared by the high heat transfer rate from the thick aluminium regions to the flow. In an effort to prove this hypothesis it is necessary to regulate the heat transfer between the flow and a model with large material thickness thereby determining whether natural transition can be seen. 
The 1.82-m span, 0.61-m chord DU00-W212 was mounted in the Open Jet Wind Tunnel as shown in Figure 3.15. This full span model was used in order to test the hypothesis that large aluminium models with significant material thicknesses require special heat transfer regulation in order to show transition temperature effects. Only the upper half of the span of this model (approximately 0.6-m) was covered in ConTact $^{\odot}$ paper because only this region would be in the flow of the Open Jet Wind Tunnel. The model was heated using Kapton polyimide film flexible heaters mounted inside the models. This, and the model cross section showing wall thicknesses, can be seen in Figure 3.7.

Figure 3.16 shows the infrared image results of the DU00-W-212 covered in only ConTact ${ }^{\circledR}$ paper, in the Open Jet Wind Tunnel at $30-\mathrm{ms}^{-1}$ at three angles of attack. The heaters are arranged an inch apart in the open regions of the

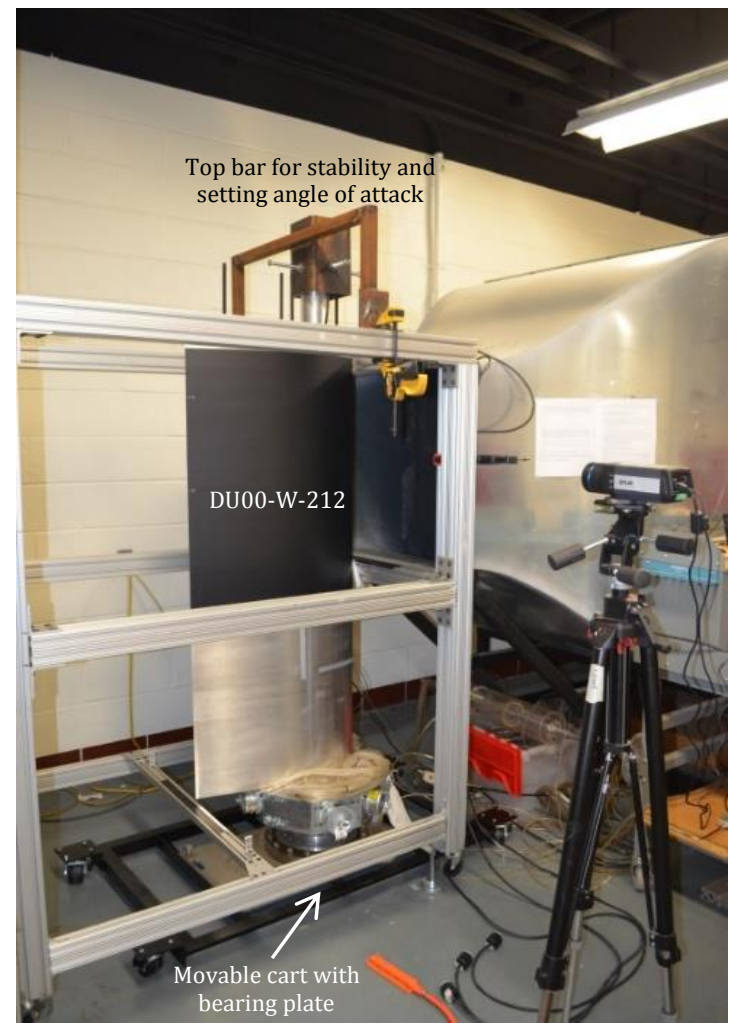

Figure 3.15 Setup of DU00-W-212 in Open Jet Wind Tunnel, Suction Side Facing Camera cross section as shown in Figure 3.7. Similar to the results of the 18\% proprietary model, the only temperature gradients observed were attributed to material heat transfer over the model. The temperature gradients show discontinuity at the leading edge insert as well as between laminates. However, there is no apparent temperature gradients due to flow phenomenon. Figure 3.16 further shows that forcing transition by attaching at a trip strip of 0.8-mm dimple tape failed to provide evidence of the temperature gradients which would be expected from transition. Finally, the power to the heaters was reduced by as much as $10 \%$ using a General Radio Type W5MT Variac (5-A maximum output) of its full capability but this technique still did not enable flow effects to be seen. 

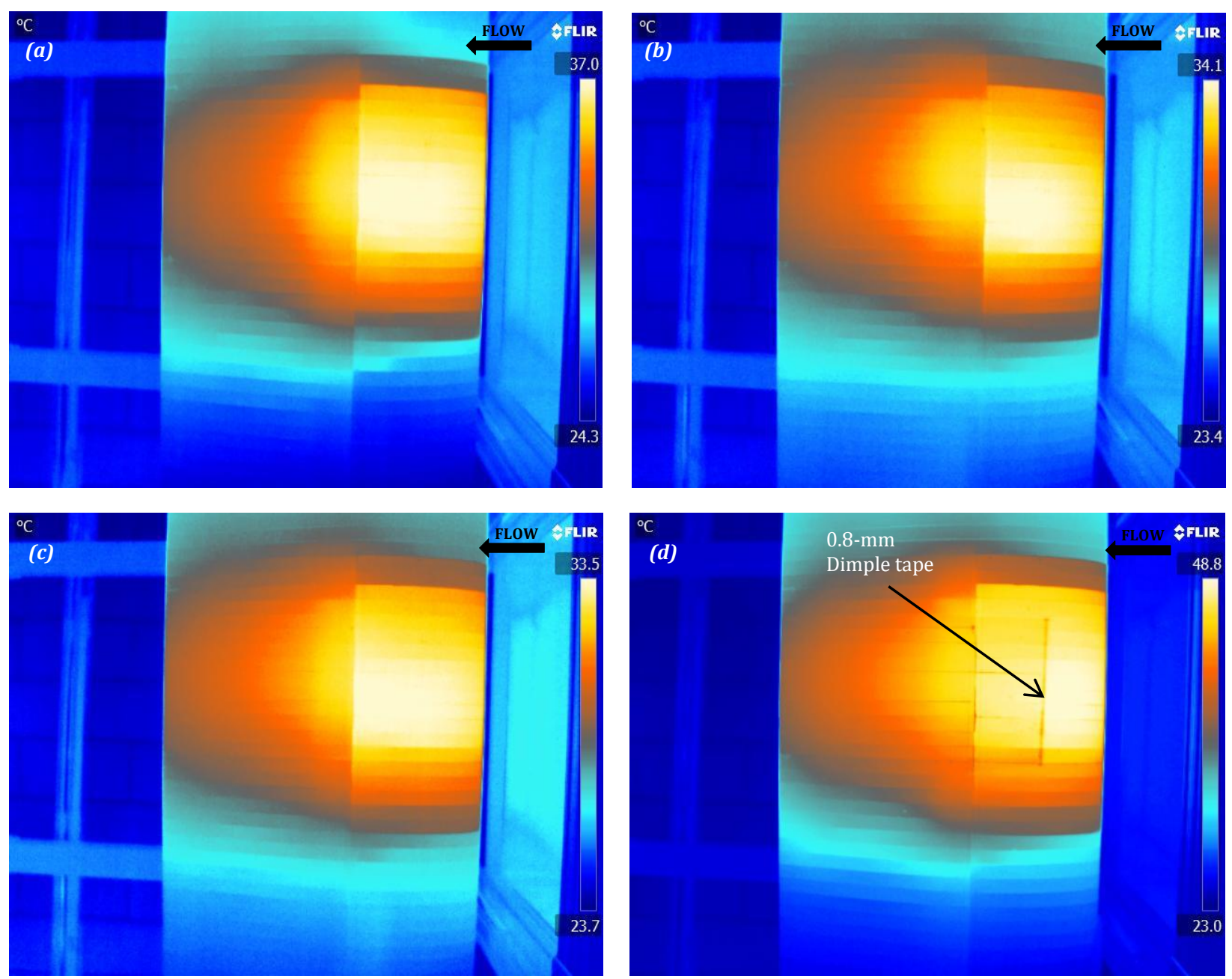

Figure 3.16 Coloured Infrared Image of Suction Side of DU00-W-212 Model at 30-ms ${ }^{-1}$ and Angle of Attacks of (a) $0^{\circ}\left(\right.$ b) $2^{\circ}$ (c) $4^{\circ}$ and (d) $0^{\circ}$ with a Trip Strip (Coldest Regions are Blue and Warmest Regions are White)

With ConTact ${ }^{\complement}$ paper still on, a 1-mm thick sheet of insulating foam was attached to the model. The insulating foam used is the same as that used as the inner lining in commonplace thermal bags. A final layer of ConTact ${ }^{\circledR}$ paper was glued to the foam to increase the surface emissivity and help smooth the surface. Again, this was only done on the portion of the model which was in the flow of the Open Jet Wind Tunnel. The foam layer and final surface condition of the insulated model is shown in Figure 3.17. As can be seen in this figure, the surface is not perfectly smooth. In fact there are several ridges in the foam which create ridges in the final model surface. However, since the goal of this investigation was to determine whether insulating the model would allow flow phenomenon occurring to appear more dominant, the surface condition was not of utmost importance. Lastly, short strips of foil tape were attached every inch along the upper edge of the DU00-W-212. These strips were very reflective in infrared allowing them to be seen on infrared images thereby producing a quick and easy estimate of the edge location of transition. 

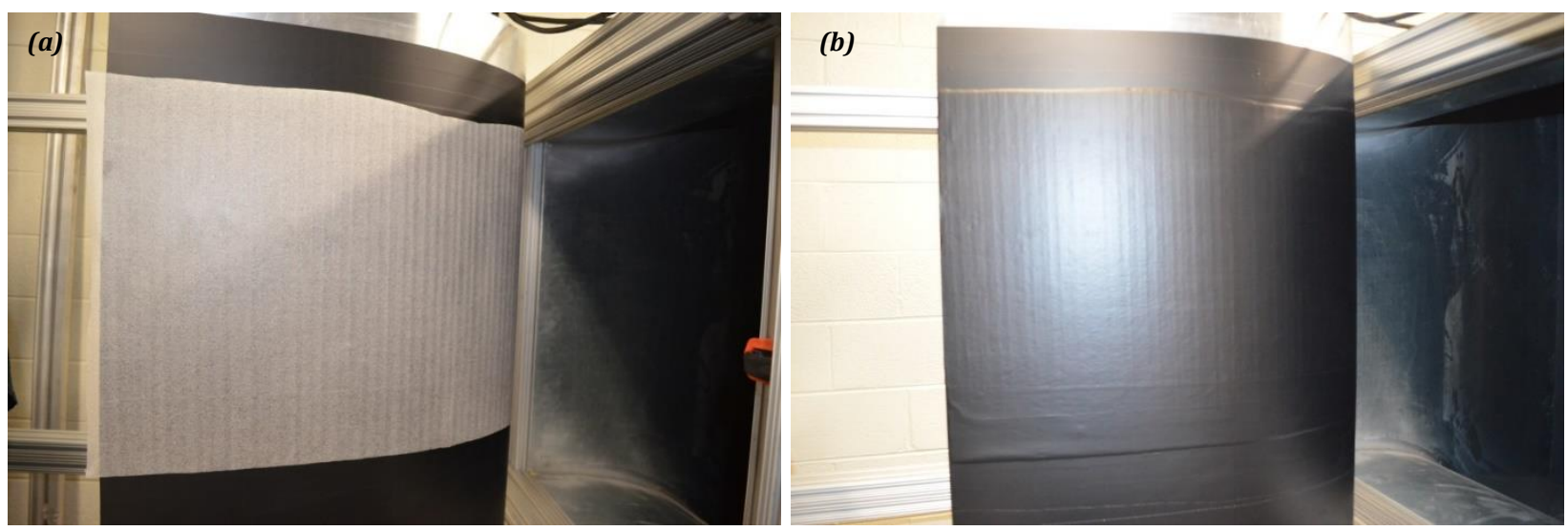

Figure 3.17 Suction Side of DU00-W-212 covered in (a) ConTact ${ }^{\odot}$ paper and Thin Layer of Foam (b) ConTact ${ }^{\odot}$ paper, Thin Layer of Foam and Final Layer of ConTact ${ }^{\circledR}$ Paper

The infrared images produced from this investigation are displayed in Figure 3.18. Looking at the first three images of this figure, it is clear that there is a distinct chord-wise temperature gradient whose location is affected by angle of attack. This would suggest that the temperature gradient is created by the flow over the model. Furthermore, the fact that the location moves toward the leading edge as the angle of attack is increased and the gradient depicts a sudden cooling of the flow over the model which correlates to a distinct rise in the rate of heat transfer, it can be interpreted that this occurrence is due to transition. Another important observation is that outside the insulated region of the model, the surface temperature does not show the distinct gradient observed over the insulated region. This gives a clear picture of the effect the insulator has on regulating the temperature and enabling the heat transfer due to flow effects to dominate.

Figure 3.18d shows the effect of adding a trip strip (0.8-mm dimple tape) to the insulated region to force transition. This was done in order to confirm that the large temperature gradient observed is in fact a consequence of transition and not a side effect of the current set up. The results show that there is a clear drop in the surface temperature at the location of the trip strip, as is expected at transition. Therefore, the gradient is most likely as a result of transition.

From this investigation, it can therefore be concluded that large aluminium models with large material thicknesses need an insulator to regulate the heat transfer between the model and the flow. If this requirement can be met while maintaining a smooth surface finish and leaving the profile of the model unchanged, aluminium would be a viable material candidate for transition detection experiments. 

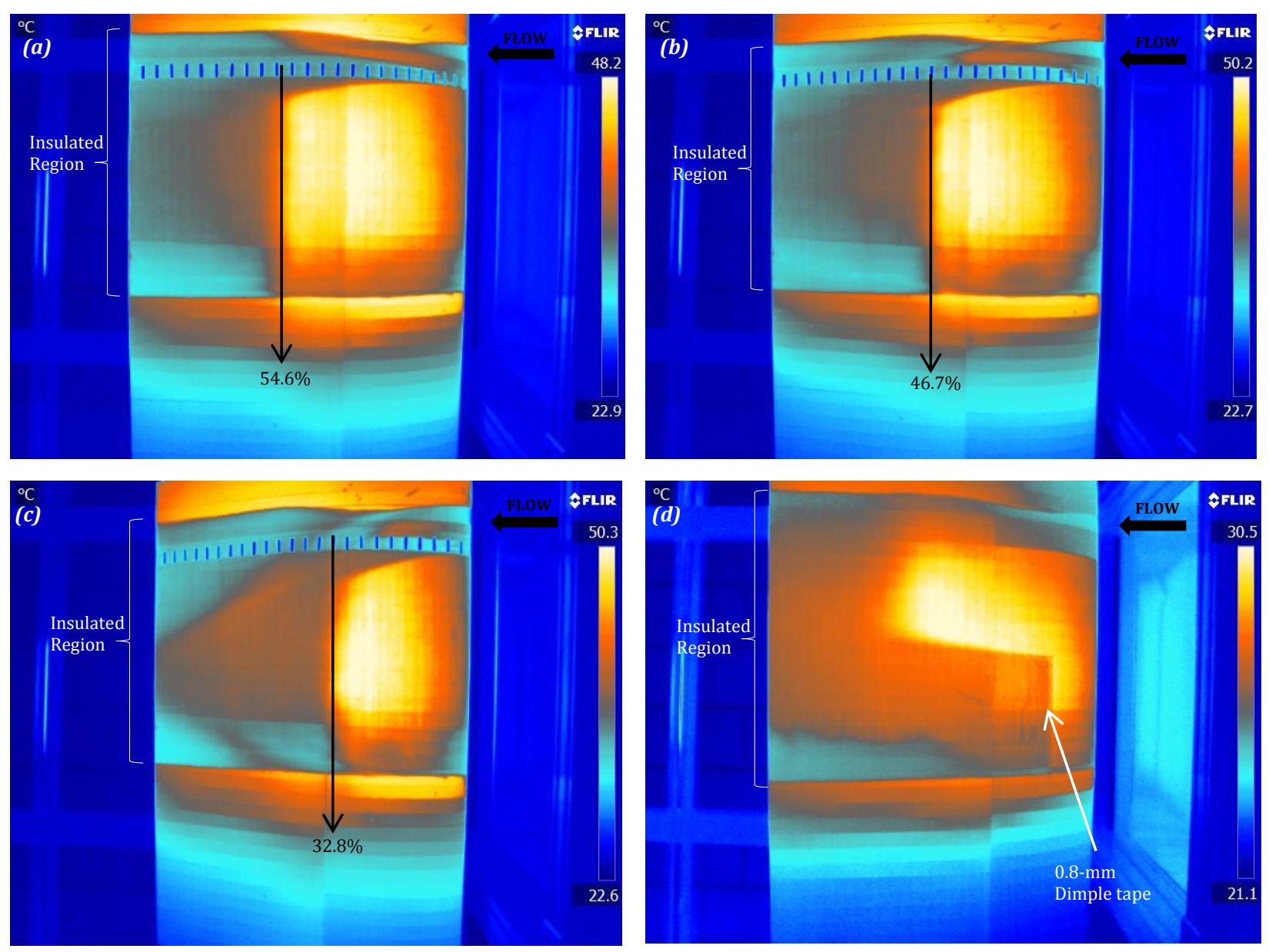

Figure 3.18 Coloured Infrared Image of Suction Side of DU00-W-212 Model Insulated with Foam at 30-ms ${ }^{-1}$ and Angle of Attacks of (a) $-4^{\circ}$ (b) $0^{\circ}$ (c) $4^{\circ}$ and (d) $-6^{\circ}$ with a Trip Strip (Coldest Regions are Blue and Warmest Regions are White)

\subsection{Aluminium Model Insulation}

As the majority of the models currently in use in the Virginia Tech Stability Wind Tunnel are constructed from aluminium and have fairly large material thicknesses, it was important to determine the best insulating materials which can be used to regulate heat transfer on the model. Good candidates must be highly insulating materials with very smooth surface finishes and thicknesses of less than $1.5-\mathrm{mm}$. The insulator must also be very easy to apply and remove without permanently damaging or altering the model.

The following is summary of the findings for each of the insulators studied. 


\section{1-mm thick, insulating foam}

As described in Section 3.4, a 1-mm thick sheet of insulating foam (from commonplace thermal grocery bags) was attached to the ConTact ${ }^{\odot}$ paper on the DU00-W-212 model followed by another layer of ConTact ${ }^{\odot}$ paper. The foam layer and final surface condition of the insulated model is shown in Figure 3.17. Despite the surface being fairly rough, natural transition-like patterns were detected, as presented in Figure 3.18.

\section{0.25-mm Mylar}

$A$ sheet of Mylar, 0.25 -mm thick, was attached to the DU00-W-212 model and covered in ConTact ${ }^{\odot}$ paper. The test was run at $30-\mathrm{ms}^{-1}$ and a few angles of attack. A sample result, at $0^{\circ}$ angle of attack, is shown in Figure 3.19. As seen in this image, there is a transition-like temperature gradient observed downstream of the leading edge module. However, due to the adhesive nature of Mylar, pockets of hot air accumulated beneath the Mylar layer. Furthermore, the adhesive beneath the Mylar could be seen on the infrared image. These occurrences can also be seen in Figure 3.19 where there is clearly a heated region downstream of the transition region. This was the case at all other angles of attack and made the transition hard to detect at some angles.

\section{Stacked Sheets of ConTact ${ }^{\circledR}$ Paper}

The model was covered in three sheets of ConTact ${ }^{\circ}$ paper to ascertain whether the insulating properties of this paper would be sufficient to regulate the heat transfer between the flow and the model. A sample result of this test is shown in Figure 3.20. In this infrared image, there is no clear transition behaviour detected.

\section{Cotton Sheets}

A 3-mm sheet of regular cotton, shown in Figure 3.21a, was glued to the DU00-W-212 model and covered in ConTact ${ }^{\odot}$ paper. The surface was not very smooth as there were issues in applying the cotton to the surface. The surface was also very compressible because of the

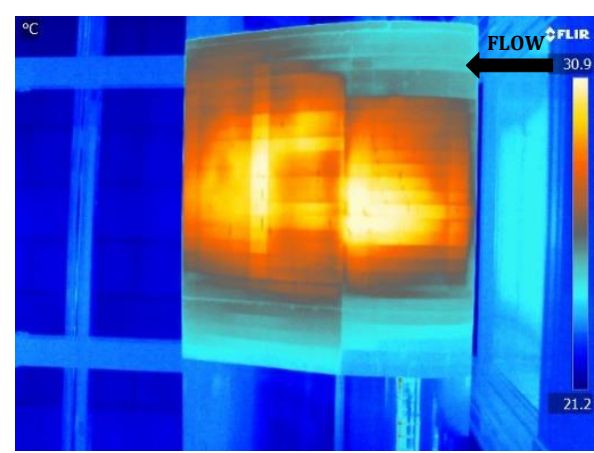

Figure 3.20 Coloured Infrared Image of Suction Side of DU00-W-212 Model, Insulted with Three Sheets of ConTact ${ }^{\odot}$ Paper, at 30-ms - $^{-1}$ and Angle of Attack of $4^{\circ}$ (Coldest Regions are Blue and Warmest Regions are White) softness of the cotton layer. The surface quality is shown in Figure 3.21b. 

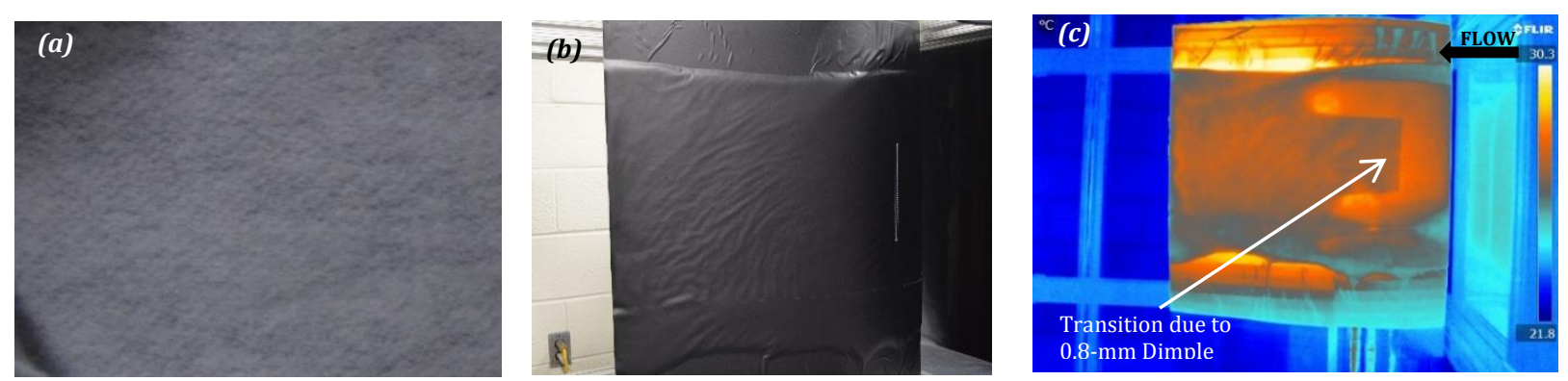

Figure 3.21 (a) Sample of 3-mm Cotton Sheet (b) Surface Quality of Suction Side of DU00-W-212 Model After Attaching Cotton Sheet and ConTact ${ }^{\circledR}$ Paper (c) Coloured Infrared Image of Suction Side of DU00-W-212 Model, Insulted with Cotton, at 30- $\mathrm{ms}^{-1}$ and Angle of Attack of $0^{\circ}$ (Coldest Regions are Blue and Warmest Regions are White)

Due to the poor surface quality and the fact that accurate transition locations were not the goal of this test, trips were added to the surface to force transition. From Figure 3.21c, which depicts one image result of this investigation, it is clear that transition forced by the trip is visible. Therefore, cotton would be a good material to use to insulate the aluminium models if a thinner, smoother, more user-friendly cotton material could be found.

\section{FDA-Compliant Silicone Rubber}

A red-orange silicone rubber sheet, $0.6-\mathrm{m} \times 0.6-\mathrm{m}$ and $0.8-\mathrm{mm}$ thick, was applied to the model. This material was obtained from McMaster-Carr, with model number 86045K21, and is made of FDA compliant materials. It also has an acrylic adhesive backing which offers easy adhesion to any surface. The material was medium soft with a durometer reading of 40A. The 0.46-m DU96-W-180 model was covered in ConTact ${ }^{\odot}$ paper and then with this insulator. This was to prevent the non-removable adhesive backing on the silicone rubber sheets from permanently bonding to the model surface. Because the rubber silicone was shiny redorange in colour, it was then covered with another sheet of ConTact $^{\complement}$ paper to increase emissivity. Strips of foil tape were placed every inch along the upper edge of the model to be used as markers. The model was heated using internally mounted flexible Kaplan heaters. Figure 3.22 shows the model covered in the red-orange silicone rubber insulator and the final surface condition after covering with a top layer of ConTact $^{\circledR}$ paper.

Figure 3.23 displays the infrared results for the DU96-W-180, insulated with rubber silicone, at $30 \mathrm{~ms}^{-1}$ and at four angles of attack. These images show a distinct chord-wise gradient in the surface temperature for each angle of attack. Moreover, as the angle is increased from negative to positive, this temperature gradient also shifts toward the leading edge. This trend is consistent with the behaviour of transition for the DU96-W-180. Therefore, the silicone rubber sheet works well for heat transfer regulation, and consequently transition detection, on large aluminium models. 

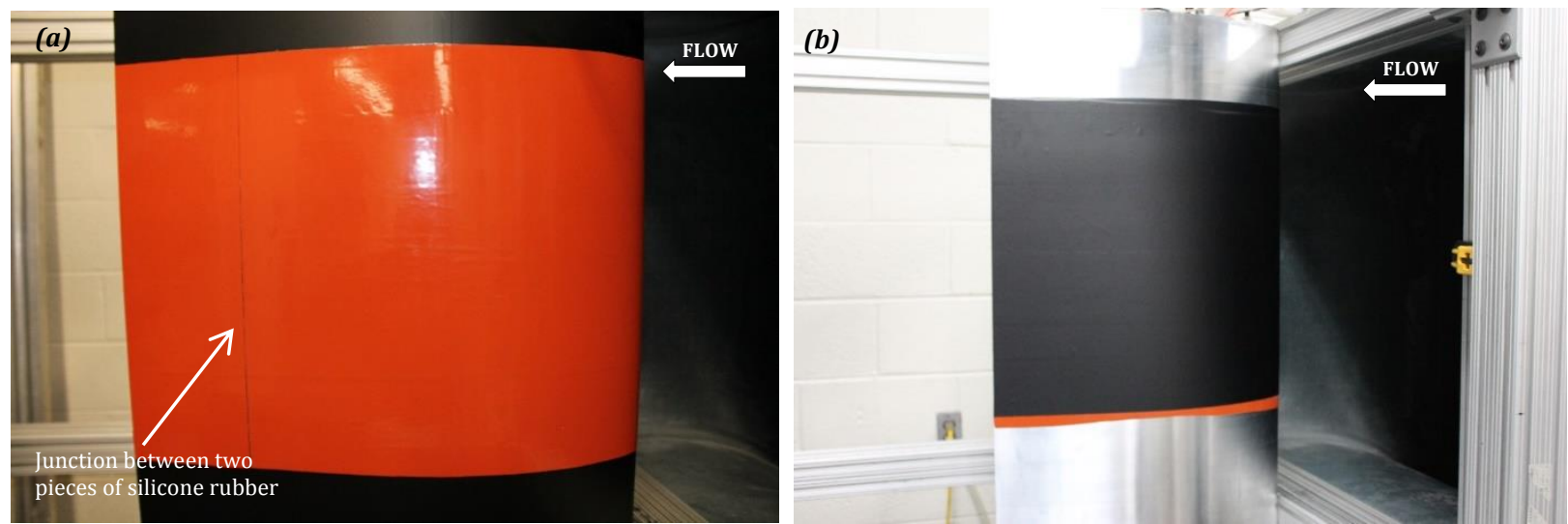

Figure 3.22 0.46-m DU96-W-180 covered in (a) FDA Compliant Silicone Rubber Sheet (b) Final layer of ConTact ${ }^{\odot}$ Paper over Silicone Rubber Insulator
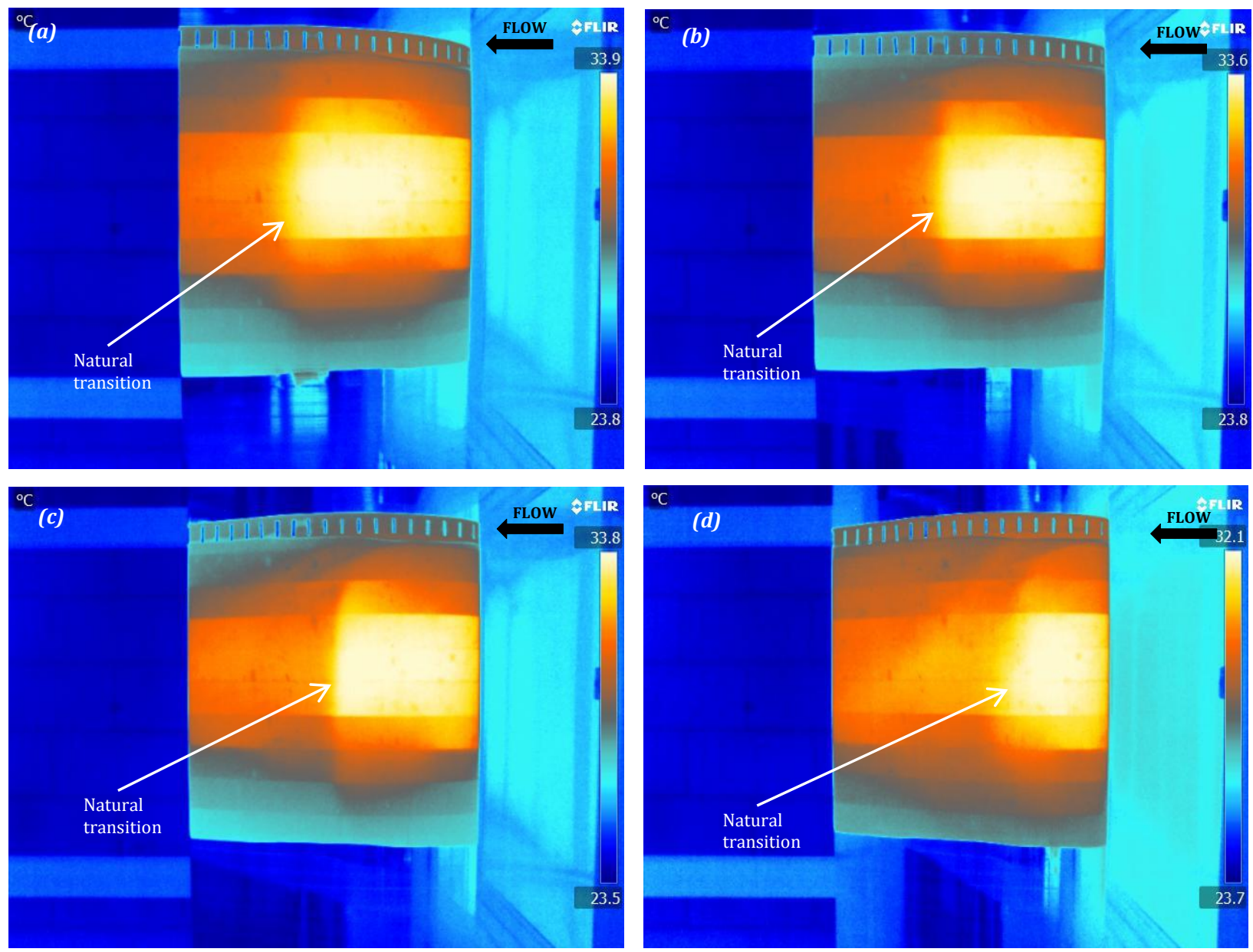

Figure 3.23 Coloured Infrared Image of Suction Side of DU96-W-180 Model, Insulated with Silicone Rubber, at 30- $\mathrm{ms}^{-1}$ and Angle of Attacks of (a) $-4^{\circ}$ (b) $-1^{\circ}$ (c) $2.5^{\circ}$ and (d) $5^{\circ}$ (Coldest Regions are Blue and Warmest Regions are White) 


\section{TemperKote INS Hi-Heat Thermal Insulating Coating}

This insulator is a composite ceramic insulation paint made up of microscopic air filled ceramic particles combined with acrylic resins. It has a flat, off white color and a maximum temperature tolerance of $505.15-\mathrm{K}\left(232^{\circ} \mathrm{C}\right)$. It was applied the upper third of the span of the DU96-W-180, 0.46-m chord, with a paint brush. There were problems applying the insulating paint evenly on the model using only a brush and hence the insulator thickness varied between $1.5-\mathrm{mm}$ to $2-\mathrm{mm}$ in some places. In order to obtain this thickness the paint was applied in consecutive layers. Each layer was left to dry for at least an hour before applying another layer, except the first layer, which was left to dry for four hours. After drying, the surface was sanded with fine grit sandpaper to improve the surface finish and even out the thickness of the paint. Figure 3.24 shows the surface finish of the insulating paint after it was applied and then after it was sanded.

Flexible heaters were used to create the temperature difference between the flow and the DU96-W-180 model covered in the TemperKote insulating paint. Figure 3.25 shows the infrared results of this model in a $30-\mathrm{ms}^{-1}$ flow at two angle of attack.

These results show that the TemperKote insulating paint is sufficiently insulating to allow the gradient associated with transition to be observed. The transition locations also moved closer to the leading edge (decreasing \% chord) as the angle of attack increases, as is expected for the suction side of the DU96-W-180.

Therefore, TemperKote insulating paint is a good insulator for use with aluminum models. It not only allows for transition detection but it is also not infrared reflective. Furthermore, the surface can be made relatively smooth, the thickness can be varied by number of coats applied, and it does not damage the model. The issues with this paint were that it has a long drying time and is difficult to apply uniformly without the use of a high power texture paint sprayer.

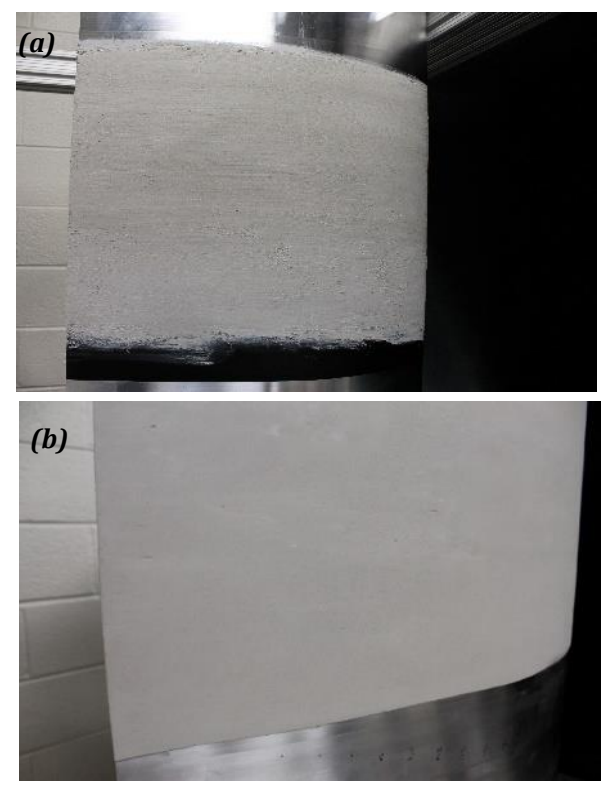

Figure 3.24 Photograph of TemperKote Insulating Coating (a) Immediately After Application to Airfoil (b) Sanded to Improve Surface Quality 

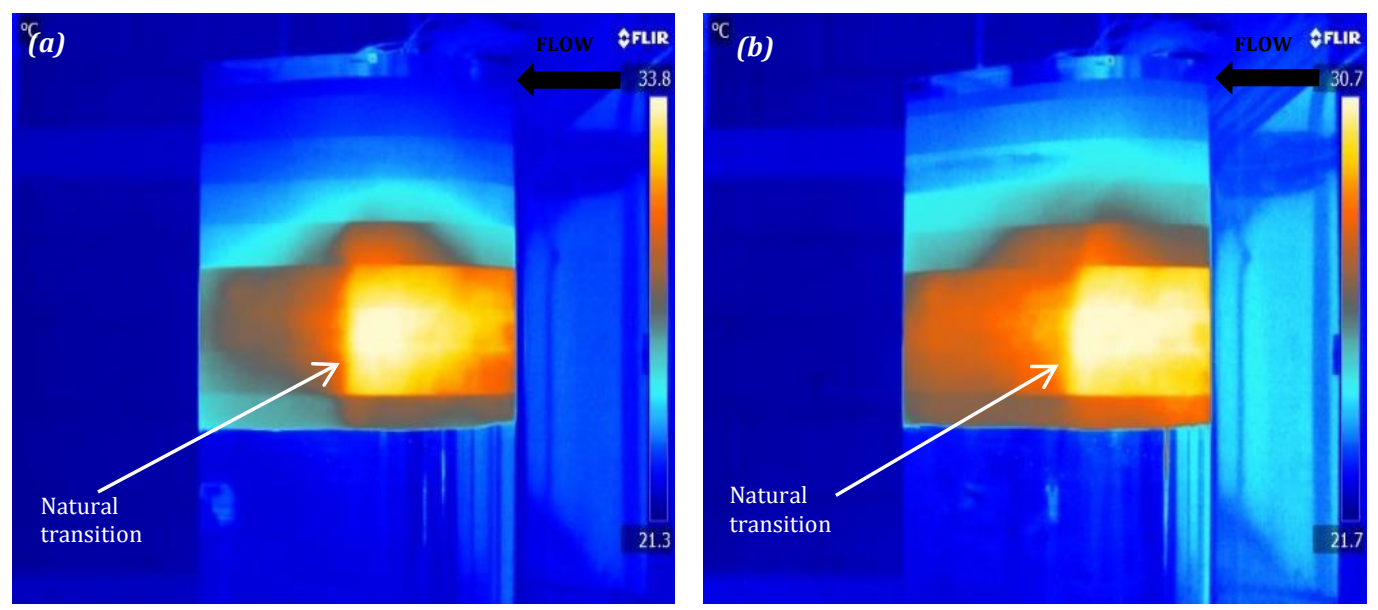

Figure 3.25 Coloured Infrared Image of Suction Side of 0.46-m DU96-W-180 Model, covered in TemperKote Insulating Paint, at 30- $\mathrm{ms}^{-1}$ and Angle of Attacks of (a) $0^{\circ}$ (b) $4^{\circ}$ (Coldest Regions are Blue and Warmest Regions are White)

The preceding comparison of insulators led to the conclusion that the best insulators for transition detection on aluminium models were those with high insulating properties, and which are closed-cell. The latter property ensures that no air pockets are created between the model and insulator. Of these, the FDA-compliant silicone rubber was the best option for insulating aluminium models in the Stability Wind Tunnel. This material was the thinnest of all those investigated and offered the best surface finish after application. It could also be applied most efficiently because it had adhesive backing and attached to the model smoothly with little effort and time (approximately fifteen minutes to cover one third of the 1.82-m span). Furthermore, the infrared transition results were very clear when observed, with no issues pertaining to air pockets or other unwanted features.

\subsection{Additional Transition Detection Measurement Systems}

While the preliminary results obtained from using an insulator with full scale aluminium models appear very promising, it was necessary to verify that the temperature gradients observed in infrared images were indeed that of transition, and not, for example, a result of camera angle, location or glare.

The transition results cannot be adequately validated solely by known numerical simulators such as XFOIL. Secondary measurement systems were therefore developed to validate the transition results which were obtained by the infrared camera. 


\subsubsection{Mean Pressure Analysis}

As laminar flow becomes turbulent, the increase in skin friction causes a measureable rise in the pressure at the point on the airfoil surface where transition occurs. This pressure rise can therefore be used to detect the location of transition. Figure 3.26 shows a typical negative coefficient of pressure (-Cp) distribution over the suction and pressure side of an airfoil (in this case, the 0.46-m DU96-W180). There is a clear drop in -Cp plots which is indicative of the sudden rise in pressure which is characteristic of transition.

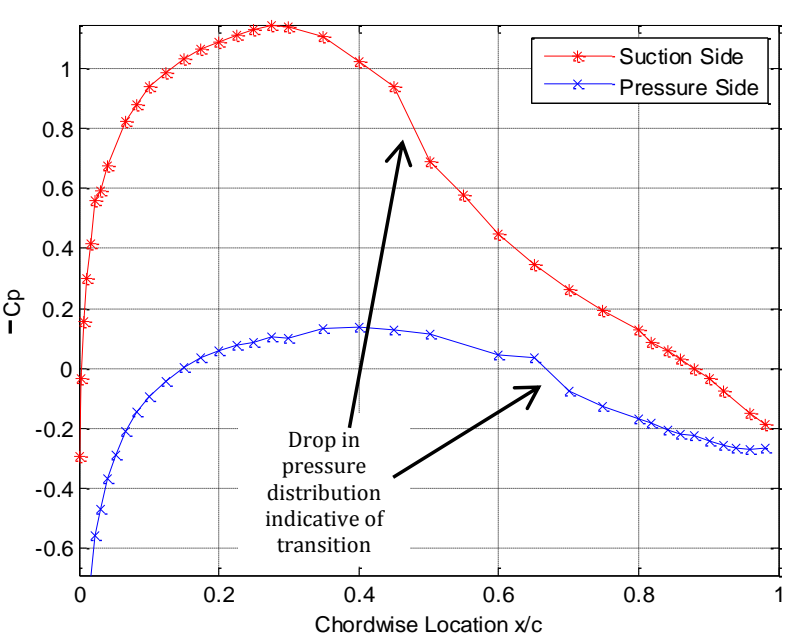

Figure 3.26 Coefficient of Pressure Distribution for the 0.46- $\mathrm{m} D U 96-W-180$ at $R e=1.5 \times 10^{6}$ and $\alpha=3^{\circ}$

The sharp drop in the -Cp distribution can be characterized by a peak in the second derivative of the -Cp distribution (Popov et al., 2008) since the second derivative is a measure of the curvature of a plot. The sharpest drop in the - $\mathrm{Cp}$ is essentially the point of highest curvature and will be the maxima of the second derivative calculation. Applying this theory to the pressure distribution in Figure 3.26, plots of the second derivative and the transition region are created and shown in Figure 3.27.
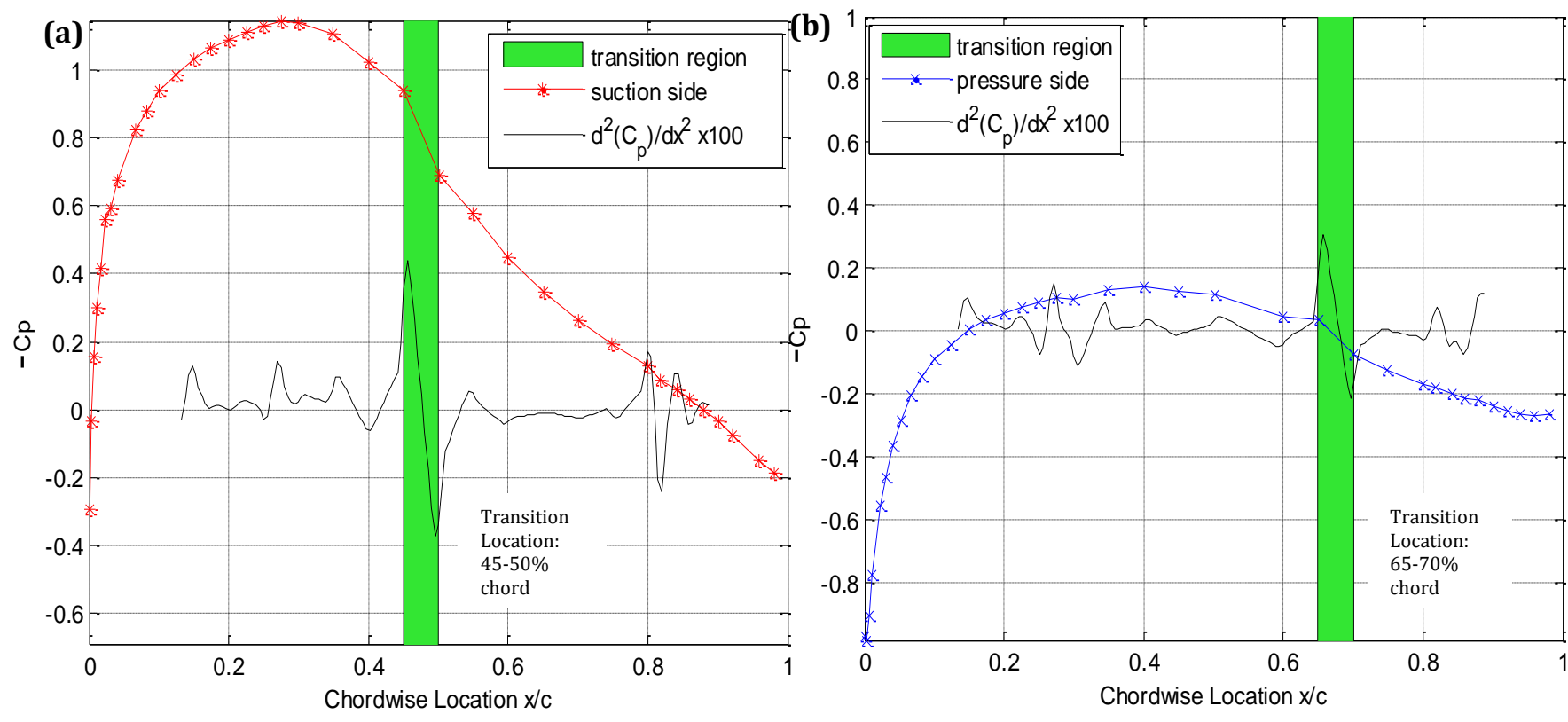

Figure 3.27 Coefficient of Pressure Distribution with Transition Region Characterized by the Second Derivative of the Negative Coefficient of Pressure for the (a) Suction Side and (b) Pressure Side of the 0.46-m DU96-W-180 at Re=1.5 $\times 10^{6}$ and $\alpha=3^{\circ}$ 
This method of transition detection, while potentially accurate, is limited by the surface pressure ports in two ways. The first is that the port distribution dictates the size of the transition region detected since the pressure drop can only be detected from port to port. Therefore, if ports are far apart, the region over which the pressure drop is seen is larger. This reduces the accuracy of the results in that the uncertainty band for each data point is larger. Furthermore, it is not possible to tell the exact point where transition begins by this method - the results only give the region within which it occurs. For example, the 0.46-m DU96-W-180 has a maximum distance between surface pressure taps of 5\% chord (23-mm) and thus the results obtained for this model can only be detected within $5 \%$ intervals. The actual transition location can be anywhere within this region. The second limitation occurs as the angle of attack changes and the transition location moves. The pressure taps are able to definitively detect the drop in pressure only when this pressure drop occurs between pressure tap locations. If the angle of attack is such that the transition occurs at the location of a pressure tap, the pressure drop will not be observed and the results will be obtained from interpolation. The mean pressure analysis method was used for validation of the infrared results and therefore its limitations did not cause significant issues. Only angles of attack which produced clear pressure drops were used in the validation. Lastly, as rightly stated by Popov (2008), the second derivative of pressure calculated close to the leading edge is very irregular. This is clear in Figure 3.28a-e where it is seen that there are large spikes in the second derivative at the leading edge as well as the actual transition location. These regions cannot therefore be considered in the mean pressure transition detection analysis. In each case, the analysis was started at $15 \%$ chord.

In the Open Jet Wind Tunnel, five angles of attack were tested with infrared thermography and mean pressure analysis to determine whether the results from each method are consistent. The 0.46-m DU96-W-180 airfoil was mounted in the Open Jet Wind Tunnel, heated using flexible heaters and insulated using red-orange silicone rubber as shown in Figure 3.22. The pressure data results, with the accompanying second derivative analysis, are shown in Figure 3.28. A comparison of the infrared thermography results and the mean pressure results for these five angle is presented in Figure 3.29. 

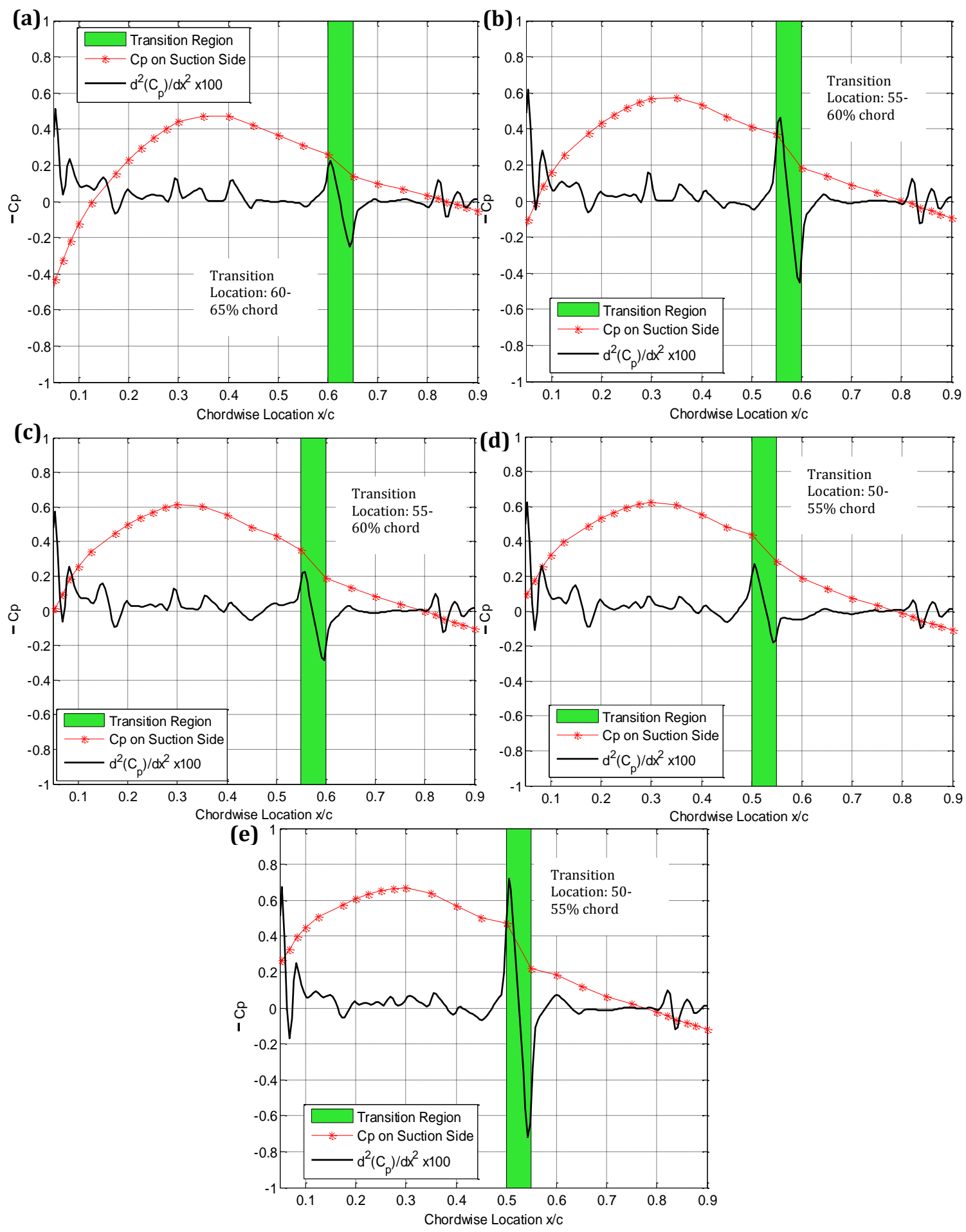

Figure 3.28 Coefficient of Pressure Distribution with Transition Region Characterized by the Maxima in Second Derivative of the -Cp for the Suction Side of the 0.46- $m$ DU96-W-180 (a) $\alpha=-2^{\circ}$ (b) $\alpha=-0^{\circ}$ (c) $\alpha=0.5^{\circ}$ (d) $\alpha=1^{\circ}(e) \alpha=2^{\circ}$ 
From Figure 3.29 it can be said that, for the small angles tested, the transition results from infrared thermography are in agreement with that obtained from the mean pressure analysis, within the uncertainties. Therefore, it can be concluded that the temperature gradients perceived on the infrared cameras are as a result of transition. However, the limitations of the Open Jet Wind Tunnel test set up affords some level of uncertainty in these results. Hence, the mean pressure analysis was also done during Phase Two of this study in the Stability Wind Tunnel, where the flow is more stable and uniform.

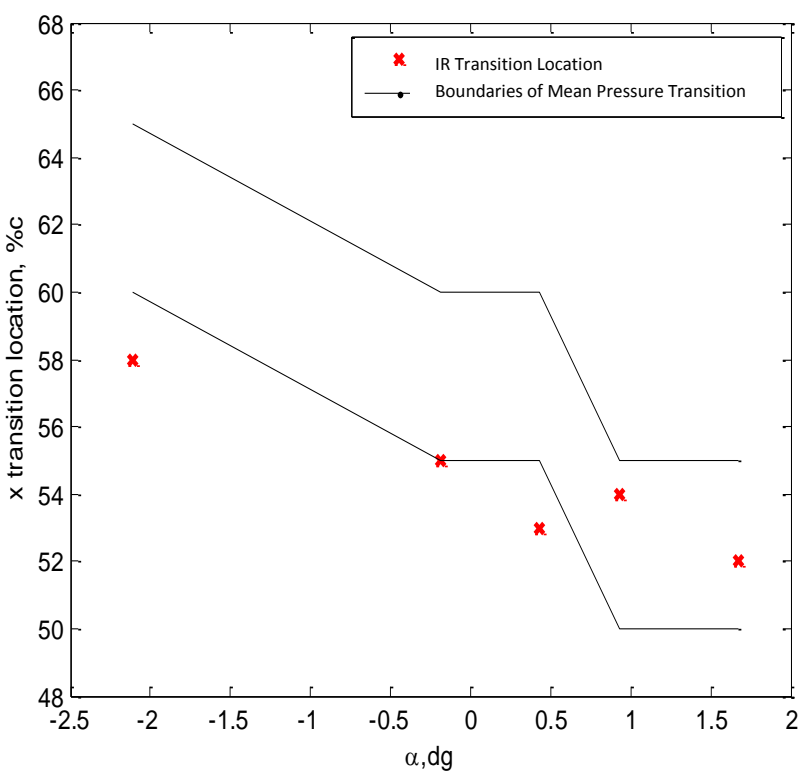

Figure 3.29 Comparison of Infrared Thermography and Mean Pressure Results for Transition Location on the Suction Side of the 0.46-m DU96-W-180

\subsubsection{Stethoscope Measurements}

The unsteady nature of turbulent flow creates increased noise levels in a stethoscope immersed in the boundary layer. Conversely, in laminar flow there are only very small fluctuations resulting a lower noise levels heard through a stethoscope. This feature allows for transition detection by measuring the increase in noise levels over the surface of the model.

An Autocraft automotive stethoscope (model AC637), attached to a Pitot probe, was used to listen to changes in air flow around model surface. The diaphragm of the stethoscope was replaced with a thin Mylar diaphragm in order to improve the sensitivity of the stethoscope to pressure fluctuations. The Pitot Probe was made of 11 gauge, stainless steel tubing, with a $3-\mathrm{mm}$ outer

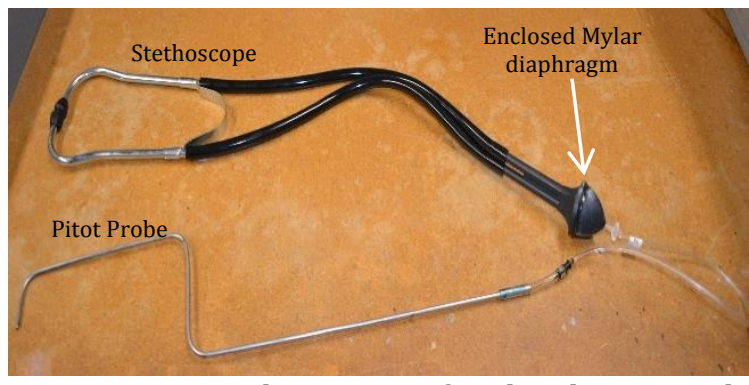

Figure 3.30 Stethoscope Outfitted with Pitot Probe for Transition Detection diameter and a $0.254-\mathrm{mm}$ inner diameter. The stethoscope with attached Pitot Probe is shown in Figure 3.30. The Pitot probe was traversed along the model surface, from leading to trailing edge, while the experimenter listened for changes in the sound levels and recorded the edge location where this occurred. 
The transition results using the stethoscope method were measured on the suction side of the insulated 0.46-m DU96-W-180 which was heated internally using Kaplan flexible heaters. At most angles of attack, it was observed that there were two distinct sound changes over the airfoil chord. The first discernable sound change was not as loud as the second sound change, which was further downstream than the first. It was concluded that, due to its location and levels, the first sound change was indicative of the onset of instabilities in the flow. The first sound was also very difficult to observe in the Open Jet Wind Tunnel, and is therefore associated with large uncertainties. The second sound was much louder and easier to detect. It was therefore determined to be the fully turbulent flow where pressure fluctuations are largest. The results obtained from this investigation are presented in Figure 3.31 .

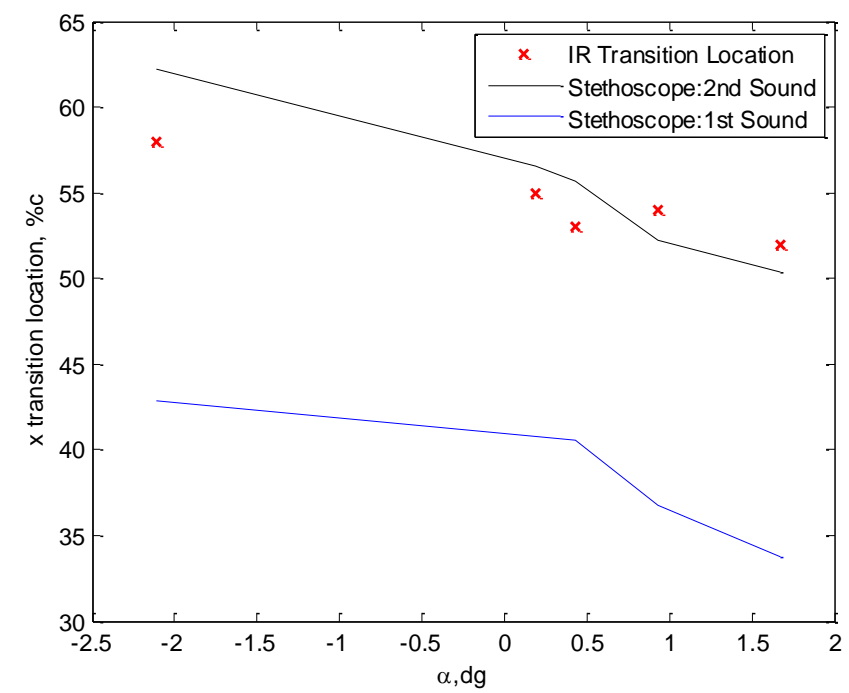

Figure 3.31 Comparison of Infrared Thermography and Stethoscope Results for Transition Location on the Suction Side of the 0.46-m DU96-W-180

The results show that the second sound detected by the stethoscope agrees with that detected through infrared thermography to within 5\% chord. This agreement is not excellent because of the errors associated with the Open Jet Wind Tunnel flow and angle of attack setting. However, the overall trend is the same and the agreement is close enough to support the theory that the gradients detected on the infrared images are as a result of transition. Testing in a more controlled facility, such as the Stability Wind Tunnel, should produce more reliable and clear results from the stethoscope.

\subsubsection{Preliminary Validation of Infrared Thermography Measurements}

Figure 3.32a-e presents the compiled transition results obtained from the mean pressure analysis, stethoscope sound tests and infrared thermography. These results consist of numerical plots overlaid on the black and white infrared images and are presented for five 
angles of attack. Chord-wise temperature gradients on the images are due to flow effects while span-wise temperature gradients are due to differences in temperature between the laminates, and between the insulated and bare model. In these figures, the blue circles are indicative of the mean pressure over the chord of the model while the black line is representative of the second derivative of this pressure. The yellow lines delineate the transition region between the first and second stethoscope sounds. The local, chord-wise maxima of the temperature gradient is depicted by the red dots.
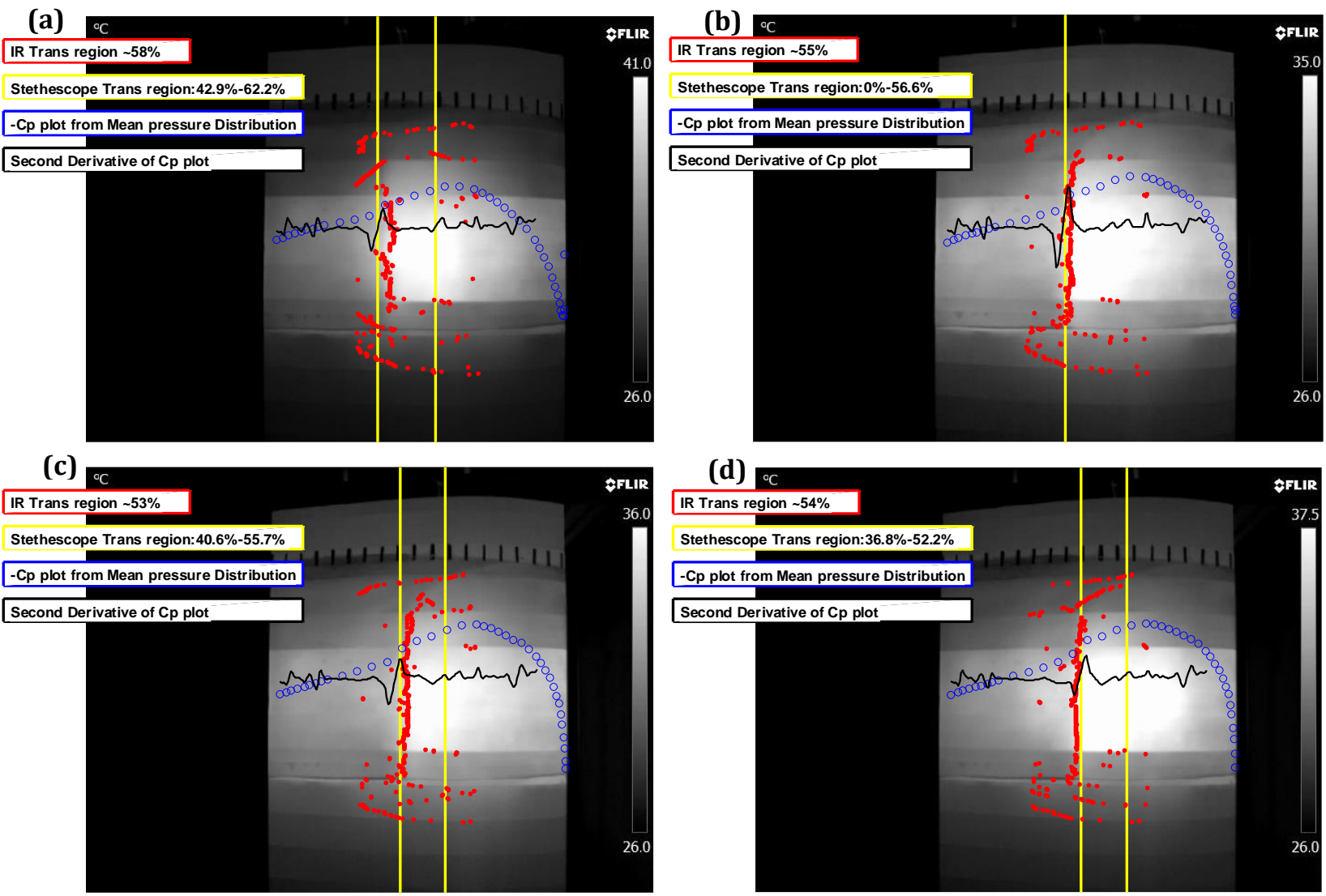

\section{(e)}

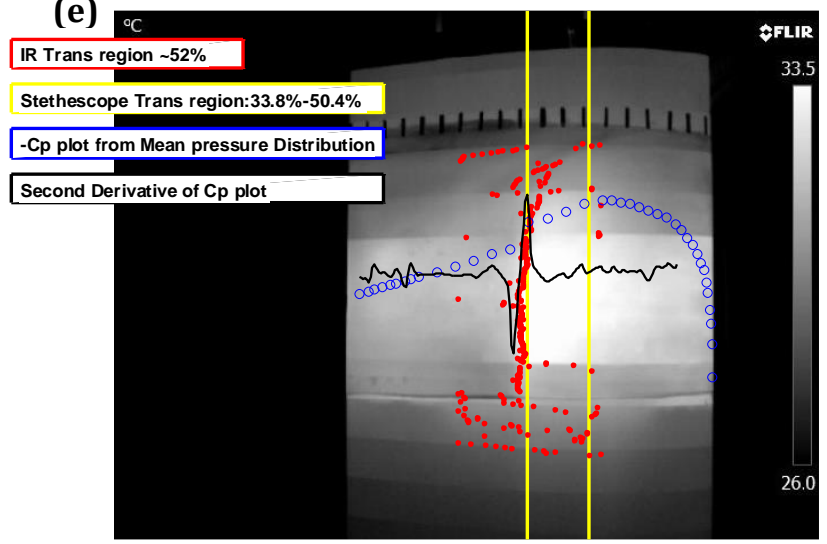

Figure 3.32 Comparison of Transition Results from Mean Pressure, Stethoscope and Infrared Thermography for the Suction Side of the 0.46-m DU96-W-180 (a) $\alpha=-2^{\circ}$ (b) $\alpha=-0^{\circ}$ (c) $\alpha=0.5^{\circ}$ (d) $\alpha=1^{\circ}$ (e) $\alpha=2^{\circ}$ 
These local maxima of the temperature gradient were extracted from the black and white infrared images using an image processing algorithm. The grey colour palette was chosen because it was observed that the transition detection algorithm worked better with black and white images. The algorithm developed computes the chord-wise gradient of each pixel in the infrared image and finds its maximum. The maximum gradient will indicate the onset of transition where the flow first starts to experience instabilities. This computation was carried out for each row of pixels and the transition location is taken as the mode of the peak gradients. This process provides the location along the chord of the airfoil where transition occurs. The pixel value is then converted to a chord-wise location using reference marks, made every $12.7-\mathrm{mm}$, on the model.

Figure 3.32a-e shows that the three results agree fairly well at all angles of attack. Wedges appeared as the flow curled over the step created between the insulator and the bare model. In these regions, the algorithm detects the wedges as the maximum gradient instead of the transition - this is observed in Figure 3.32 as the diagonal rows of red dots toward the top and bottom of the infrared images.

The agreement of the transition results from three different measurements strongly suggests that the current system - infrared camera and insulated aluminium model heated internally - is able to reliably detect transition. However, the accuracy of these results requires further validation because of the inaccuracies associated with the flow and the angle of attack in the Open Jet Wind Tunnel.

\subsection{System Implementation in Stability Wind Tunnel}

The system was adapted to be used in the Stability Wind Tunnel by constructing camera mounts, special windows and upgrading the test models with silicone rubber insulators and internally mounted flexible heaters.

For this system to work, it was necessary to install windows in the tunnel test section wall which allow at least $80 \%$ infrared light penetration. These windows were built in panels 4 and 5 shown in Figure 2.14. They are located approximately 2.85-m and 3.62-m (respectively) from the upstream end of the test section, on both starboard and port sides of the test section. It was decided that to obtain the largest field of view at the most angles of attack without compromising the integrity of the flow, one window would be fitted into each of these four panels. Figure 3.33a shows a diagram of the window locations in both panels. 

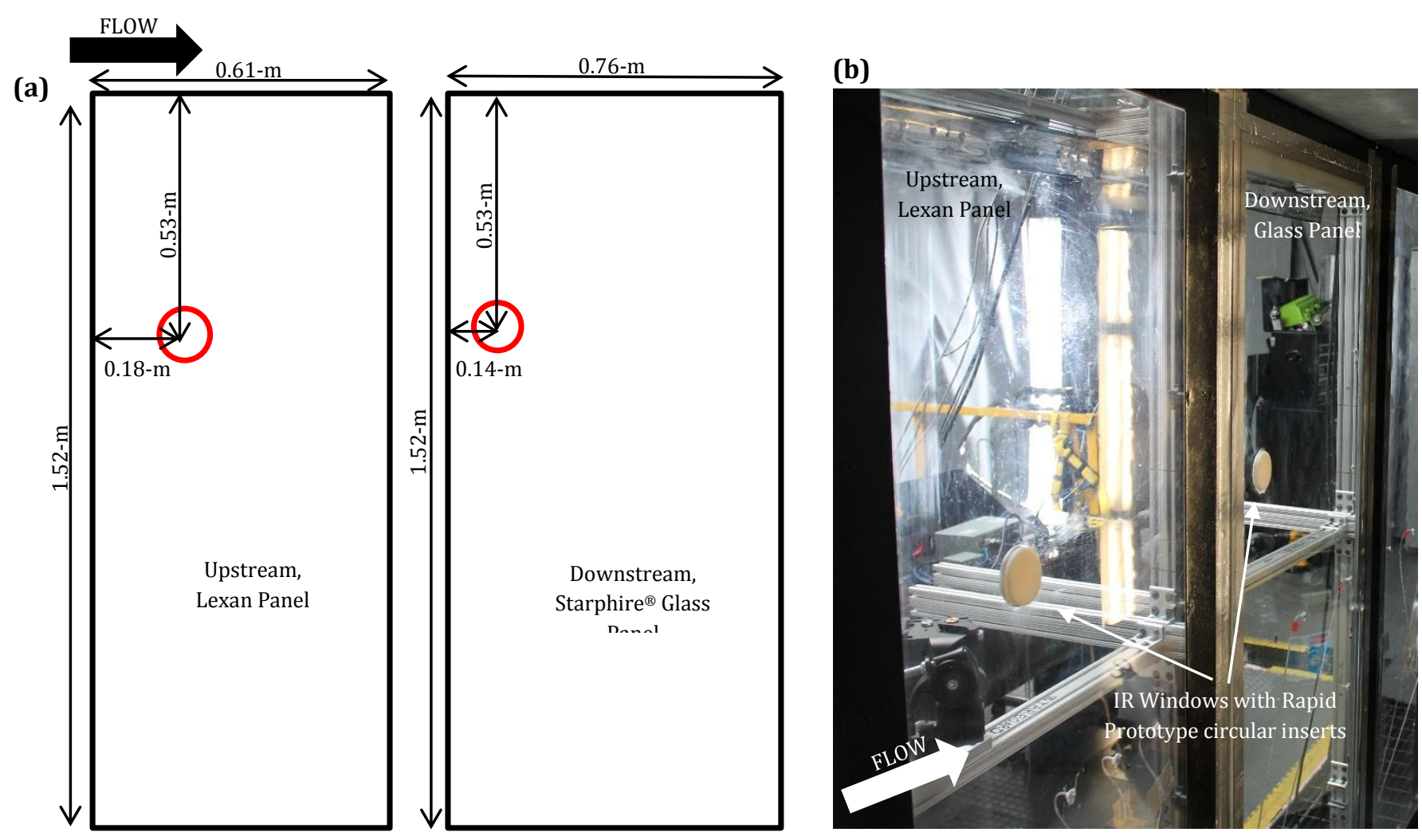

Figure 3.33 (a) Diagram (b) Picture Showing Location of IR Windows in Starboard Glass and Lexan Panels

Based on previous research, the best materials for such a window were likely Zinc Selenide, Crystalized sapphire, Germanium, and infrared Plexiglass (a picture of the latter is shown in Figure A3). Yet, after further research and preliminary testing, it was found that only Germanium was within the operating infrared spectrum of the camera $(7.5-14-\mu \mathrm{m})$. However, an in depth look at Germanium revealed that this material is extremely brittle and toxic if altered in any way after purchase. Moreover, the maximum thickness available is 2$\mathrm{mm}$, which will not be able to withstand the forces which will be experienced during testing. This led to the decision that an open circular window, with rapid-prototype inserts for when the camera is not in use, was the best option for this system. The panels with cut holes for infrared camera are shown in Figure 3.33b.

Since the outer diameter of the camera lens is approximately $65-\mathrm{mm}$, a circular hole of 63.5-mm (closest pre-set drill bit size) was cut in the larger two of the four Starphire ${ }^{\circledR}$ glass panels and two Lexan panels. The original intention was to cut holes in all the glass panels since they were closest to the model when mounted and this would also enable the flow visualization system and the infrared thermography system to be operated simultaneously. However, since the Starphire ${ }^{\circledR}$ panels are made of laminated safety glass, cracks began to form in the inner layers of the glass while cutting. Hence, only the two larger glass panels were cut, one for each of the port and starboard sides of the wind tunnel. The 
locations which allowed for the best and largest range of view for a 0.6-m chord model, from angles of attack of $-20^{\circ}$ to $20^{\circ}$, were determined.

During runs when infrared images are not being taken, the rapid prototype pieces are inserted and foil tape used to create a flush surface with the test section wall and preserve the fidelity of the wall pressure data. This can be seen in Figure 3.34.

The camera mounting system is the same as that used for the DSLR cameras used in the wind tunnel tuft flow visualization system. It comprises of a framework of $80 / 20$ aluminium beams bolted to the outer flanges of

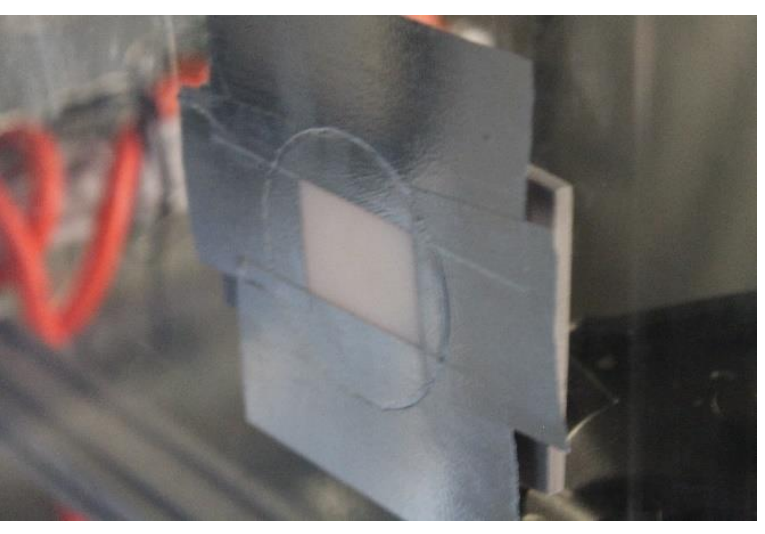

Figure 3.34 Infrared Window Sealed with Rapid Prototype Insert and Foil Tape the test section. Four pairs of Manfrotto 808RC4 Quick Release tripod heads were attached to the beams so that the cameras were able to precisely tilt, pan and rotate as needed. It was of great importance to have the capability to move the camera both in the stream-wise (horizontal) and span-wise (vertical) directions so that as much of the model could be viewed at all angles of attack. The mounting system accomplishes this by allowing experimenters to loosen a few screws on the beams so that bars could easily be slid in either direction.

Lastly, to protect the camera lens during testing and to minimize camera vibrations, 2-mm thick foam tape was applied to the region around the circular holes where the camera would make contact with the test sections panels. The complete infrared camera set up is shown in Figure 3.35.

The flexible silicone rubber heaters shown in Figure 3.8 were attached to the inner surface of both models. The heaters were only attached in the top half section of the span (approximately 0.15-m above the pressure taps), covering approximately 0.46-m of the span. They were mounted approximately $38.1-\mathrm{mm}$ apart inside the model and PVC pipe was used to protect the electrical wires and the Tygon tubes for the pressure ports from the effect of the heaters. For the 0.8-m DU96-W-180 model a 50.8-mm diameter PVC pipe was used while a 38.1-mm diameter pipe was used for the $0.46-\mathrm{m}$ model. The electrical wires connected to the heaters (and the pressure tubes) were passed through these PVC pipes then up through the centre shaft of the model which allows access to the external power supply. The heaters on each side of the model were controlled independently during testing using two General Radio Type W5MT Variacs, shown in Figure A4. 

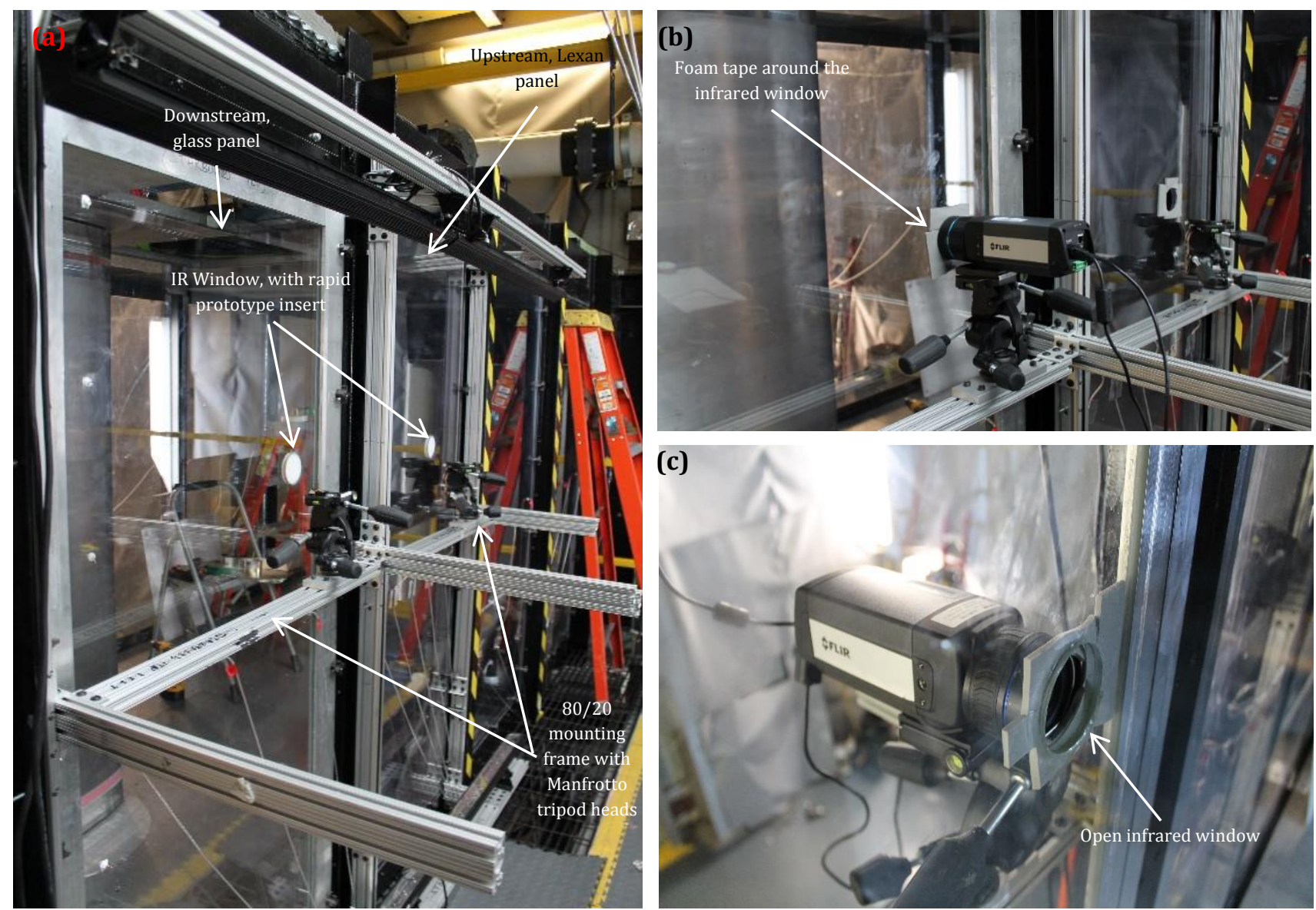

Figure 3.35 (a) Aluminium Framework for Camera Mounting on Suction Side of Test Section (b) View of Mounted Camera from Outside Suction Side of Test Section (c) View of Mounted Camera from Inside Test Section

Figure 3.36 shows the arrangement of the heaters on the inside of each of the $0.8-\mathrm{m}$ and the $0.46-\mathrm{m}$ DU96-W-180 models. These diagrams are not drawn to scale and the heater locations are approximate but the general arrangement of the heaters for each model is depicted. The wall thickness are also shown in Figure 3.36.

Figure 3.37 is a picture of the heaters fixed inside the $0.8-\mathrm{m}$ DU96-W-180 (model is laying pressure side up). The heater arrangement and the PVC pipe are visible as well as the heater to wall thickness ratio. 
(a)

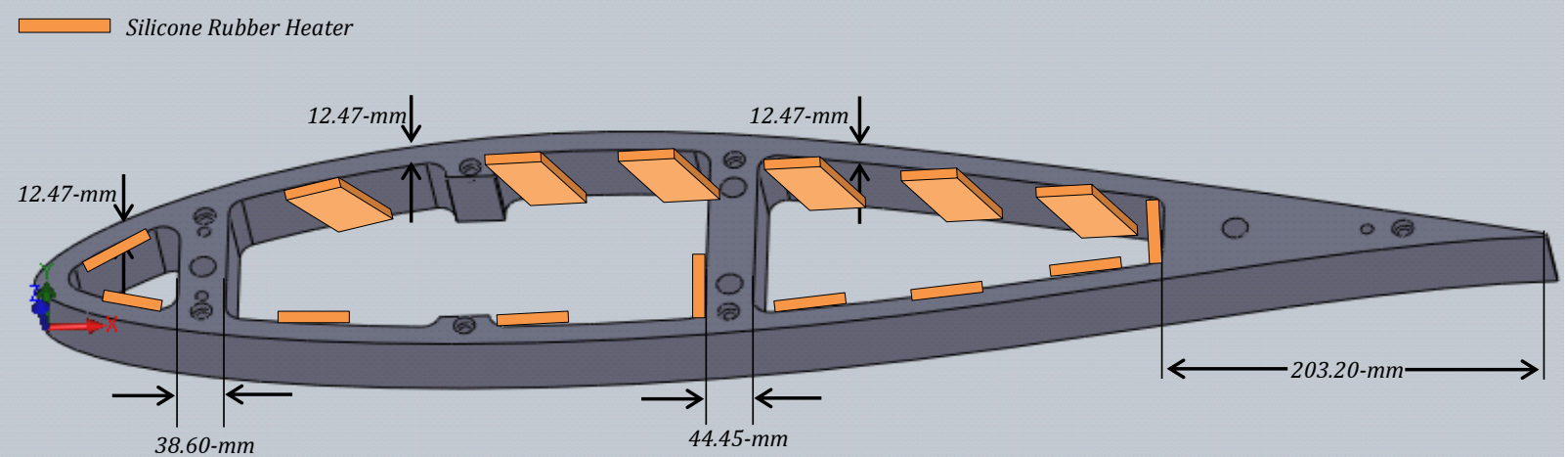

(b)

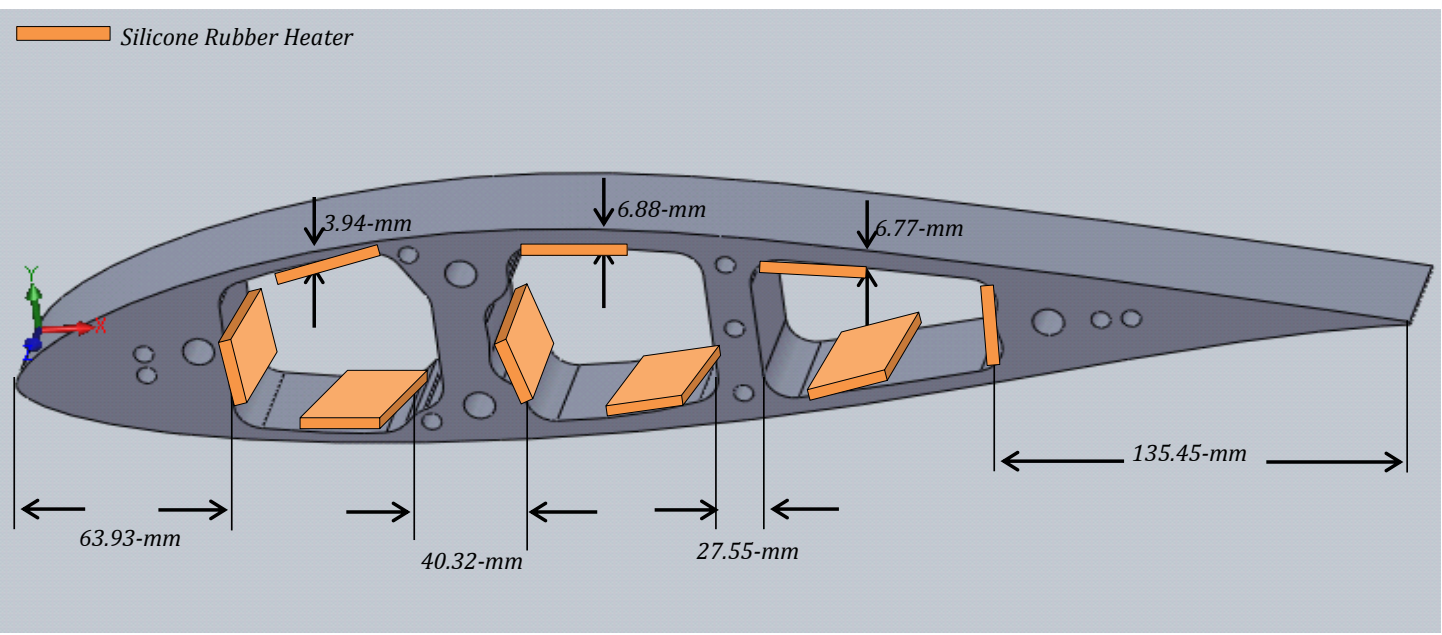

Figure 3.36 Diagram of Heater Arrangement inside the Model for the (a) 0.8-m and (b) 0.46-m DU96-W-180 (Locations are approximate and Drawings not to scale)

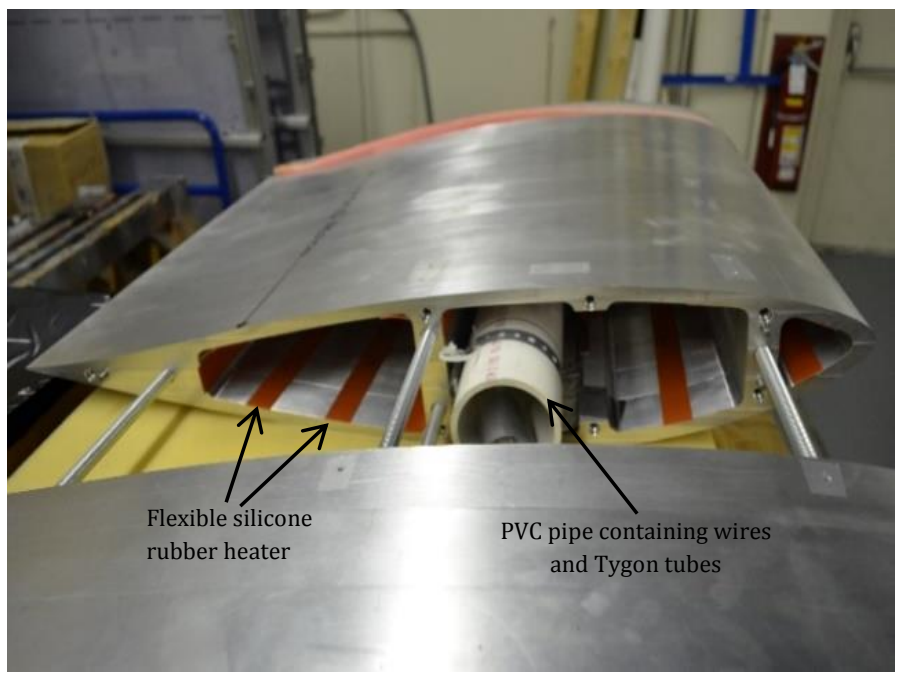

Figure 3.37 0.8-m DU96-W-180, Pressure Side Up, with Flexible Silicone Rubber Heaters Installed Inside 
The FDA-Compliant Silicone Rubber insulation used in the Stability Wind Tunnel tests was purchased from McMaster-Carr (model number 8991K999) and is black in colour with 0.8 -mm thickness. It is glossy and had an estimated emissivity of 0.98 . Apart from the colour, it is the same as the red-orange silicone rubber used in the Phase One tests, having an acrylic adhesive backing and medium softness (durometer reading of 40A). It was applied to the model, which was already covered in a layer of ConTact ${ }^{\odot}$ paper to prevent the nonremovable adhesive on the silicone rubber sheets from permanently bonding to the model surface. An additional layer of ConTact $^{\circledR}$ paper was pasted over the insulator to ensure that no reflections affected the results, since the silicone rubber is glossy. The final surface was very smooth with no visible imperfections. Figure 3.38 shows the final surface condition, with the insulator on, of the $0.8-\mathrm{m}$ DU96-W-180 and the relative thickness of the insulator to the model. The process and outcome was the same for the 0.46-m DU96-W-180 model. It should be noted that the $0.46-\mathrm{m}$ section which contained the heaters and was covered by the insulator, was located at approximately $0.15-\mathrm{m}$ above the pressure taps. The relative locations can be seen in Figure 2.23 and Figure 2.26 which are pictures of each model. This allowed both pressure and infrared measurements to be taken simultaneously, thereby affording the capability to investigate the effects that the insulator or the heated surface has on the flow.
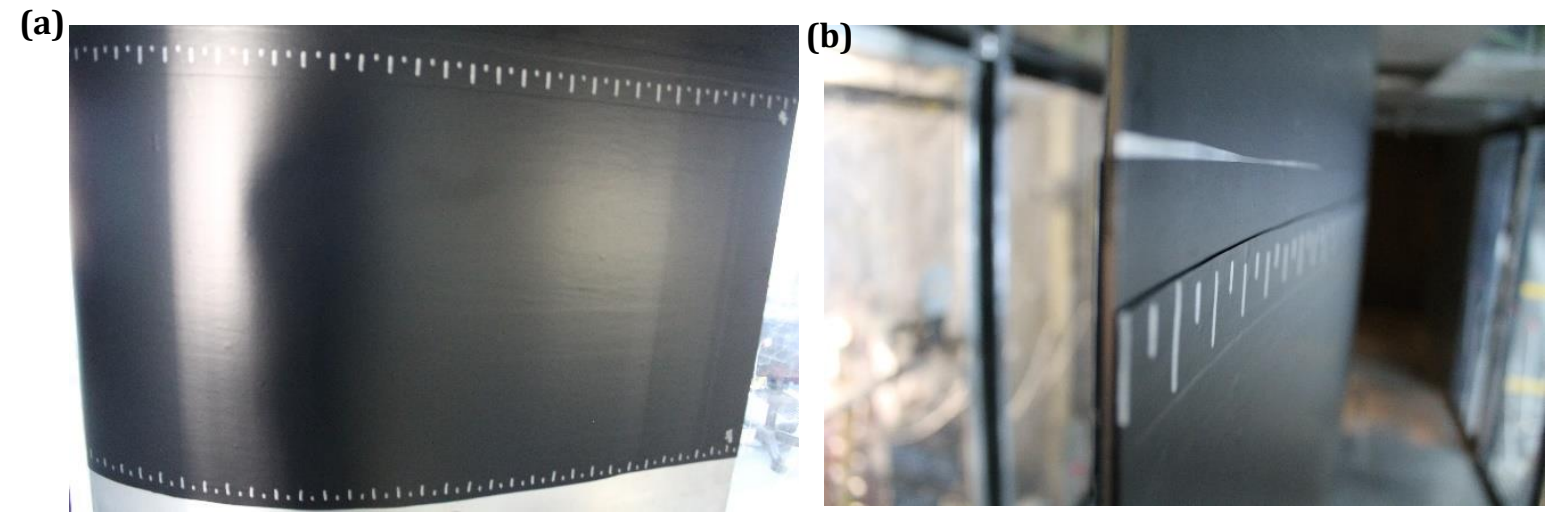

Figure 3.38 (a) Suction Side of the 0.8-m DU96-W-180 covered in 0.8-mm Black Silicone Rubber Insulator (b) Thickness of Insulator

The complete infrared thermography system, with 0.8-m DU96-W180, is portrayed from downstream in Figure 3.39. The set up for the 0.46-m DU96 was identical to that of the larger chord as is shown in Figure 3.40. 


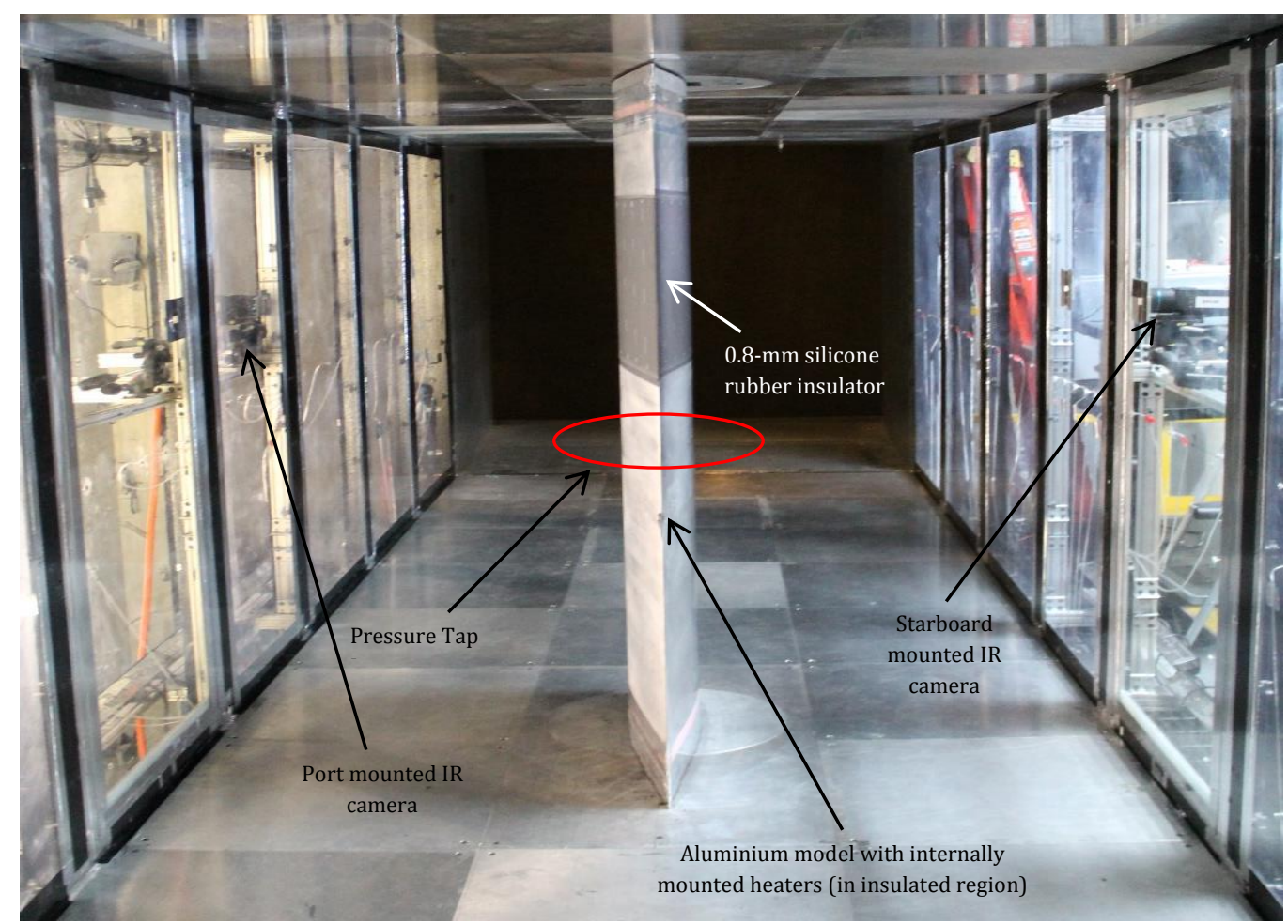

Figure 3.39 Downstream View of 0.8-m DU96-W-180 Mounted in Wind Tunnel with Infrared Thermography System

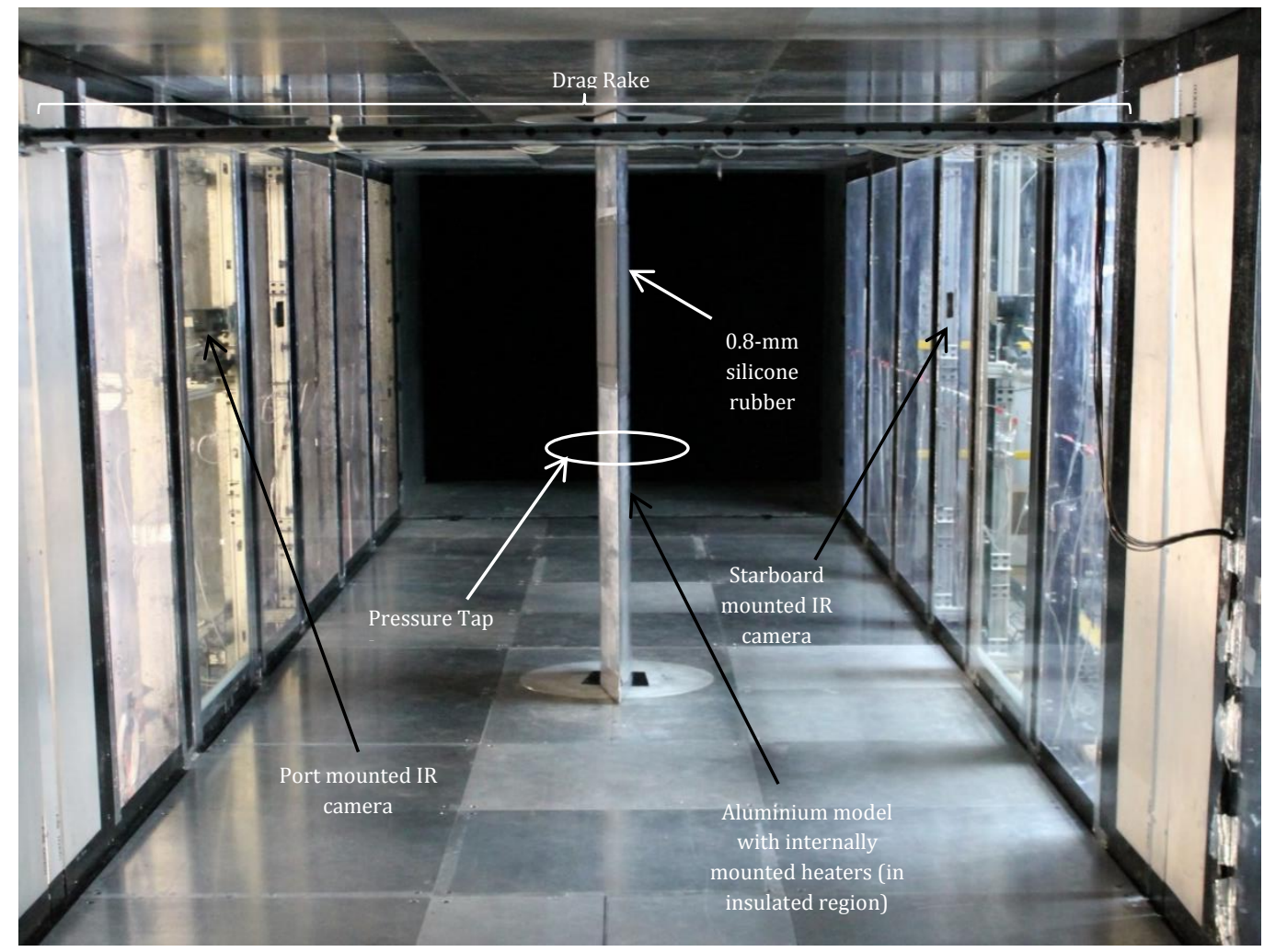

Figure 3.40 Downstream View of 0.46-m DU96-W-180 Mounted in Wind Tunnel with Infrared Thermography System 
The stethoscope measurement system was also adapted for use in the Stability Wind Tunnel. This was accomplished by adding a metal tube extension to the Pitot probe. The metal extension was made from hollow stainless steel and is $10-\mathrm{mm}$ in diameter, 1.1-m long. The Pitot probe was made of Precision Miniature 11 gauge stainless steel tubing (316 stainless steel) from McMaster-Carr (product number 89935K44). It has an outer diameter of 3.05-mm, and inner diameter of $2.54-\mathrm{mm}$ and a wall thickness of $0.25-\mathrm{mm}$. This tube was bent to approximately $90^{\circ}$ to form the Pitot probe which would traverse the airfoil surface. The bent region was covered in a thin layer of tape to prevent the metal tube from damaging the airfoil surface during testing. 1.8-mm diameter Tygon tubing was connected to the end of the Pitot probe and run through the length of the stainless steel extension to finally connect with the stethoscope. This device allowed the probe to be extended into the wind tunnel through slots made in the rapid prototype inserts which covered the camera ports when the infrared system was not in use. The stethoscope and the slot in the rapid prototype insert are shown in Figure 3.41 and the measurement technique is shown in Figure 3.42.

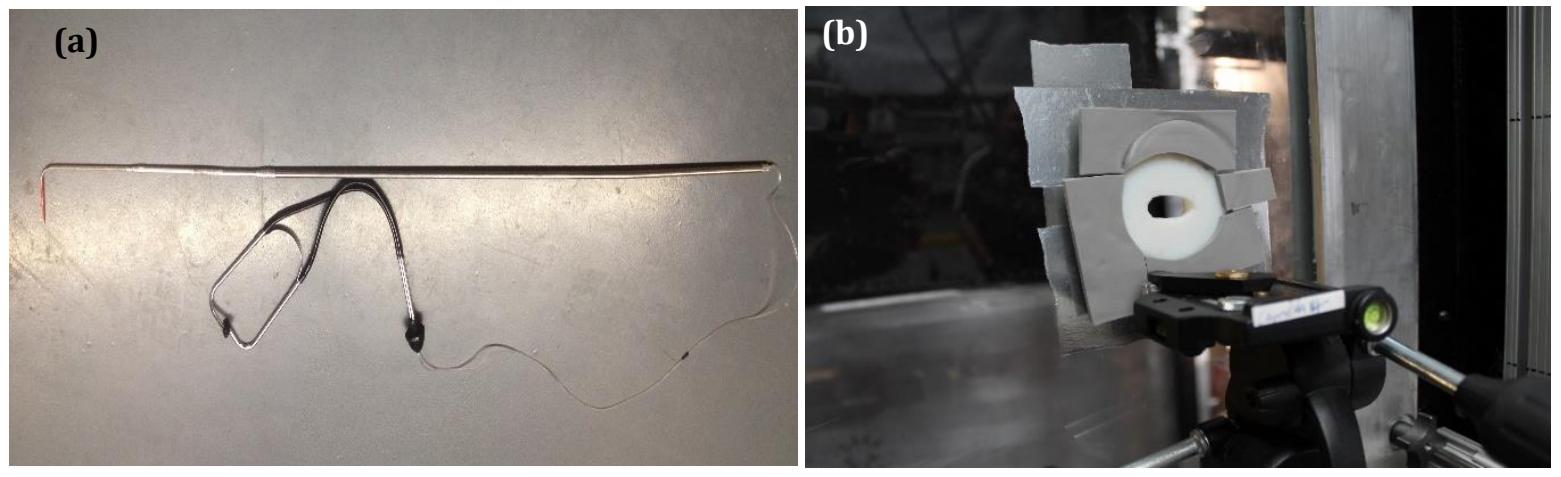

Figure 3.41 (a) Stethoscope Adapted for Use in the Stability Wind Tunnel (b) Slot in Rapid Prototype Insert for Inserting the Pitot Probe into Test Section

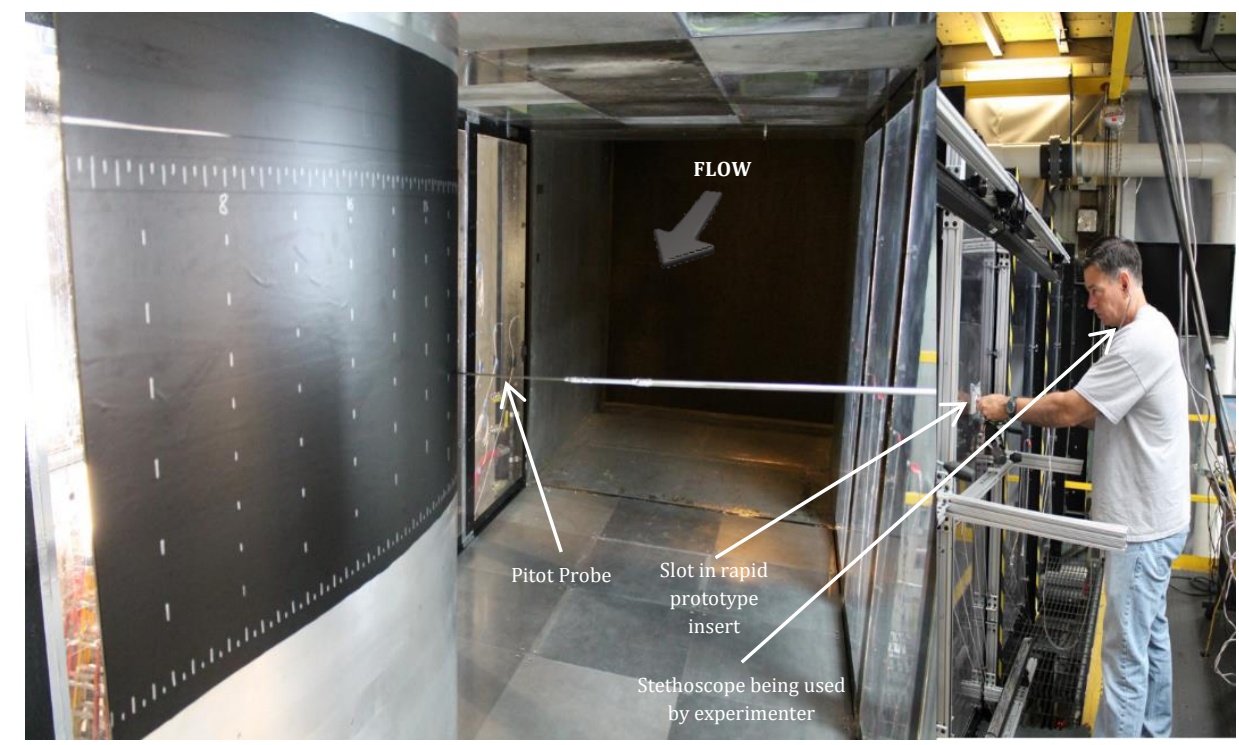

Figure 3.42 Experimenter Taking Stethoscope Measurements on the Pressure Side of the Insulated 0.8-m DU96$W$-180 in the Stability Wind Tunnel (use of likeness with permission of experimenter) 


\section{Chapter 4: TRANSITION DETECTION SYSTEM VALIDATION}

Phase Two testing was carried out in the Virginia Tech Stability Wind Tunnel to obtain transition results from the infrared system, the stethoscope and the mean pressure. The premise of validation is that if the transition results of these three measurement systems agree, the infrared system can be deemed reliable and sound.

The models used for validation tests are the 0.8-m DU96-W-180 - typically tested at $\mathrm{Re}=1.5 \times 10^{6}, 2 \times 10^{6}$ and $3 \times 10^{6}$, and the $0.46-\mathrm{m}$ DU96-W- $180-$ typically tested at $\mathrm{Re}=1.5 \times 10^{6}$ and $2 \times 10^{6}$. For the $0.46-\mathrm{m}$ model higher Reynolds Numbers could not be obtained because the shorter chord length means a higher flow speed is required to achieve high Reynolds Numbers, which is limited by the facility capabilities. All lift data presented were obtained from the surface pressure tap measurements. The drag data was obtained from measurements of the drag rake, which was located at a vertical height of $1.32-\mathrm{m}$ in the test section. Both the lift and drag have been corrected by the method developed by Allen and Vincenti (1947), discussed in Section 2.2.4.5. Before delving into the validation analysis, it is important to first examine the performance of each of these models and to verify the neutral impact of the infrared system on the flow.

\subsection{Airfoil Performance}

Both the $0.8-\mathrm{m}$ and the $0.46-\mathrm{m}$ DU96-W-180 models have the same profile but different chord length, therefore it is expected that the normalized, corrected lift and the drag on the models will be the same. These distributions, obtained from pressure measurements in the Virginia Tech Stability Wind Tunnel, are shown for the 0.8-m model in Figure 4.1.
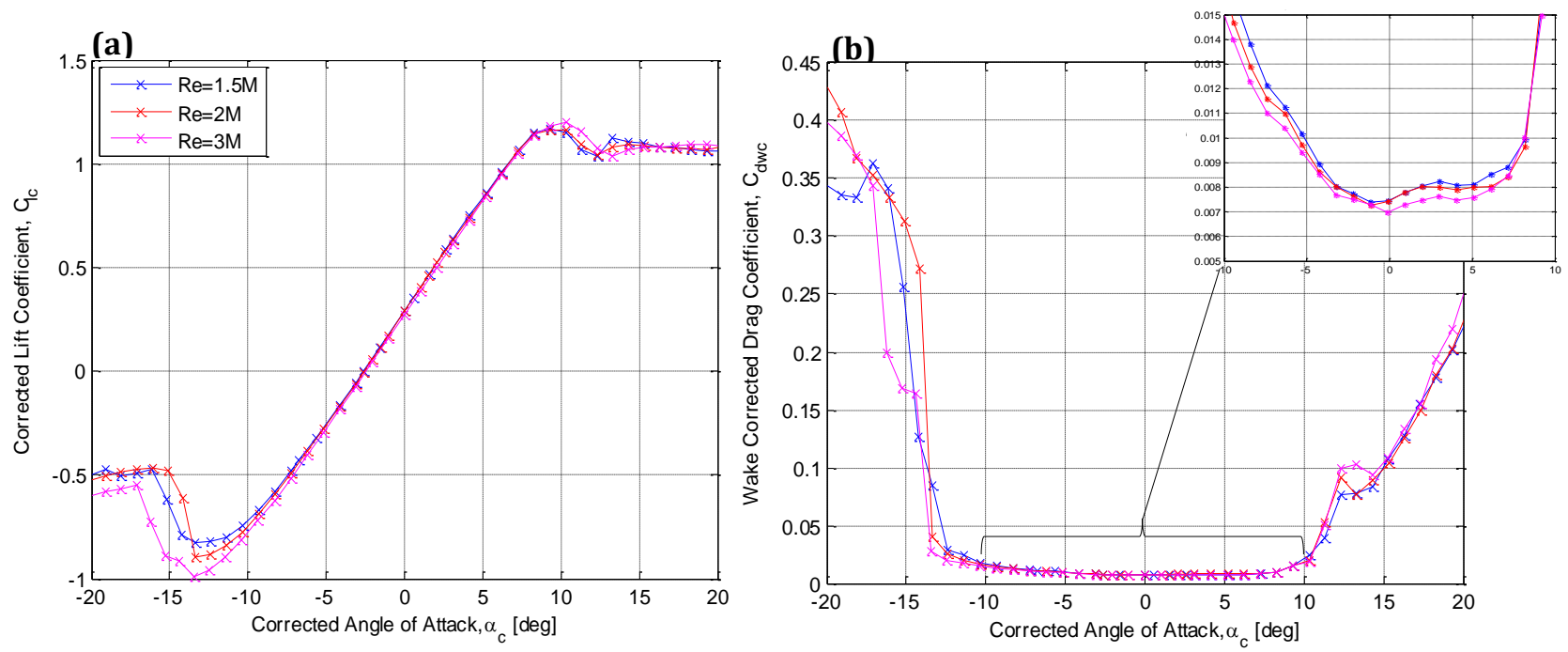

Figure 4.1 Corrected Coefficient of (a) Lift (from integrated surface pressure) and (b) Drag Distribution over the 0.8-m DU96-W-180 at Three Reynolds Numbers 
Figure $4.1 a$ is the coefficient of lift variation with angle of attack for the untripped airfoil. Corrected angle of attack is plotted on the $\mathrm{x}$-axis and the corrected coefficient of lift is on the y-axis. This distribution is plotted on the same axes for three different Reynolds numbers: $1.5 \times 10^{6}, 2 \times 10^{6}$ and $3 \times 10^{6}$, each represented by the blue, red and magenta lines, respectively. The lift distribution shown has a typical shape, having a linear region and two non-linear regions occurring at larger negative and larger positive angles. In the linear region, the lift increases with angle of attack from $\alpha_{c} \approx-13^{\circ}$ to $\alpha_{c} \approx 10^{\circ}$. At $\alpha_{c} \approx 10^{\circ}$ there is maximum lift of $C_{1} \approx 1.2$, after which there is a distinct drop in the lift, indicating stall. The same phenomenon occurs at $\alpha_{c} \approx-13^{\circ}$ where there is another break in the lift curve coinciding with negative stall. For this model, the linear region has an approximate slope of $0.044 \mathrm{dg}^{-1}$ and the zero lift angle of attack is $\alpha_{\mathrm{c} 0} \approx-2^{\circ}$.

The variation of drag coefficient over the $0.8-\mathrm{m}$ DU96-W-180 is illustrated in Figure 4.1b. As in item (a) $1.5 \times 10^{6}$ is represented by the blue line, $2 \times 10^{6}$ is represented by the red line and $3 \times 10^{6}$ is represented by the magenta line. At all three speeds, there is a clear horizontal region in which the drag is relatively small. At the angles when stall occurs, $\alpha_{c} \approx-$ $13^{\circ}$ and $\alpha_{c} \approx 10^{\circ}$, the distribution becomes non-linear and there is a sudden and large increase in the drag. As Reynolds number increases, it is seen that there is a slight widening of the drag bucket as the drag increases at $\alpha_{c} \approx-14^{\circ}$.

The performance for the 0.46-m DU96-W-180 was found to be similar to the 0.8-m chord DU96-W-180 discussed and shown in Figure 4.1. A comparison of the lift and drag distributions for both models is presented in Figure 4.2, with the blue lines representing the 0.8-m chord model and the red line signifying the performance of the 0.46-m chord model. For both plots, the corrected angle of attack is plotted on the $\mathrm{x}$-axis and the corrected lift or drag coefficient is on the y-axis. Figure $4.2 a$ shows that the linear regions of the lift plot are
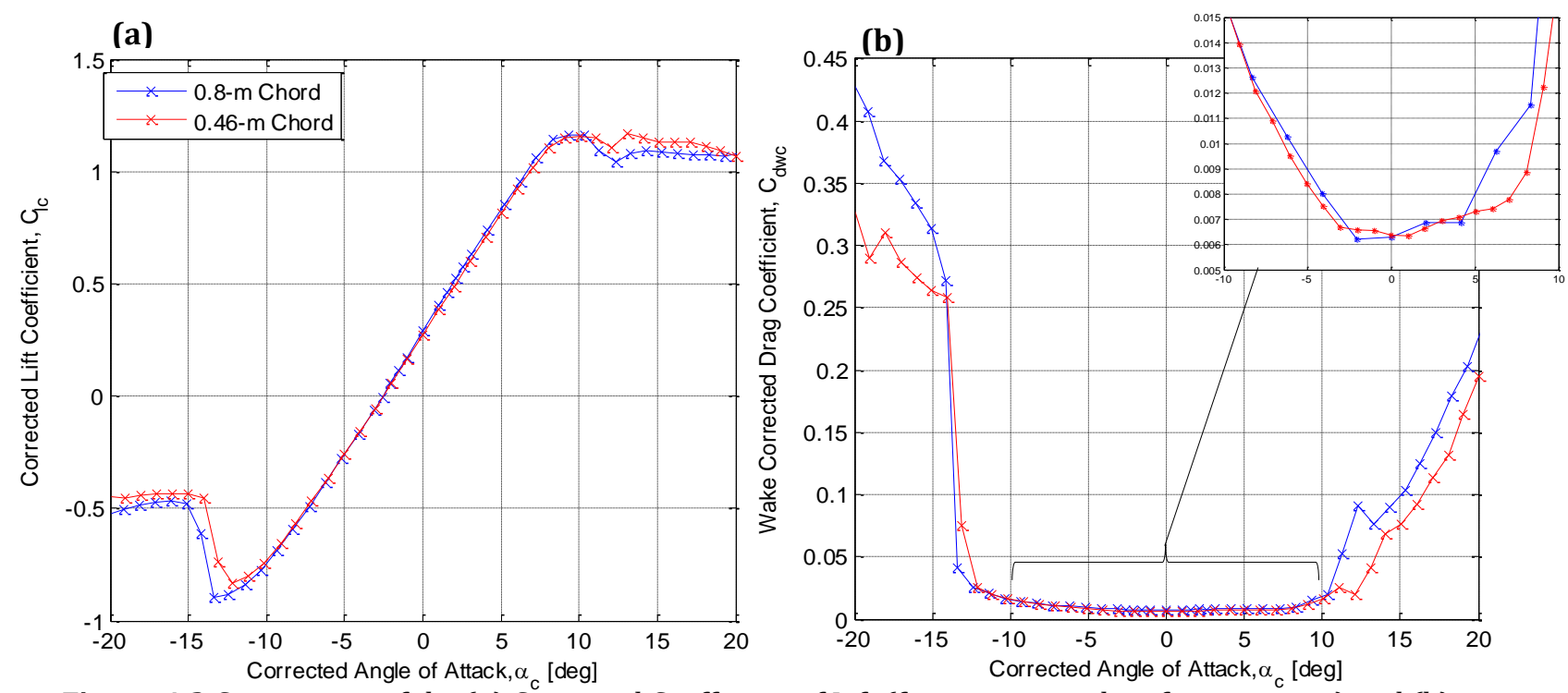

Figure 4.2 Comparison of the (a) Corrected Coefficient of Lift (from integrated surface pressure) and (b)

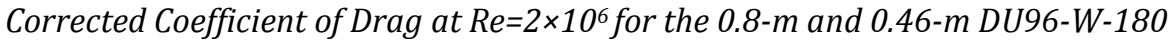


almost identical for both chord lengths. Both slopes are again $0.044 \mathrm{dg}^{-1}$. The drag comparison in Figure $4.2 \mathrm{~b}$ similarly exhibits close agreement for both models. In fact, in the horizontal region of the plot between the stall angles there is an average difference of $5.6 \%$ between the drag for the $0.8-\mathrm{m}$ and $0.46-\mathrm{m}$ chord. Outside the linear region, there is some variation in the stall patterns as drag increases. Small differences in both lift and drag are not uncommon after stall occurs when three-dimensional effects are dominant. These variations may also be due to uncertainty in measurements and repeatability of experiments.

In order to determine how much of the variations observed in the lift and drag plots are due to repeatability and how much are due to actual changes in the flow or model conditions, a repeatability study was done. Repeatability tests were done for the $0.8-\mathrm{m}$ DU96-W-180 at $\operatorname{Re}=1.5 \times 10^{6}, \mathrm{Re}=2 \times 10^{6}$ and $\mathrm{Re}=3 \times 10^{6}$. Figure 4.3 shows the results for the $\mathrm{Re}=1.5 \times 10^{6}$ case, with the first run denoted by the blue line and the second run represented by the red line.
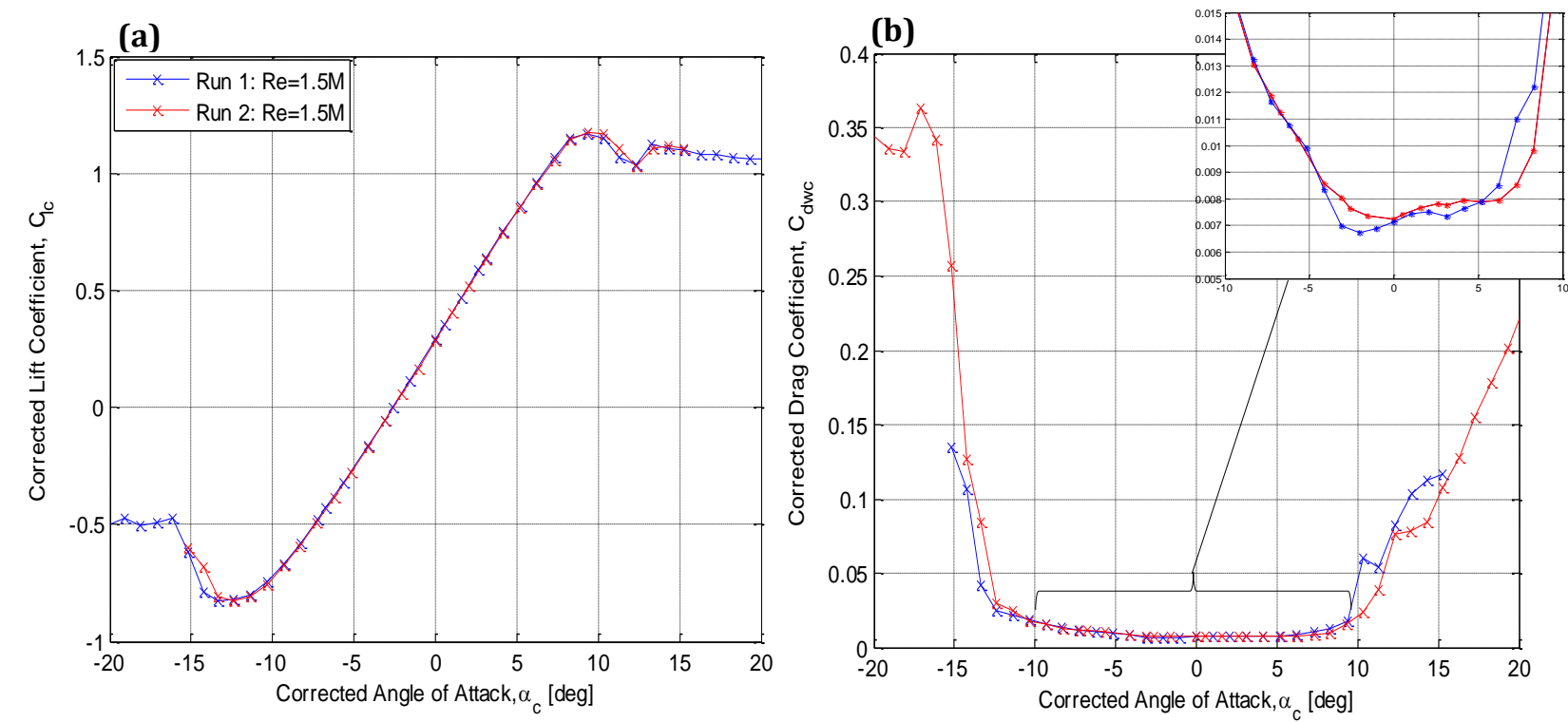

Figure 4.3 Corrected Coefficient of (a) Lift from integrated surface pressure) and (b) Drag at Re $=1.5 \times 10^{6}$ for two Runs of the 0.8-m DU96-W-180

Studying Figure 4.3, it is seen that there are variations in both the lift and drag distributions between the two runs. The variations occur in the region after stall occurs, as was observed in Figure 4.1 and Figure 4.2, while the region between stall angles show almost identical results. Variations for the lift appear to be on the order of $\mathrm{C}_{1} \approx 0.01$ or $1.4 \%$ in the linear region. The maximum error observed occurs after stall when differences of $\mathrm{C}_{1} \approx 0.1$ or $14 \%$ are observed - but this occurs only at one angle of attack. The drag variations are harder to quantify as they vary extensively after stall. The average difference due to repeatability in this region is $9 \%$ but variations can be larger at some angles of attack. However, in the horizontal region between stall angles, there is close agreement for both runs with an average difference of $5 \%$. Therefore, it can be said that the linear region is most important 
when examining performance data, and this region should be the focus of any analysis conducted. The extent of the repeatability effects will become increasingly important as the effect of the infrared system is explored.

\subsection{Effect of Infrared System on Airfoil Performance}

Before an in depth examination of the transition results is undertaken, the effect that each component of the infrared transition detection system has on the flow must be determined. Heating a model can change the behaviour of the boundary layer and affect the overall flow on the model. Adding an insulator to the model adds thickness to the profile and thus, can change the performance. To ascertain the extent of these effects, the corrected lift (obtained from integrating the surface pressure) and corrected drag distributions for the clean 0.8-m DU96-W-180 will be compared with those obtained from the insulated or heated model.

\subsubsection{Effect of Heated Model}

The clean 0.8-m DU96-W-180 was heated and maintained at about 5-K to $6-\mathrm{K}\left(10^{\circ} \mathrm{F}\right)$ above the temperature of the flow. Pressure data was taken for angles between $-20^{\circ}$ and $20^{\circ}$ for three Reynolds Numbers: $1.5 \times 10^{6}, 2 \times 10^{6}$, and $3 \times 10^{6}$. These results were then compared to those obtained from the clean, unheated model in order to determine the effect of heating the model. The lift and drag data from these pressure measurements were compared and is presented in Figure 4.4.

Figure 4.4a-b presents the corrected coefficient of lift (from surface pressure) curves and the corrected coefficient of drag plots, respectively, for the heated and unheated 0.8-m DU96-W-180 at a Reynolds number of $1.5 \times 10^{6}$. The corrected angle of attack is shown on the $\mathrm{x}$-axis and the corrected lift coefficient and corrected drag is plotted on the $\mathrm{y}$-axis. The blue markers indicate the data obtained from the unheated, clean model while the red markers represents the data obtained from the heated model. The unheated lift and drag curves in items (a) and (b) of this plot are the same as those presented in Figure 4.1 and discussed in depth in Section 4.1; there is a clear linear region in the lift and a flat region in the drag occurring from $\alpha_{c} \approx-13^{\circ}$ to $\alpha_{c} \approx 10^{\circ}$ and a non-linear region outside these angles. The lift curve for the heated data in Figure $4.4 a$ agrees well with the data from the clean airfoil. In the linear region, there is no indication of disagreement as both curves align very well having a slope of $0.044 \mathrm{dg}^{-1}$. In the non-linear region there is some small divergence observed, approximately 7\% error. These differences are small enough to be attributed to uncertainty in pressure measurements and the repeatability effects discussed at the end of Section 4.1. 
(a)

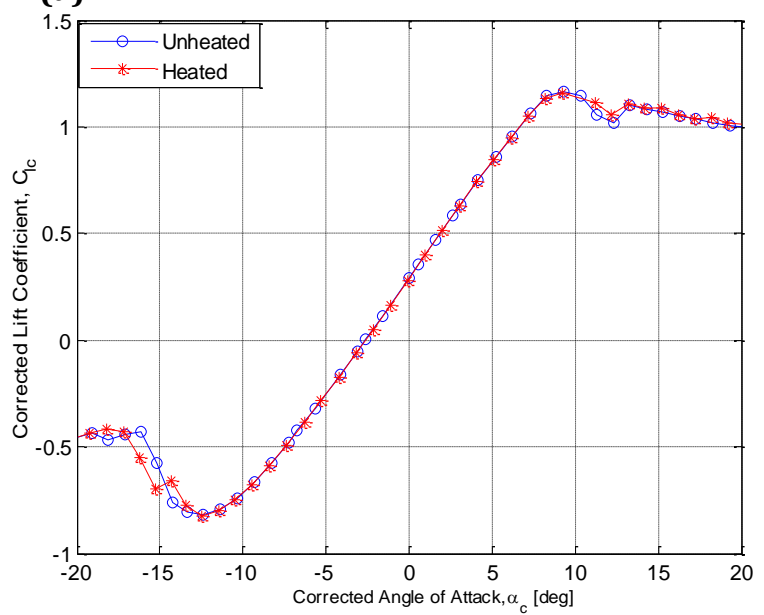

(c)

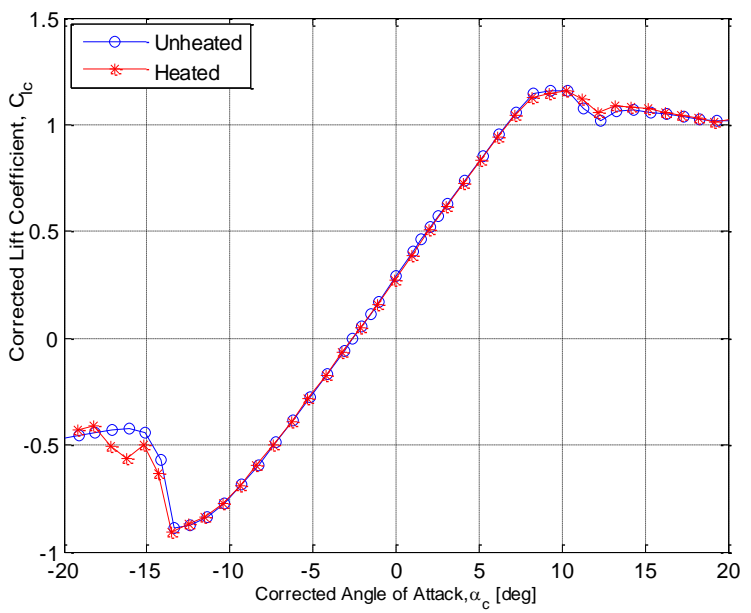

(e)

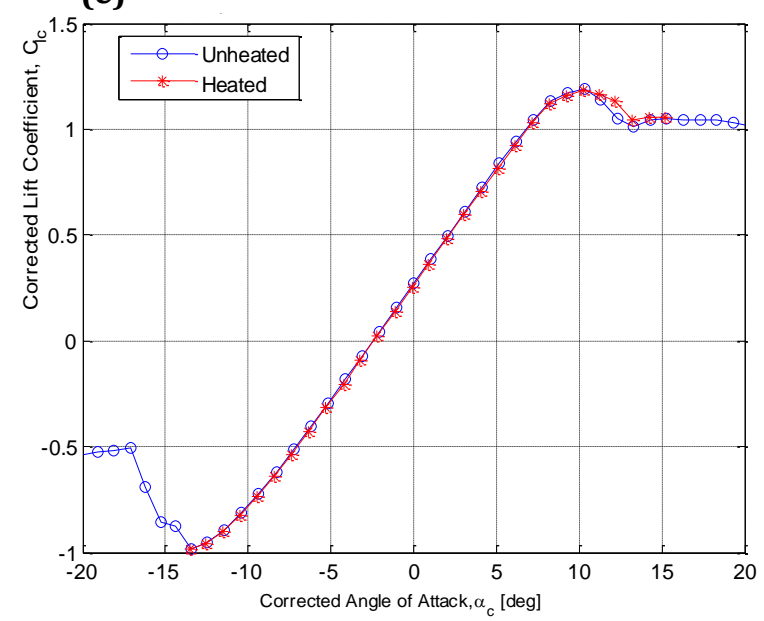

(b)
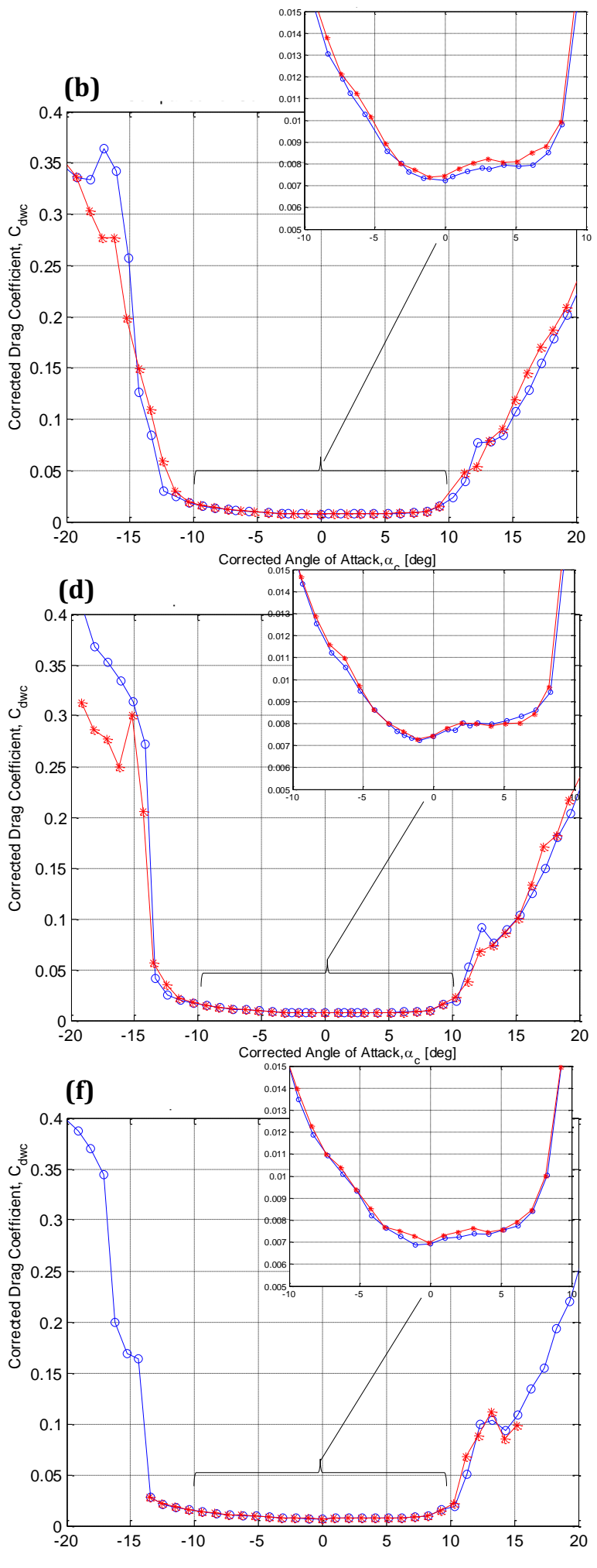

Figure 4.4 Effect of Heating on (a) Corrected Coefficient of Lift at Re $=1.5 \times 10^{6}$ (b) Corrected Coefficient of Drag at $R e=1.5 \times 10^{6}(\mathrm{c})$ Corrected Coefficient of Lift and Drag at Re $=2 \times 10^{6}(\mathrm{~d})$ Corrected Coefficient of Drag at $R e=2 \times 10^{6}(\mathrm{e})$ Corrected Coefficient of Lift Re $=3 \times 10^{6}(\mathrm{f})$ Corrected Coefficient of Drag at Re $=3 \times 10^{6}$ for the 0.8- $\mathrm{m}$ DU96-W-180 
Similarly to the results for the lift, the drag coefficient curves graphed in Figure $4.4 b$ also exhibit close agreement with each other. In the flat region there appears to be no differences between the heated and unheated data while there exists some variations in the non-linear region - 5\% error on an average. Figure 4.4c-f, which show the lift and drag distributions at higher speeds, also show excellent agreement within the linear regions. In the non-linear regions there are again small variations, typically $4 \%$ or less. What is interesting here is that the errors become smaller as the flow speed (and therefore Reynolds Number) is increased. This is seen even in the regions between stall angles (see magnified drag plots) where there is closer agreement at higher Reynolds Numbers. This variation strongly suggests that errors are primarily due to uncertainties in the pressure measurements. Repeatability effects also add to these variations.

As a final check, the half span cross sections through the wake of the airfoil (measured by drag rake) were analysed to ascertain the effects of heating the model on the wake. Cross sections for the $0.8-\mathrm{m}$ DU96-W-180 at $\mathrm{Re}=1.5 \times 10^{6}$ and $\alpha=-5^{\circ}$ and $0^{\circ}$ are shown in Figure 4.5 as contour plots of the pressure distribution in the wake. The span-wise distance of the test

(a) $\alpha=-5^{\circ}$

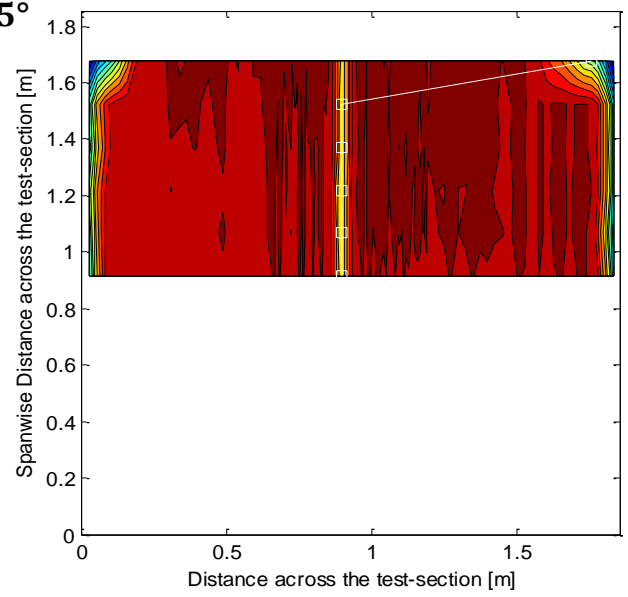

(b) $\alpha=0^{\circ}$

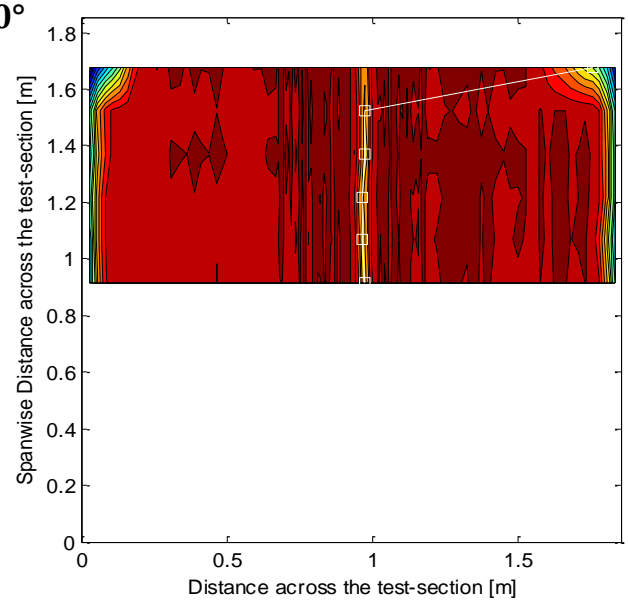

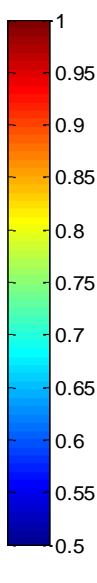

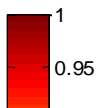

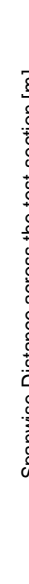
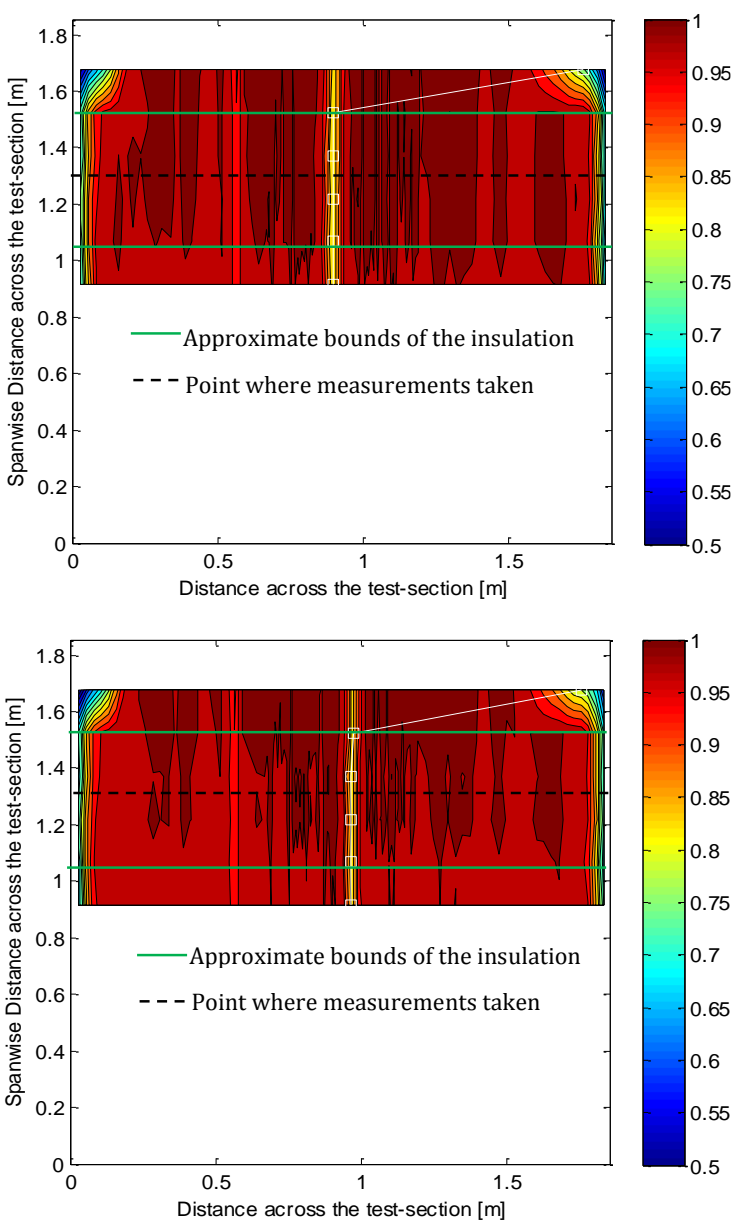

Figure 4.5 Comparisons of the Wake Cross Sections for the Clean, Unheated 0.8-m DU96-W-180 (left) and the Clean, Heated Model (right) at Re $=1.5 \times 10^{6}$ and (a) $\alpha=-5^{\circ}$ and (b) $\alpha=0^{\circ}$ 
section (vertical height) is on the y-axis and distance across the test section (width of test section) is on the x-axis; as a result each plot provides a scaled view from downstream looking upstream at the wake of the airfoil. The test section wall boundary layers can be seen as reduced pressure regions at the left and right edges of each plot. Likewise the meshing of the boundary layers of the test section walls and roof form the pressure 'bubbles' at the top left and right corners of the plots. The airfoil wake is the vertical band of lower pressure close to the middle of the plot, the centre of which is delineated by a white vertical line. The results of this analysis proved that heating the model has no significant effect on the wake because the wake for the clean, unheated model is seen to be identical to the wake for the clean, heated model for both angles. This was also the case at $\mathrm{Re}=2 \times 10^{6}$ and $\mathrm{Re}=3 \times 10^{6}$, the results of which are not detailed in this thesis.

These results suggest that heating the model to $5-\mathrm{K}$ or $6-\mathrm{K}$ does not change the boundary layer such that the performance of the airfoil is affected. Therefore, the results obtained when the model is heated are valid and representative of the airfoil performance and no other variable.

\subsubsection{Effect of Silicone Rubber Insulator}

Figure 4.6 shows the relative thickness of the insulator on the normalized DU96-W180.

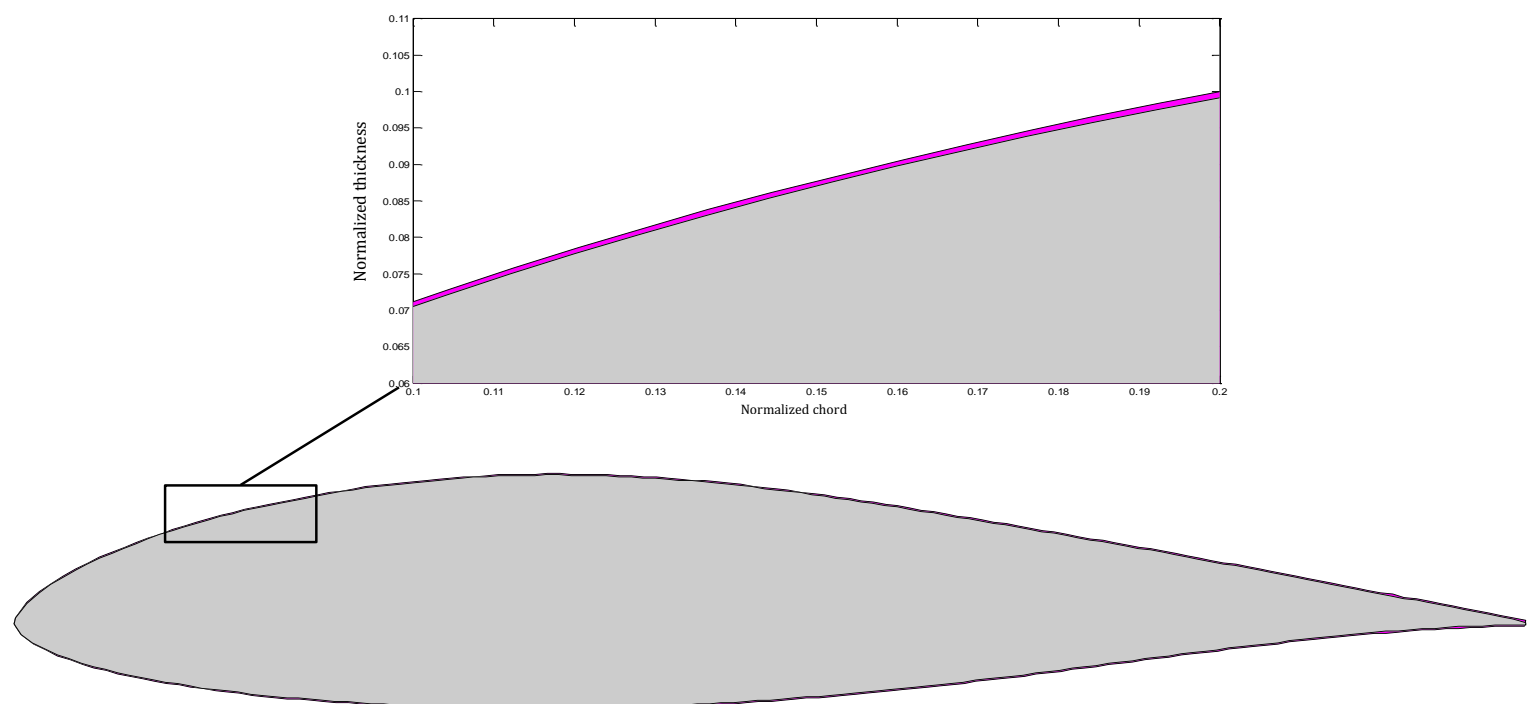

Figure 4.6 Normalized Profile of DU96-W-180 showing the Insulator Thickness Relative to the Model Thickness

A $0.45-\mathrm{m}$ section above mid-span of the $0.8-\mathrm{m}$ DU96-W-180 was covered in $0.8-\mathrm{mm}$ thick, black FDA-Compliant silicone rubber from McMaster-Carr, shown in Figure 3.38. Pressure data was taken for angles between $-20^{\circ}$ and $20^{\circ}$ for three Reynolds Numbers: $1.5 \times 10^{6}, 2 \times 10^{6}$, and $3 \times 10^{6}$. These results were then compared to those obtained from the 
clean model in order to determine the effect of adding the insulator to the model. The comparison is presented in Figure 4.7. The corrected angle of attack is shown on the x-axis and the corrected lift coefficient is plotted on the y-axis. The blue line indicates the data obtained from the clean model while the red line represents the data obtained from the insulated model.

Figure 4.7a-b portray the coefficient of lift curves and the coefficient of drag plots, respectively, for the clean and insulated 0.8-m DU96-W-180 at a Reynolds number of $1.5 \times 10^{6}$. The two lift curves in graph (a) show agreement in the linear region with both curves having a slope of $0.044 \mathrm{dg}^{-1}$. In graph (b) the two drag plots likewise show great agreement with each other. After stall, in the non-linear region, there is less agreement between the curves. For both the lift and drag the difference between the plots is typically $5 \%$ or less. Comparisons for higher Reynolds Numbers are denoted in items (c) through (f). All the coefficient of lift plots show very close agreement in the linear region and small divergence in the non-linear region, typically $3 \%$ or less. The drag plots show a similar trend in that they agree closely between stall angles and show small variation in the non-linear region, on average $5 \%$ or less. As with the heated/unheated comparisons, it is observed that errors diminish with increasing flow speed, suggesting the errors are due to uncertainties in the pressure measurement. Errors are also in part due to repeatability effects.

As with the study on heating effects, the half span cross sections through the wake of the airfoil were analysed to ascertain the effects of the insulator. The results for the $0.8-\mathrm{m}$ DU96-W-180 at $\mathrm{Re}=1.5 \times 10^{6}$ and $\alpha=-5^{\circ}, 0^{\circ}$ and $5^{\circ}$ are shown in Figure 4.8. The wake of the clean, unheated model appears identical to the wake of the insulated, unheated model in Figure 4.8a, at $\alpha=-5^{\circ}$. At $\alpha=0^{\circ}$, shown in Figure 4.8b, there is an increase in width of the wake around the junction between the bottom of the insulator and the bare model. This is expected due to the folding of the flow over the step created by the 0.8 - $\mathrm{mm}$ insulator. What is important is that the wake profile in the region where the bulk of the insulator is installed (including at 1.32-m where pressure measurements was actually taken) is approximately the same as that over the bare model. In other words, the increases in thickness of the wake are localized to the junction between the insulator and the clean model. This is also observed in Figure 4.8c, at $\alpha=5^{\circ}$, where growth in the wake profile is evident at both the top and bottom junctions of the insulator and bare model. This is expected because on the suction side of the model, at positive angles of attack, the separation effects of the step are more pronounced. Again, the wake width at the point where measurements are taken is the same as that over the bare model suggesting that the insulator does not affect the flow in this region of interest. The behaviour described in Figure 4.8 was also observed at $\operatorname{Re}=2 \times 10^{6}$ and $\operatorname{Re}=3 \times 10^{6}$, but is not presented here. 

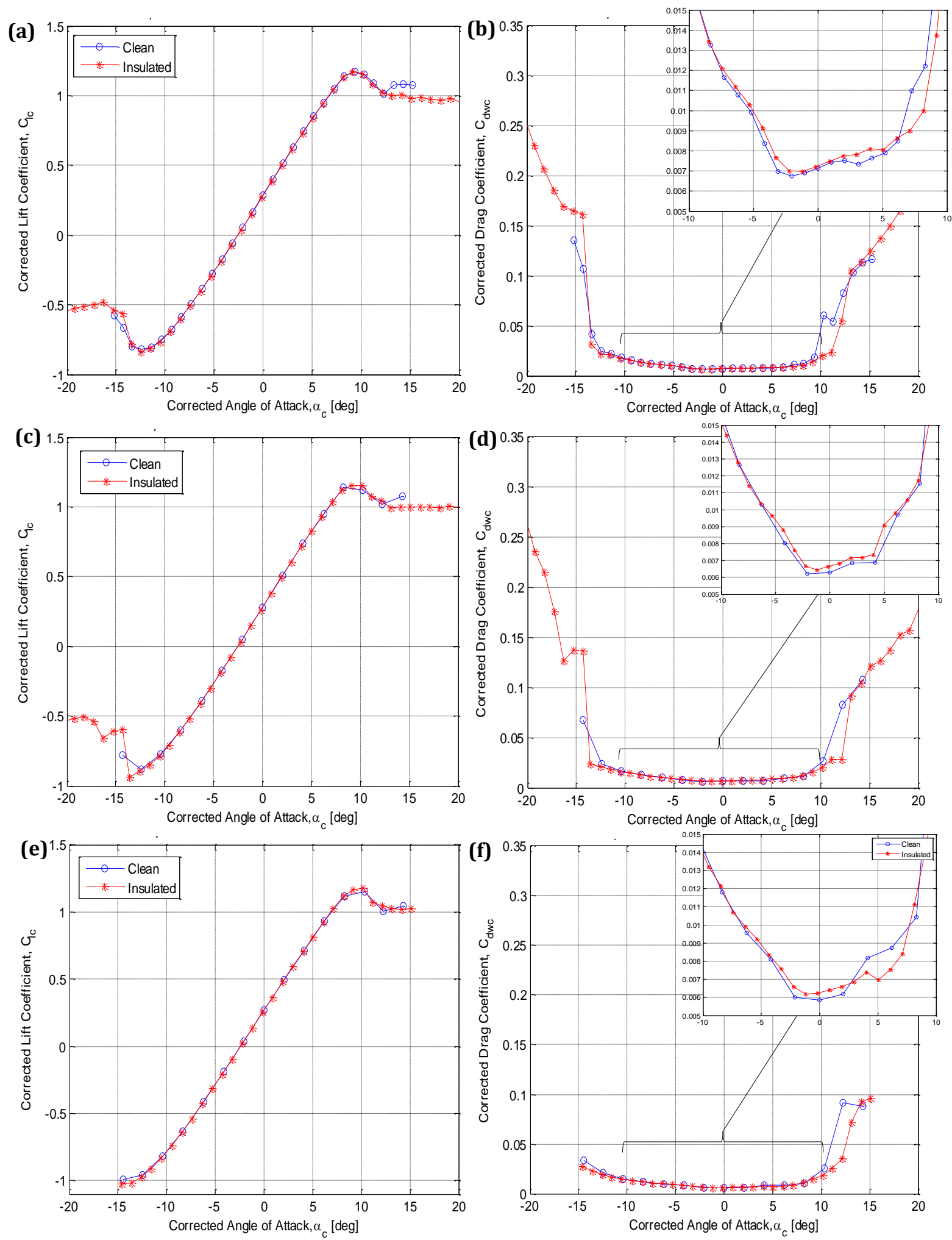

Figure 4.7 Effect of Insulator on (a) Corrected Coefficient of Lift at Re $=1.5 \times 10^{6}$ (b) Corrected Coefficient of Drag at Re $=1.5 \times 10^{6}$ (c) Corrected Coefficient of Lift at Re $=2 \times 10^{6}(\mathrm{~d})$ Corrected Coefficient of Drag at Re $=2 \times 10^{6}$ (e) Corrected Coefficient of Lift Re $=3 \times 10^{6}(f)$ Corrected Coefficient of Drag at Re $=3 \times 10^{6}$ for the 0.8-m DU96-W- 
(a) $\alpha=-5^{\circ}$

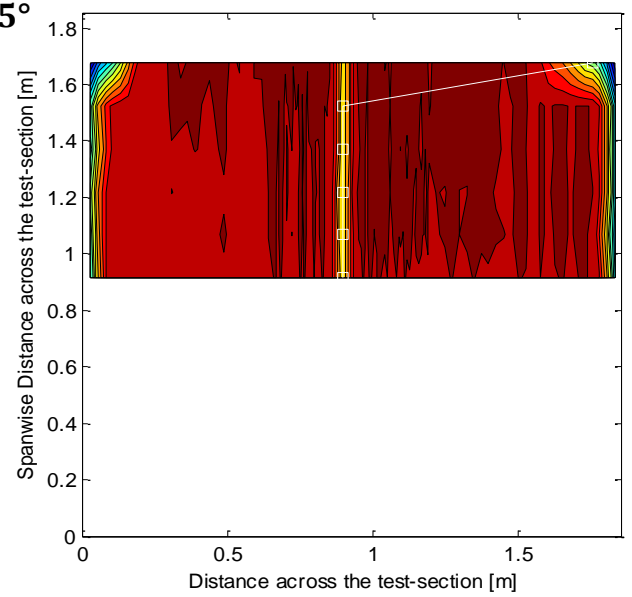

(b) $\alpha=0^{\circ}$

(c) $\alpha=5^{\circ}$

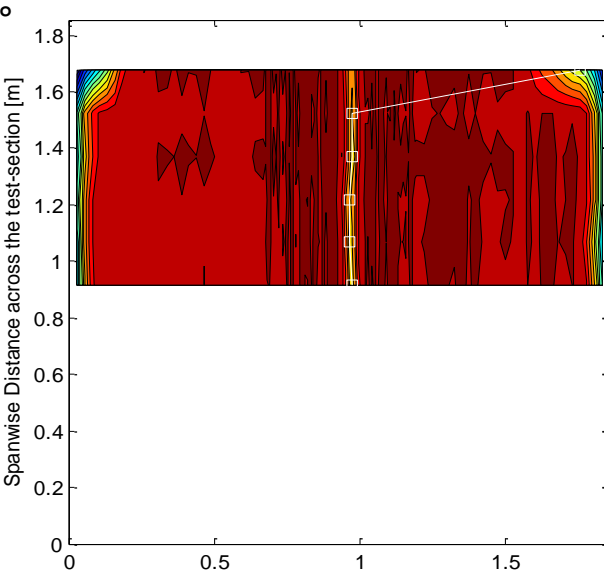

Distance across the test-section [m]

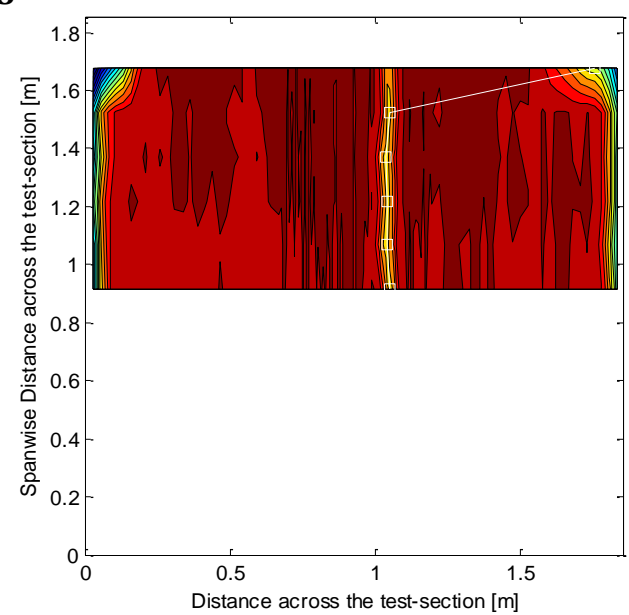

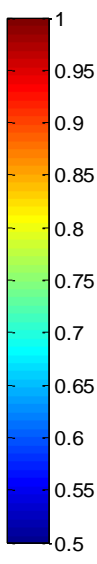
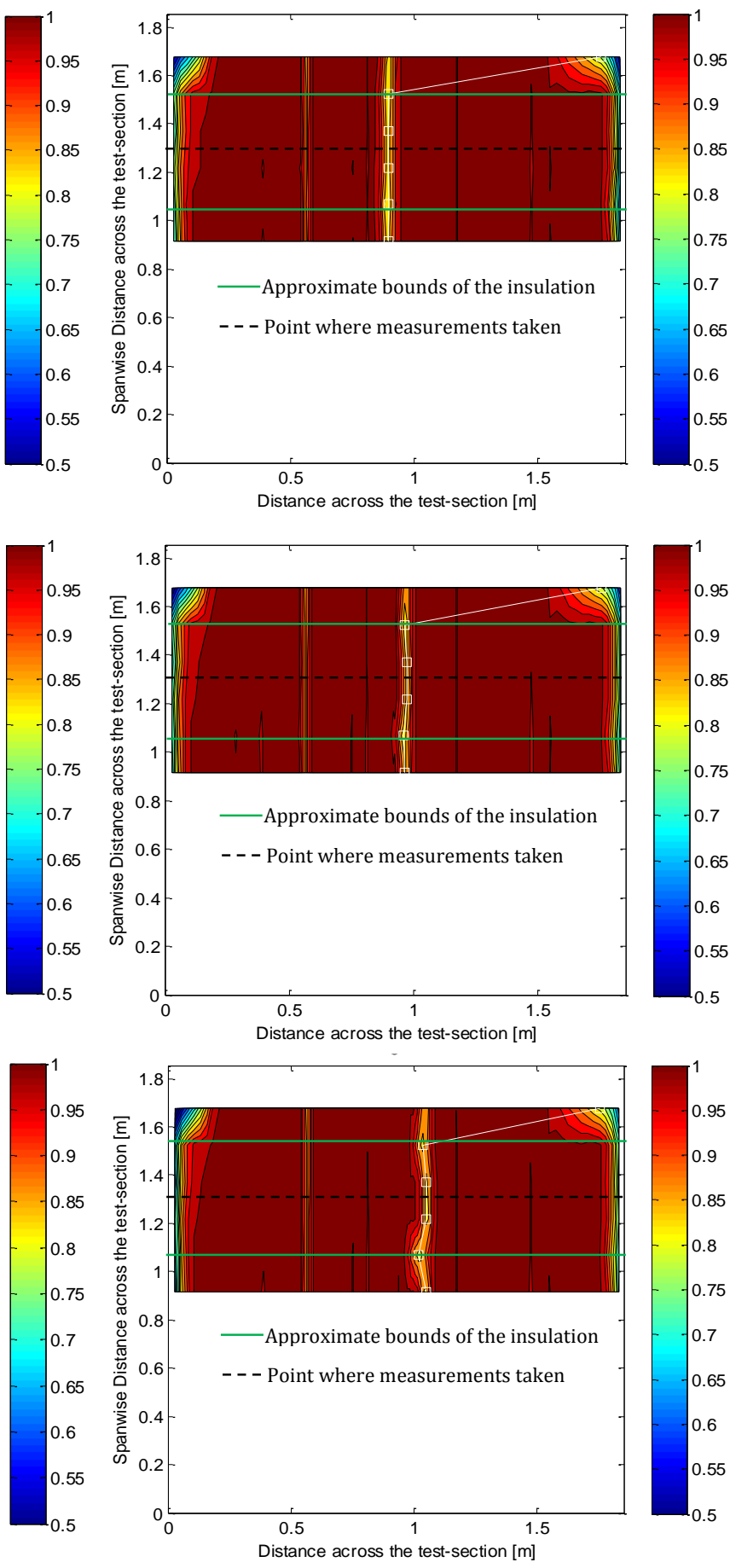

Figure 4.8 Comparisons of the Wake Cross Sections for the Clean, Unheated 0.8-m DU96-W-180 (left) and the Insulated, Unheated Model (right) at $\operatorname{Re}=1.5 \times 10^{6}$ and (a) $\alpha=-5^{\circ}$ (b) $\alpha=0^{\circ}$ and (c) $\alpha=5^{\circ}$ 
The close agreement between both the lift and drag plots for the insulated and clean 0.8-m DU96-W-180, at three Reynolds Numbers, suggests that the 0.8-mm insulator has negligible effect on the performance of the model. This is further proven by the agreement of the wake data for the clean and insulated model. Therefore, it can be stated that using the 0.8-mm silicone rubber as part of the infrared transition detection system will not affect the results of the system.

\subsubsection{Combined Effect of Heated Model and Silicone Rubber Insulator}

After the effects of the FDA Compliant rubber silicone insulator and heating the model were proven to be negligible on their own, a test was conducted to determine the effect of both components on airfoil performance. This entailed heating the insulated $0.8-\mathrm{m}$ chord DU96-W-180 to approximately 5-K - 6-K and measuring pressure over the chord. Since the pressure taps are located at least 12.4-mm lower down the span than the insulated region, it was possible to take useful pressure measurements while the transition system was installed and in use.

The corrected lift and corrected drag plots are shown in Figure $4.9 a-b$ for $\mathrm{Re}=$ $1.5 \times 10^{6}$. Examining these plots it appears that using the insulator and the heaters simultaneously does not have any significant effect on the flow, as was the case with the individual components. The agreement between the baseline and the heated/insulated results is very good. The results at $\operatorname{Re}=2 \times 10^{6}$ and $3 \times 10^{6}$ are presented in Figure $4.9 c-f$ and these also show good agreement between the clean, unheated model and the heated, insulated model. This agreement at three Reynolds Numbers leads to the conclusion that the transition detection system does not negatively impact the performance of the DU96-W-180 and therefore, any temperature gradients observed are due to flow phenomenon and not effects of the system itself. Furthermore, the fact that the airfoil tap pressure measurements made when the infrared system is installed are the same as that when the infrared system is not installed means that both pressure and transition measurements can be made at the same time. 

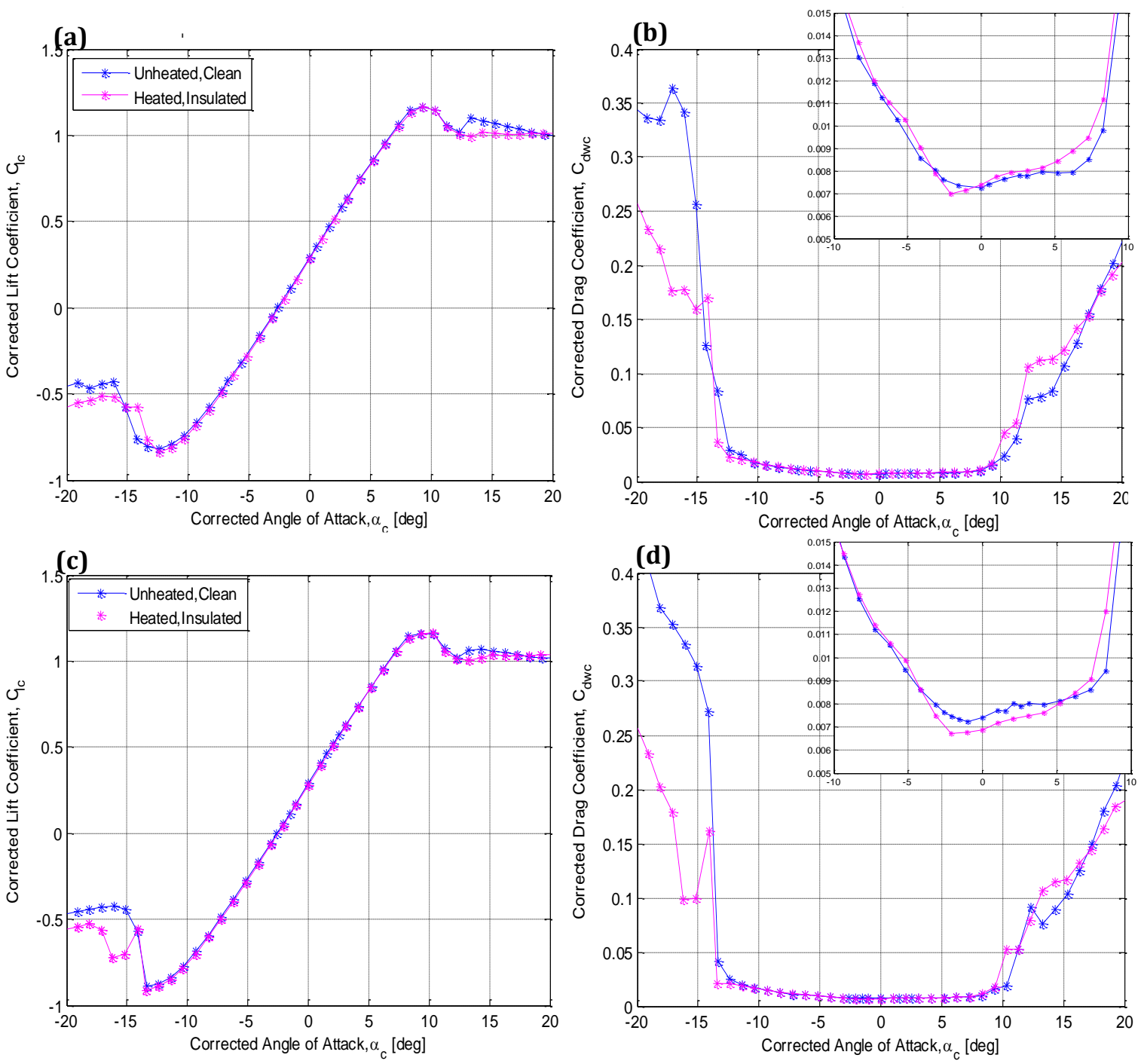

(e)
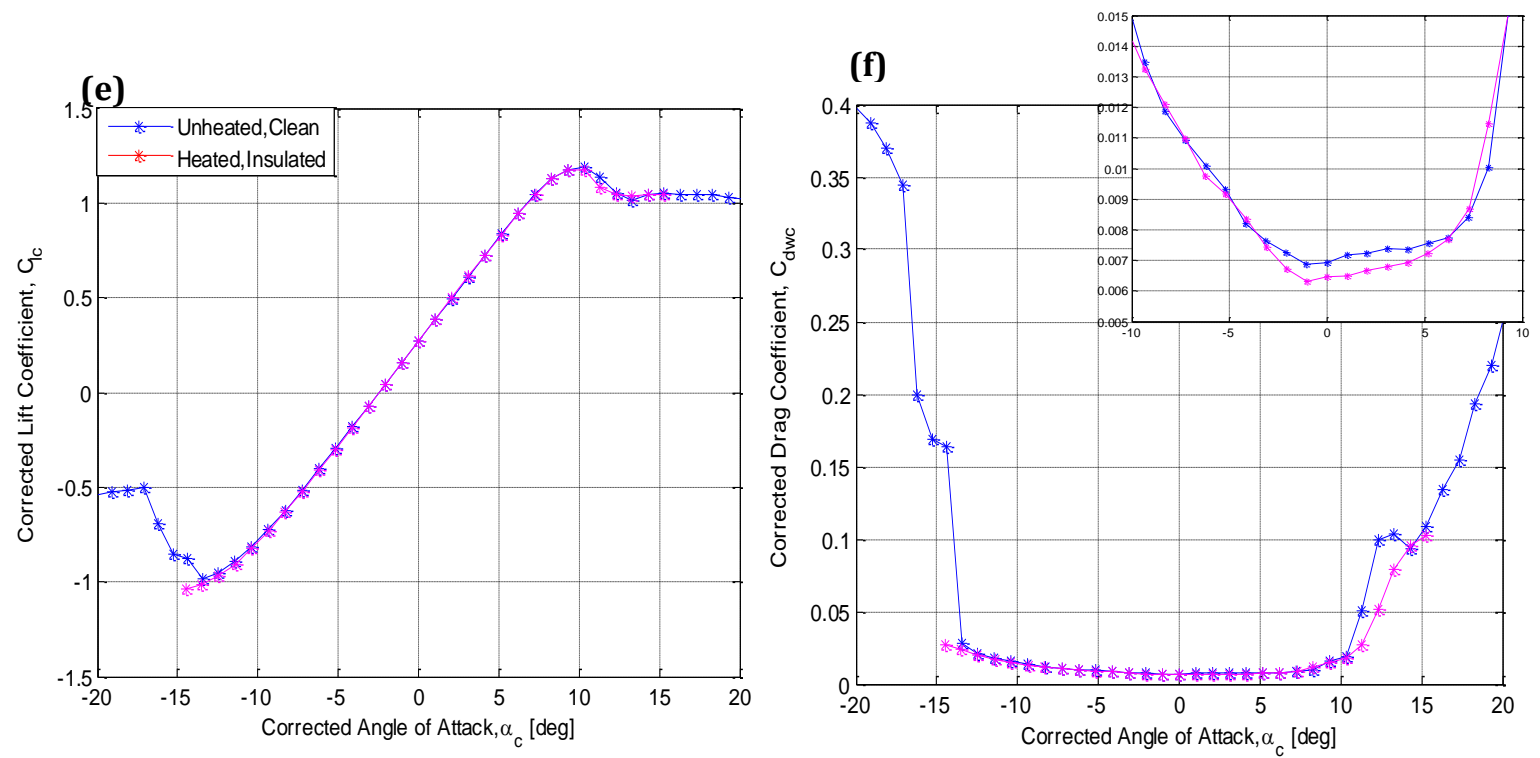

Figure 4.9 Combined Effects of Insulation and Heat on (a) Corrected Coefficient of Lift at Re $=1.5 \times 10^{6}$ (b) Corrected Coefficient of Drag at Re $=1.5 \times 10^{6}$ (c) Corrected Coefficient of Lift at Re $=2 \times 10^{6}$ (d) Corrected Coefficient of Drag at Re $=2 \times 10^{6}$

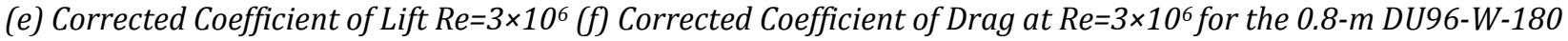




\subsection{Transition Detection Results}

Transition results obtained during the Phase Two study, from the infrared thermography system, mean pressure analysis and stethoscope sound levels will be described in this section. XFOIL was used as a prediction method for the transition results. XFOIL predicts natural transition using the simplified envelope version of the $\mathrm{e}^{\mathrm{N}}$ criterion. For this wind tunnel study the user-specified parameter (log of the amplification factor of the most-amplified frequency which triggers transition) was set to 8 . The results obtained from XFOIL will be used as a third validation method for the infrared thermography transition detection system and will be a guide when analysing the results of all methods. It should be noted that XFOIL is known to be unreliable outside the region between positive and negative stall because of increased viscous behaviour which it cannot numerically take into account. This must be kept in mind when analysing the results observed in this region, or in any region where viscous effects begin to grow and become significant.

\subsubsection{Mean Pressure Results}

Transition causes a sharp drop in the -Cp distribution that can be characterized by a peak in its second derivative. Therefore, second derivative of the mean pressure will be investigated for the $0.8-\mathrm{m}$ and the $0.46-\mathrm{m}$ DU96-W-180 models, at three Reynolds Numbers: $1.5 \times 10^{6}, 2 \times 10^{6}$, and $3 \times 10^{6}$. It is important to note that the maximum range within which the transition can be detected by this method is $5 \%$, since this is the resolution of the pressure taps on the models.

Figure 4.10 shows the negative coefficient of pressure distribution on the suction side of the $0.8-\mathrm{m}$ DU96-W-180 at two angles, at $\mathrm{Re}=2 \times 10^{6}$. Normalized chord locations are plotted on the $\mathrm{x}$-axis and negative coefficient of pressure is plotted on the $y$-axis. The red circles indicate the pressure on the suction side of the model while the black curve is the second
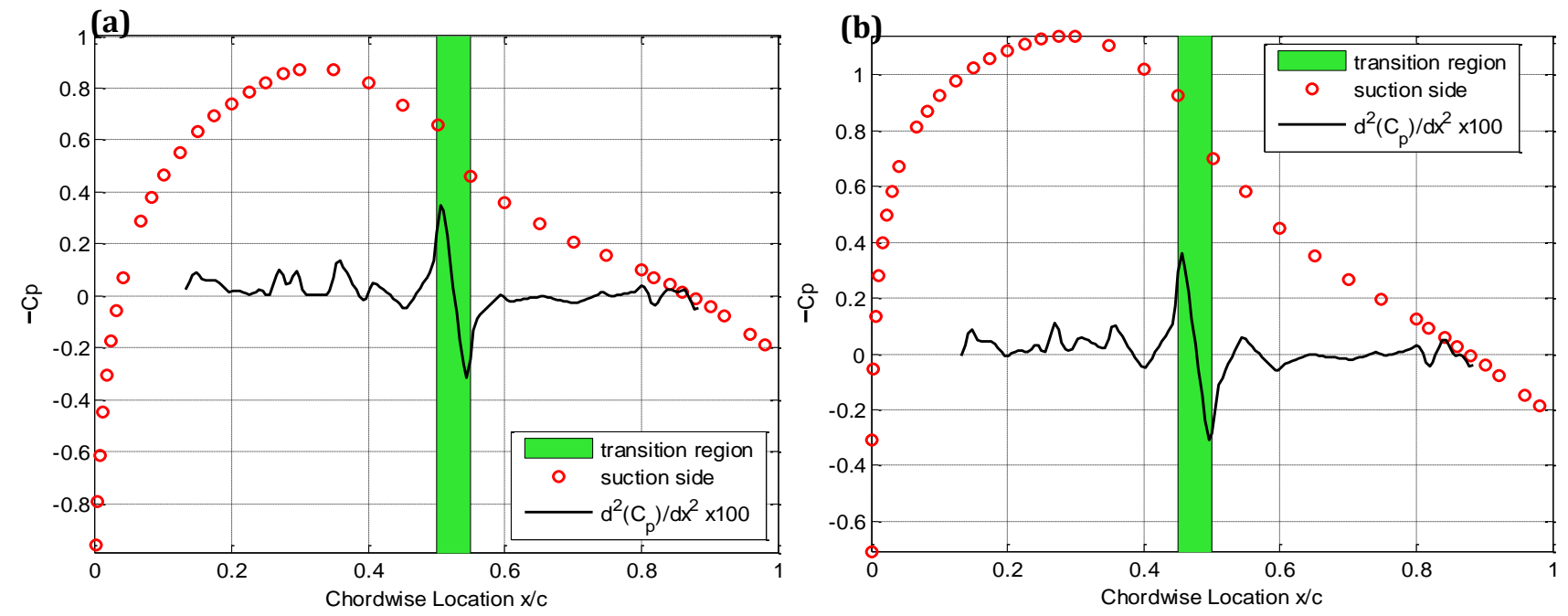

Figure 4.10 Coefficient of Pressure for Suction Side of 0.8-m DU96-W-180, at $\operatorname{Re}=2 \times 10^{6}$ and (a) $\alpha=0^{\circ}$ and (b) $\alpha=3^{\circ}$ 
derivative of that mean pressure, multiplied by 100 for scaling purposes. The green highlighted section is indicative of the $5 \%$ transition region coinciding with peaks in the second derivative and the maximum curvature in the mean pressure. Figure 4.10a shows the results obtained at $0^{\circ}$ angle of attack. In this graph, the -Cp curve rises smoothly from the leading edge to peak at the quarter chord location. It then begins to decrease smoothly until $50 \%$ chord when there is a sharp drop in the -Cp. After this drop, at 55\% chord, the $-\mathrm{Cp}$ continues to decrease smoothly until the trailing edge. This trend is reflected in the second derivative plot which shows a series of small peaks with the largest and most distinct peak occurring between $50 \%$ and $55 \%$ chord. This result suggests that natural transition for this model at $0^{\circ}$ occurs between $50 \%$ and $55 \%$ chord, within the green highlighted area.

Figure $4.10 \mathrm{~b}$ depicts the $-\mathrm{Cp}$ on the suction side of the model at $3^{\circ}$ and $\operatorname{Re}=2 \times 10^{6}$. Similarly to the case at $0^{\circ}$, the negative coefficient of pressure increases from the leading edge to a maximum at the quarter chord. After this point there is a smooth decrease until $45 \%$ chord when -Cp drops significantly. The decrease in - $\mathrm{Cp}$ then continues to the trailing edge. The -Cp drop in this plot again coincides with the peak of the second derivative of pressure plot. Hence, transition occurs between $45 \%$ and $50 \%$ of the chord at $3^{\circ}$ angle of attack. Since this result suggests that as angle of attack increases, transition on the suction side decreases which is the expected result. Therefore, this analysis was completed for all angles of attack on the suction side of the model, but the details of this are not shown in this report.

Figure 4.11 depicts the distribution of the negative coefficient of pressure over the pressure side of the $0.8-\mathrm{m}$ DU96-W-180, for two angles at $\mathrm{Re}=2 \times 10^{6}$. The normalized chord locations are plotted on the $\mathrm{x}$-axis and negative coefficient of pressure is plotted on the $y$ axis. Blue circles depict the - $\mathrm{Cp}$ on the pressure side of the model while the black curve is the second derivative of that pressure (multiplied by 100). The green highlighted section is the
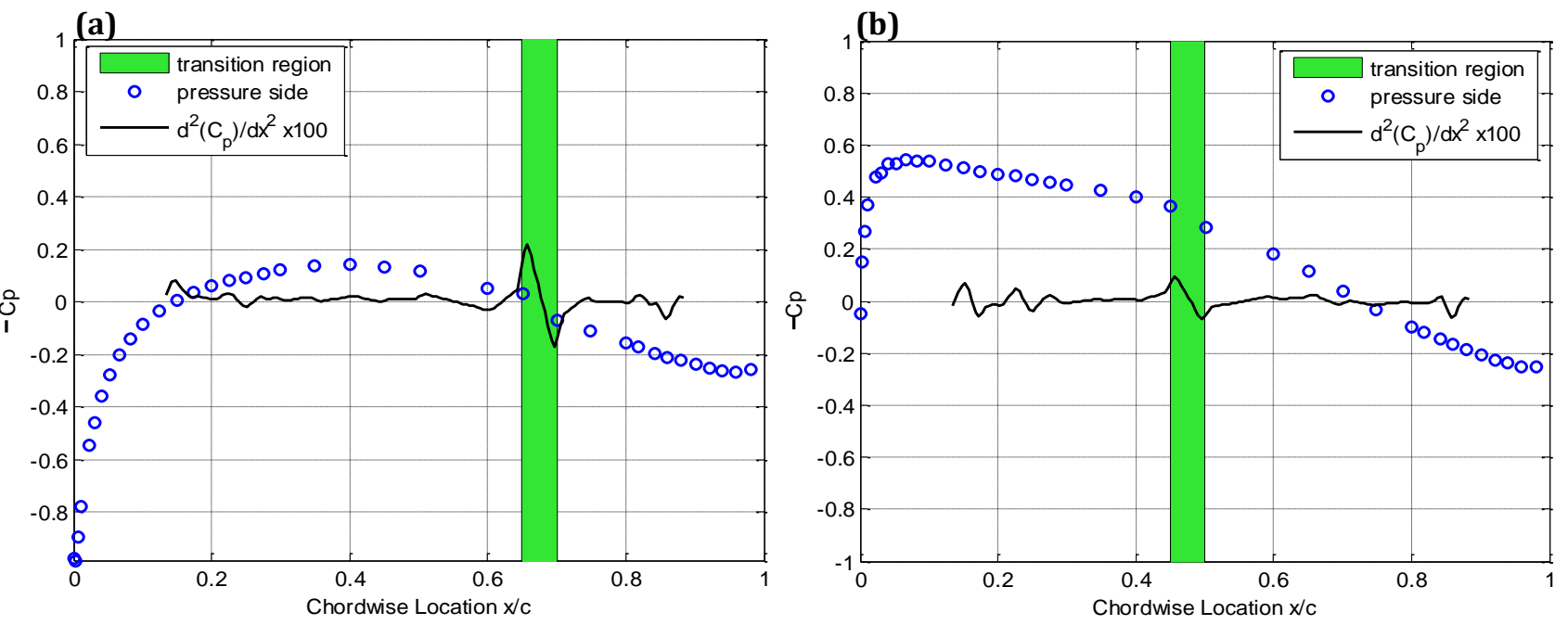

Figure 4.11 Coefficient of Pressure for Pressure Side of 0.8-m DU96-W-180, at $\operatorname{Re}=2 \times 10^{6}$ and (a) $\alpha=3^{\circ}$ and (b) $\alpha=-2^{\circ}$ 
$5 \%$ transition region. Results at an angle of attack of $3^{\circ}$ are presented in Figure 4.11a. This graph shows that the -Cp rises smoothly up to $40 \%$ chord at which point it decreases just as smoothly. At $65 \%$ chord there is a sharp drop which creates a peak in the second derivative plot. This is the transition region: $65 \%$ to $70 \%$. After this point the -Cp continues to decrease to the trailing edge. Plot (b) shows the -Cp distribution at $-2^{\circ}$ on the pressure side of the model. In this case, the -Cp rises up to the $10 \%$ cord location after which it begins to decrease smoothly. However, at $45 \%$ chord there is a distinct drop in the pressure distribution. This is the transition location, and is reflected in the second derivative plot by a clear peak. This analysis was repeated for the pressure side of the model for all angles of attack.

The results of the mean pressure analysis are shown in Figure 4.12; the suction side in part (a) and the pressure side in part (b). In this figure, the angle of attack is plotted on the $\mathrm{x}$-axis and the chord-wise location of transition is plotted on the $y$-axis. The blue lines denote the upper limit of the transition region indicated by the mean pressure results and the red line denotes the lower limit of this region. The black x's are indicative of the transition result obtained from XFOIL for the $0.8-\mathrm{m}$ DU96-W- 180 at Re $=2 \times 10^{6}$. Examining part (a) of Figure 4.12 , it is clear that the overall trend of the coefficient of pressure results for transition agrees with that produced by XFOIL: the transition location moves toward the leading edge as the angle of attack is increased. It is also observed that the XFOIL results for transition are within the limits ( $5 \%$ range) measured from the mean pressure analysis. Similarly, the overall trend of the plot in Figure $4.12 \mathrm{~b}$ agrees with that of the XFOIL predictions for the pressure side of the model: as angle of attack increases, the transition location moves toward the trailing edge. In fact, for most angles for which pressure results were obtained, the XFOIL results were well within the transition region predicted by the pressure analysis. At the angles between -10 and 10 when pressure results are not shown, there was no distinct peak in the second derivative of - $\mathrm{Cp}$, which is due to the limitations of this method discussed in Section 3.6.1. However, the fact that the results agree for both the pressure and suction side, for more than 10 angles on each side, strongly suggests that the transition results from mean pressure are accurate for the $0.8-\mathrm{m}$ DU96-W- 180 at $\mathrm{Re}=2 \times 10^{6}$.

Compiling all the mean transition results obtained from mean pressure, Figure 4.12c$e$ were produced. These figures show the variation of the transition location with angle of attack, at the two Reynolds numbers. It should be noted that results could not be obtained for several angles, mostly on the pressure side of the model, because no distinct -Cp drop could be detected. As discussed in Section 3.6.1, this is due to the location of transition between the pressure taps. For these reasons Figure $4.12 \mathrm{c}$ shows only the results on the suction side of the model at $\mathrm{Re}=1.5 \times 10^{6}$. Results could only be obtained on the pressure side at two angles and therefore a useful plot was not developed. However, the results on the suction side show excellent agreement with the predictions of XFOIL for transition. In fact, useful results were extracted at 13 angles of attack, all of which agree with the XFOIL results. 
(a)

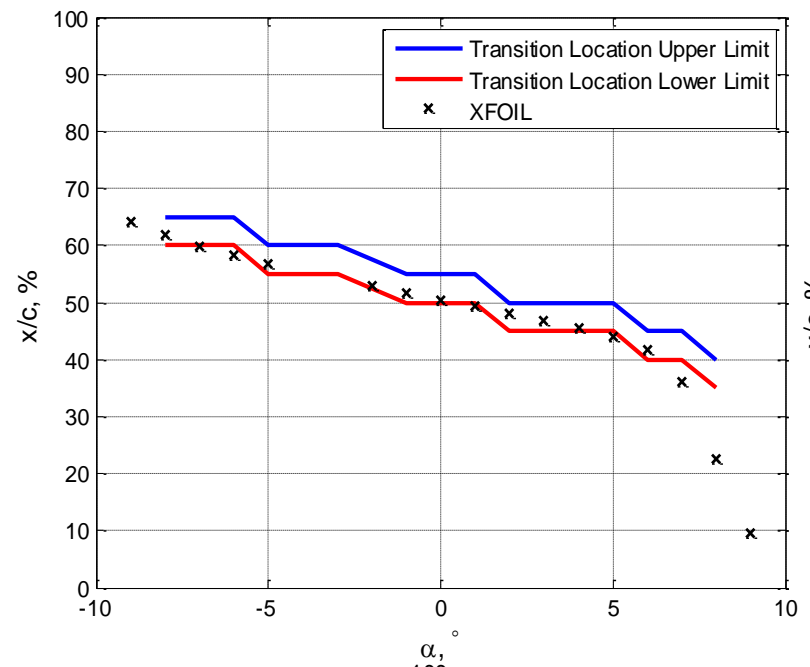

(b)

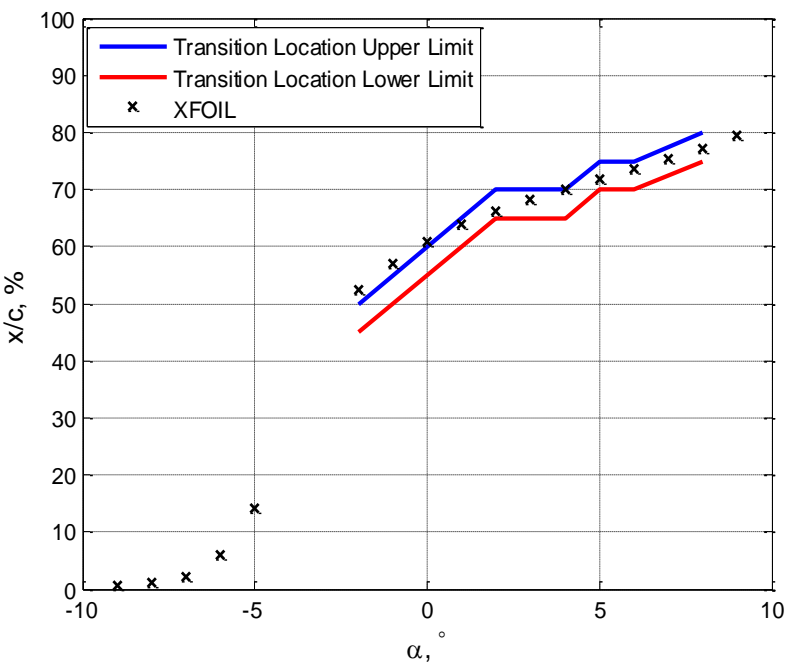

\section{(c)}

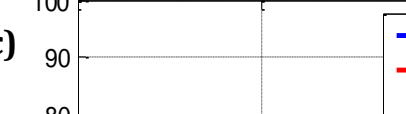

Transition Location Upper Limit

$\times \quad$ XFOIL

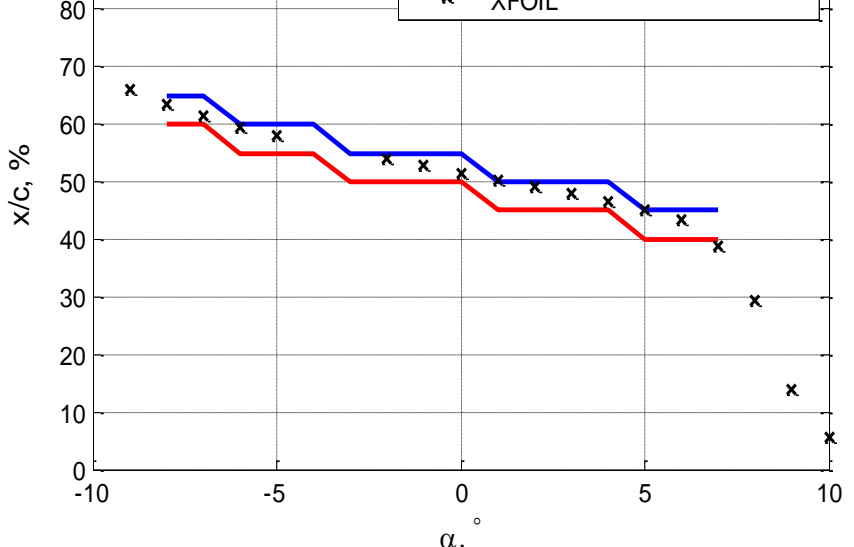

$\alpha$,
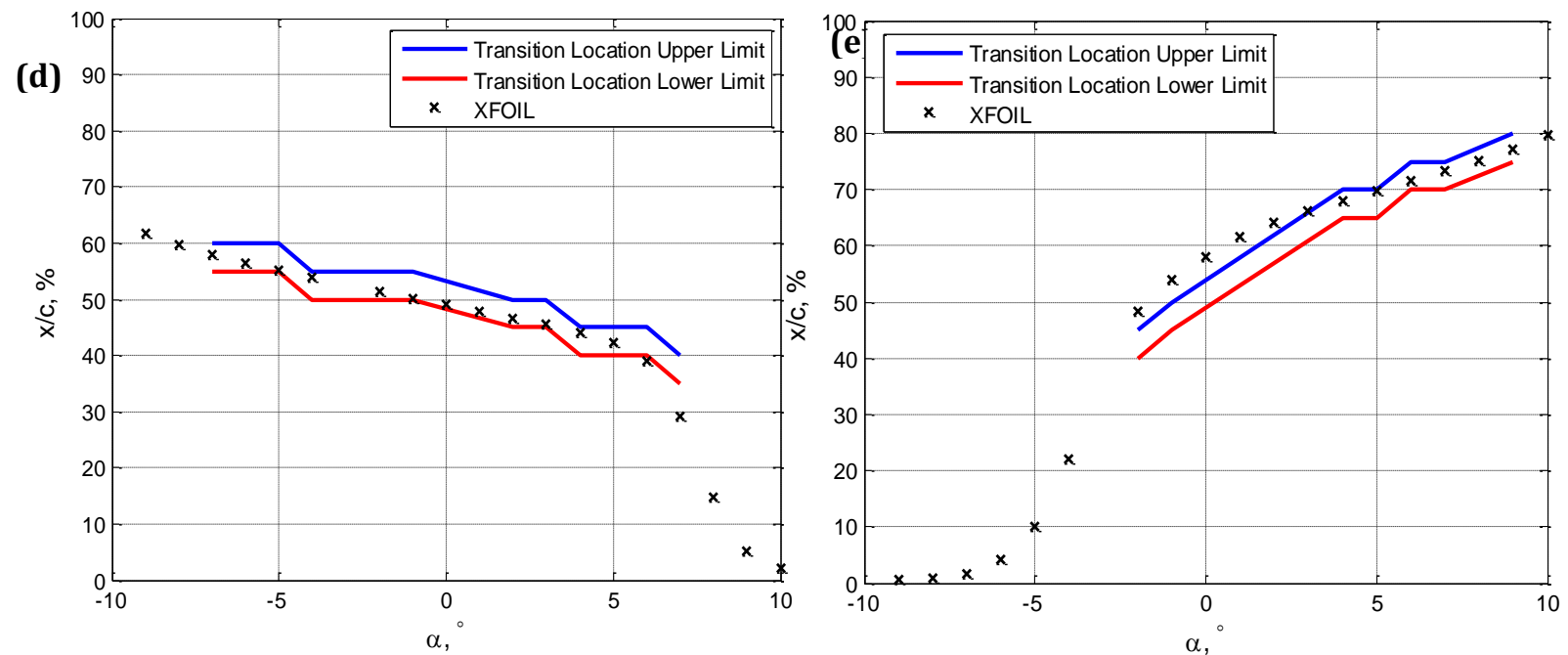

Figure 4.12 Coefficient of Pressure Prediction of Transition Range for 0.8-m DU96-W-180: (a) Suction Side at $R e=2 \times 10^{6}$ (b) Pressure Side at $R e=2 \times 10^{6}$ (c) Suction Side at $R e=1.5 \times 10^{6}$ (d) Suction Side at Re $=3 \times 10^{6}$ (b) Pressure Side at $\operatorname{Re}=3 \times 10^{6}$ 
While this speaks toward the limited range of usability of the mean pressure analysis method discussed in Section 3.6.1, it does prove that the results which are obtained can be trusted. The results at $\mathrm{Re}=3 \times 10^{6}$ in Figure 4.12d-e shows the same close agreement between XFOIL and the mean pressure results. On the suction side, shown in part (d) of the figure, the XFOIL predictions occur well within the mean pressure transition region for 13 angles. On the pressure side, there is less agreement between the two sets of data. While the XFOIL results for 7 angles are within the mean pressure transition range, the results for 5 angles are 2.5\% chord downstream of this range. These differences are most likely due the limitations of XFOILs ability to predict transition accurately, as discussed previously. However, variations can also be due to issues with the accuracy to which the mean pressure transition results can be given. The mean pressure results corresponds to the downstream edge of the transition. On the other hand, the XFOIL transition is the location when the turbulence levels become large enough to satisfy the $\mathrm{e}^{\mathrm{N}}$ criteria. This means that it is possible for the $5 \%$ chord range in which the mean pressure transition occurs to disagree with the results of XFOIL because fully turbulent flow develops in the downstream 5\% range, which would not be shown as a pressure drop. This occurrence is highly dependent on the model profile, the Reynolds Number (or speed) and the angle of attack. In cases where the XFOIL result is within 2.5\% chord of the limits of the mean pressure transition results, this is most likely what is occurring and does not necessarily mean that the mean pressure results are inaccurate.

To further ensure that the pressure data method is sound, mean pressure results for the 0.46-m DU96-W-180 are compiled and presented in Figure 4.13a- $d$ for $\mathrm{Re}=1.5 \times 10^{6}$ and $\mathrm{Re}=2 \times 10^{6}$. In both figures, angle of attack is on the $\mathrm{x}$-axis and chord-wise transition location is on the y-axis. The red lines again represent the lower limit of the $5 \%$ transition region while the blue lines represent the upper limit and the black x's are the XFOIL predictions. As with the $0.8-\mathrm{m}$ model, the transition location moves upstream on the suction side, at both Reynolds Numbers. Conversely, the transition location on the pressure side moves downstream with angle of attack. Furthermore, the XFOIL results agree with the mean pressure results (they fall within the upper and lower limits of the mean pressure result) at all but three of the angles of attack shown in Figure $4.13\left(2^{\circ}\right.$ and $4^{\circ}$ at $\operatorname{Re}=1.5 \times 10^{6}$ and $2^{\circ}$ at $\operatorname{Re}=2 \times 10^{6}$ ). As these discrepancies less than $2.5 \%$ chord, they are again most likely due to the limiting effects of the pressure tap location.

The mean pressure transition results have shown that for three Reynolds numbers, for both the suction and the pressure side of two models, and for several angles of attack, the mean pressure results agree with XFOIL predictions. This proves that XFOIL results for transition are likely accurate within 5\% chord (the resolution of the pressure taps). It is important to note that the mean pressure analysis method is not able to produce results at every angle of attack nor is it able to give results more accurately than $5 \%$ chord of the actual transition region. However, when it does produce results they appear to be reliable. 


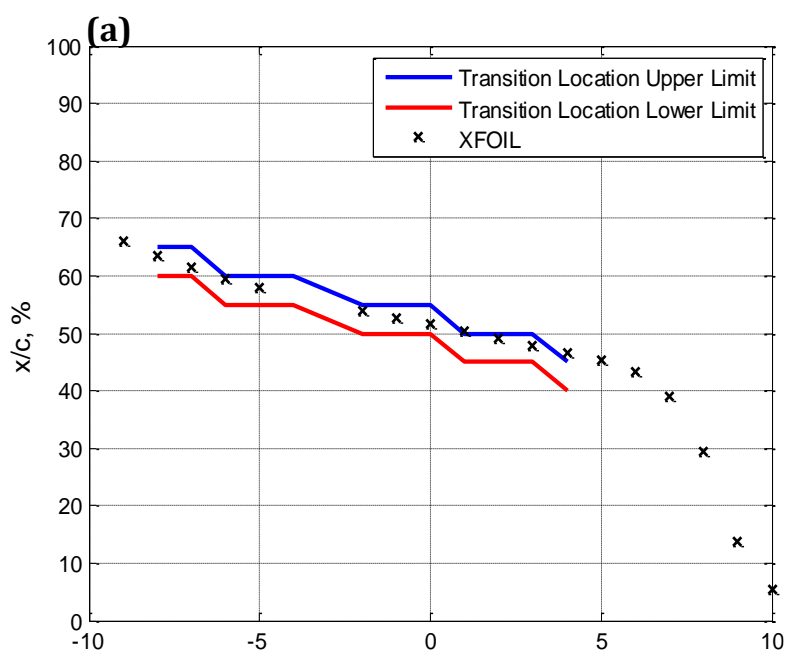

(c)

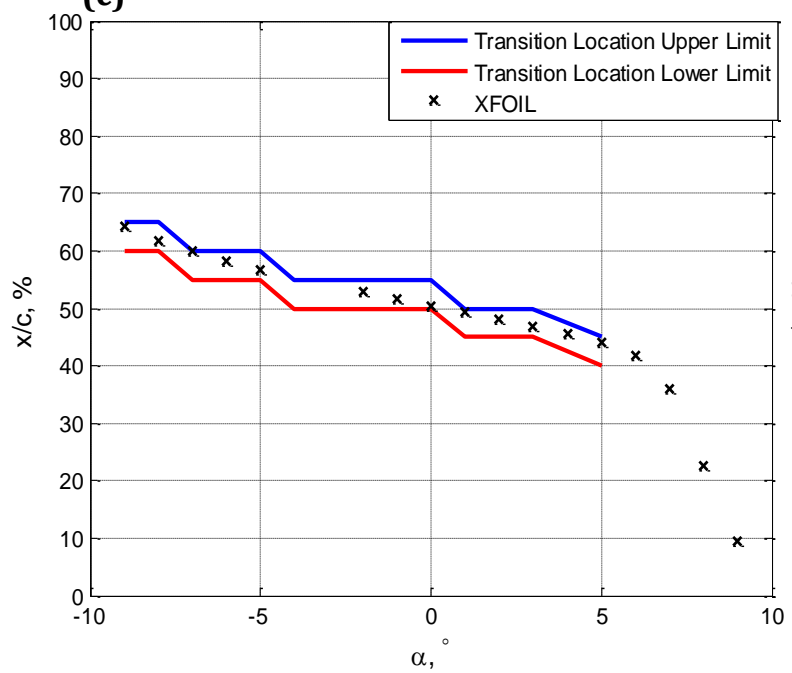

(b)

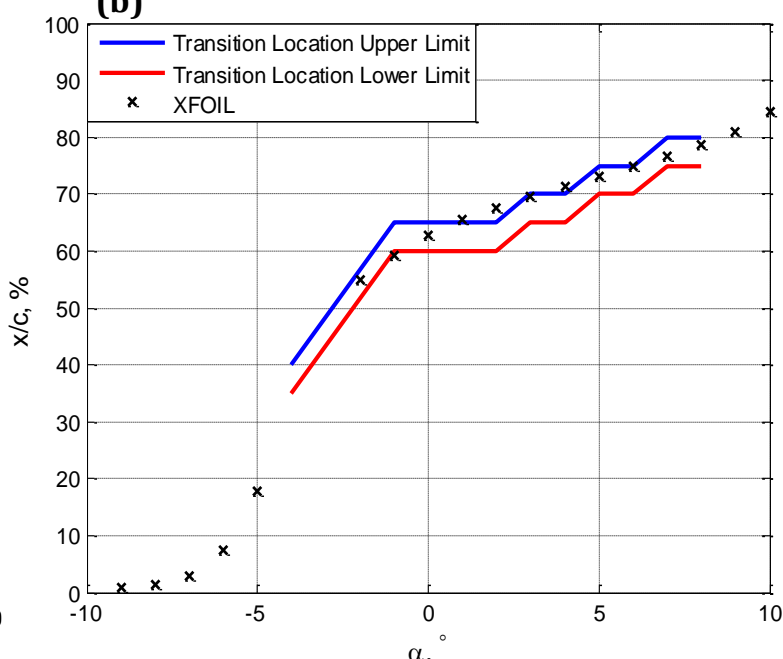

(d)

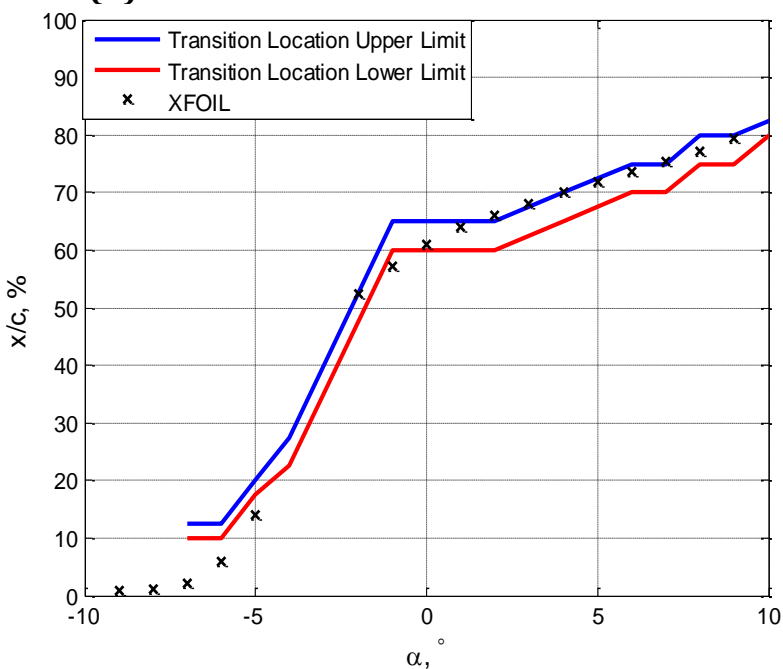

Figure 4.13 Coefficient of Pressure Prediction of Transition Range for 0.46-m DU96-W-180 (a) Suction Side at $R e=1.5 \times 10^{6}$ (b) Pressure Side at $R e=1.5 \times 10^{6}$ (c) Suction Side at $R e=2 \times 10^{6}$ and (d) Pressure Side at $\operatorname{Re}=2 \times 10^{6}$

\subsubsection{Stethoscope Results}

Due to high mixing and unsteadiness, turbulent flow produces significantly greater pressure fluctuations than laminar flow. There are also distinct frequency levels between the two flow regimes which can be detected by the human ear through a stethoscope. This technique, discussed in detail in Section 3.6.2, was used to observe the flow over the clean, unheated 0.8-m DU96-W-180 at two Reynolds Numbers $\left(1.5 \times 10^{6}\right.$ and $\left.2 \times 10^{6}\right)$ and the clean, unheated $0.46-\mathrm{m}$ DU96-W-180 at one Reynolds Number $\left(1.5 \times 10^{6}\right)$. The range of Reynolds numbers was limited by the forces exerted on the Pitot probe during testing, an unavoidable shortcoming of this method.

Recordings of the sounds heard at different flow regimes were obtained using a 
Standard Bruel \& Kjaer, 4182 probe microphone attached to Pitot probe at one end (much like the stethoscope set up described in Section 3.6.2), and computer at other end. This device is shown in Figure A5. The sound changes observed can be heard below, with the laminar sound level magnified by a factor of two.
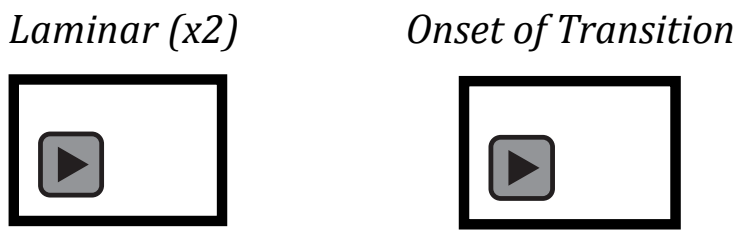

Transition

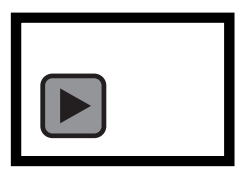

Turbulence

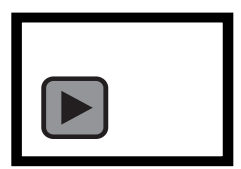

For this method, the estimated uncertainty in detecting the sound changes is $\pm 13-\mathrm{mm}$ along the edge of the model. For the 0.8 -m model, this is $\pm 3 \%$ chord and for the 0.46 -m model this uncertainty translates to $\pm 5.4 \%$ chord. It is important to note that these uncertainties were converted from the edge length uncertainty using an algorithm which accounts for the surface curvature. Since surface curvature changes over the chord length of the model and on both the pressure and suction side, the uncertainty at each point along the chord will be slightly different. Another important finding is that inserting the Pitot Probe into the flow induces transition ahead of the natural transition location as was cautioned by Kuklova (2012) who discussed the effect of invasive methods on the flow. The effect of the probe on the flow is presented in Figure 4.14 which shows infrared images before and after inserting the Pitot Probe close to the transition location. This effect adds another level of uncertainty to the stethoscope measurements, which cannot be precisely quantified because it varies with angle of attack. However, what is certain is that the transition location detected by the stethoscope will be further upstream than the actual transition.
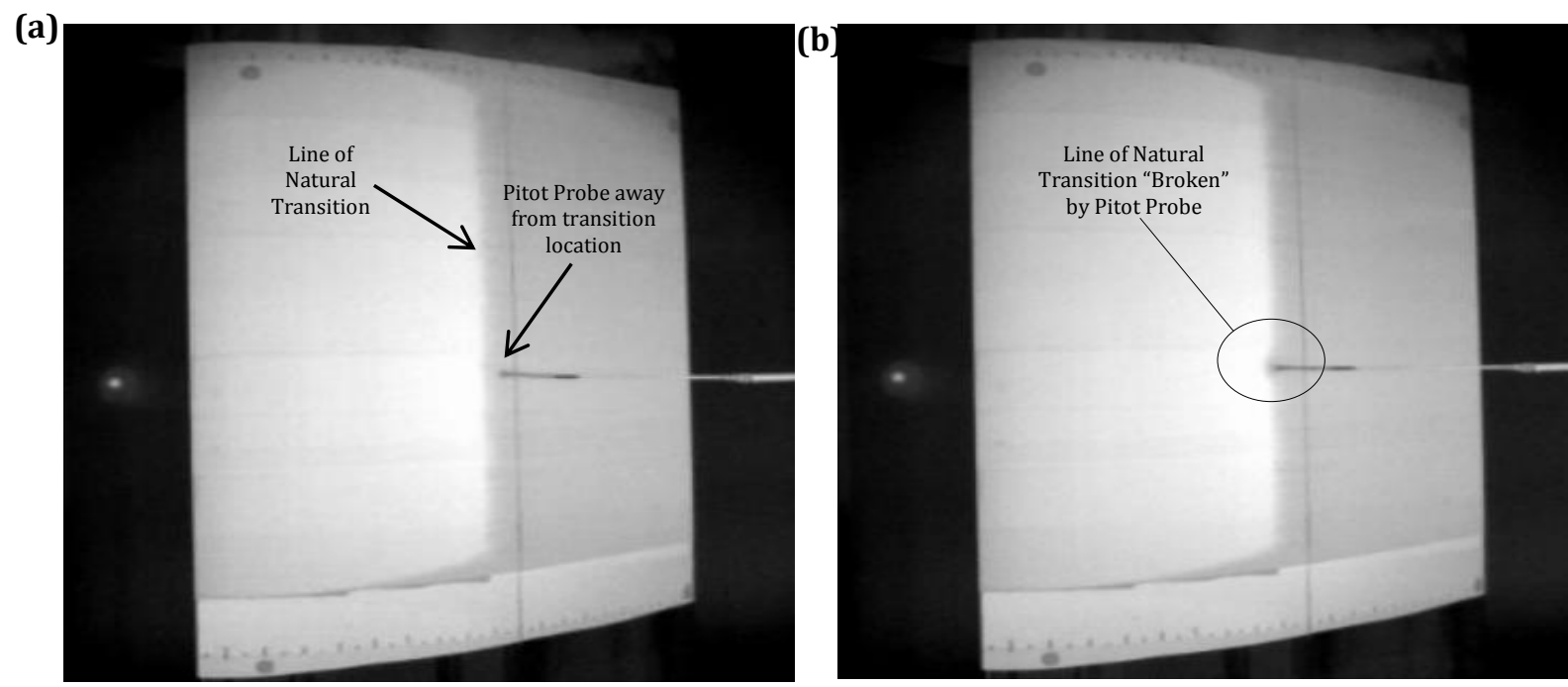

Figure 4.14 Infrared Images showing the (a) Before and (b) After Interference Effect of Inserting a Pitot Tube into the Boundary Layer of the 0.48-m DU96-W180 (at $\left.\alpha=8^{\circ}, \operatorname{Re}=2 \times 10^{6}\right)$ 
Figure 4.15 is a composite of two plots of the stethoscope results for transition, on the suction and pressure side of the $0.8-\mathrm{m}$ DU96-W-180 and $\mathrm{Re}=1.5 \times 10^{6}$. On the $\mathrm{x}$-axis of both plots is the angle of attack and on the y-axis is the chord-wise transition location. The red lines represents the first sound change observed (discussed in Section 3.6.2) from the leading edge and the associated uncertainty as red error bars. The blue line is the second sound change heard downstream of the first sound change, and the associated uncertainty as blue error bars. The black x's are the XFOIL predictions for this model at $\operatorname{Re}=1.5 \times 10^{6}$.
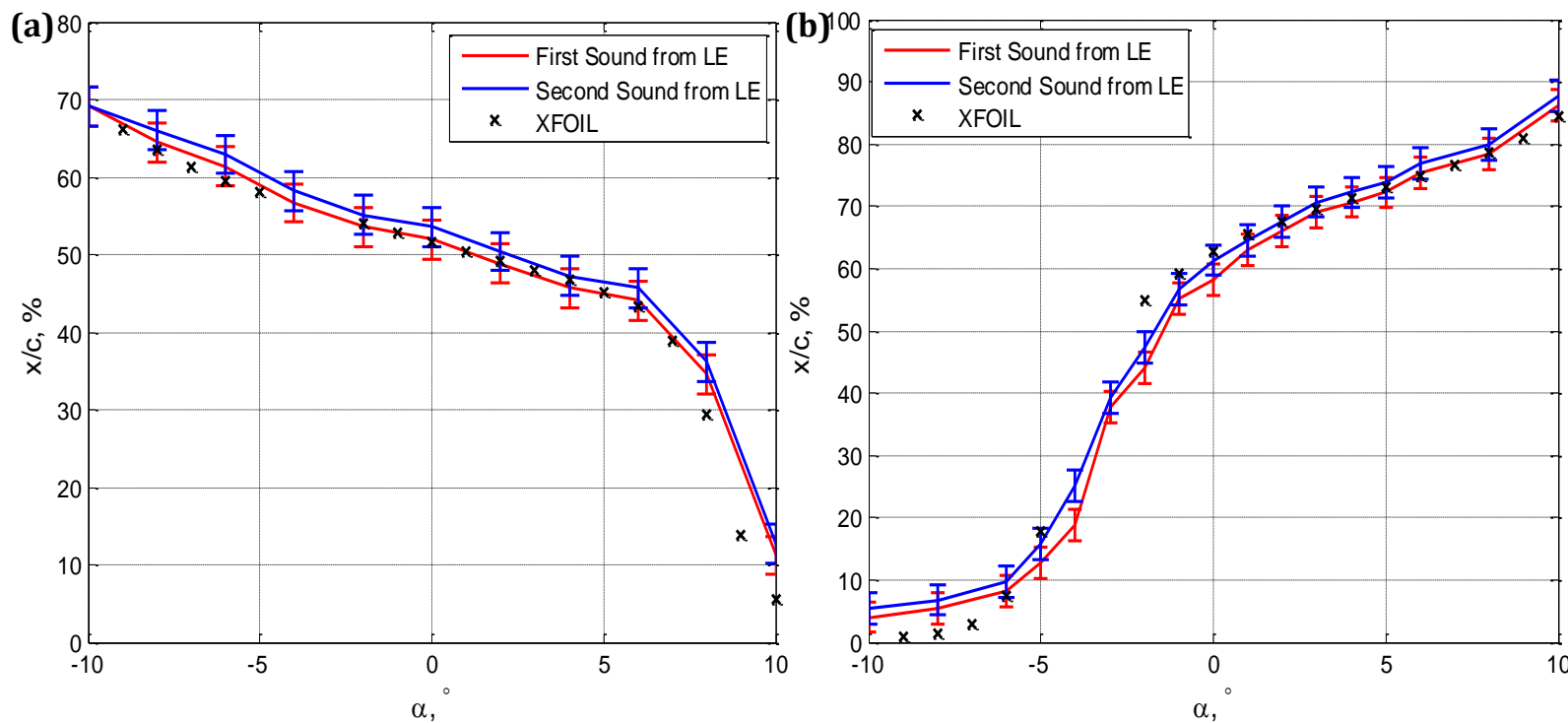

Figure 4.15 Stethoscope Results for Transition Range for (a) Suction Side (b) Pressure Side of 0.8-m DU96-W180 at $\operatorname{Re}=1.5 \times 10^{6}$

Examining the results for the suction side of the model shown in Figure 4.15a, the transition location moves toward the leading edge of the model as the angle of attack increases. It is clear that the stethoscope results are in accordance with the XFOIL predictions, within the stated uncertainty. The only variations occur outside the linear region capped by stall, where XFOIL is known to be unreliable. Slight variations are also observed on the suction side as the positive stall angle is approached (upwards of $\alpha=6^{\circ}$ ) but are likely due to three dimensional effects in the transition (wedge like transition) at these angles. XFOIL cannot predict these effects. The results for the pressure side, in part (b) of the figure, shows similar agreement. There is no apparent agreement between XFOIL and either the first or second sound, instead XFOIL appears to predict transition somewhere between these two sounds. As was the case with the mean pressure analysis, it makes sense that XFOIL would predict transition after the initial onset of instability because it uses the $\mathrm{e}^{\mathrm{N}}$ method for prediction.

Similarly, stethoscope results for the $0.8-\mathrm{m}$ DU96-W-180 at $\mathrm{Re}=2.0 \times 10^{6}$ show the same agreement with XFOIL on the suction side, as seen in Figure 4.16a. The measurements on the pressure side, presented in Figure 4.16b, were collected by a different experimenter than the 
results obtained on the suction side and at $\mathrm{Re}=1.5 \times 10^{6}$. These results clearly show less agreement with XFOIL and thus, while still within the stated uncertainty in most cases, strongly speaks toward the dependency of the stethoscope result on the user. In this case, the user could not hear the first sound change at most angles of attack and reported only the second sound change.
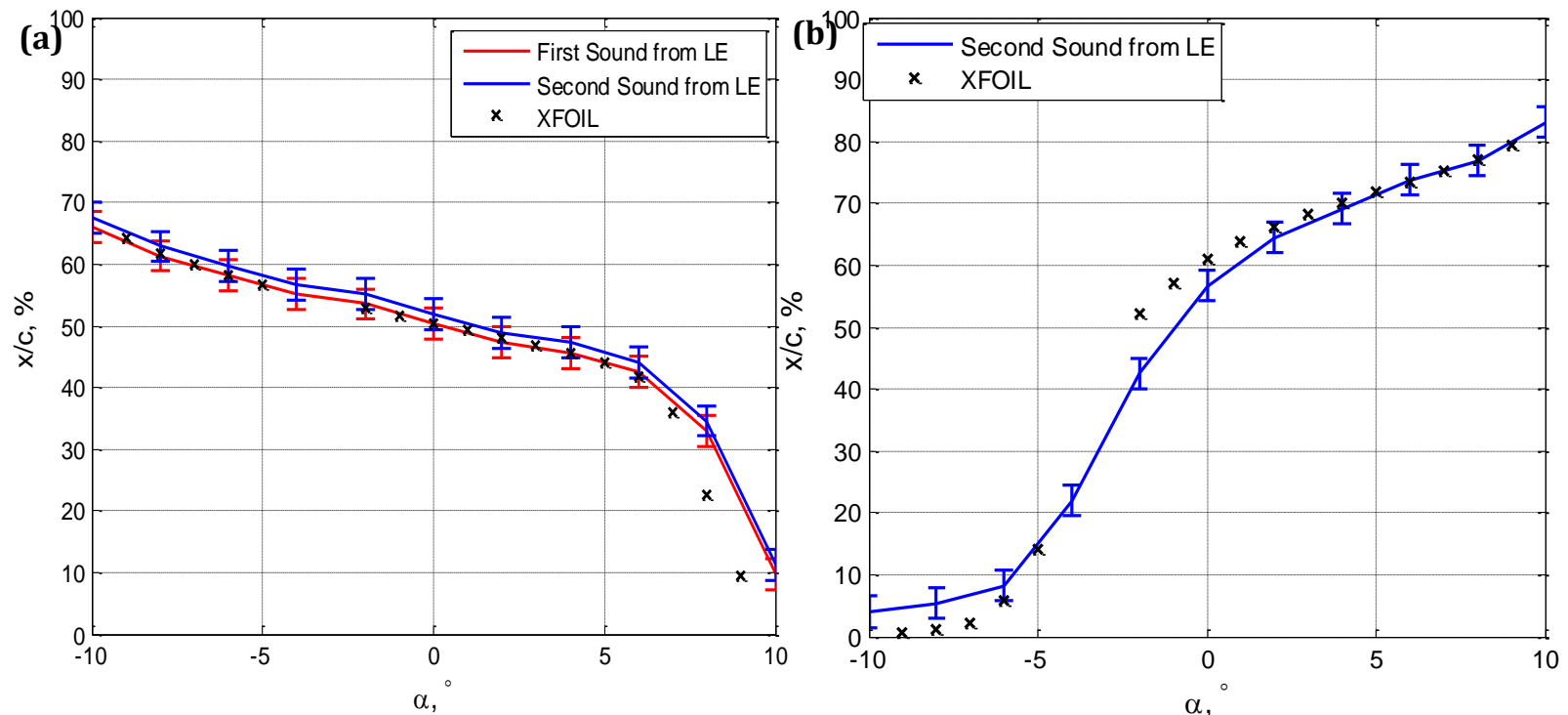

Figure 4.16 Stethoscope Results for Transition Range for (a) Suction Side (b) Pressure Side of 0.8-m DU96$W-180$ at $\operatorname{Re}=2 \times 10^{6}$

Stethoscope tests on the $0.46-\mathrm{m}$ DU96-W- 180 at $\mathrm{Re}=1.5 \times 10^{6}$ produced results that are in agreement with XFOIL. These are plotted using the same convention as the larger chord models and are presented in Figure 4.17. As in the case of the larger chord model, the result agree very well in the linear region between stall, where XFOIL can be trusted.
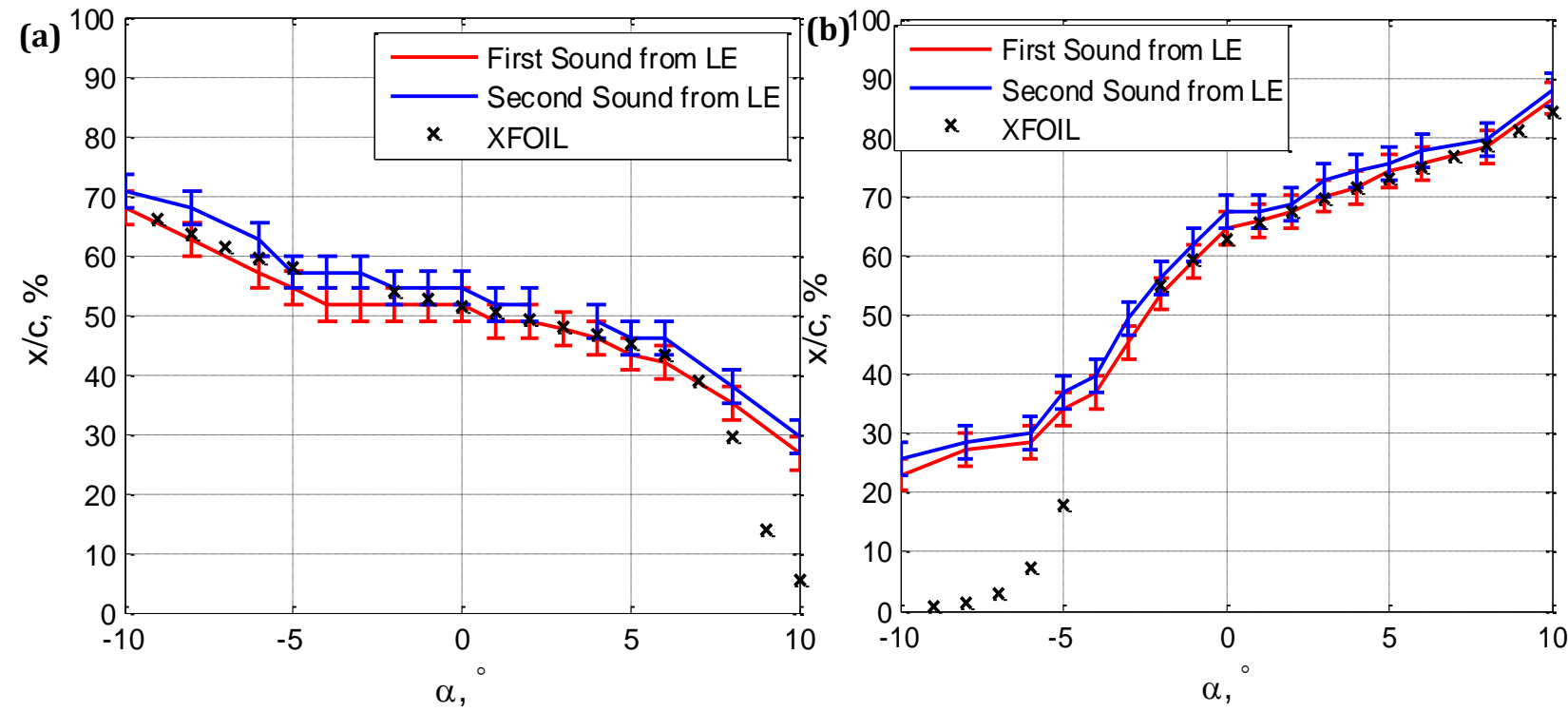

Figure 4.17 Stethoscope Results for Transition Range for (a) Suction Side (b) Pressure Side of 0.46-m DU96-W180 at $\operatorname{Re}=1.5 \times 10^{6}$ 
The fact that useful results were obtained for the two different models and at three different speeds, it can be stated that this method is useful to produce accurate transition results at most angles of attack. However, it is limited by the Reynolds number and the forces on the Pitot probe as well as the curvature of the model and the access to the model from the test section wall. It is also highly dependent on the skills of the user and results tend to vary slightly between experimenters.

Stethoscope measurements were also collected on the insulated, unheated $0.8-\mathrm{m}$ DU96-W-180 so that comparisons could be made with the results of the clean, unheated model. A sample of these results is presented in Figure 4.18 for the case of $\mathrm{Re}=2 \times 10^{6}$, where the results for the insulated model are in red and those of the clean model are in blue. On both the suction and pressure sides there is close agreement, within the stated uncertainty, between stethoscope results at all angles. This agreement was also observed at $\operatorname{Re}=1.5 \times 10^{6}$, which is not presented here. The fact that the transition results from the stethoscope are almost identical on both the insulated and clean model strongly supports the earlier conclusion that the insulator does not affect the airfoil performance. This findings also leads to the conclusion that the quality of the laminates and their assembly is very good, creating a continuous and smooth surface finish.
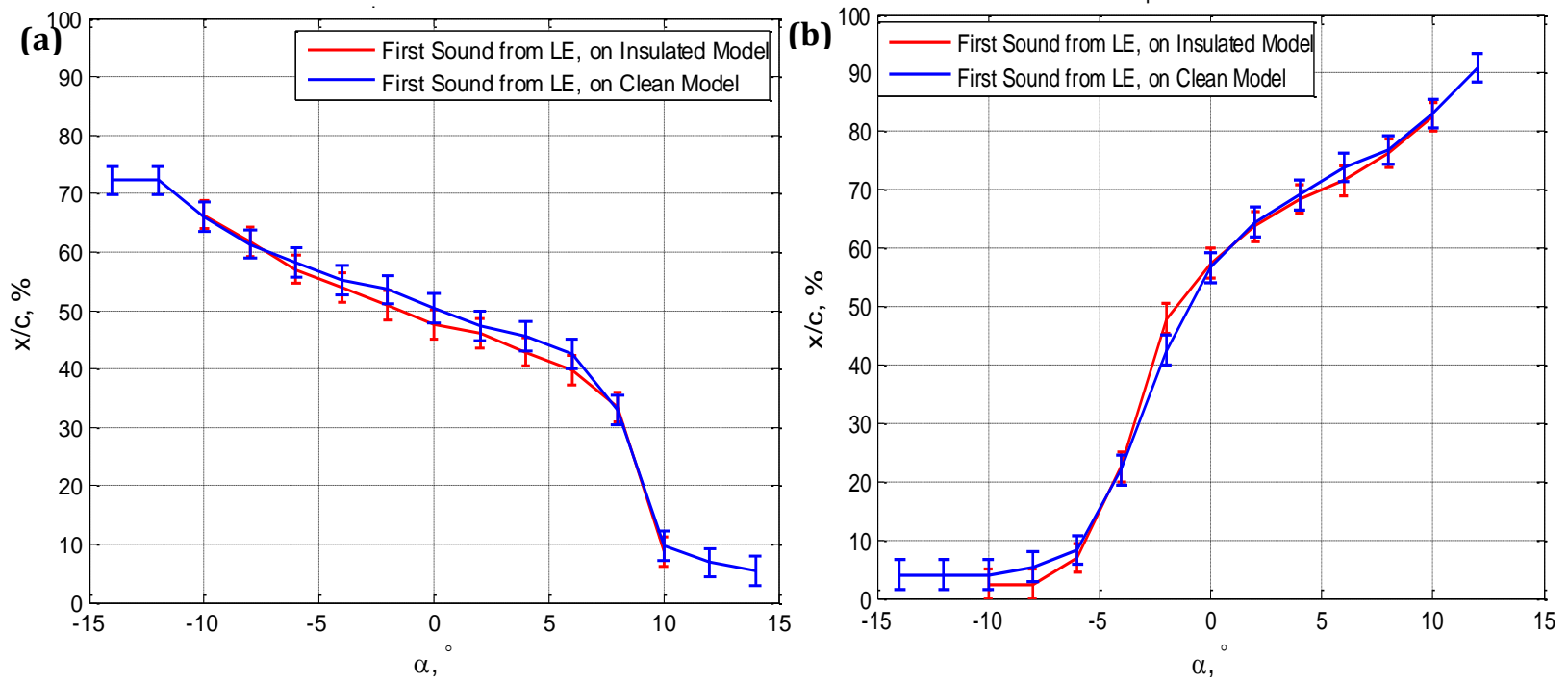

Figure 4.18 Comparison of Stethoscope Results on Insulated versus Clean (a) Suction Side (b) Pressure Side of 0.8-m DU96-W-180 at $\operatorname{Re}=2 \times 10^{6}$ 


\subsubsection{Infrared Thermography Results}

The 0.8-m and the 0.46-m DU96-W-180 aluminium airfoils were mounted in the Virginia Tech Stability Wind Tunnel and set up using the infrared thermography transition detection system described in Section 3.7. The 0.8-m model was tested at three Reynolds numbers: $1.5 \times 10^{6}, 2 \times 10^{6}$ and $3 \times 10^{6}$ and angles from $-20^{\circ}$ to $20^{\circ}$. The $0.46-\mathrm{m}$ model was tested at two Reynolds numbers: $1.5 \times 10^{6}$ and $2 \times 10^{6}$ and angles from $-20^{\circ}$ to $20^{\circ}$.

A composite of sample infrared images obtained from the infrared thermography transition detection test done on the 0.8-m DU96-W-180 is presented in Figure 4.19a-e. The images were taken on the suction side of the model at $\operatorname{Re}=1.5 \times 10^{6}$ and angles of attack of $\alpha$ $=\left[-10^{\circ},-5^{\circ}, 0^{\circ}, 5^{\circ}, 10^{\circ}\right]$ (results for all angles are included in Appendix $D$ ). In these images the flow is moving from left to right and the areas of lower temperature coincides with darker/blacker colour on the image while the regions of higher temperatures are whiter. Since the model is being heated and it was established that laminar regions have low heat transfer rates, the large white regions (hotter regions) of the image represent the laminar flow regime. Conversely the darker regions on the images are the areas in which the high flow mixing dissipates the heat away from the model resulting in significantly lower surface temperature. These are therefore turbulent regions. Hence, the beginning of the transition region will be the region of highest chord-wise temperature gradient on these pictures when the flow becomes significantly cooler. Figure 4.19 also displays green coloured plots of the temperature distribution, averaged over the mid-section of the insulated area, along the chord of the model. This data was extracted from the infrared image itself and plotted over it to show how the average temperature varies with the chord-wise location.

It should be stated that the expected flow regime-temperature relationship is true only in the chord-wise direction and over the insulated area of the model shown in the image. Outside this insulated area, other factors come into play and affect the observed temperature gradient. The temperature gradients occurring in the span-wise direction are as a result of the stacking of laminates which make up the airfoil itself, a method which naturally creates small gaps between each laminates. The largest span-wise gradients, which occur close to the top and bottom of the images, are indicative of the end of the rubber silicone insulated portion of the airfoil. There are small, turbulent wedges evident in this region as separation occurs over the 0.8 -mm step produced.

Figure 4.19 shows that, for the suction side, as angle of attack moves from negative to positive the peak temperature gradient moves closer to the leading edge. This is the overall trend observed on the suction side from the mean pressure analysis, stethoscope data and XFOIL. What is interesting to note here is the clear contrast between laminar and turbulent regions. With this system, the transition is very hard to miss thereby reducing the uncertainty in the measurements to that related to the image processing method. Another point of importance is that the numerical temperature gradient over the model (plotted in 
green) also reflects the marked fall in temperature. However, this drop is not step-like (vertical) but occurs progressively over a region of the chord. This finding clearly confirms the known theory that transition occurs over a region of flow and suggests that the infrared
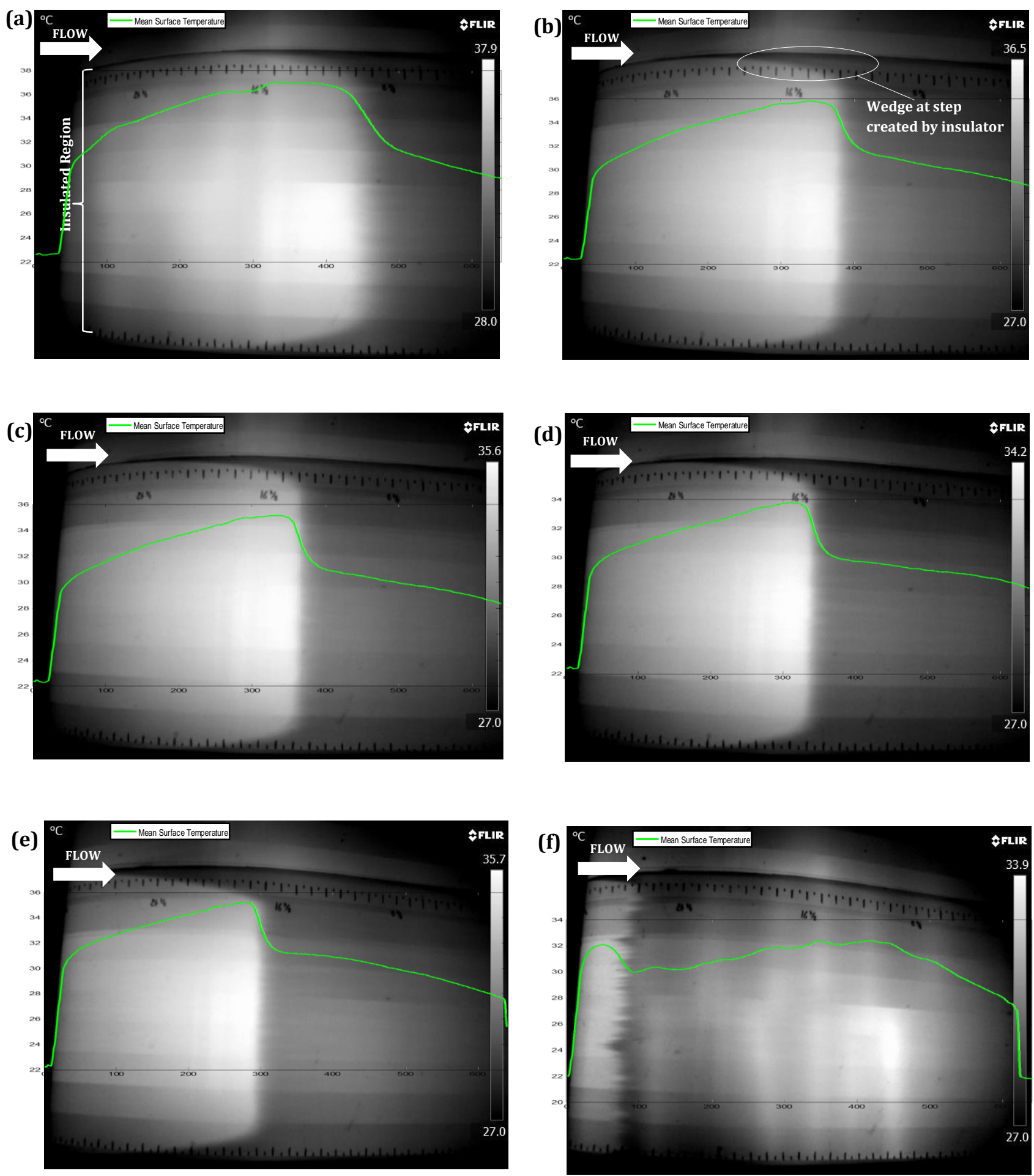

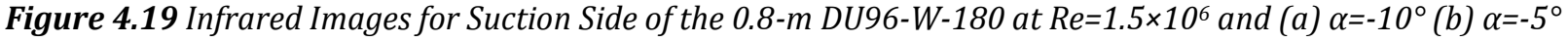
(c) $\alpha=-3^{\circ}$ (d) $\alpha=0^{\circ}$ (e) $\alpha=5^{\circ}$ (f) $\alpha=10^{\circ}$ 
system reveals true flow behaviour. Furthermore, the transition occurs as a relatively straight line for angles within the linear region between stall. This is clear from Figure 4.19a$f$ where the transition line is clean and clear. Figure 4.19f however, shows a transition which is not linear but which contains small wedge-like patterns reminiscent of threedimensionality. Recalling the discussion in Section 4, the 0.8-m DU96-W-180 at $\operatorname{Re}=1.5 \times 10^{6}$ experiences positive stall at about $10^{\circ}$, which is the case depicted in Figure $4.19 e$. The fact that the increase in viscous nature associated with stall can be seen on the infrared image is an added advantage of the infrared thermography system and implies that the infrared system is both clear and sensitive. This, however, introduced higher uncertainties in extracting transition locations at these angles - the presence of wedges means that the transition location must be an average over the chord-wise length of the wedges.

Infrared images of the pressure side of the $0.8-\mathrm{m}$ DU96-W-180, at $\mathrm{Re}=1.5 \times 10^{6}$ and angles of attack of $\alpha=\left[-5^{\circ},-3^{\circ}, 0^{\circ}, 5^{\circ}, 8^{\circ}\right]$, are presented in Figure 4.20a-e. As was the case on the suction side, the transition between stall $\left(\alpha=-5^{\circ},-3^{\circ}, 0^{\circ}\right)$ is clean and observed as a relatively straight (span-wise) temperature difference. However, at angles approaching stall, $\left(\alpha=5^{\circ}, 8^{\circ}\right)$ three dimensional effects are evident on the infrared image by the appearance of wedge-like features. Again, this shows the sensitivity of the system and its ability to detect three dimensional effects, a phenomenon none of the other methods could clearly capture. Item (f) of this figure shows the numerical temperature distribution over the chord at $\alpha=0^{\circ}$, where the clear drop is seen for transition. Similar results were observed at $\operatorname{Re}=2 \times 10^{6}$ and $\mathrm{Re}=3 \times 10^{6}$ but will not be discussed in detail here; these are included in Appendix E and Appendix $F$.

The $0.46-\mathrm{m}$ DU96-W-180 produced infrared results very similar to those discussed for the $0.8-\mathrm{m}$ model. Figure 4.21 shows infrared images for this model at $\mathrm{Re}=1.5 \times 10^{6}$ and angles of attack of $\alpha=\left[-10^{\circ},-5^{\circ}, 0^{\circ}, 5^{\circ}, 10^{\circ},-7^{\circ}\right]$ (results at all angles are included in Appendix $G)$. In this case, the full chord length is always visible in the images. In addition to the edge length markers on the model, there is also a vertical line drawn 228.6-mm (9 inches) from the trailing edge to be used by the image processing scheme. As with the larger chord model, the transition is very clear and linear between stall with three dimensional effects only observed close to stall (at $\alpha=10^{\circ}$ and $\alpha=10^{\circ}$ ). Figure 4.21c, which shows the infrared image at $\alpha=0^{\circ}$, also presents the plot of the mean chord-wise temperature (averaged over midsection of insulator) showing the numerical value of the drop in temperature at transition.

Item (f) of Figure 4.21 shows a sample processed infrared image at $\alpha=-7^{\circ}$, showing the final transition result. The algorithm used finds the highest chord-wise temperature gradients in the image which coincides with the leading edge, the trailing edge, the line drawn 228.6-mm from the trailing edge, and the transition location. It then uses the first three of these locations to calculate the edge location of the transition location. This is reported in inches from the trailing edge. This is then converted to a chord wise location 

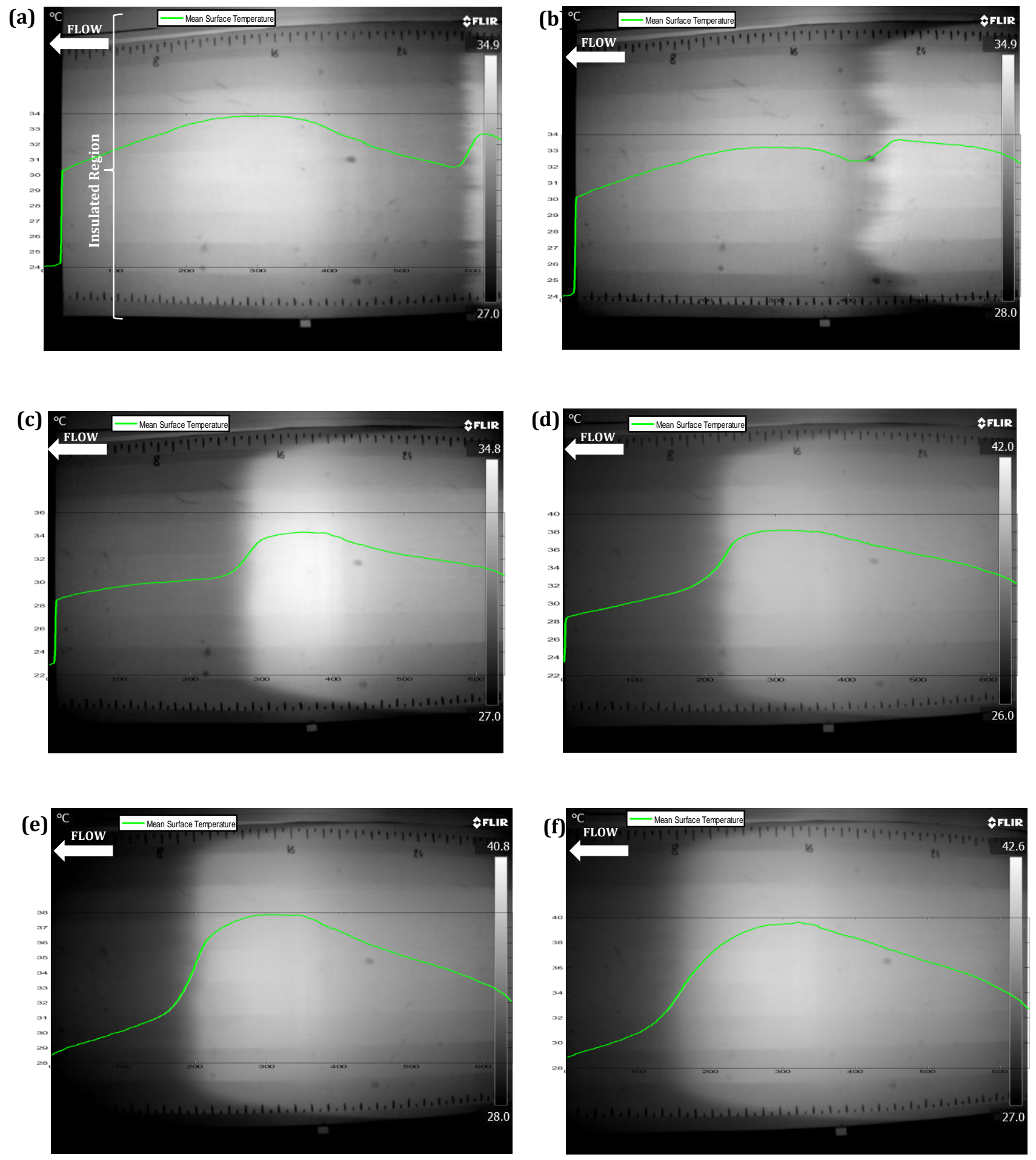

Figure 4.20 Infrared Images for Pressure Side of the 0.8- $m$ DU96-W-180 at Re $=1.5 \times 10^{6}$ and (a) $\alpha=-5^{\circ}$ (b) $\alpha=-3^{\circ}$ (c) $\alpha=0^{\circ}(d) \alpha=3^{\circ}(e) \alpha=5^{\circ}(f) \alpha=8^{\circ}$

using an algorithm which accounts for the surface curvature. The transition results produced by this algorithm is estimated to be accurate within $\pm 5-\mathrm{mm}$. The local transition location is 
marked in Figure 4.21f by red dots, and the final location is taken as the mode of these locations, which is the black line on the image.

Figure 4.22 shows the results for of the pressure side of the $0.46-\mathrm{m}$ DU96-W-180, at $\operatorname{Re}=1.5 \times 10^{6}$ and angles of attack of $\alpha=\left[-5^{\circ},-3^{\circ}, 0^{\circ}, 5^{\circ}, 8^{\circ}\right]$. These results agree very closely with that of the larger model. Figure $4.22 f$ is the processed image on the pressure side of the model again reporting the transition location in \% chord and in inches from the trailing edge. Results for $\mathrm{Re}=2 \times 10^{6}$ are not discussed in detail but were in accordance with the behaviour observed at the lower speed. These are included in Appendix $H$.

The infrared images examined prove that transition was detected by a distinct temperature gradient along the chord of both airfoil, for all angles of attacks before stall and even some after stall, at all Reynolds Numbers. The resulting images were clear and obtained very efficiently. It should also be noted that using this system, there were no issues in observing the temperature gradient throughout an entire angle of attack sweep because as the pre-set temperature difference began to decay, the heaters were turned on to reintroduce it. Additionally, the response of the system to changes in flow speed and angle of attack was almost instantaneous - changes in the live view of the infrared camera were observed within 1 seconds of changing the angle of attack. Video C1 and Video C2 are infrared videos of full angle of attack sweeps of both airfoils capturing the response time and effectiveness of the system. This not only allows for highly efficient testing, but it also enables the user to detect, in real time, changes in the flow quality and flow contaminants. Therefore, in cases where there are measures can be taken to improve the flow sooner rather than later. While very promising, this does not necessarily mean that the results were accurate. 
(a)

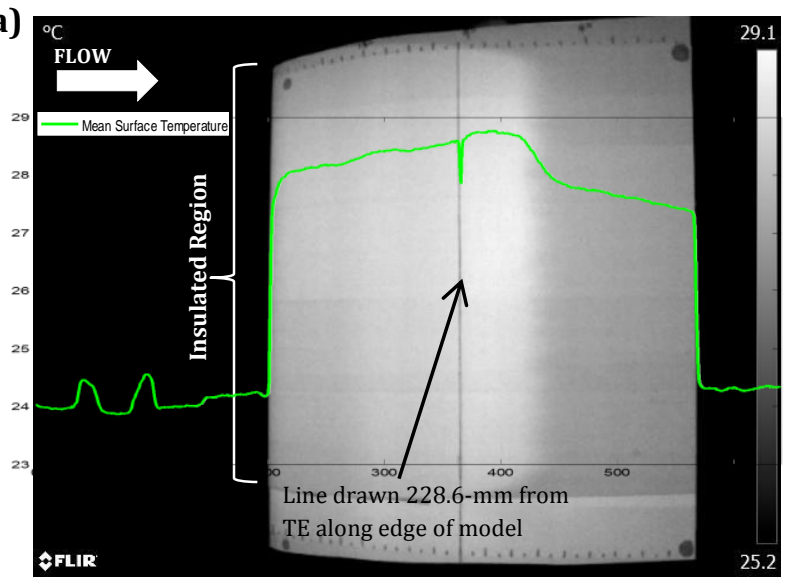

(c)

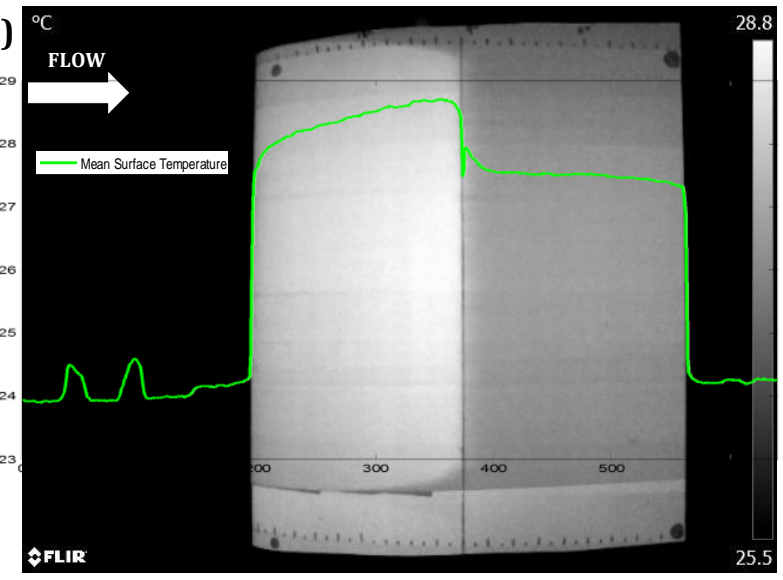

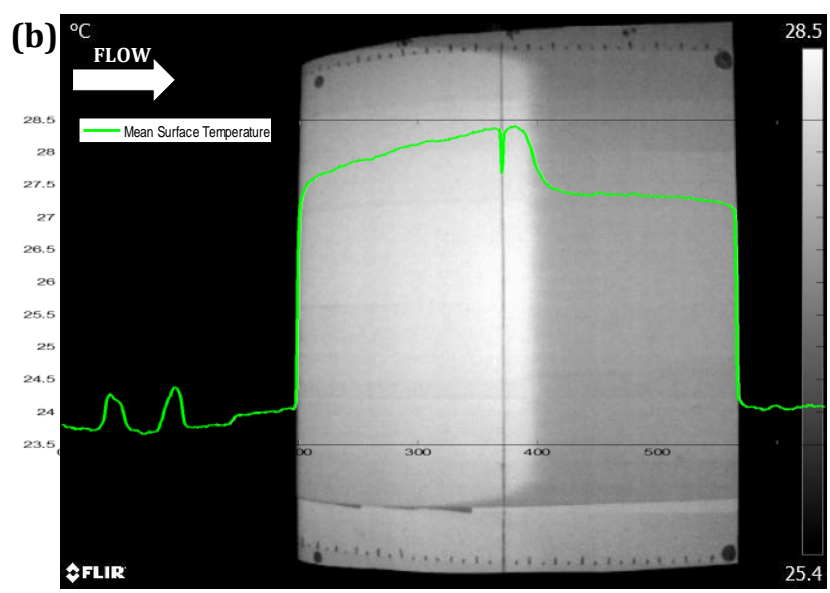

(d)

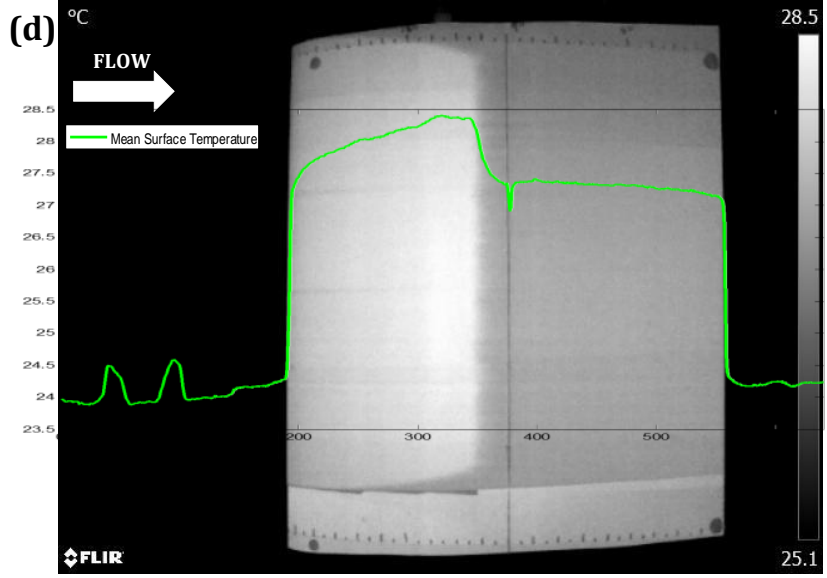

(e)

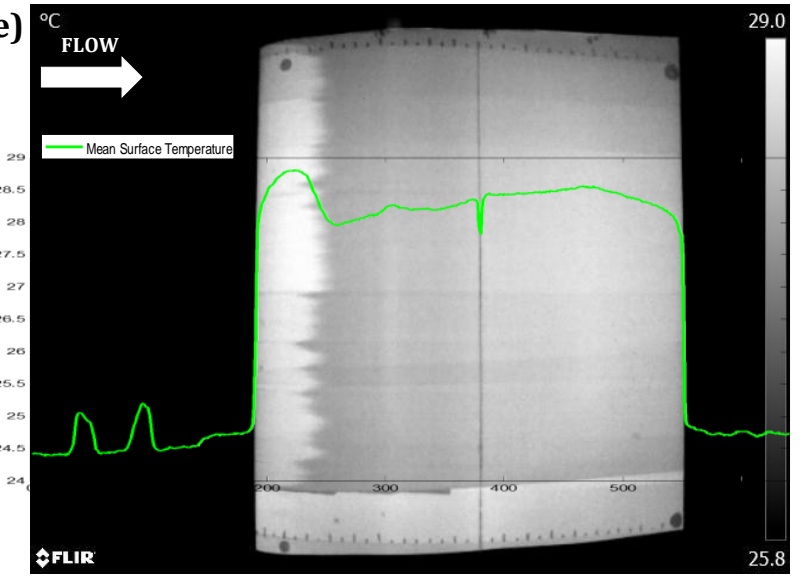

(f)

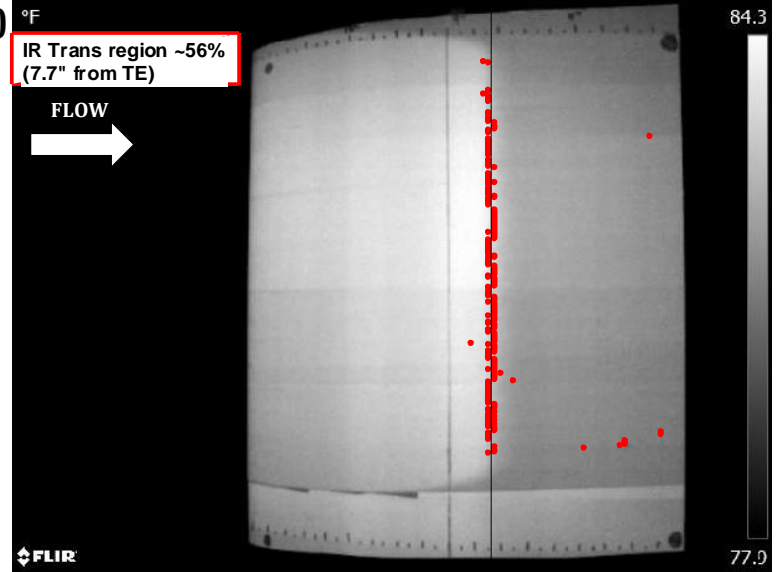

Figure 4.21 Infrared Images for Suction Side of the 0.46- $\mathrm{m}$ DU96-W-180 at $R e=1.5 \times 10^{6}$ and $(\mathrm{a}) \alpha=-10^{\circ}(\mathrm{b}) \alpha=-5^{\circ}$ (c) $\alpha=0^{\circ}$ with Plot of Local Mean Chord-wise Temperature (d) $\alpha=5^{\circ}(e) \alpha=10^{\circ}(f) \alpha=-7^{\circ}$ with Highest Temperature Gradient as Identified by the Image Processing Algorithm 

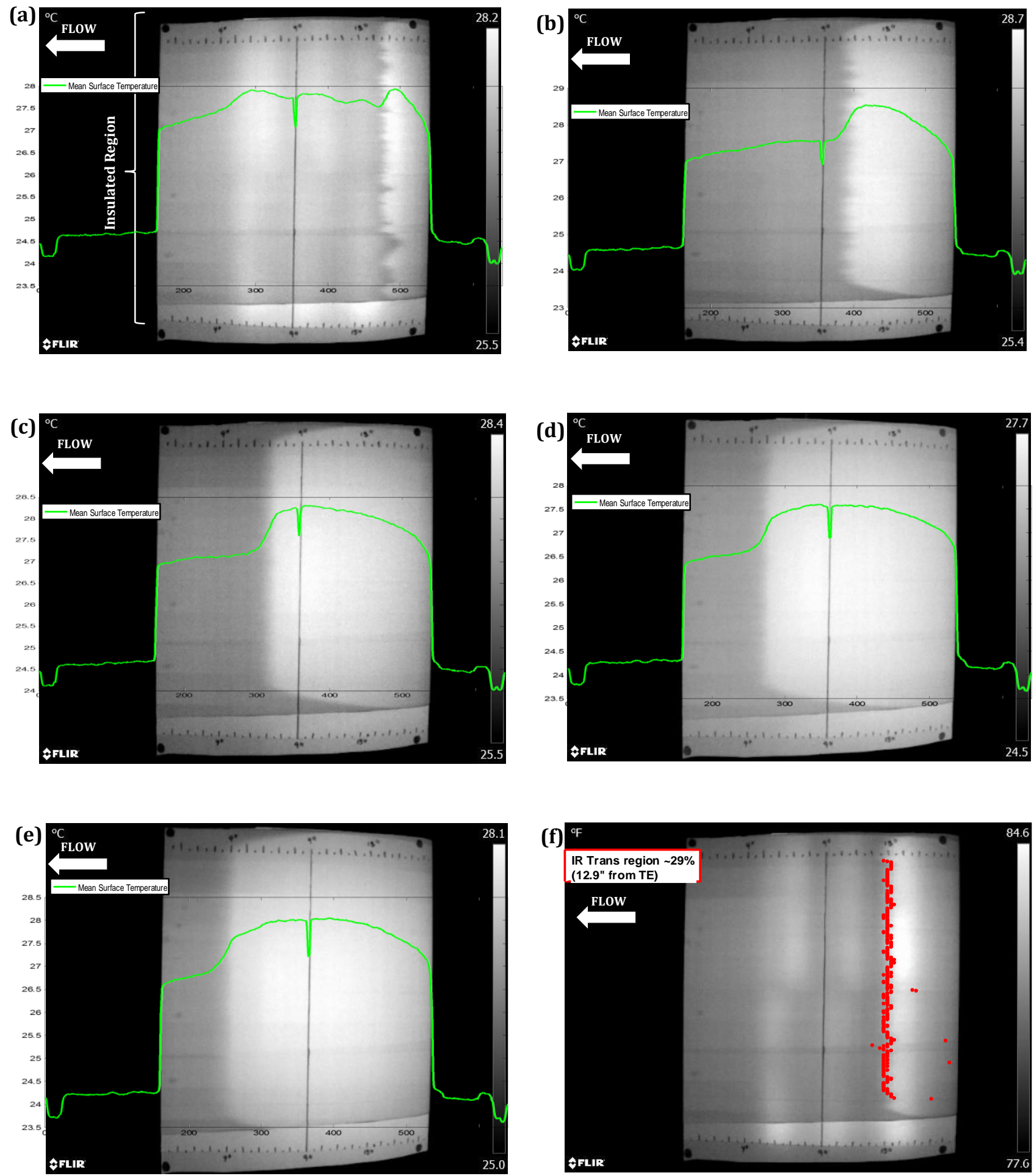

Figure 4.22 Infrared Images for Pressure Side of the 0.46-m DU96-W-180 at Re=1.5 $\times 10^{6}$ and (a) $\alpha=-5^{\circ}$ (b) $\alpha=-3^{\circ}$ (c) $\alpha=0^{\circ}(d) \alpha=5^{\circ}$ (e) $\alpha=8^{\circ}$ (f) $\alpha=-4^{\circ}$ with Highest Temperature Gradient as Identified by the Image Processing Algorithm 


\subsubsection{Validation of Infrared Results}

Validation of the infrared results was investigated by comparing them with the results of the mean pressure analysis, the stethoscope measurements and XFOIL. The premise is that if all the experimental results agree, within the stated uncertainties, the system is validated. These comparisons are shown in Figure 4.23 for the pressure and suction side of the 0.8-m DU96-W-180 and three Reynolds Numbers.

In Figure 4.23 the plots on the left are of the suction side while the plots on the right are of the pressure side. In these plots, the black, solid line represents XFOILs predictions, the blue x's depict the first sound from the stethoscope, the green triangles are indicative of the infrared transition results and the red circles with error bars are the 5\% region determined from the mean pressure. Inspecting these results, it is clear that for all speeds and on both sides of the airfoil, the results produced by the infrared thermography system show excellent agreement with both the mean pressure and stethoscope results for transition. In most cases, the results are within $\pm 1 \%$ chord of each other suggesting that the infrared results are indeed indicative of transition and no other flow phenomenon. An interesting observation is that transition results can be obtained for more angles, even past stall, from the infrared system than any of the other experimental methods. Additionally, the results also agree closely with the XFOIL predictions made in the linear regions between stall, where these predictions can be trusted. However, at some angles outside the stall regions there is marked variations between the IRT transition results and the XFOIL results, for example on the pressure side at $\operatorname{Re}=3 \times 10^{6}$ and $\alpha=\left[-3^{\circ}: 3^{\circ}\right]$ shown in Figure 4.23f. The infrared images of the pressure side at two such angles $\left(\alpha=-3^{\circ},-1^{\circ}\right)$ are presented in Figure 4.24. These images show that the transition region has three dimensional nature which explains why the XFOIL prediction, which does not account for 3-D effects, are different from the IRT transition results.

Figure 4.25 presents these comparisons for the pressure and suction side of the 0.46m DU96-W-180 at two Reynolds Numbers. As was the case with the 0.8-m model, the transition location results from the three experimental methods and XFOIL agree with each other very well at most angles of attack. In Figure 4.25 it is seen that the stethoscope data deviates from the infrared thermography results at angles higher than $5^{\circ}$ on the suction side and at angles less than $-5^{\circ}$ on the pressure side. This is due to the increased difficulty associated with traversing the stethoscope over the curved leading edge of the shorter chord model. 

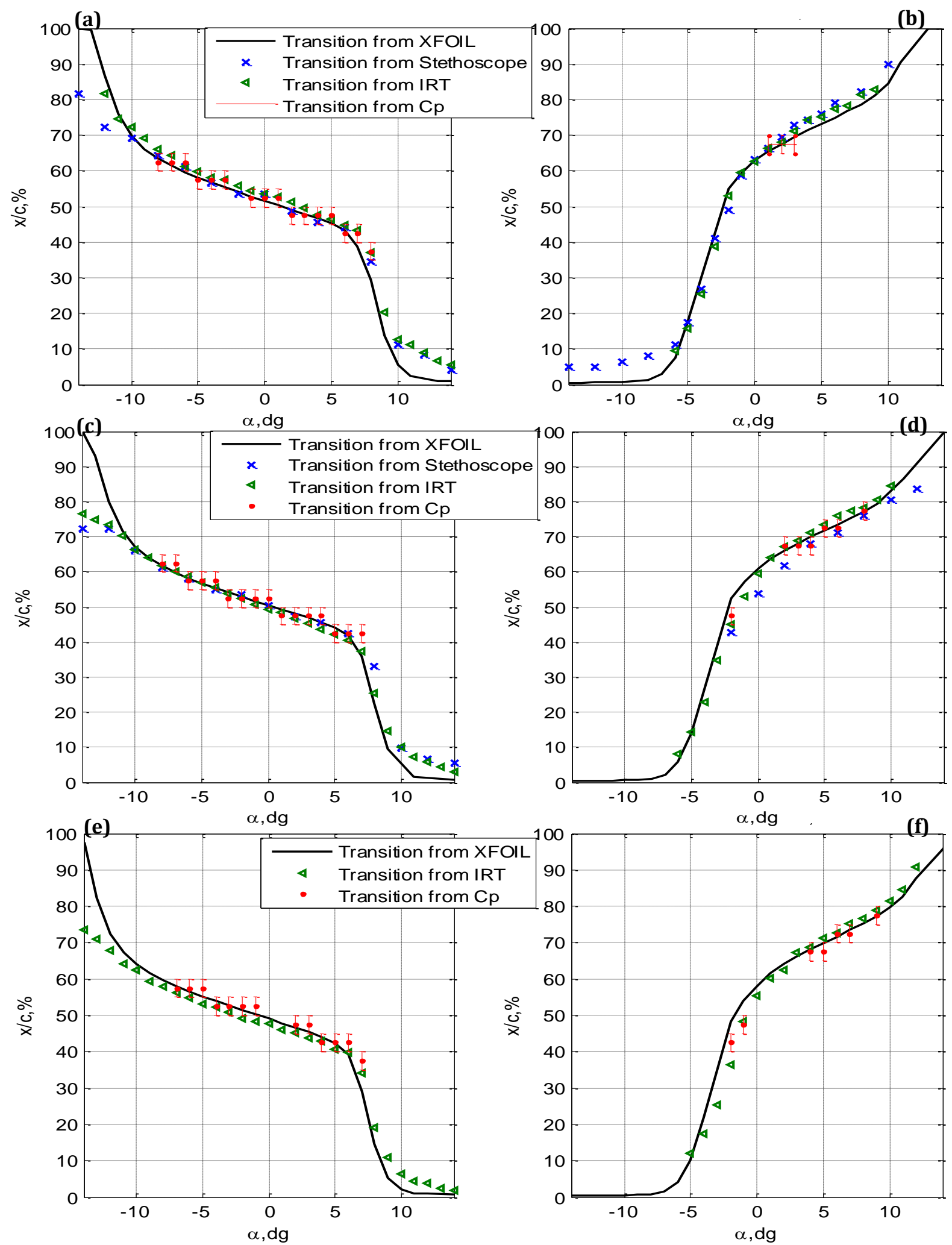

Figure 4.23 Comparison of Transition Results Obtained from Four Methods for 0.8-m DU96-W-180 (a) Suction Side at $R e=1.5 \times 10^{6}$ (b) Pressure Side at $R e=1.5 \times 10^{6}$ (c) Suction Side at $R e=2 \times 10^{6}$ (d) Pressure Side at Re $=2 \times 10^{6}$ (e) Suction Side at $R e=3 \times 10^{6}$ (f) Pressure Side at $R e=3 \times 10^{6}$ 

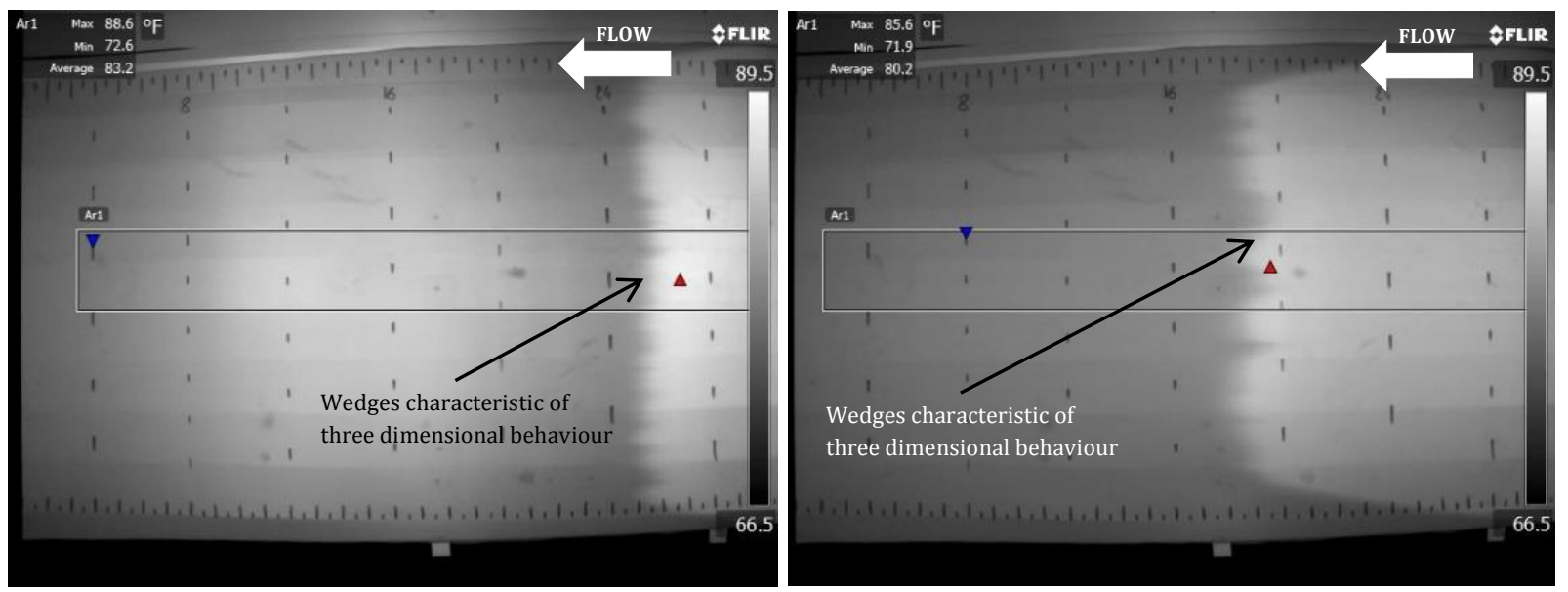

Figure 4.24 Infrared Images showing Three Dimensionality of Transition for Pressure Side of the 0.8-m DU96$W-180$ at $\operatorname{Re}=3 \times 10^{6}$ and (a) $\alpha=-3^{\circ}$ (b) $\alpha=-1^{\circ}$
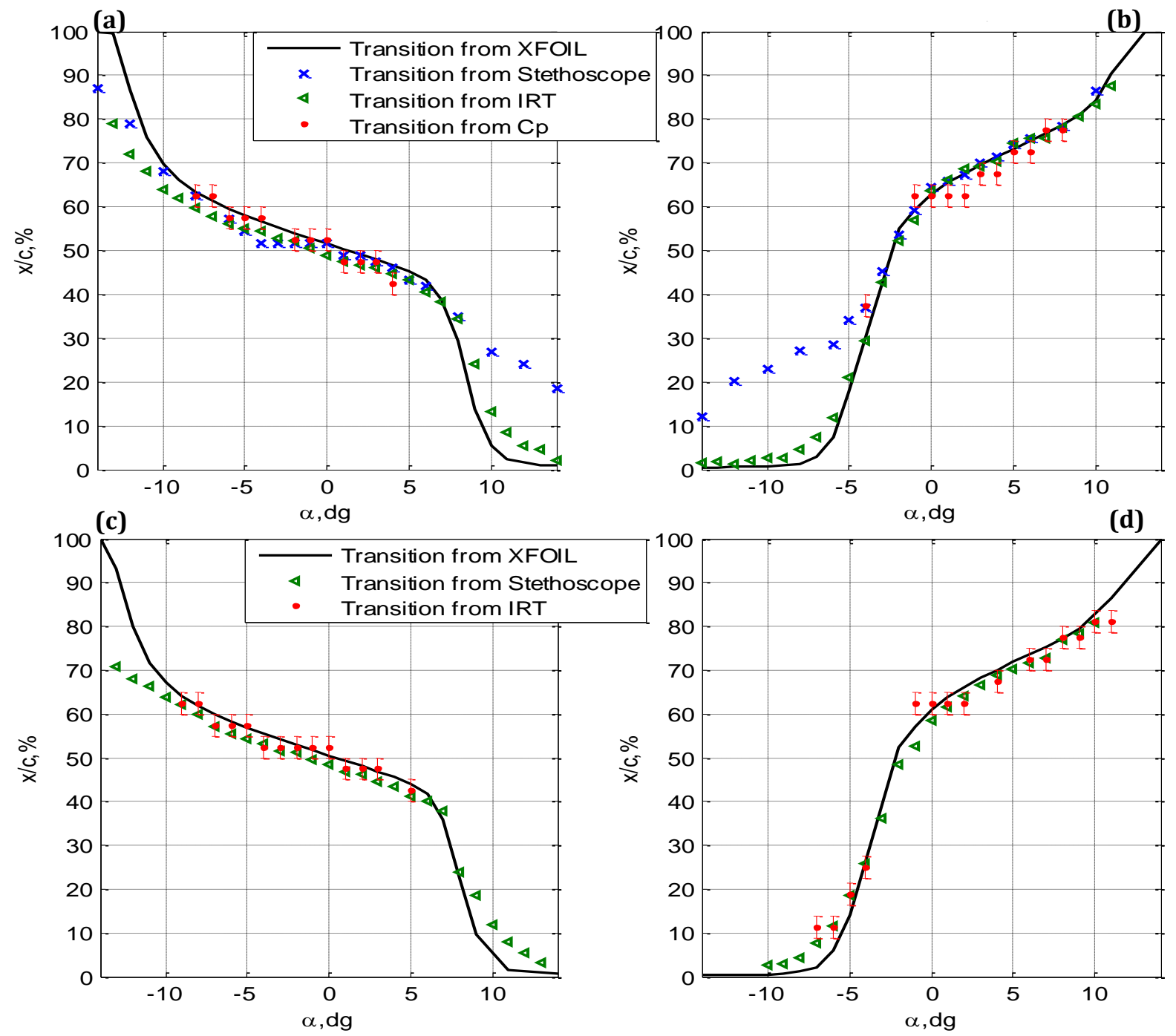

Figure 4.25 Comparison of Transition Results Obtained from Four Methods for 0.46-m DU96-W-180 (a) Suction Side at $\operatorname{Re}=1.5 \times 10^{6}$ (b) Pressure Side at $R e=1.5 \times 10^{6}$ (c) Suction Side at $\operatorname{Re}=2 \times 10^{6}$ (d) Pressure Side at Re $=2 \times 10^{6}$ 
The stethoscope results plotted in these comparisons is that of the first sound change heard when traversing from leading to trailing edge. This is because it was found that the temperature gradient on the infrared images coincide with the first sound. The second, louder sound occurs further downstream. This investigation was done on both models and both sets of results support this conclusion. The results for two sample angles of attacks are shown in Figure 4.26 to demonstrate the relationship between each sound and the infrared results.
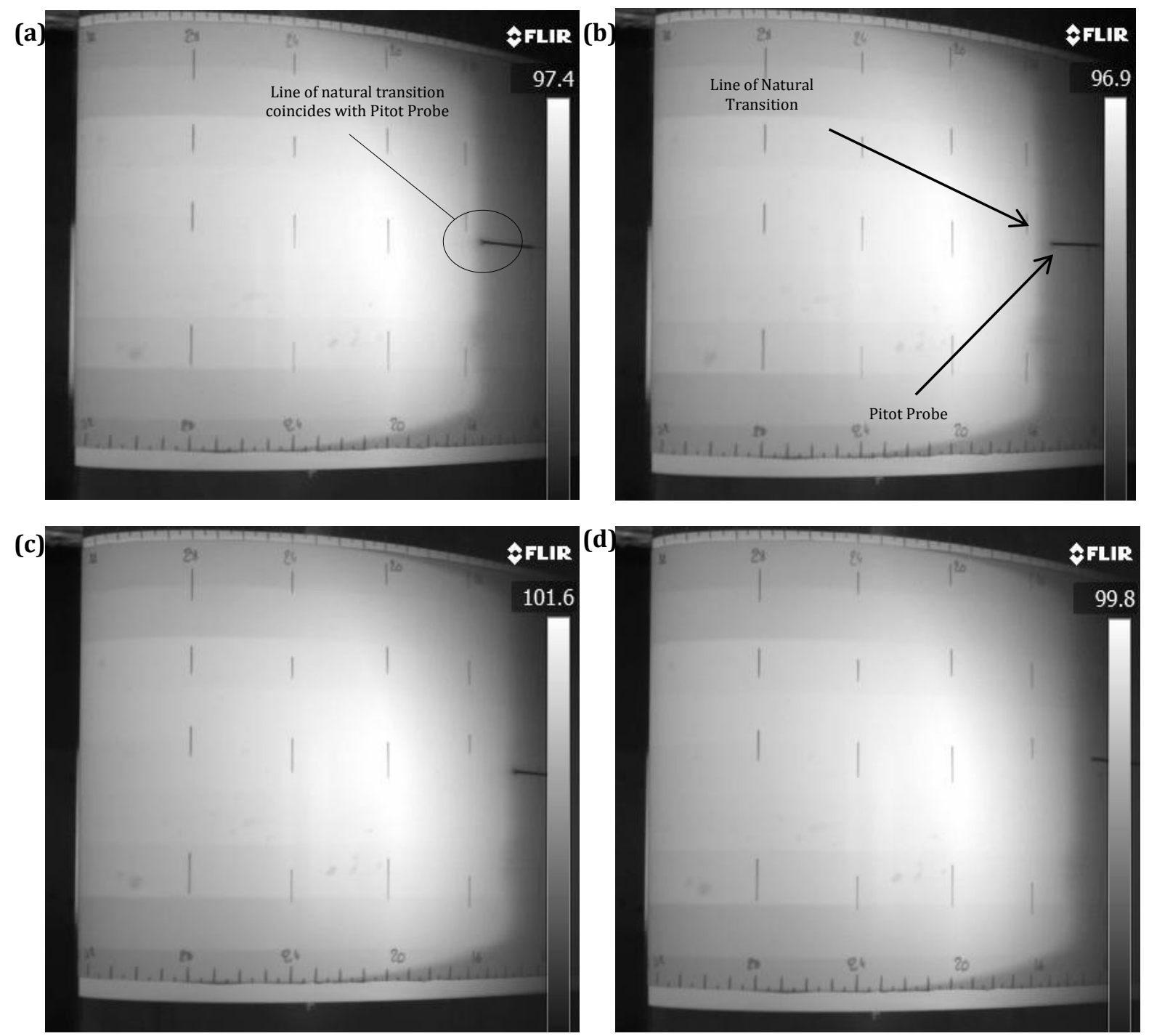

Figure 4.26 Infrared Images showing the Stethoscope Location when Detecting the (a) First Sound at $\alpha=-2^{\circ}(b)$ Second Sound at $\alpha=-2^{\circ}$ (c) First Sound at $\alpha=-4^{\circ}$ (d) Second Sound at $\alpha=-4^{\circ}$ on the Suction Side of the 0.8-m DU96-W180 
As a final validation the results of the two models, $0.46-\mathrm{m}$ and $0.8-\mathrm{m}$ chord, were compared against each other. Results are presented in Figure 4.27.

Overall, the data from both models appear to agree well. On average, differences between results are about $2 \%$ chord but are observed to reach a maximum variation of $5 \%$ chord on the suction side at $\mathrm{Re}=1.5 \times 10^{6}$. These variations are most likely due to increases in turbulence levels associated with increases in flow speed (higher speeds are needed for the shorter chords to be able to reach the same Reynolds Number as the larger chord). Increased turbulence levels will results in earlier transition. An XFOIL study was done to simulate the effect that increasing turbulence levels has on the suction and pressure side transition locations. The result of this study is shown in Figure 4.28, with suction side results on the left and pressure side results on the right. The turbulence level is increased by decreasing the $\mathrm{N}$ value in XFOIL (used in $\mathrm{e}^{\mathrm{N}}$ method). The results show that, for both the suction and pressure side, as the turbulence is increased the transition location moves toward the leading edge. This confirms that the increased turbulence levels associated with increased flow speed in the Stability Wind tunnel are most likely to cause the shift in transition location toward the leading edge (earlier transition). From examination of Figure 4.27 and Figure 4.28 it would appear that flow over the $0.8-\mathrm{m}$ model correspond to an $\mathrm{N}$ value of $\sim 8$ while flow over the 0.46 -m model has an $\mathrm{N}$ value closer to 6 . Another possible source of error is the wall interference which tends to increase the pressure on the side of the model producing positive pressure (suction side at negative angles and pressure side at positive angles).

Overall, it can be stated that this level of repeatability between models of different chord length, at different speeds, tested months apart, is excellent considering the many variables which influence boundary layer transition. This finding strongly suggests that the transition detection system produces trustworthy results. 

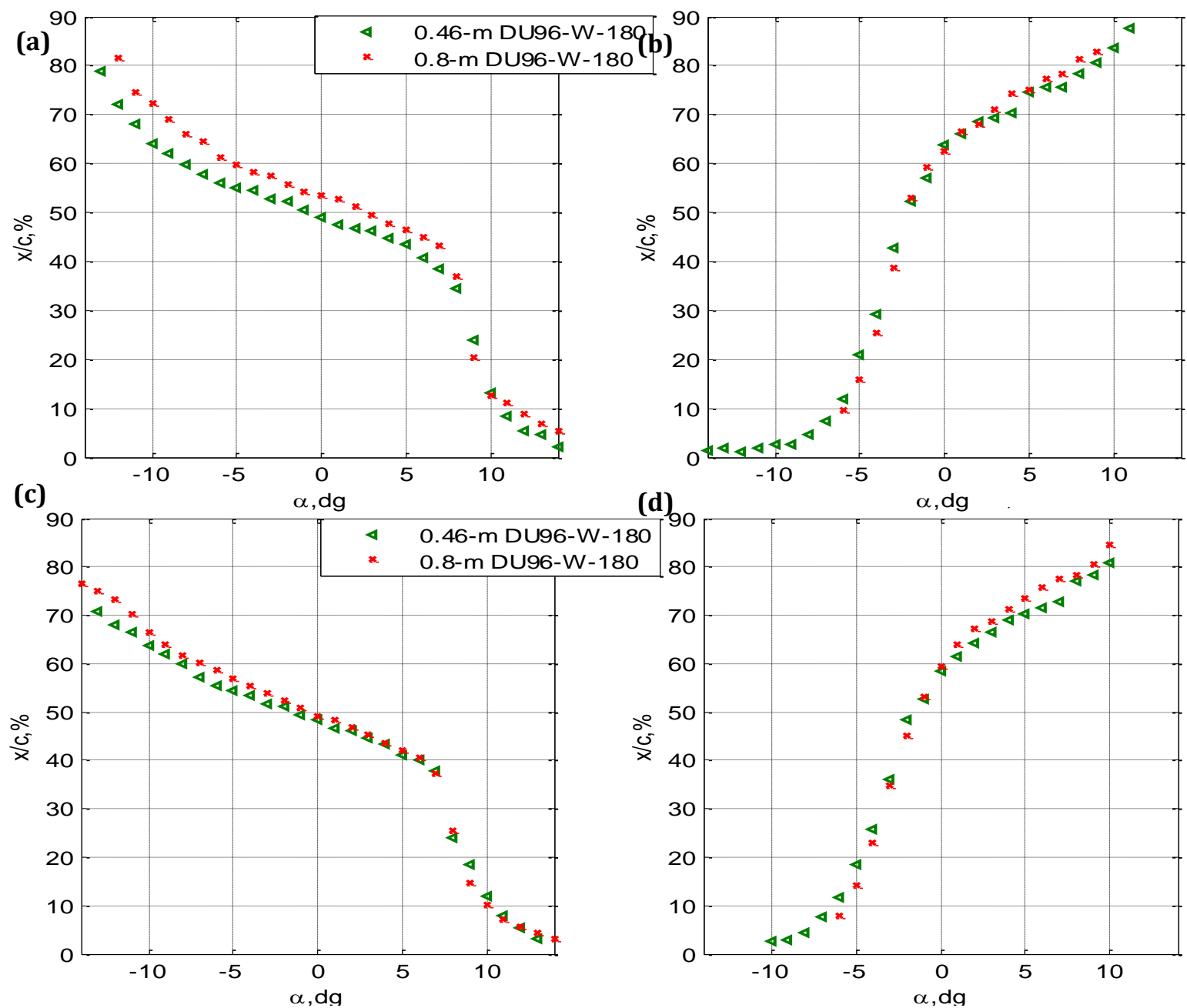

Figure 4.27 Effect of Chord Length of DU96-W-180 on the Infrared Thermography Transition Results, Investigated on (a) Suction Side at Re $=1.5 \times 10^{6}$ (b) Pressure Side at $R e=1.5 \times 10^{6}$ (c) Suction Side at Re $=2 \times 10^{6}$ (d) Pressure Side at
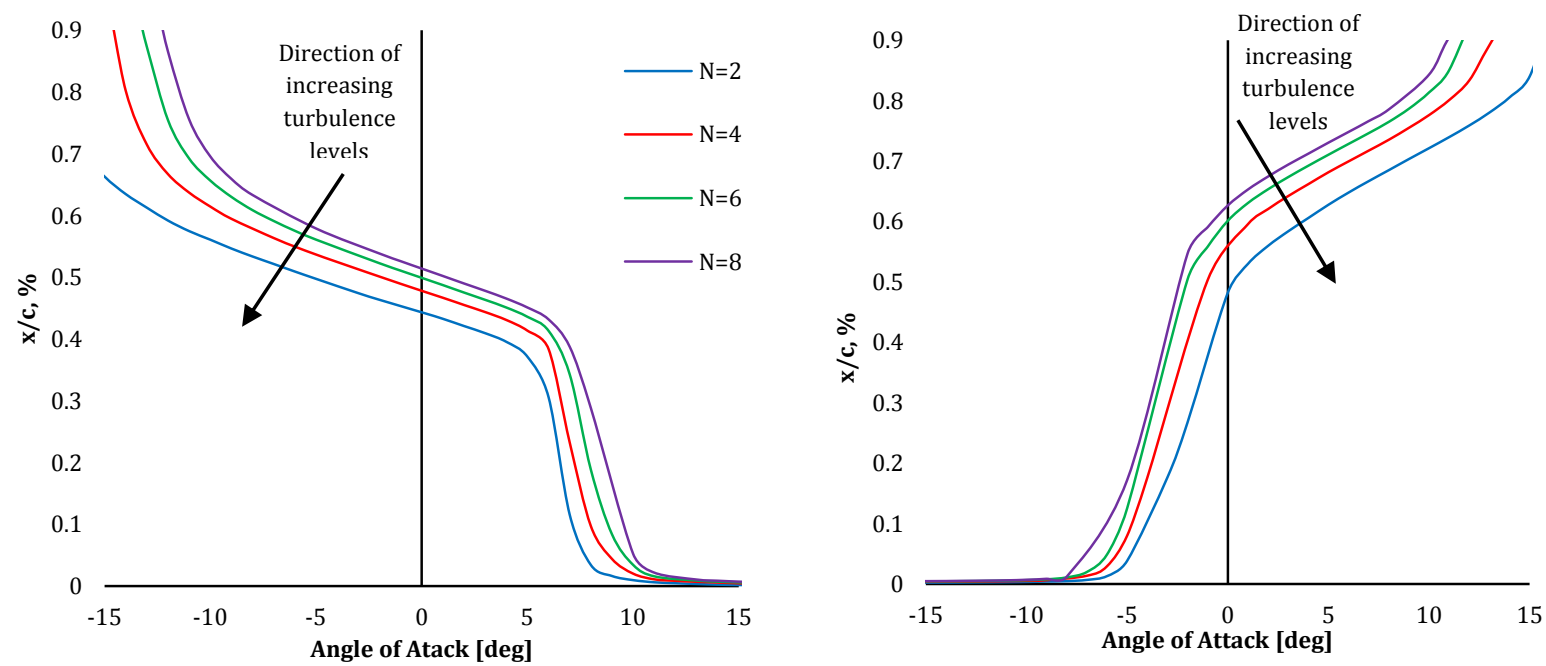

Figure 4.28 Variation of Transition Location with Increasing Turbulence Levels, as Predicted by XFOIL, for the DU96-W-180 at $R e=2 \times 10^{6}$ 


\subsection{Boundary Layer Flow Diagnostics}

During testing, distinct turbulent wedges were observed periodically on the infrared images. As this was observed in real time, as they appeared, the tests were paused and the source of the wedges were investigated. In all cases, it was found that some surface imperfection had initiated local transition thereby producing the wedge. In some cases the imperfection was due to contaminants in the flow which were transferred to the model surface while in most cases it was due to small air bubbles forming beneath the ConTact $\odot$ paper as the temperature was varied. Figure 4.29 shows a 5 -mm air bubble which formed on the $0.46-\mathrm{m}$ DU96-W-180 model at $\alpha=5^{\circ}$ and $\mathrm{Re}=1.5 \times 10^{6}$ and the wedge that resulted.

(a)

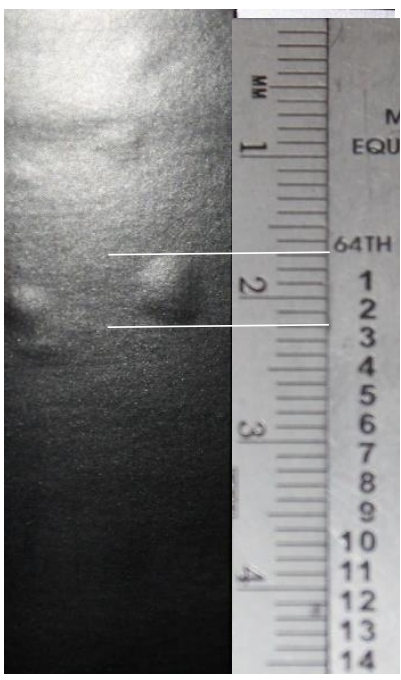

(b)

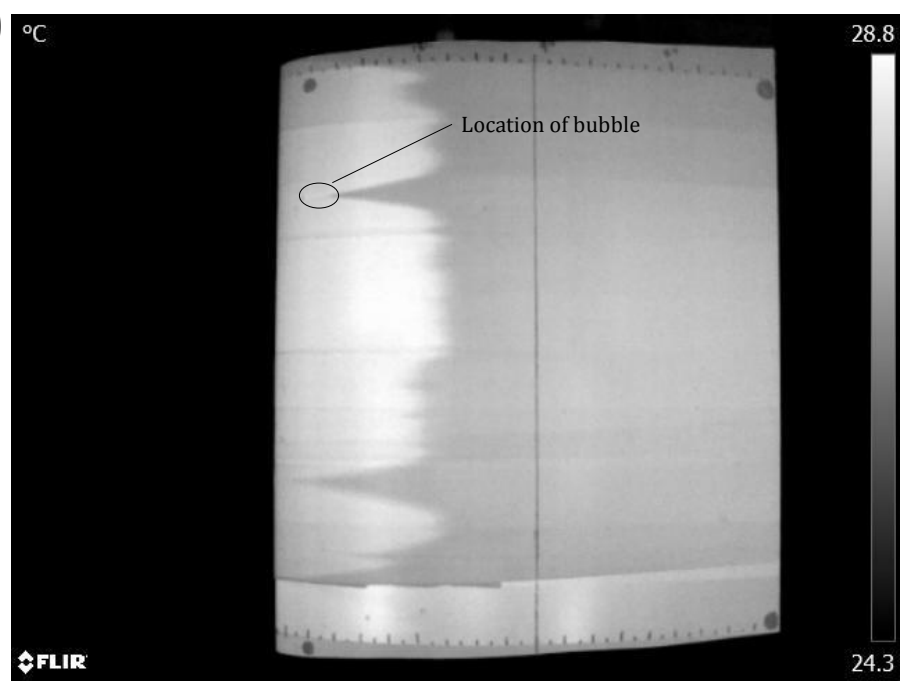

Figure 4.29 (a) 5-mm Air Bubble Formed in ConTact ${ }^{\odot}$ Paper on Model Surface and (b) Infrared Image showing the Turbulent Wedge Produced by the Air Bubble

Similarly, Figure 4.30 shows the effect of two $2-\mathrm{mm}$ long, $0.05-\mathrm{mm}$ high surface imperfections on the surface of the $0.8-\mathrm{m}$ DU96-W-180, at $\mathrm{Re}=1.5 \times 10^{6}$. This feature, shown in Figure 4.30a, caused early transition and produced distinct turbulent wedges on the infrared images, as seen in Figure 4.30b. Early detection of this feature allowed measures to be taken to improve the surface condition so that the fidelity of the results is preserved. After removing the surface imperfection, the wedges were no longer present, as depicted in Figure $4.30 c$, thereby proving that its source was indeed the imperfection and not the infrared system itself. These findings support Zuccher's (2008) observations that infrared thermography is very sensitive to surface conditions. 


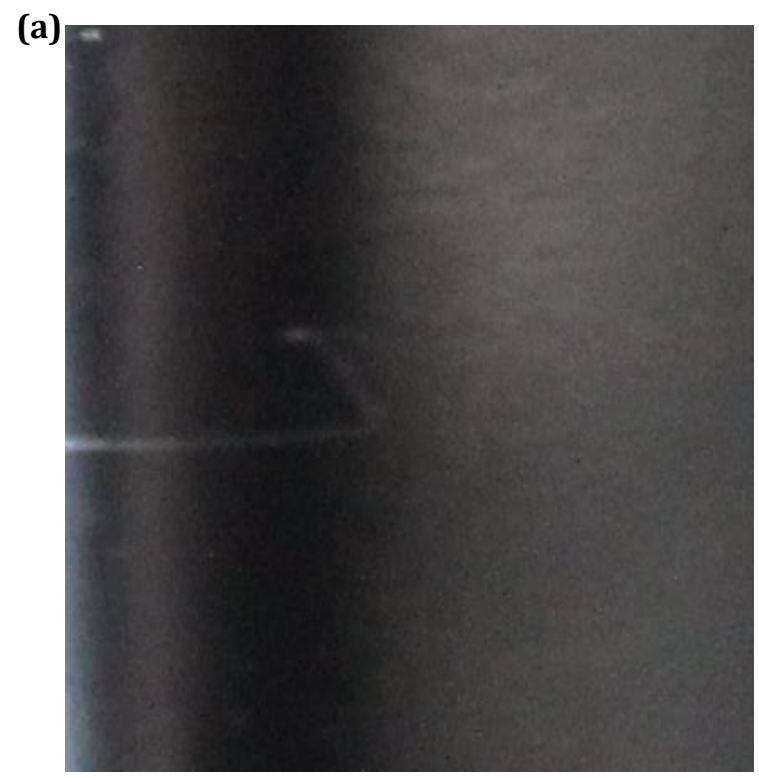

(b)

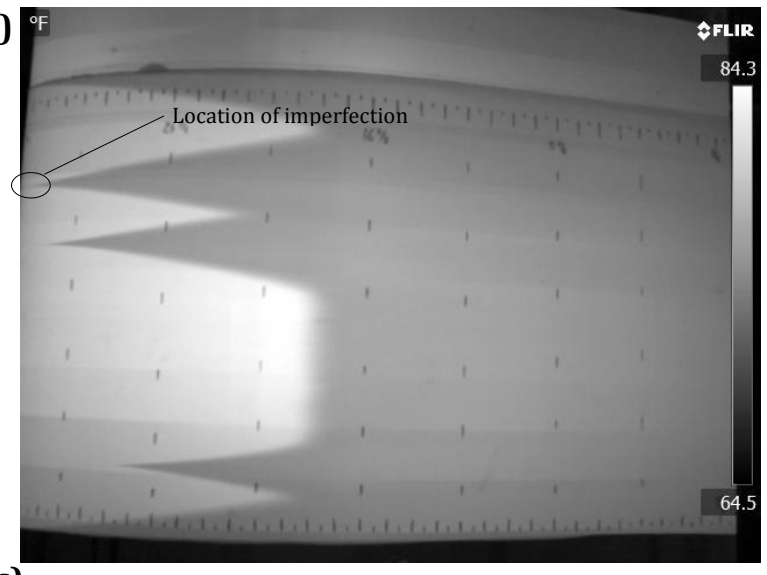

(c)

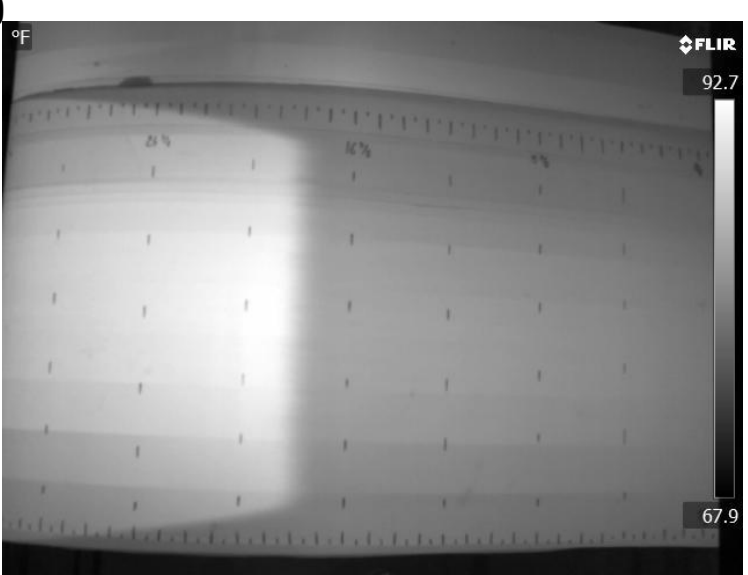

Figure 4.30 (a) 2-mm Tear in ConTact ${ }^{\odot}$ Paper on Model Surface (b) Infrared Image showing the Turbulent Wedge Produced by the Tear (c) Infrared Image after Tear is removed

These observations demonstrate the capability of the infrared thermography system to serve as a flow diagnostic tool in real time, during wind tunnel tests. This capability is invaluable in large wind tunnel tests as it would enable experimenters to investigate issues in flow quality as they arise, as opposed to after the test is completed and issues are reflected in the final results. Another important point to note is that even in the presence of these turbulent wedges, the natural transition location can still be extracted from the image suggesting that the system is very reliable in detecting transition wherever it occurs over the model. 


\section{Chapter 5:CONCLUSIONS}

A new boundary layer transition detection system was developed, implemented and validated in the Virginia Tech Stability Wind Tunnel. This system utilizes infrared thermography to visualize the inherent rise in heat transfer which occurs as laminar flow becomes turbulent, and then extracts the chord-wise transition location through image processing techniques.

The developmental phase of the system was conducted in the Virginia Tech Open Jet Wind Tunnel and involved tests to determine the best model material; the best method of creating a temperature difference; the best model insulation and additional transition detection methods for validation. During the developmental phase it was proved that:

1. Models must have a low surface emissivity (dull and dark in colour) to reduce infrared reflections which can extensively affect results.

2. Large metal models (such as aluminium) will not show IR transition results because material heat transfer will overshadow the heat transfer occurring in the flow.

3. Insulated aluminium models are excellent candidates for IR transition detection.

4. The best insulation for metal models must be closed-cell as well as insulating.

5. Wooden models as well as plastic models are acceptable for IR transition detection.

6. An active means of creating a temperature difference is integral to an efficient and reliable system as the experimenter must be able to control and sustain the temperature difference during the tests.

7. Special infrared windows are needed to allow camera to view the model since glass, Lexan and Plexiglas are opaque in the infrared range of the camera.

Based on these findings, the final infrared thermography transition detection system is comprised of:

1. Two high resolution FLIR A655sc infrared cameras, one mounted on each of the port and starboard sides of the wind tunnel. The cameras are each fixed to a tripod head which is mounted on a framework of $80 / 20$ aluminium bars bolted to the outer test section walls. Cameras and image acquisition are computer controlled.

2. A total of four $63.5-\mathrm{mm}$ diameter circular windows, two on each side of the test section, cut in the side walls.

3. Aluminium models covered in 0.8-mm FDA Compliant Rubber Silicone and a final top layer of ConTact $^{\circledR}$ paper.

4. A series of 25.4-mm wide rubber silicone fiberglass insulated heaters mounted inside the model and controlled externally by experimenters. 
5. An image processing software which extracts the transition location from the infrared images by detecting the maximum stream-wise colour gradient, converting the image pixel location to an edge length location and finally to chord length location.

The additional transition detection measurement schemes developed for validation include:

1. The use of a stethoscope attached to a Pitot probe to traverse along the airfoil chord observing changes in noise levels from leading and trailing edge. Transition is marked by a change in sound frequency and level. This is done simultaneously on both pressure and suction side of the model.

2. Analysis of the mean pressure distribution over the chord of the model to find the sudden drop which is associated with transition. This analysis was done by ascertaining the maxima in the second derivative of the coefficient of pressure distribution.

The validation phase of the system development, conducted in the Virginia Tech Stability Wind Tunnel, involved comparing the results of these additional transition detection methods with that produced by the infrared thermography system and XFOIL predictions. This was done on two DU96-W-180 airfoils, one with a chord of 0.46-m and the other with a chord of $0.8-\mathrm{m}$, two months apart.

The effect of the infrared system on the flow was investigated on the 0.8-m DU96-W-180 model and it was found that:

1. Heating the model to $\Delta \mathrm{T} \sim 16.67-\mathrm{K}\left(20^{\circ} \mathrm{F}\right)$ does not significantly affect the flow. The lift and drag plots agree closely for both the heated and unheated model.

2. Experimental and simulated results (from XFOIL) prove that an insulator of 0.8-mm thickness, such as that used in the current system, does not significantly affect the flow.

The tests of the validation study also revealed that the mean pressure analysis method for transition detection is efficient and produces accurate transition results. It also has the added advantage of being applicable to any model for which a surface pressure distribution is available. However, there are significant limitations to this method arising from the high sensitivity of the technique to the pressure tap arrangement on the model. To summarize it can be conclusively stated that the mean pressure analysis:

1. is an easy way to determine the transition region for any model with an associated pressure surface distribution.

2. does not produce useful results at high angles of attack.

3. is limited by the distance between consecutive pressure taps such that the transition region can only be reported as being within this region. 
4. will not show the distinct drop indicative of the transition if the transition region occurs sufficiently close to or directly over a pressure tap, an occurrence which is inevitable in an angle of attack sweep (especially for models with small chords).

The stethoscope tests involved in the system validation proved to be a quick and easy means of estimating the transition location. However, this technique was highly userdependent and, of all the transition detection methods, it has the largest uncertainty (on average $\sim \pm 12.7-\mathrm{mm}$ ). It was also proven that the stethoscope technique:

1. possesses an inherent and unchanging uncertainty due to the effect of the probe once it is inserted into the flow - it was shown that transition is moved upstream once the probe is inserted

2. produces consistent results for both the clean and insulated 0.8-m DU96-W-180 further proving that the $0.8-\mathrm{mm}$ insulator does not affect the flow

3. could not be used at high Reynolds Numbers because the probe cannot withstand increased forces

4. is limited by issues with aligning the probe with model surface when airfoil curvature is large or when the angle of attack is high

5. is not efficient for large scale testing because it requires at least 30 seconds per angle, and at least two experimenters, to collect useful data

6. cannot detect three dimensional effects and can only detect non-uniformity if several points are taken along the airfoil span for each angle.

The infrared transition detection system developed is robust and efficient. Additionally, the set-up is minimal and does not affect any other system in use in the wind tunnel. The combined transition results from all four methods (XFOIL, IRT, mean pressure, stethoscope) showed good agreement with each other for the two models, at least two Reynolds numbers and several angles of attack. This agreement, of under several different conditions, suggests the infrared thermography system implemented in the Virginia Tech Stability Wind Tunnel has the capability to efficiently and accurately detect transition over a large range of angles of attack, at low speeds. It was further proven that:

1. IRT results are in agreement with XFOIL, mean pressure analysis and stethoscope results at 3 different Reynolds Numbers and for two models, suggesting that infrared thermography gives accurate results for transition location.

2. Of the three transition detection methods, IRT is most efficient and reliable method, producing results at all Reynolds numbers and every angle of attack between $-20^{\circ}$ to $20^{\circ}$.

3. The IRT results correspond to the onset of transition, when two dimensional instabilities (Tollmien Schlichting waves) first develop. 
4. Infrared transition results are seen instantaneously (in less than 1-s of changing any flow condition), and with as small a $\Delta \mathrm{T}$ as $0.56-\mathrm{K}\left(1^{\circ} \mathrm{F}\right)$.

5. IR images on both sides of the airfoil and the complete pressure distribution can be acquired simultaneously.

6. Infrared thermography detects three-dimensional effects in the flow: including the small effects associated with transition at angles of attack just before stall, as well as the fully three dimensional flow observed after stall. This is not possible with other methods.

7. The infrared system is able to consistently produce useful results for every angle of attack and every Reynolds number tested, even angles of attack past the stall angle.

8. The results of the infrared system can be estimated within an uncertainty of \pm 5 - $\mathrm{mm}$ using the algorithm developed for image processing and transition location extraction. This is better than both the stethoscope and the mean pressure analysis methods.

9. IRT is an excellent flow diagnostics tool as it detects imperfections in the model surface condition in real time, an outcome which other techniques cannot achieve. This enables experimenters to maintain the fidelity of testing if and when imperfections develop. 


\section{References}

Aerospace Engineering Department. "Stability Wind Tunnel Aerodynamic Qualities." Virginia Tech, n.d. Web. December 2013.

Allen H.J. and Vincenti W.G., 1947, "Wall Interference in a Two-Dimensional-Flow Wind Tunnel, with Consideration of the Effect of Compressibility" NACA Report, No. 782.

Astarita T., Cardone G., Carlomagno G.M., Meola C., 2000, "A Survey on Infrared Thermography for Convective Heat Transfer Measurements", Optics \& Laser Technology, vol. 32, pp. 593-610.

Baek, P., Fuglsang, P., "Experimental Detection of Transition on Wind Turbine Airfoils", European Wind Energy Conference, 2009.

Brooks, T. F., Pope, D. S., Marcolini, M. A., Airfoil Self-Noise and Prediction, NASA Reference Publication 1218, pp. 5-8, 1989.

Crawford, B. K., Duncan G. T. Jr., West, D. E., Saric, W. S, "Quantitative Boundary-Layer Transition Measurements Using IR Thermography", AIAA SciTech, 13-17 January 2014, National Harbour, MD.

De Luca L, Guglieri G., Cardone G., Carlomagno G. M., 1995, “Experimental Analysis of Surface Flow on a Delta Wing by Infrared Thermography”, AIAA Journal, vol. 33, no. 8.

De Luca L., Carlomagno G. M., Buresti, G., 1990, "Boundary Layer Diagnostics by Means of an Infrared Scanning Radiometer", Experiments in Fluids, vol 9, 121-128.

Ehrmann, R. S., White, E. B., "Influence of 2D Steps and Distributed Roughness on Transition on a NACA 633-418”, AIAA SciTech, 13-17 January 2014, National Harbor, MD

Freels, J. R., 2012, "An Examination of Configurations for Using Infrared to Measure Boundary Layer Transition", M.S. Thesis, Aerospace Engineering, Texas A\&M University.

Gaidos, E. J., 1990, "Remote Infrared Thermography for Boundary Layer Measurements", M.S. Thesis, Aeronautics and Astronautics, MIT.

Gompertz Kyle, Bons, J. P., Gregory, J. W., Passive Trip Evaluation Using Infrared Thermography, American Institute of Aeronautics and Astronautics, Inc., 2012.

Kuklova J., Popelka, L., Souckova, N., Vitu, T., "Visualization of Airfoil Boundary Layer by Infrared Thermography to Determine Influence of Roughness-Due-to-Insect", 11th International Conference on Quantitative InfraRed Thermography, Naples Italy, 2012. 
Le Sant, Y., Marchand, M., Millan, P., Fontaine, J., 2002, "An overview of infrared thermography techniques used in large wind tunnels", Aerospace Science and Technology, vol. 6, pp. 355-366.

Lutz, Th., Wurz, W., Wagner, S., 2001, "Numerical Optimization and Wind Tunnel Testing of Low Reynolds Number Airfoils", Fixed and Flapping Wing Aerodynamics for Micro-Air Vehicle Applications, Vol. 195, AIAA.

Maughmer, M. D., Coder, J. G., "Comparisons of Theoretical Methods for Predicting Airfoil Aerodynamic Characteristics", Airfoils, Incorporated, Port Matilda, August 2010.

Mueller, T. J., 2001, "Fixed and Flapping Wing Aerodynamics for Micro Air Vehicle Applications", American Institute of Aeronautics and Astronautics.

Patorski, J., Bauer, G. S., Dementjev, S., 2000, "Two-Dimensional and Dynamic (2DD) Method of Visualization of the Flow Characteristics in a Convection Boundary Layer using Infrared Thermography", Thermosense XXII, edited by R. B. Dinwiddie, D. H. LeMieux, vol. 4020.

Popov, A. V., Botez, R. M, Labib, M., 2008, "Transition Point Detection from the Surface Pressure Distribution for Controller Design", Journal of Aircraft, vol 45, no. 1, pp. 23-28.

Quast, A., 1987, "Detection of Transition by Infrared Image Technique", 12th International Congress on Instrumentation in Aerospace Simulation Facilities, Williamsburg, VA, June 22 25.

Ricci, R., Sergio Montelpare, S., 2009, "Analysis of Boundary Layer Separation Phenomena by Infrared Thermography - Use of Acoustic and/or Mechanical Devices to Avoid or Reduce the Laminar Separation Bubble Effects", Quantitative InfraRed Thermography Journal, vol. 6, no. 1, pp. 101-125.

Schmitt, R. L. and Chanetz, B. P., 1985, "Experimental Investigation of Three Dimensional Separation on an Ellipsoid-Cylinder Body at Incidence", AIAA 18th Fluid Dynamics and Plasmadynamics and Lasers Conference, Cincinatti OH, July 16-18. AIAA-85-1686.

Yokokawa, Y., 2005, "Transition Measurement on Metallic Aircraft Model in Typical Lowspeed Wind Tunnel", Transactions of the Japan Society of Aeronautical and Space Sciences, vol. 48(161), pp. 175-176.

Zuccher, S., Saric, W. S., 2008, "Infrared Thermography Investigations in Transitional Supersonic Boundary Layers", Experiments in Fluids, vol. 44, pp. 145-157. 


\section{Appendix A Additional Instrumentation \& Equipment}

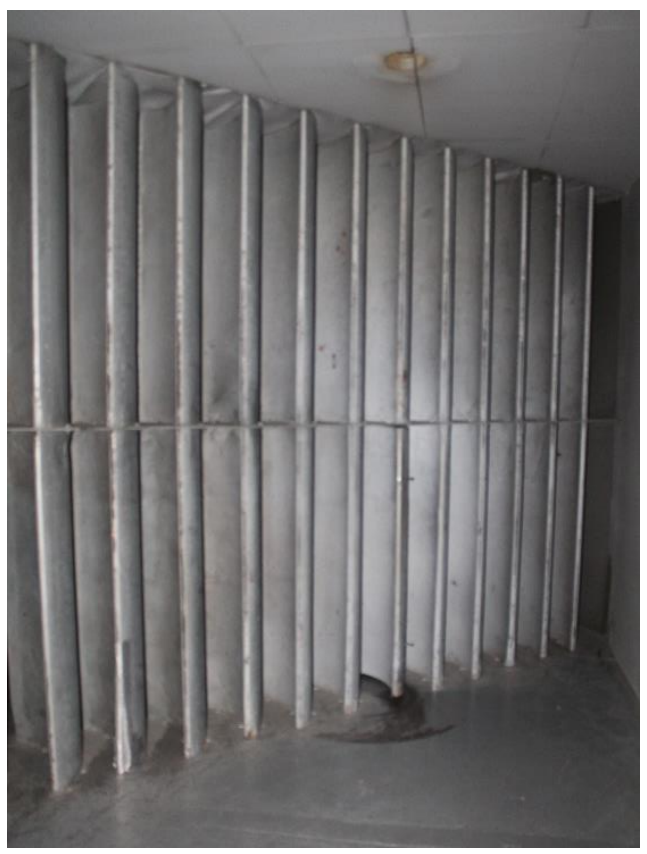

Figure A1: Close up of Turning Vanes between Diffuser and Fan of Virginia Tech Stability Wind Tunnel

(a)

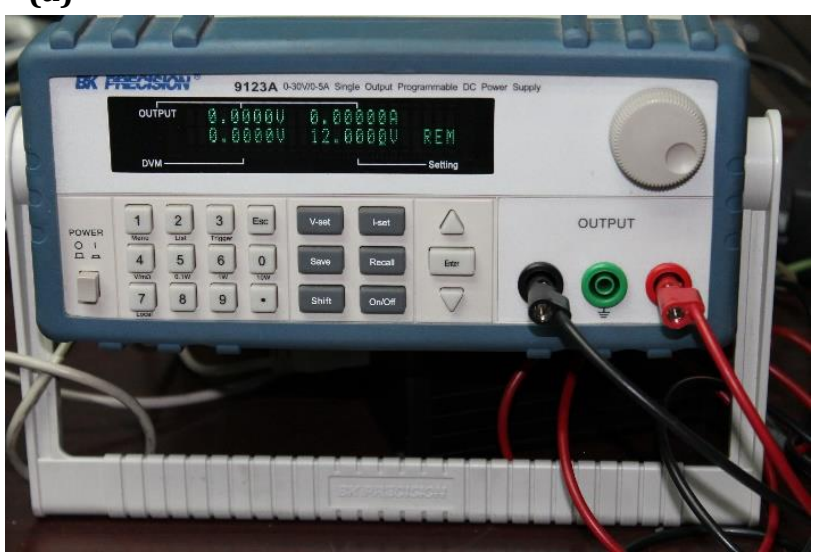

(b)

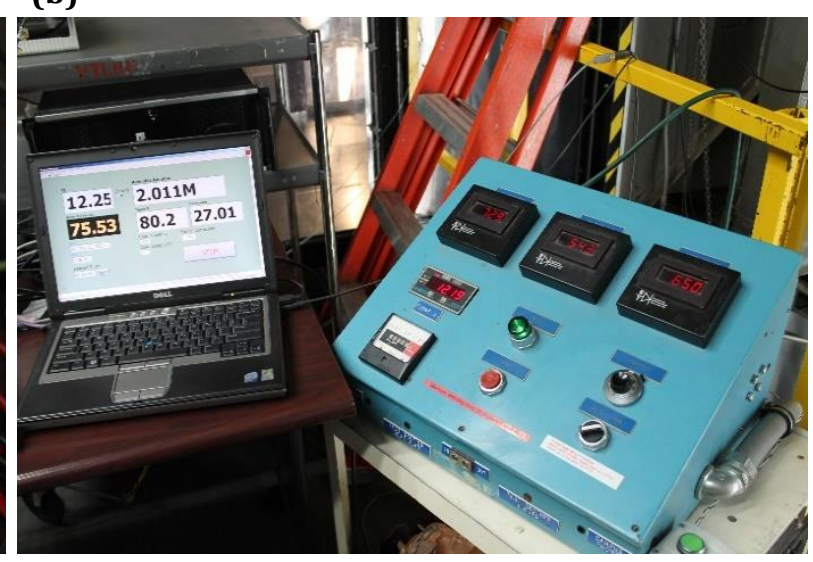

Figure A2: Instrumentation to Control the (a) Tunnel Turntable and (b) Wind Tunnel Speed 


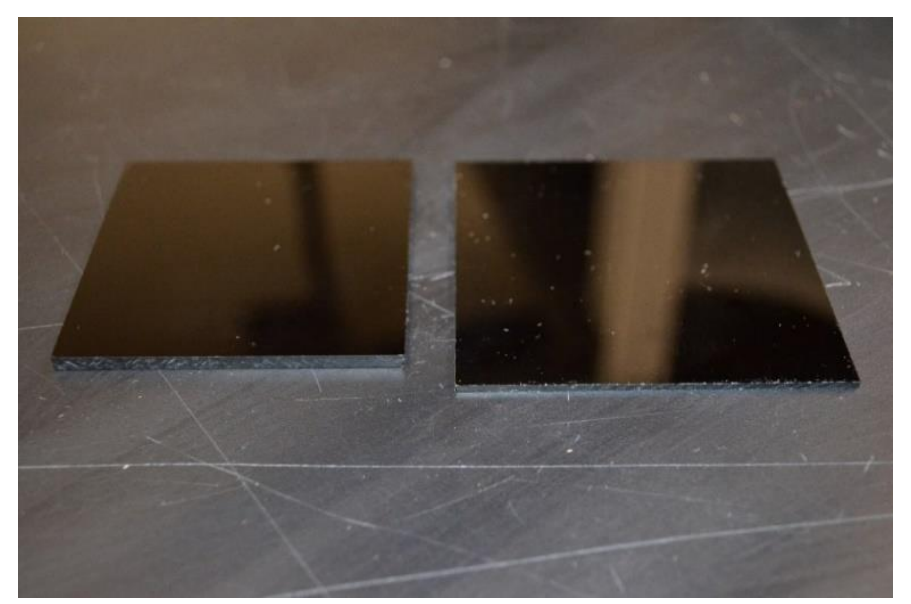

Figure A3: Samples of Infrared Plexiglass of Different Thicknesses
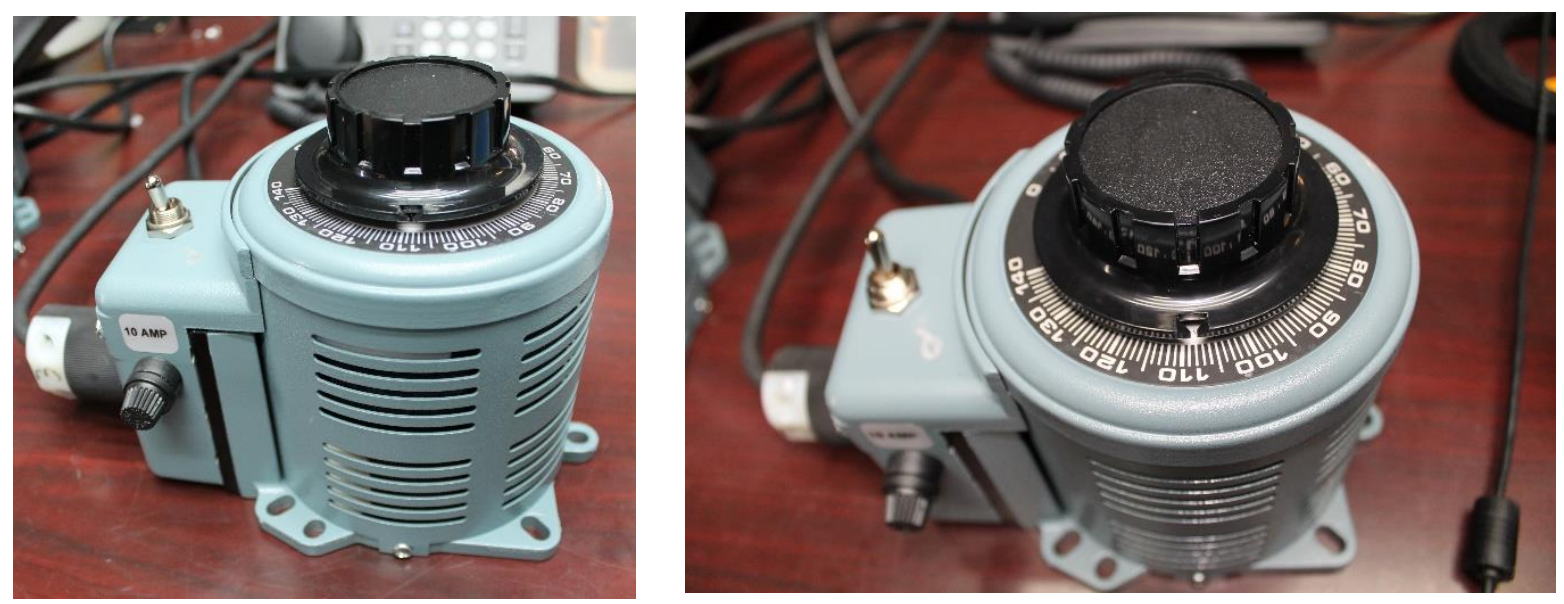

Figure A4: General Radio Type W5MT Variac used to Control Heaters during Tests

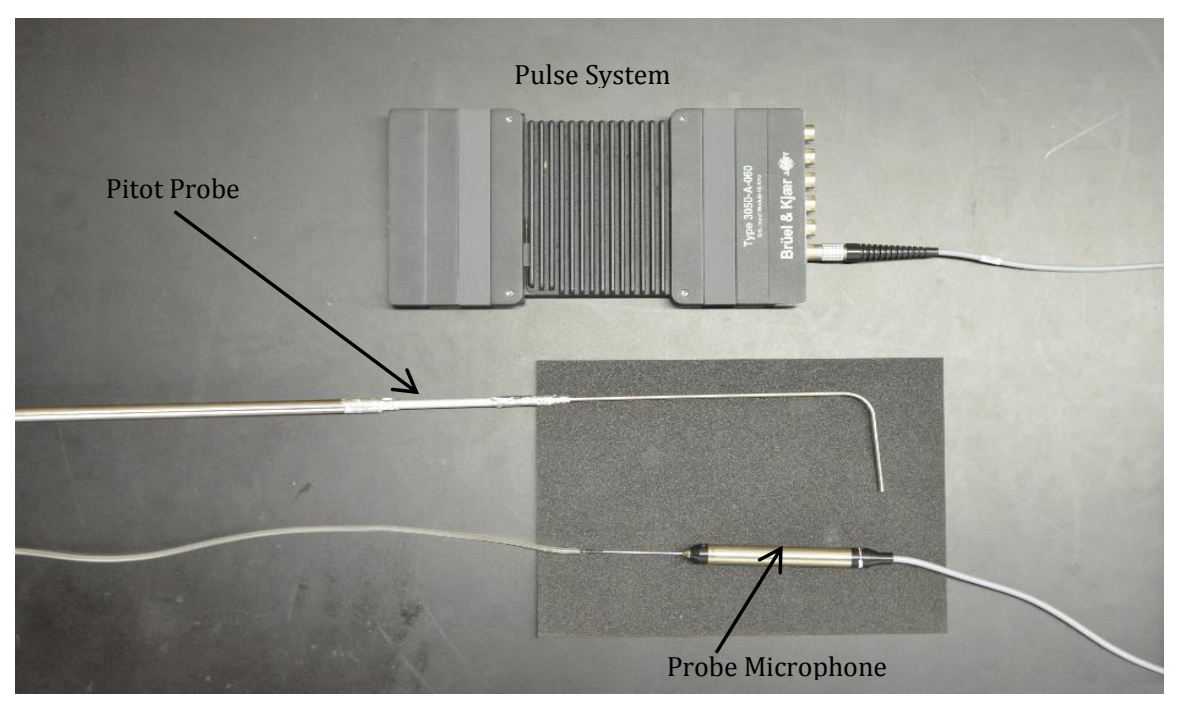

Figure A5: Probe Microphone used to Record Sounds Heard by Stethoscope 


\section{Appendix B Airfoil Pressure Port Locations}

\begin{tabular}{|c|c|c|c|c|c|c|}
\hline$x$ [in] & $y$ [in] & $z$ [in] & \multirow{5}{*}{$\begin{array}{l}z=1: \text { Suction Side } \\
z=-1: \text { Pressure Side }\end{array}$} & $x$ [in] & $y$ [in] & $z$ [in] \\
\hline 0.00000 & 0 & 1 & & 0.03150 & 0.00844 & -1 \\
\hline 0.03150 & 0.00844 & 1 & & 0.11025 & 0.029541 & -1 \\
\hline 0.11025 & 0.029541 & 1 & & 0.22050 & 0.059083 & -1 \\
\hline 0.22050 & 0.059083 & 1 & & 0.36225 & 0.097065 & -1 \\
\hline 0.36225 & 0.097065 & 1 & & 0.53550 & 0.143487 & -1 \\
\hline 0.53550 & 0.143487 & 1 & & 0.74025 & 0.198349 & -1 \\
\hline 0.74025 & 0.198349 & 1 & & 0.97650 & 0.261652 & -1 \\
\hline 0.97650 & 0.261652 & 1 & & 1.29150 & 0.346056 & -1 \\
\hline 1.29150 & 0.346056 & 1 & & 1.66950 & 0.447341 & -1 \\
\hline 1.66950 & 0.447341 & 1 & & 2.11050 & 0.565507 & -1 \\
\hline 2.11050 & 0.565507 & 1 & & 2.61450 & 0.700553 & -1 \\
\hline 2.61450 & 0.700553 & 1 & & 3.15000 & 0.84404 & -1 \\
\hline 3.15000 & 0.84404 & 1 & & 3.93750 & 1.05505 & -1 \\
\hline 3.93750 & 1.05505 & 1 & & 4.72500 & 1.26606 & -1 \\
\hline 4.72500 & 1.26606 & 1 & & 5.51250 & 1.47707 & -1 \\
\hline 5.51250 & 1.47707 & 1 & & 6.30000 & 1.68808 & -1 \\
\hline 6.30000 & 1.68808 & 1 & & 7.08750 & 1.89909 & -1 \\
\hline 7.08750 & 1.89909 & $\frac{1}{1}$ & & 7.87500 & 2.1101 & -1 \\
\hline $\begin{array}{l}7.87500 \\
866250\end{array}$ & $\begin{array}{r}2.1101 \\
232111\end{array}$ & $\frac{1}{1}$ & & 8.66250 & 2.32111 & -1 \\
\hline $\begin{array}{l}8.66250 \\
9.45000\end{array}$ & $\begin{array}{l}2.32111 \\
2.53212\end{array}$ & $\frac{1}{1}$ & & 9.45000 & 2.53212 & -1 \\
\hline 11.02500 & 2.95414 & $\frac{1}{1}$ & & 11.02500 & 2.95414 & -1 \\
\hline 12.60000 & 3.37616 & 1 & & 12.60000 & 3.37616 & -1 \\
\hline 14.17500 & 3.79818 & 1 & & 14.17500 & 3.79818 & -1 \\
\hline 15.75000 & 4.2202 & 1 & & 15.75000 & 4.2202 & -1 \\
\hline 17.32500 & 4.64222 & 1 & & 17.32500 & 4.64222 & -1 \\
\hline 18.90000 & 1.46424 & 1 & & 18.90000 & 1.46424 & -1 \\
\hline 20.47500 & 1.88626 & 1 & & 20.47500 & 1.88626 & -1 \\
\hline 22.05000 & 2.30828 & 1 & & 22.05000 & 2.30828 & -1 \\
\hline 23.62500 & 2.7303 & 1 & & 23.62500 & 2.7303 & -1 \\
\hline 25.20000 & 3.15232 & 1 & & 25.20000 & 3.15232 & -1 \\
\hline 25.83000 & 3.321128 & 1 & & 25.83000 & 3.321128 & -1 \\
\hline 26.46000 & 3.489936 & 1 & & 26.46000 & 3.489936 & -1 \\
\hline 27.09000 & 3.658744 & 1 & & 27.09000 & 3.658744 & -1 \\
\hline 27.72000 & 3.827552 & 1 & & 27.72000 & 3.827552 & -1 \\
\hline 28.35000 & 3.99636 & 1 & & 28.35000 & 3.99636 & -1 \\
\hline 28.98000 & 4.165168 & 1 & & 28.98000 & 4.165168 & -1 \\
\hline 29.61000 & 4.333976 & 1 & & 29.61000 & 4.333976 & -1 \\
\hline 30.24000 & 4.502784 & 1 & & 30.24000 & 4.502784 & -1 \\
\hline 30.87000 & 4.671592 & 1 & & 30.87000 & 4.671592 & -1 \\
\hline
\end{tabular}

Table B1: Pressure Port Locations on 0.8-m chord, DU96-W-180 Airfoil 


\begin{tabular}{|c|c|c|c|c|c|c|}
\hline$x$ [in] & $y$ [in] & $z$ [in] & & $x$ [in] & $y$ [in] & $z$ [in] \\
\hline 0.00000 & 0 & 1 & & 0.01800 & 0.004823 & -1 \\
\hline 0.01800 & 0.004823 & 1 & \multirow{2}{*}{$\begin{array}{l}z=1: \text { Suction Side } \\
z=-1: \text { Pressure Side }\end{array}$} & 0.06300 & 0.016881 & -1 \\
\hline 0.06300 & 0.016881 & 1 & & 0.12600 & 0.033762 & -1 \\
\hline 0.12600 & 0.033762 & 1 & & 0.20700 & 0.055465 & -1 \\
\hline 0.20700 & 0.055465 & 1 & & 0.30600 & 0.081992 & -1 \\
\hline 0.30600 & 0.081992 & 1 & & 0.42300 & 0.113343 & -1 \\
\hline 0.42300 & 0.113343 & 1 & & 0.55800 & 0.149516 & -1 \\
\hline 0.55800 & 0.149516 & 1 & & 0.73800 & 0.197747 & -1 \\
\hline 0.73800 & 0.197747 & 1 & & 0.95400 & 0.255624 & -1 \\
\hline 0.95400 & 0.255624 & 1 & & 1.20600 & 0.323147 & -1 \\
\hline 1.20600 & 0.323147 & 1 & & 1.49400 & 0.400316 & -1 \\
\hline 1.49400 & 0.400316 & 1 & & 1.80000 & 0.482309 & -1 \\
\hline 1.80000 & 0.482309 & 1 & & 2.25000 & 0.602886 & -1 \\
\hline 2.25000 & 0.602886 & 1 & & 2.70000 & 0.723463 & -1 \\
\hline 2.70000 & 0.723463 & 1 & & 3.15000 & 0.84404 & -1 \\
\hline 3.15000 & 0.84404 & 1 & & 3.60000 & 0.964617 & -1 \\
\hline 3.60000 & 0.964617 & 1 & & 4.05000 & 1.085194 & -1 \\
\hline 4.05000 & 1.085194 & 1 & & 4.50000 & 1.205771 & -1 \\
\hline 4.50000 & 1.205771 & 1 & & 4.95000 & 1.326349 & -1 \\
\hline $\begin{array}{l}4.95000 \\
540000\end{array}$ & $\begin{array}{l}1.326349 \\
1446926\end{array}$ & $\frac{1}{1}$ & & 5.40000 & 1.446926 & -1 \\
\hline $\begin{array}{l}5.40000 \\
6.30000\end{array}$ & $\begin{array}{c}1.446926 \\
1.68808\end{array}$ & $\frac{1}{1}$ & & 6.30000 & 1.68808 & -1 \\
\hline 7.20000 & 1.929234 & 1 & & 7.20000 & 1.929234 & -1 \\
\hline 8.10000 & 0.000388 & 1 & & 8.10000 & 0.000388 & -1 \\
\hline 9.00000 & 0.241543 & 1 & & 9.00000 & 0.241543 & -1 \\
\hline 9.90000 & 0.482697 & 1 & & 9.90000 & 0.482697 & -1 \\
\hline 10.80000 & 0.723851 & 1 & & 10.80000 & 0.723851 & -1 \\
\hline 11.70000 & 0.965006 & 1 & & 11.70000 & 0.965006 & -1 \\
\hline 12.60000 & 1.20616 & 1 & & 12.60000 & 1.20616 & -1 \\
\hline 13.50000 & 1.447314 & 1 & & 13.50000 & 1.447314 & -1 \\
\hline 14.40000 & 1.688468 & 1 & & 14.40000 & 1.688468 & -1 \\
\hline 14.76000 & 0.00493 & 1 & & 14.76000 & 0.00493 & -1 \\
\hline 15.12000 & 0.101392 & 1 & & 15.12000 & 0.101392 & -1 \\
\hline 15.48000 & 0.197853 & 1 & & 15.48000 & 0.197853 & -1 \\
\hline 15.84000 & 0.294315 & 1 & & 15.84000 & 0.294315 & -1 \\
\hline 16.20000 & 0.390777 & 1 & & 16.20000 & 0.390777 & -1 \\
\hline 16.56000 & 0.487239 & 1 & & 16.56000 & 0.487239 & -1 \\
\hline 16.92000 & 0.5837 & 1 & & 16.92000 & 0.5837 & -1 \\
\hline 17.28000 & 0.680162 & 1 & & 17.28000 & 0.680162 & -1 \\
\hline 17.64000 & 0.776624 & 1 & & 17.64000 & 0.776624 & -1 \\
\hline
\end{tabular}

Table B2: Pressure Port Locations on 0.46-m chord, DU96-W-180 Airfoil 


\section{Appendix C Infrared Videos of Flow}
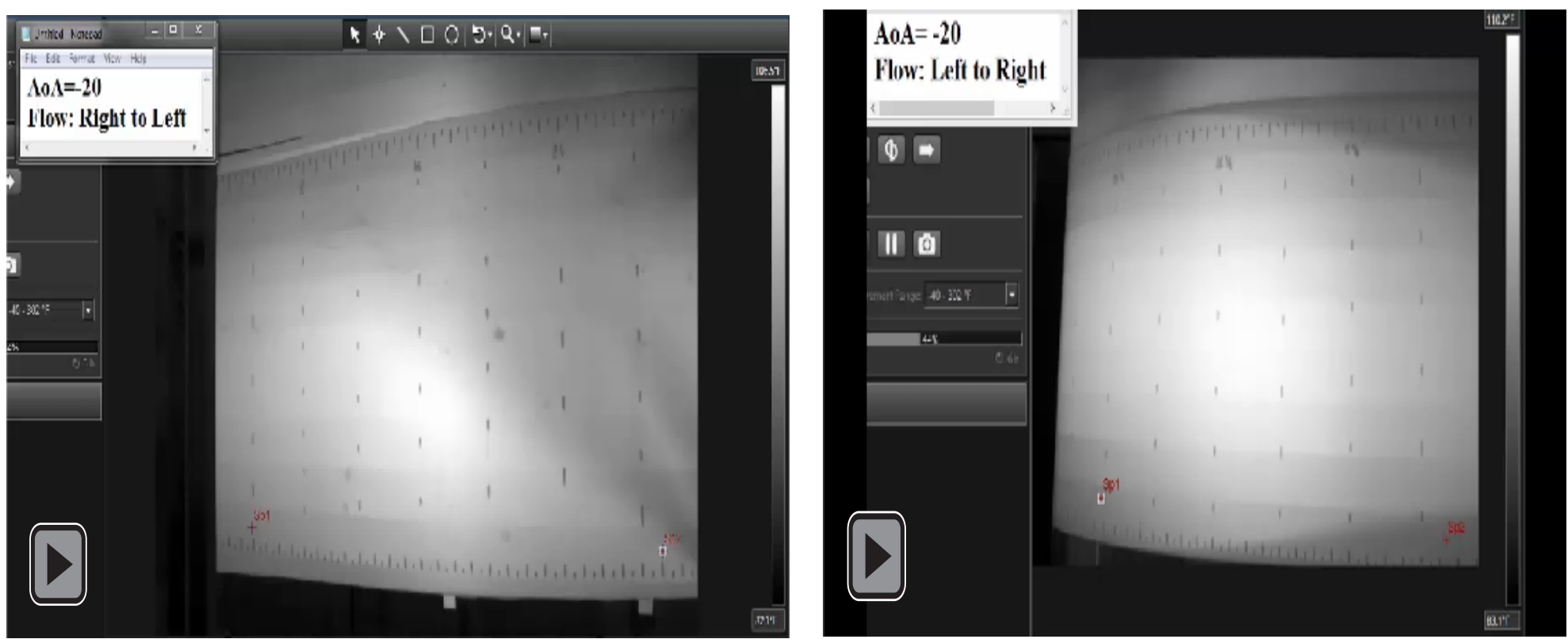

Video C1: Flow over (a) Pressure and (b) Suction Sides of 0.8- $m$ DU96-W-180 at Re $=2 \times 10^{5}$, Rotating from $\alpha=$ $20^{\circ}$ to $20^{\circ}$, as seen through Infrared Camera
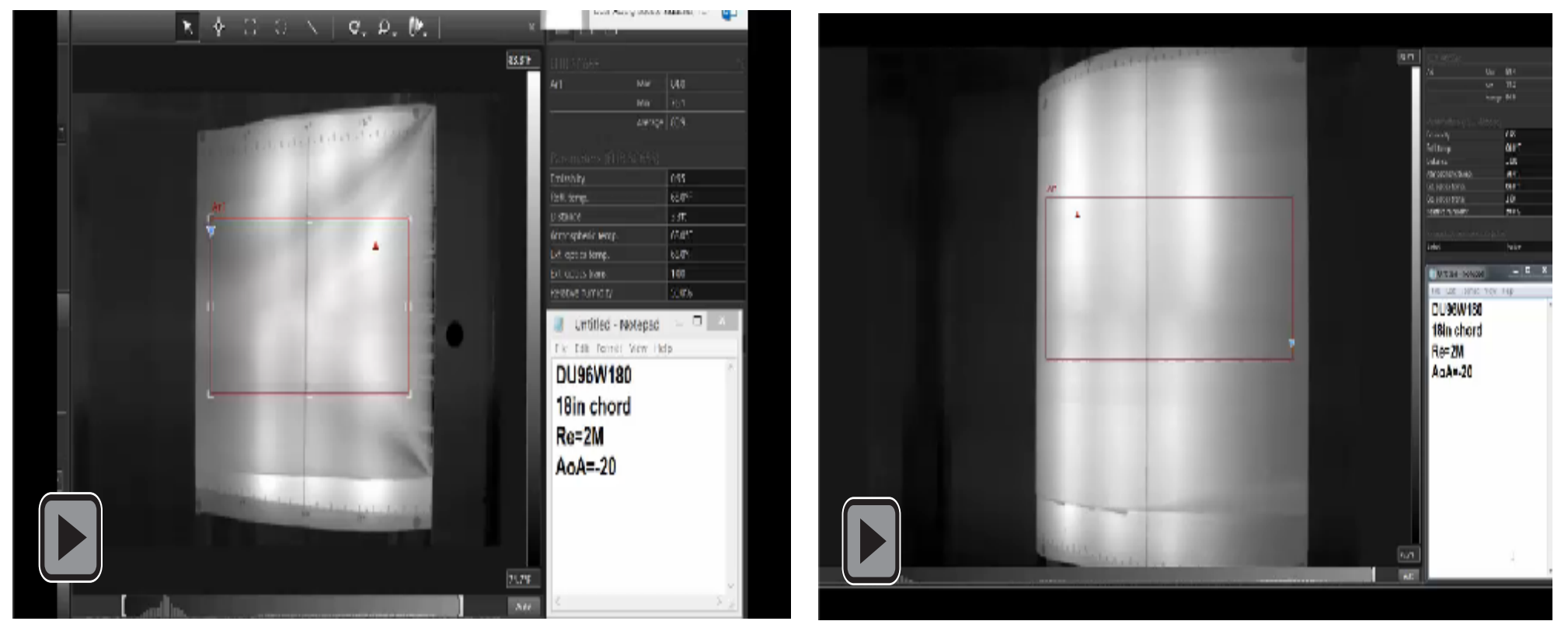

Video C2: Flow over (a) Pressure and (b) Suction Sides of 0.46- $\mathrm{m}$ DU96-W-180 at Re $=2 \times 10^{5}$, Rotating from $\alpha=$ $20^{\circ}$ to $20^{\circ}$, as seen through Infrared Camera 
Appendix D Infrared Images of 0.8-m DU96-W-180 at Re $=1.5 \times 10^{5}$ (From $\alpha=20^{\circ}$ to -20 , Pressure Side Images are on Left and Suction Side Images are on Right.) 


\section{8- $\mathrm{m}$ DU96-W-180 at $R e=1.5 \times 10^{5}$}

\section{PRESSURE SIDE}
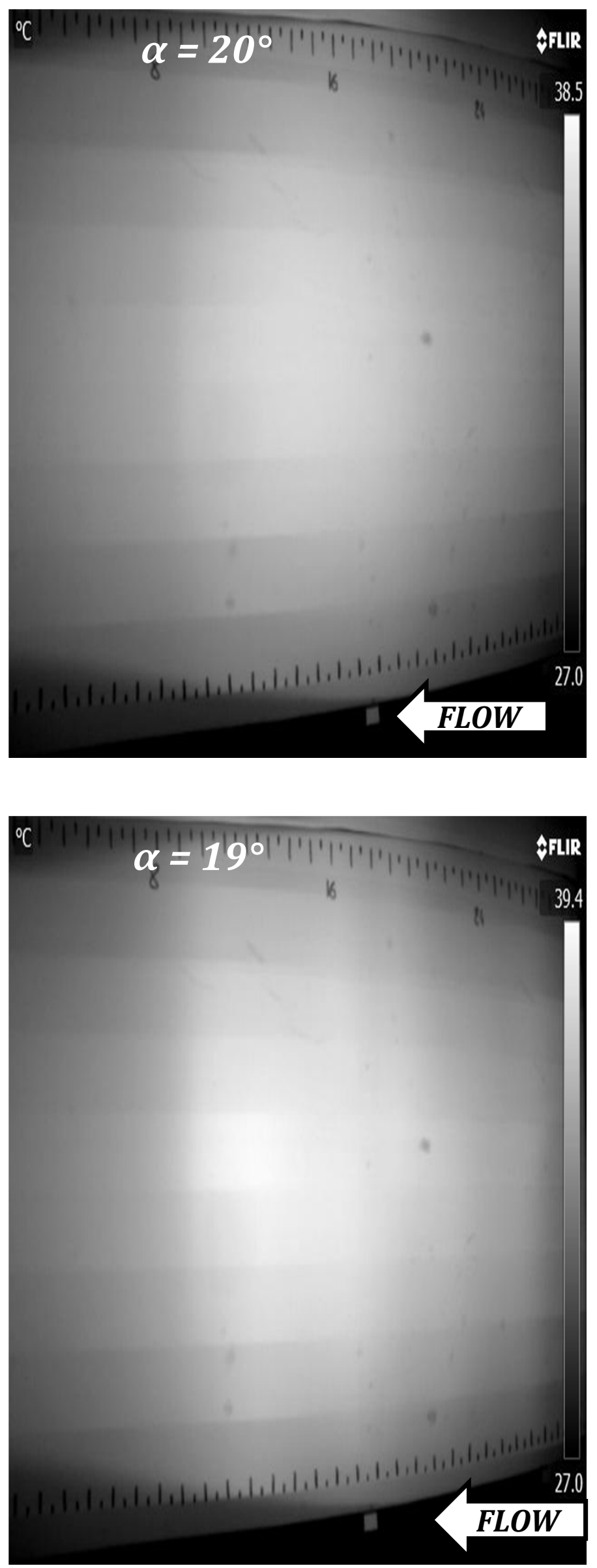

SUCTION SIDE
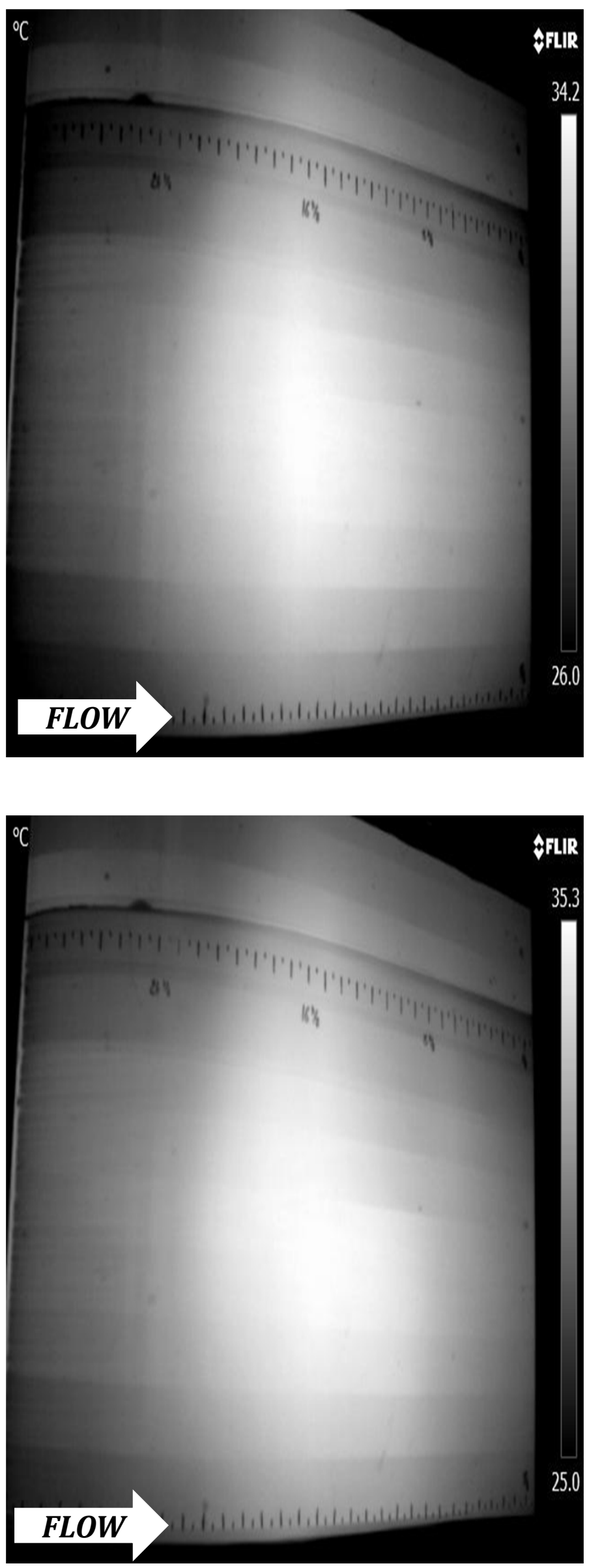


\section{8- $\mathrm{m}$ DU96-W-180 at $R e=1.5 \times 10^{5}$}

\section{PRESSURE SIDE}
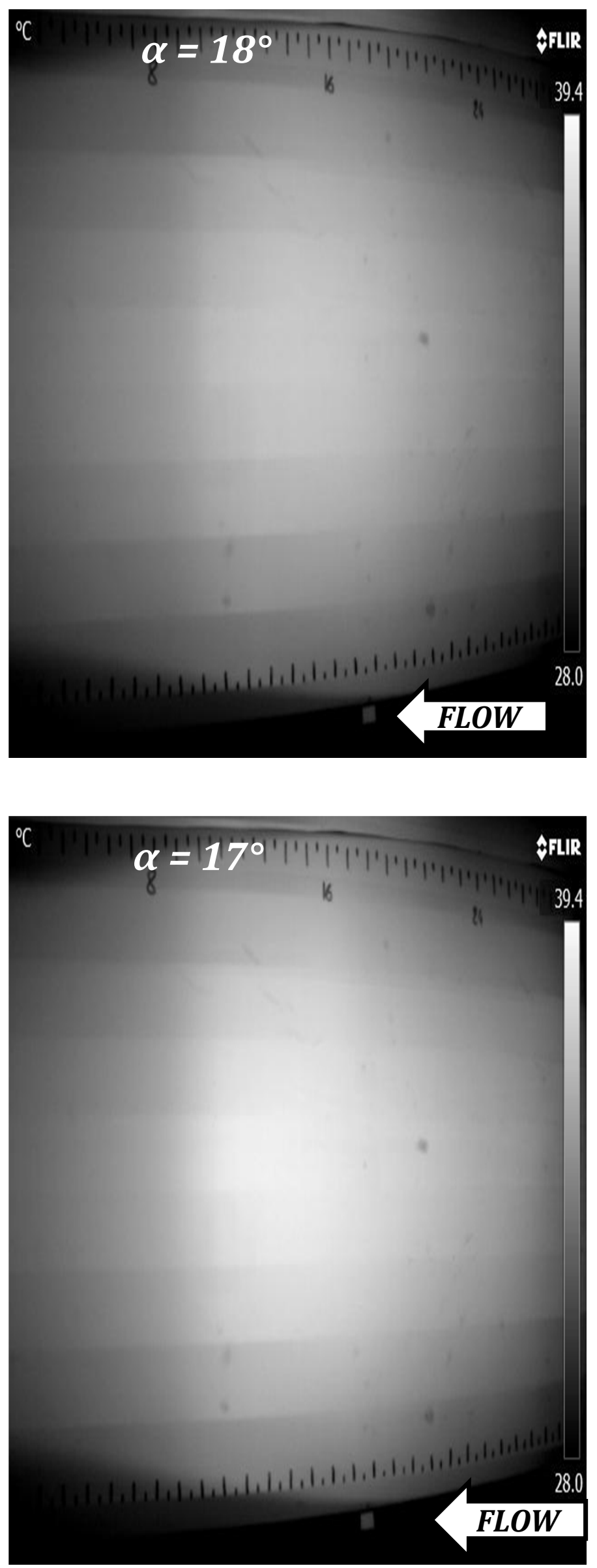

SUCTION SIDE
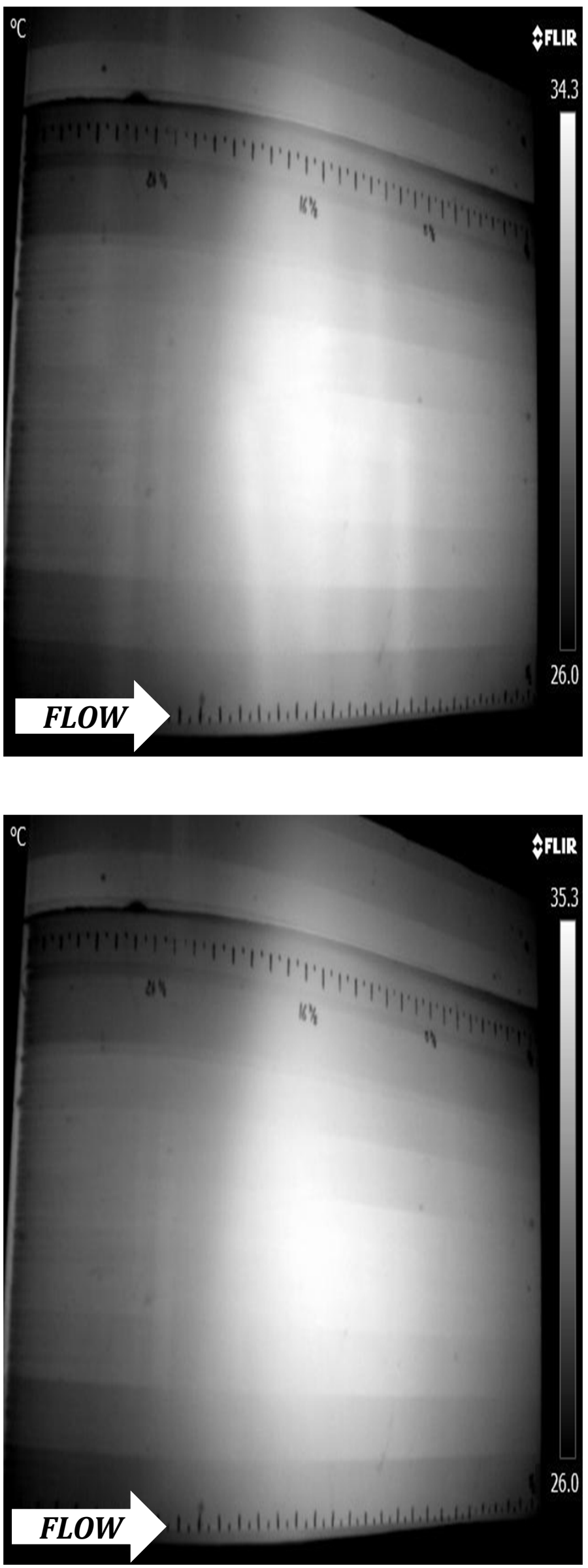


\section{8- $\mathrm{m}$ DU96-W-180 at $R e=1.5 \times 10^{5}$}

\section{PRESSURE SIDE}
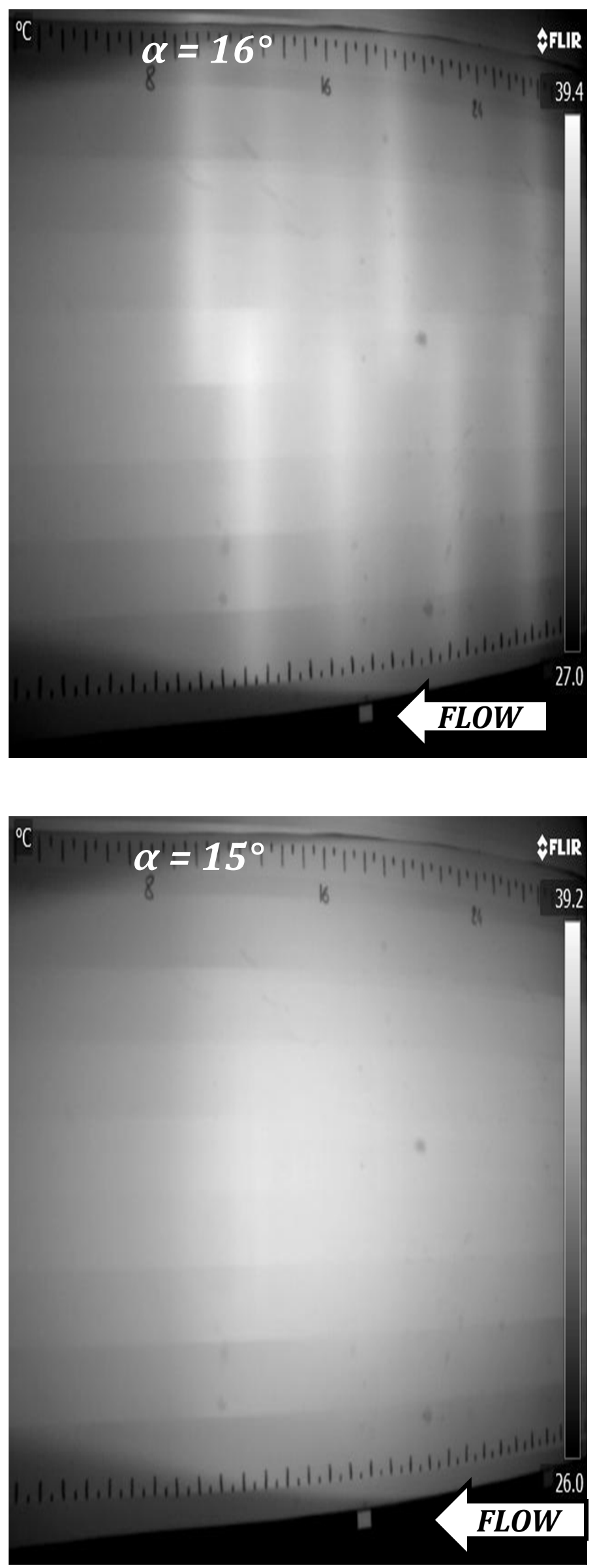

SUCTION SIDE
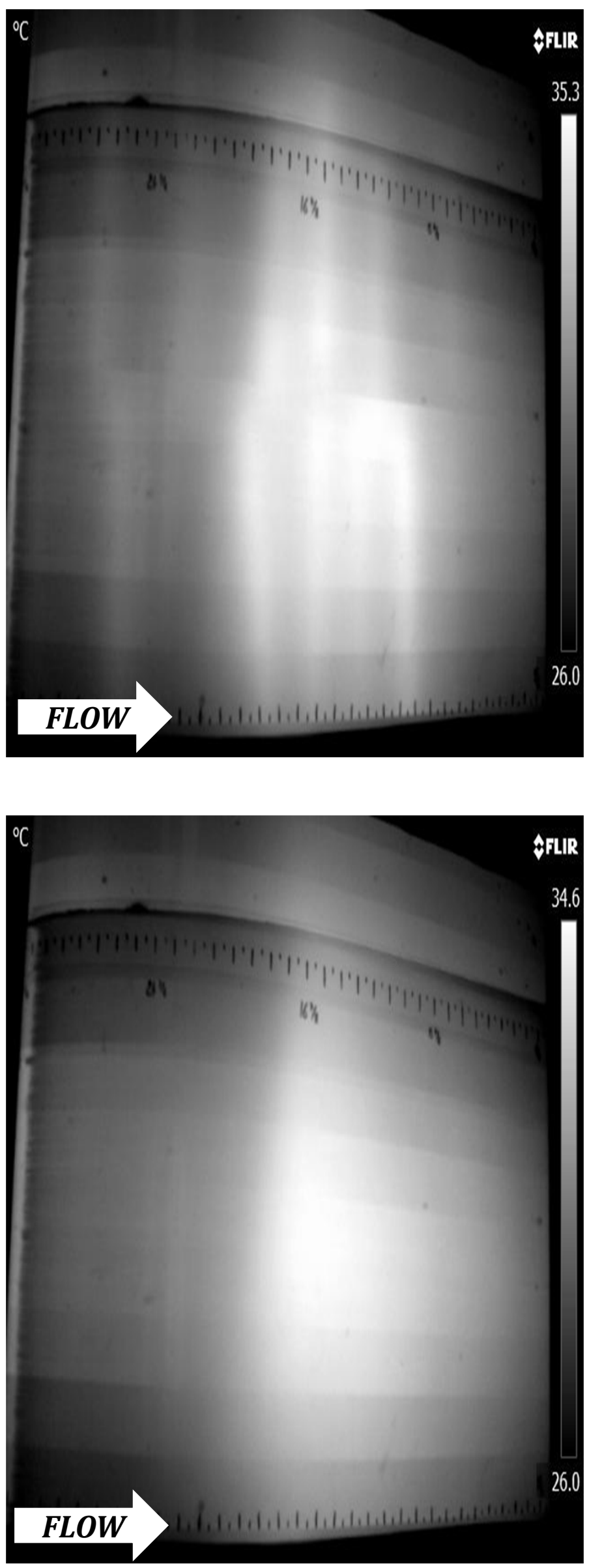


\section{8- $\mathrm{m}$ DU96-W-180 at $R e=1.5 \times 10^{5}$}

\section{PRESSURE SIDE}
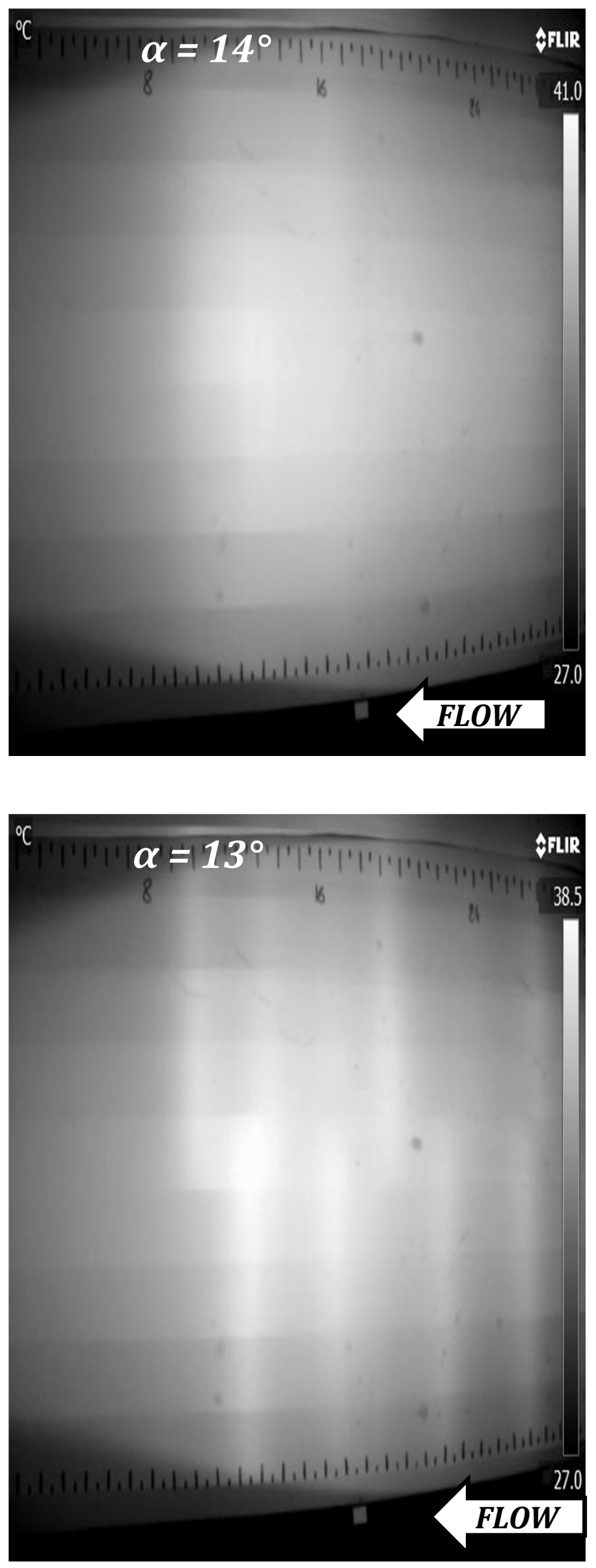

SUCTION SIDE
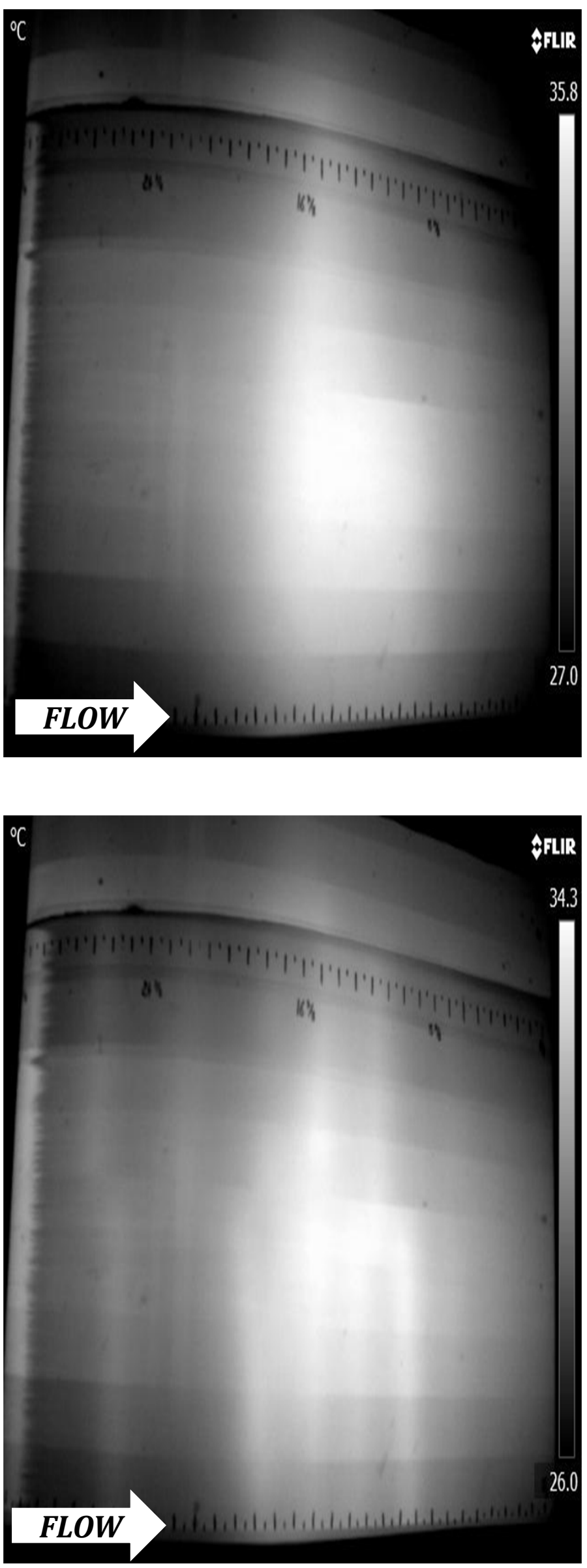


\section{8- $\mathrm{m}$ DU96-W-180 at $R e=1.5 \times 10^{5}$}

PRESSURE SIDE
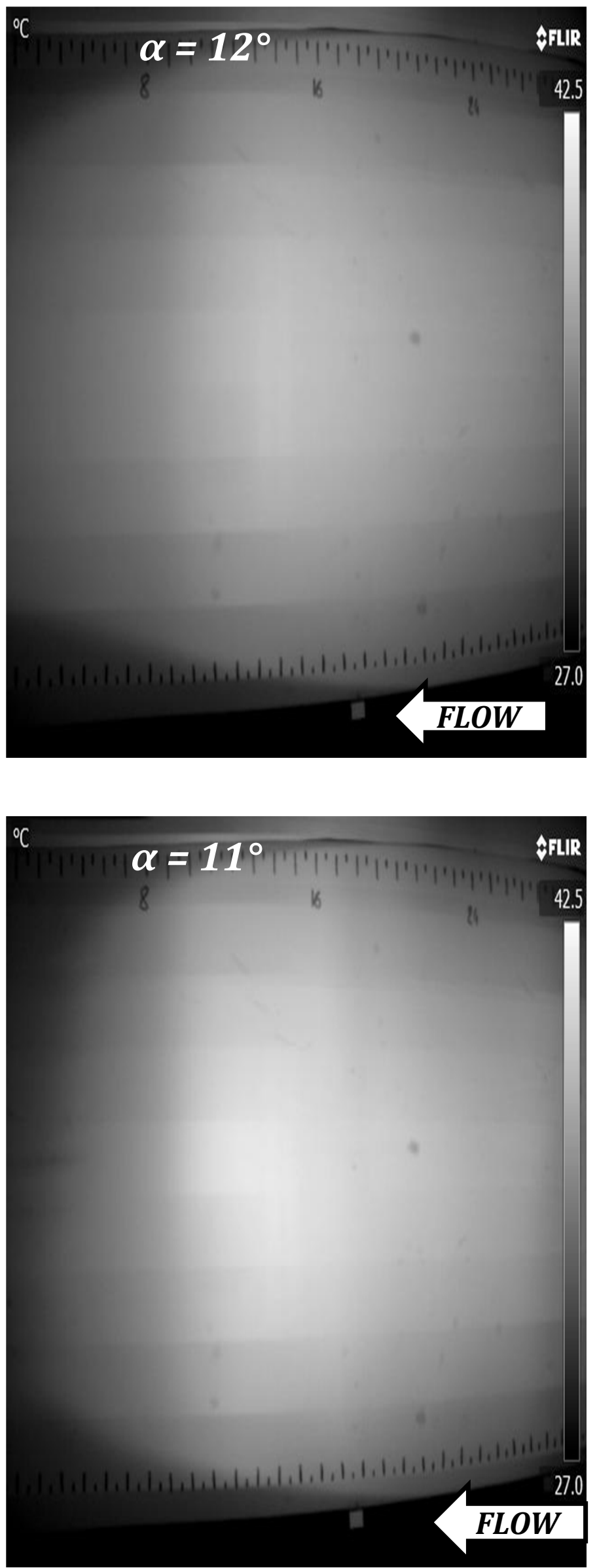

SUCTION SIDE
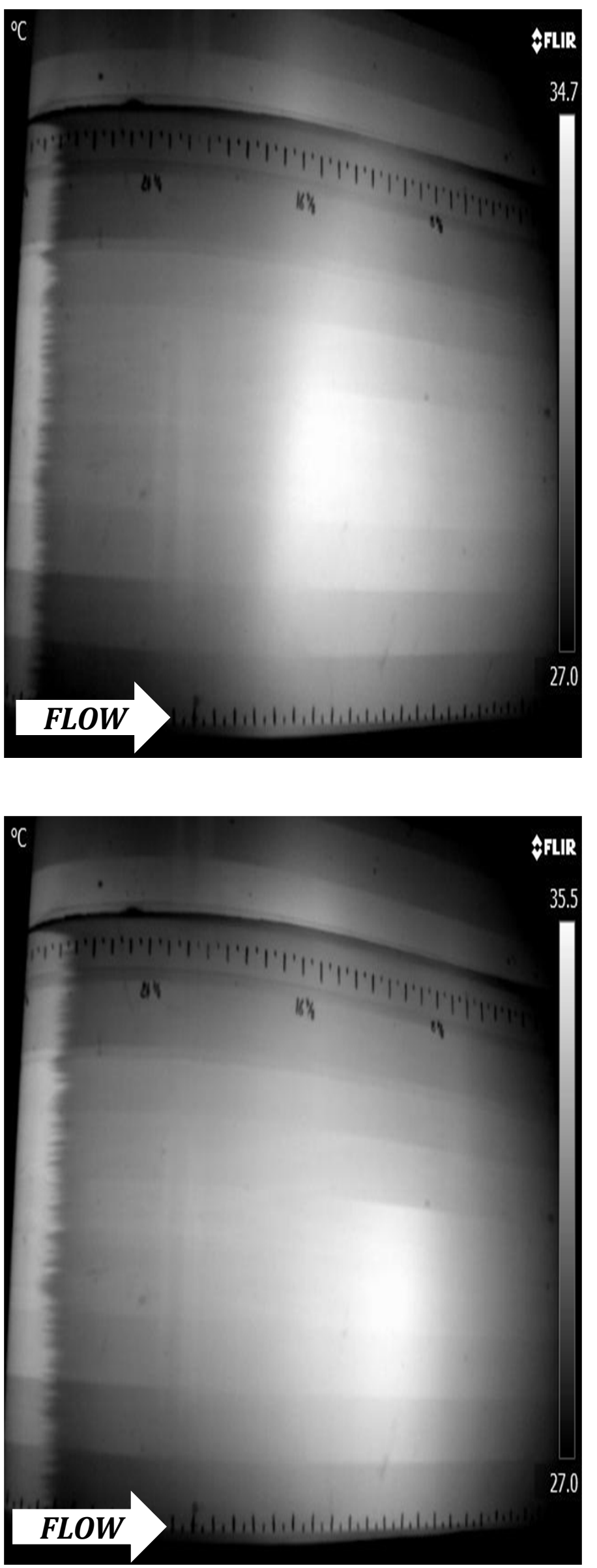


\section{8- $\mathrm{m}$ DU96-W-180 at $R e=1.5 \times 10^{5}$}

\section{PRESSURE SIDE}
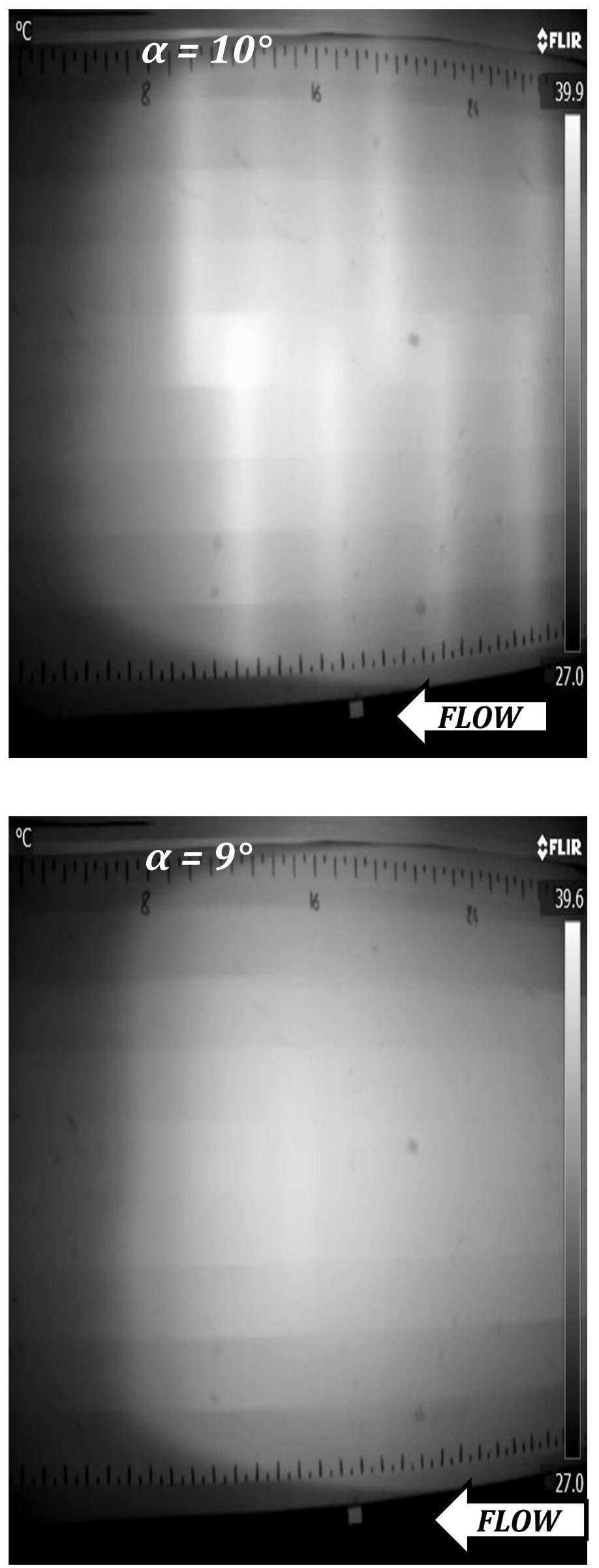

SUCTION SIDE
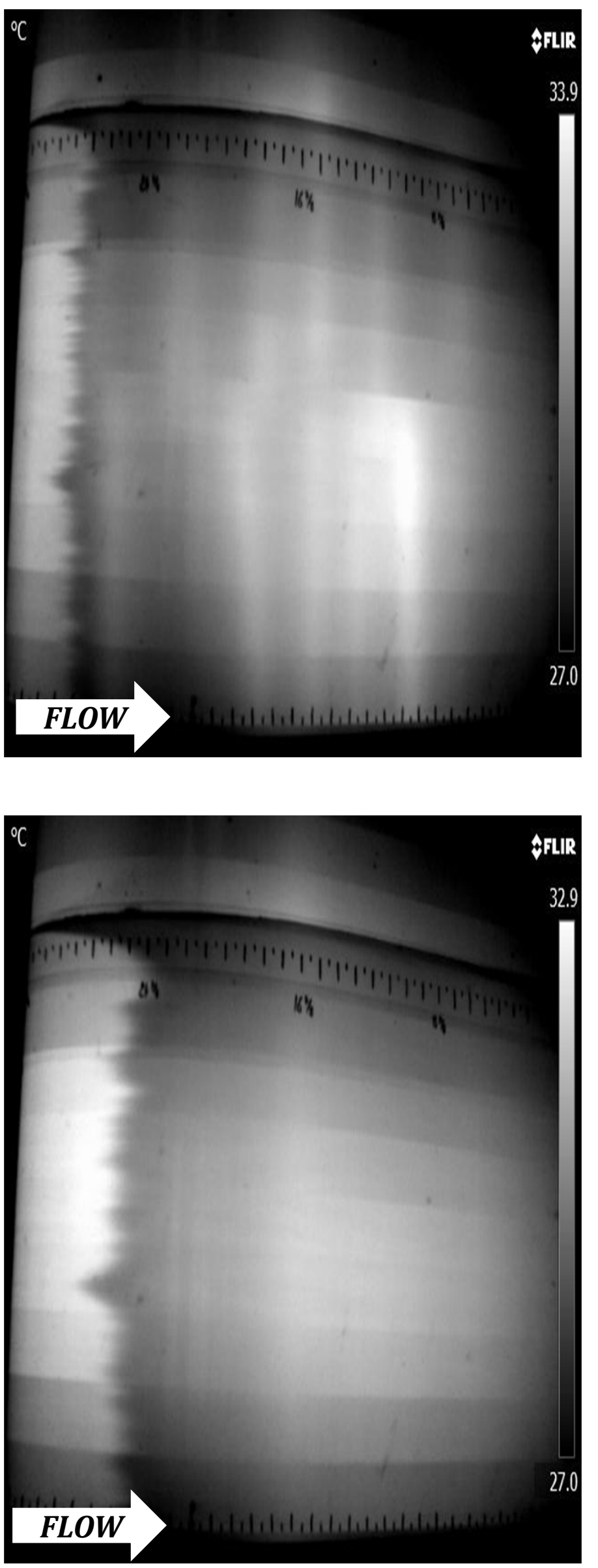


\section{8-m DU96-W-180 at $R e=1.5 \times 10^{5}$}

\section{PRESSURE SIDE}
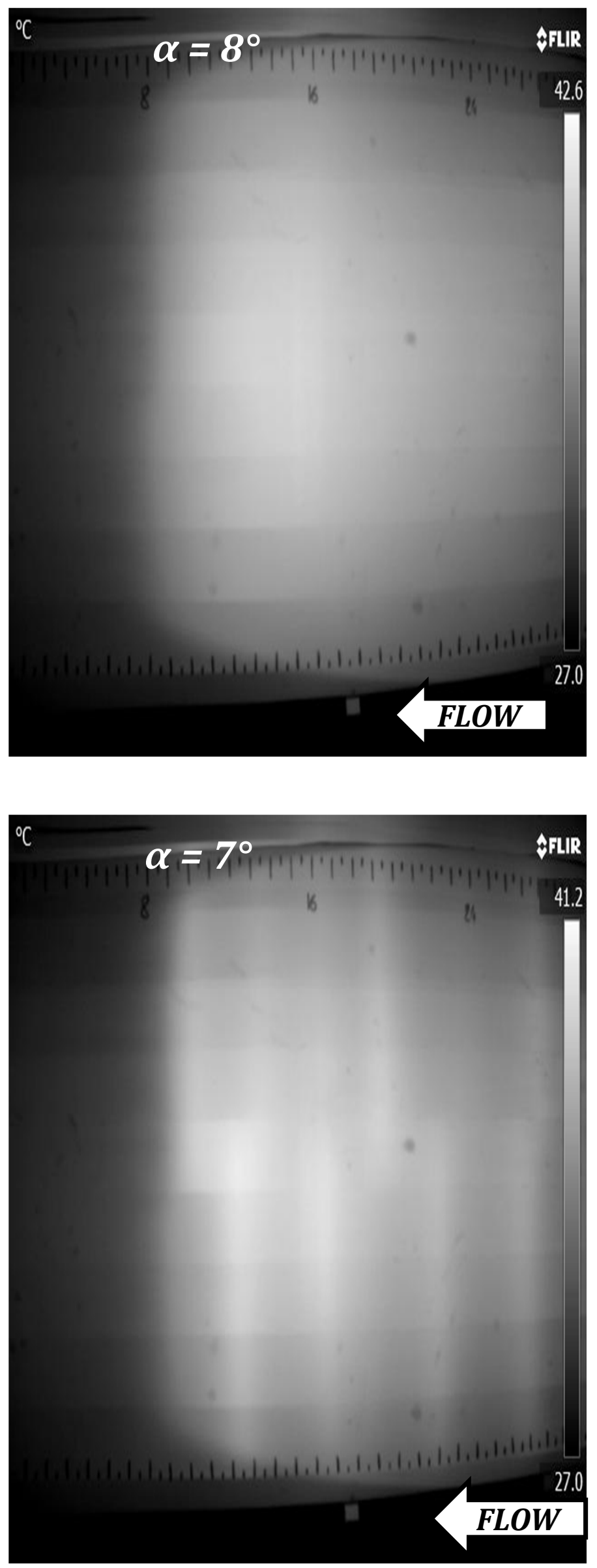

SUCTION SIDE
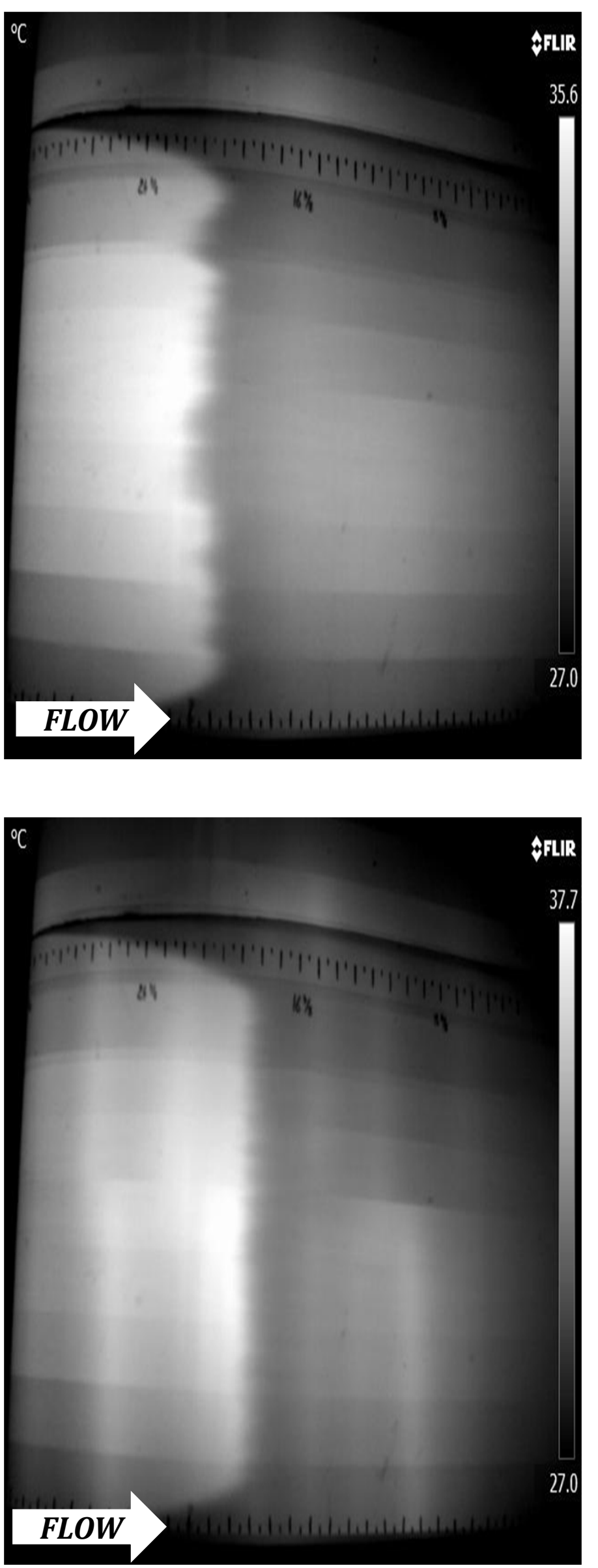


\section{8- $\mathrm{m}$ DU96-W-180 at $R e=1.5 \times 10^{5}$}

\section{PRESSURE SIDE}
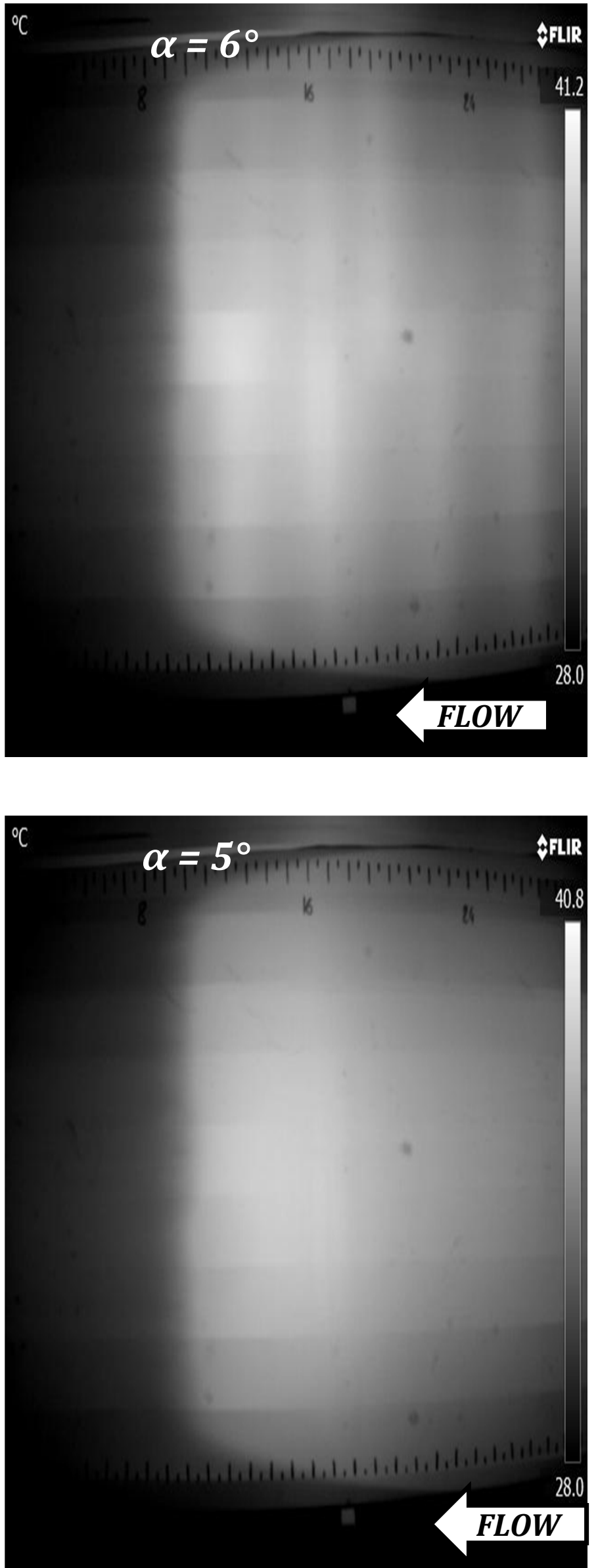

SUCTION SIDE
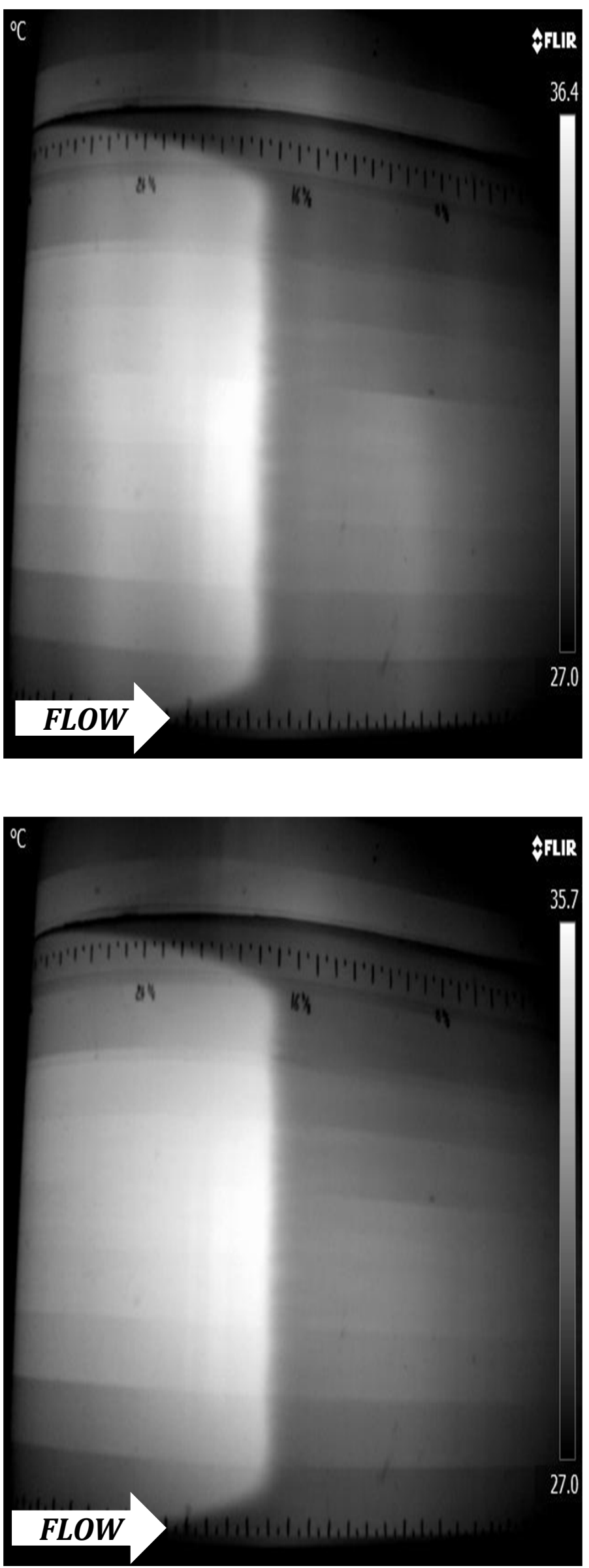


\section{8-m DU96-W-180 at $R e=1.5 \times 10^{5}$}

\section{PRESSURE SIDE}
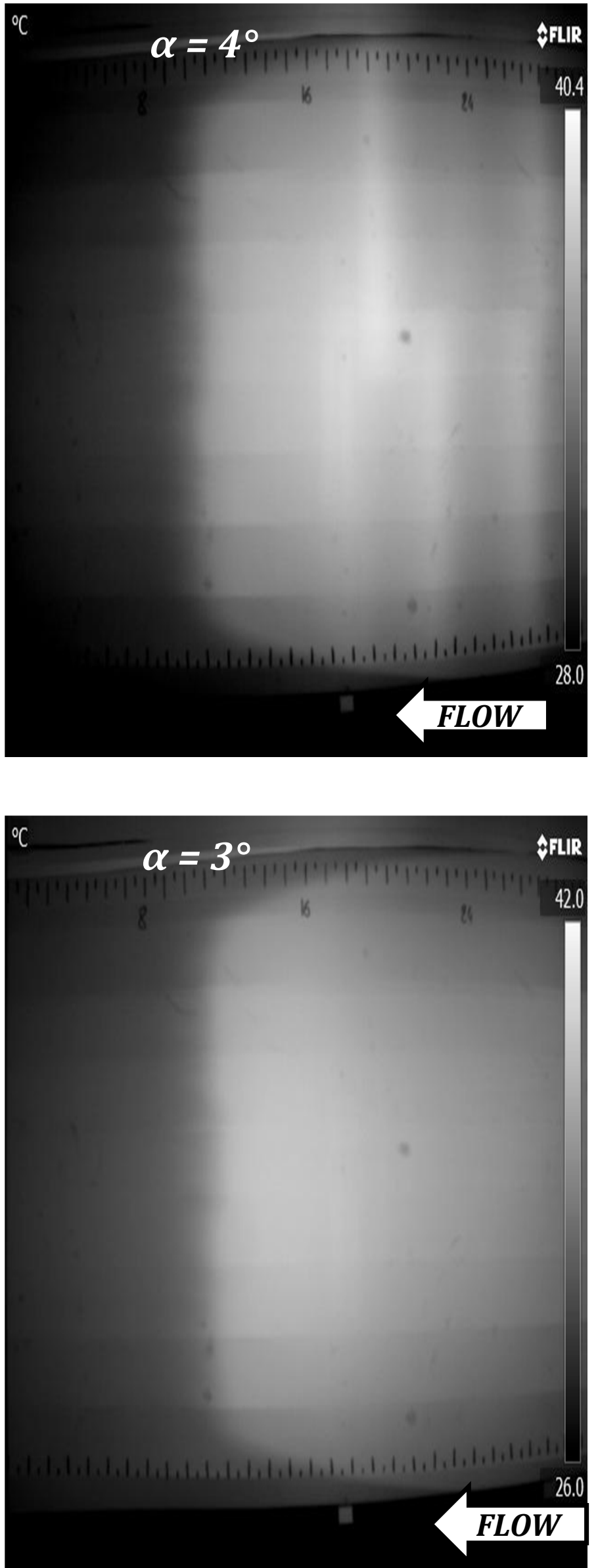

SUCTION SIDE
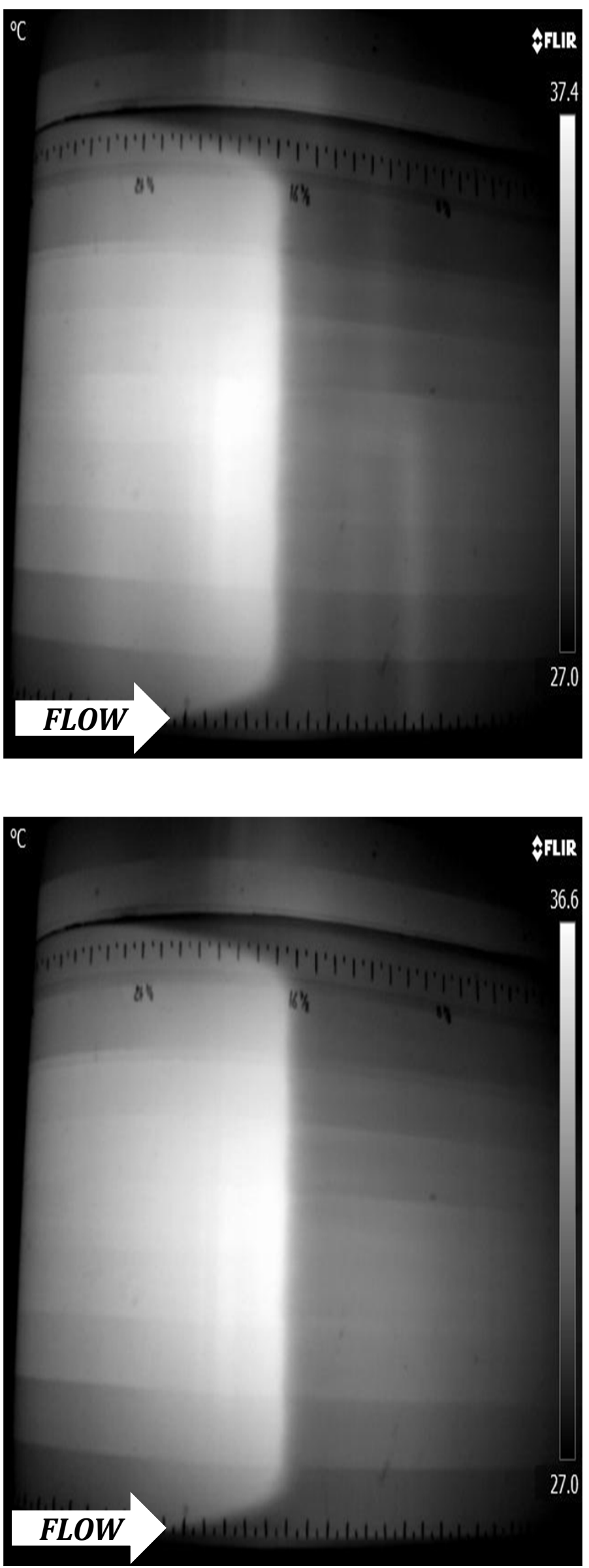


\section{8- $\mathrm{m}$ DU96-W-180 at $R e=1.5 \times 10^{5}$}

\section{PRESSURE SIDE}
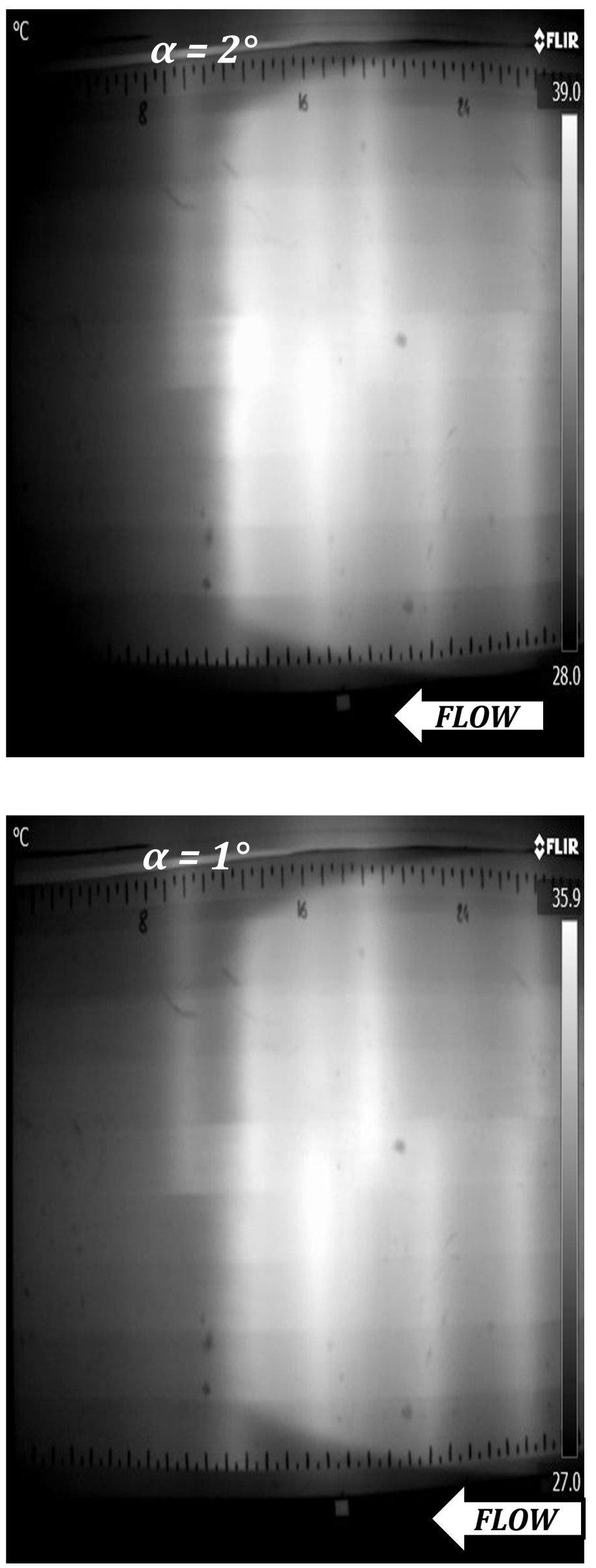

SUCTION SIDE
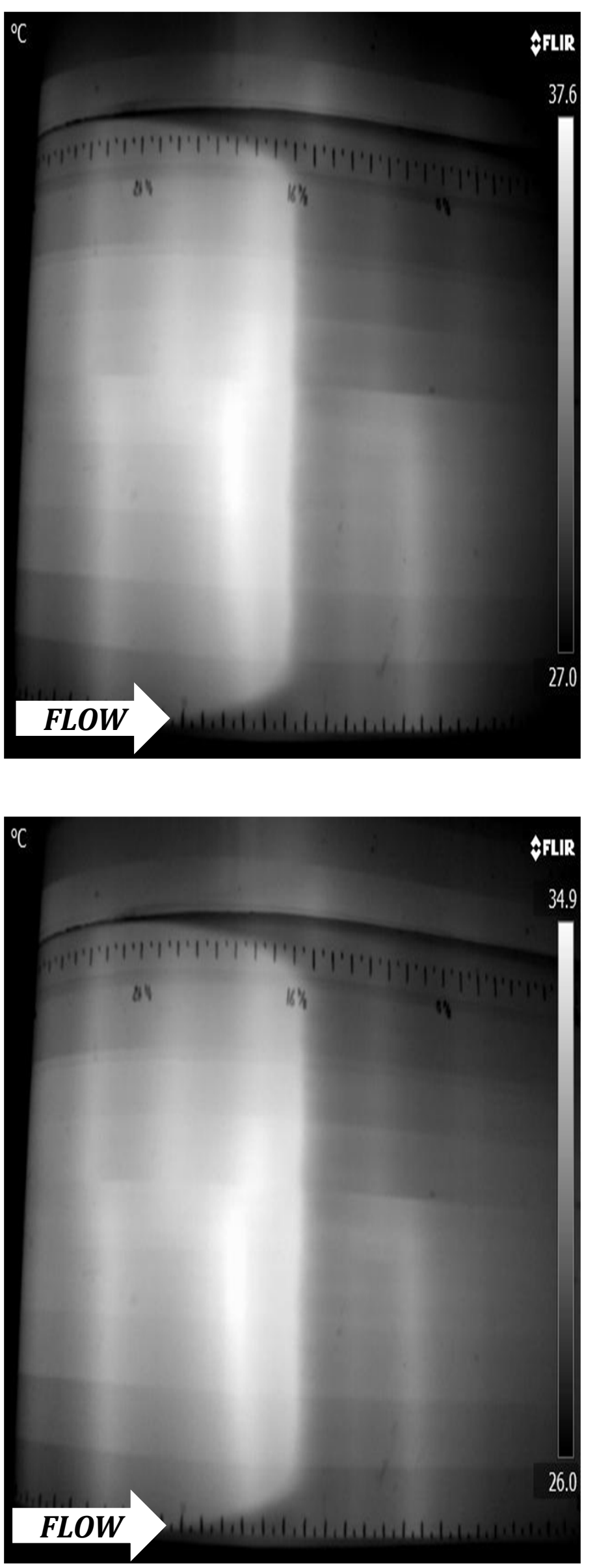


\section{8- $\mathrm{m}$ DU96-W-180 at $R e=1.5 \times 10^{5}$}

\section{PRESSURE SIDE}

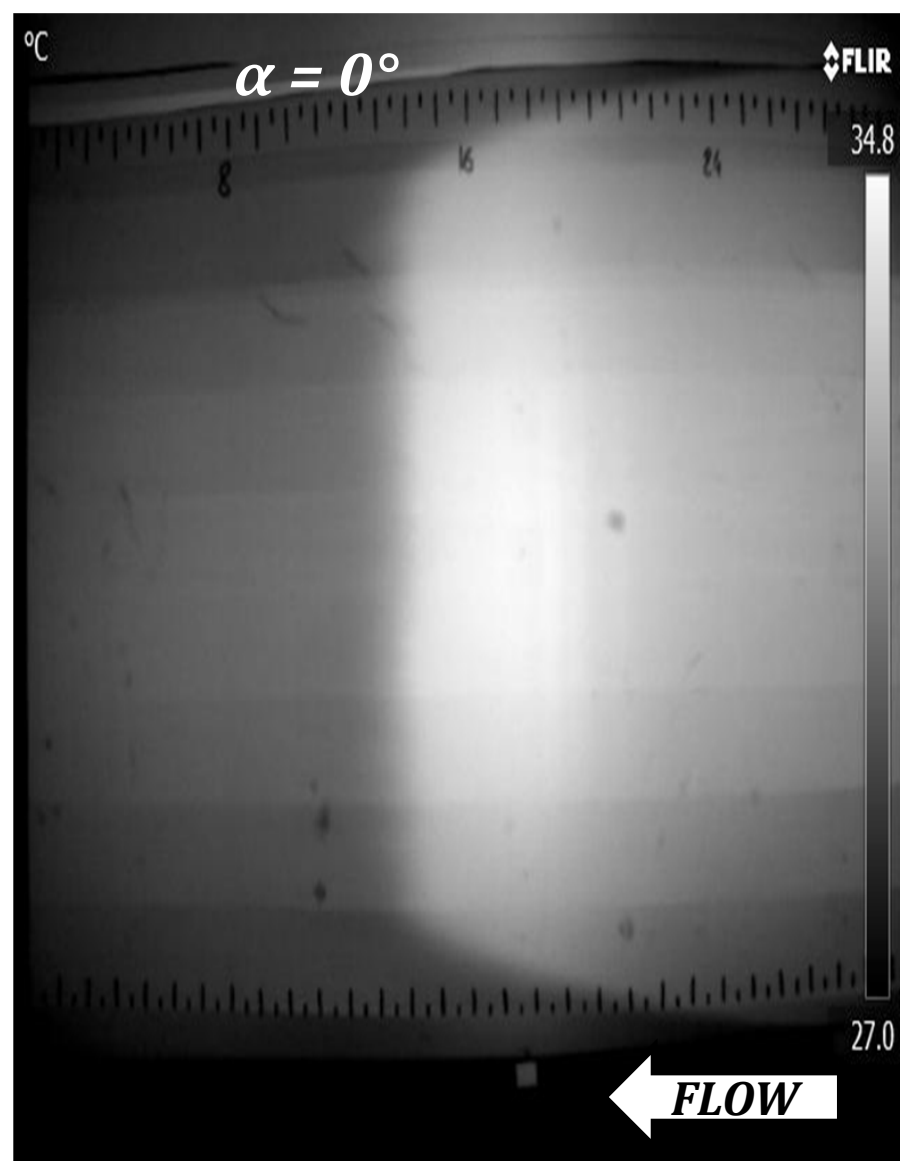

${ }^{\circ} \mathrm{C}$

$$
\alpha=-1^{\circ}
$$

SUCTION SIDE
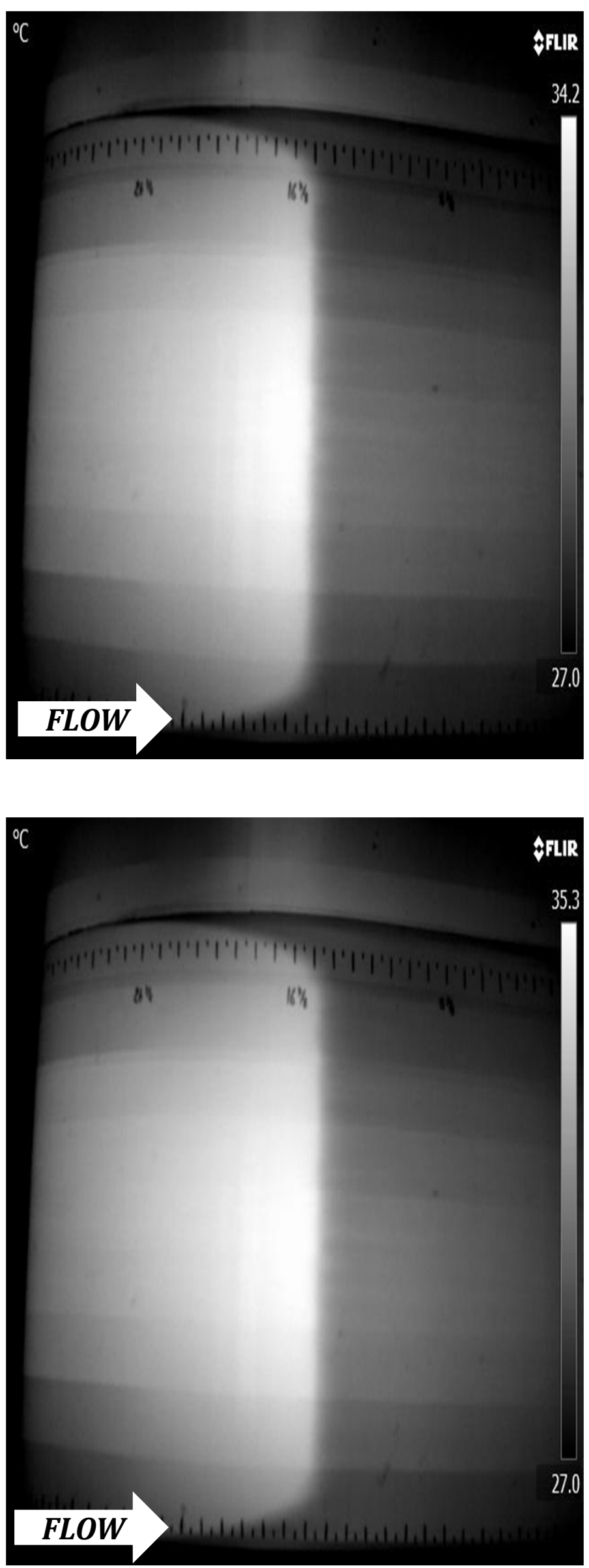


\section{8- $\mathrm{m}$ DU96-W-180 at $R e=1.5 \times 10^{5}$}

\section{PRESSURE SIDE}
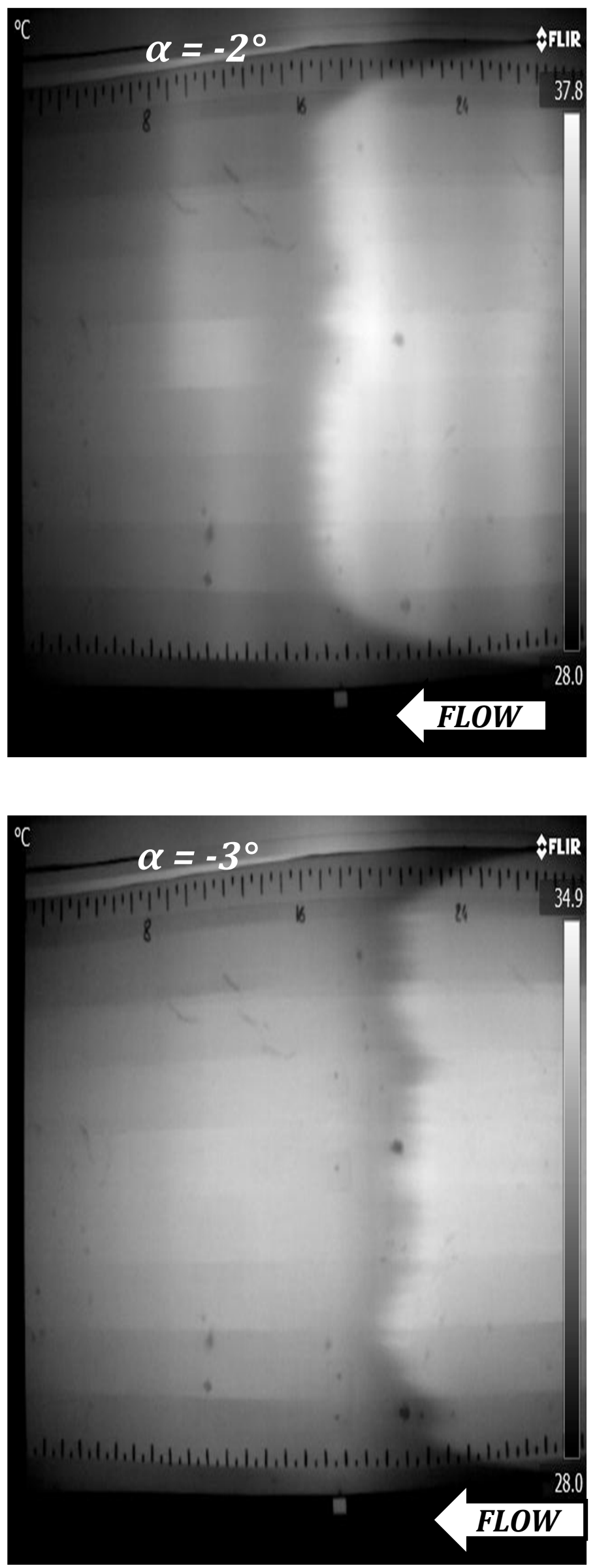

SUCTION SIDE
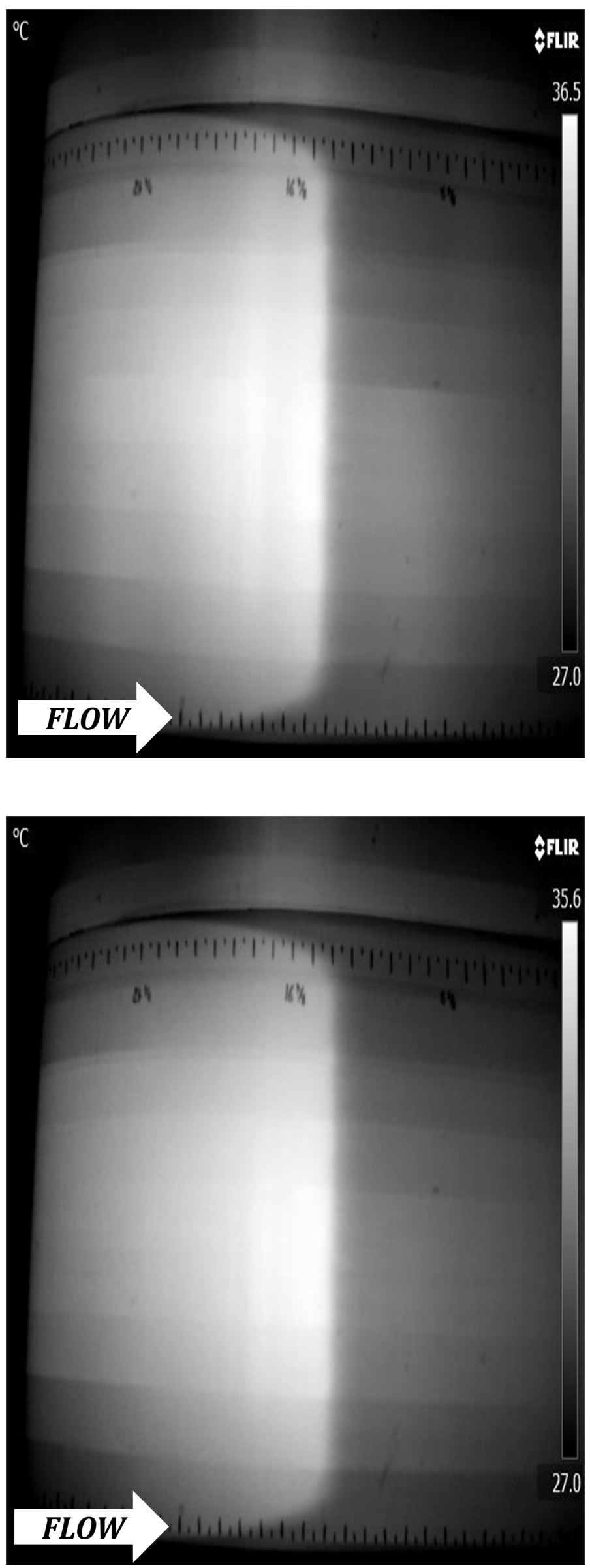


\section{8- $\mathrm{m}$ DU96-W-180 at $R e=1.5 \times 10^{5}$}

\section{PRESSURE SIDE}
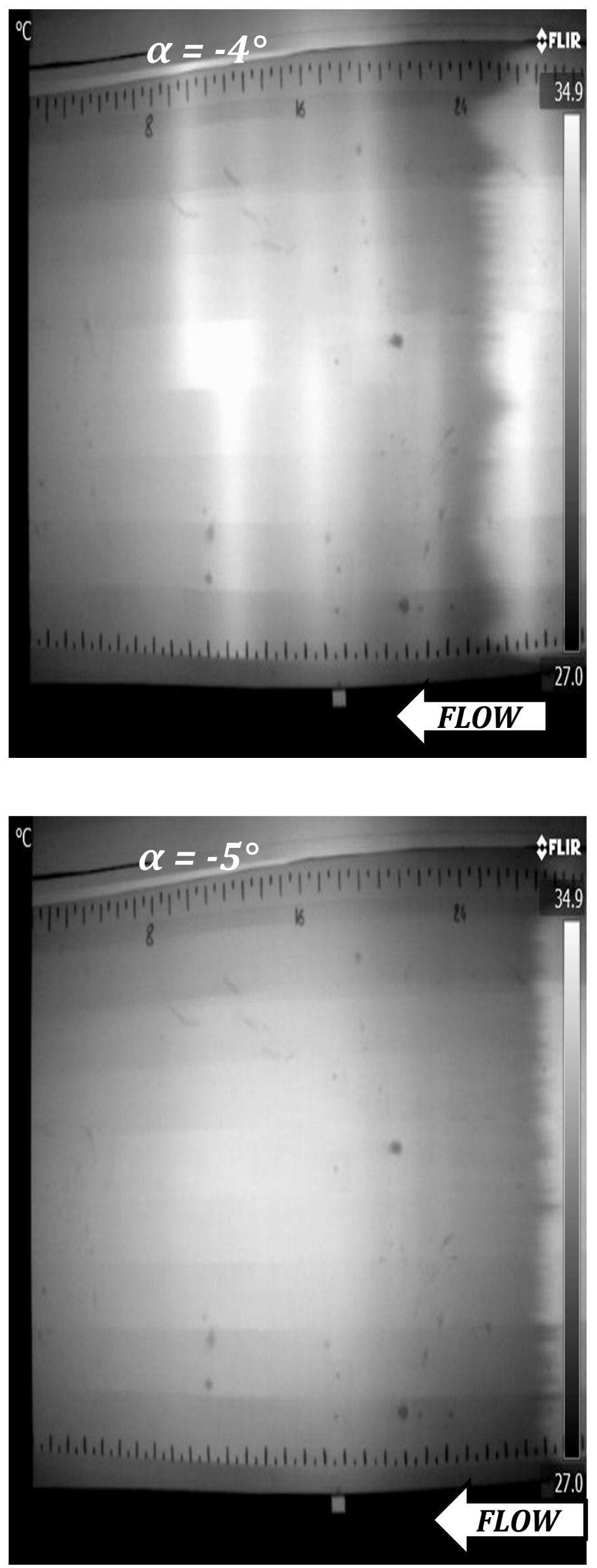

SUCTION SIDE
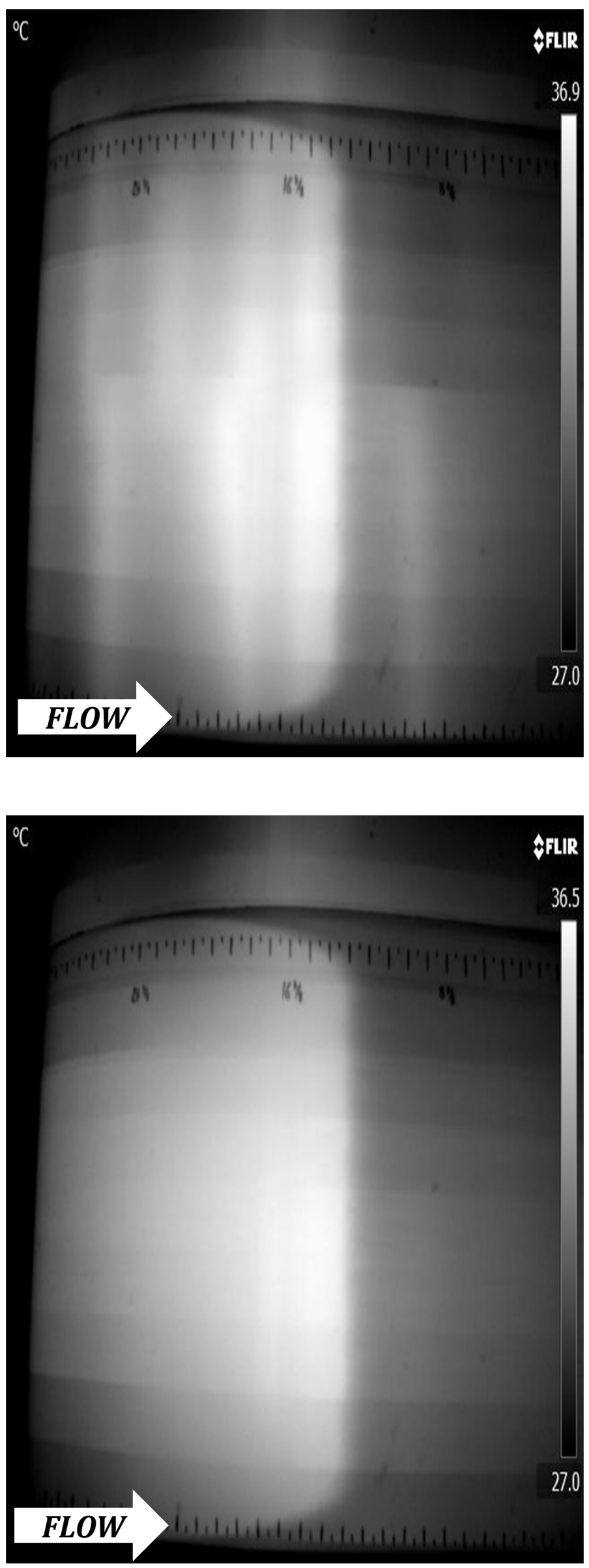


\section{8- $m$ DU96-W-180 at $R e=1.5 \times 10^{5}$}

\section{PRESSURE SIDE}
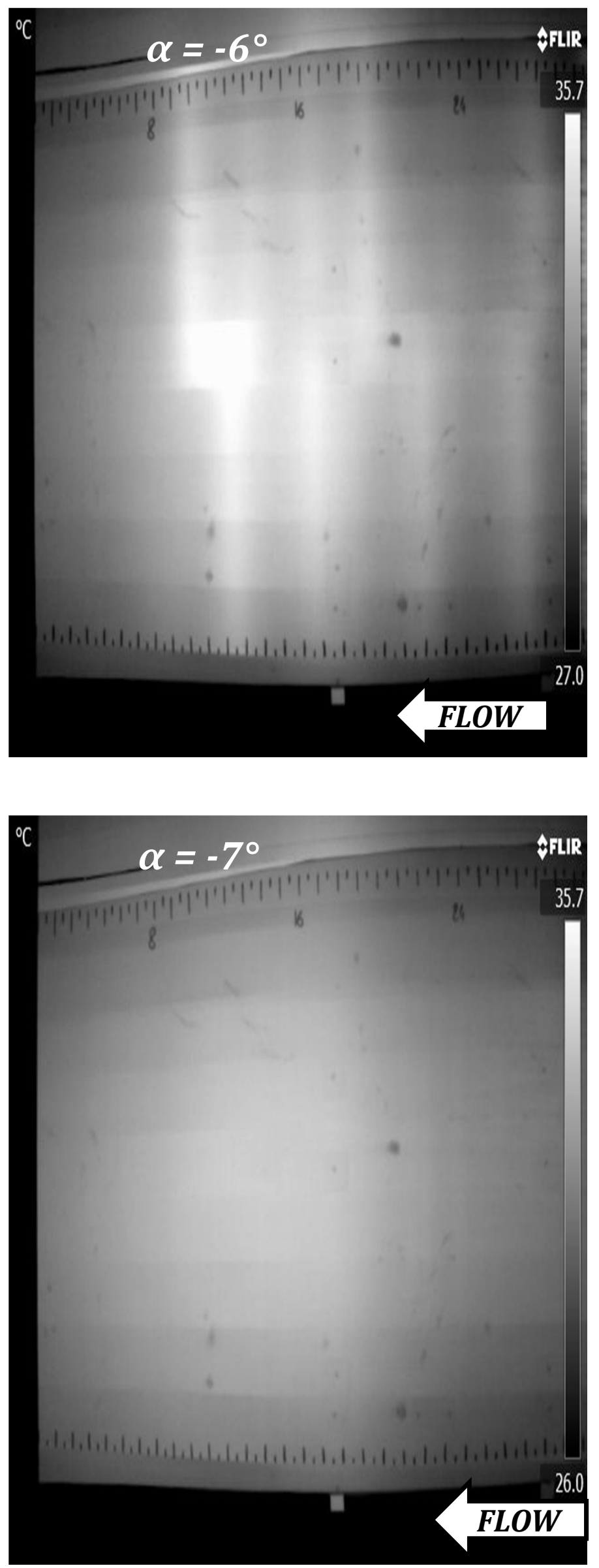

SUCTION SIDE
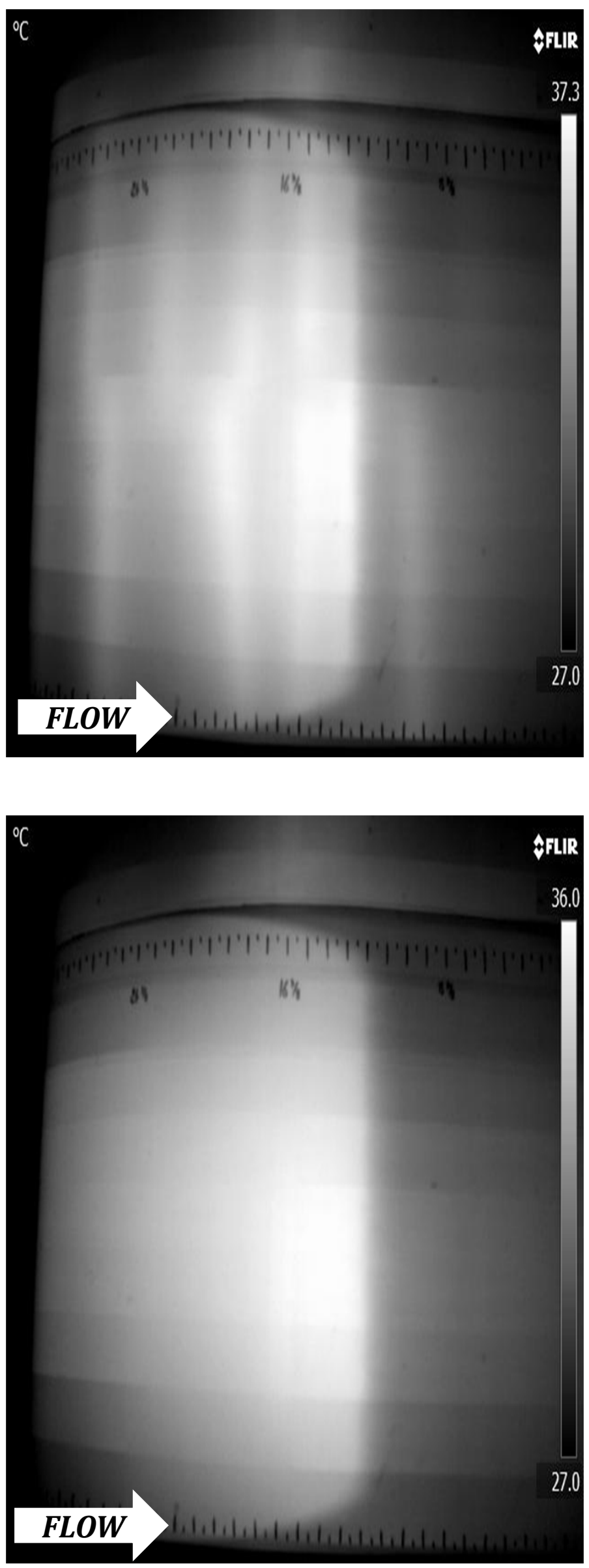


\section{8- $\mathrm{m}$ DU96-W-180 at $R e=1.5 \times 10^{5}$}

\section{PRESSURE SIDE}
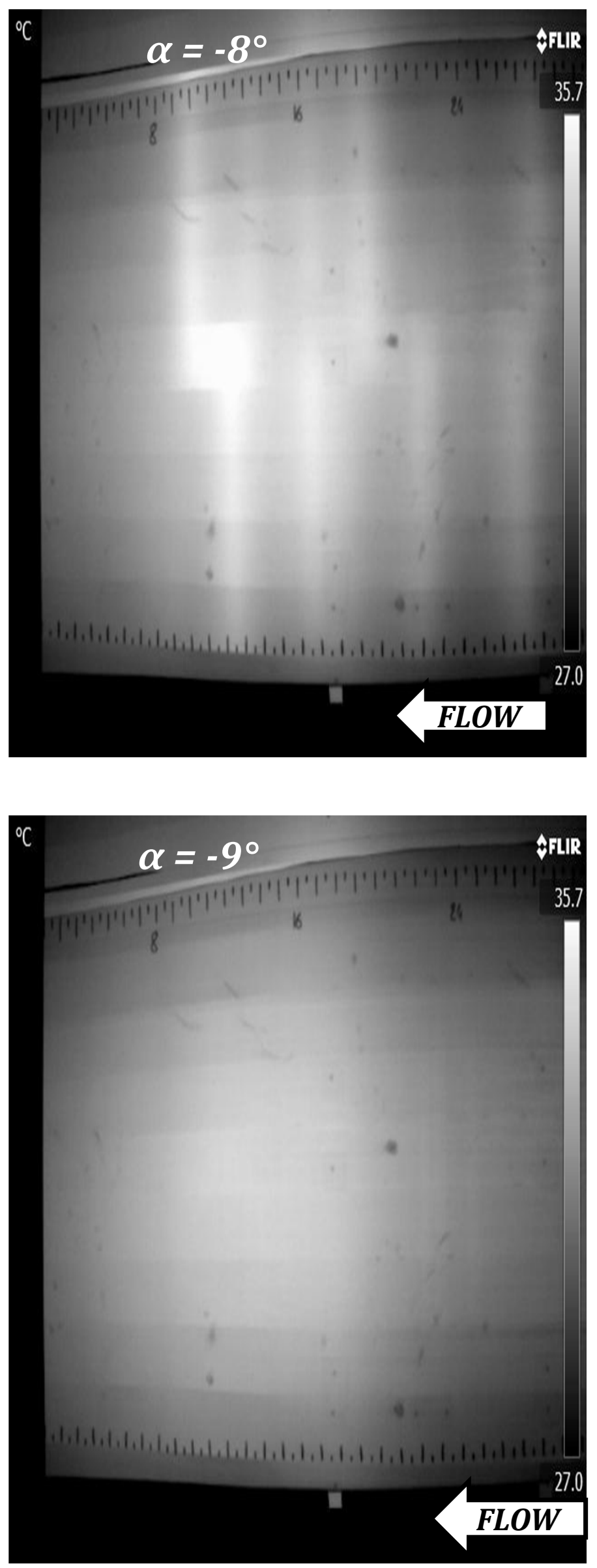

SUCTION SIDE
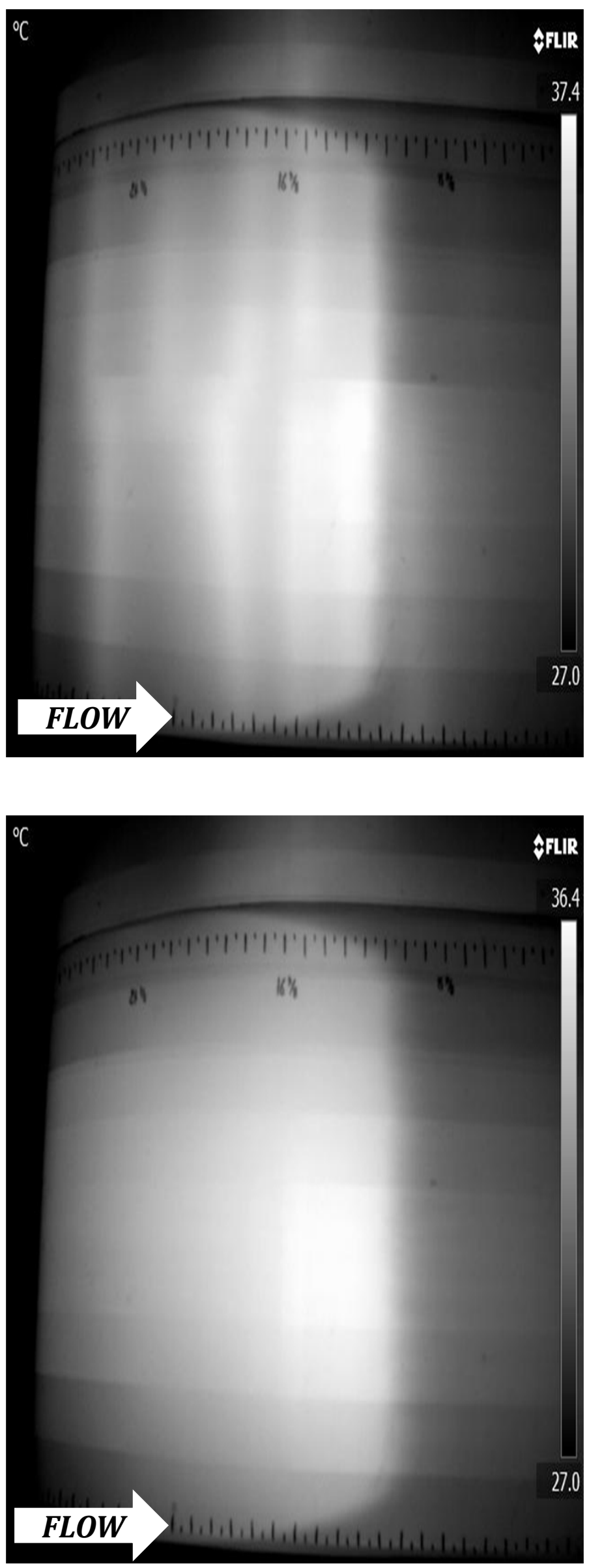


\section{8- $\mathrm{m}$ DU96-W-180 at $R e=1.5 \times 10^{5}$}

\section{PRESSURE SIDE}
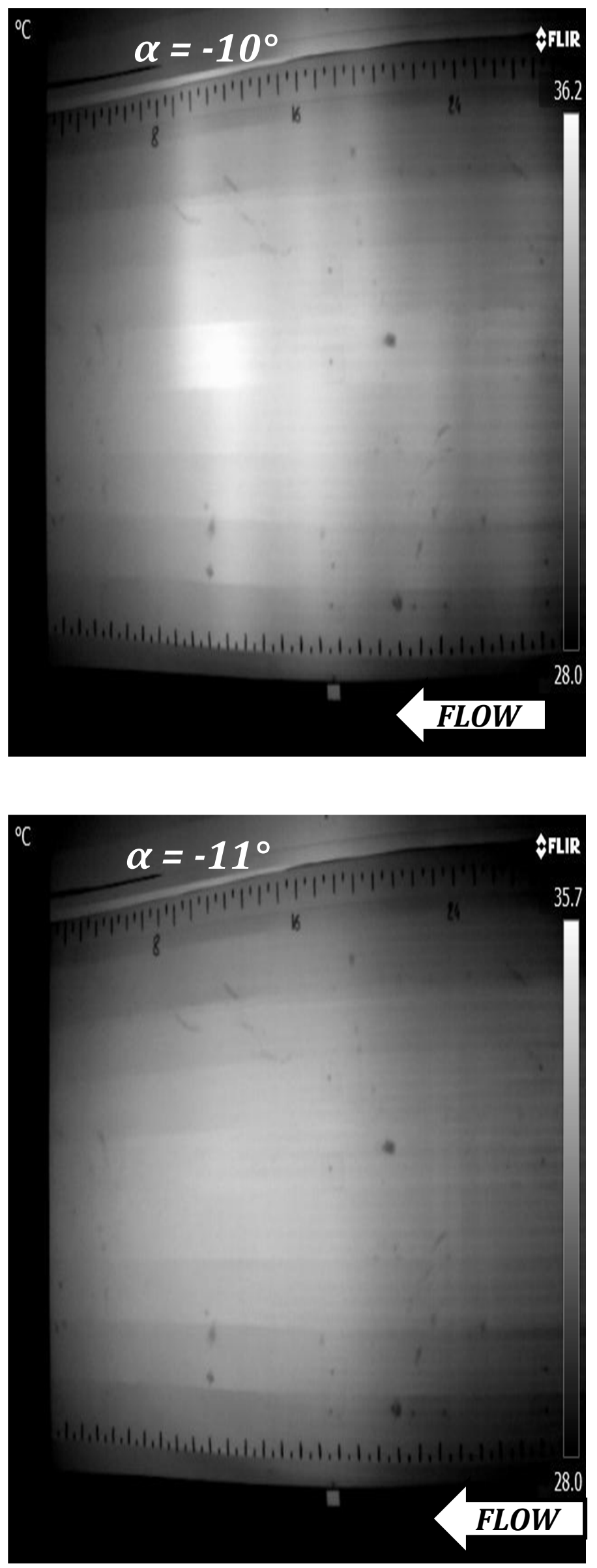

SUCTION SIDE
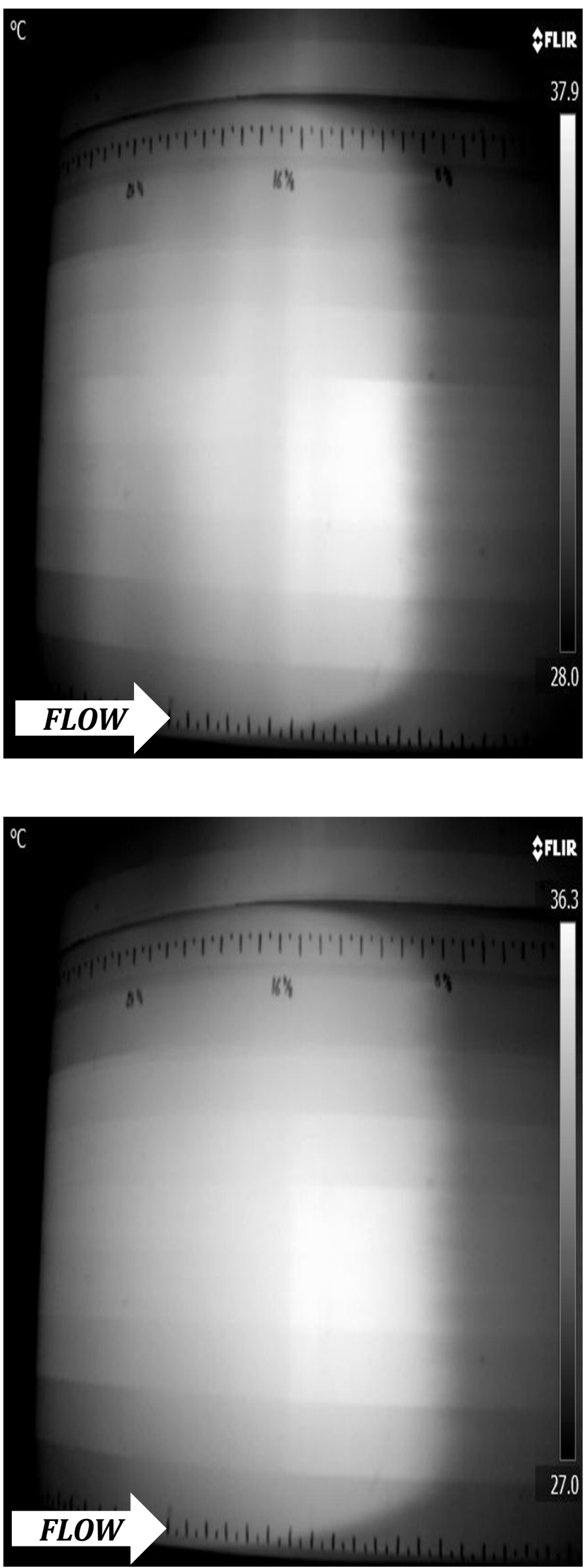


\section{8- $\mathrm{m}$ DU96-W-180 at $R e=1.5 \times 10^{5}$}

\section{PRESSURE SIDE}
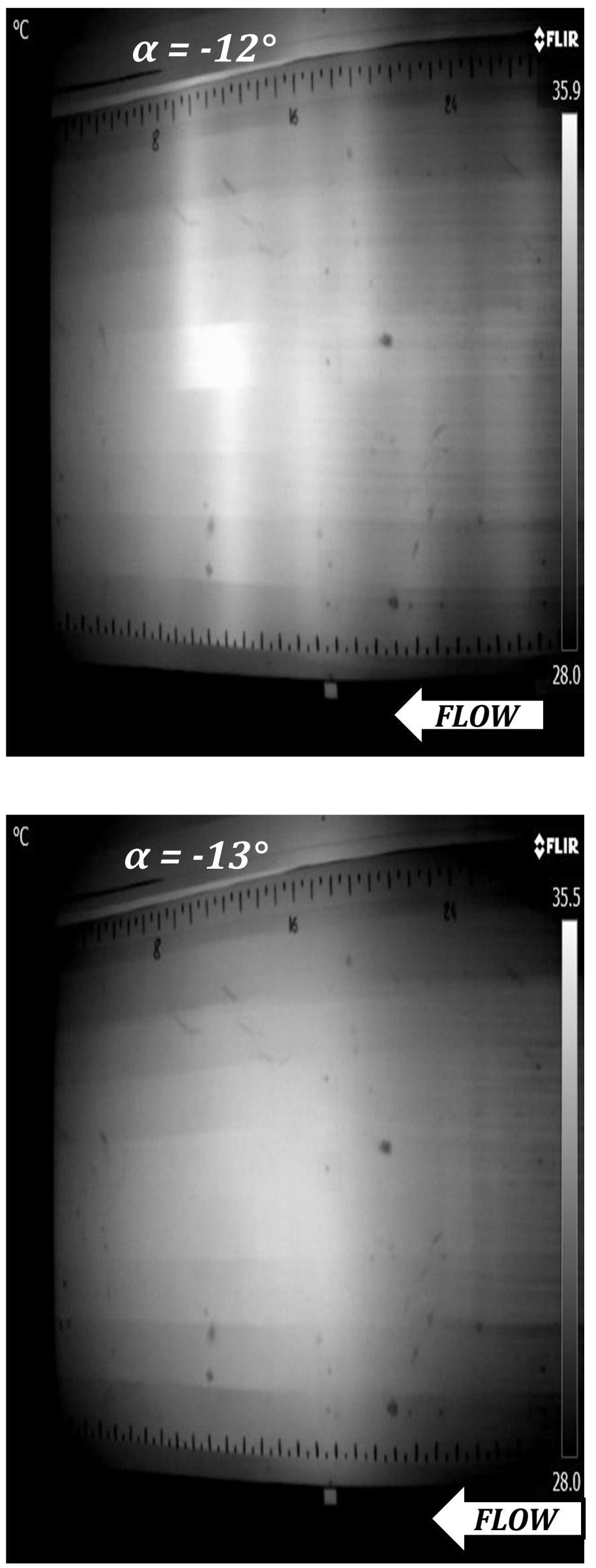

SUCTION SIDE
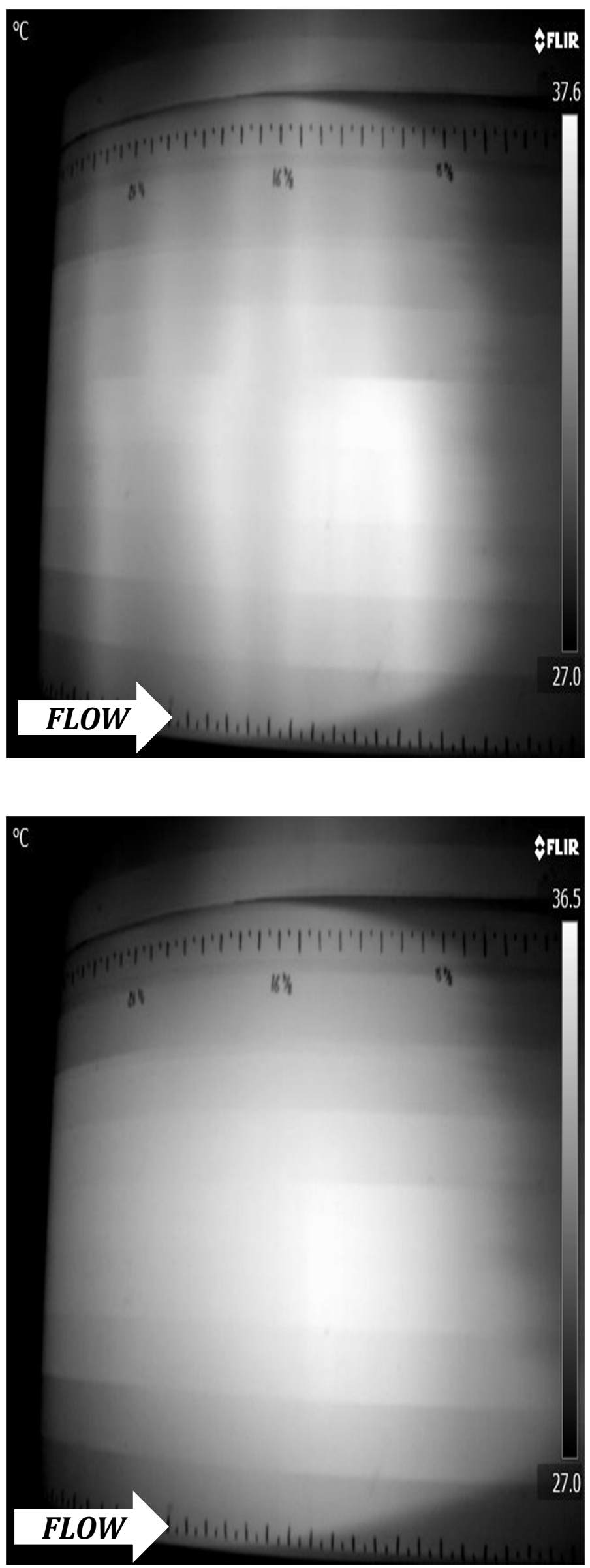


\section{8- $\mathrm{m}$ DU96-W-180 at $R e=1.5 \times 10^{5}$}

\section{PRESSURE SIDE}
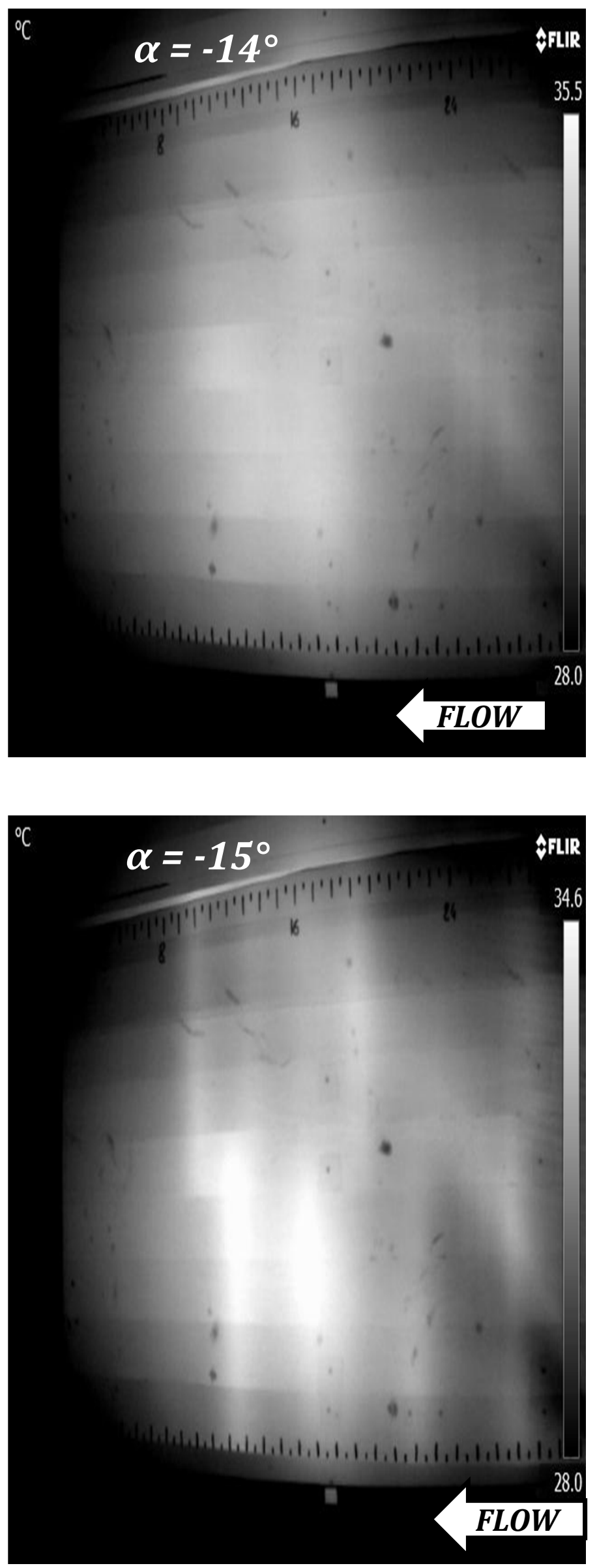

SUCTION SIDE
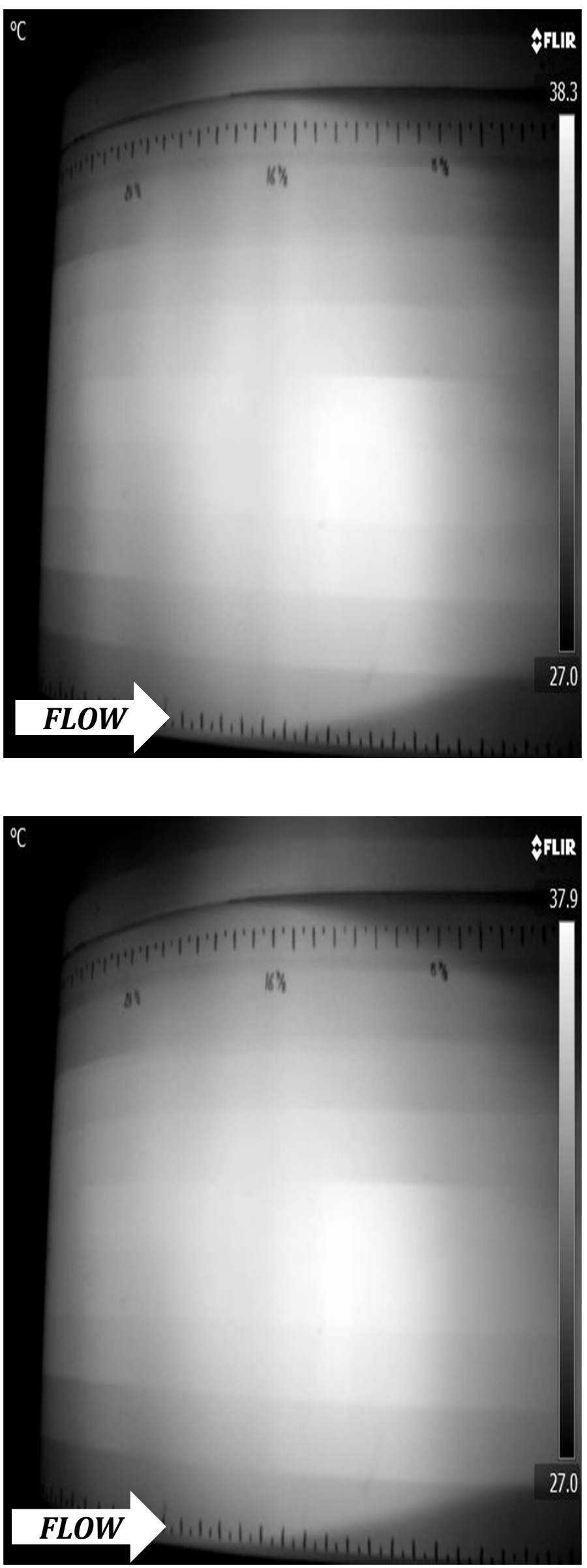


\section{8- $\mathrm{m}$ DU96-W-180 at $R e=1.5 \times 10^{5}$}

\section{PRESSURE SIDE}
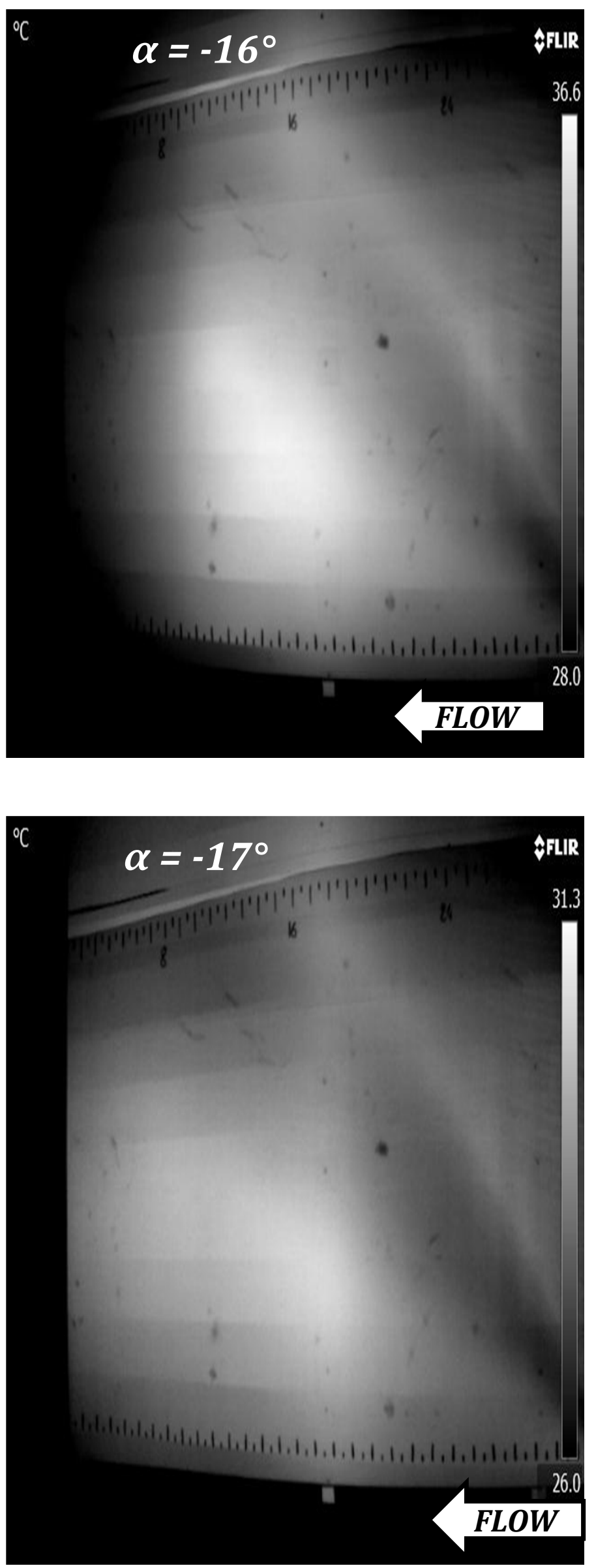

SUCTION SIDE
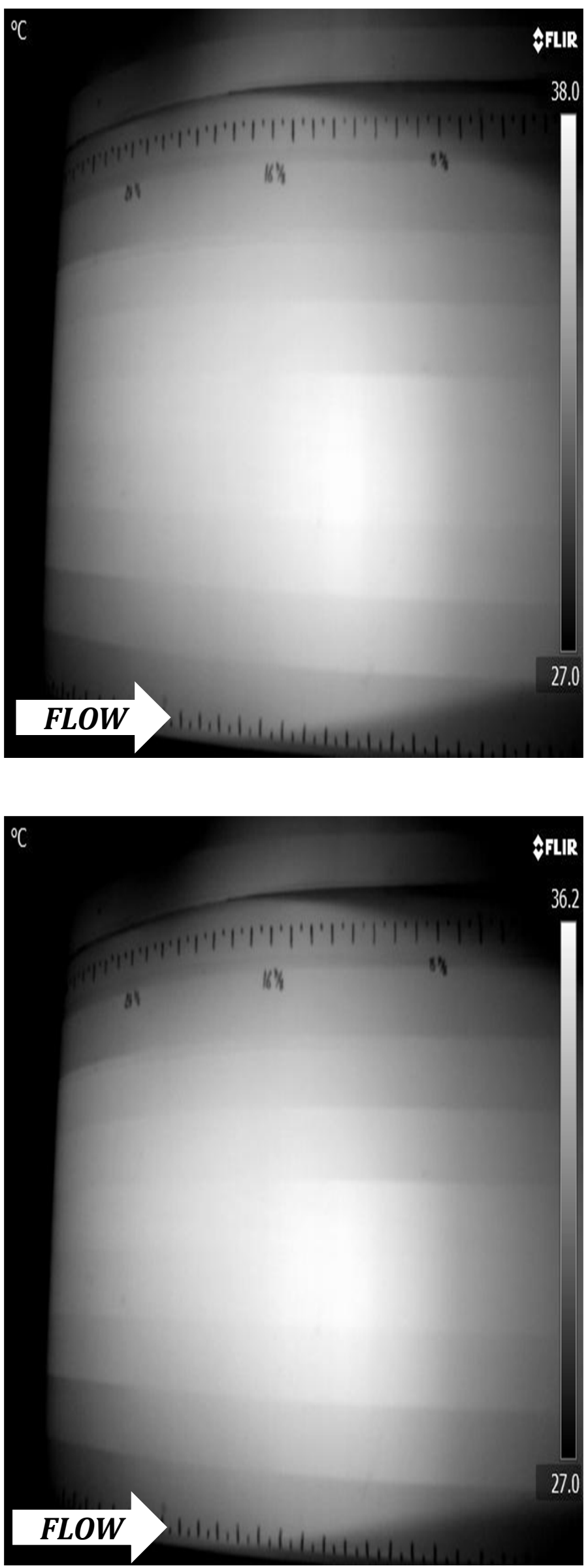


\section{8- $\mathrm{m}$ DU96-W-180 at $R e=1.5 \times 10^{5}$}

\section{PRESSURE SIDE}
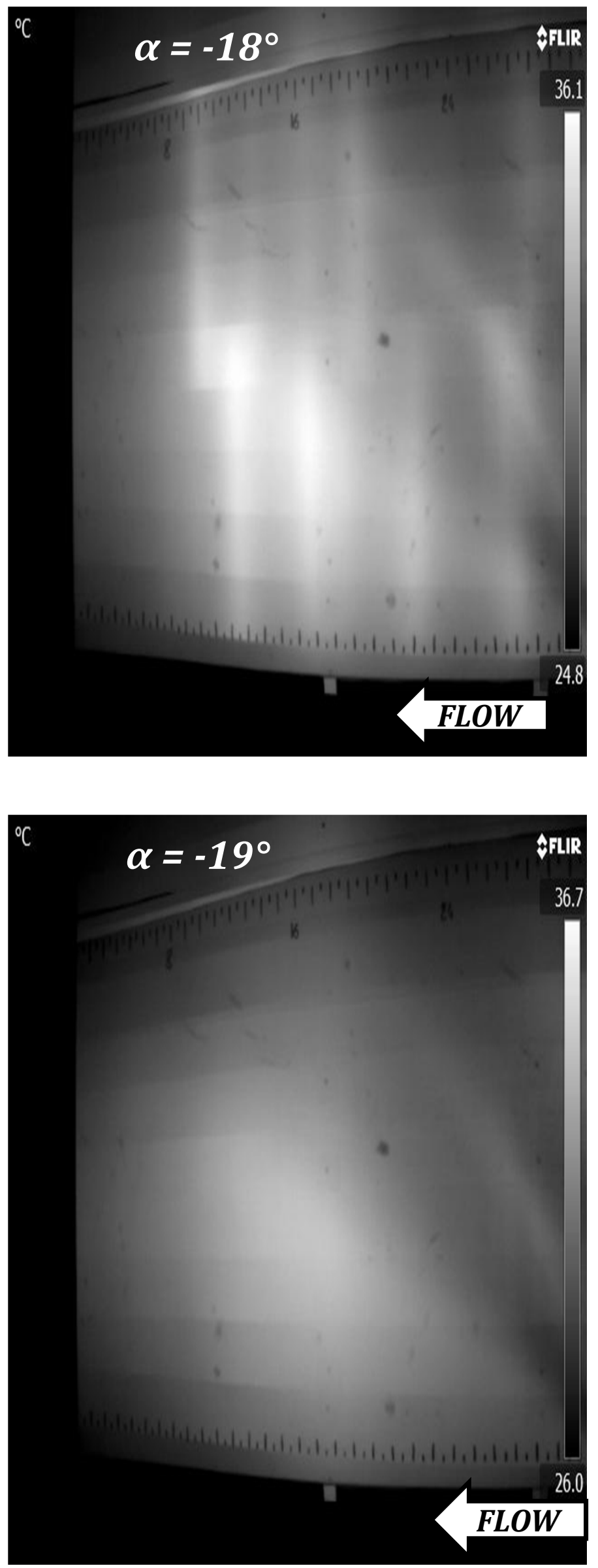

SUCTION SIDE
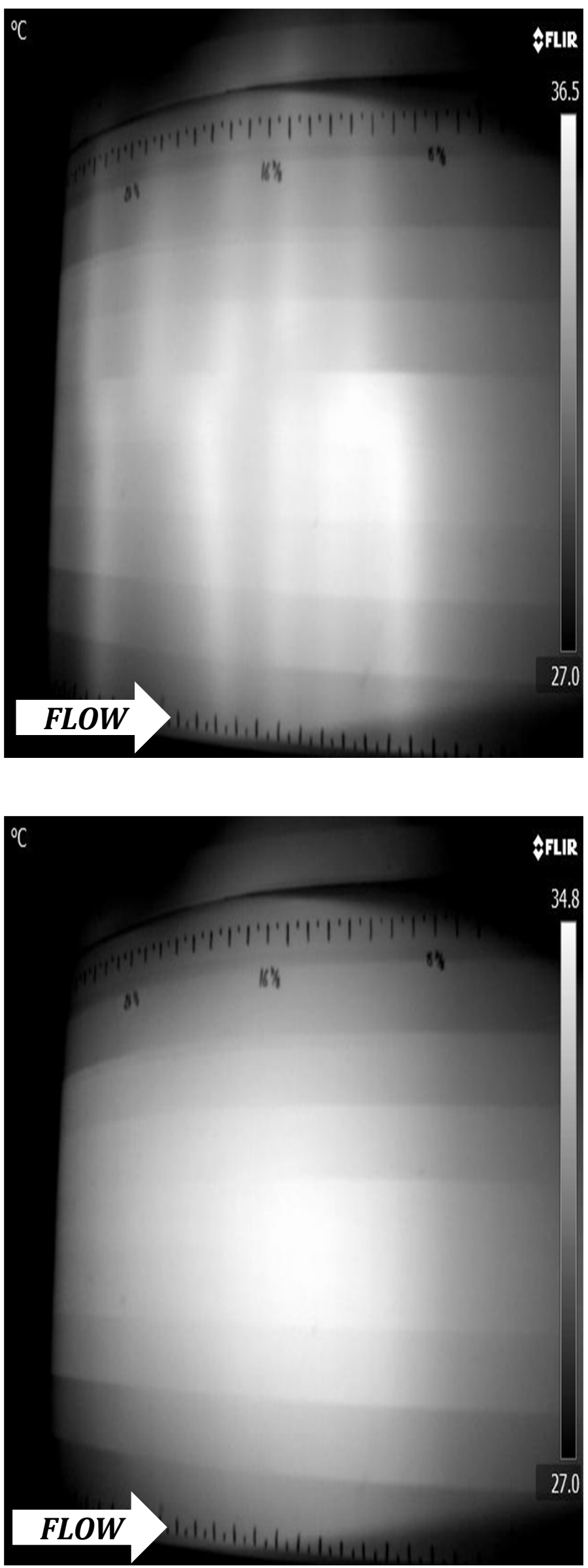


\section{8-m DU96-W-180 at $R e=1.5 \times 10^{5}$}

\section{PRESSURE SIDE}

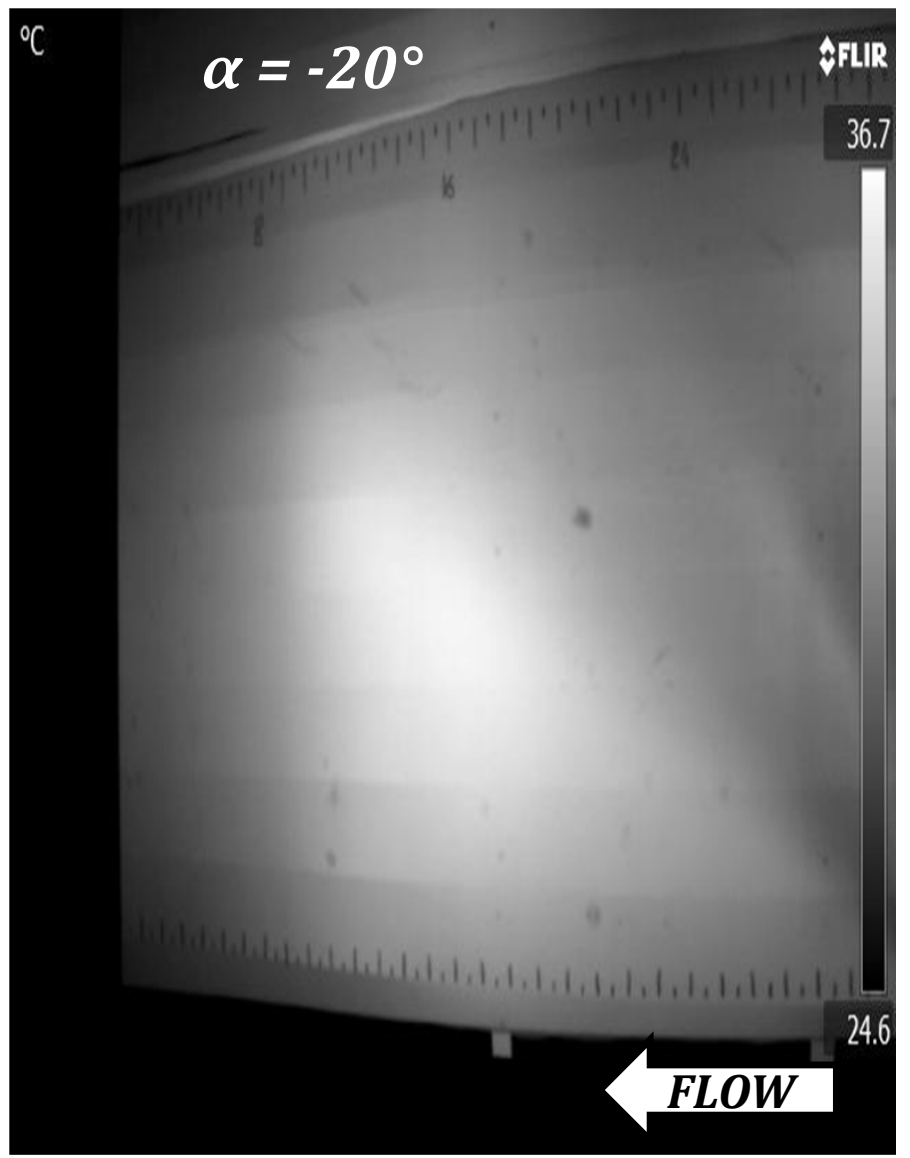

SUCTION SIDE

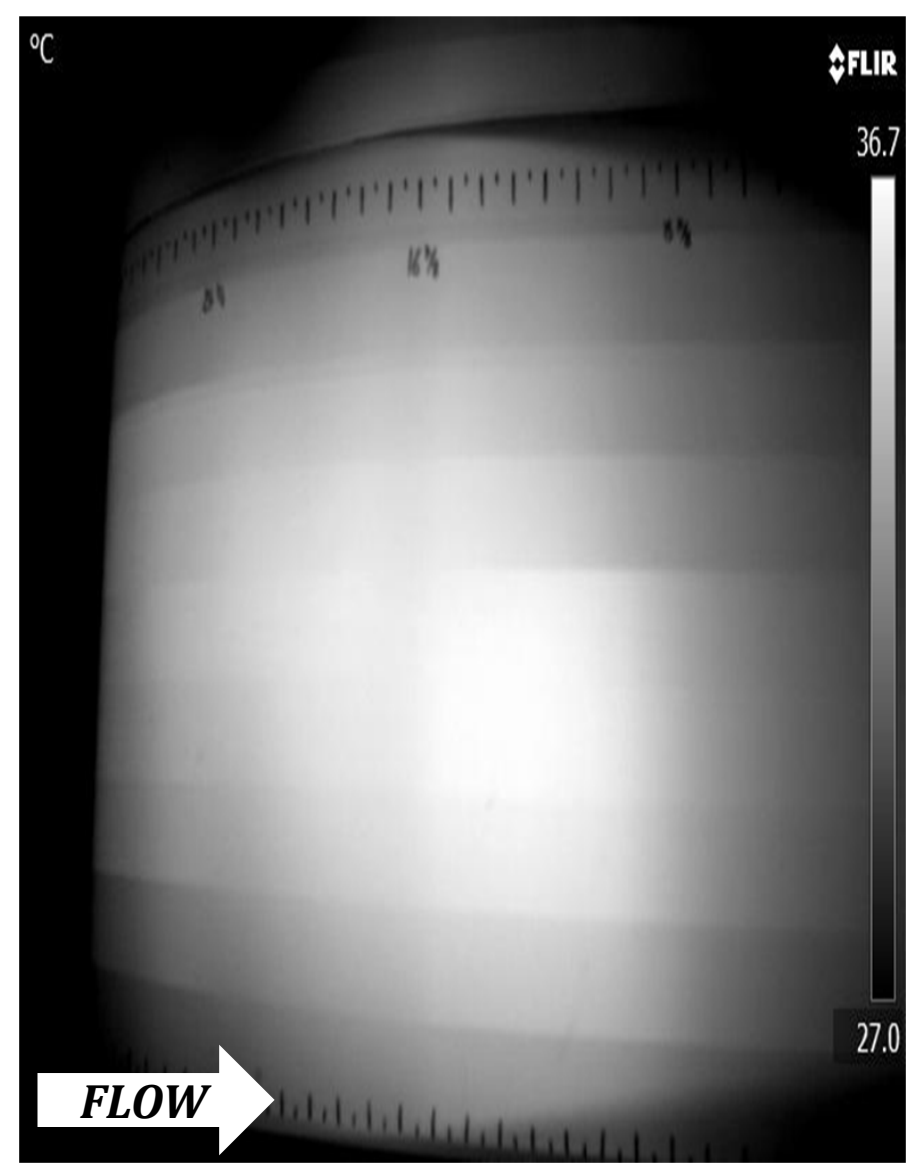


Appendix E Infrared Images of 0.8-m DU96-W-180 at $\mathrm{Re}=2 \times 10^{5}$

(From $\alpha=20^{\circ}$ to -20 , Pressure Side Images are on Left and Suction Side Images are on Right.) 


\section{8-m DU96-W-180 at $R e=2 \times 10^{5}$}

\section{PRESSURE SIDE}
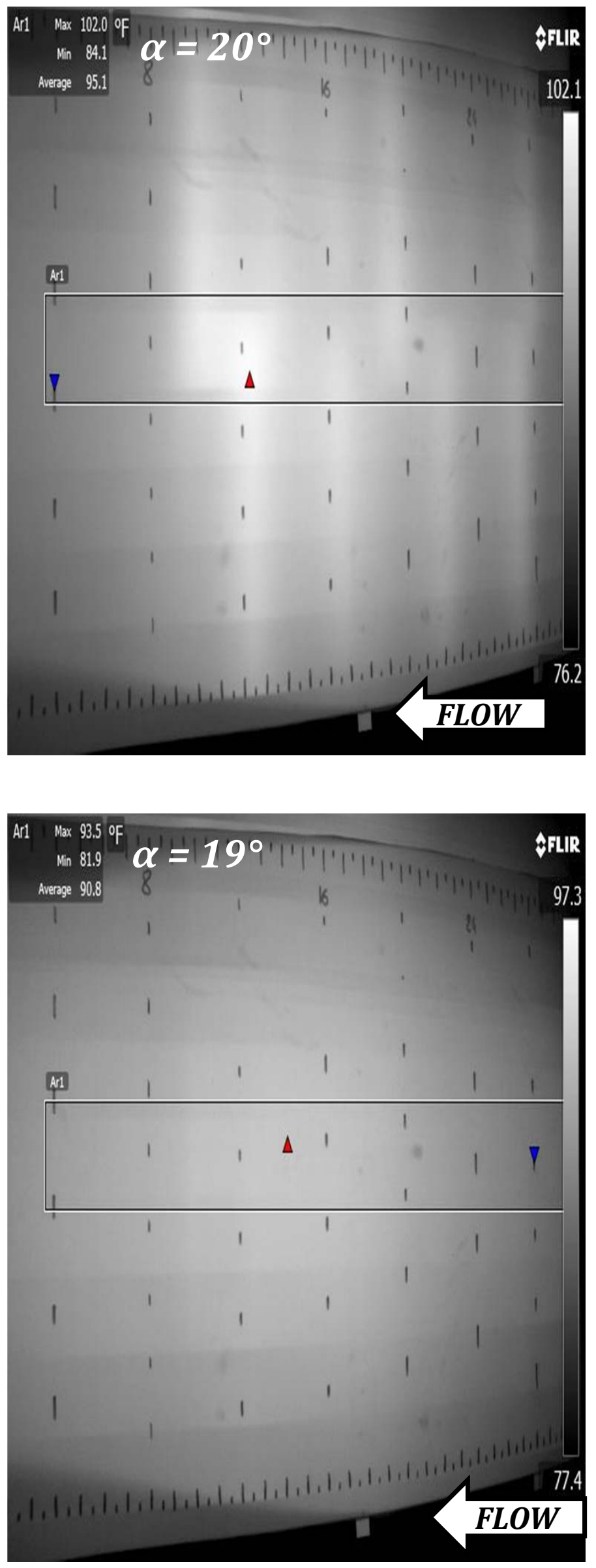

SUCTION SIDE
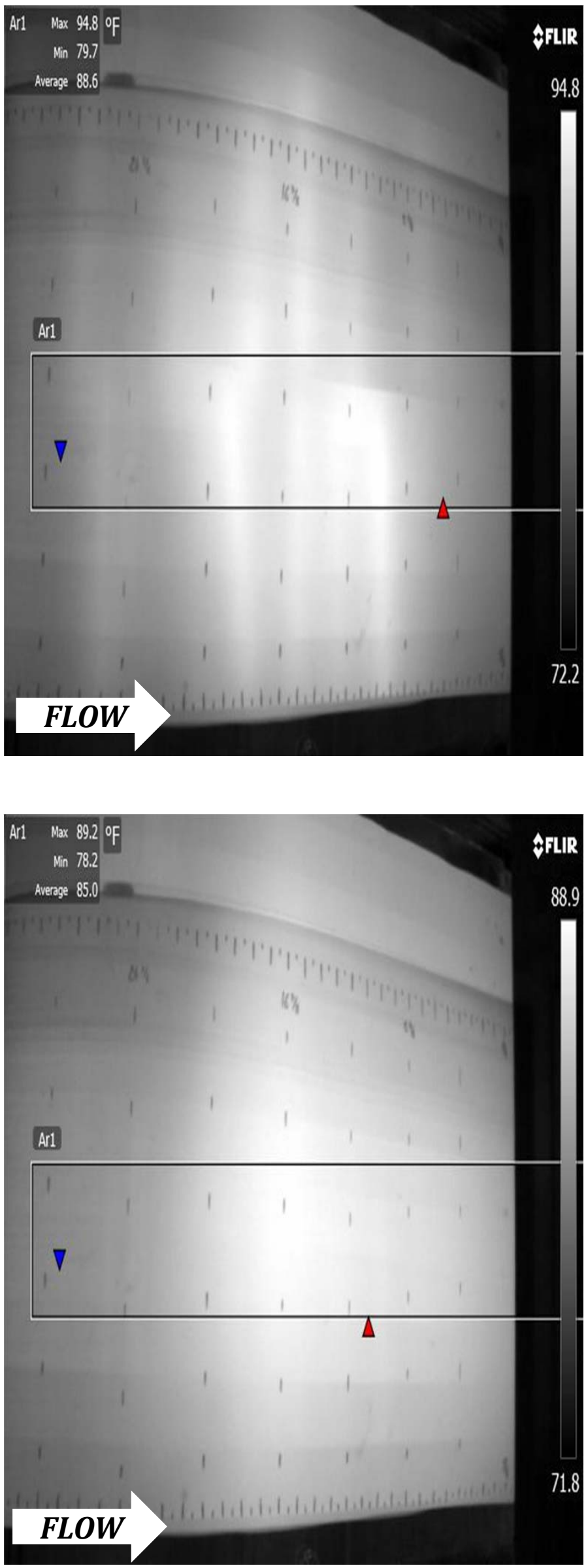


\section{8-m DU96-W-180 at $R e=2 \times 10^{5}$}

\section{PRESSURE SIDE}
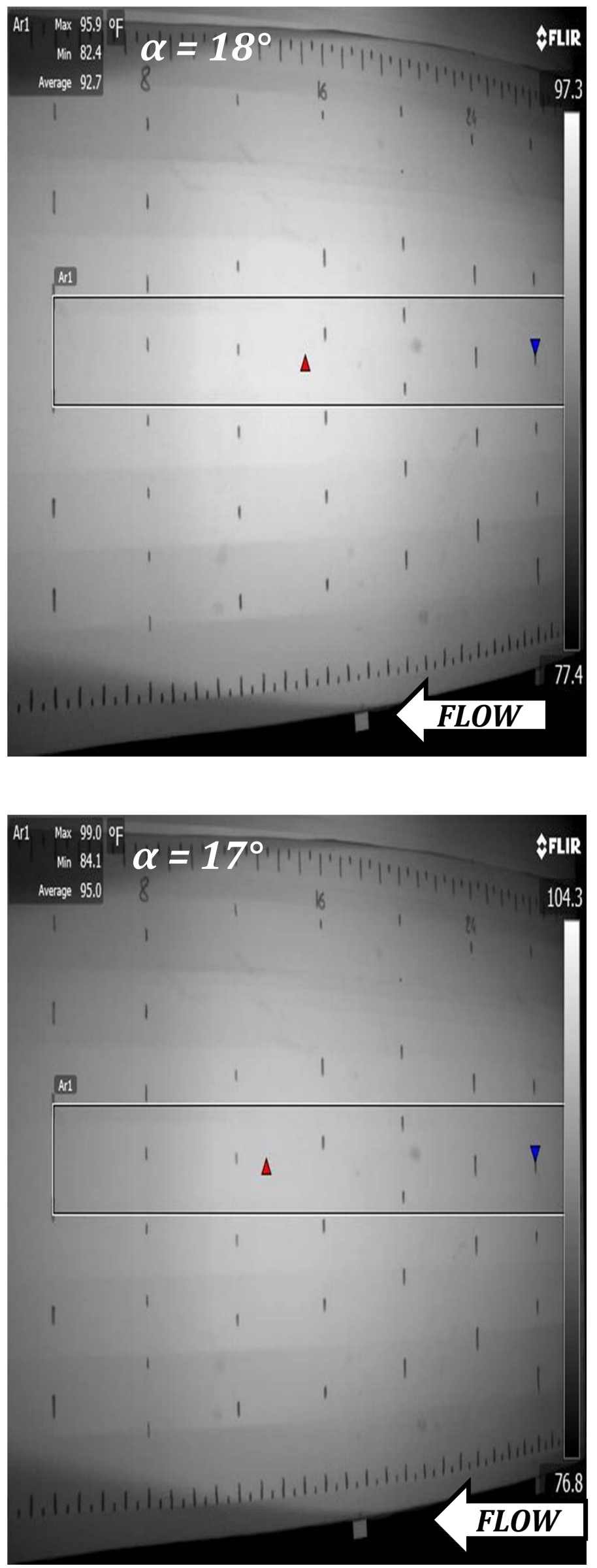

SUCTION SIDE
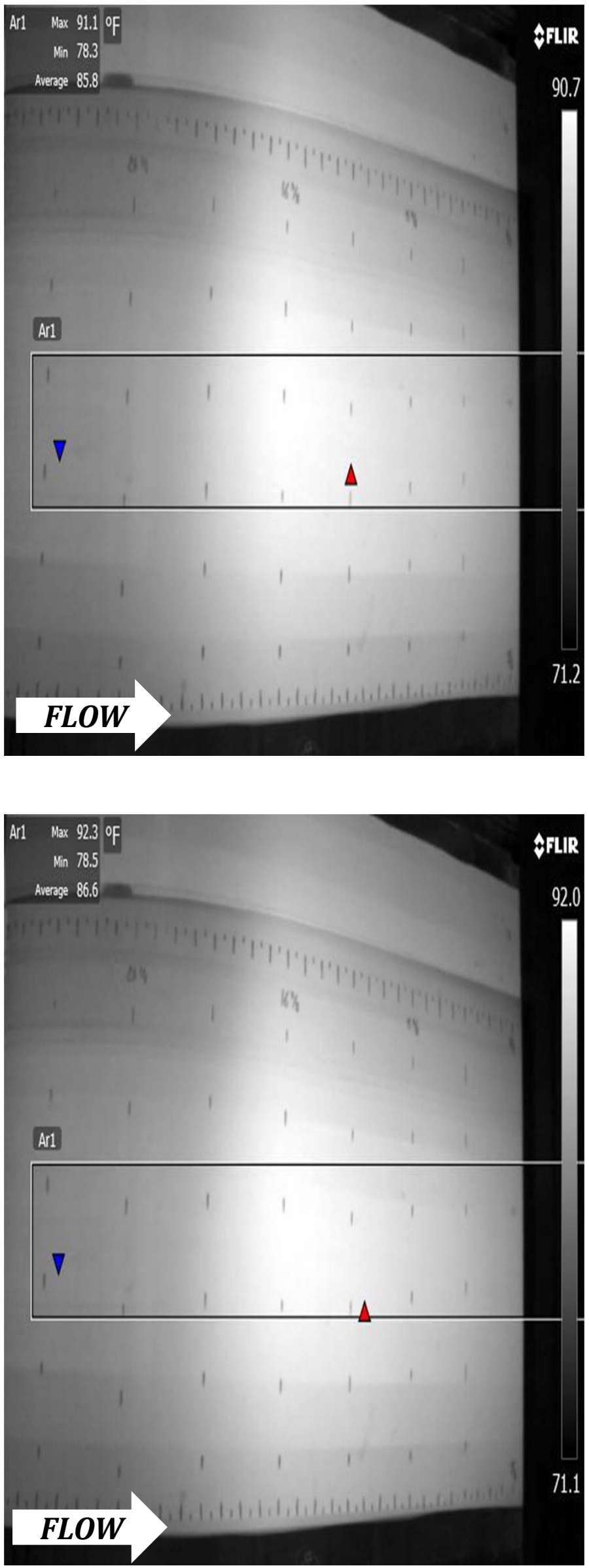


\section{8-m DU96-W-180 at $R e=2 \times 10^{5}$}

\section{PRESSURE SIDE}
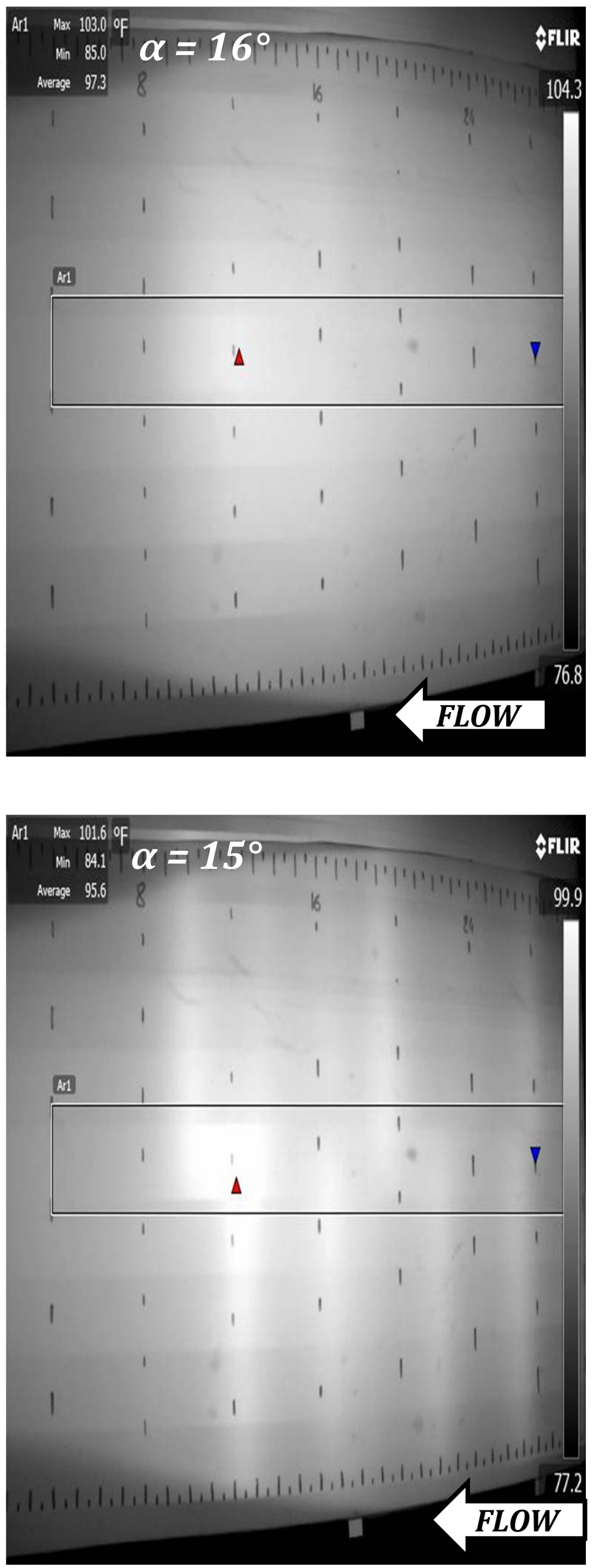

SUCTION SIDE
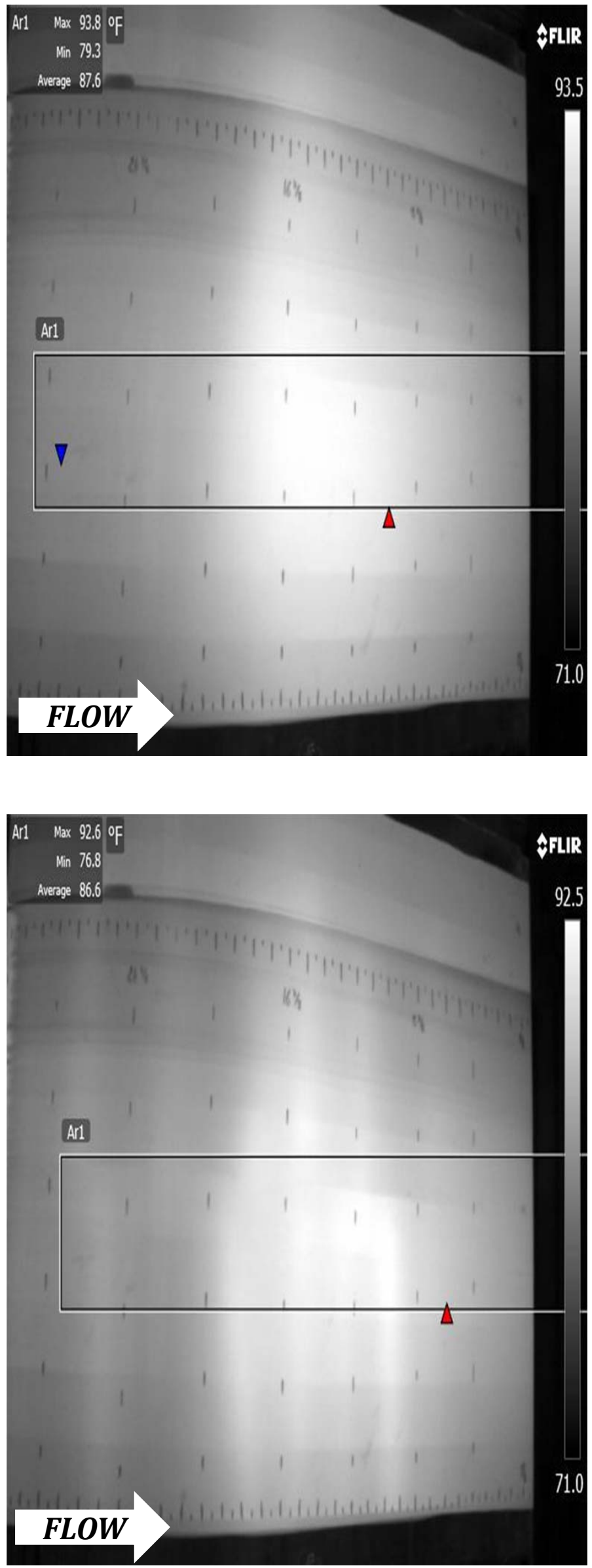


\section{8-m DU96-W-180 at $R e=2 \times 10^{5}$}

\section{PRESSURE SIDE}
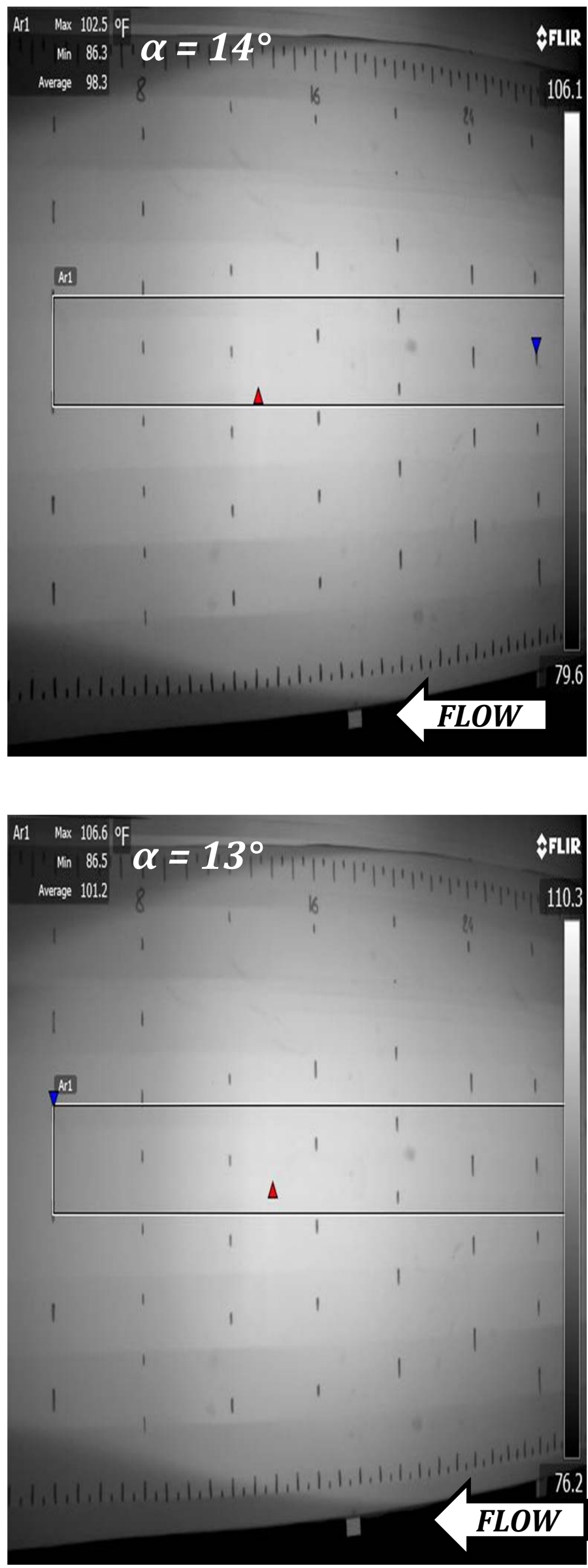

SUCTION SIDE
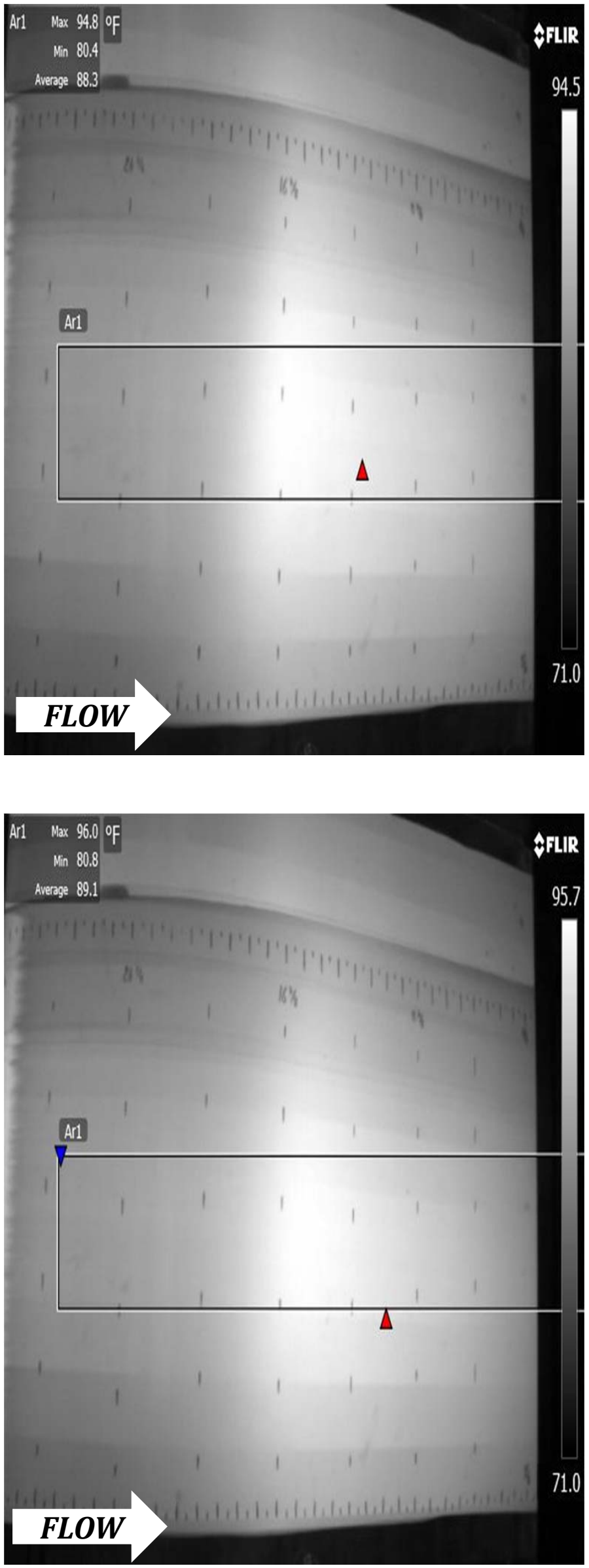


\section{8-m DU96-W-180 at $R e=2 \times 10^{5}$}

\section{PRESSURE SIDE}
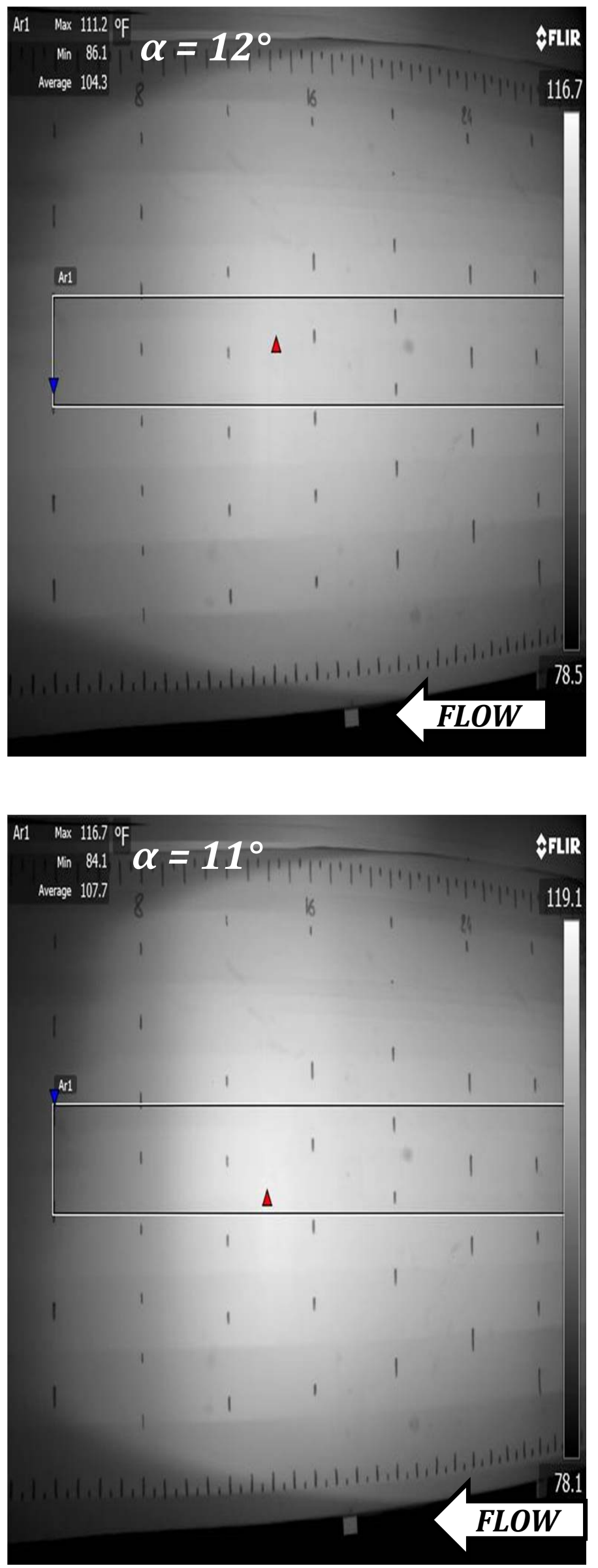

SUCTION SIDE
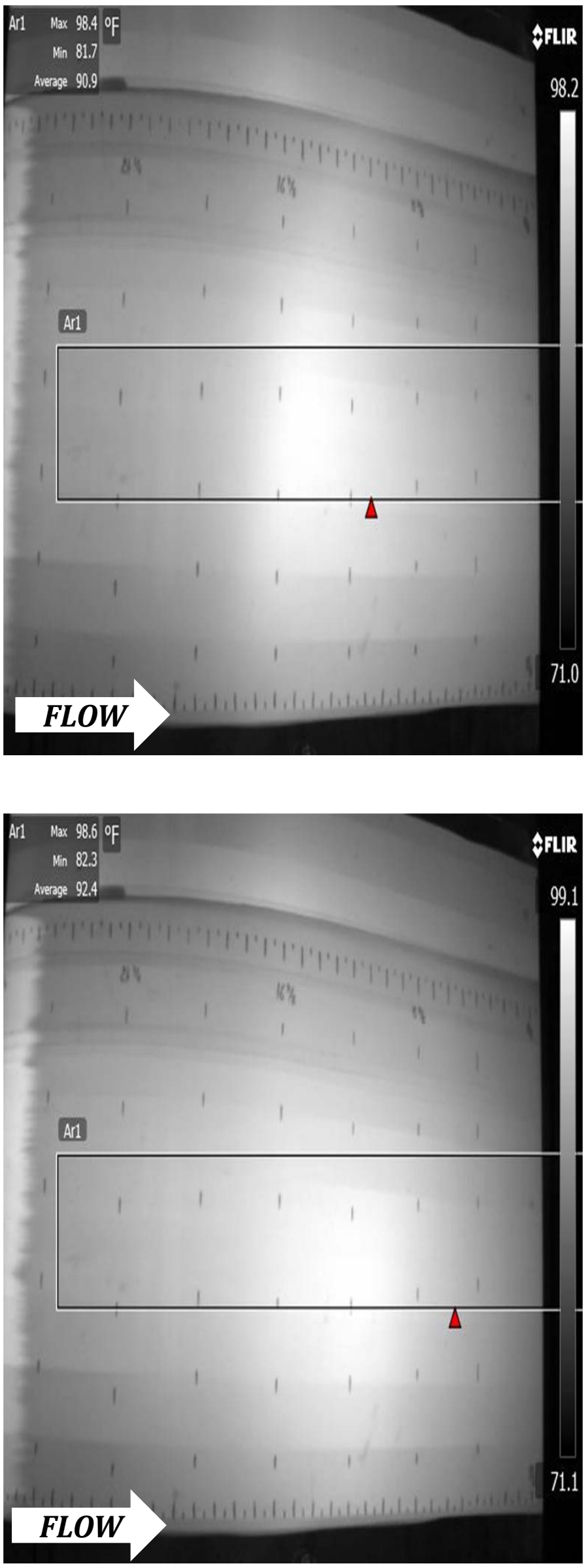


\section{8-m DU96-W-180 at $R e=2 \times 10^{5}$}

\section{PRESSURE SIDE}
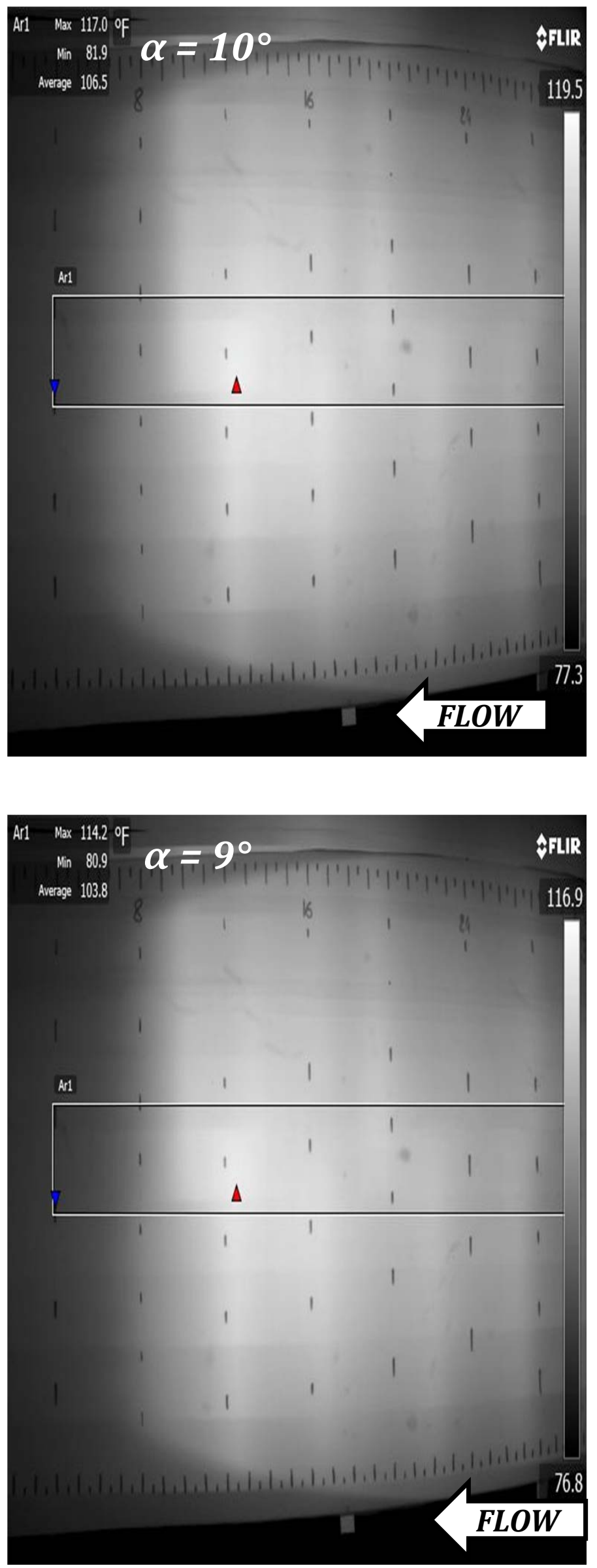

SUCTION SIDE
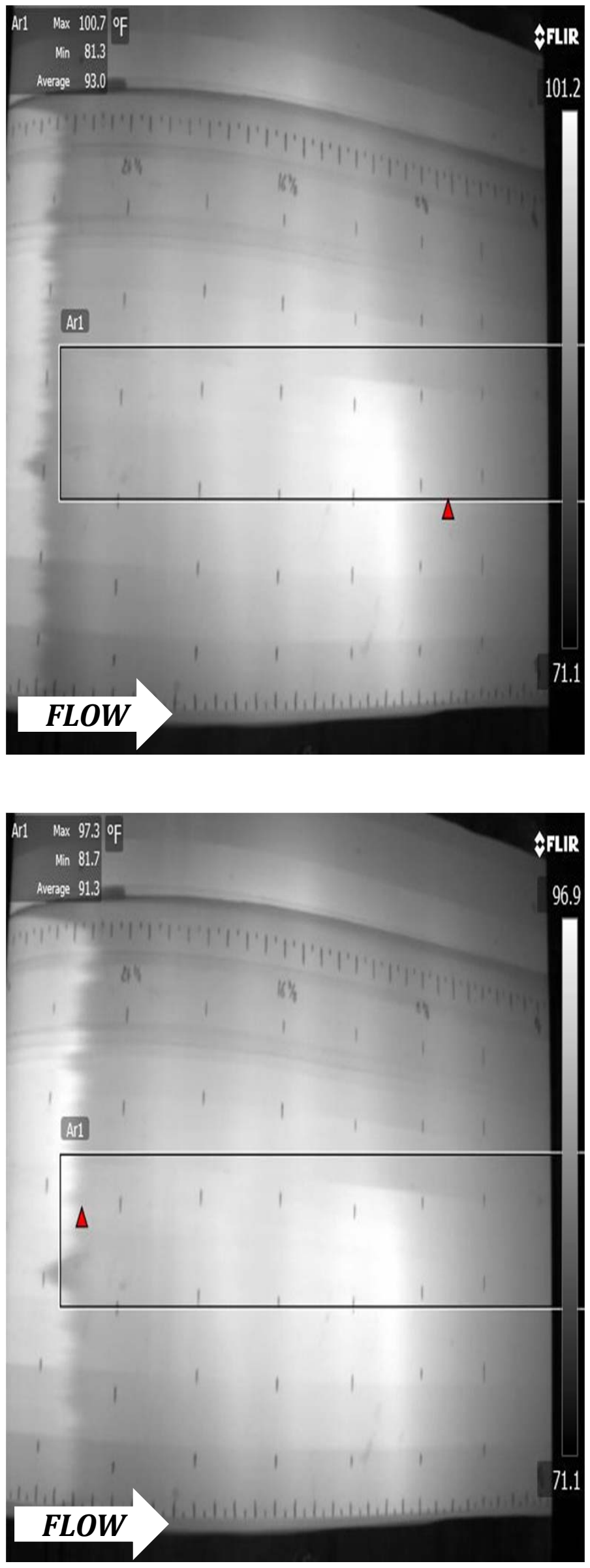


\section{8-m DU96-W-180 at $R e=2 \times 10^{5}$}

\section{PRESSURE SIDE}
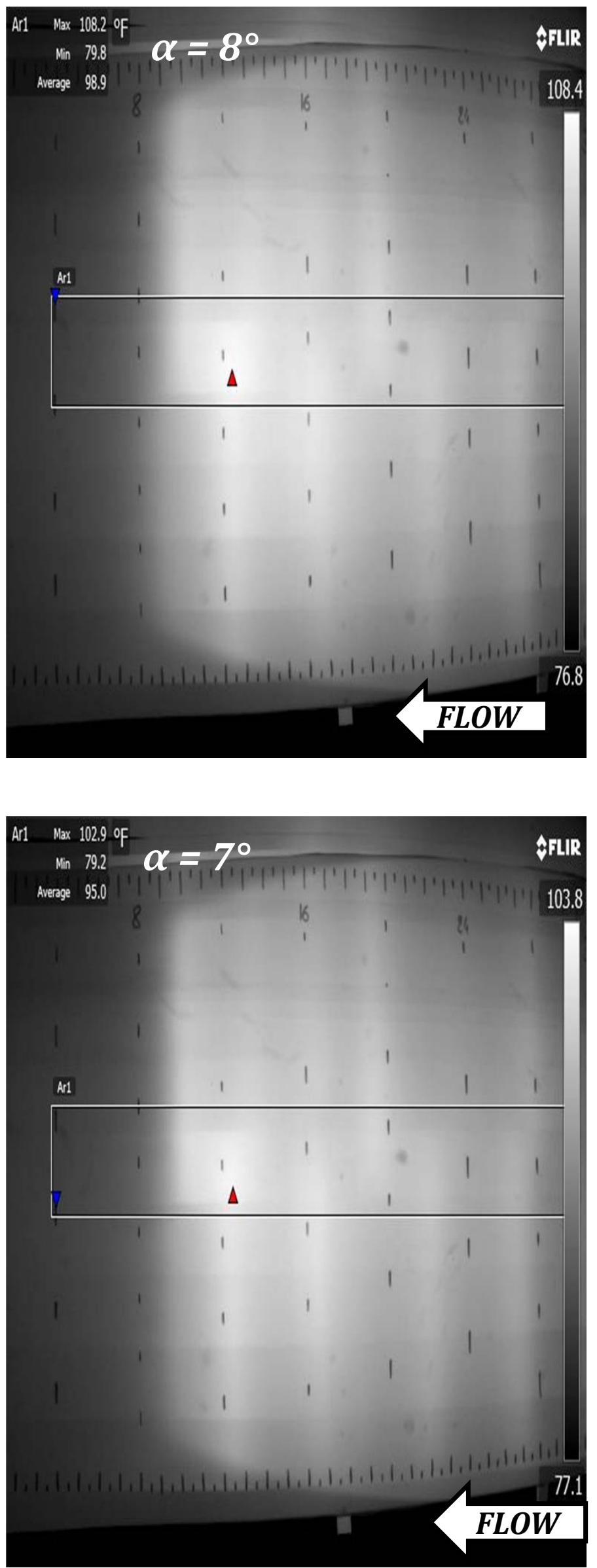

SUCTION SIDE
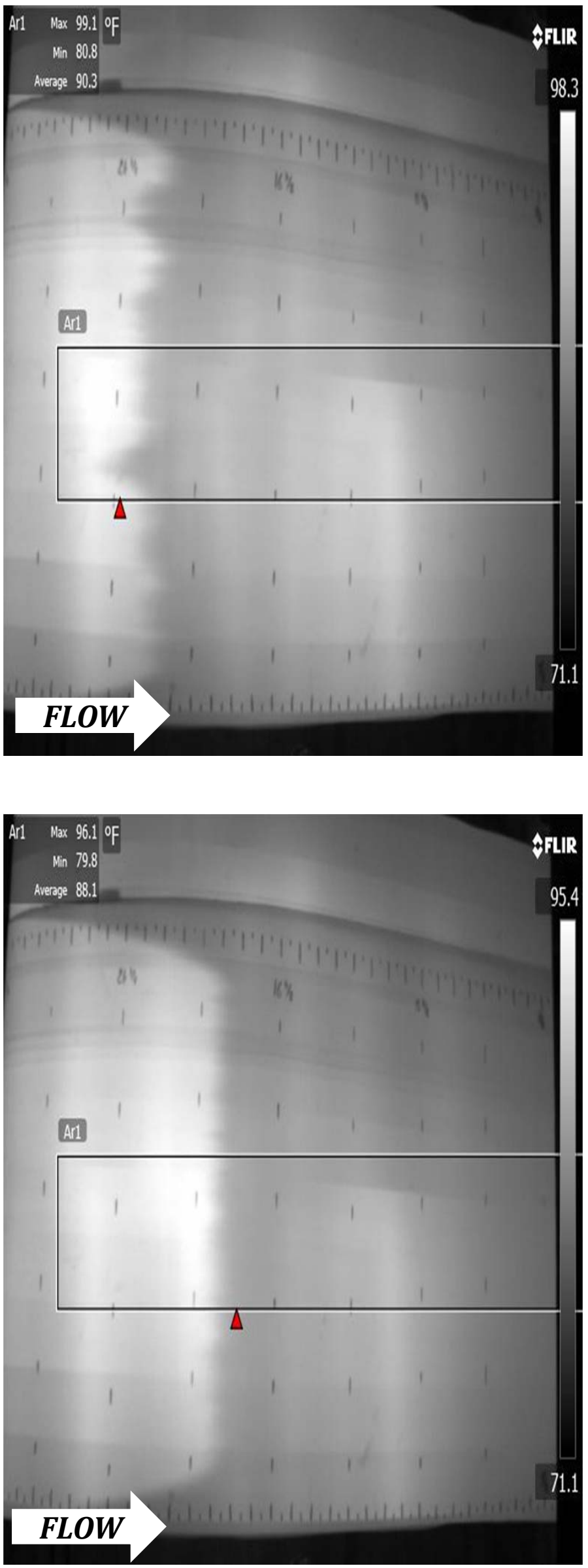


\section{8-m DU96-W-180 at $R e=2 \times 10^{5}$}

\section{PRESSURE SIDE}
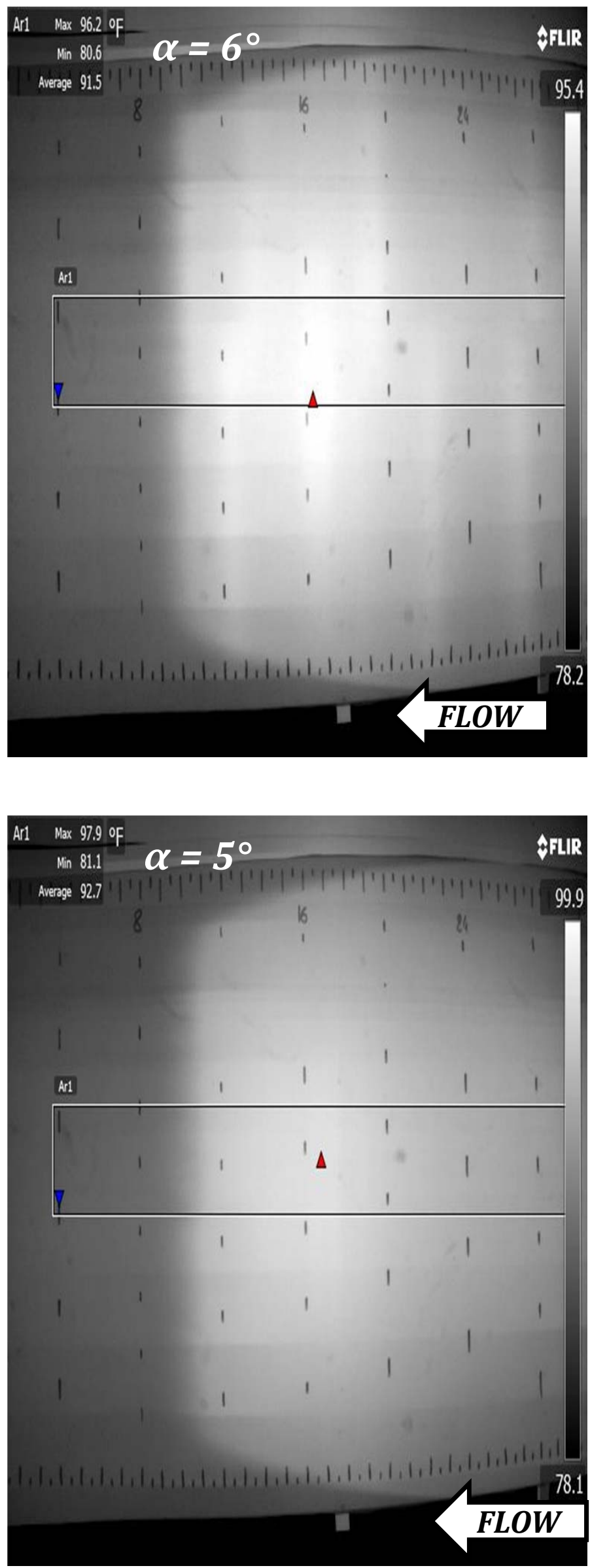

SUCTION SIDE
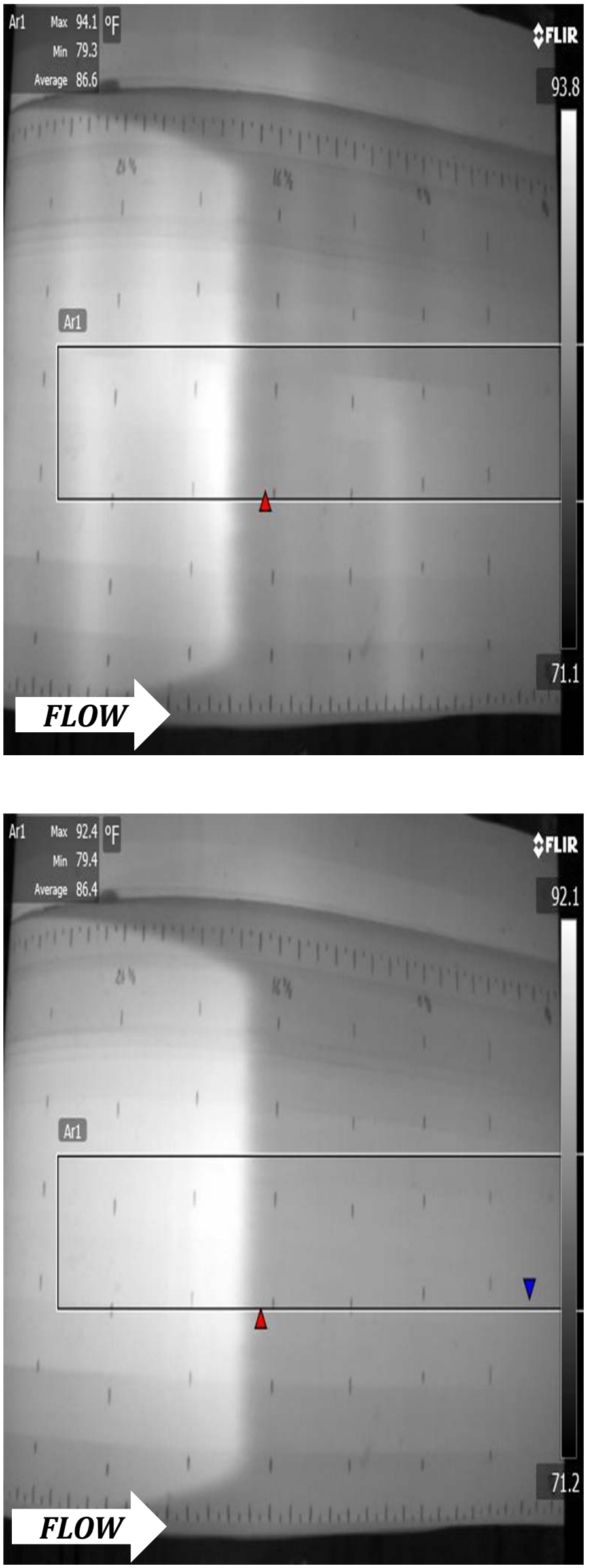


\section{8-m DU96-W-180 at $R e=2 \times 10^{5}$}

\section{PRESSURE SIDE}
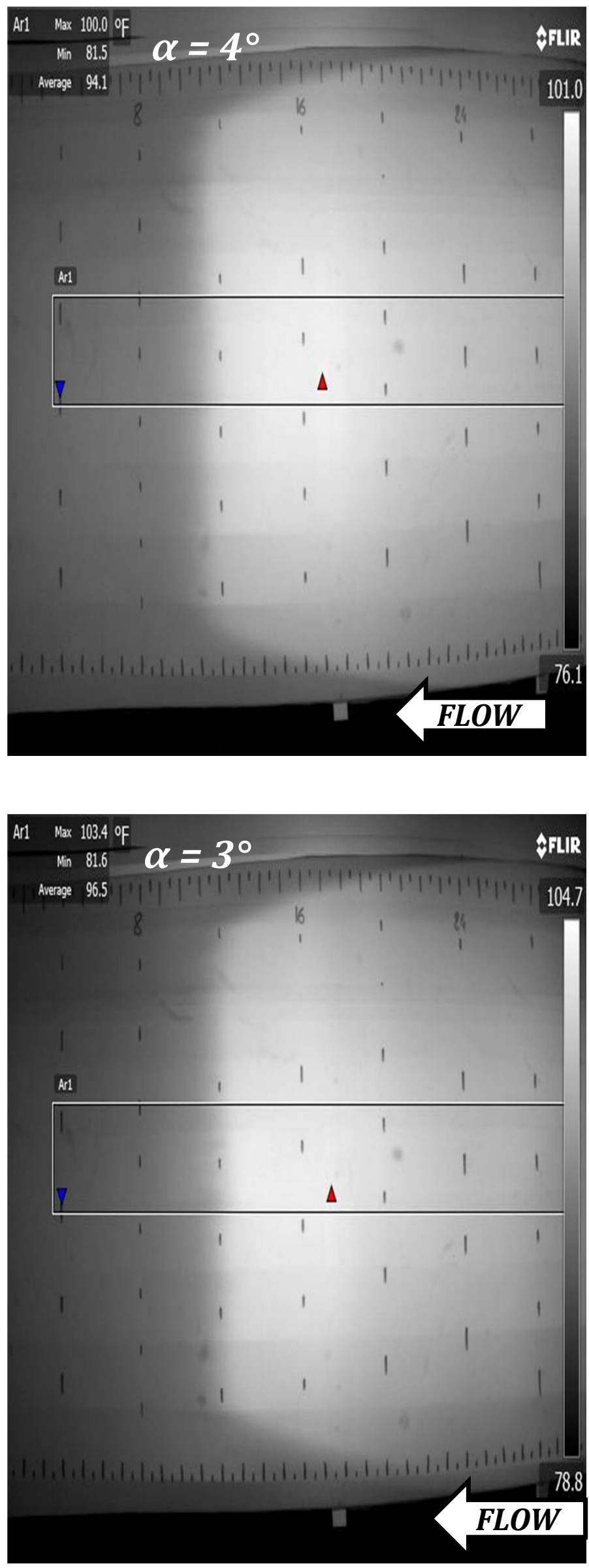

SUCTION SIDE
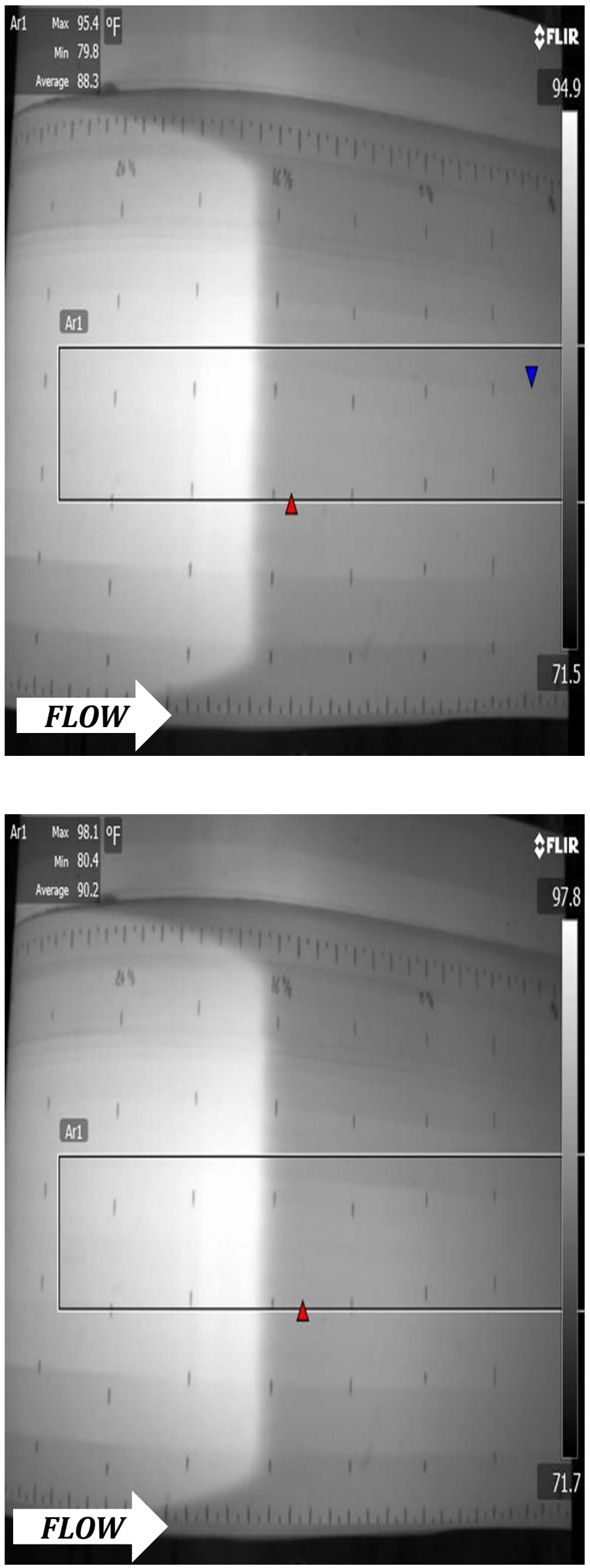


\section{8-m DU96-W-180 at $R e=2 \times 10^{5}$}

\section{PRESSURE SIDE}

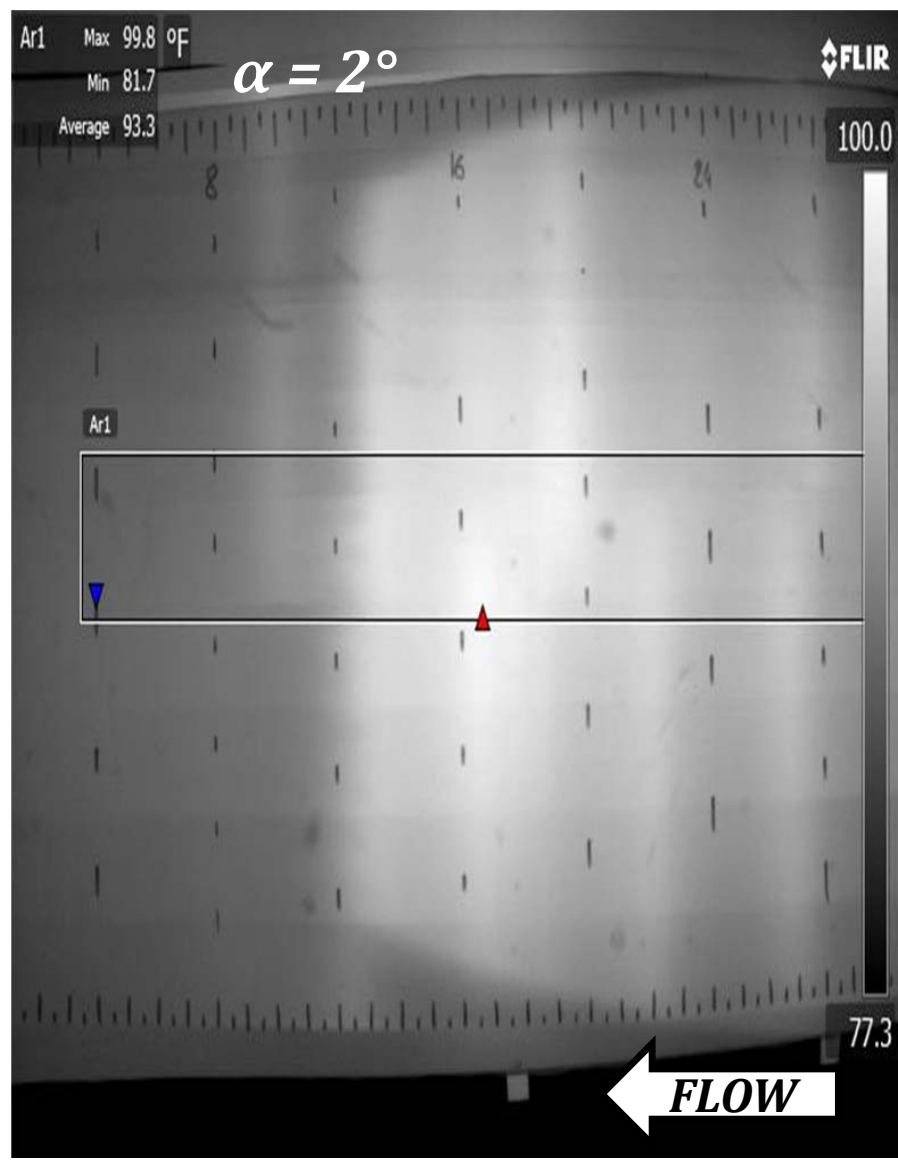

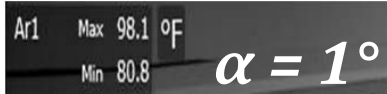

AFLIR

Averge 92.1

Arr

7

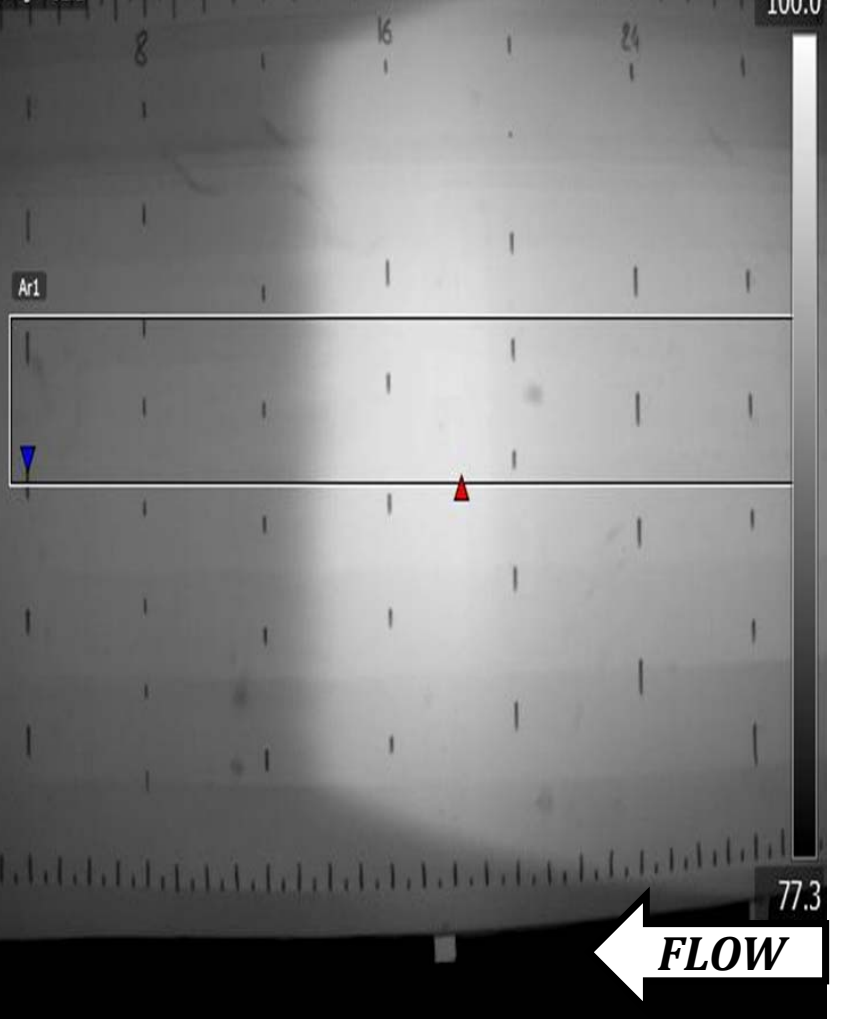

SUCTION SIDE
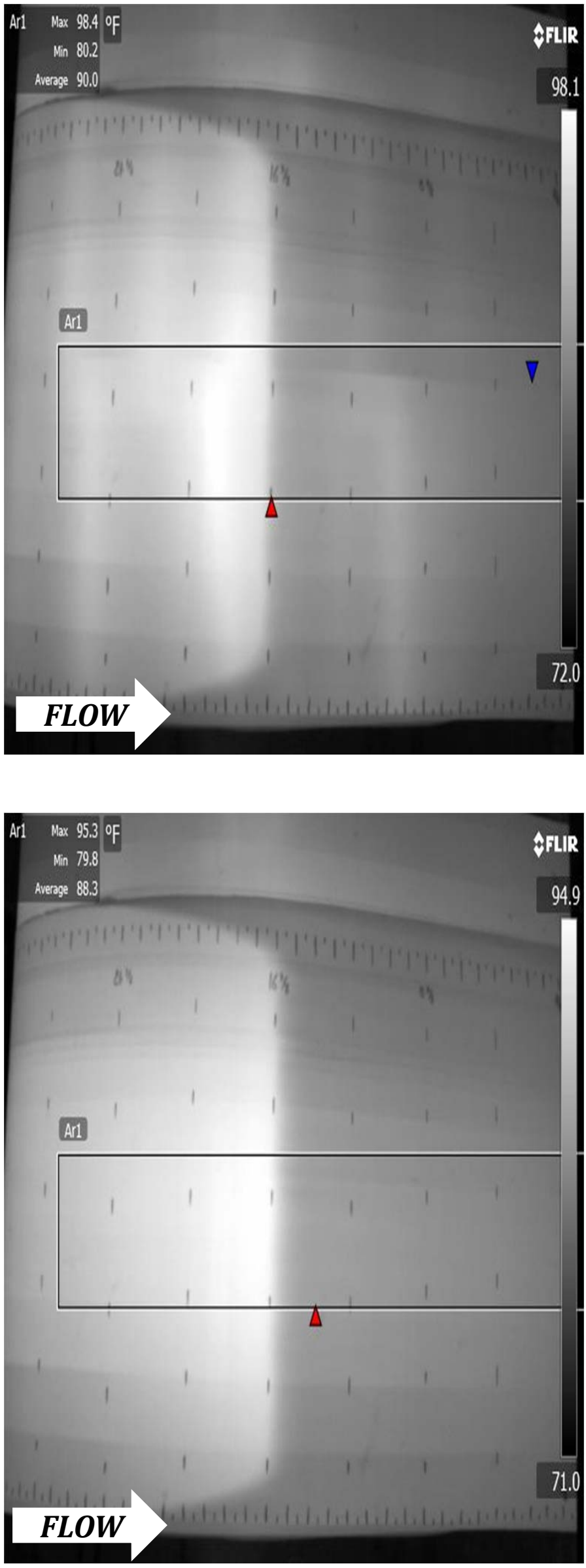


\section{8-m DU96-W-180 at $R e=2 \times 10^{5}$}

\section{PRESSURE SIDE}

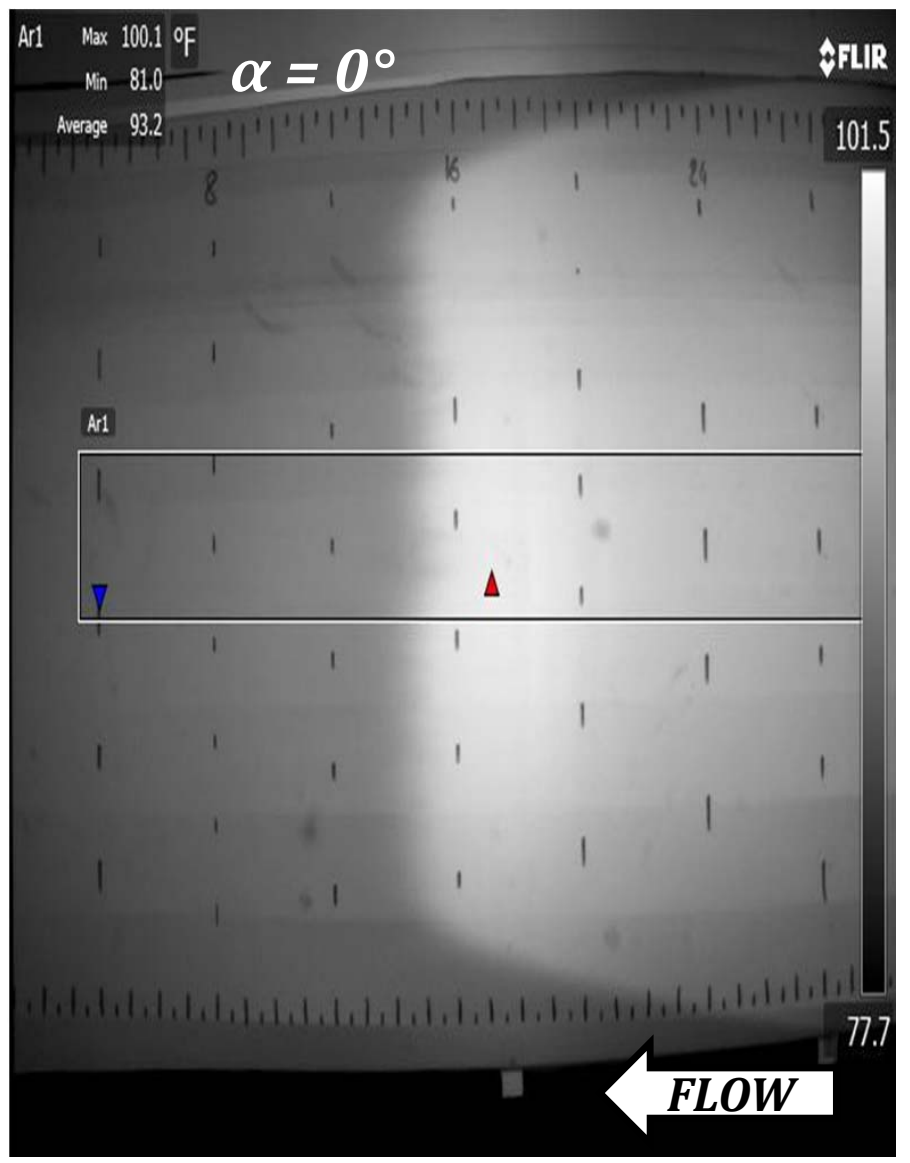

Ar1 $\quad \operatorname{Max} 103.1$ of $\alpha=-1^{\circ}$

AFLIR

Average 95.3

ArI

$\Delta$

7
SUCTION SIDE
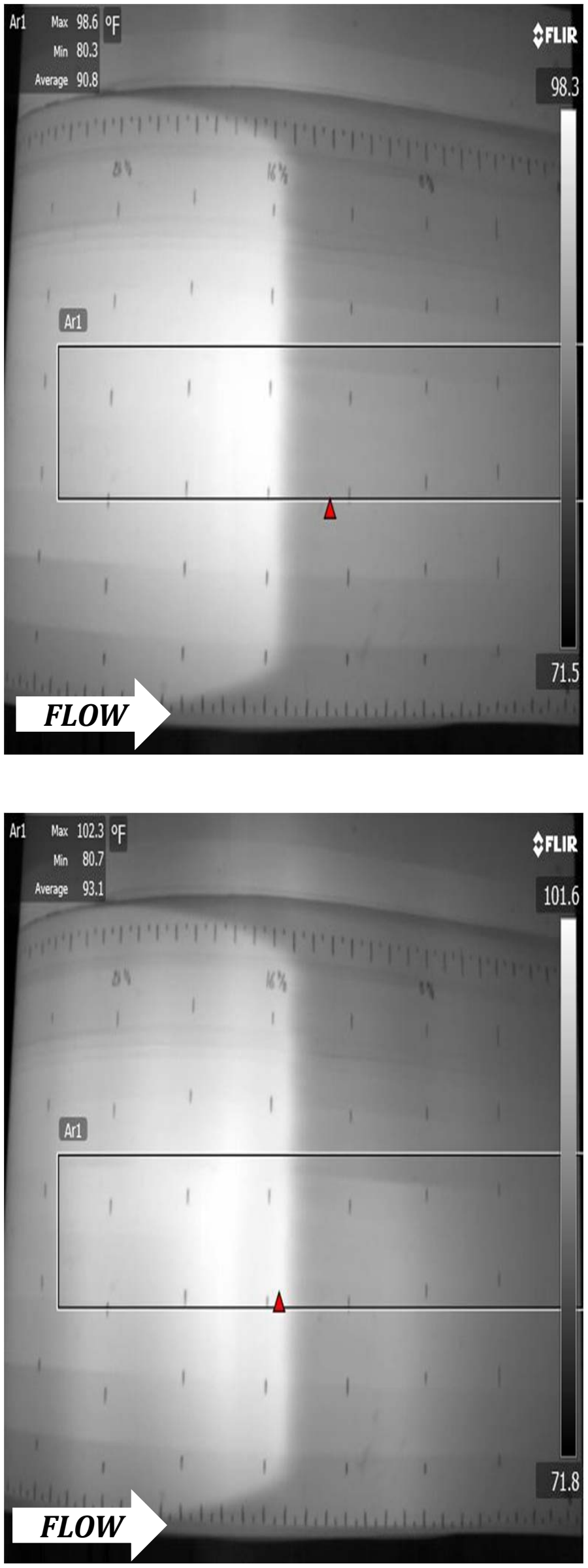


\section{8-m DU96-W-180 at $R e=2 \times 10^{5}$}

\section{PRESSURE SIDE}
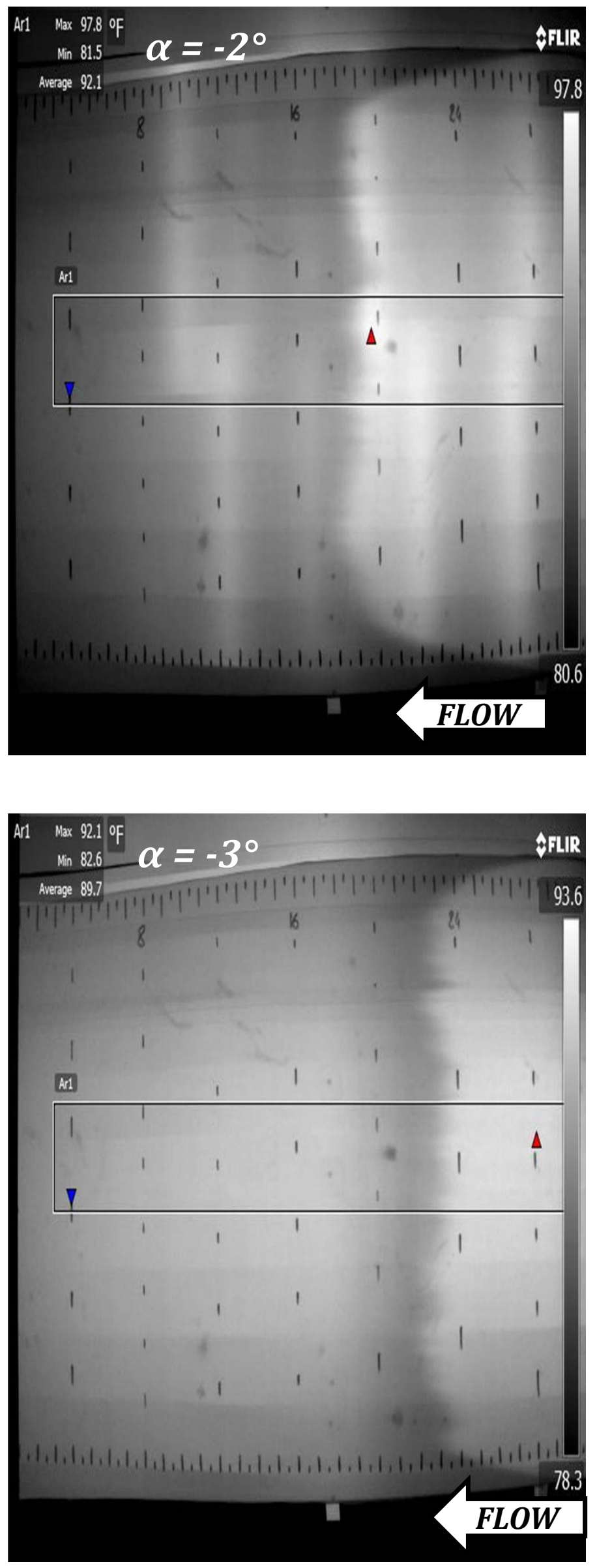

SUCTION SIDE
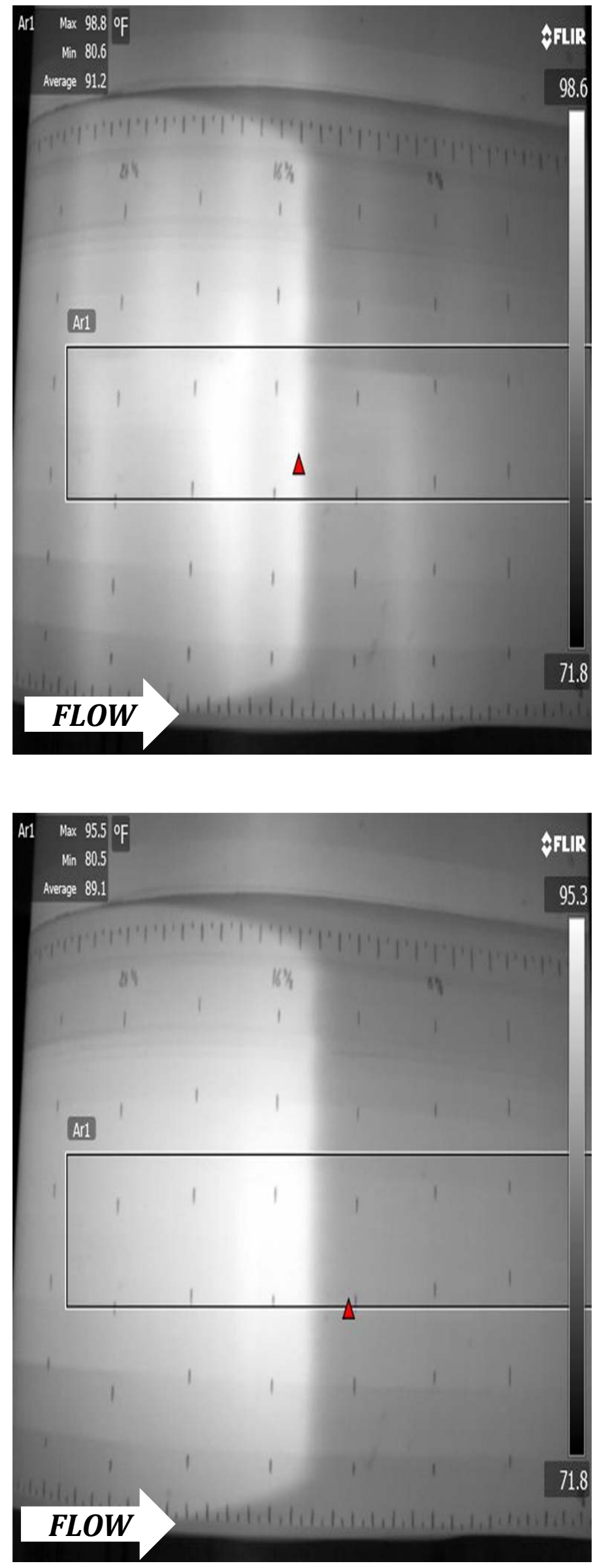


\section{8-m DU96-W-180 at $R e=2 \times 10^{5}$}

\section{PRESSURE SIDE}
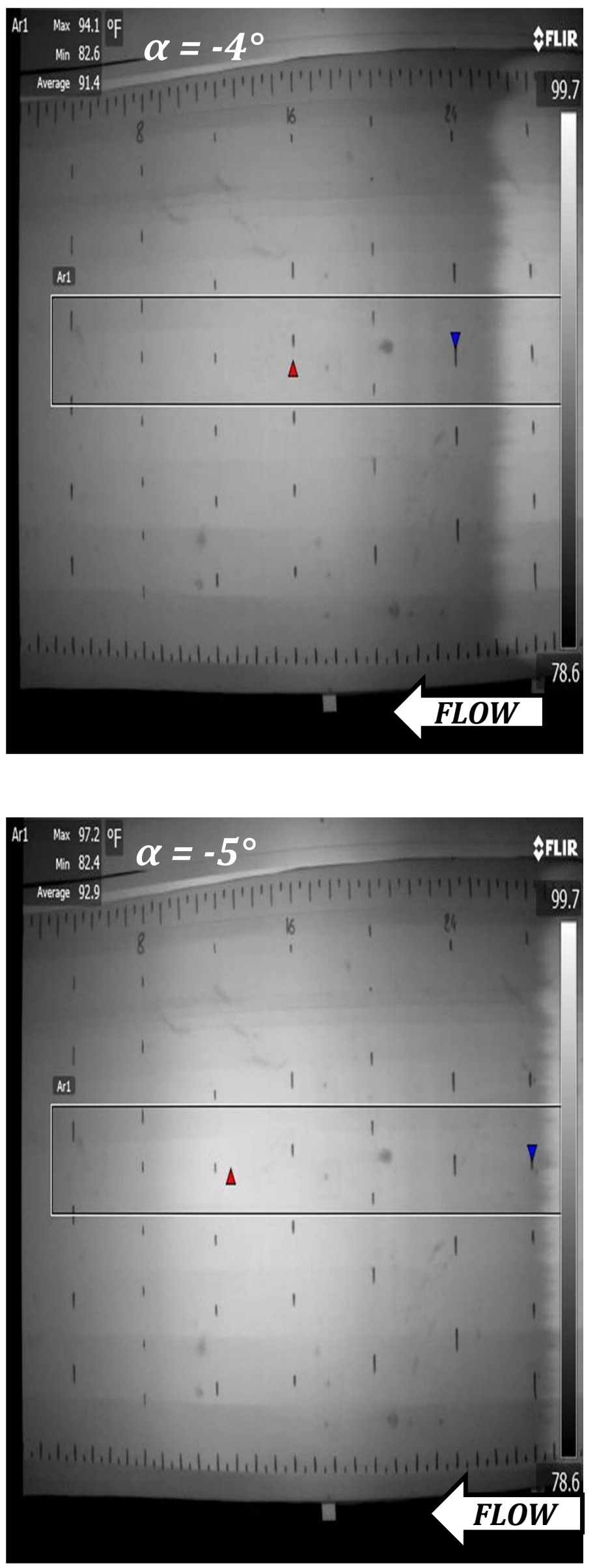

SUCTION SIDE
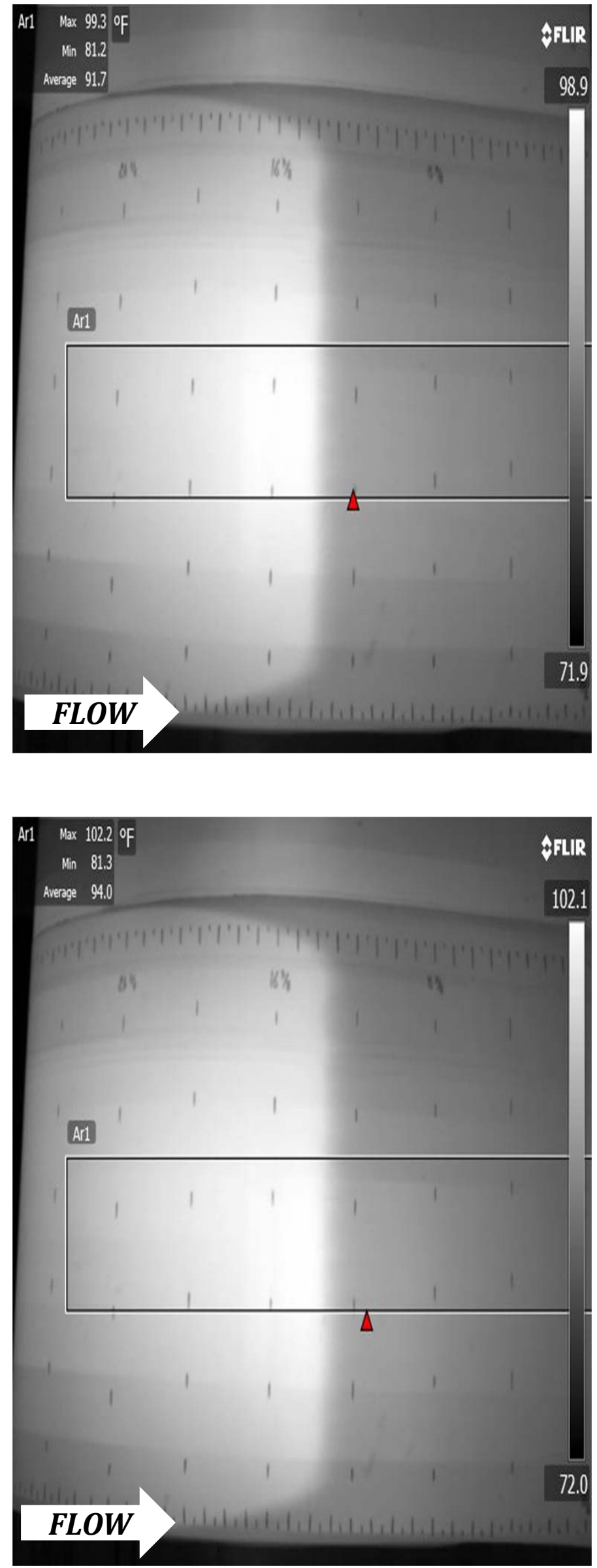


\section{8-m DU96-W-180 at $R e=2 \times 10^{5}$}

\section{PRESSURE SIDE}
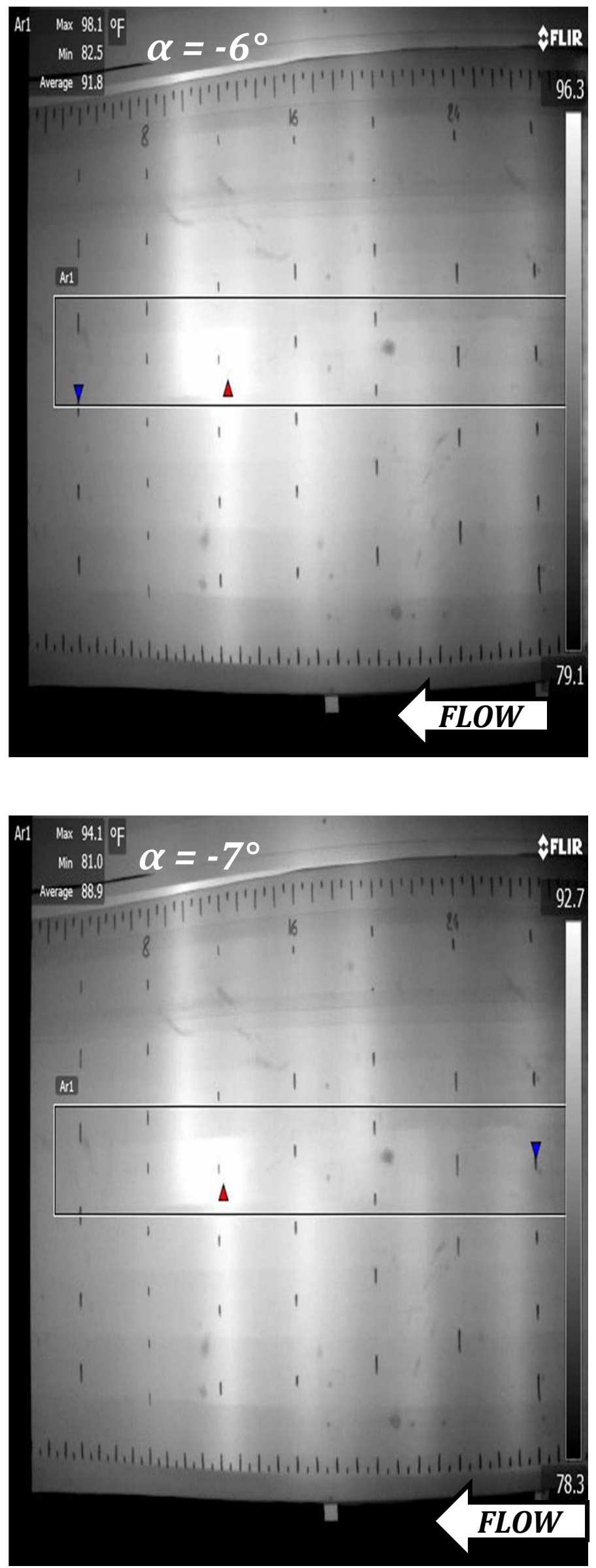

SUCTION SIDE
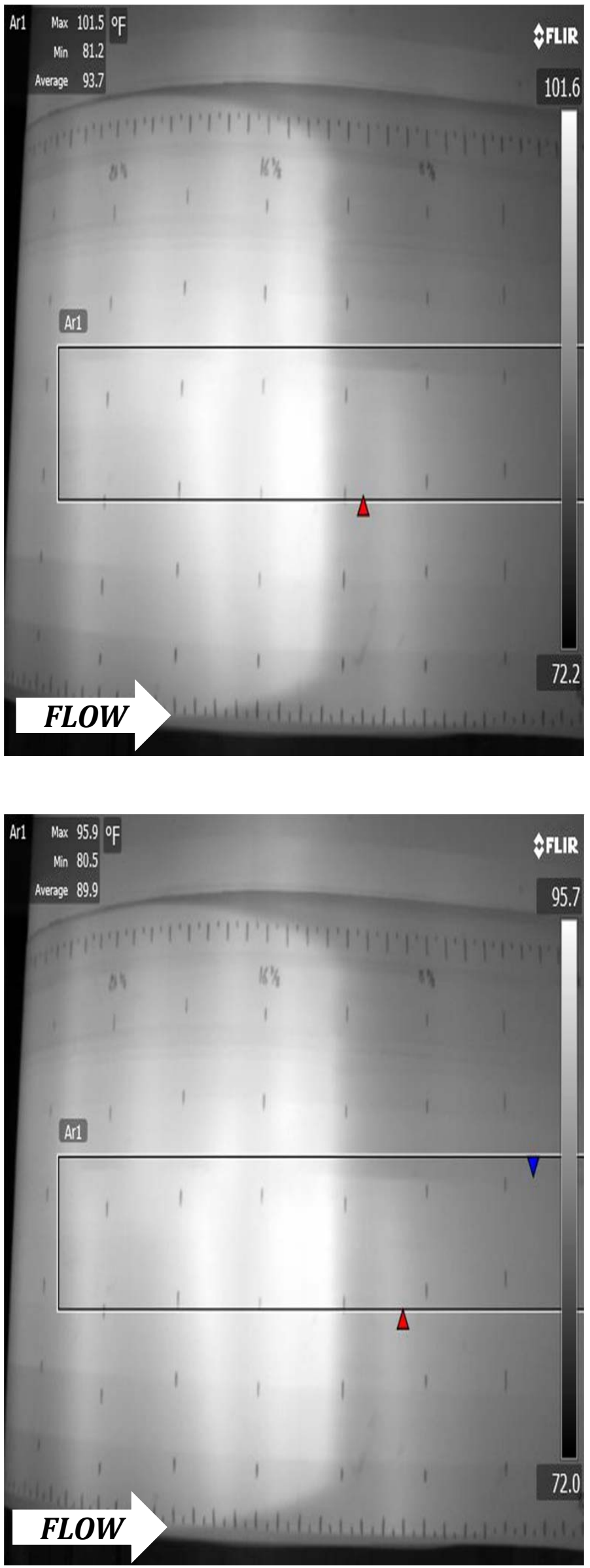


\section{8-m DU96-W-180 at $R e=2 \times 10^{5}$}

\section{PRESSURE SIDE}
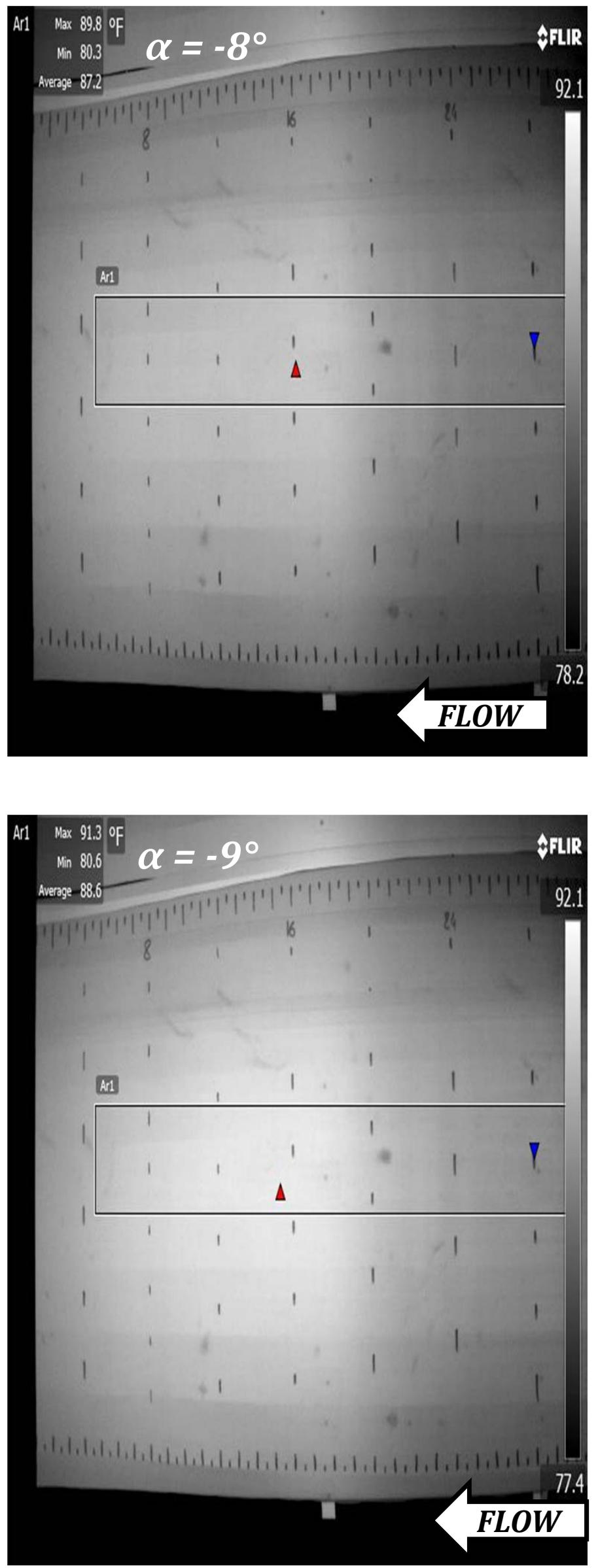

SUCTION SIDE
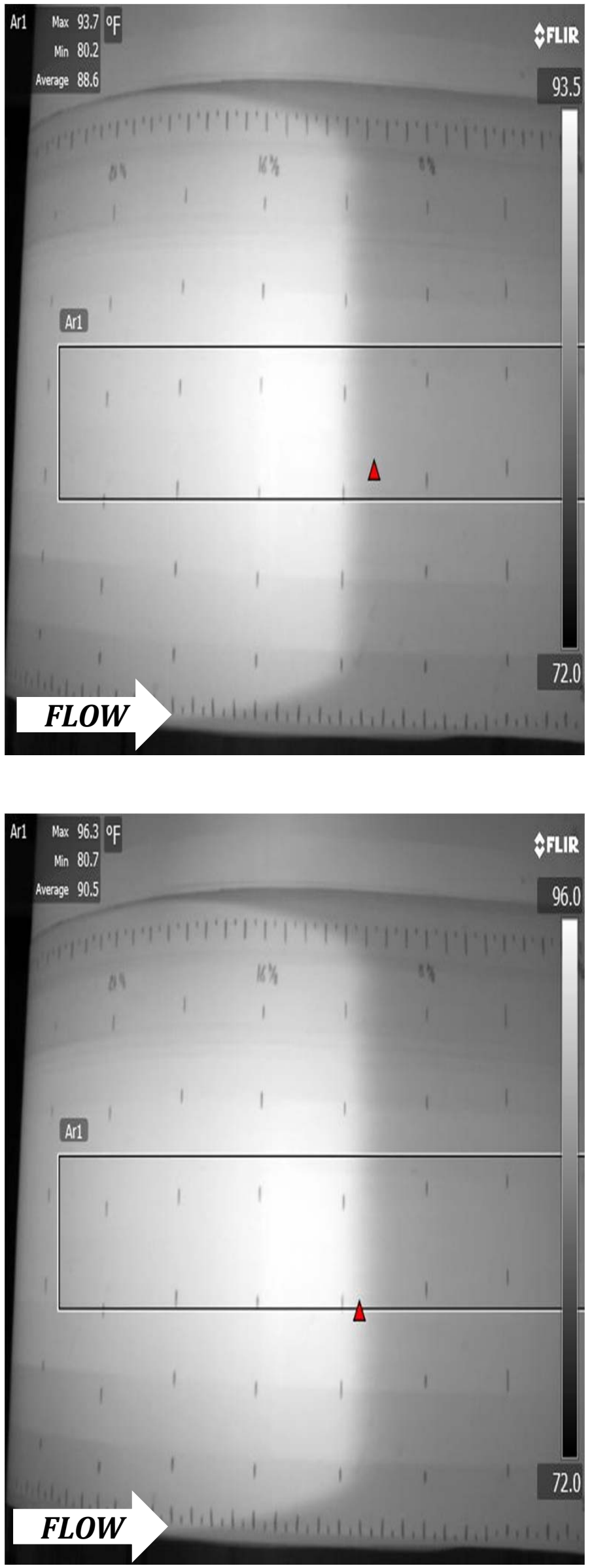


\section{8-m DU96-W-180 at $R e=2 \times 10^{5}$}

\section{PRESSURE SIDE}

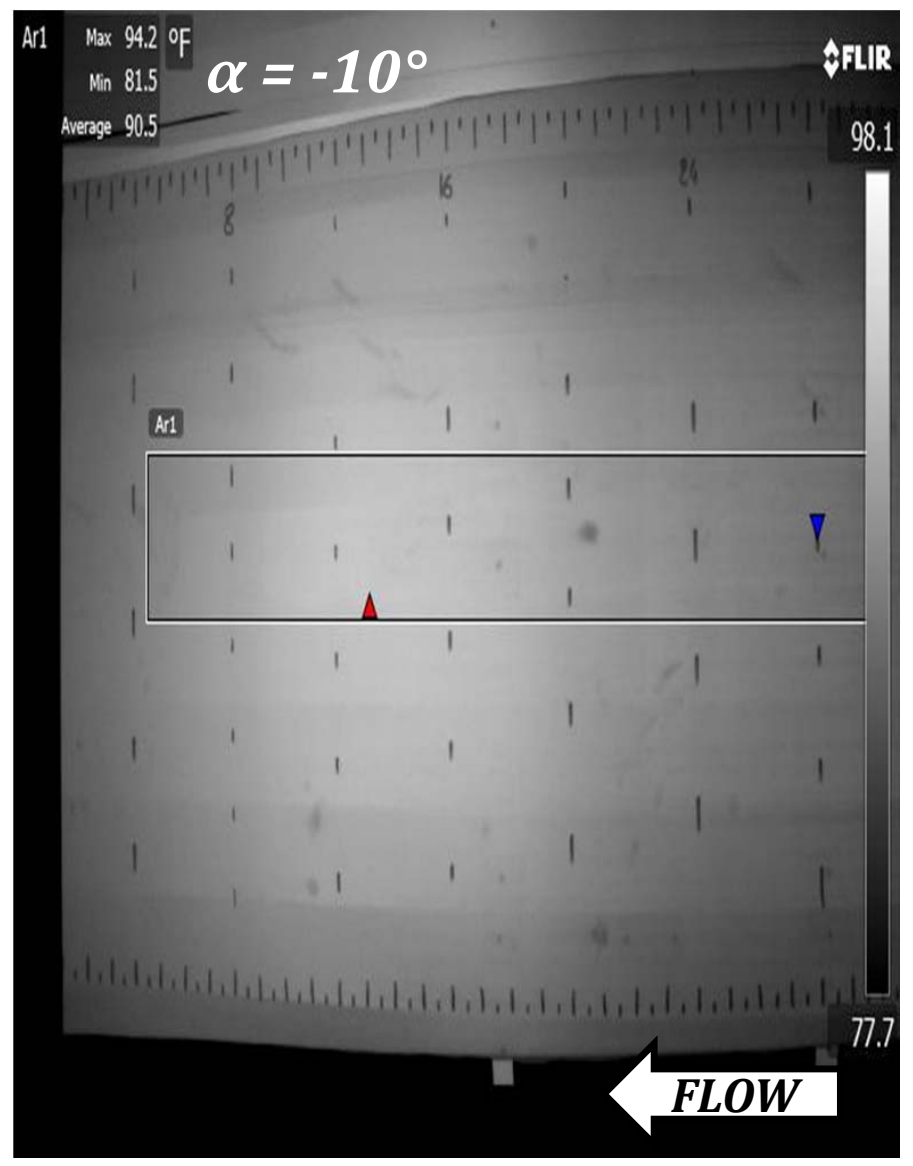

Ar1 Max 98.6 of

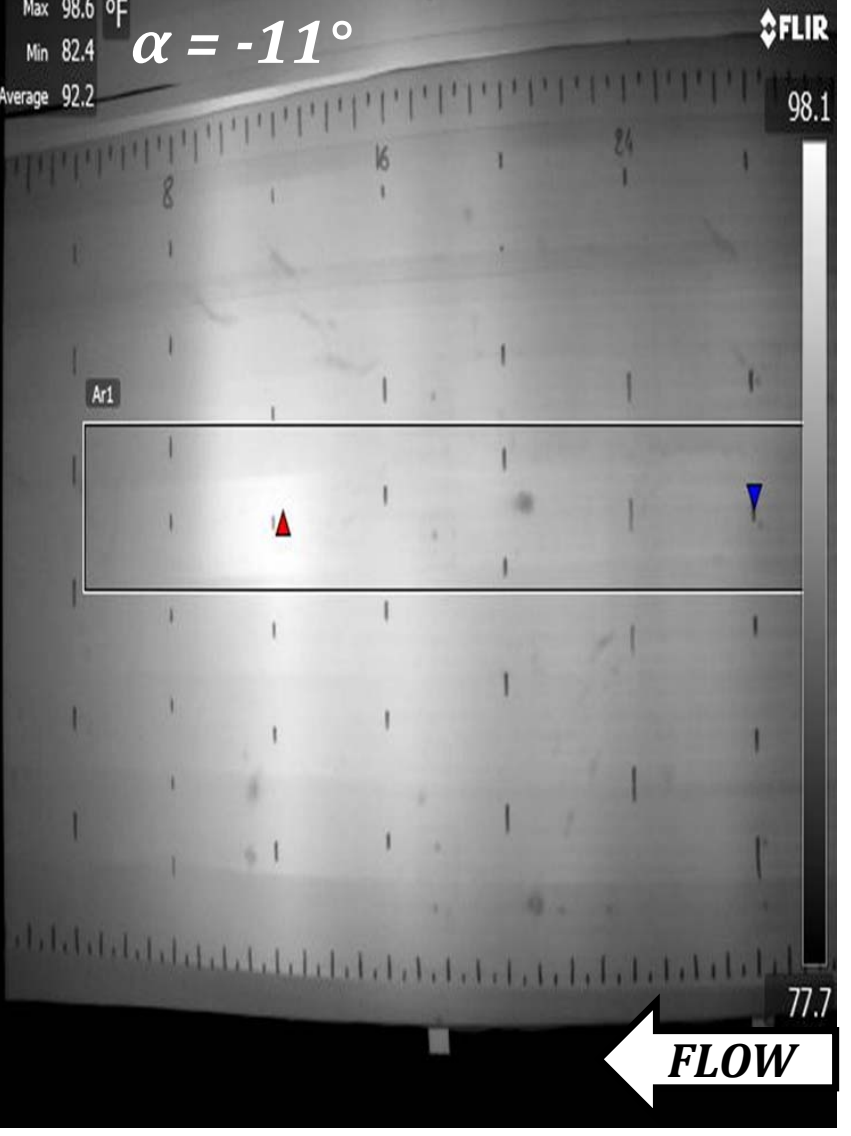

SUCTION SIDE
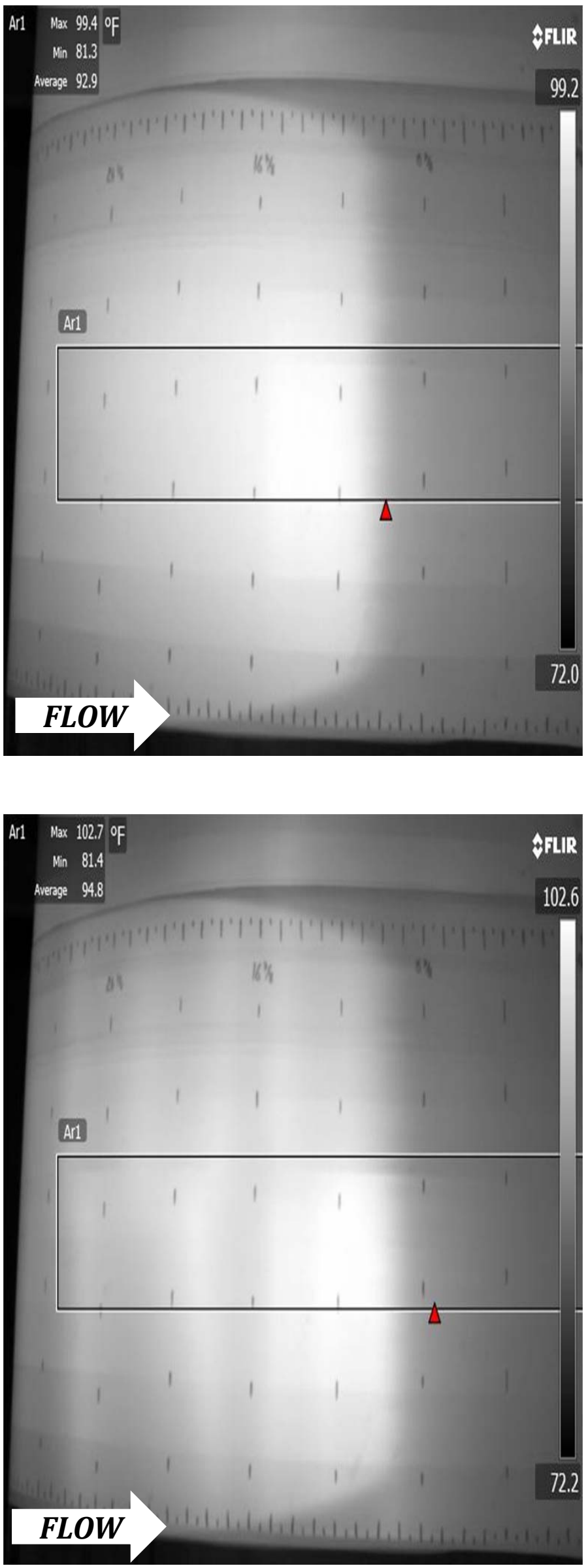


\section{8-m DU96-W-180 at $R e=2 \times 10^{5}$}

\section{PRESSURE SIDE}
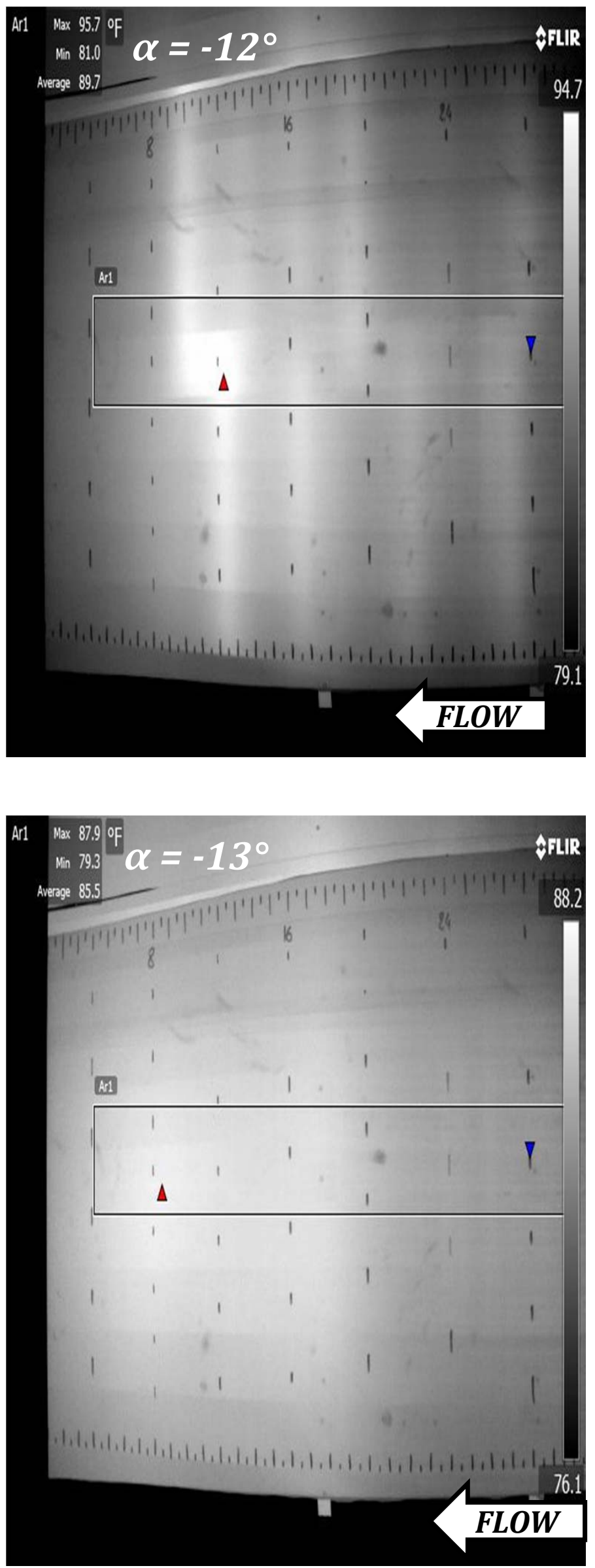

SUCTION SIDE
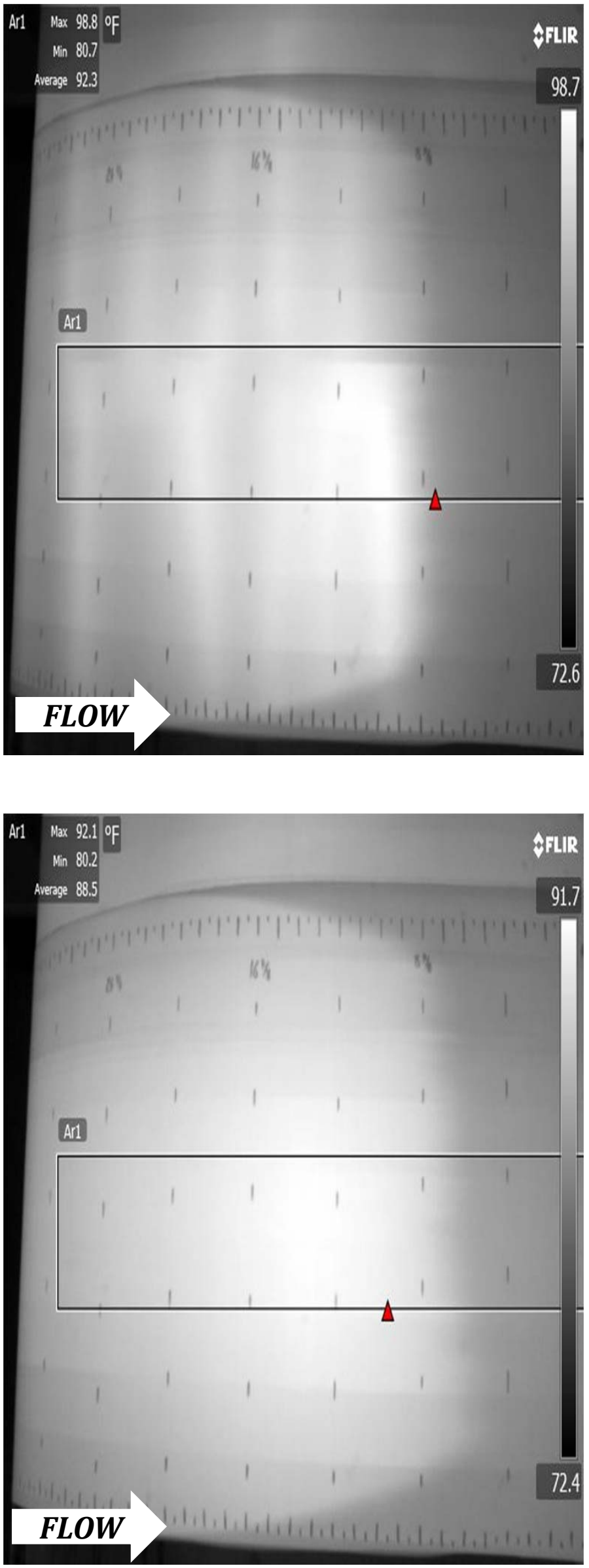


\section{8-m DU96-W-180 at $R e=2 \times 10^{5}$}

\section{PRESSURE SIDE}
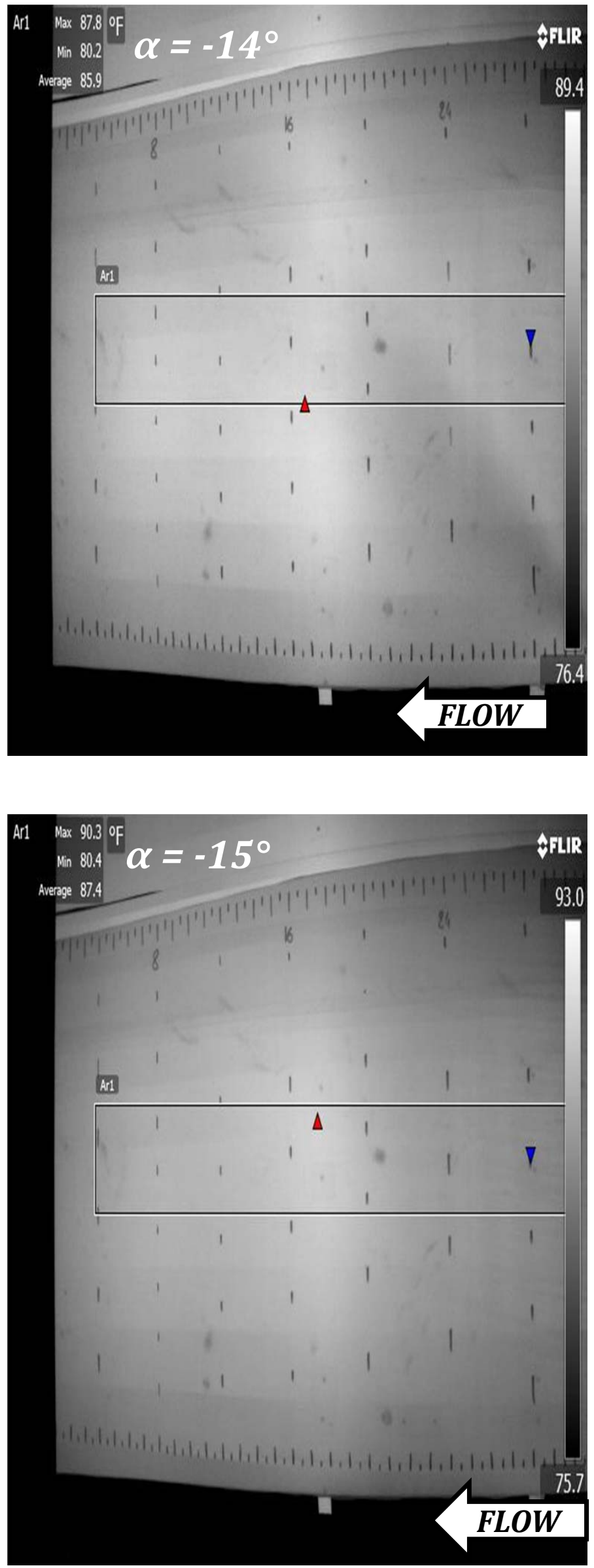

SUCTION SIDE
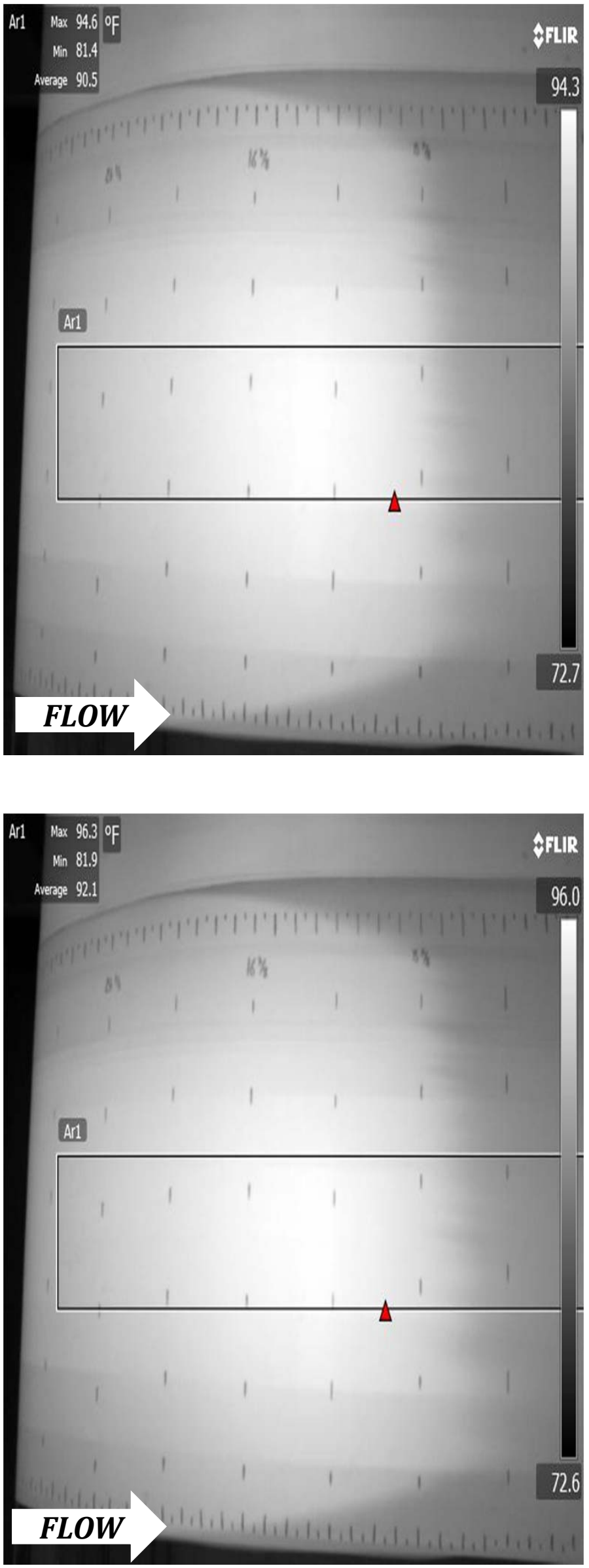


\section{8-m DU96-W-180 at $R e=2 \times 10^{5}$}

\section{PRESSURE SIDE}
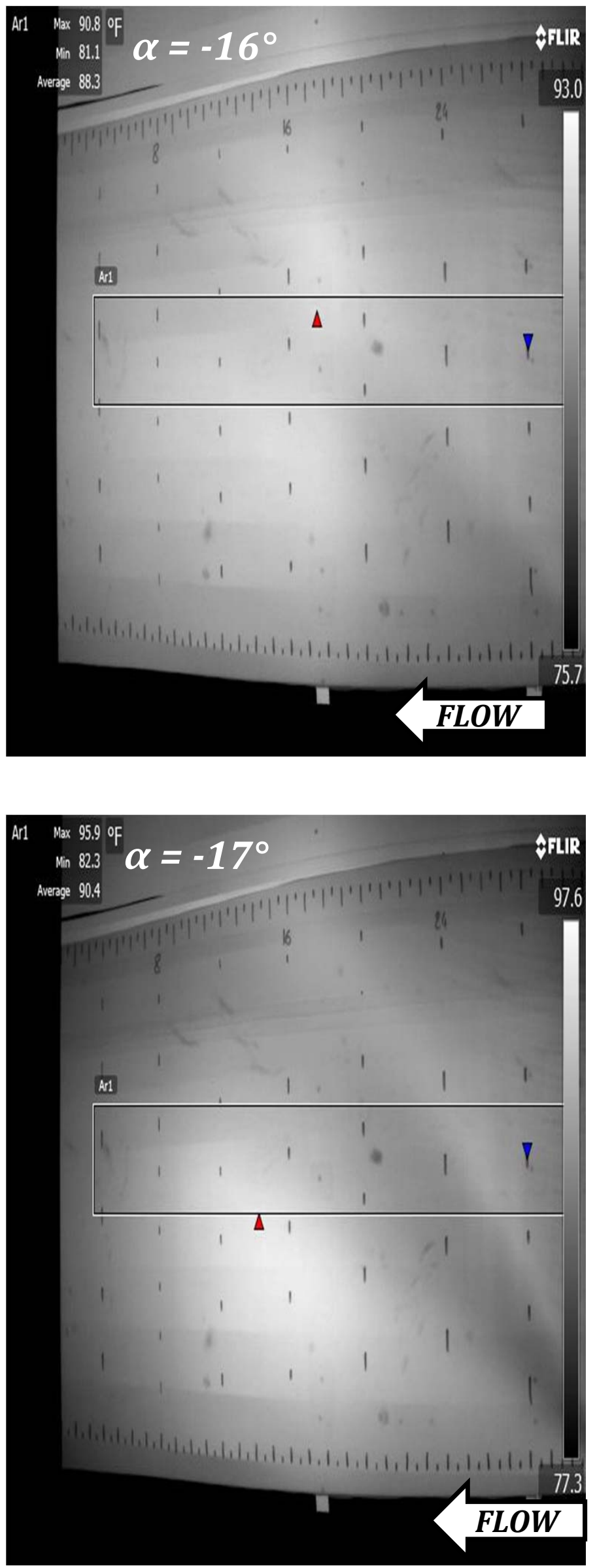

SUCTION SIDE
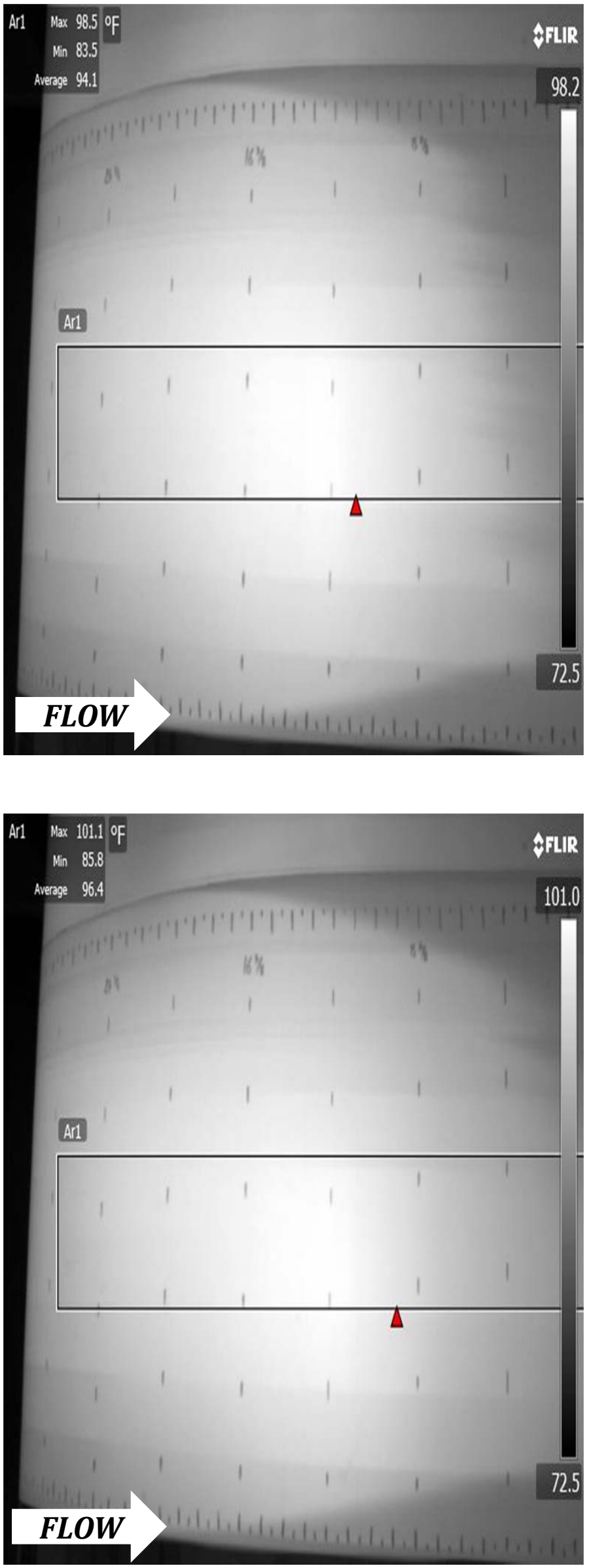


\section{8-m DU96-W-180 at $R e=2 \times 10^{5}$}

\section{PRESSURE SIDE}
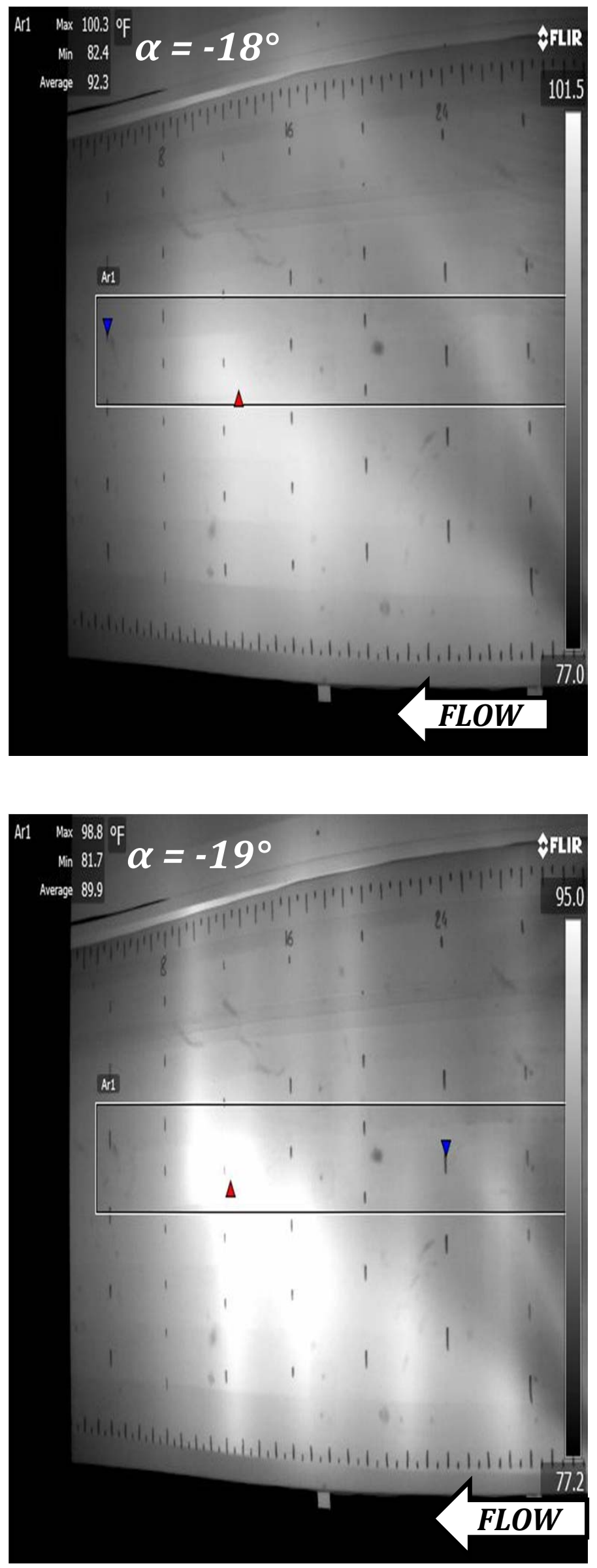

SUCTION SIDE
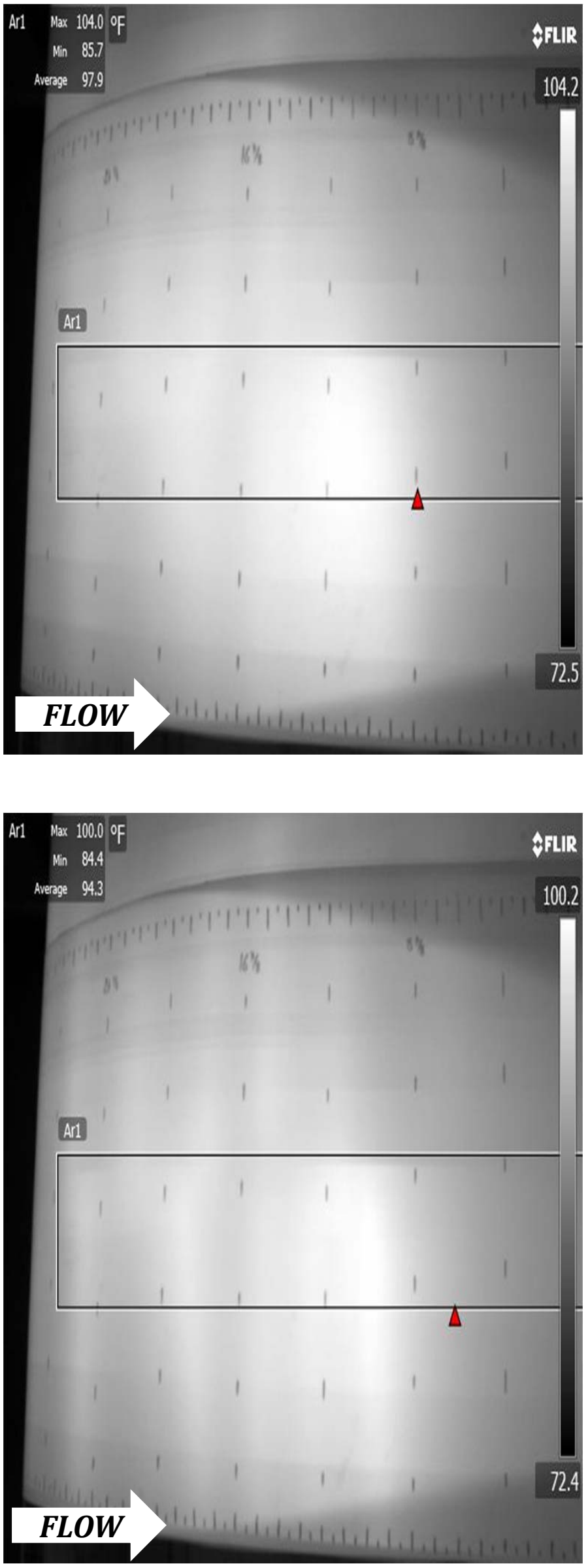


\section{8-m DU96-W-180 at $R e=2 \times 10^{5}$}

\section{PRESSURE SIDE}

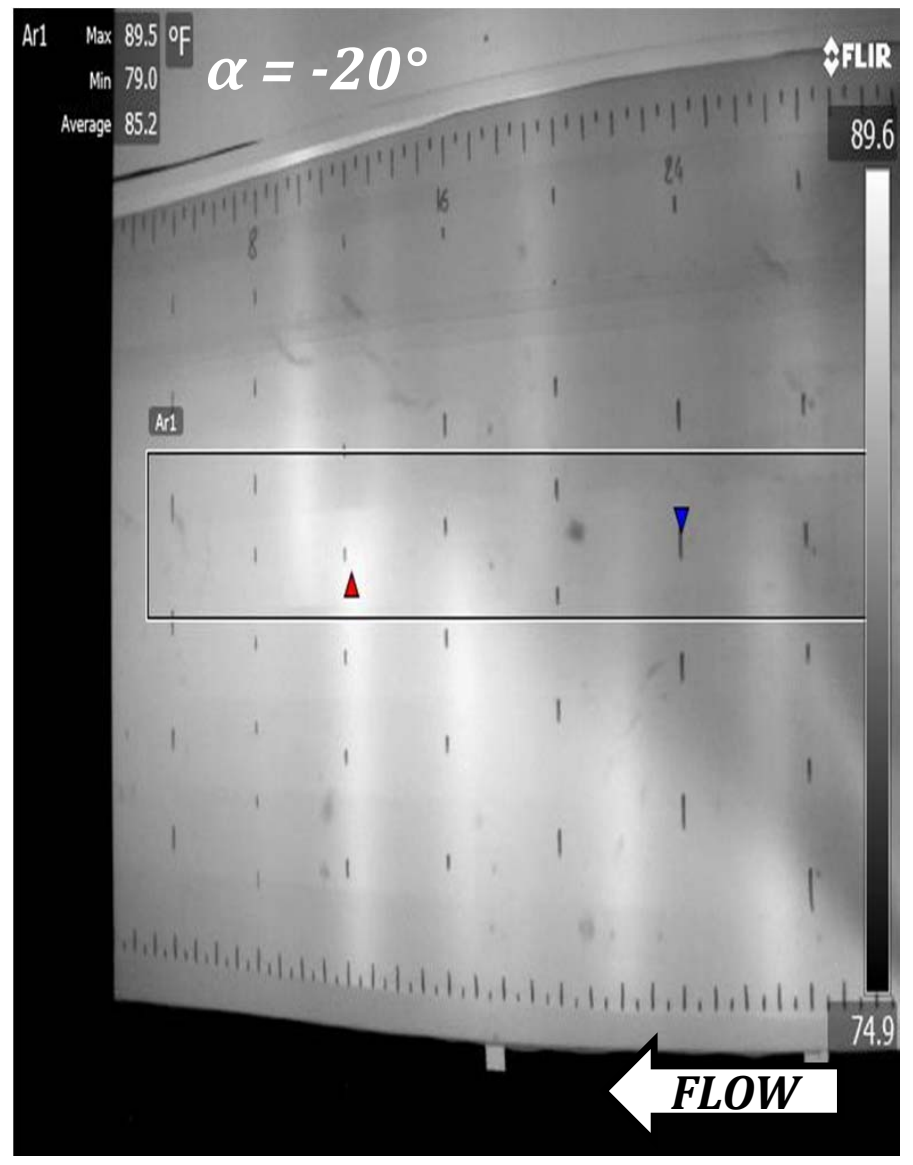

SUCTION SIDE

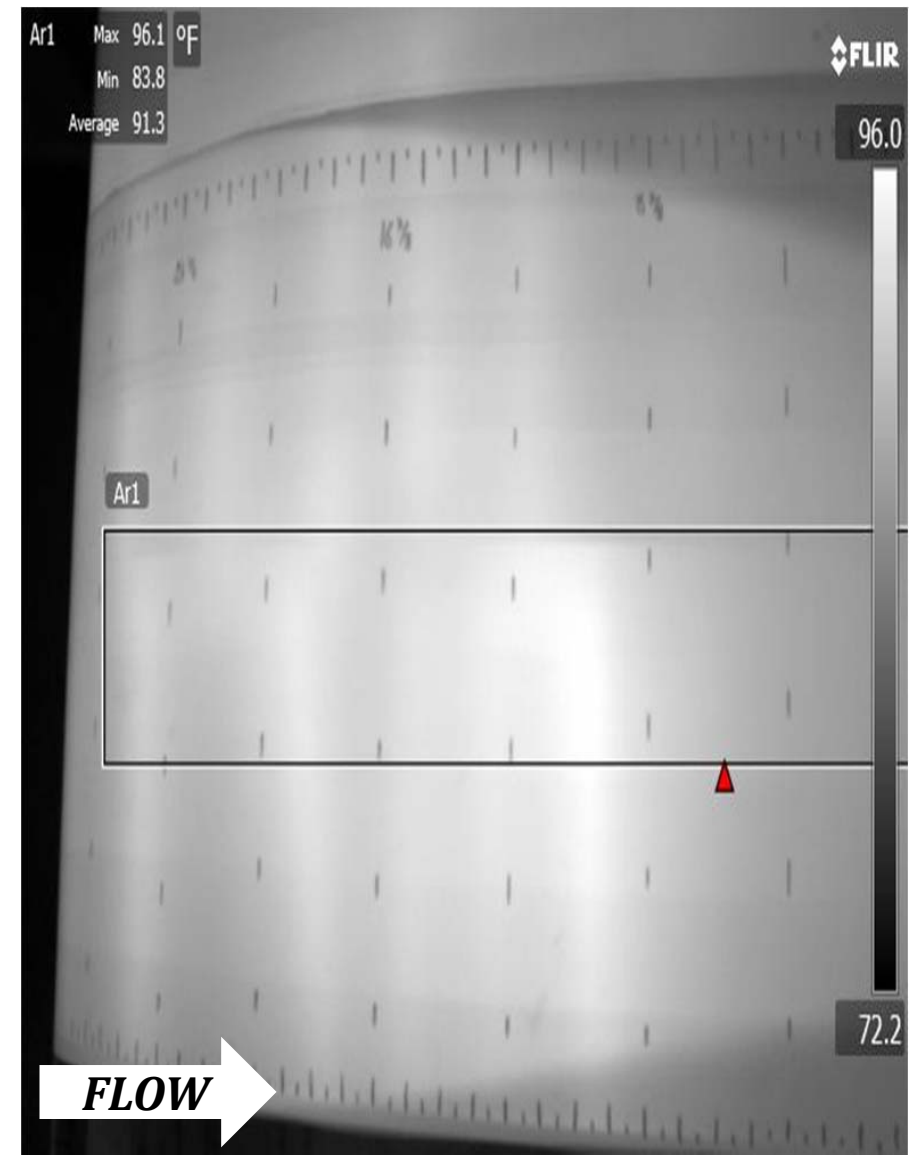


Appendix F Infrared Images of 0.8-m DU96-W-180 at $\mathrm{Re}=3 \times 10^{5}$

(From $\alpha=20^{\circ}$ to -20 , Pressure Side Images are on Left and Suction Side Images are on Right.) 


\section{8-m DU96-W-180 at $R e=3 \times 10^{5}$}

\section{PRESSURE SIDE}
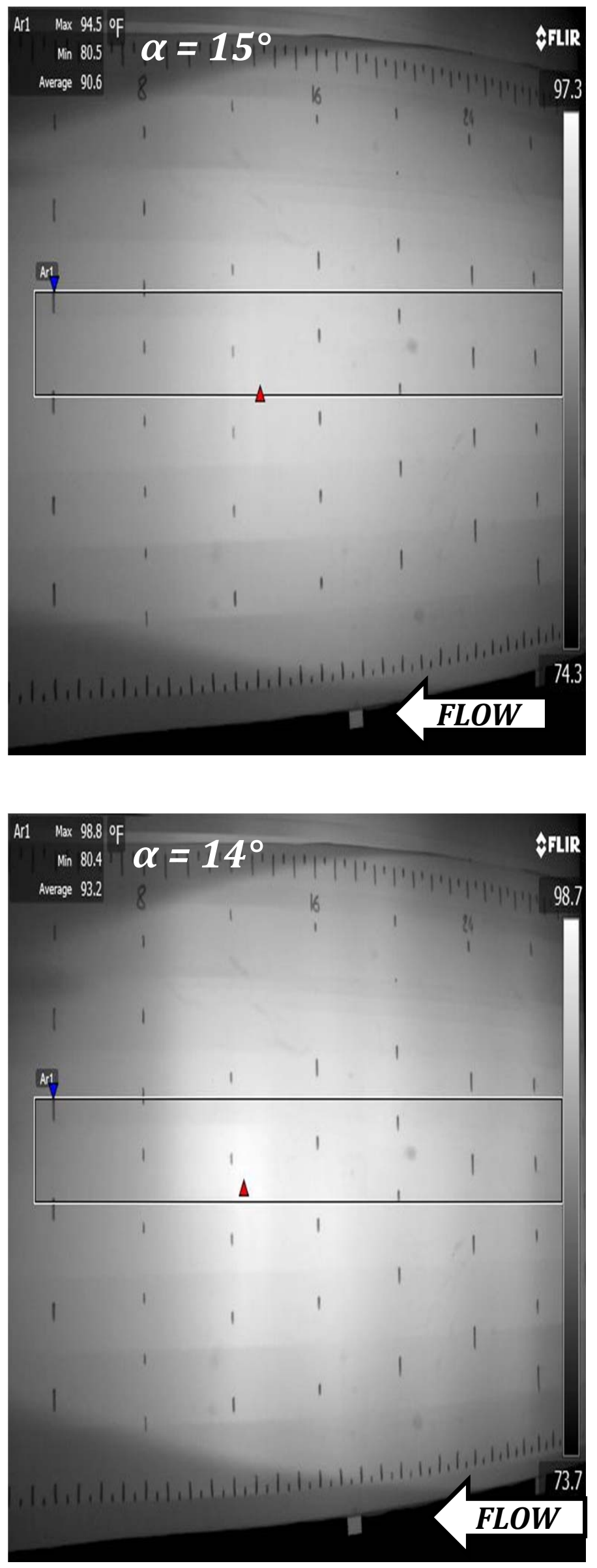

SUCTION SIDE
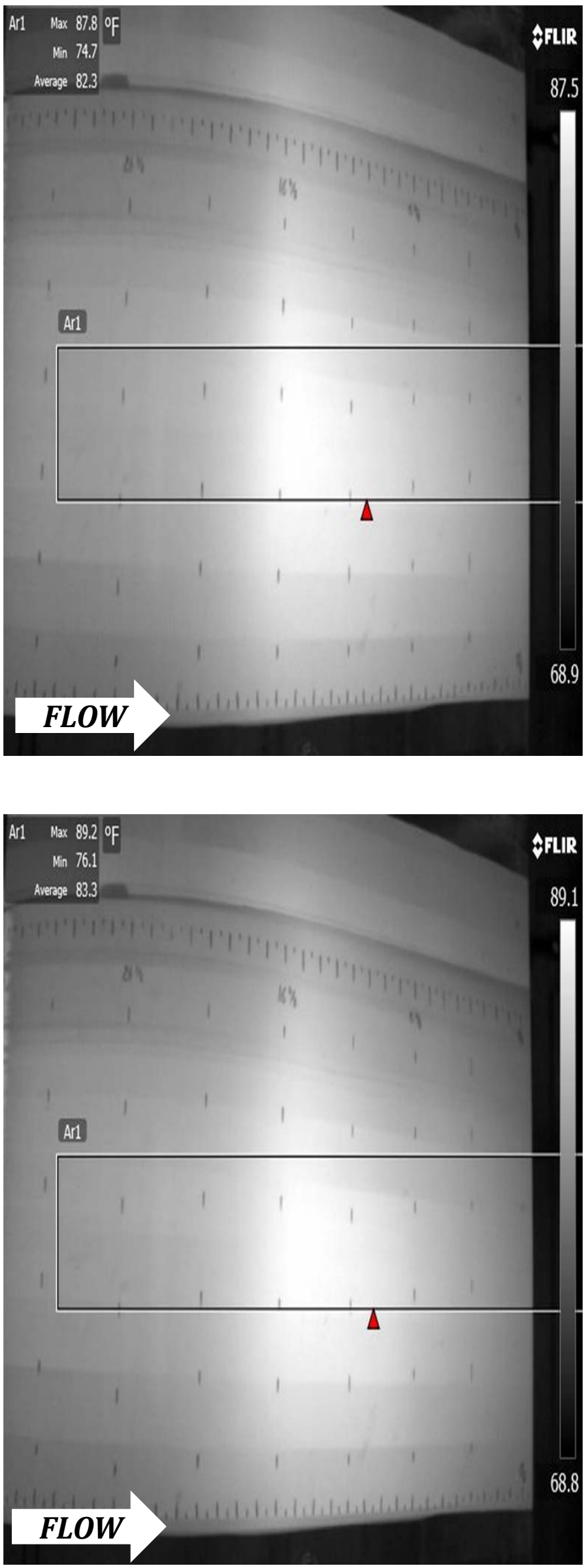


\section{8-m DU96-W-180 at $R e=3 \times 10^{5}$}

\section{PRESSURE SIDE}
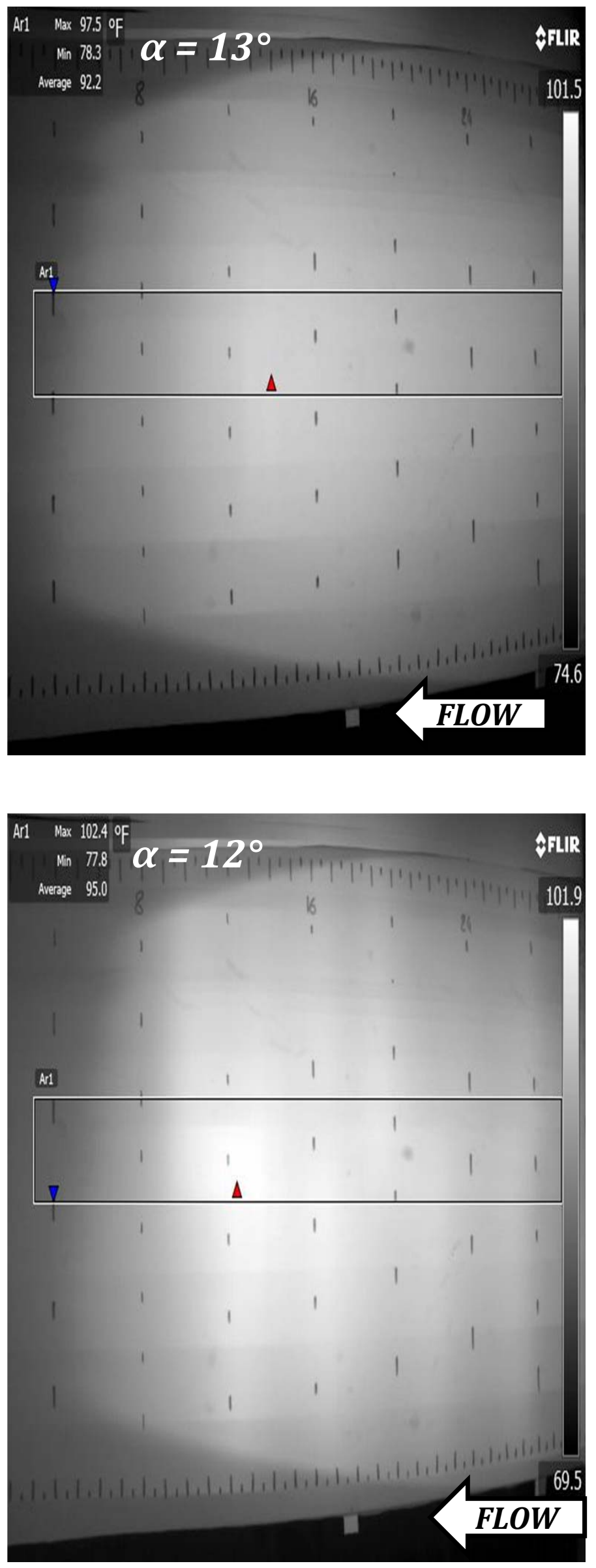

SUCTION SIDE
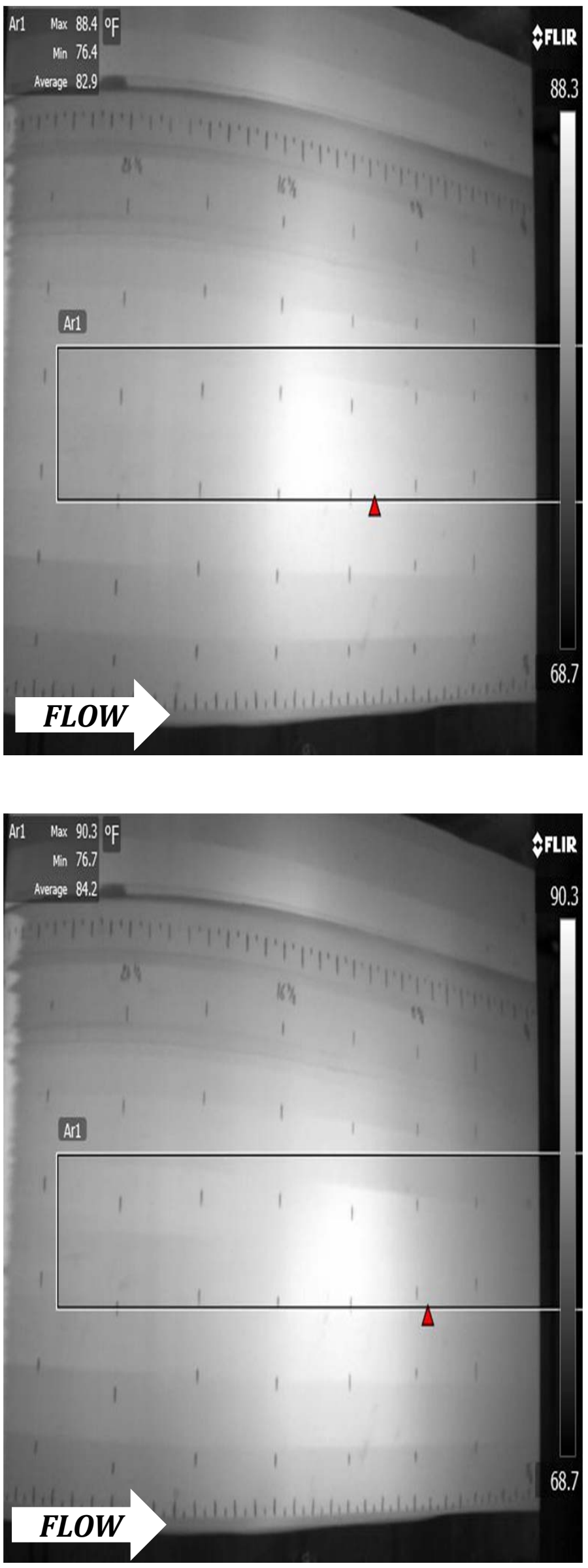


\section{8-m DU96-W-180 at $R e=3 \times 10^{5}$}

\section{PRESSURE SIDE}
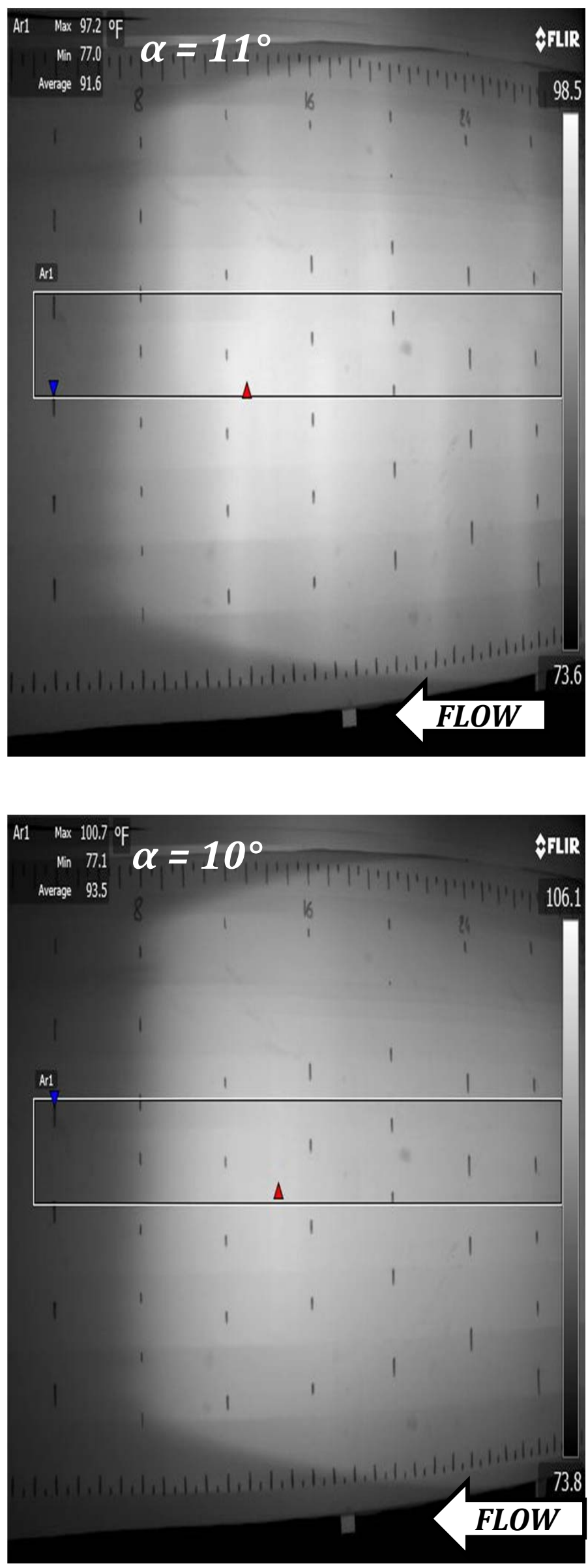

SUCTION SIDE
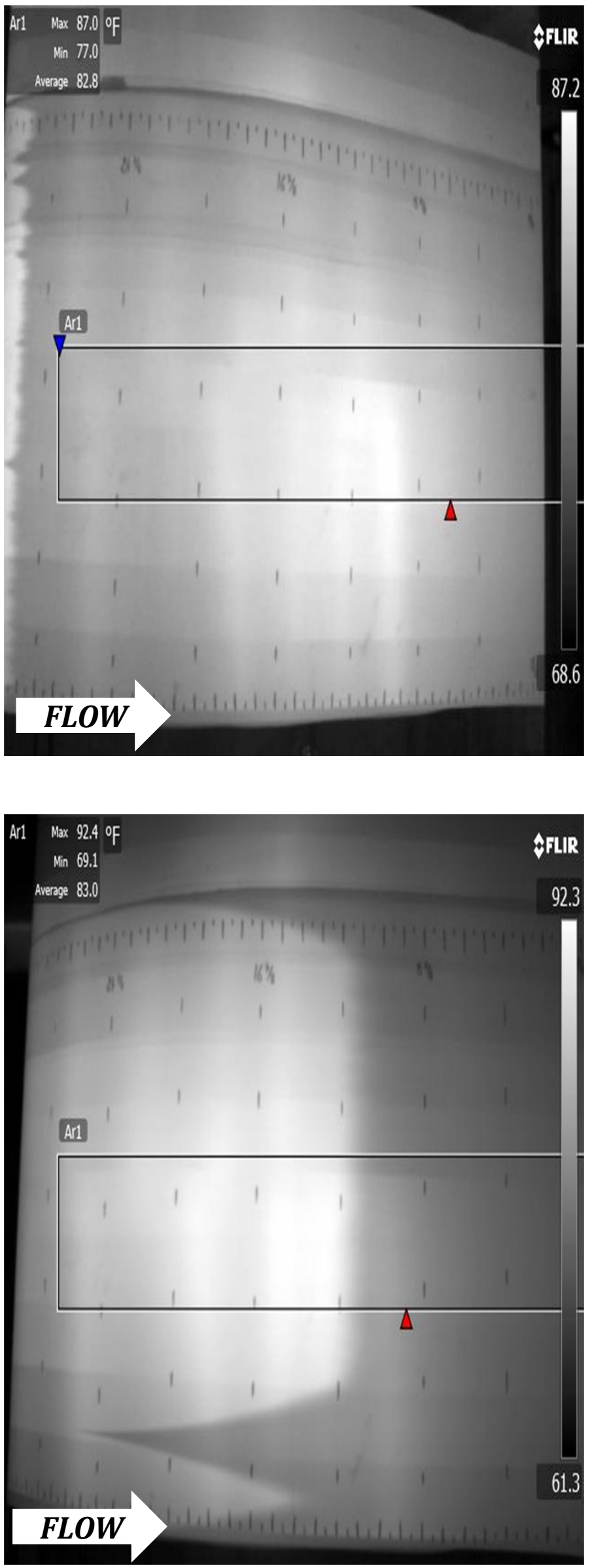


\section{8-m DU96-W-180 at $R e=3 \times 10^{5}$}

\section{PRESSURE SIDE}
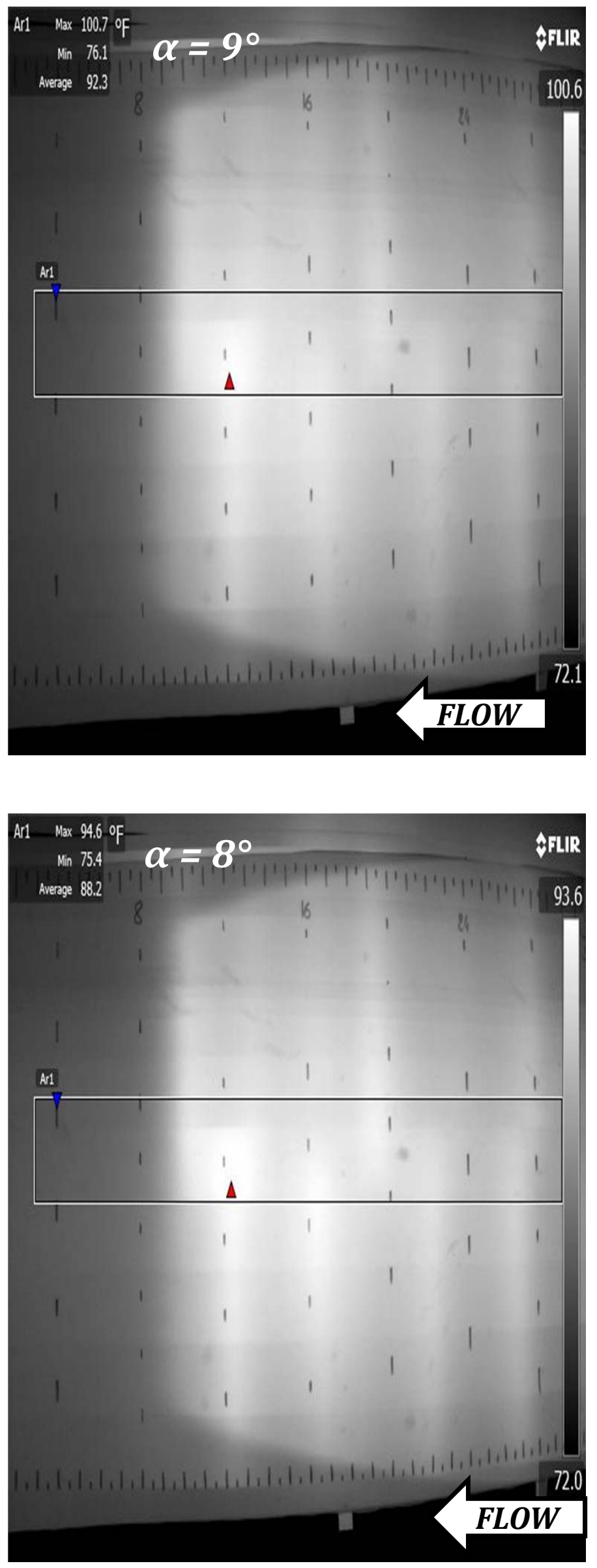

SUCTION SIDE
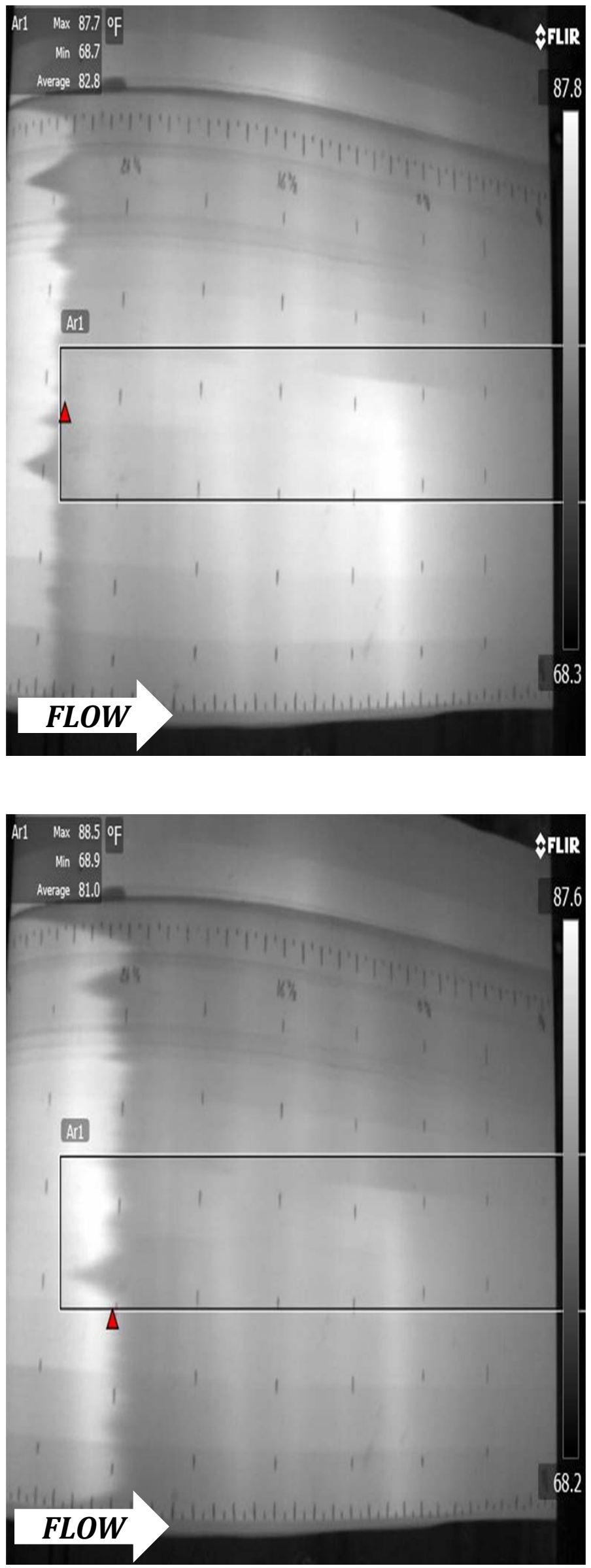


\section{8-m DU96-W-180 at $R e=3 \times 10^{5}$}

\section{PRESSURE SIDE}

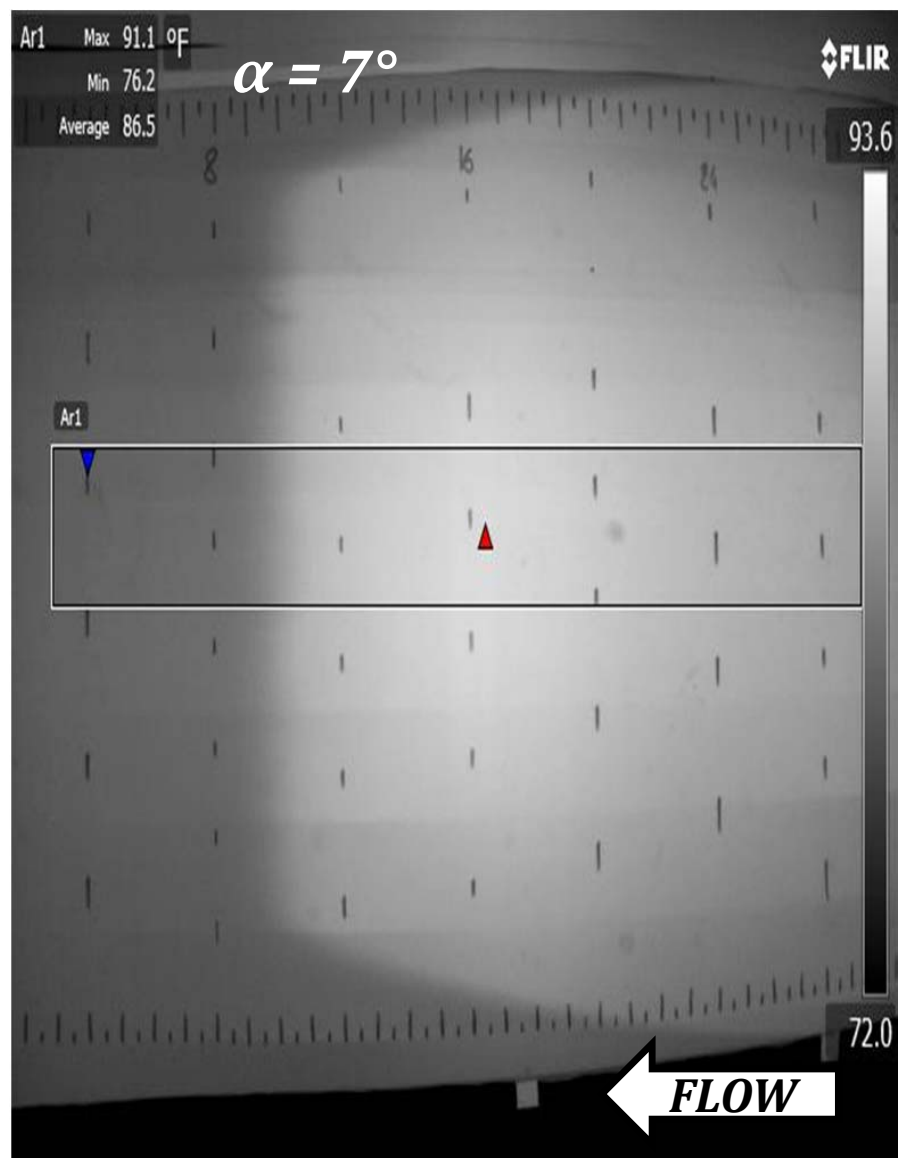

Ar1 $\operatorname{Max}_{\operatorname{Mn}} 73.8$ of $-\alpha=6^{\circ}$

AFIIR

Averge 88.1

Ar1

$\Delta$

\section{SUCTION SIDE}
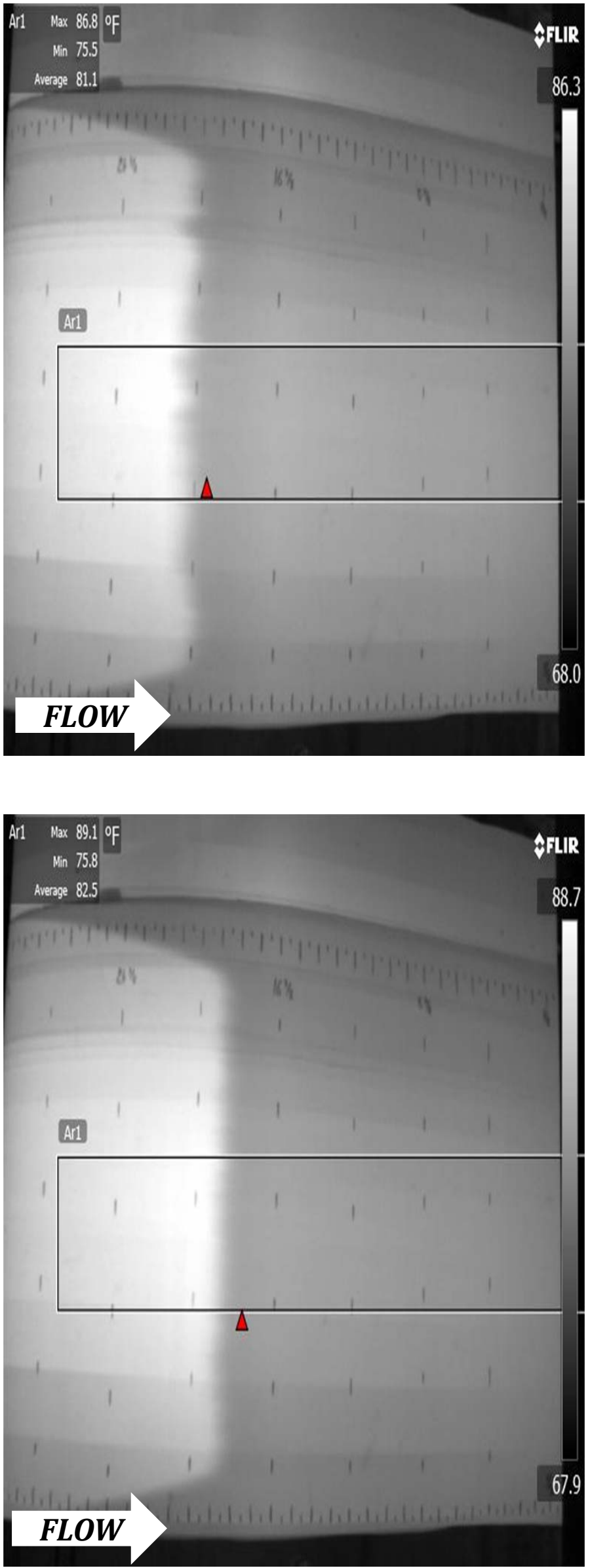


\section{8-m DU96-W-180 at $R e=3 \times 10^{5}$}

\section{PRESSURE SIDE}
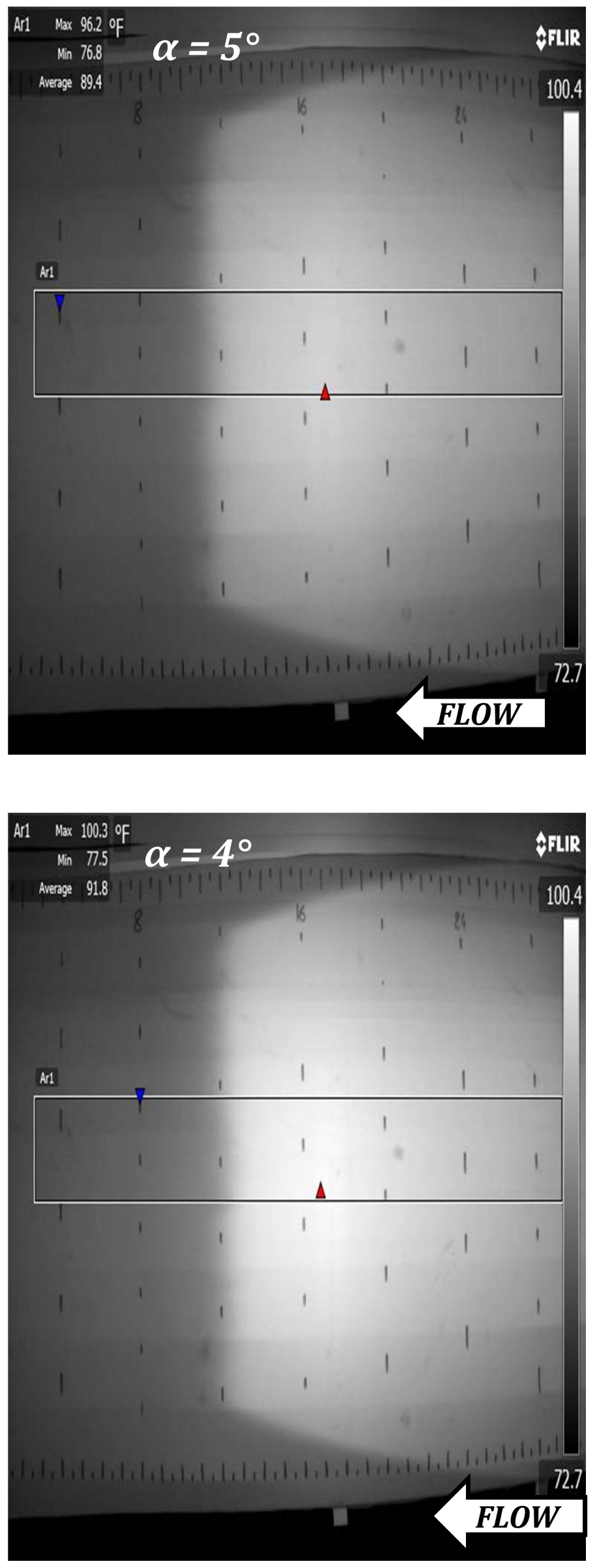

SUCTION SIDE
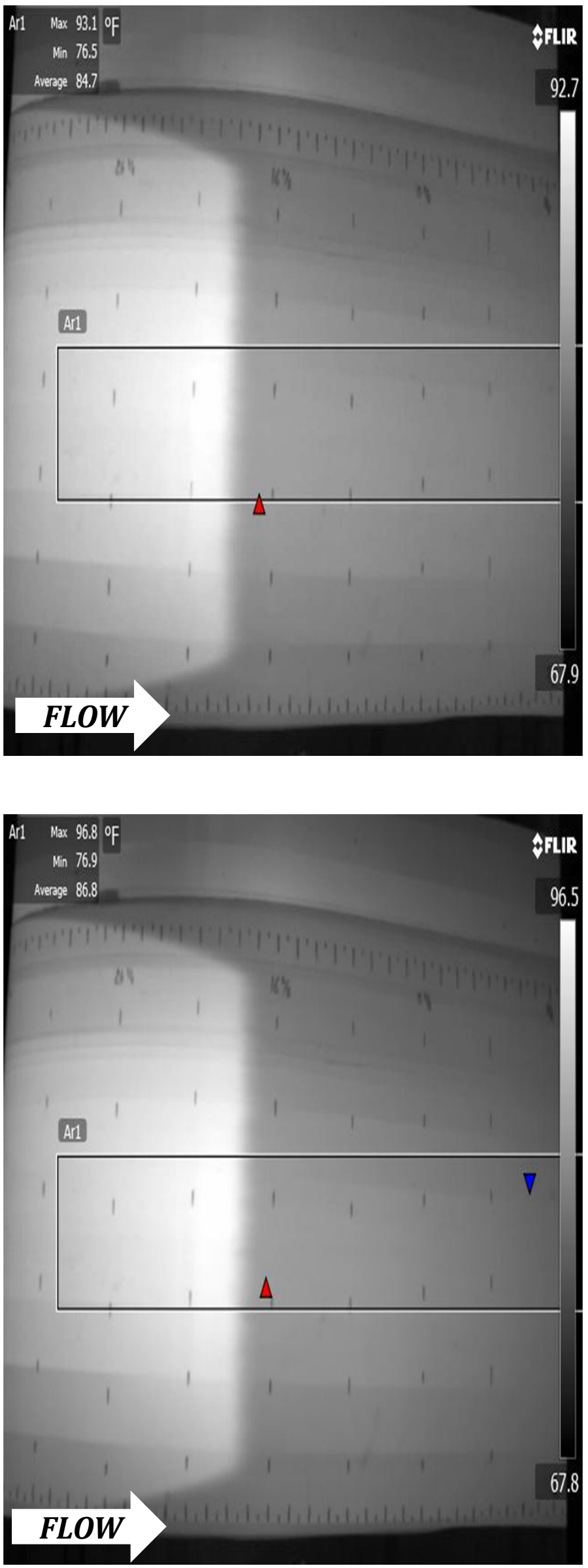


\section{8-m DU96-W-180 at $R e=3 \times 10^{5}$}

\section{PRESSURE SIDE}
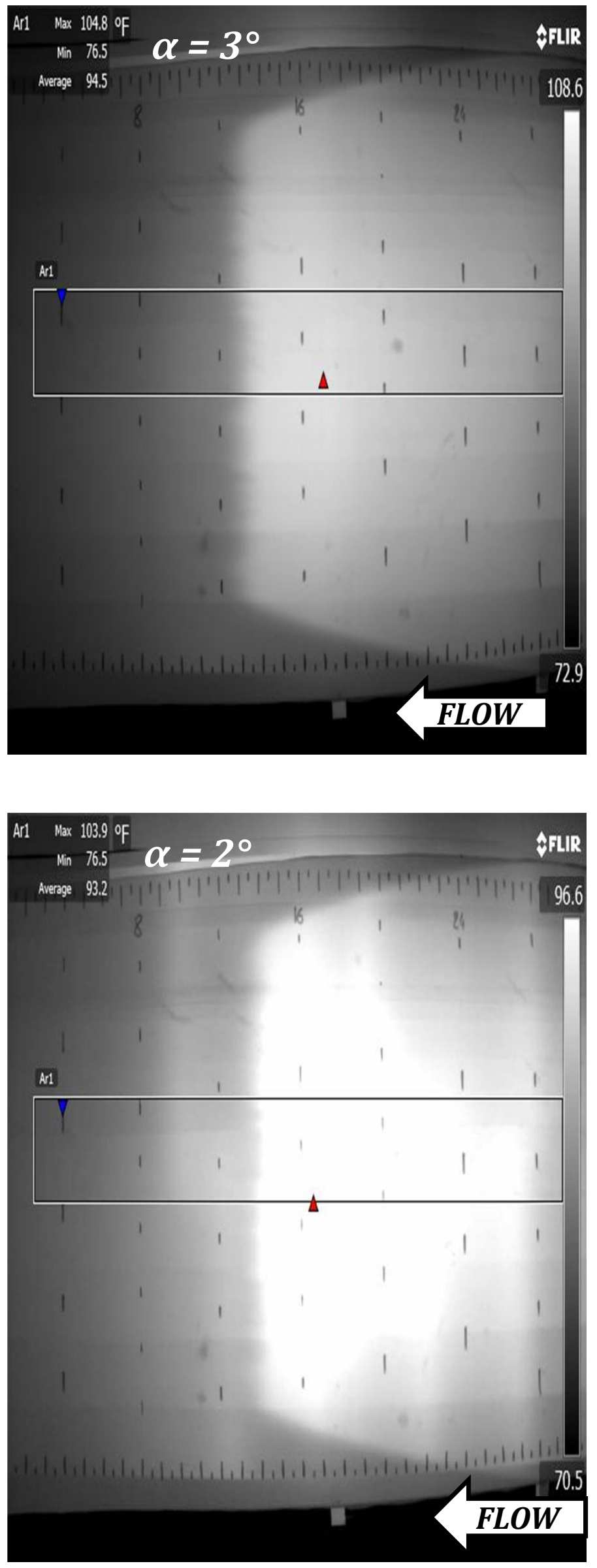

SUCTION SIDE
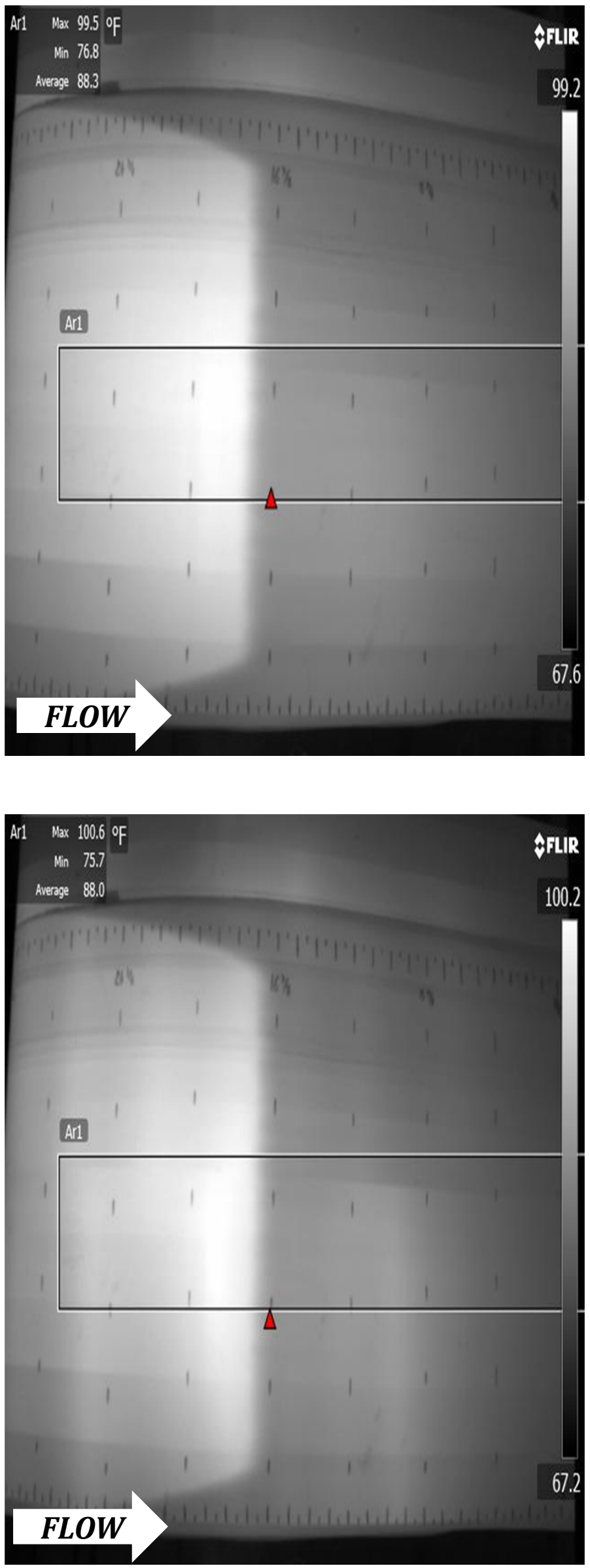


\section{8-m DU96-W-180 at $R e=3 \times 10^{5}$}

\section{PRESSURE SIDE}
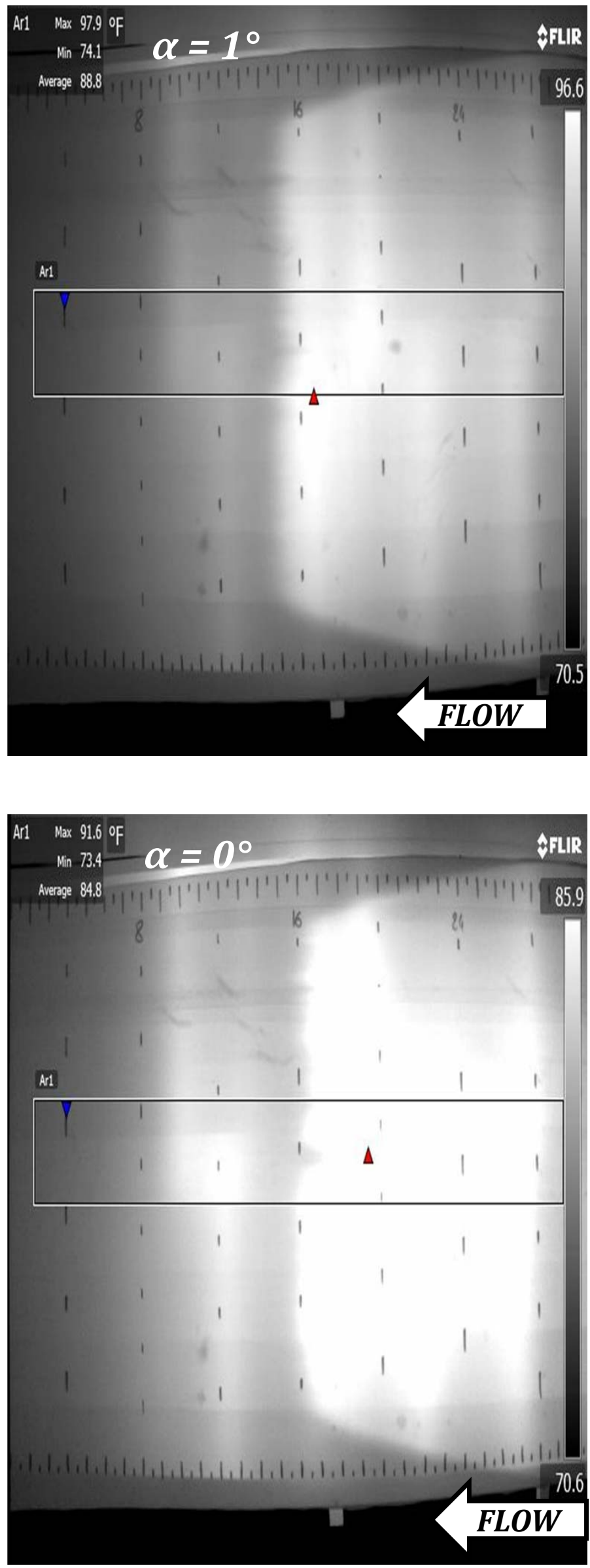

SUCTION SIDE
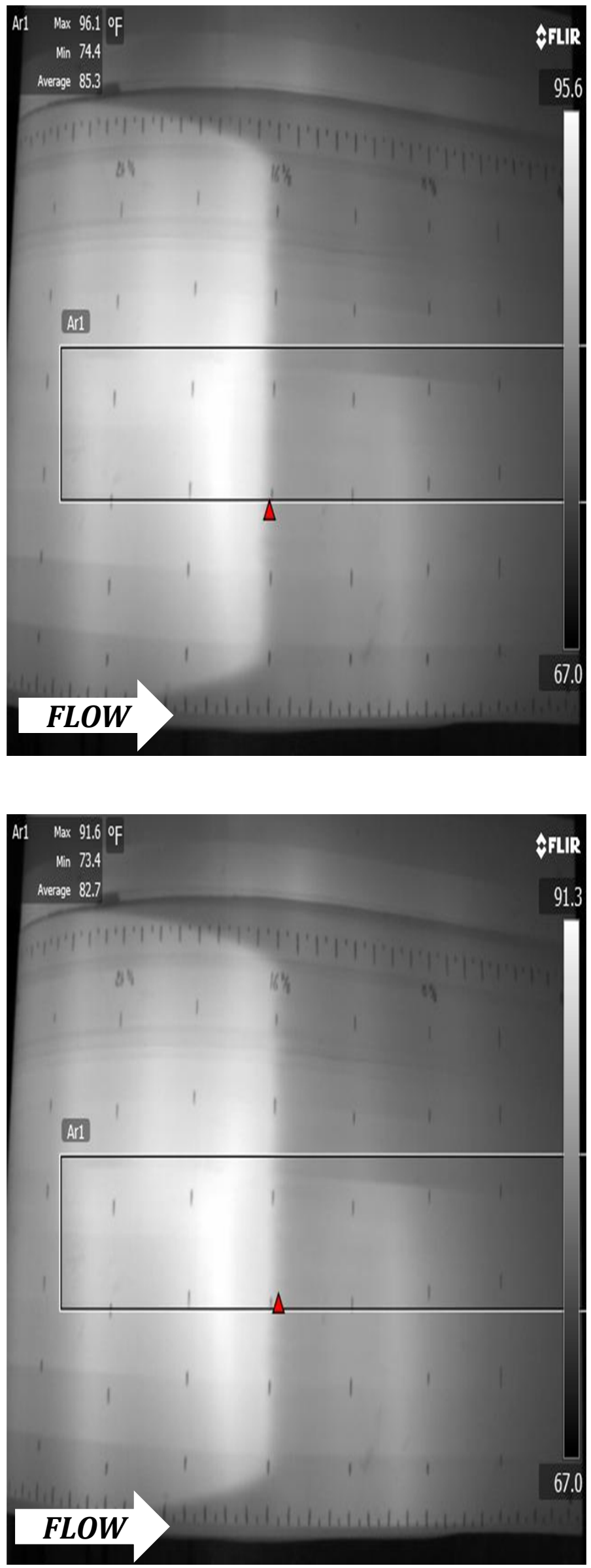


\section{8-m DU96-W-180 at $R e=3 \times 10^{5}$}

\section{PRESSURE SIDE}
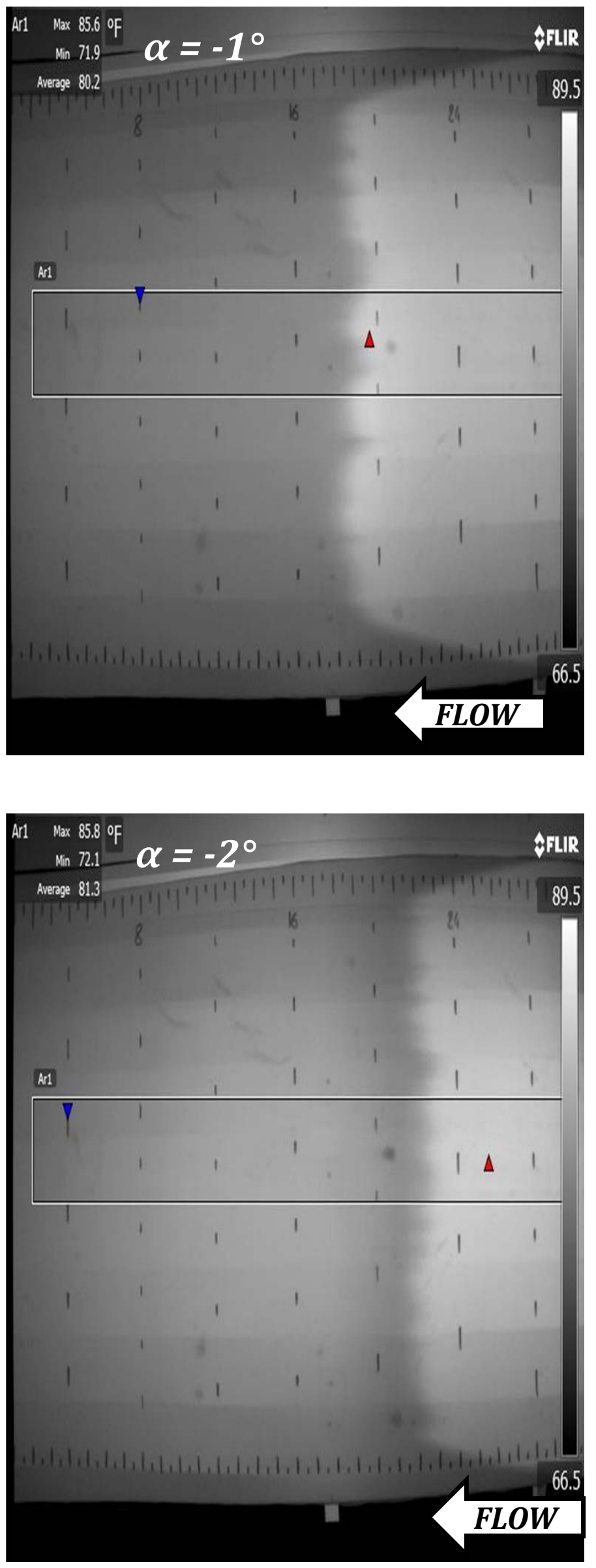

SUCTION SIDE
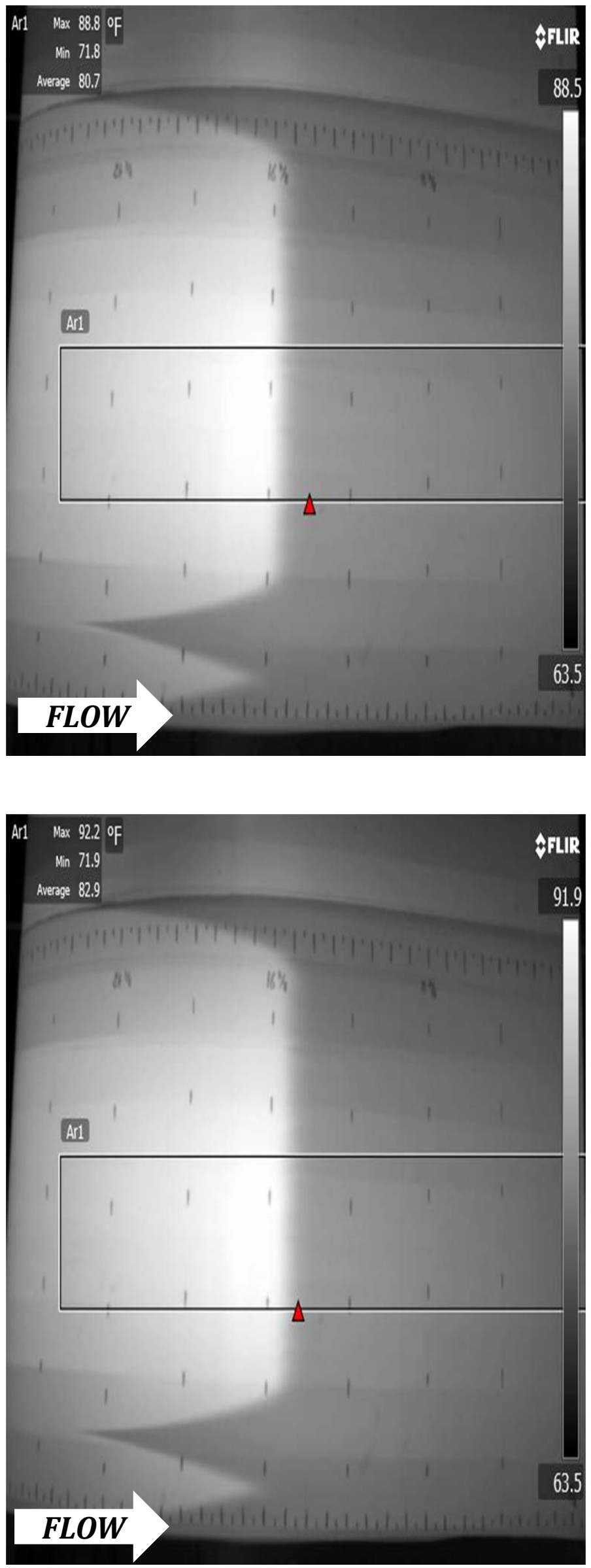


\section{8-m DU96-W-180 at $R e=3 \times 10^{5}$}

\section{PRESSURE SIDE}
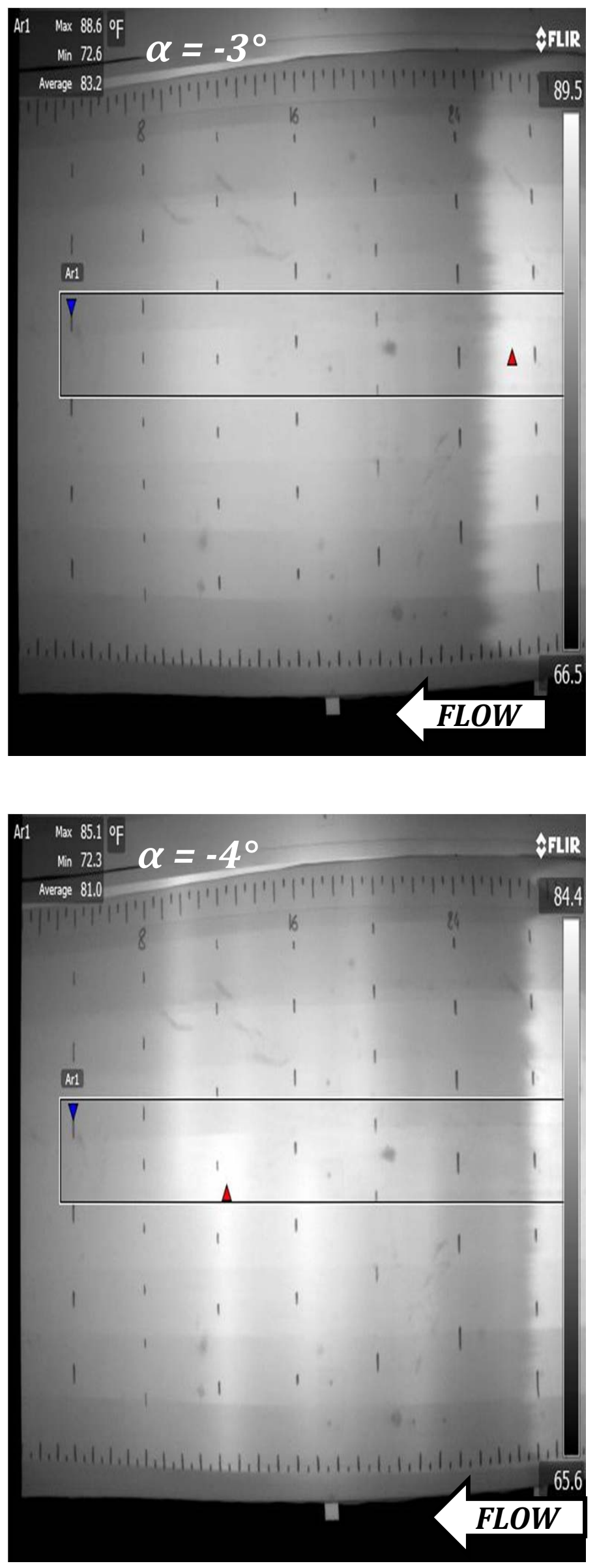

SUCTION SIDE
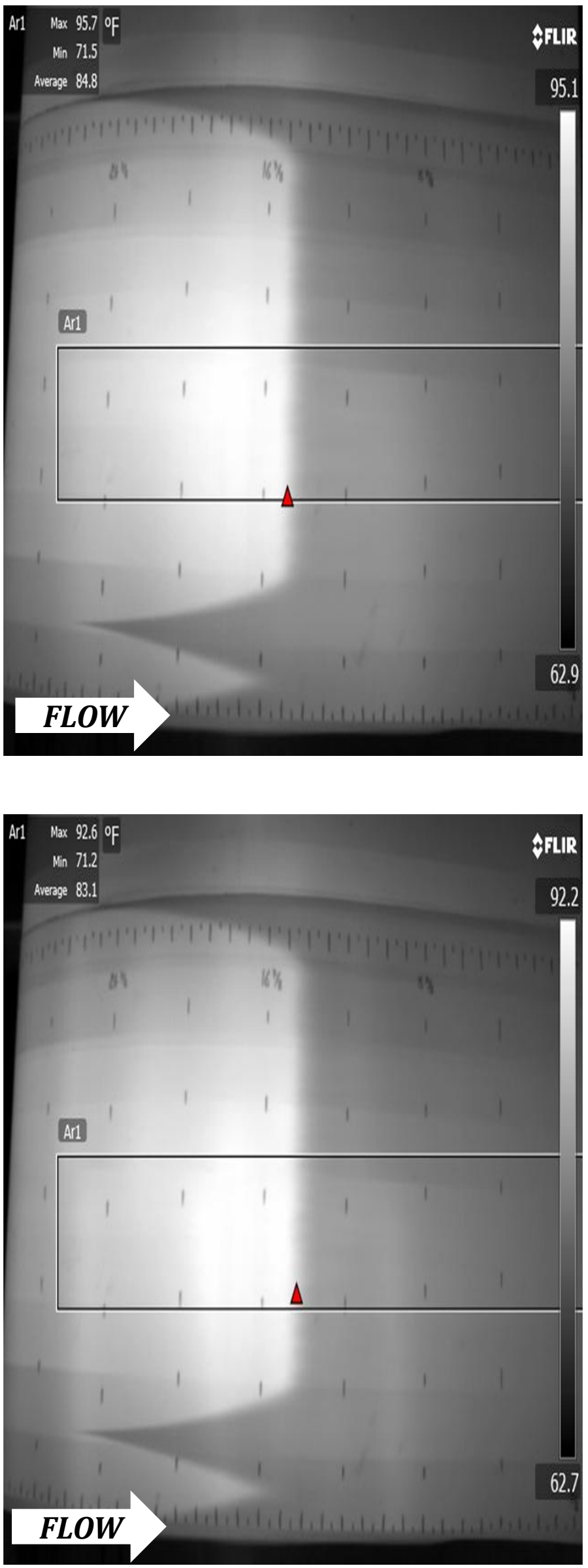


\section{8-m DU96-W-180 at $R e=3 \times 10^{5}$}

\section{PRESSURE SIDE}
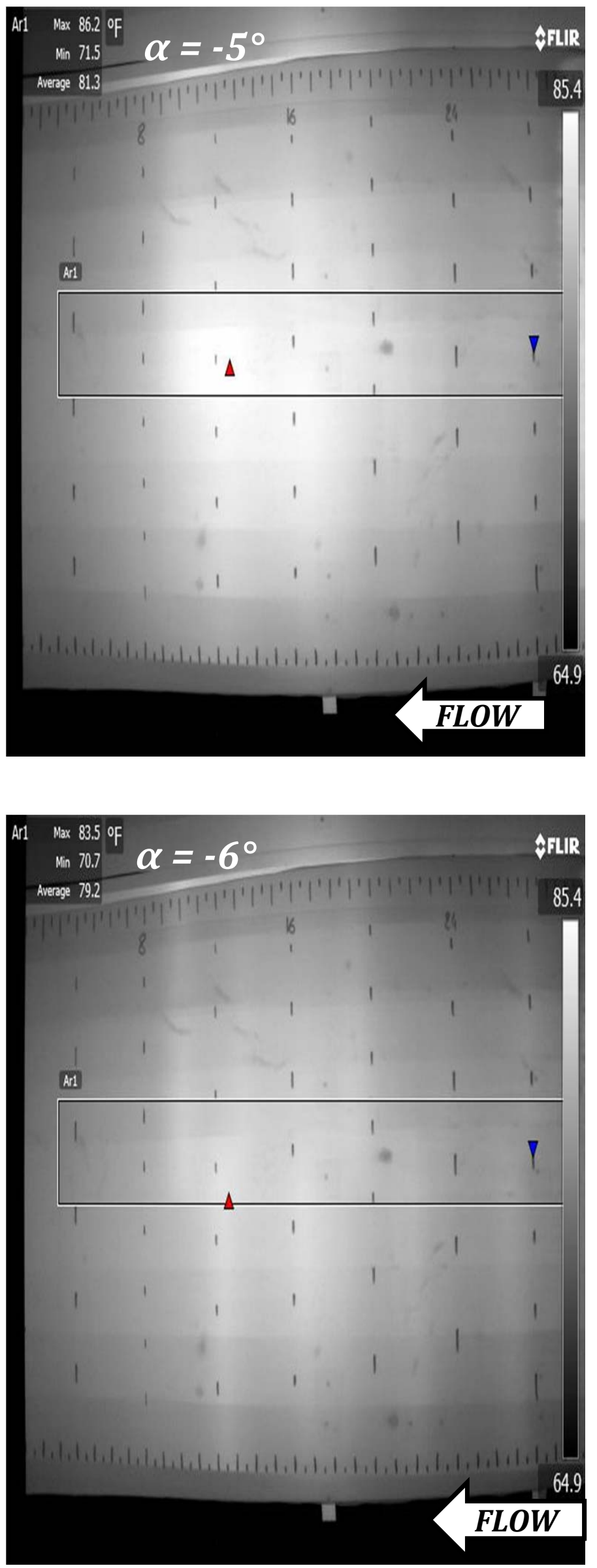

SUCTION SIDE
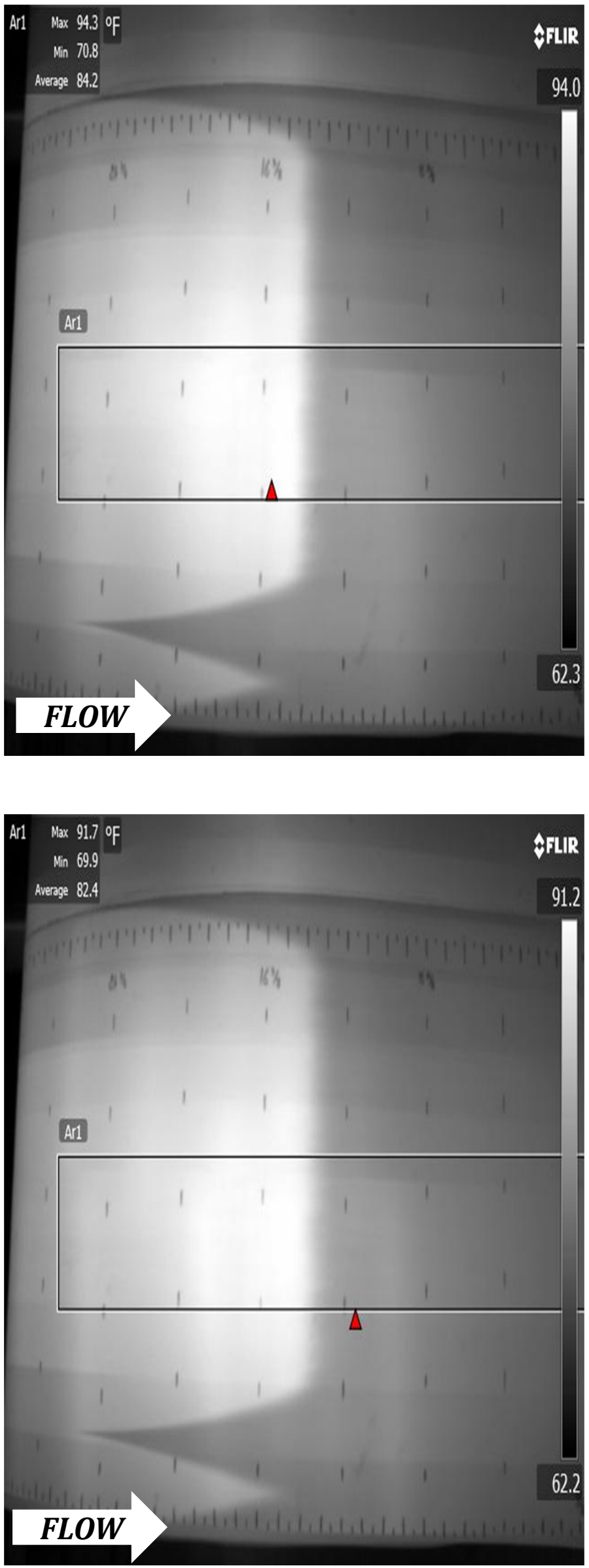


\section{8-m DU96-W-180 at $R e=3 \times 10^{5}$}

\section{PRESSURE SIDE}
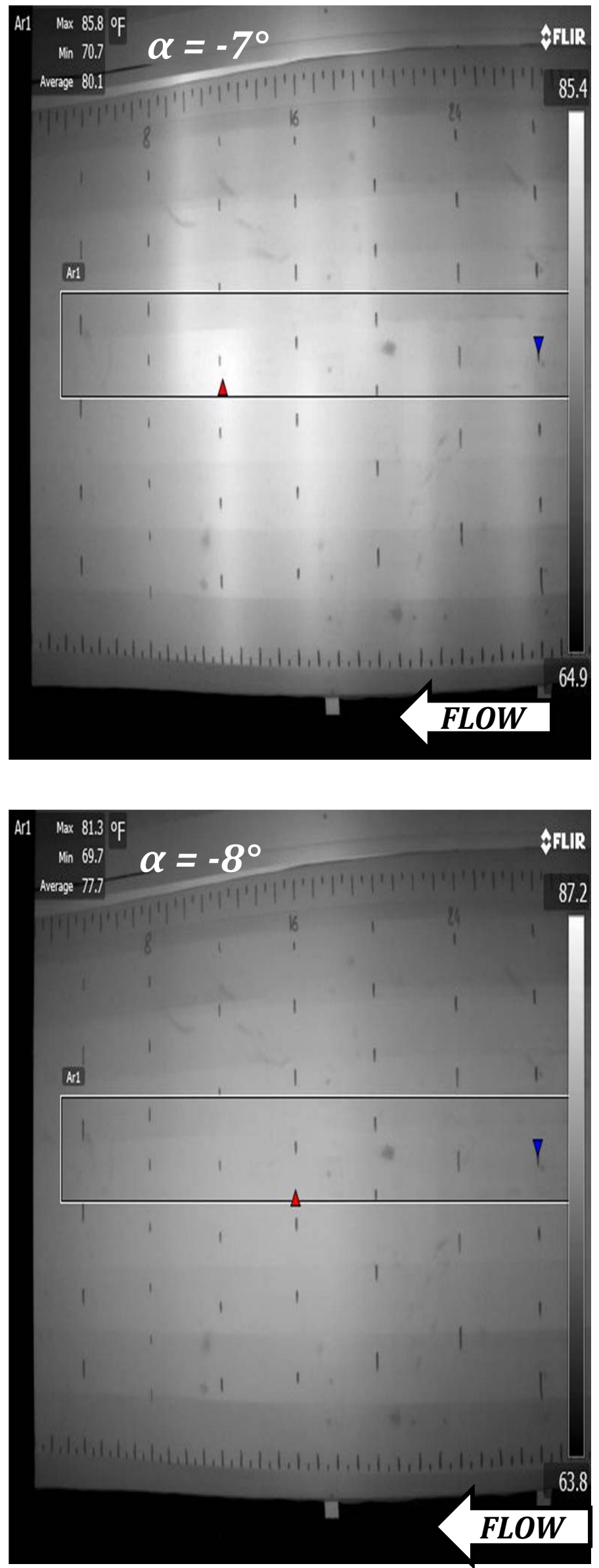

SUCTION SIDE
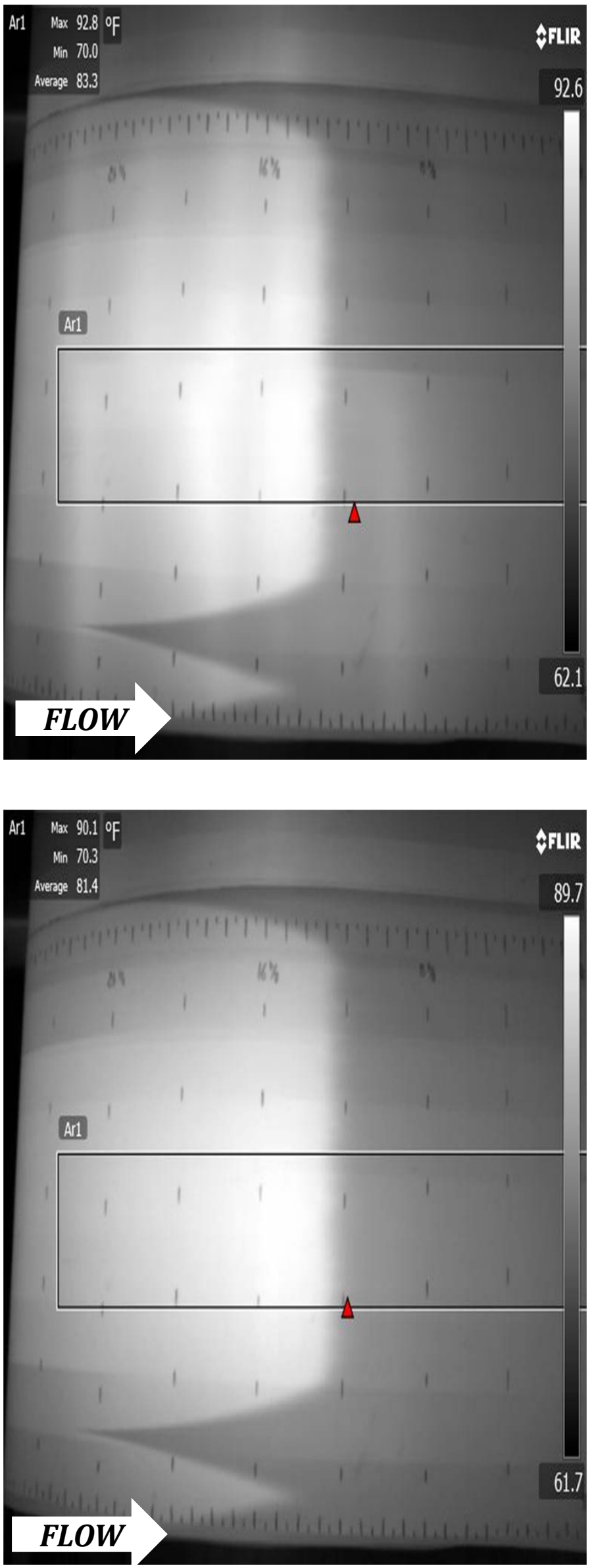


\section{8-m DU96-W-180 at $R e=3 \times 10^{5}$}

\section{PRESSURE SIDE}
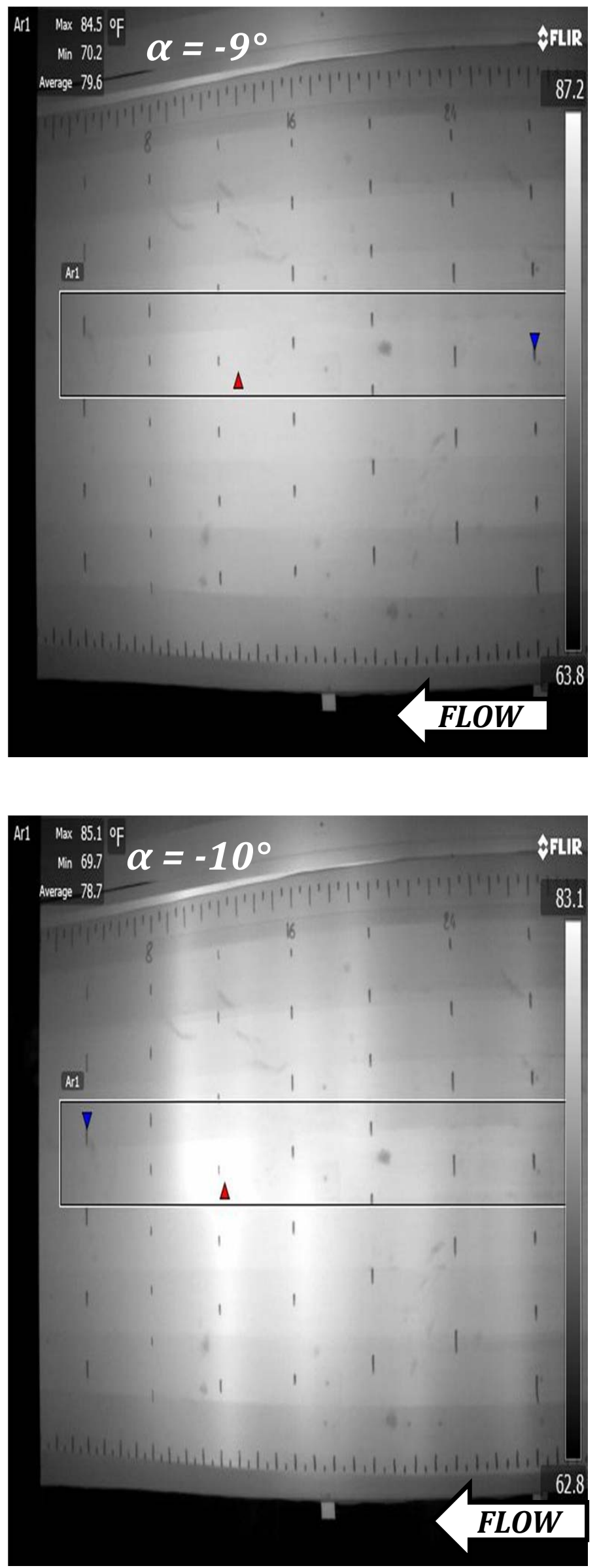

SUCTION SIDE
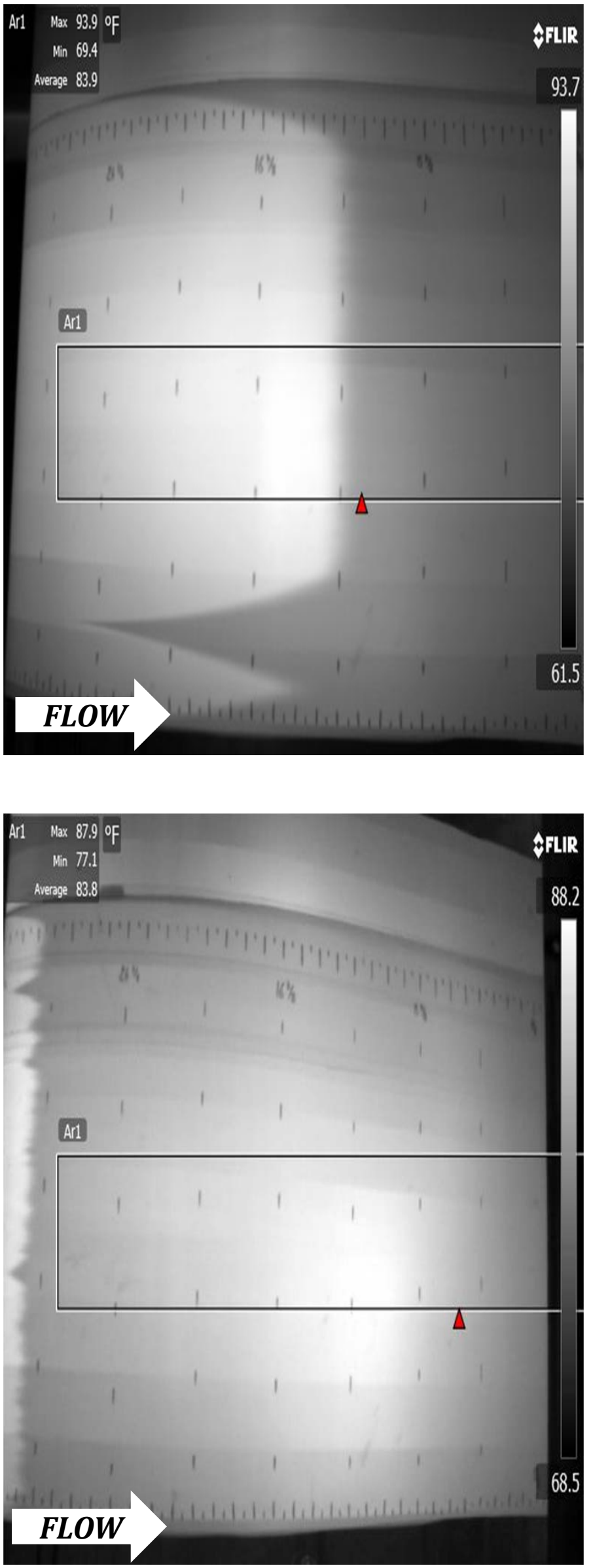


\section{8-m DU96-W-180 at $R e=3 \times 10^{5}$}

\section{PRESSURE SIDE}

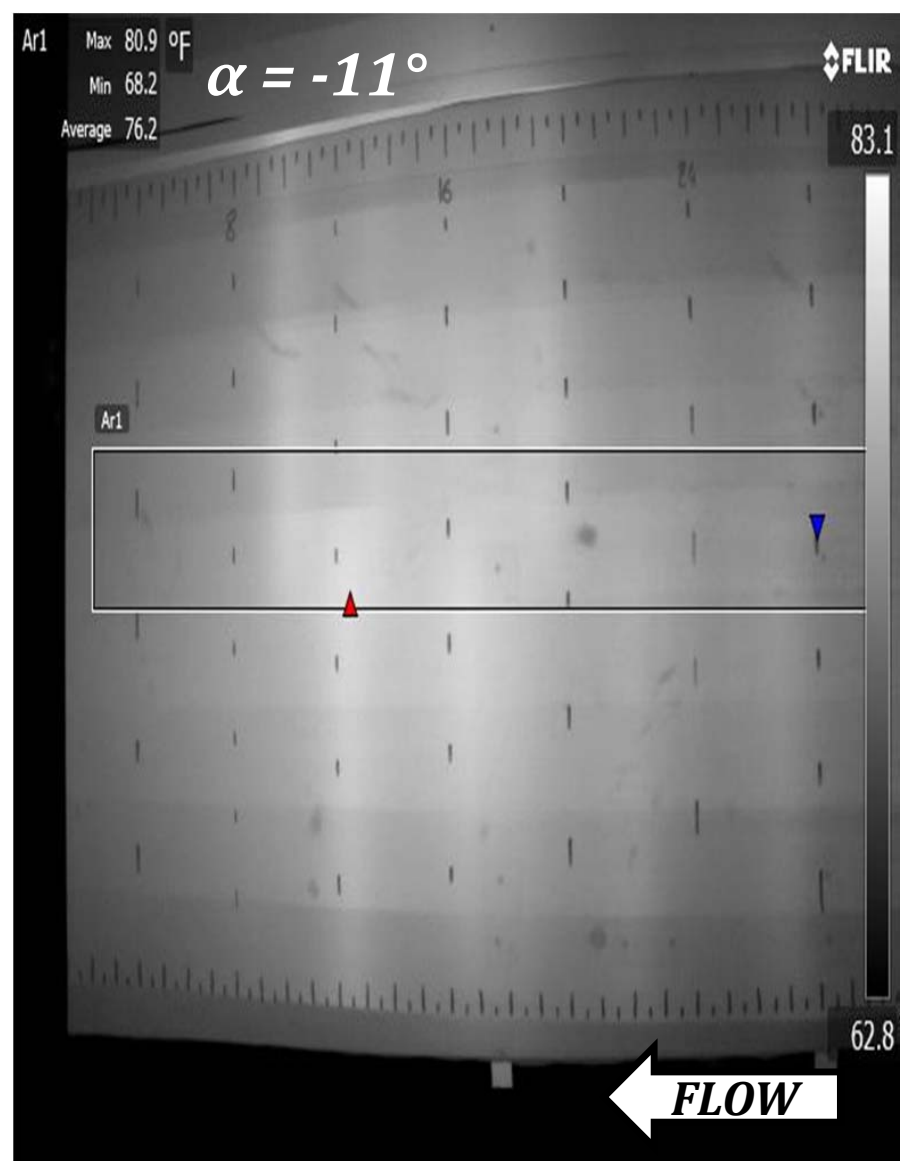

Ar1 Max 81.8 of

Max 81.8 of $\alpha=-122^{\circ}$
Averge 76.9

Ar1

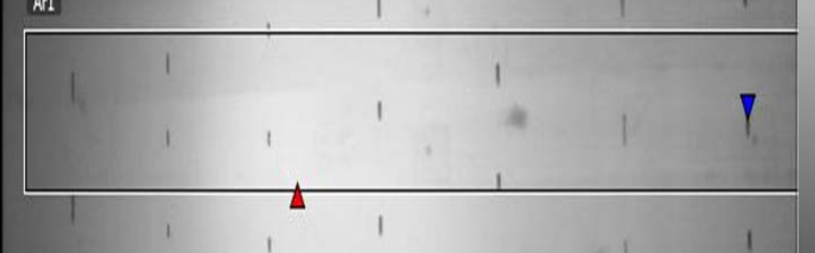

SUCTION SIDE
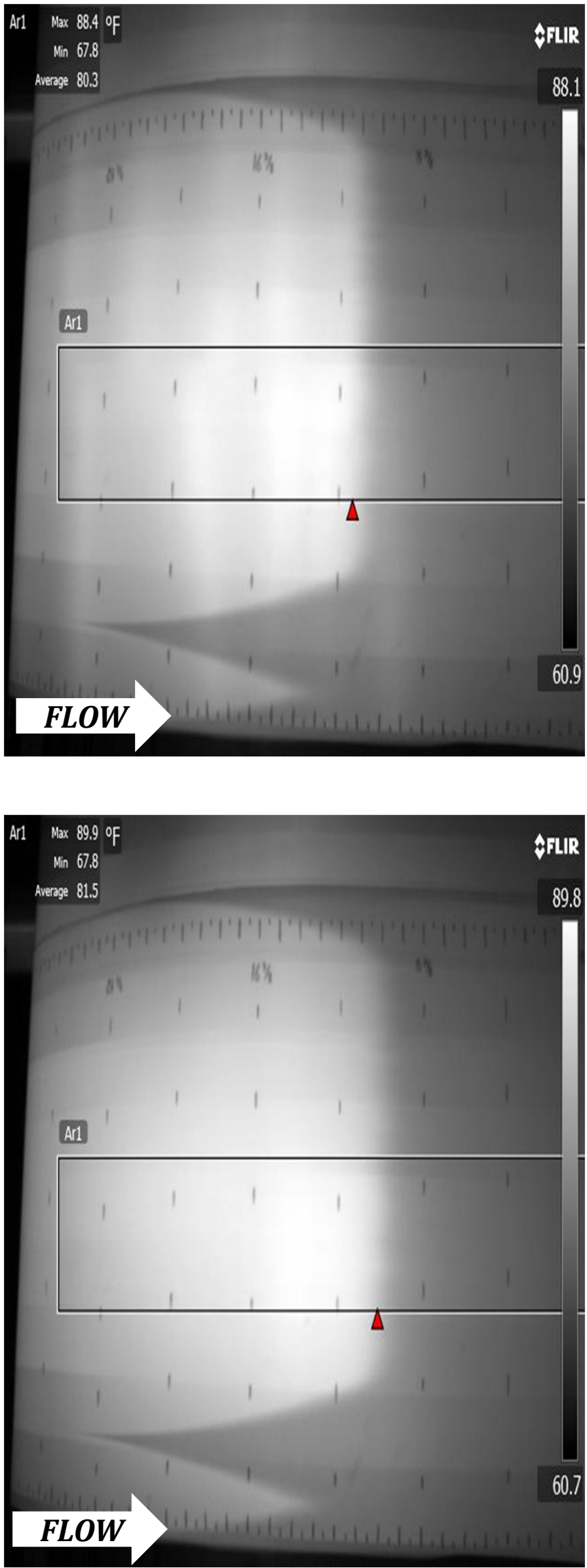


\section{8-m DU96-W-180 at $R e=3 \times 10^{5}$}

\section{PRESSURE SIDE}

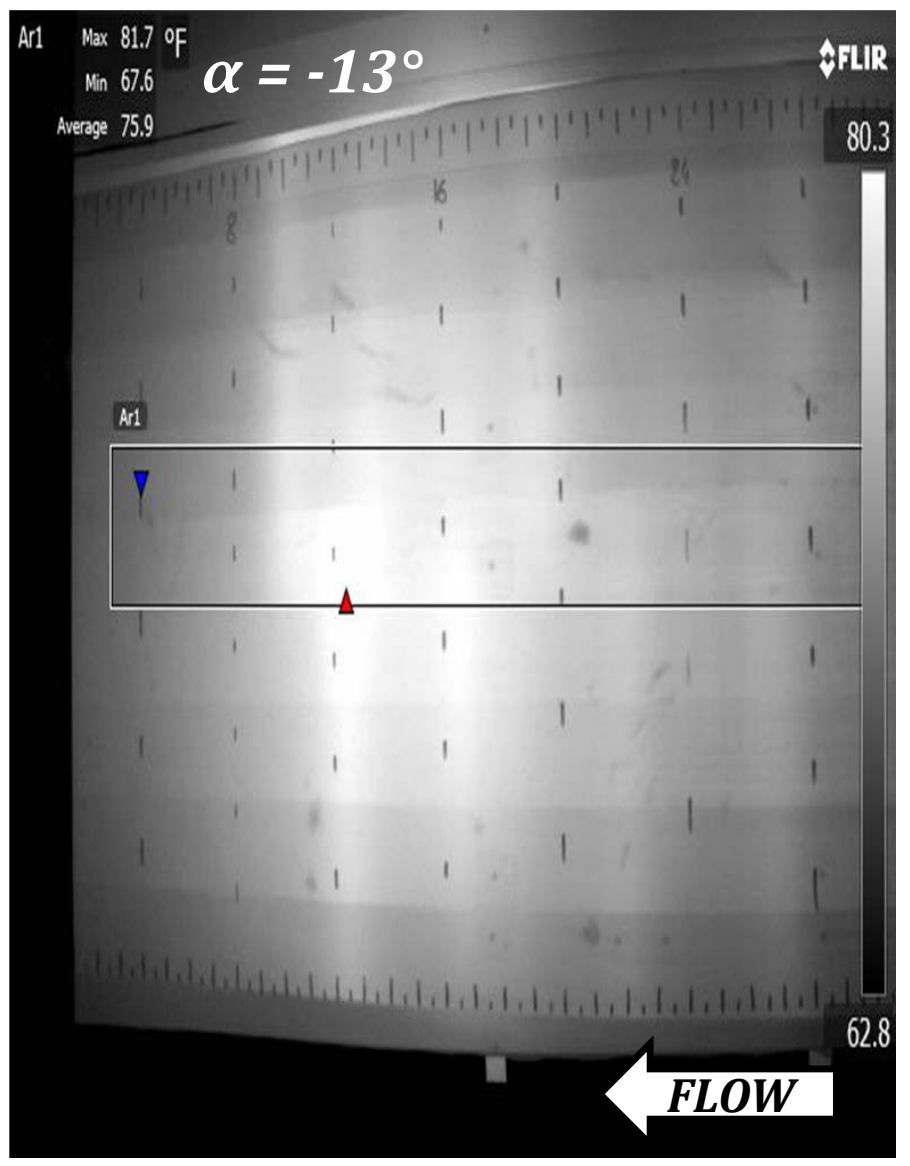

\section{Ar1 $\operatorname{Max} 84.2$ of}

Min $66.4^{\circ} \alpha=-14^{\circ}$

AFLIR

Avergege 76.6

ArI

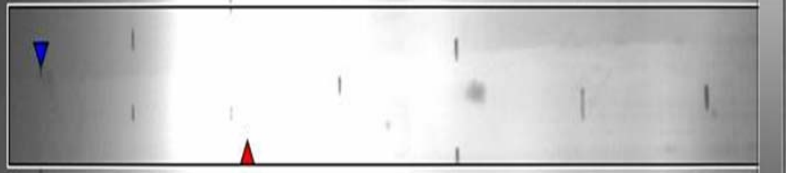

SUCTION SIDE
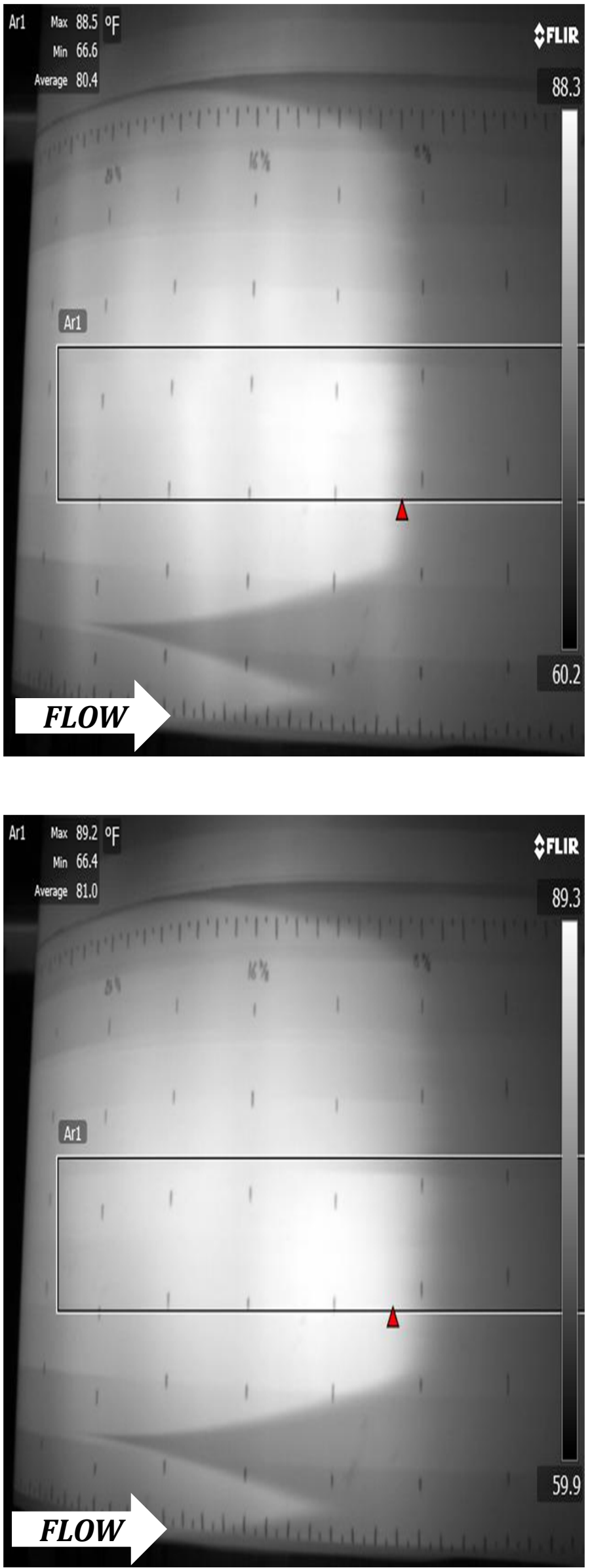
Appendix G Infrared Images of 0.46-m DU96-W-180 at Re $=1.5 \times 10^{5}$

(From $\alpha=20^{\circ}$ to -20 , Pressure Side Images are on Left and Suction Side Images are on Right.) 


\subsection{6-m DU96-W-180 at $R e=1.5 \times 10^{5}$}

\section{PRESSURE SIDE}
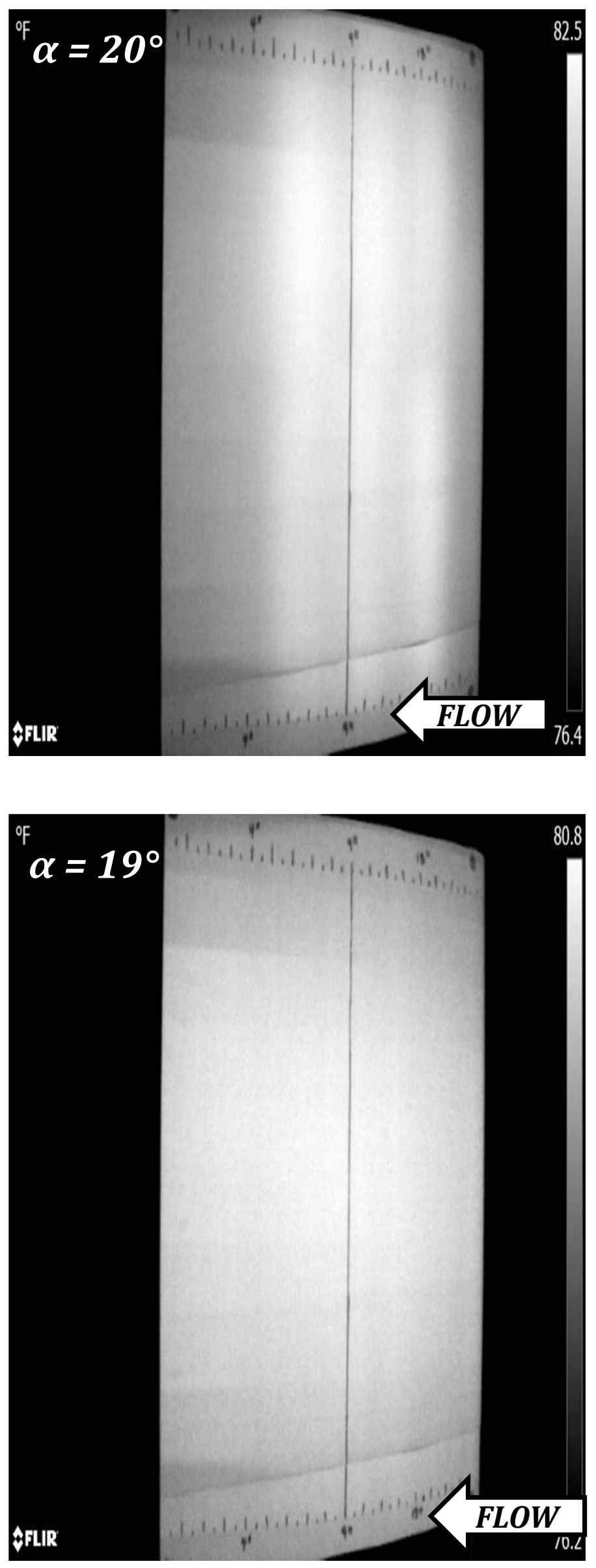

SUCTION SIDE
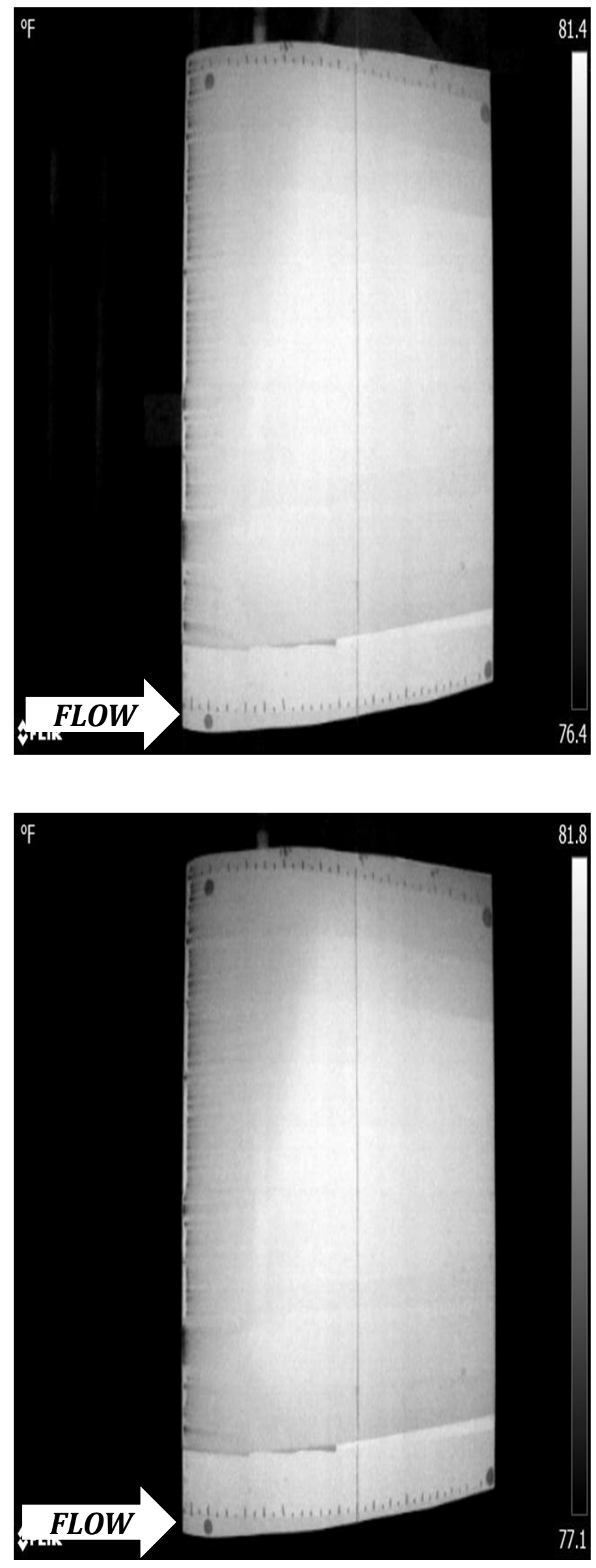


\subsection{6- $m$ DU96-W-180 at $R e=1.5 \times 10^{5}$}

\section{PRESSURE SIDE}
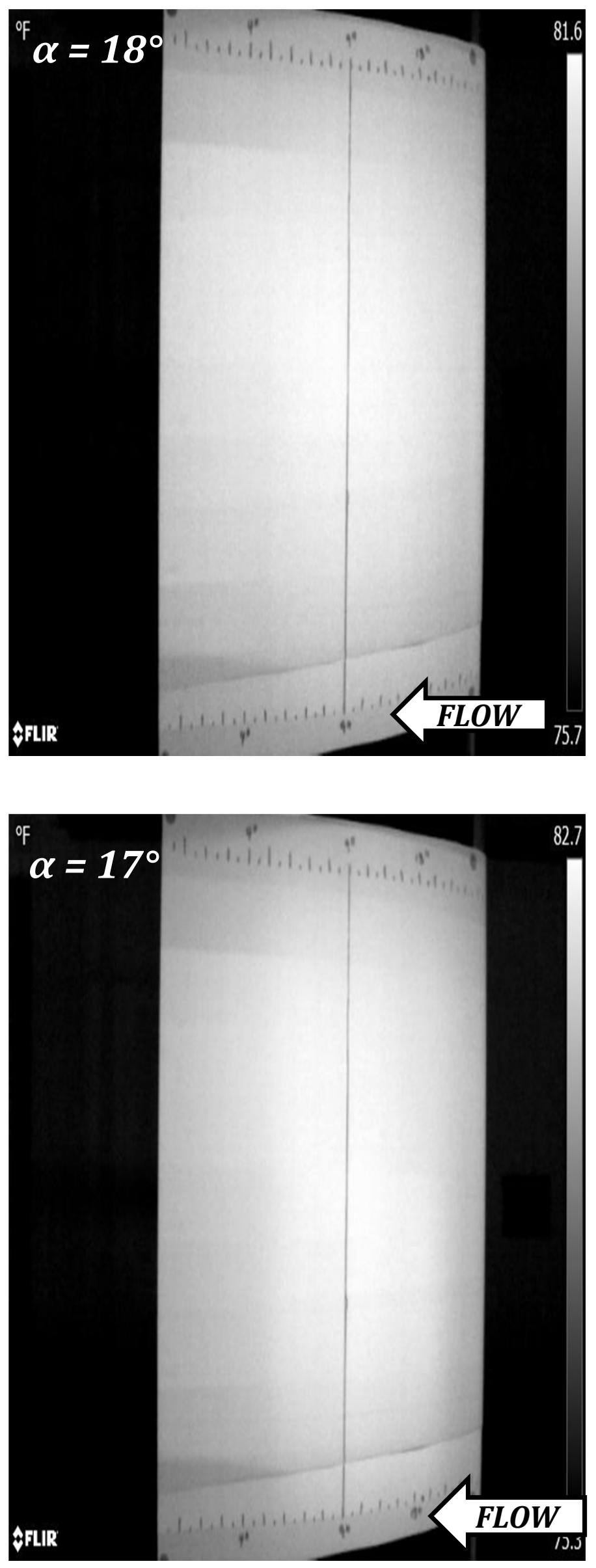

SUCTION SIDE
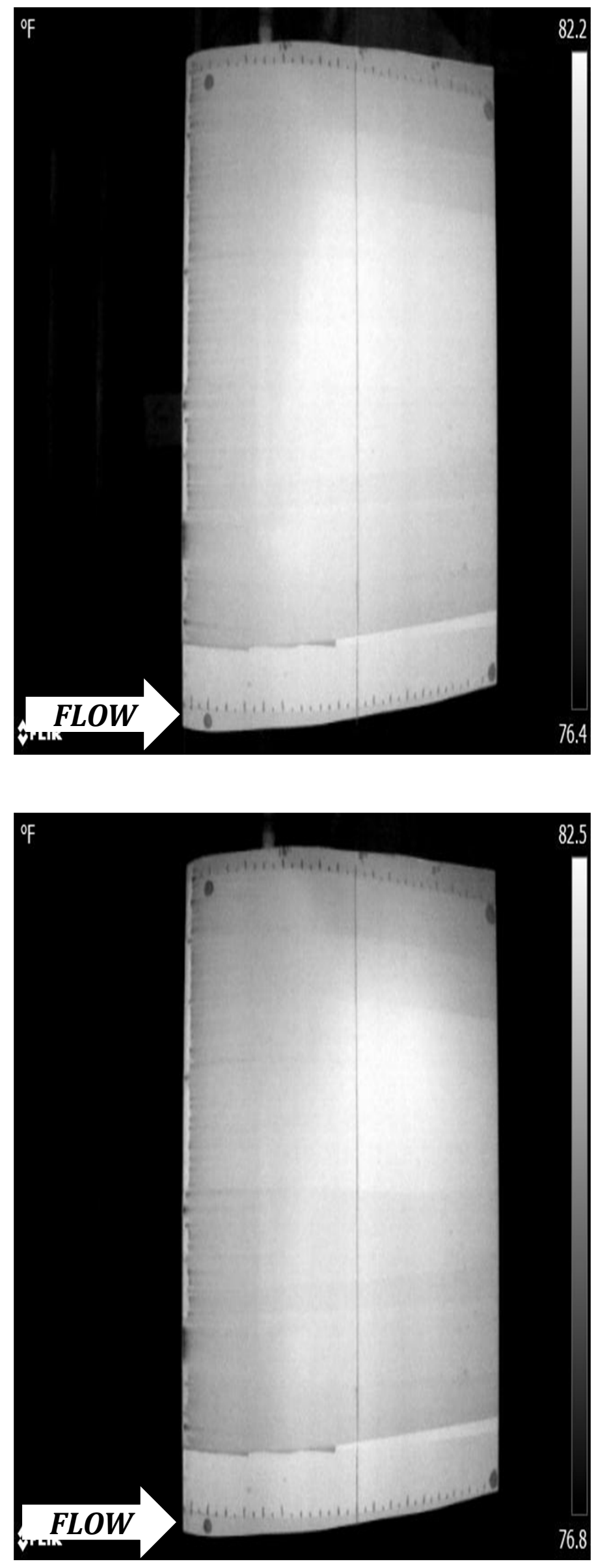


\subsection{6-m DU96-W-180 at $R e=1.5 \times 10^{5}$}

\section{PRESSURE SIDE}
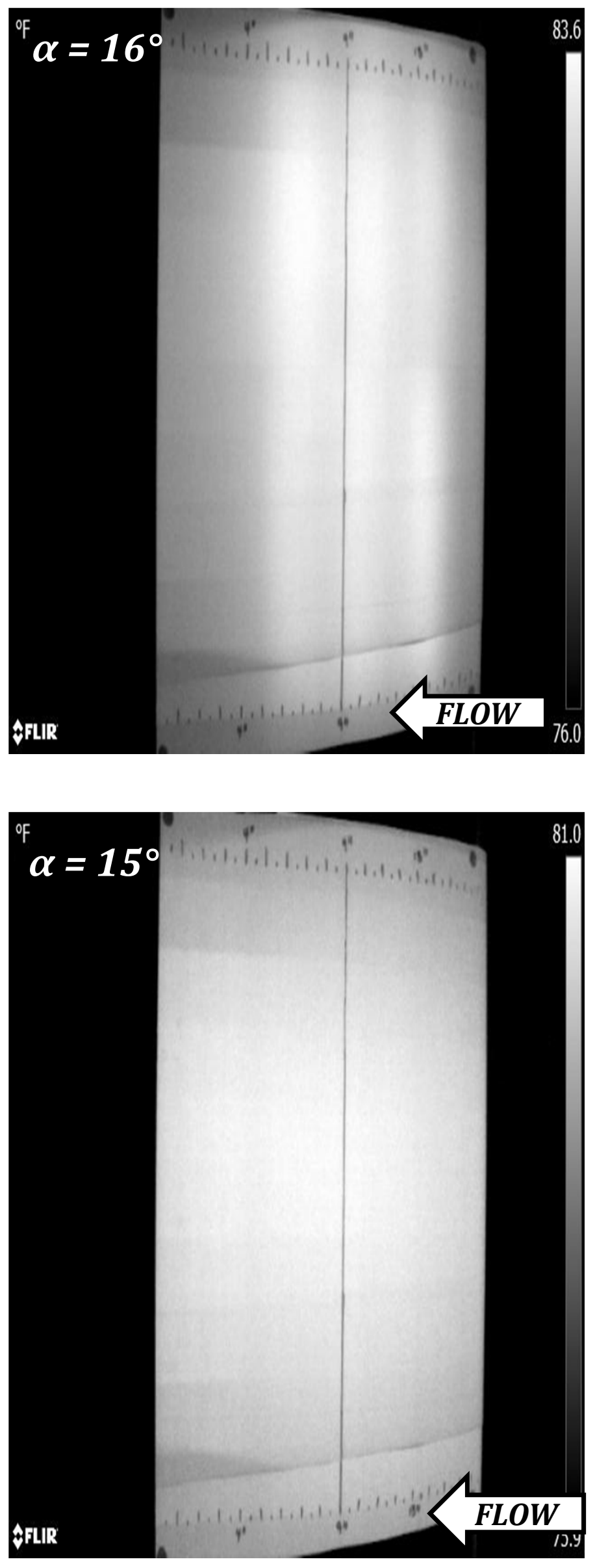

\section{SUCTION SIDE}
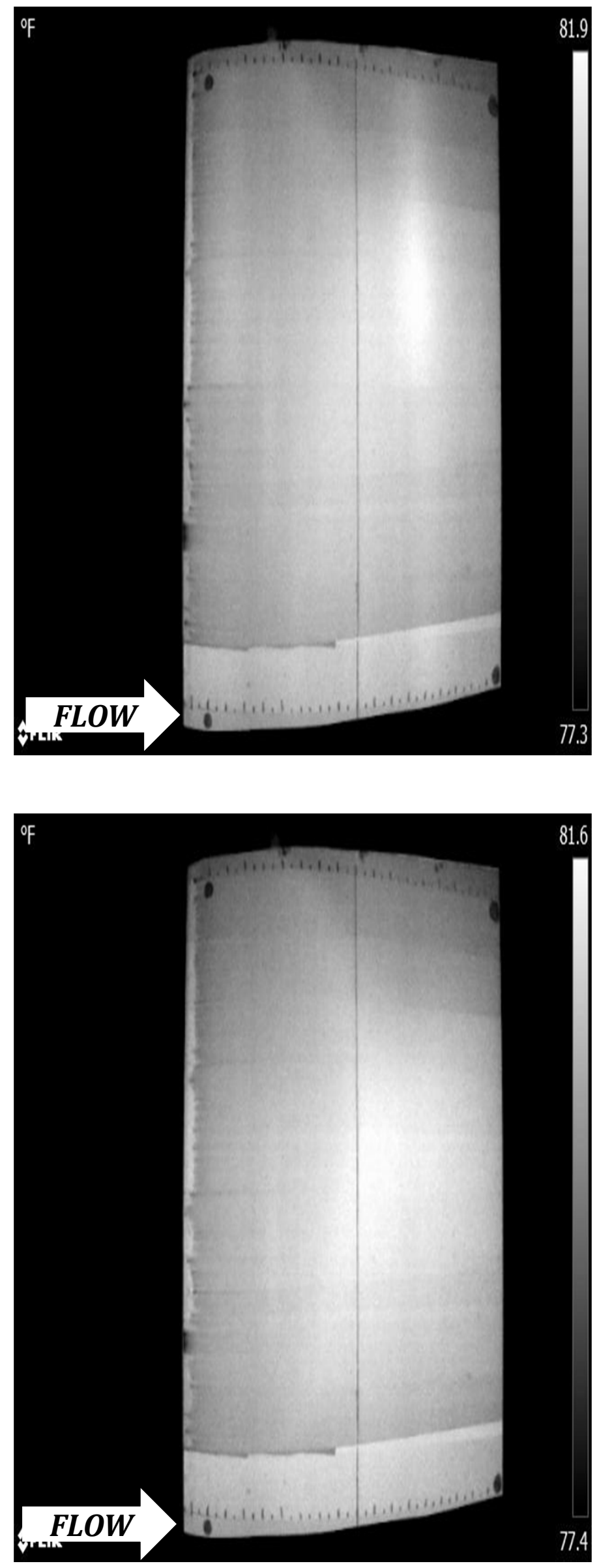


\subsection{6-m DU96-W-180 at $R e=1.5 \times 10^{5}$}

\section{PRESSURE SIDE}
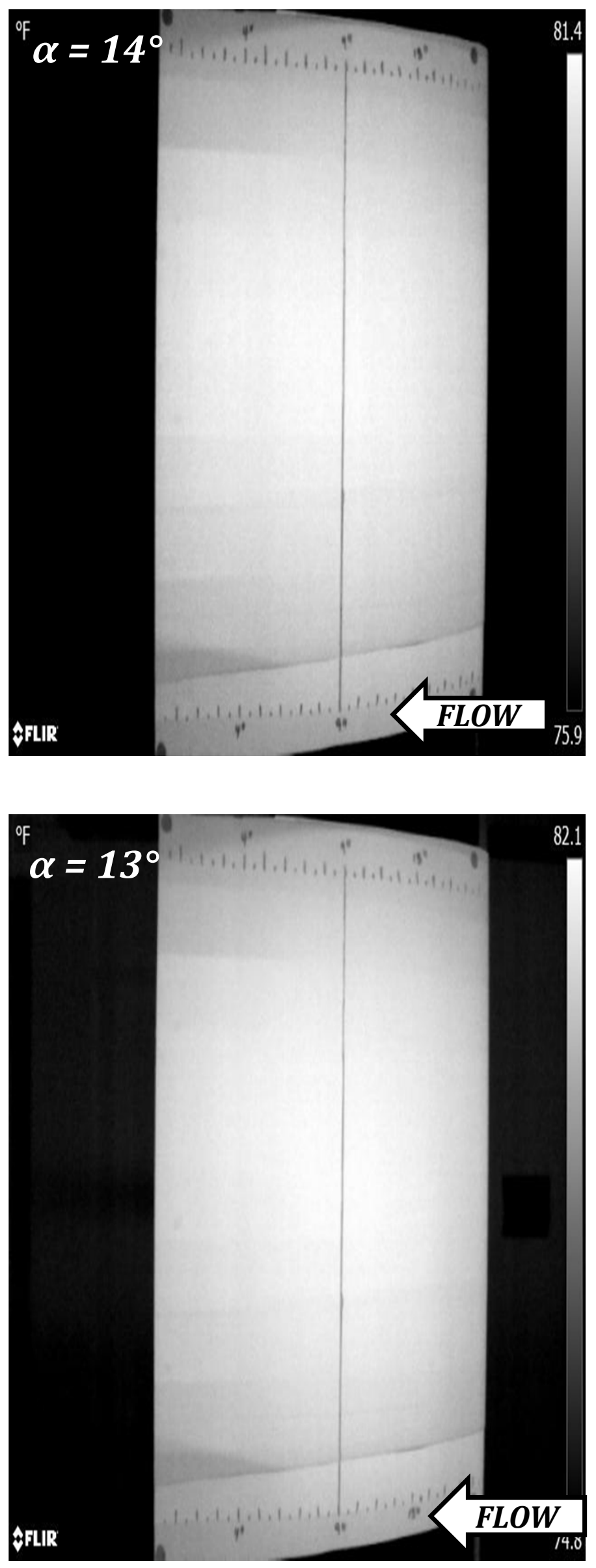

SUCTION SIDE
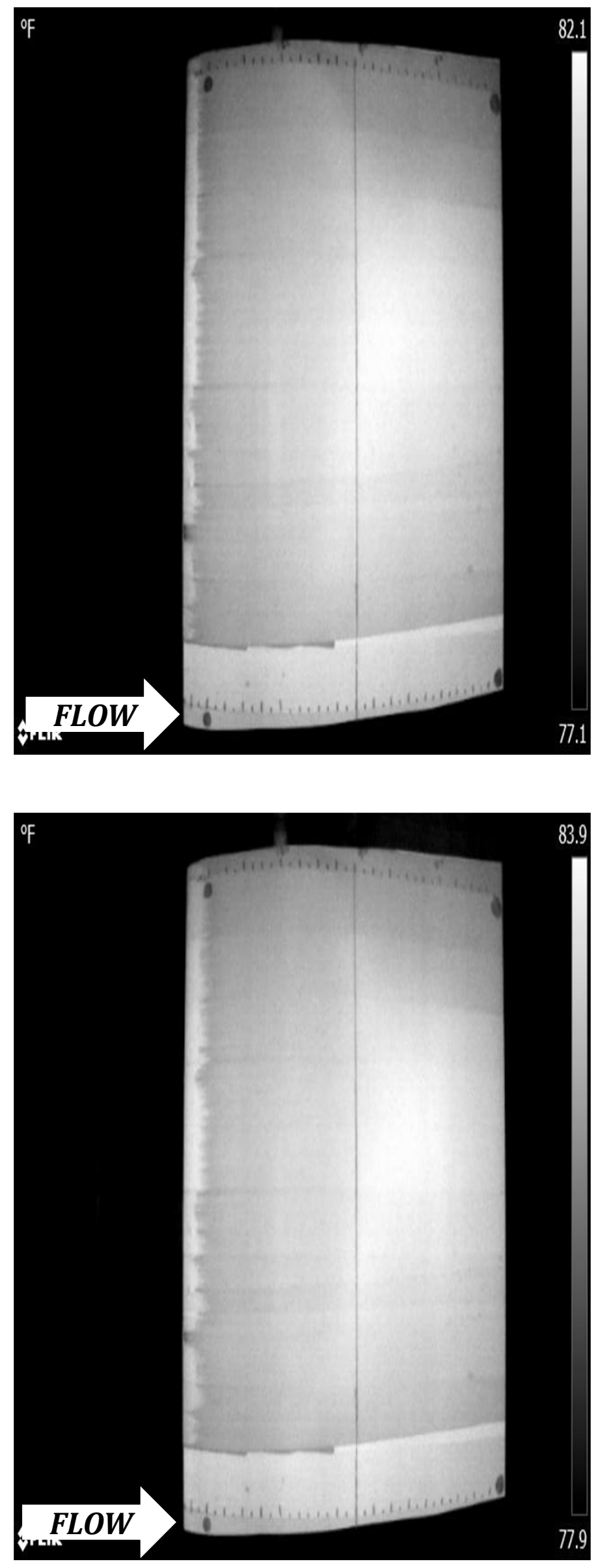


\subsection{6-m DU96-W-180 at $R e=1.5 \times 10^{5}$}

\section{PRESSURE SIDE}
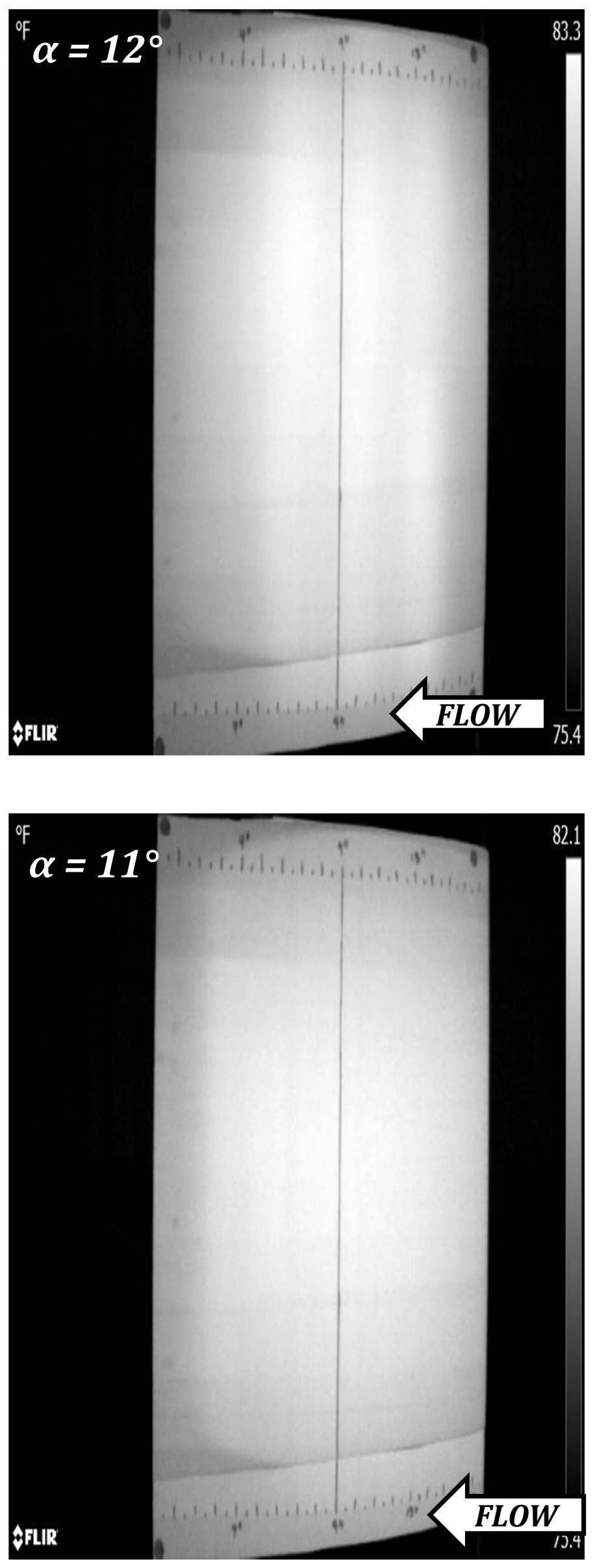

\section{SUCTION SIDE}
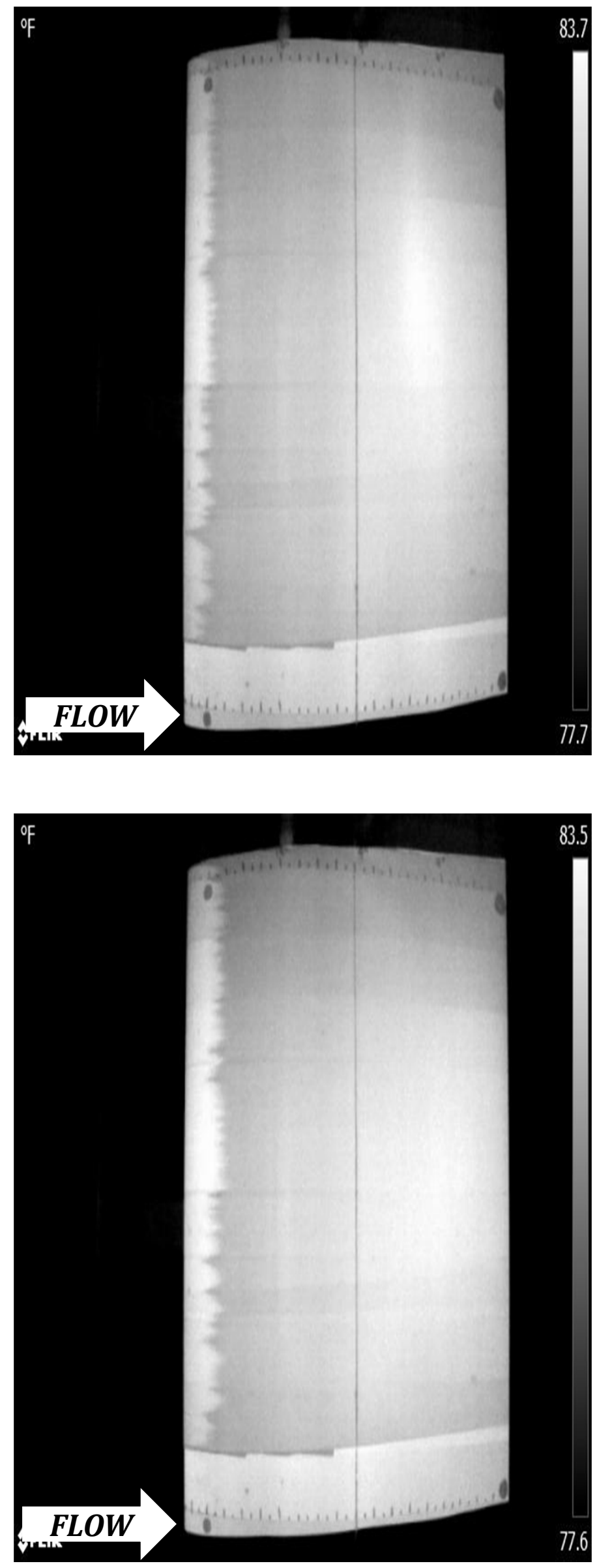


\subsection{6-m DU96-W-180 at $R e=1.5 \times 10^{5}$}

\section{PRESSURE SIDE}
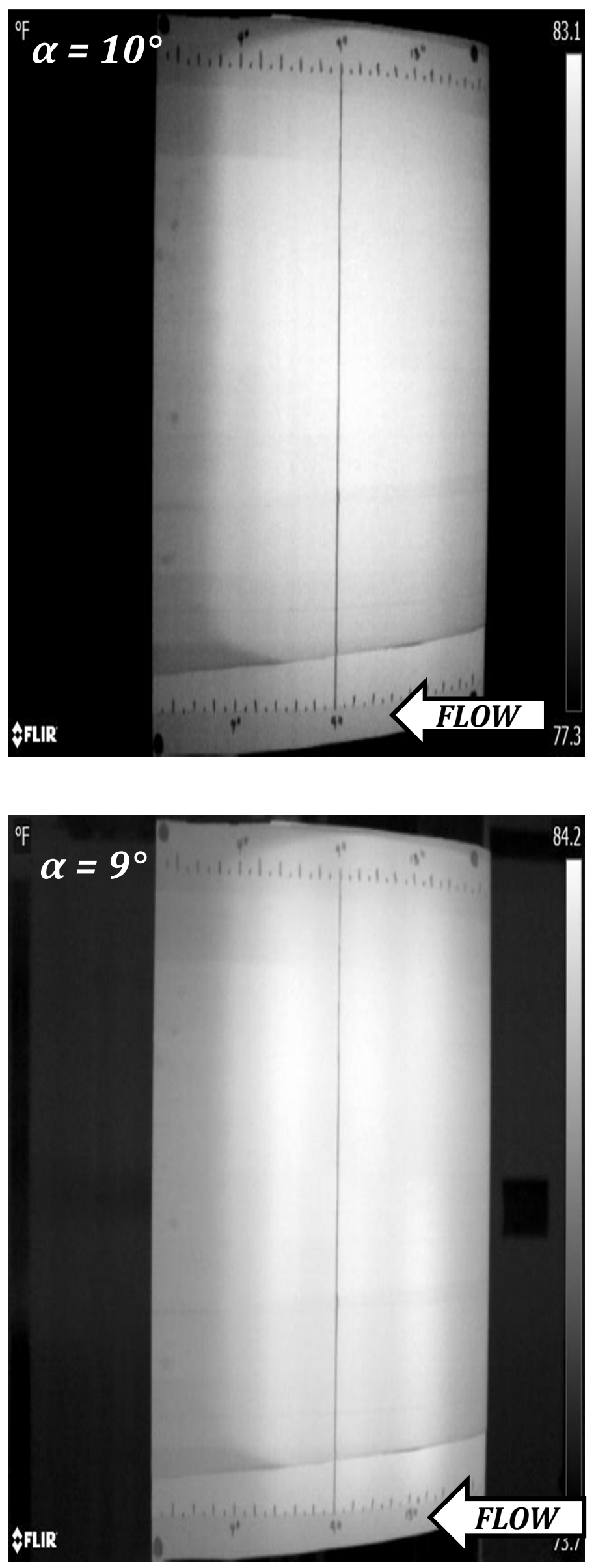

\section{SUCTION SIDE}
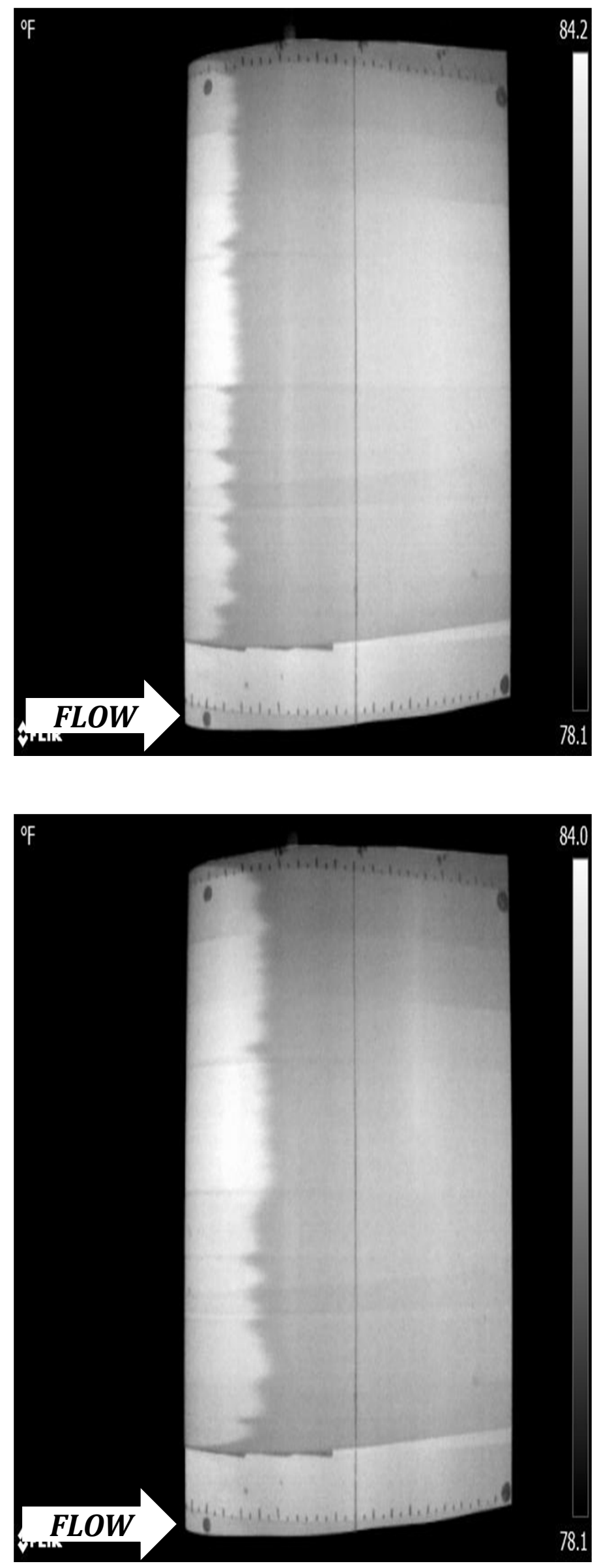


\subsection{6- $m$ DU96-W-180 at $R e=1.5 \times 10^{5}$}

\section{PRESSURE SIDE}
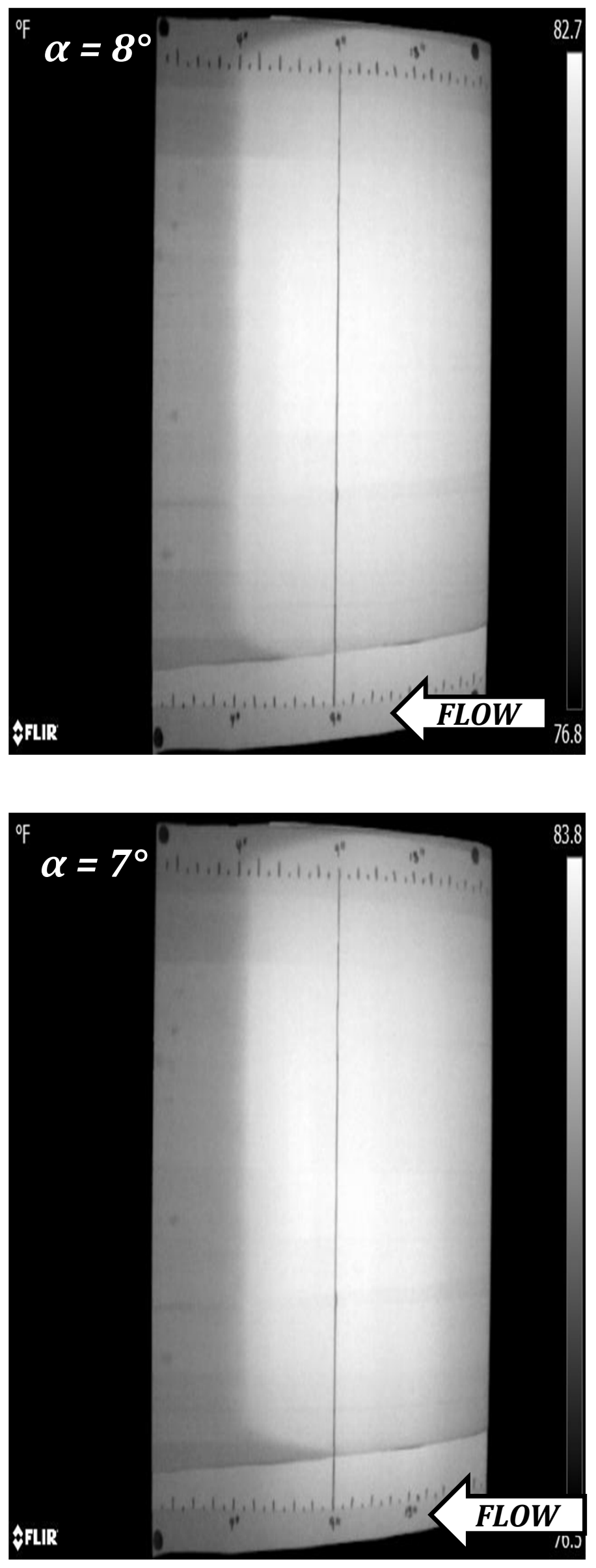

\section{SUCTION SIDE}
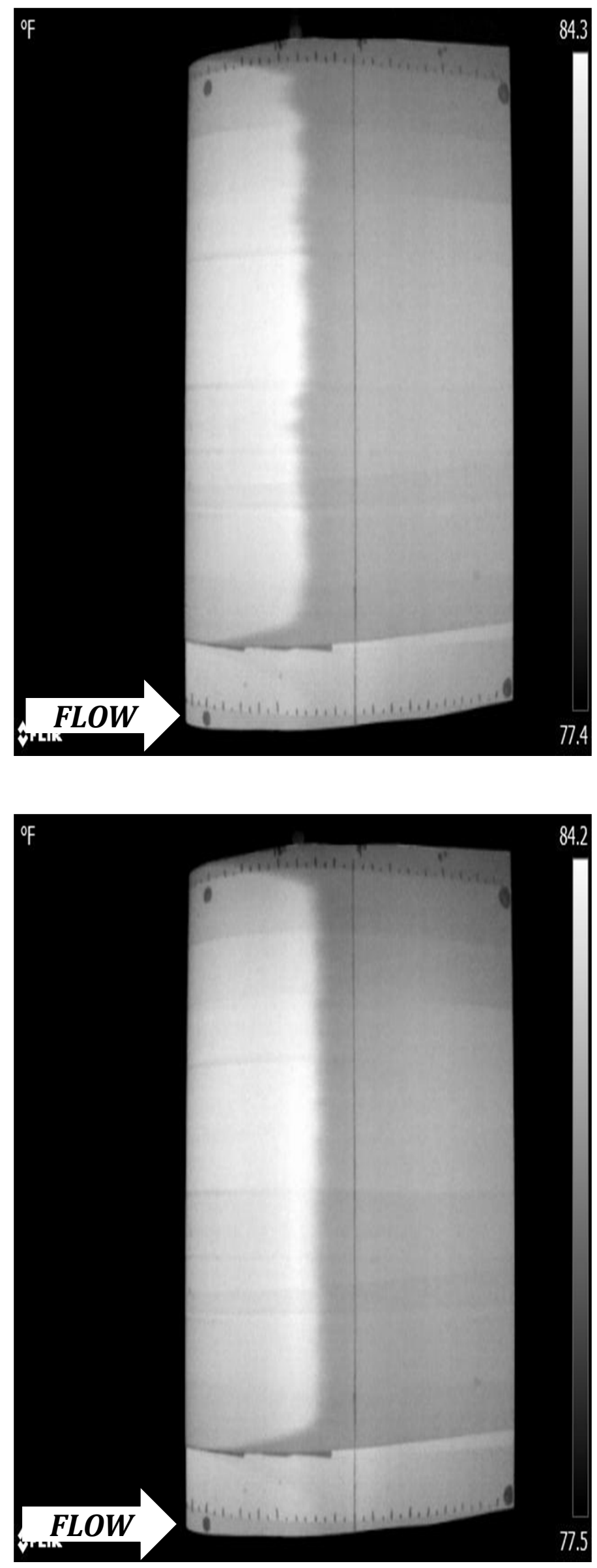


\subsection{6-m DU96-W-180 at $R e=1.5 \times 10^{5}$}

\section{PRESSURE SIDE}
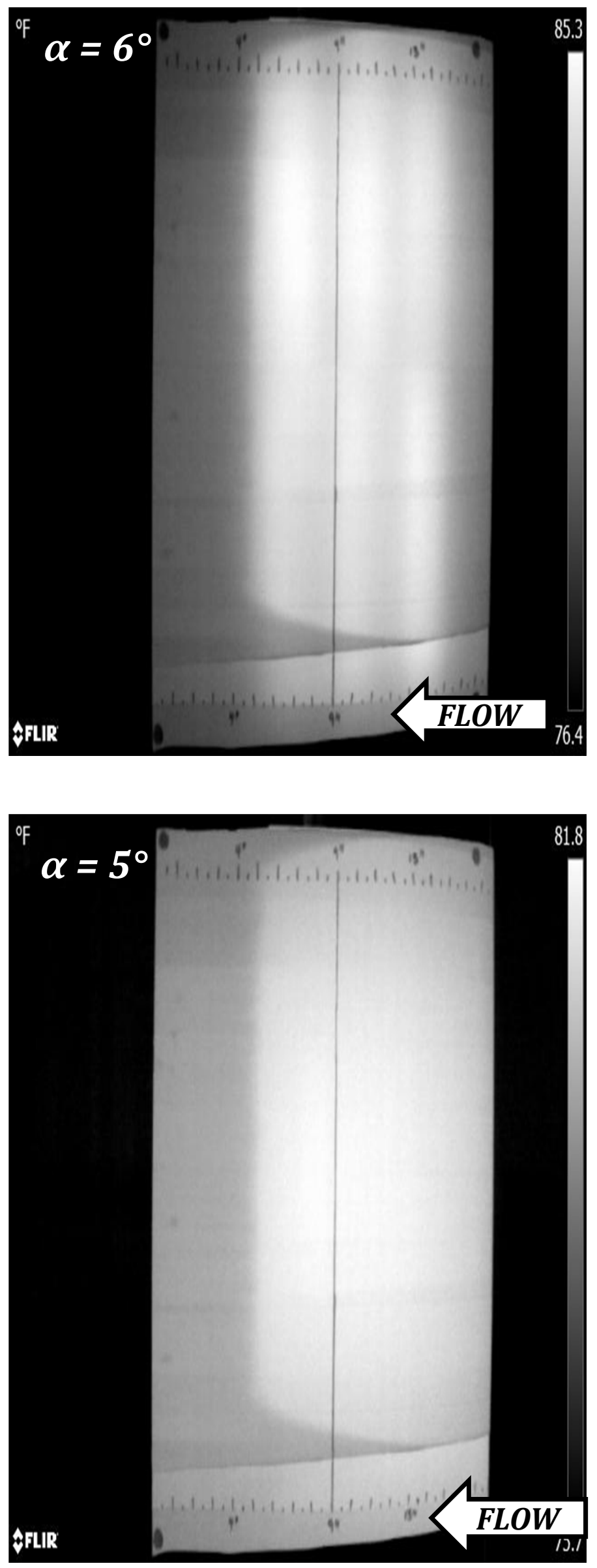

\section{SUCTION SIDE}
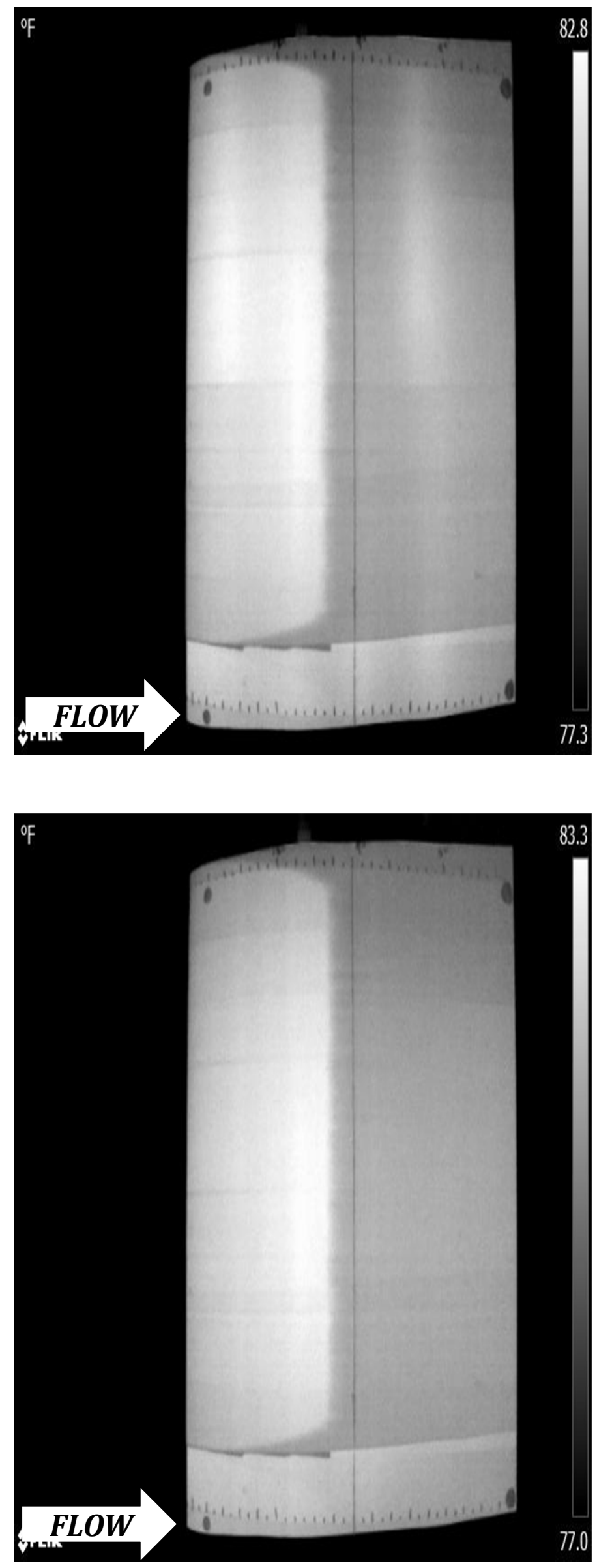


\section{PRESSURE SIDE}
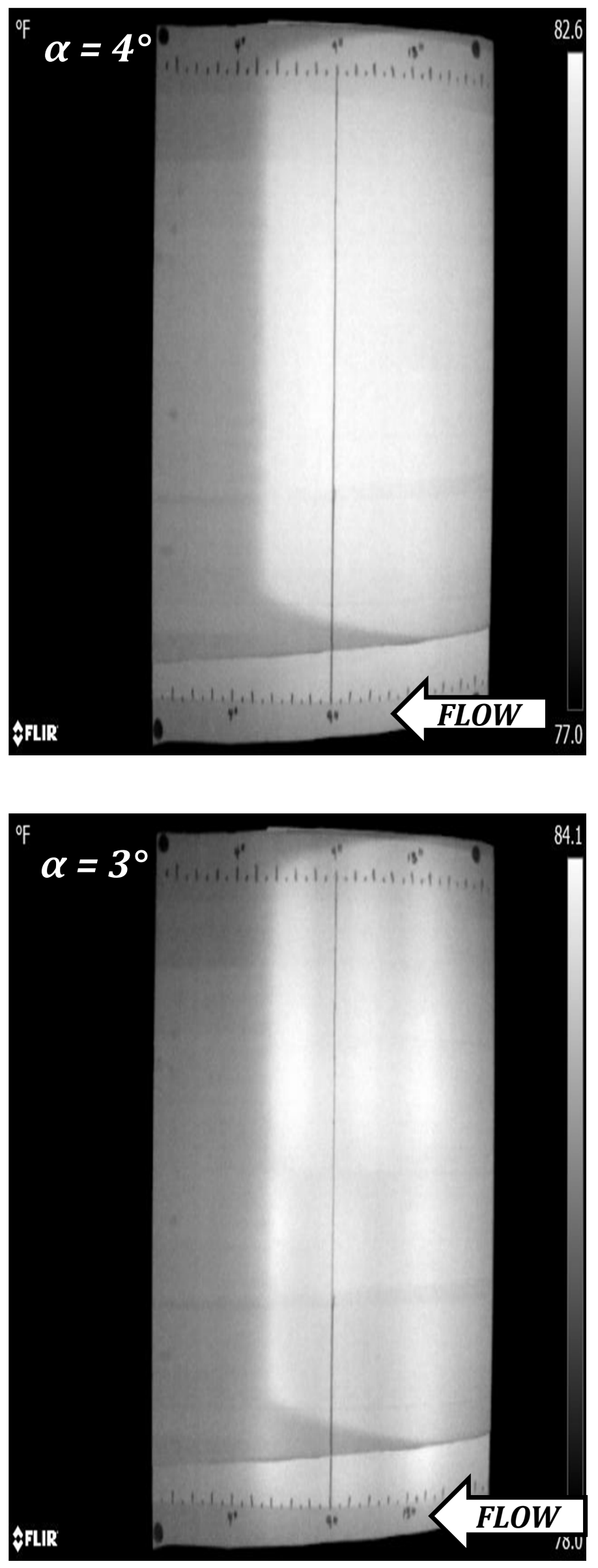

SUCTION SIDE
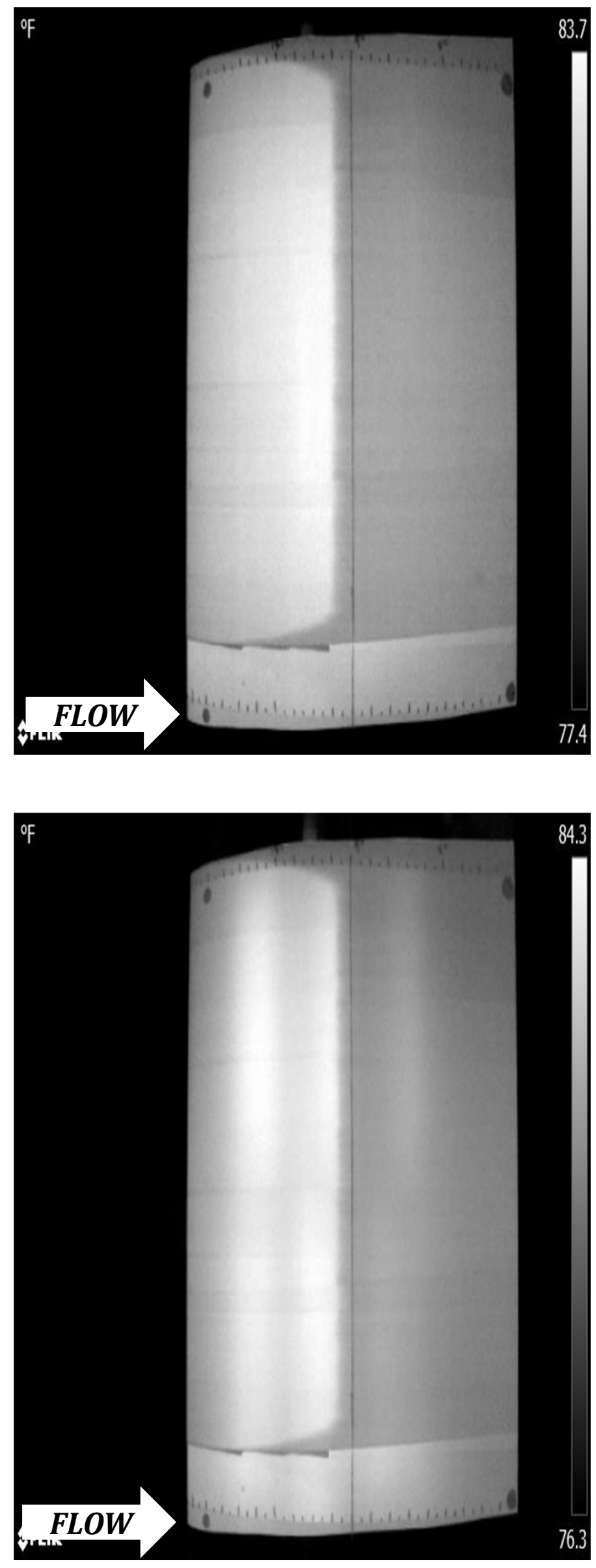


\section{PRESSURE SIDE}
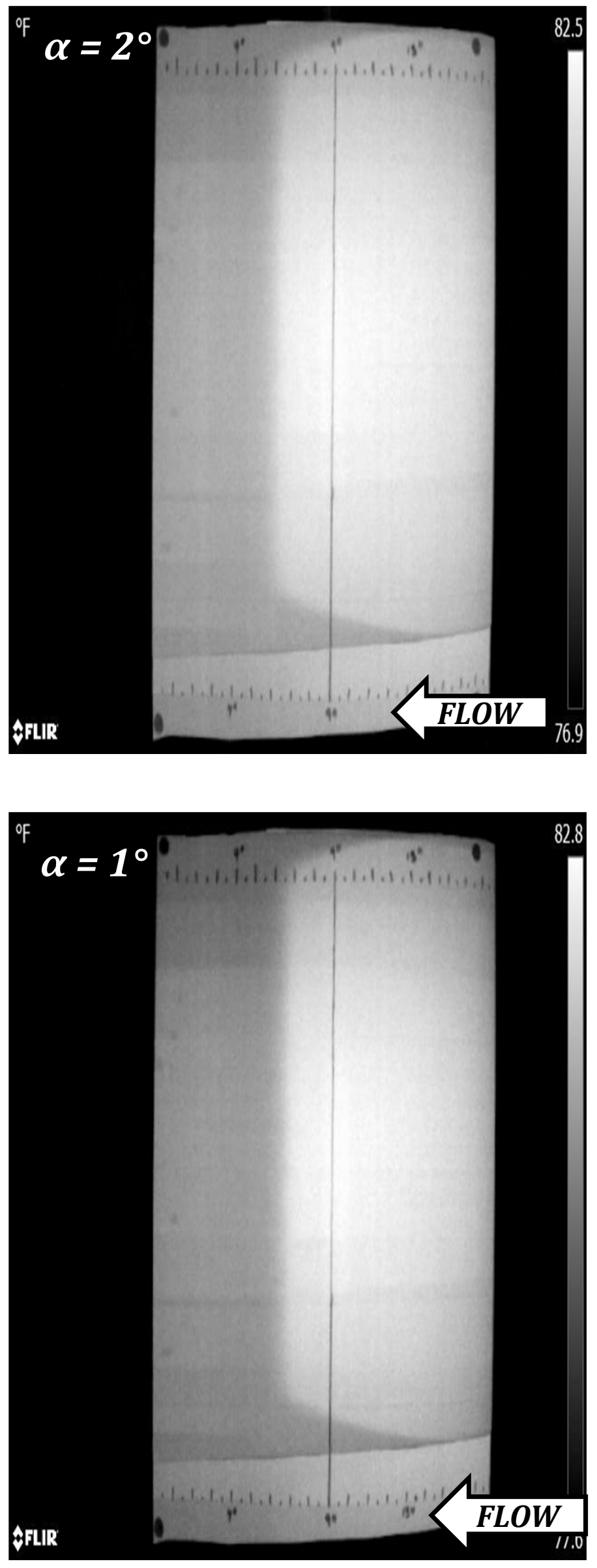

\section{SUCTION SIDE}
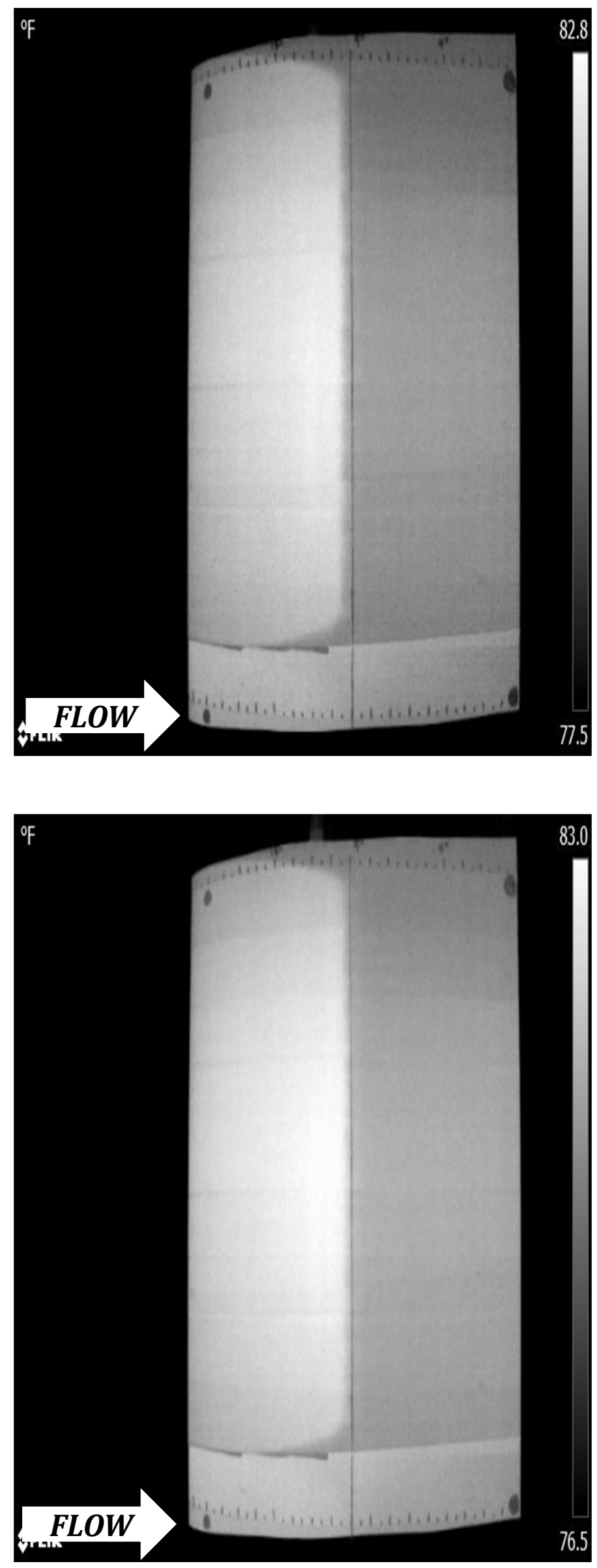


\subsection{6-m DU96-W-180 at $R e=1.5 \times 10^{5}$}

\section{PRESSURE SIDE}
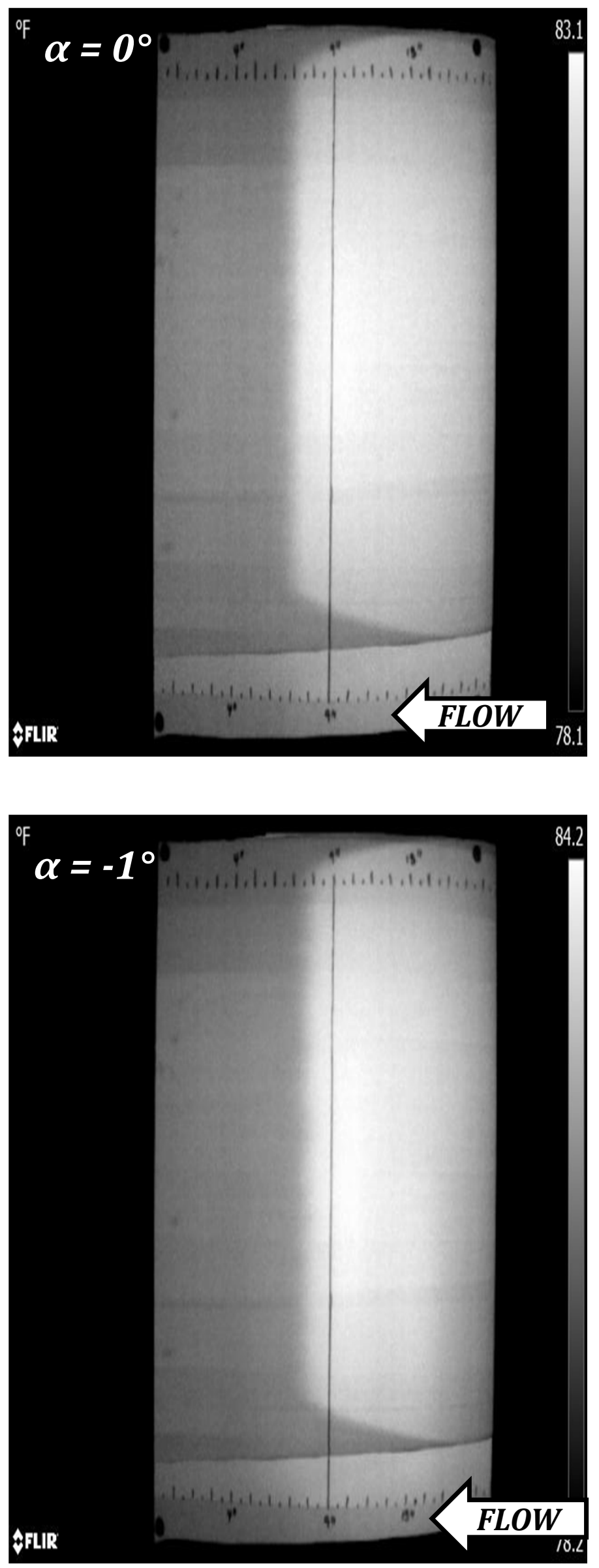

SUCTION SIDE
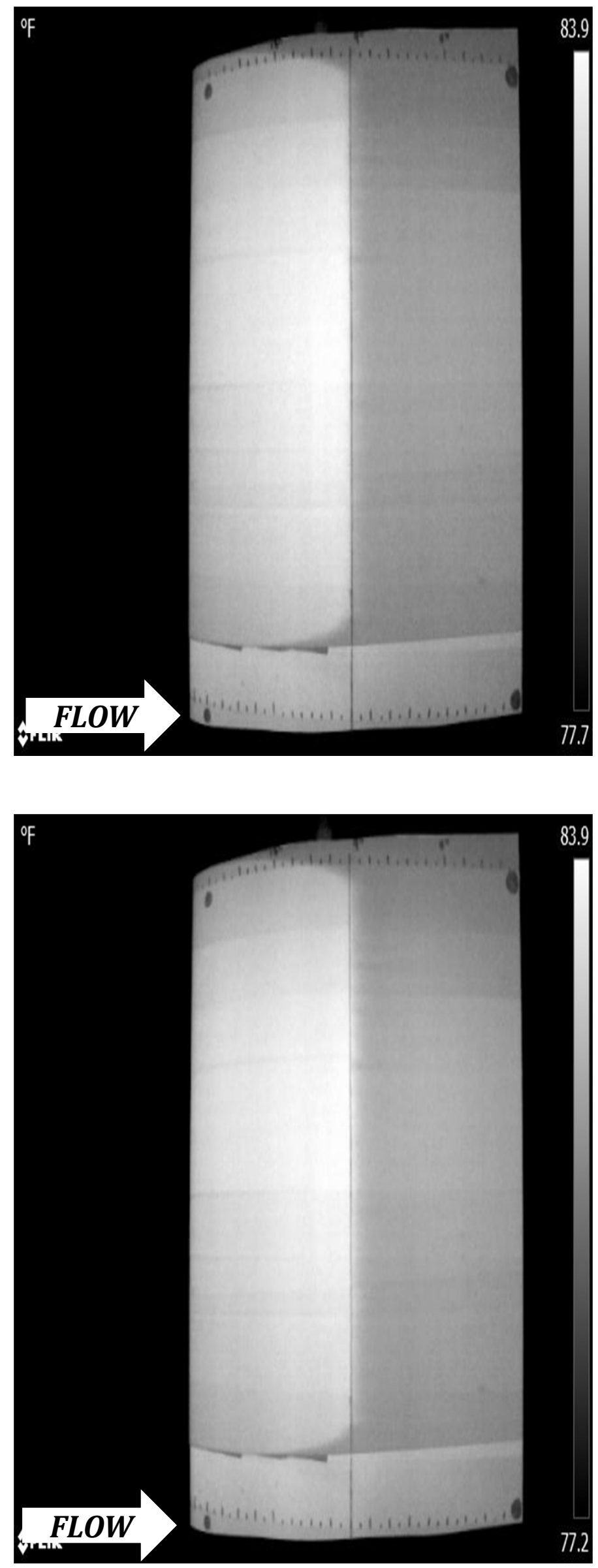


\section{PRESSURE SIDE}
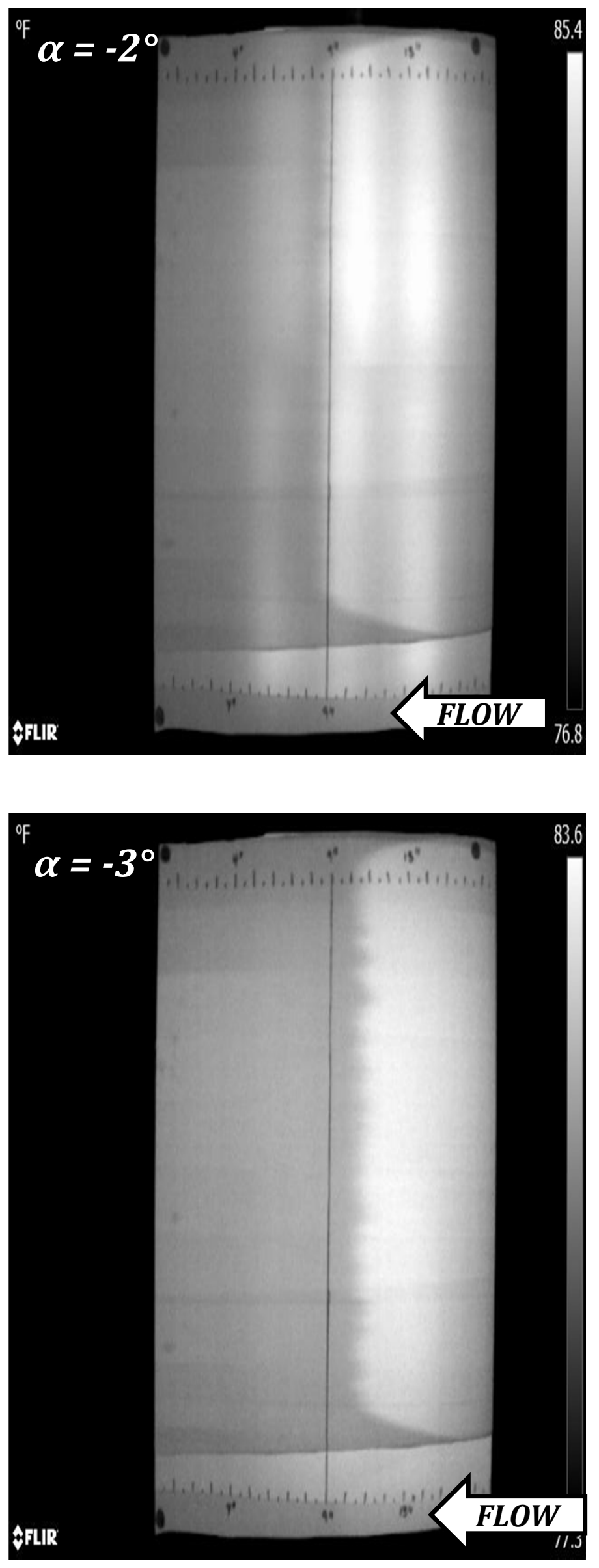

\section{SUCTION SIDE}
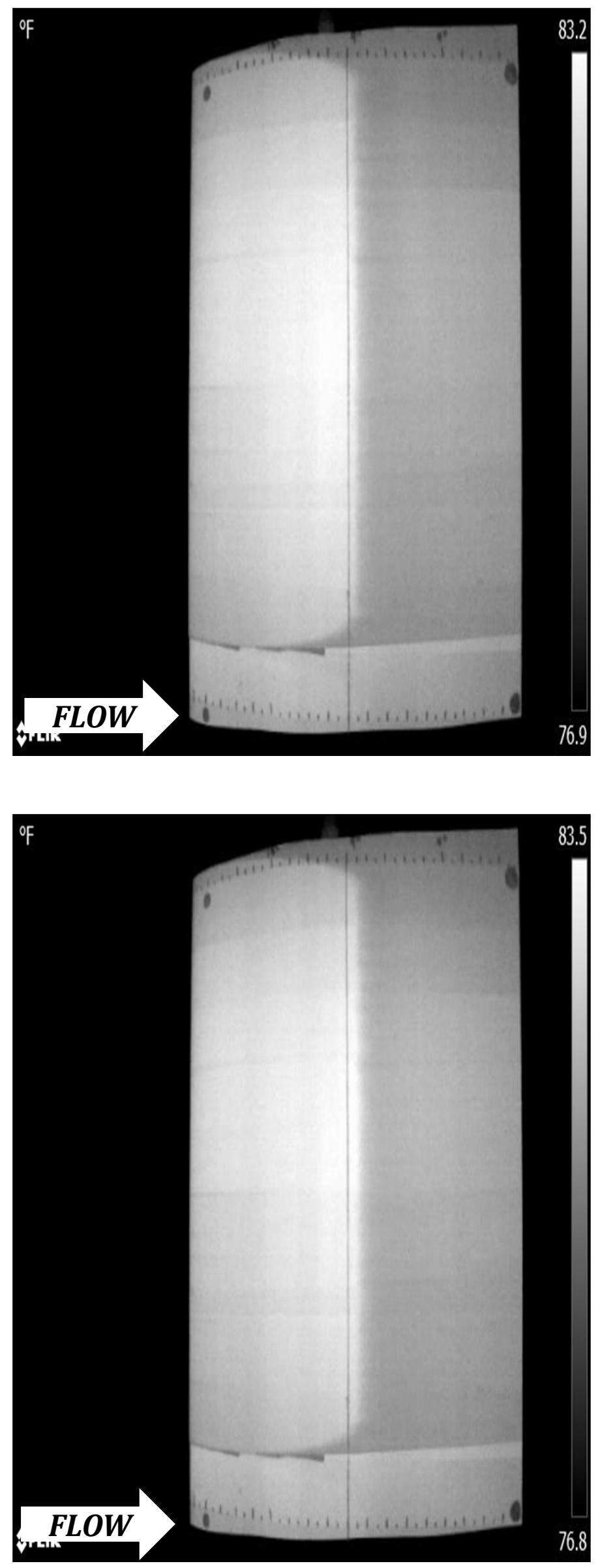


\subsection{6-m DU96-W-180 at $R e=1.5 \times 10^{5}$}

\section{PRESSURE SIDE}
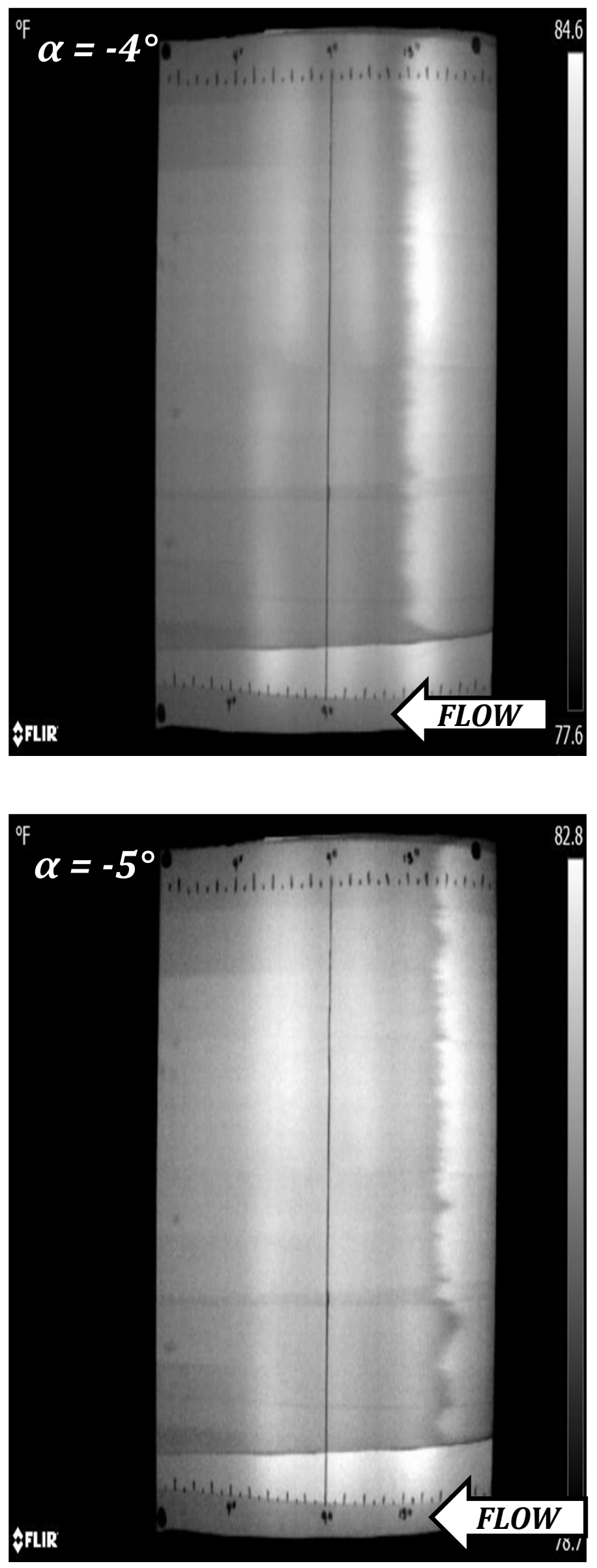

\section{SUCTION SIDE}
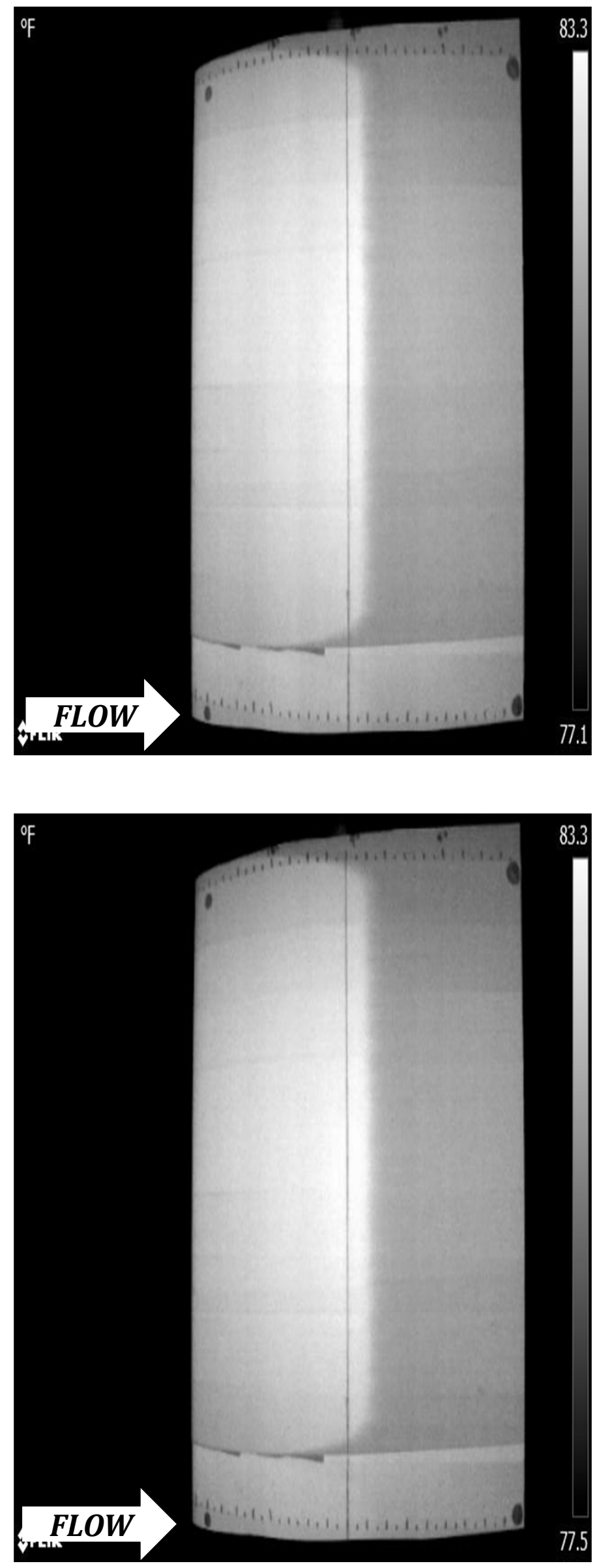


\subsection{6- $m$ DU96-W-180 at $R e=1.5 \times 10^{5}$}

\section{PRESSURE SIDE}
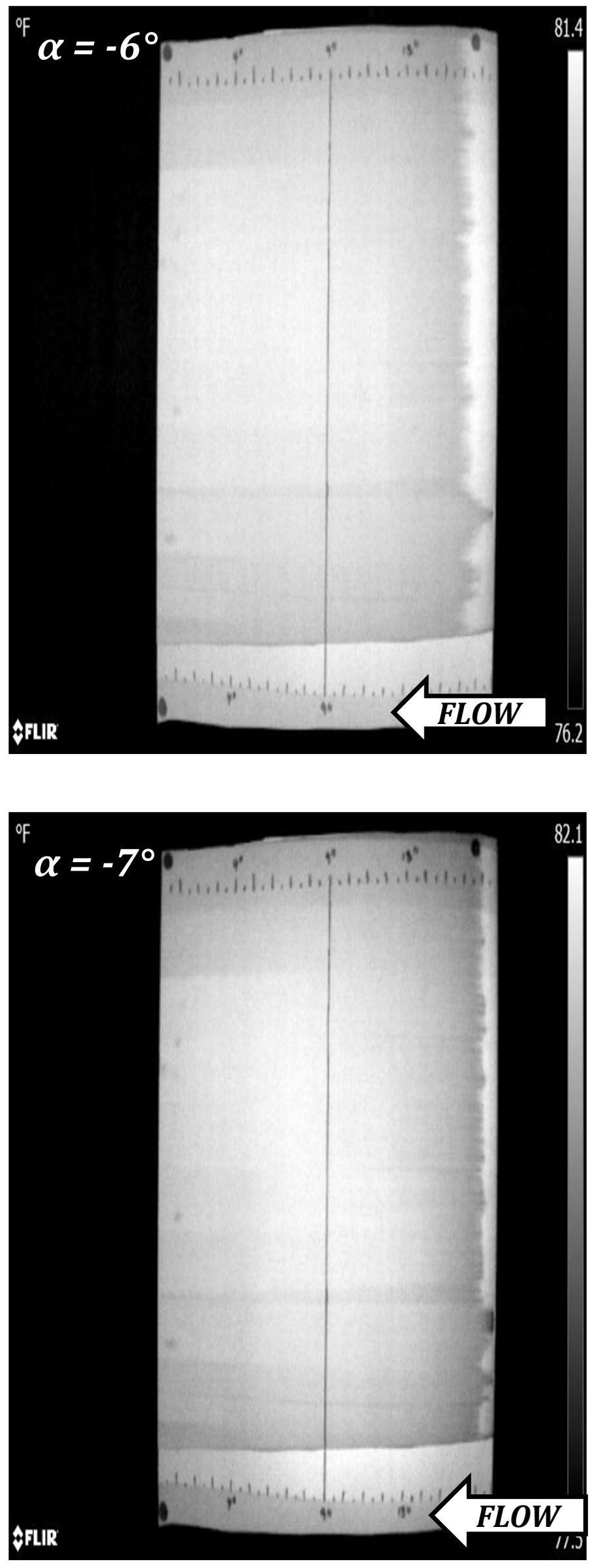

\section{SUCTION SIDE}
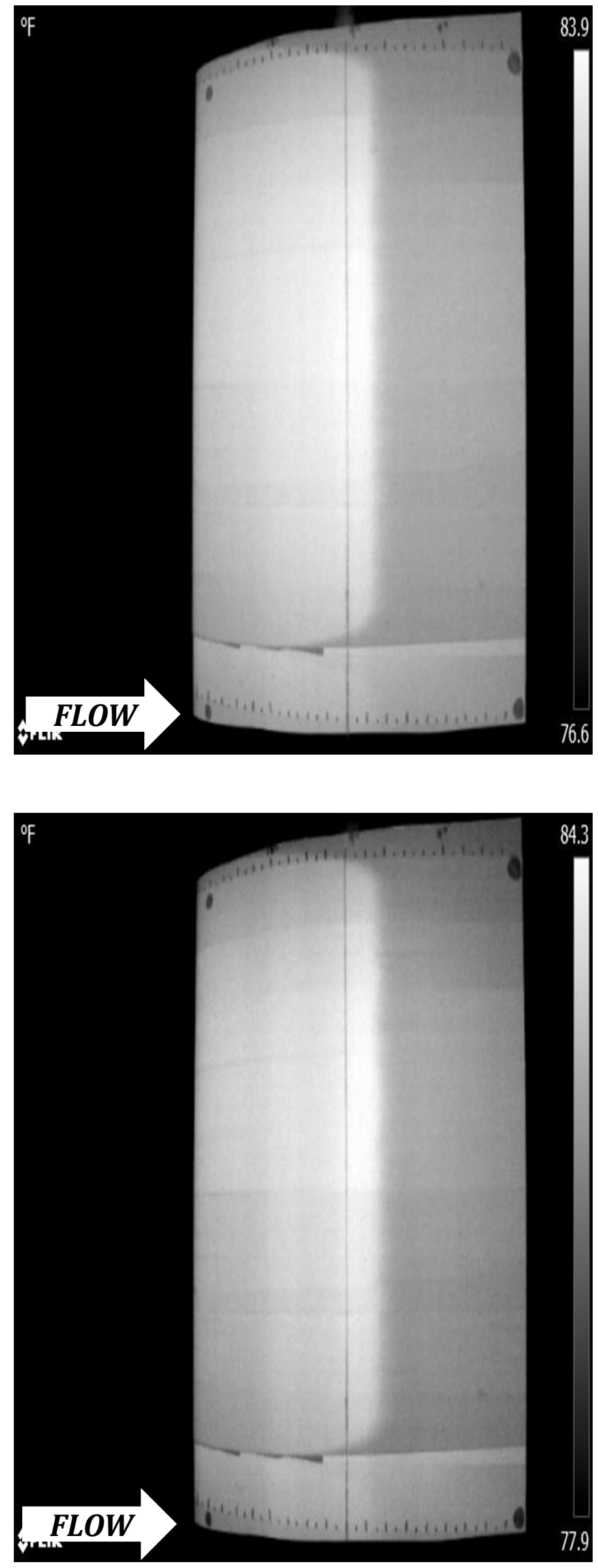


\subsection{6-m DU96-W-180 at $R e=1.5 \times 10^{5}$}

\section{PRESSURE SIDE}
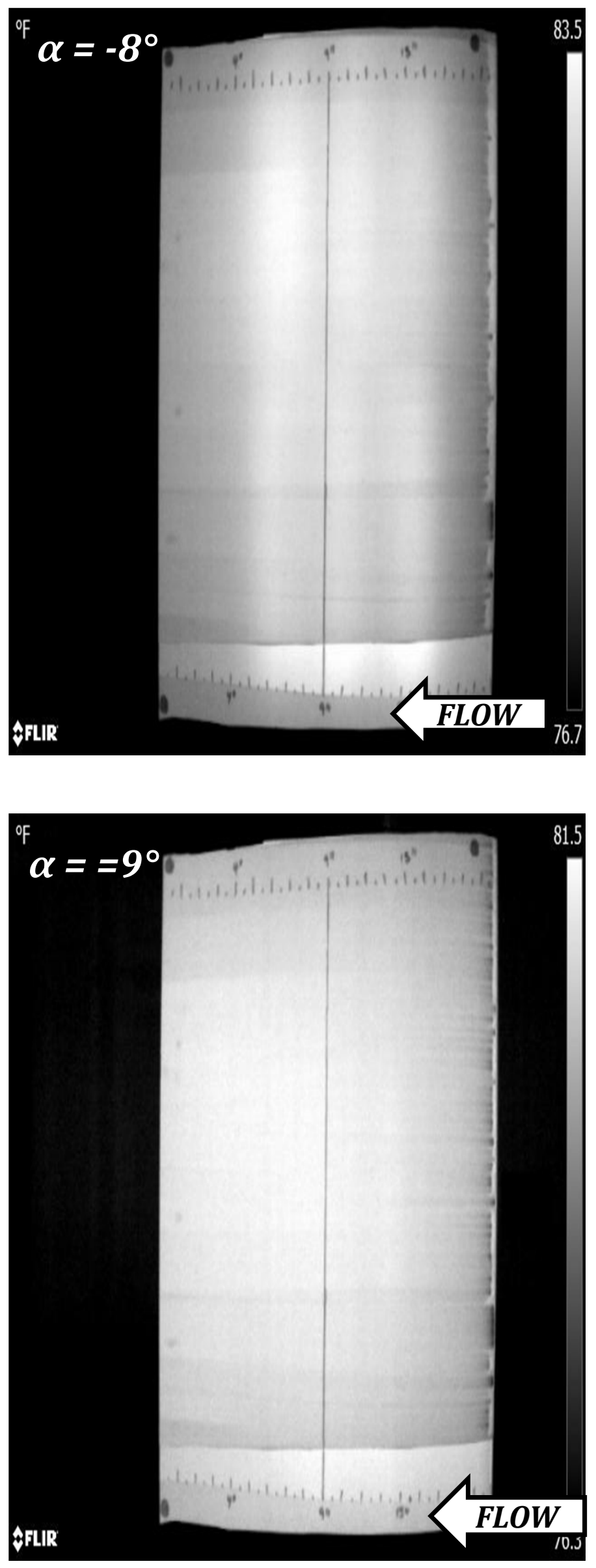

\section{SUCTION SIDE}
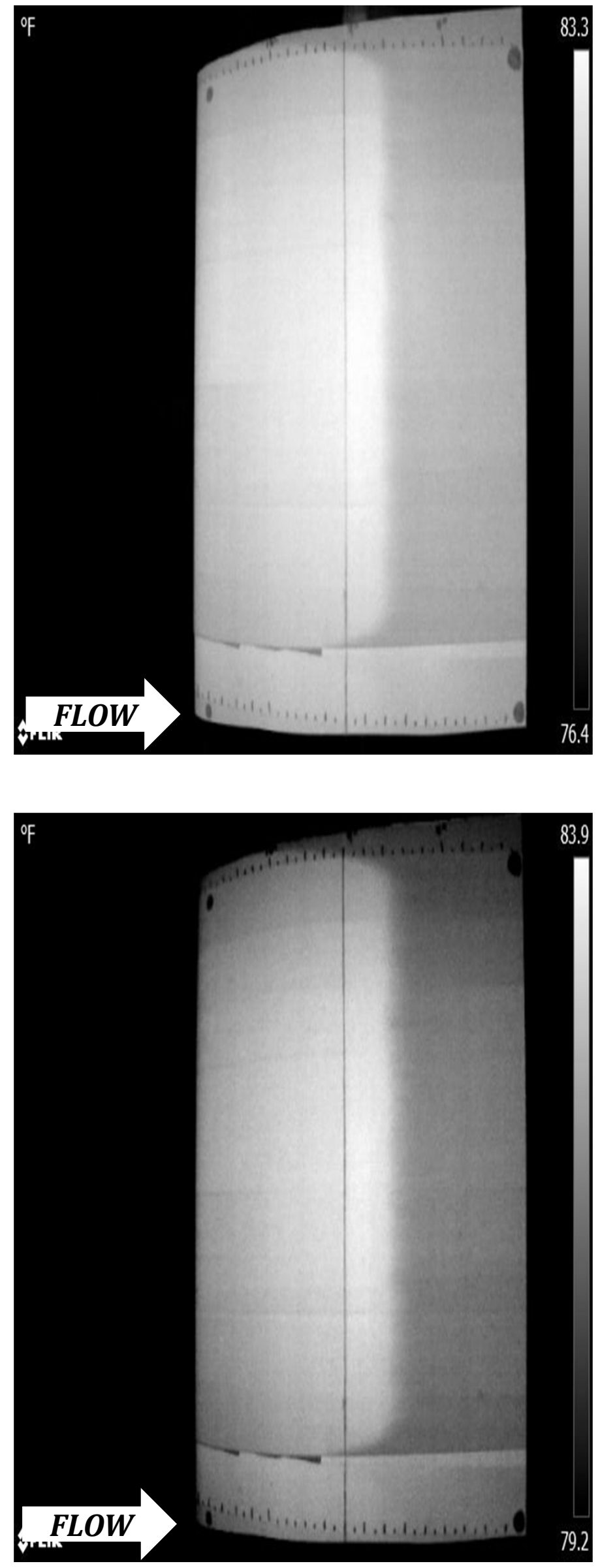


\section{PRESSURE SIDE}
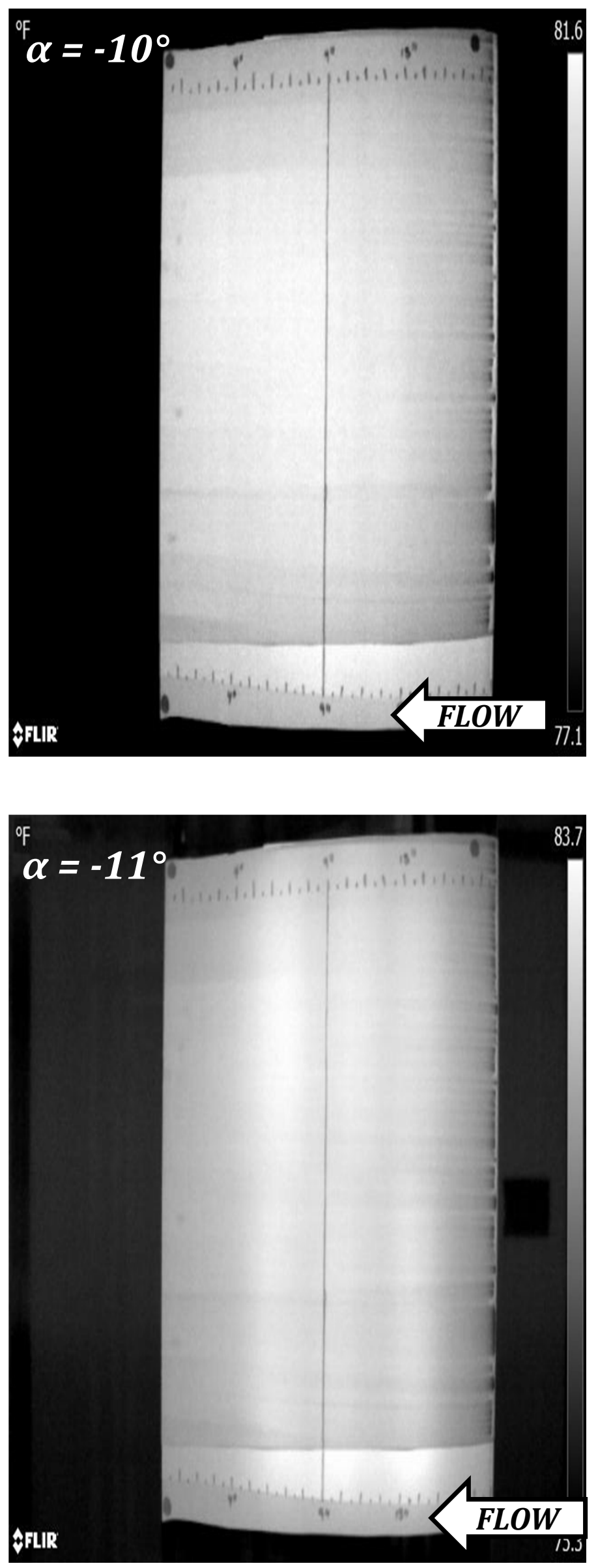

\section{SUCTION SIDE}
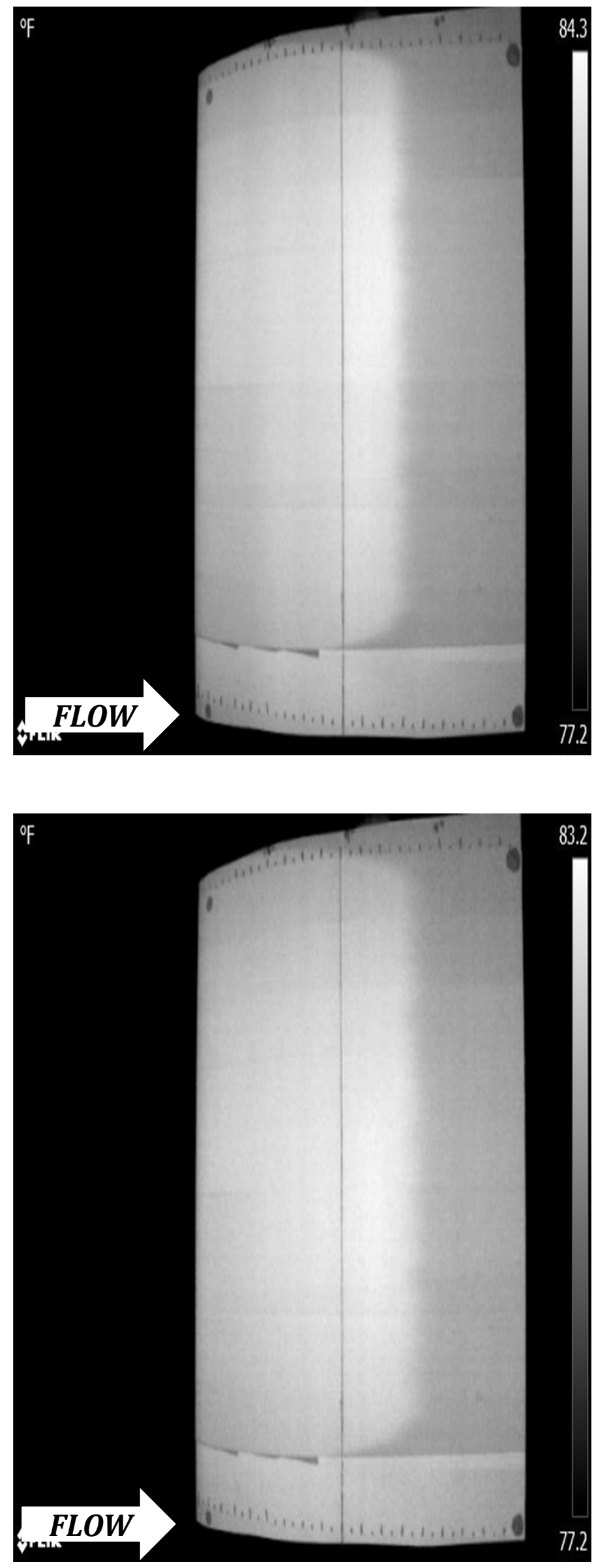


\subsection{6-m DU96-W-180 at $R e=1.5 \times 10^{5}$}

\section{PRESSURE SIDE}
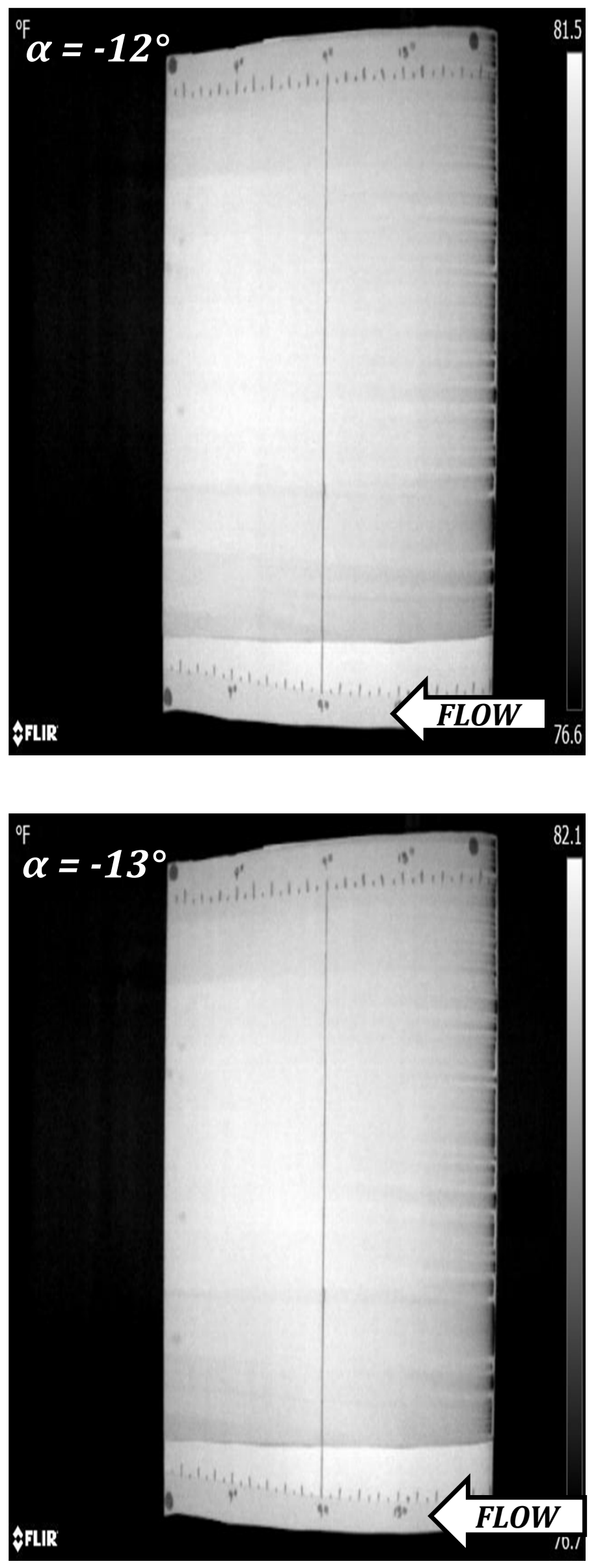

\section{SUCTION SIDE}
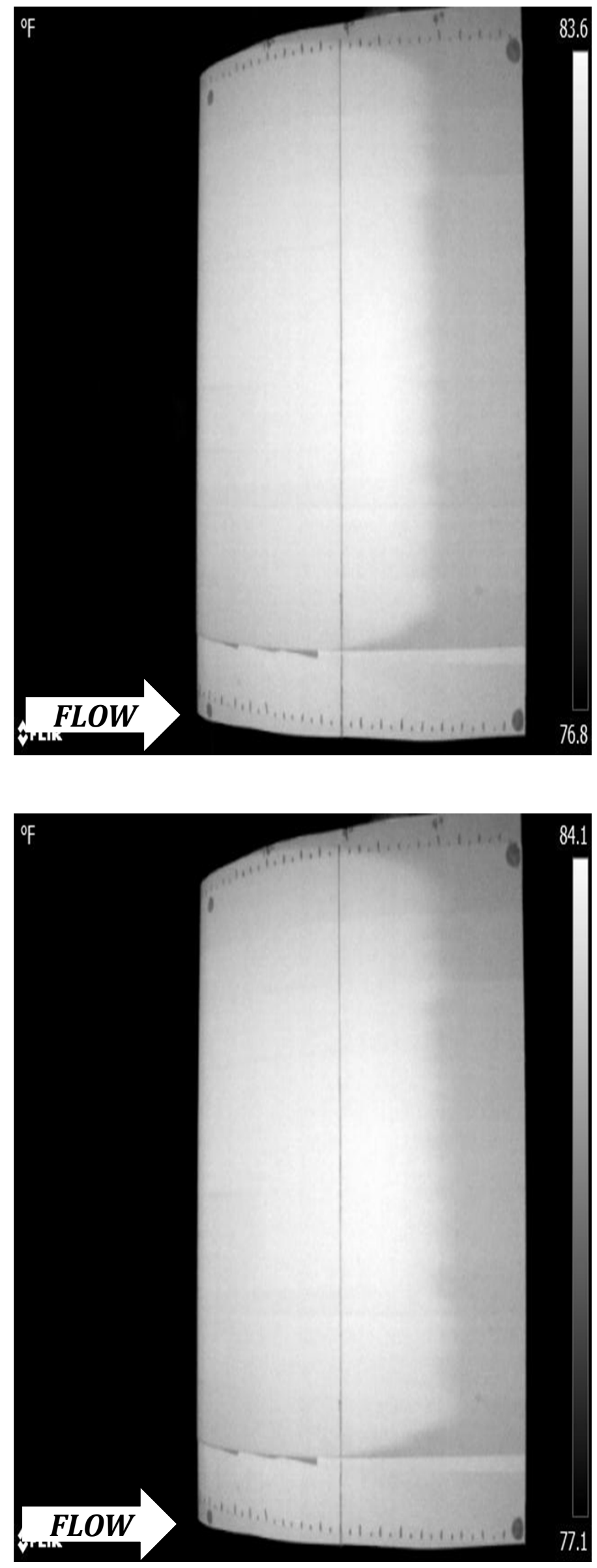


\subsection{6-m DU96-W-180 at $R e=1.5 \times 10^{5}$}

\section{PRESSURE SIDE}
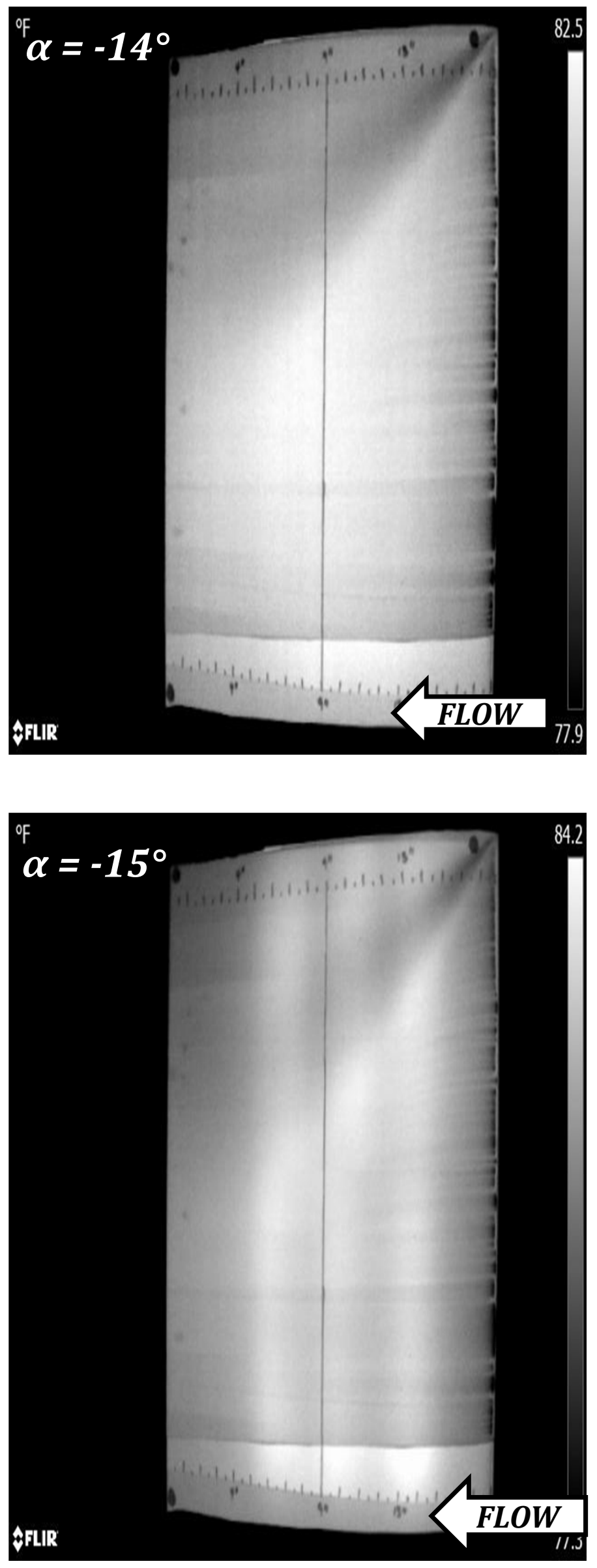

\section{SUCTION SIDE}
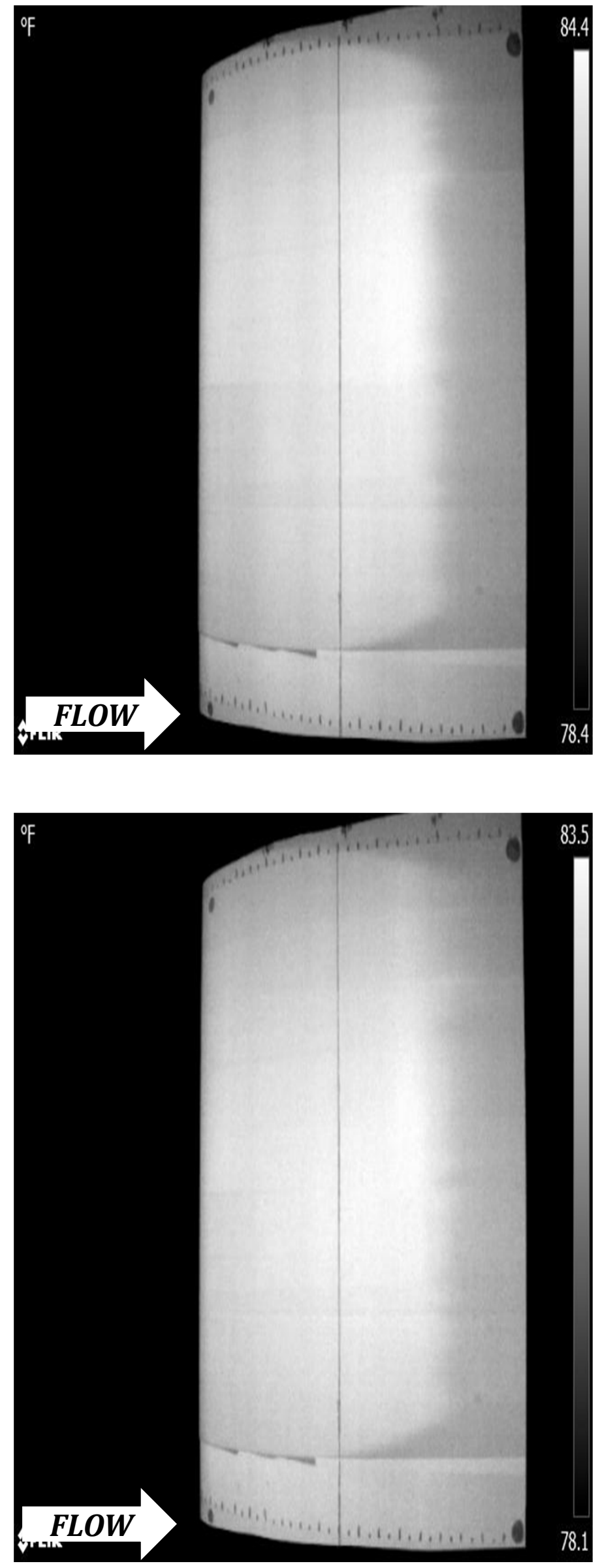


\subsection{6-m DU96-W-180 at $R e=1.5 \times 10^{5}$}

\section{PRESSURE SIDE}
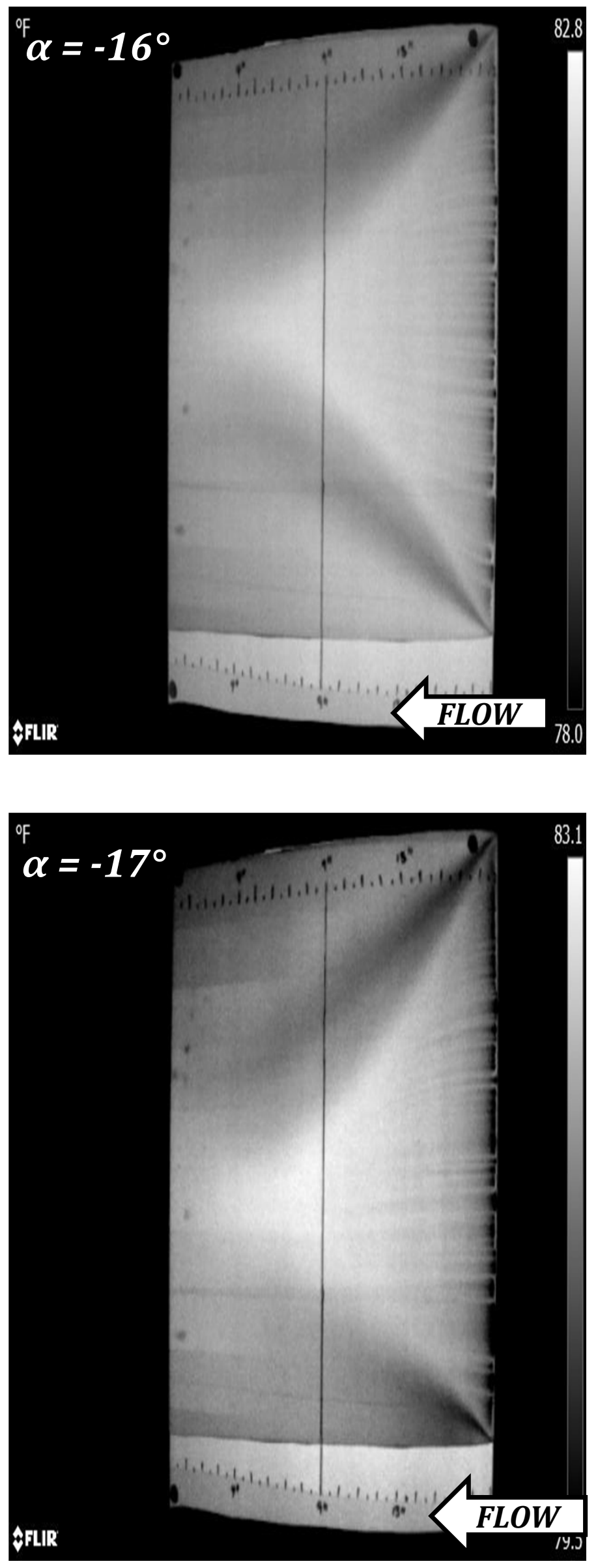

\section{SUCTION SIDE}
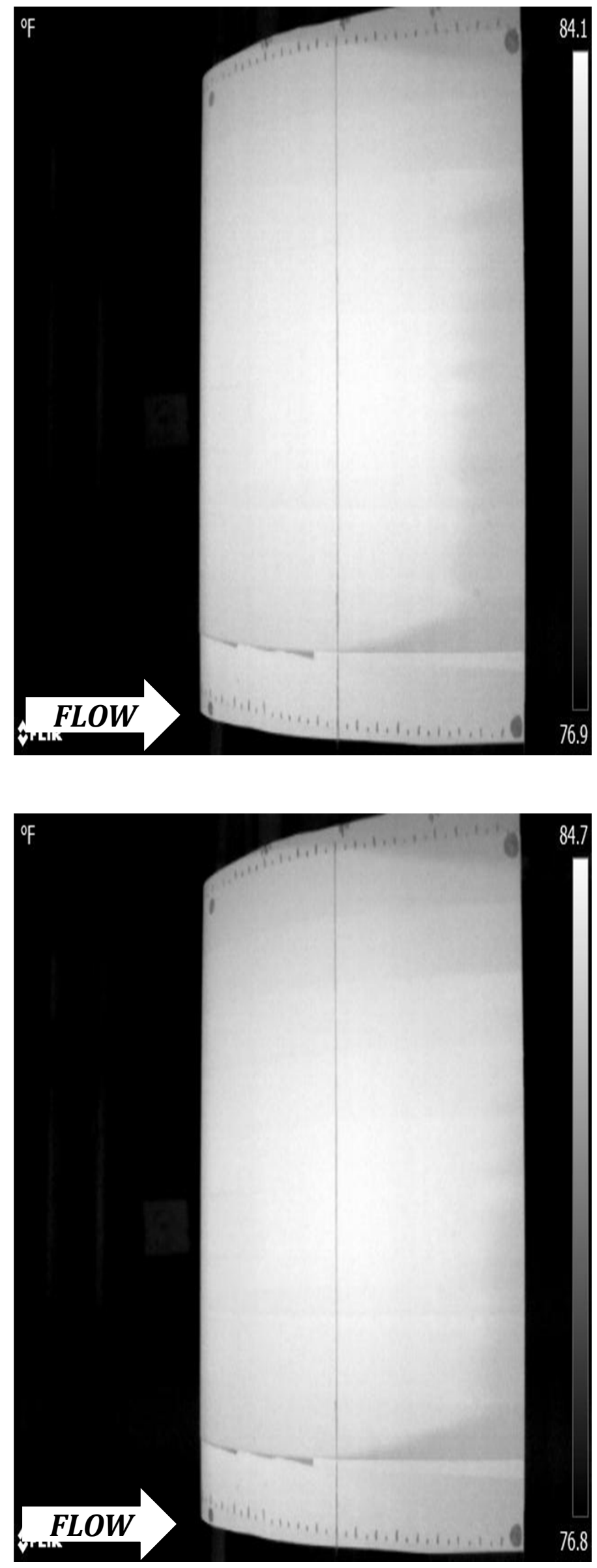


\subsection{6-m DU96-W-180 at $R e=1.5 \times 10^{5}$}

\section{PRESSURE SIDE}
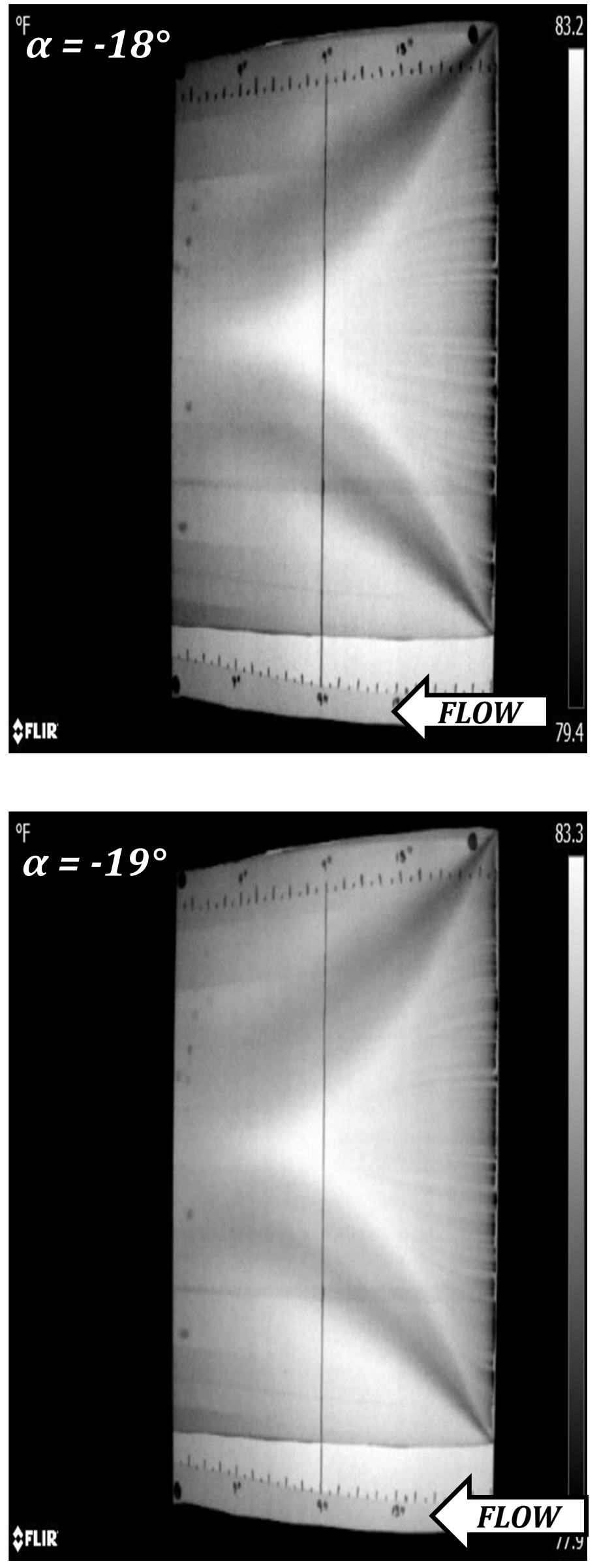

\section{SUCTION SIDE}
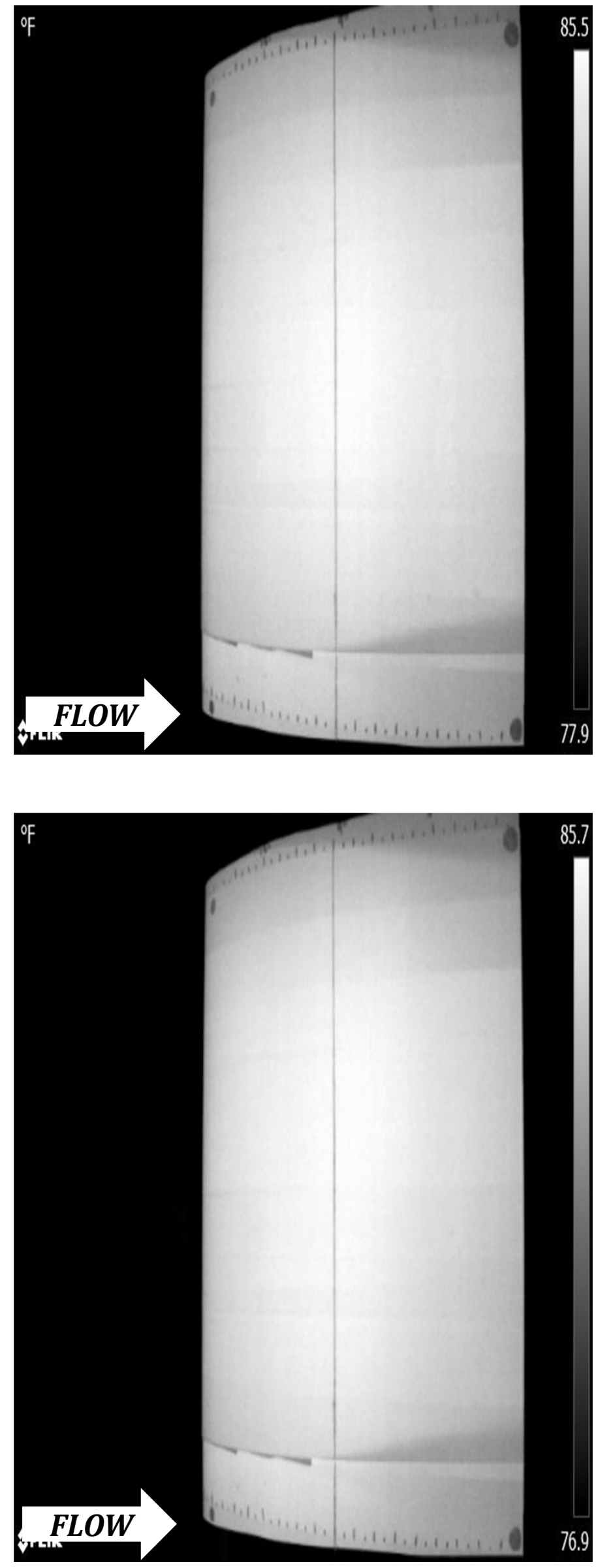


\section{$0.46-m$ DU96-W-180 at $R e=1.5 \times 10^{5}$}

\section{PRESSURE SIDE}

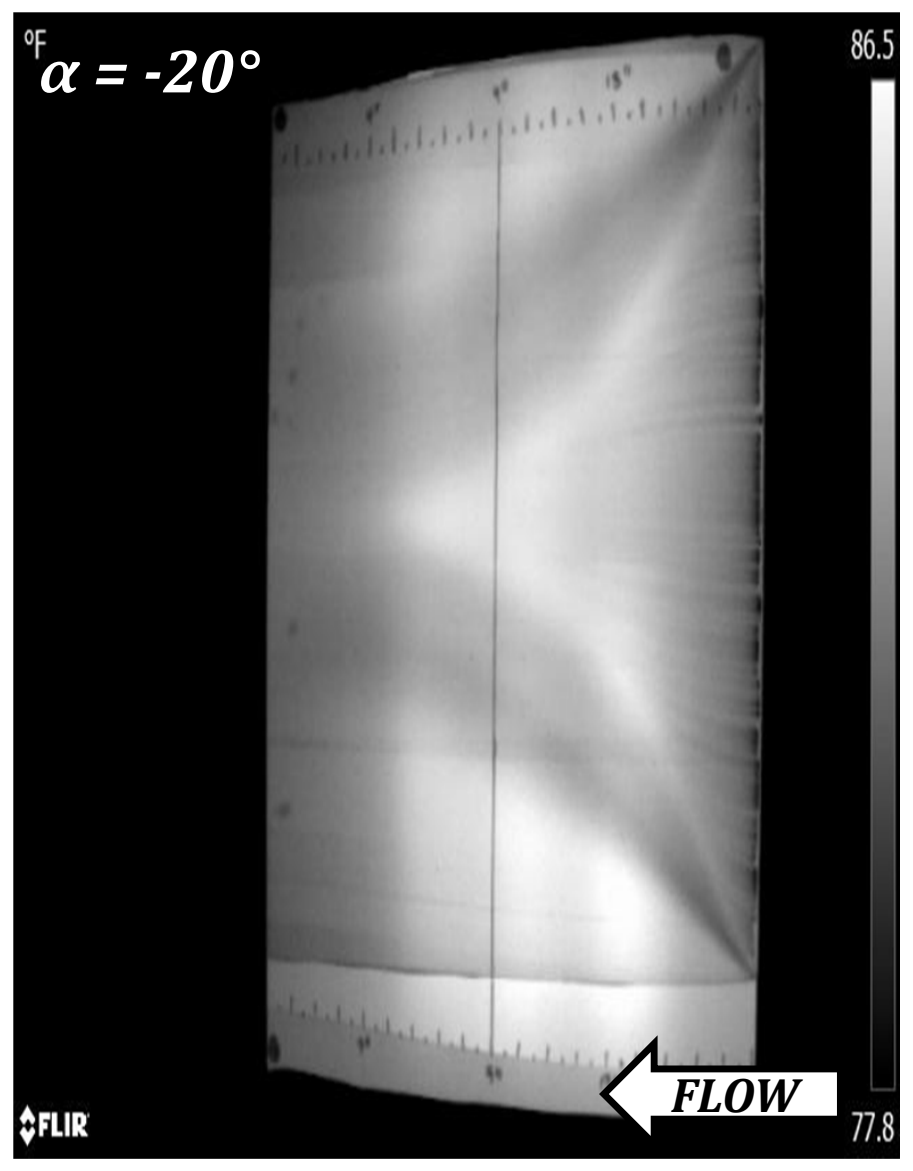

SUCTION SIDE

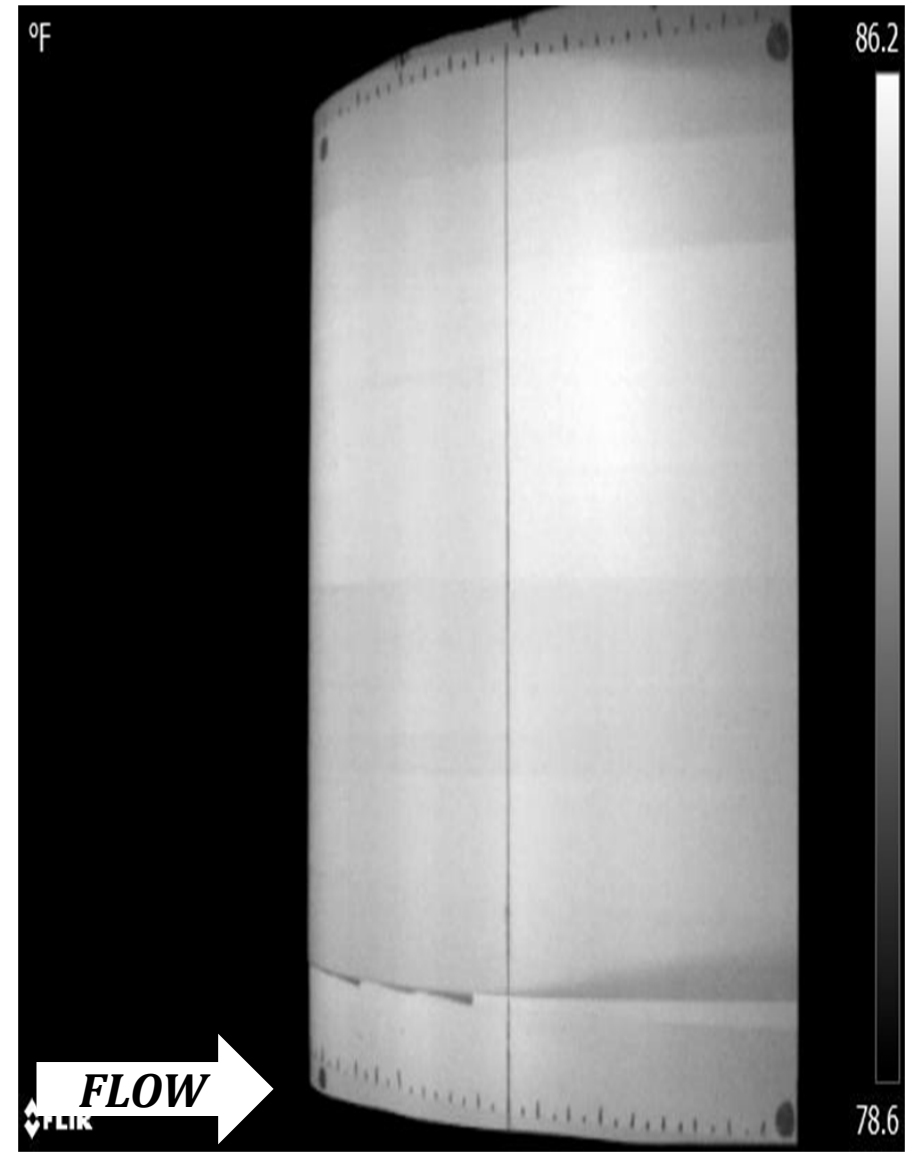


Appendix H Infrared Images of 0.46-m DU96-W-180 at Re $=2 \times 105$

(From $\alpha=20^{\circ}$ to -20 , Pressure Side Images are on Left and Suction Side Images are on Right.) 


\subsection{6-m DU96-W-180 at $R e=2 \times 10^{5}$}

\section{PRESSURE SIDE}
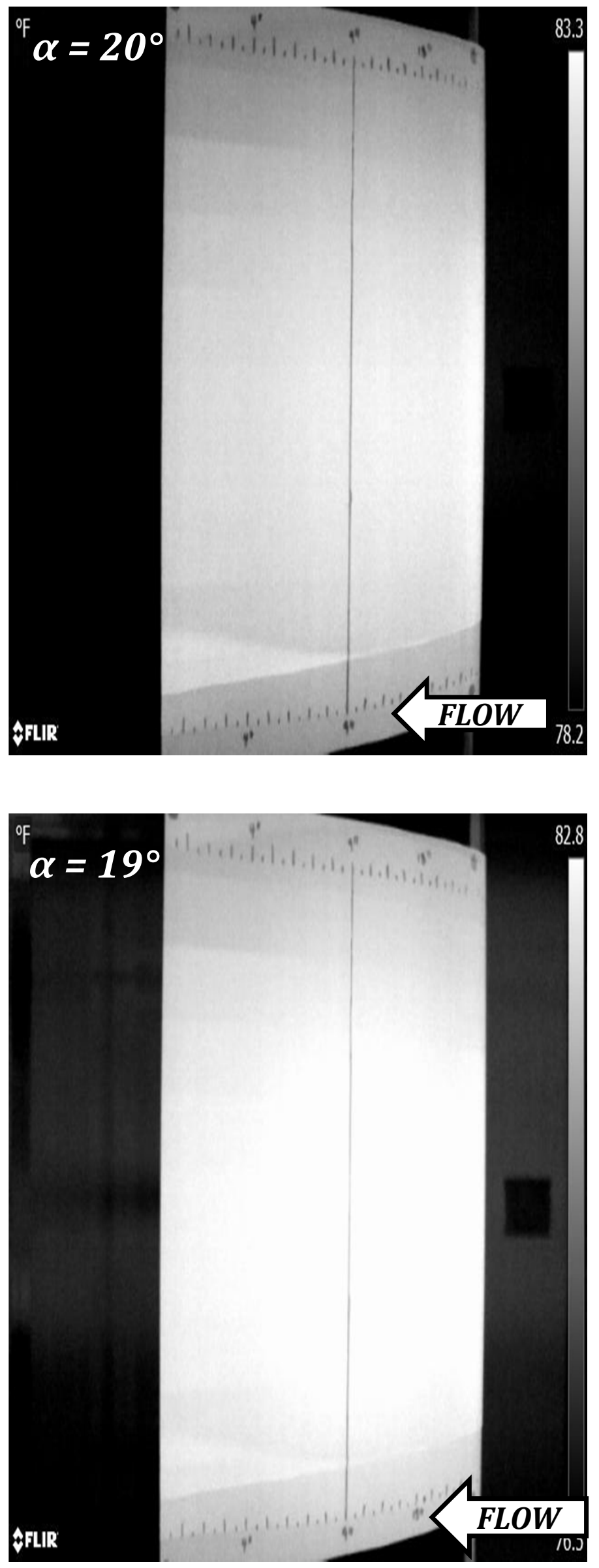

SUCTION SIDE
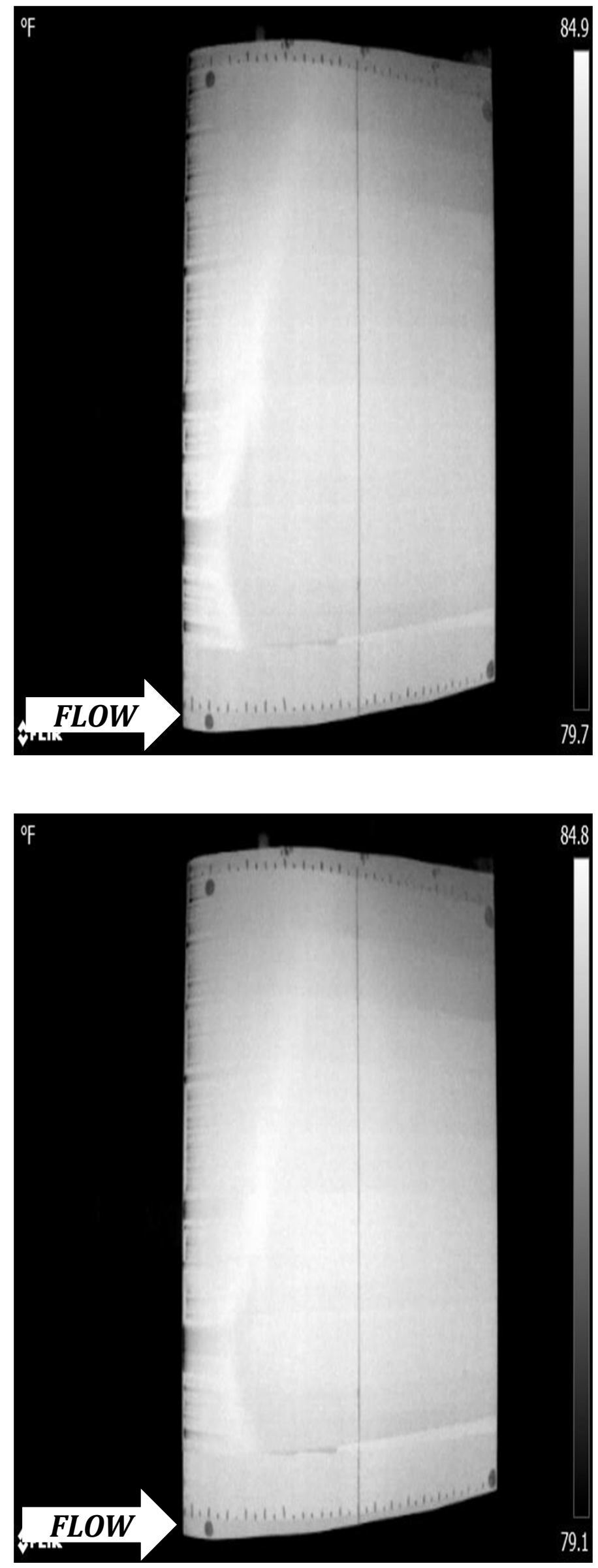


\subsection{6-m DU96-W-180 at $R e=2 \times 10^{5}$}

\section{PRESSURE SIDE}
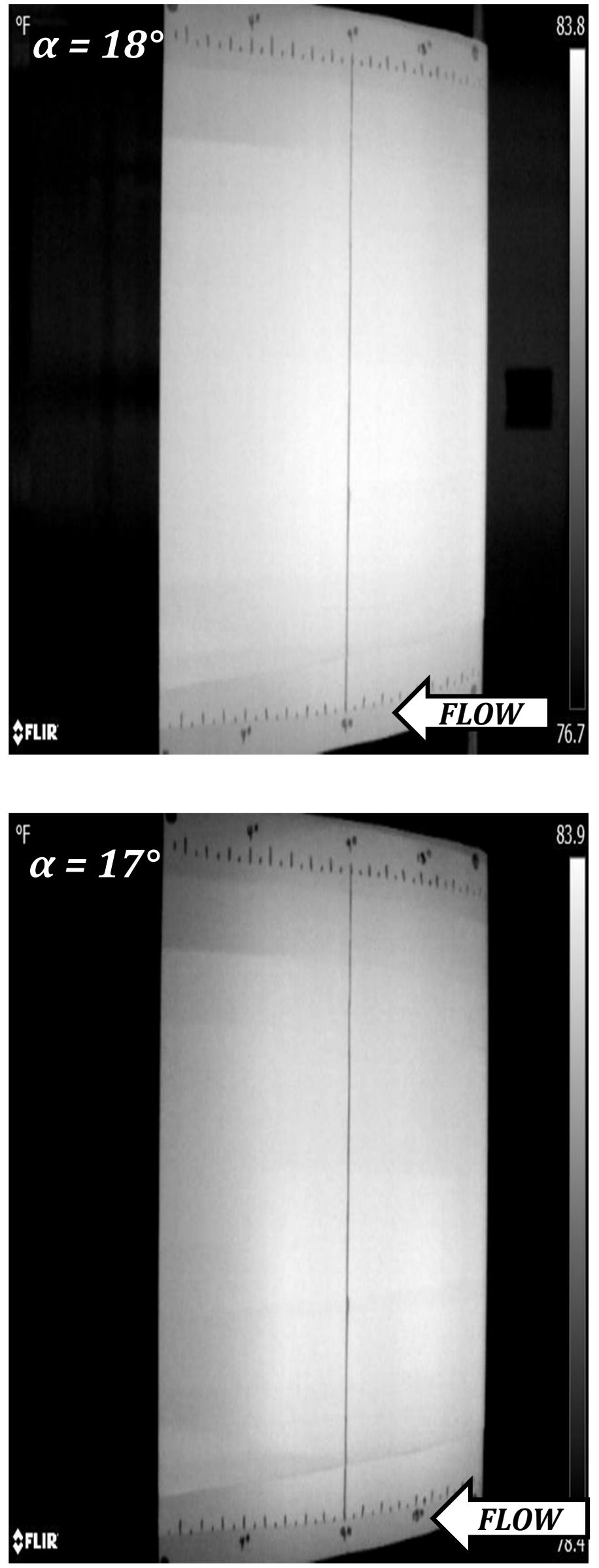

SUCTION SIDE
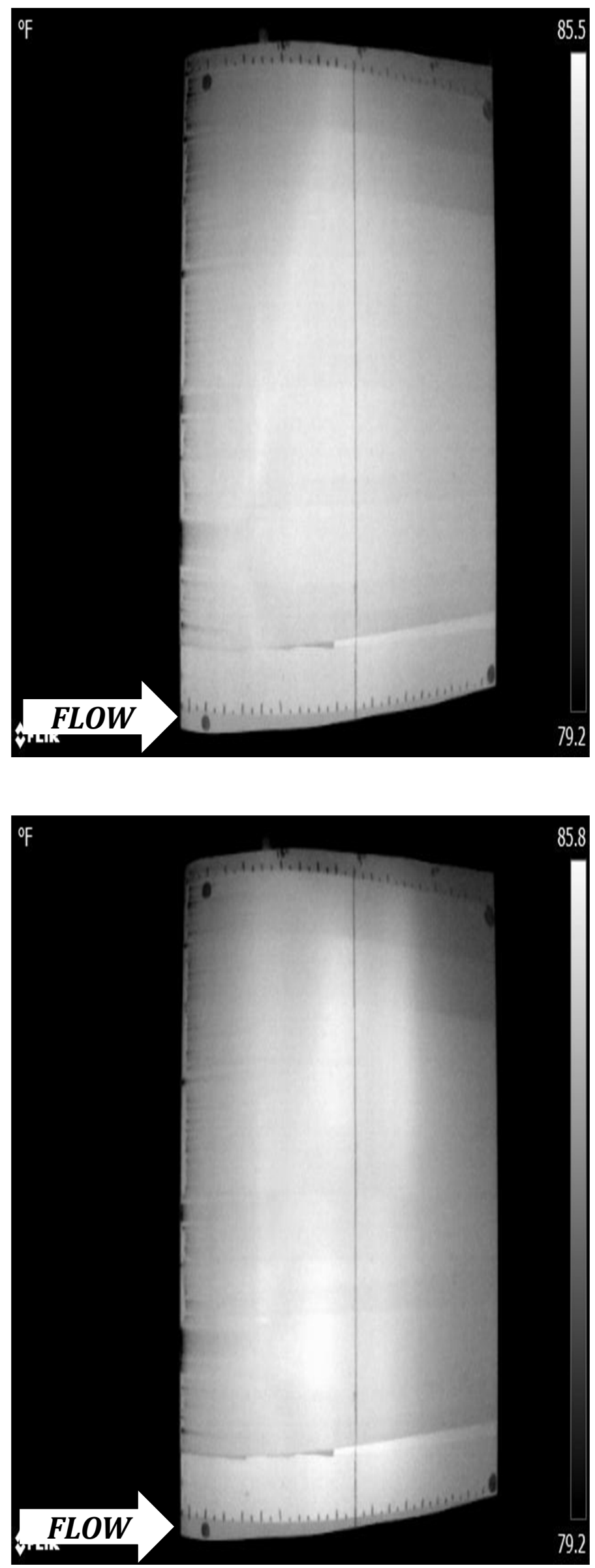


\subsection{6-m DU96-W-180 at Re $=2 \times 10^{5}$}

\section{PRESSURE SIDE}
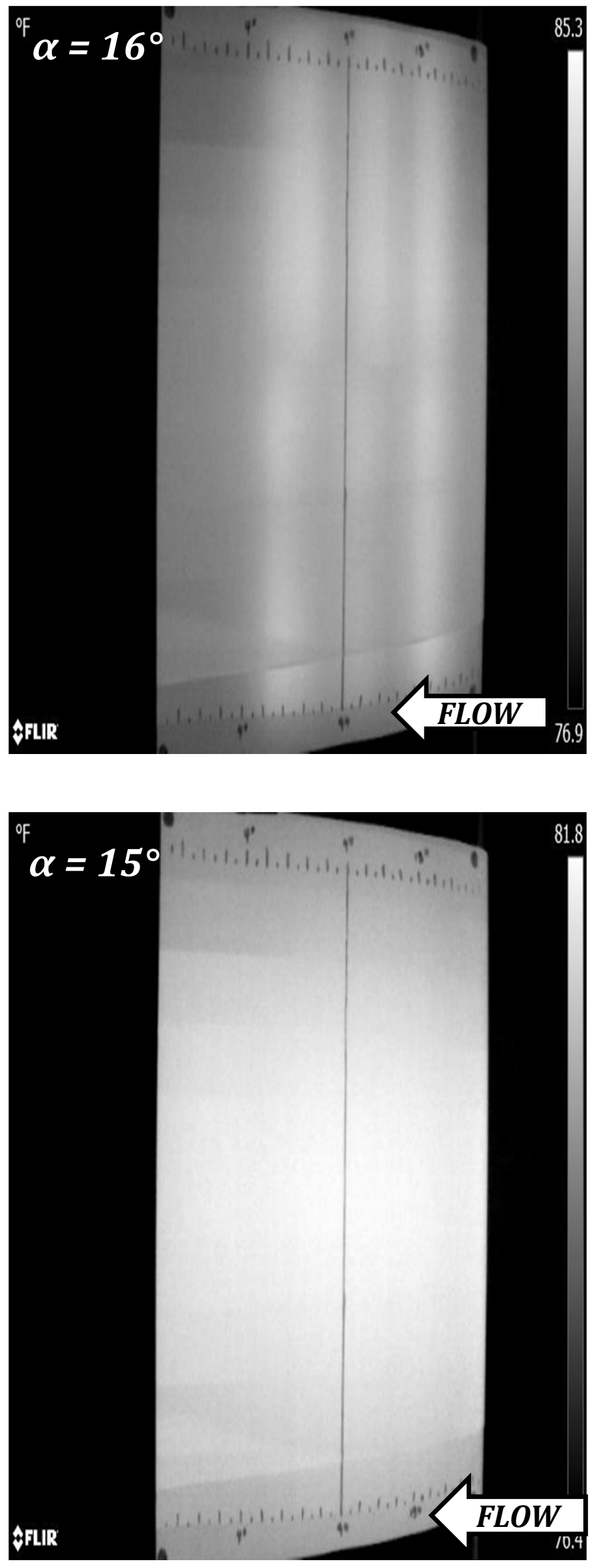

\section{SUCTION SIDE}
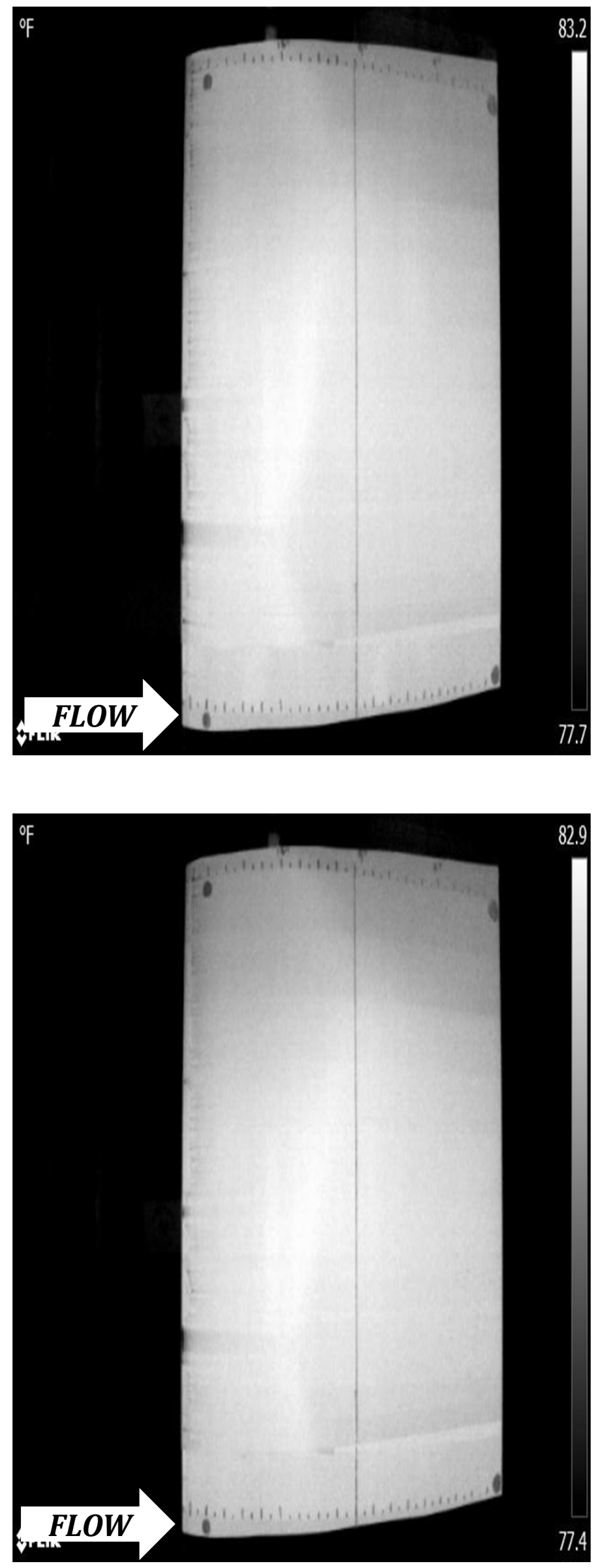


\subsection{6-m DU96-W-180 at Re $=2 \times 10^{5}$}

\section{PRESSURE SIDE}
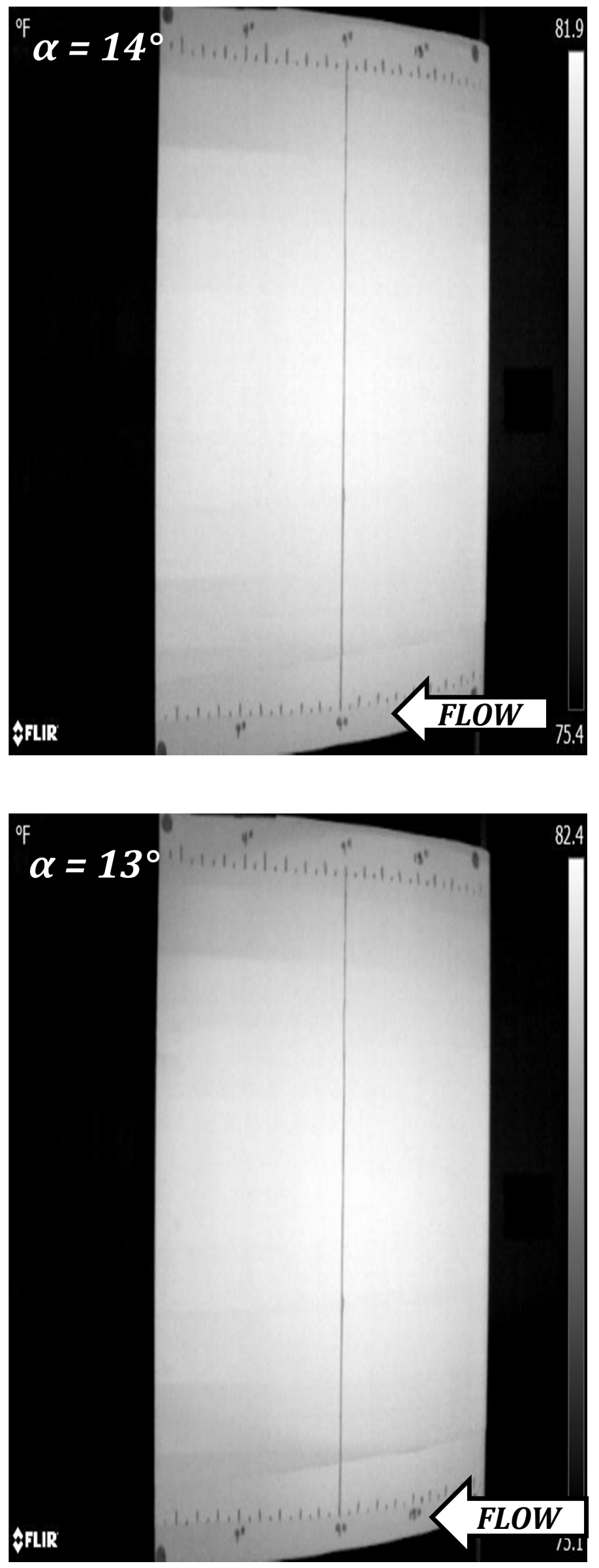

SUCTION SIDE
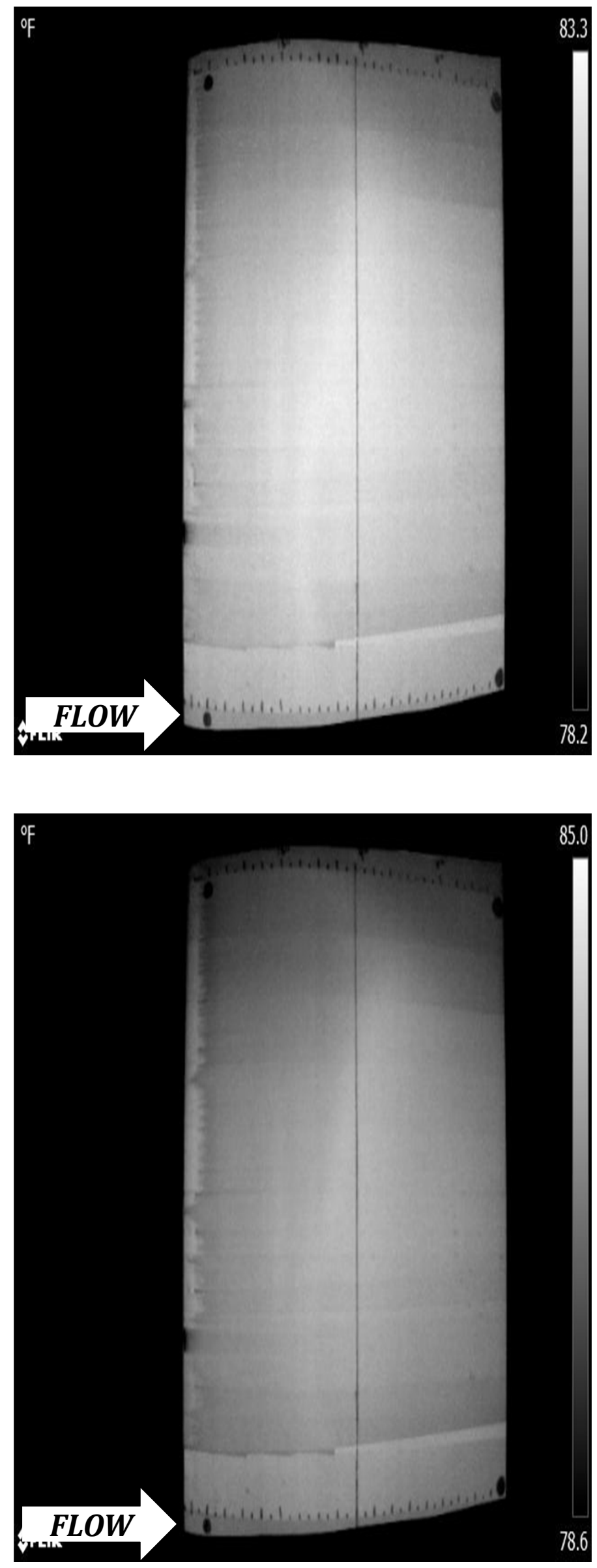


\subsection{6- $\mathrm{m}$ DU96-W-180 at $R e=2 \times 10^{5}$}

\section{PRESSURE SIDE}
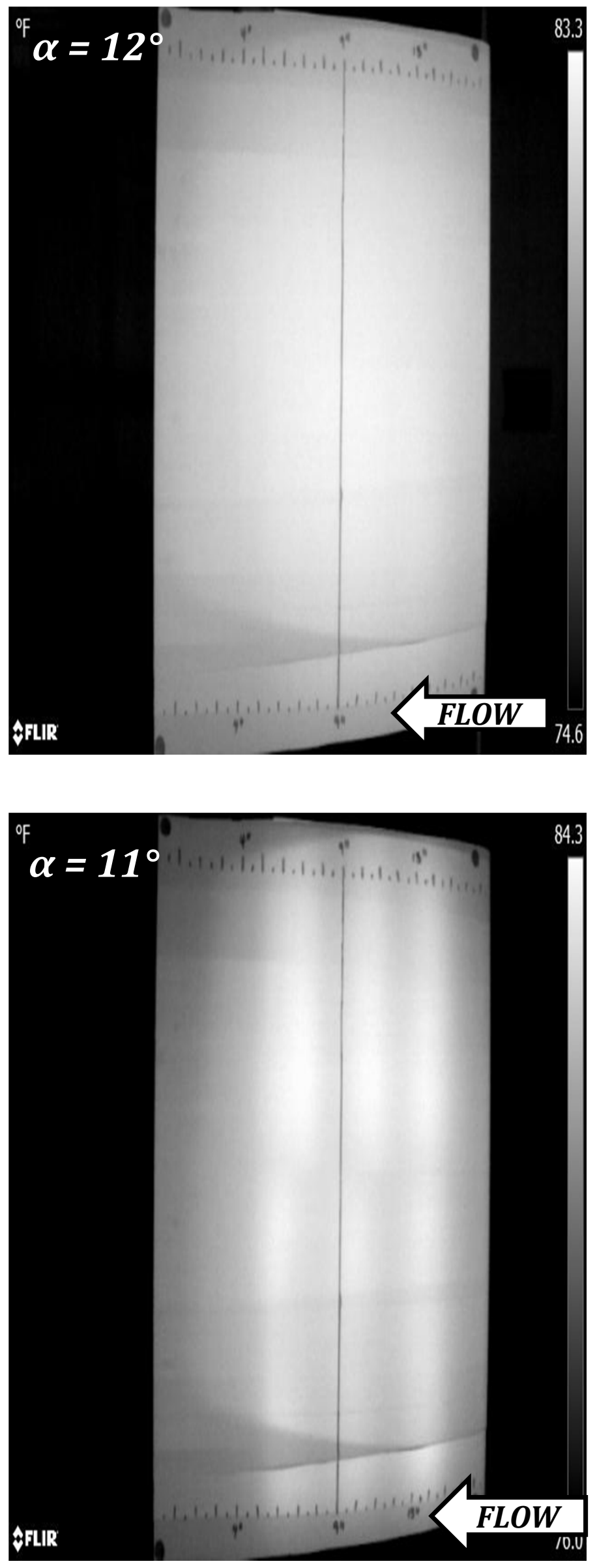

SUCTION SIDE
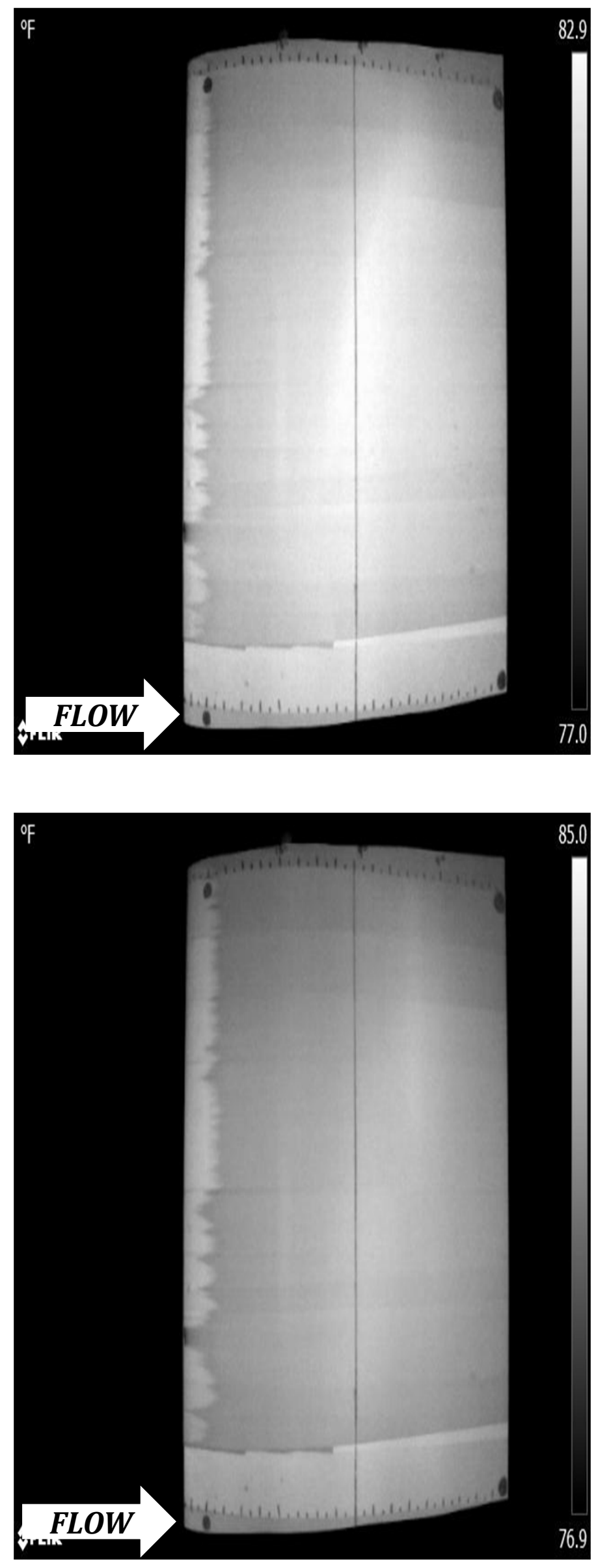


\subsection{6- $\mathrm{m}$ DU96-W-180 at $R e=2 \times 10^{5}$}

\section{PRESSURE SIDE}
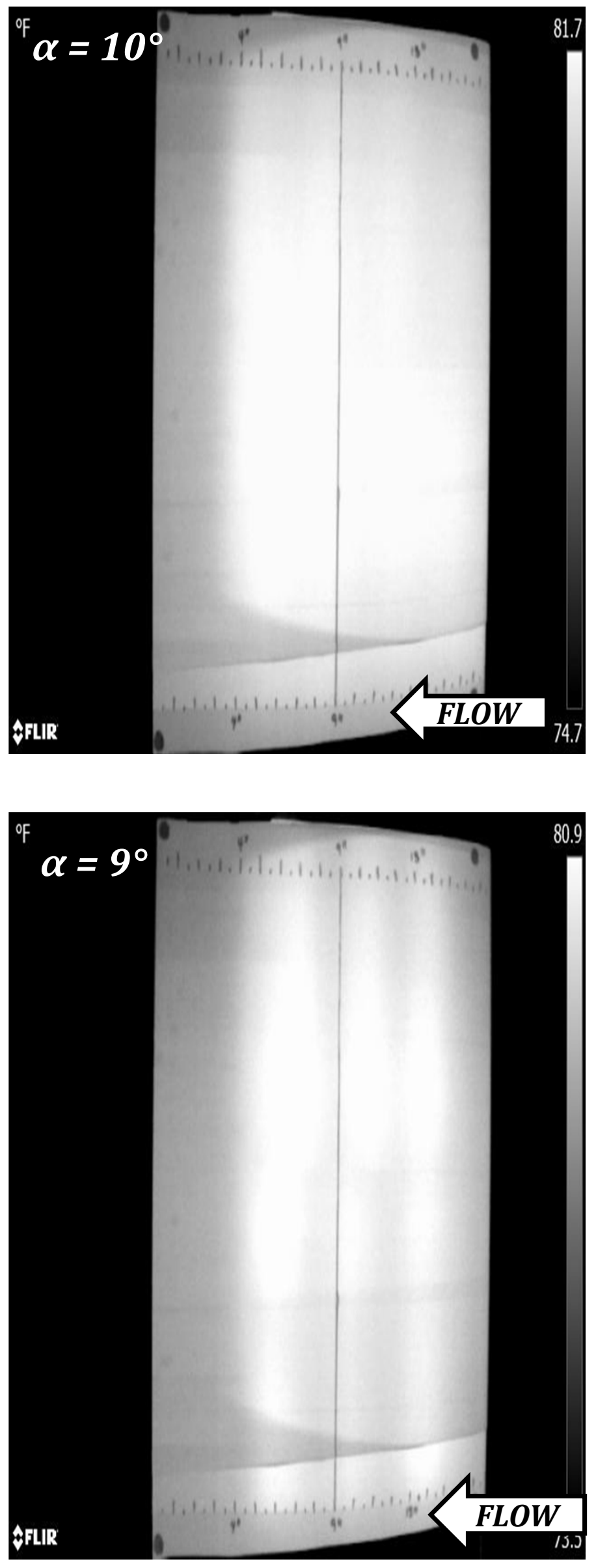

\section{SUCTION SIDE}
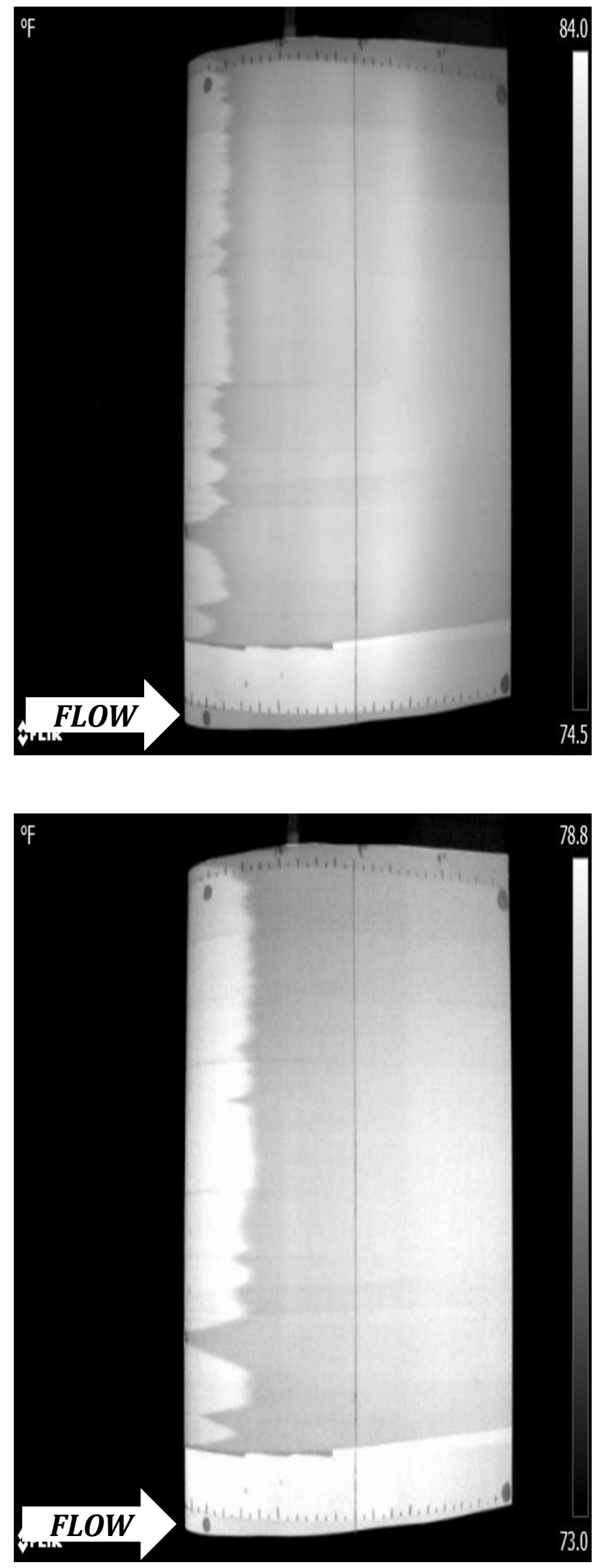


\subsection{6- $\mathrm{m}$ DU96-W-180 at $R e=2 \times 10^{5}$}

\section{PRESSURE SIDE}
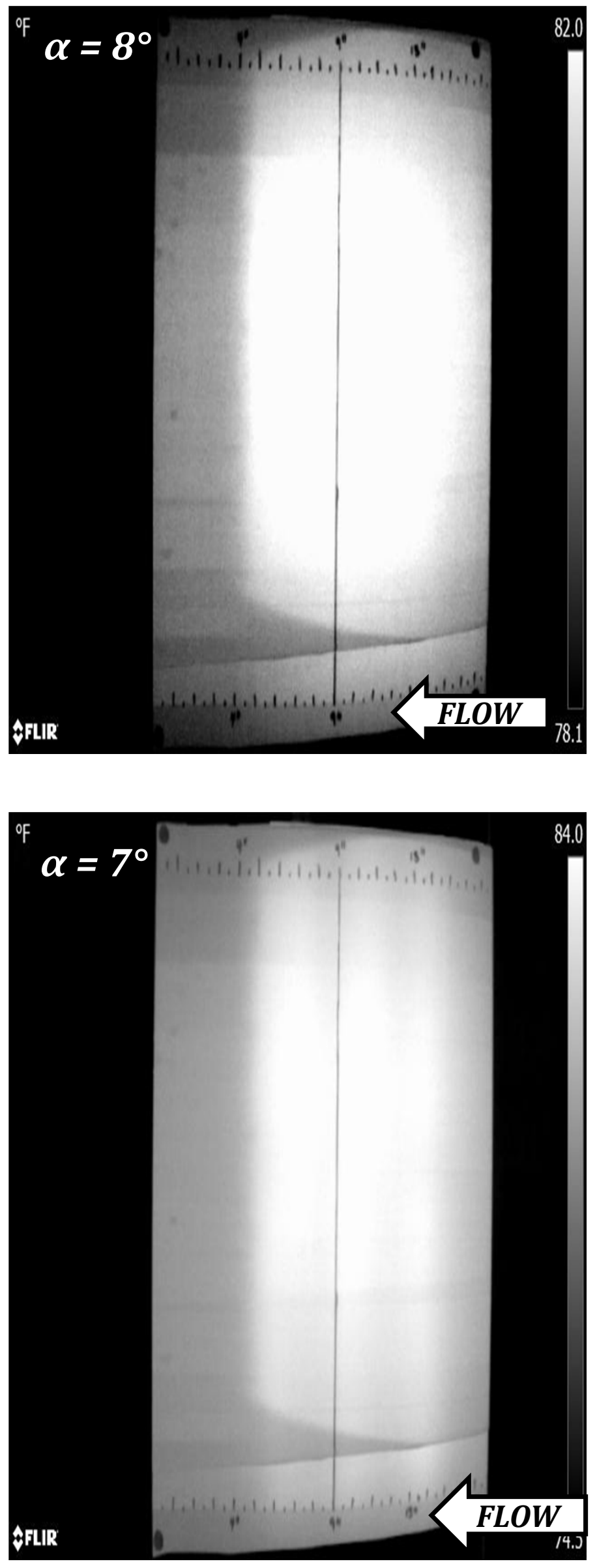

\section{SUCTION SIDE}
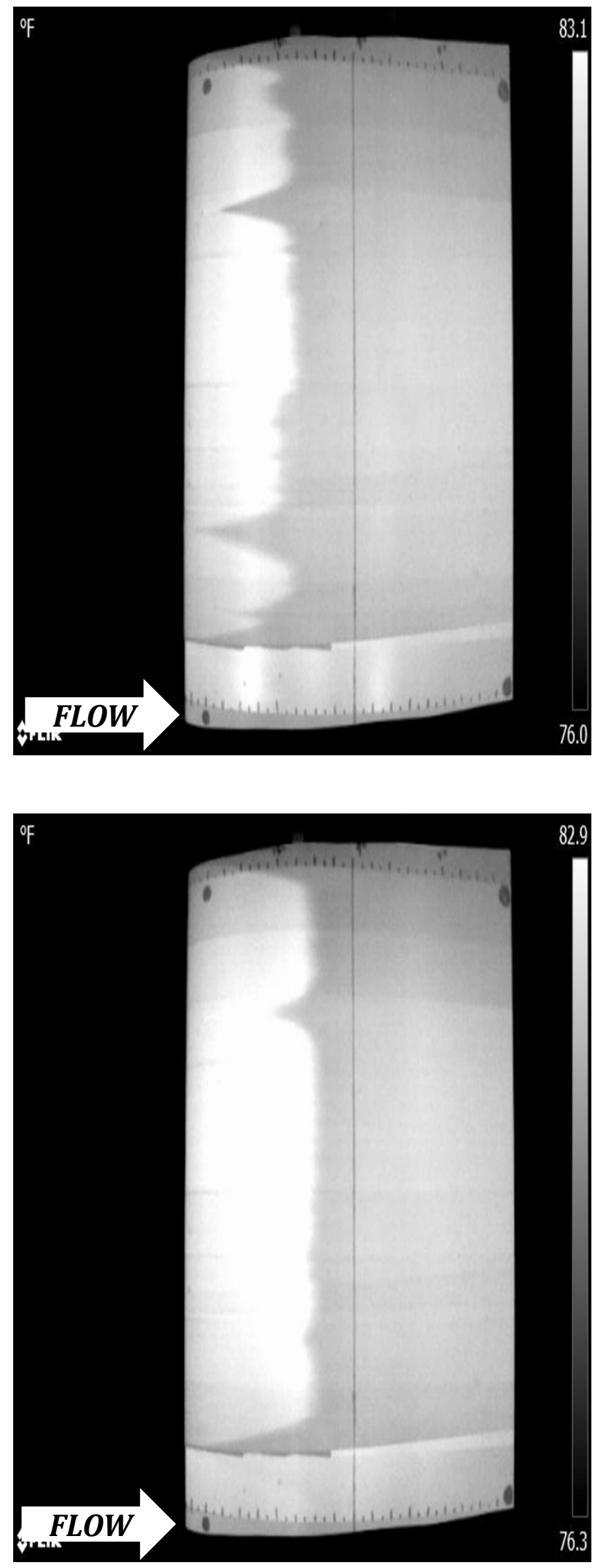


\subsection{6- $\mathrm{m}$ DU96-W-180 at $R e=2 \times 10^{5}$}

\section{PRESSURE SIDE}
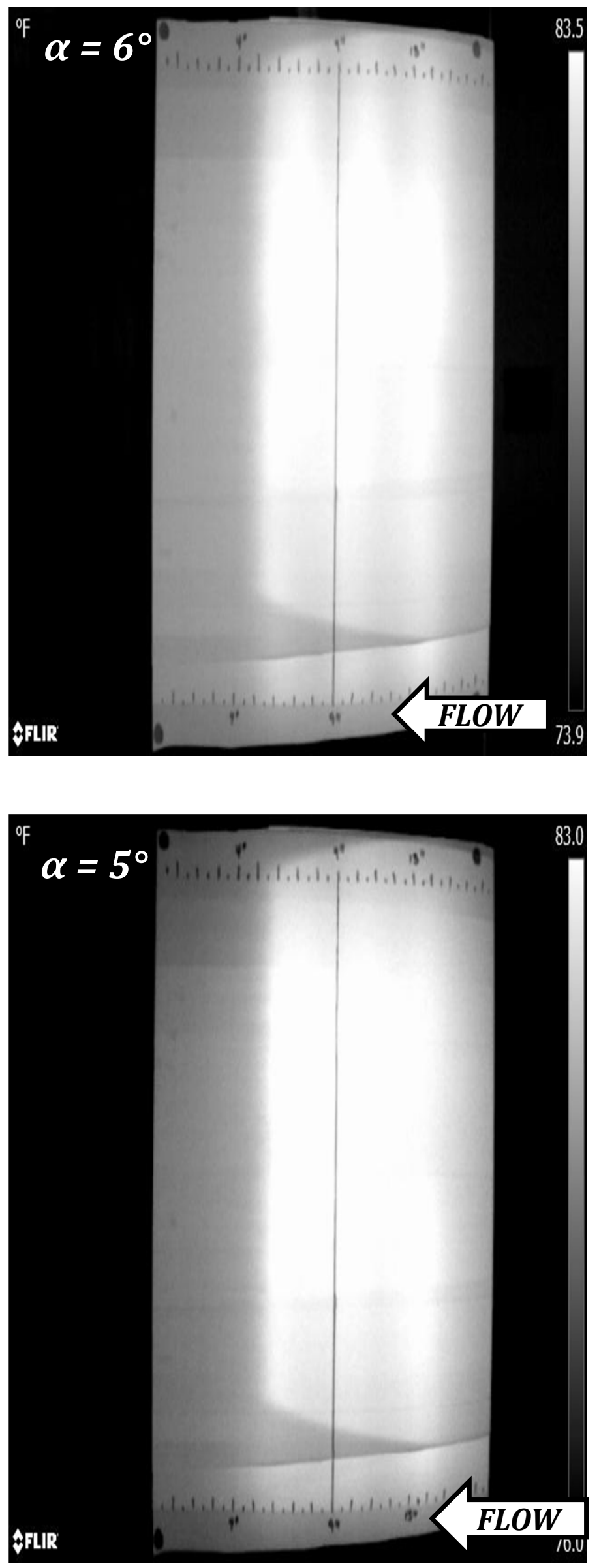

SUCTION SIDE
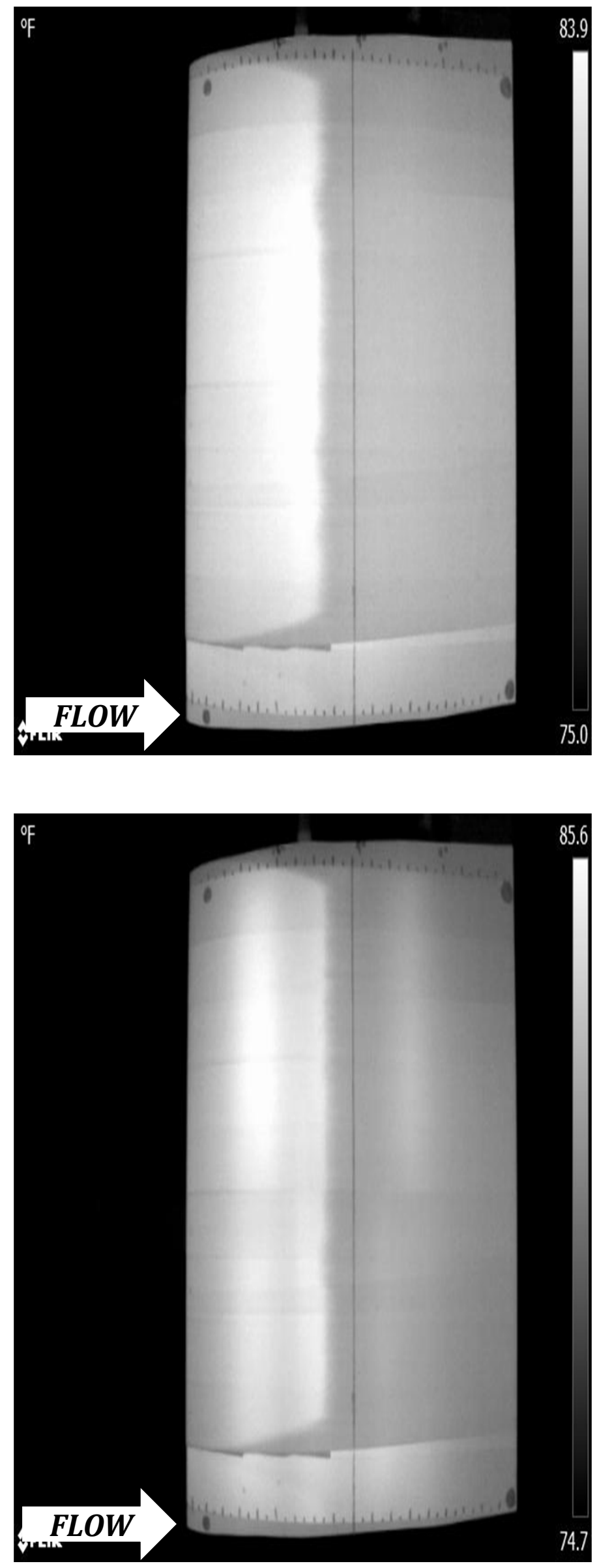


\subsection{6- $\mathrm{m}$ DU96-W-180 at $R e=2 \times 10^{5}$}

\section{PRESSURE SIDE}
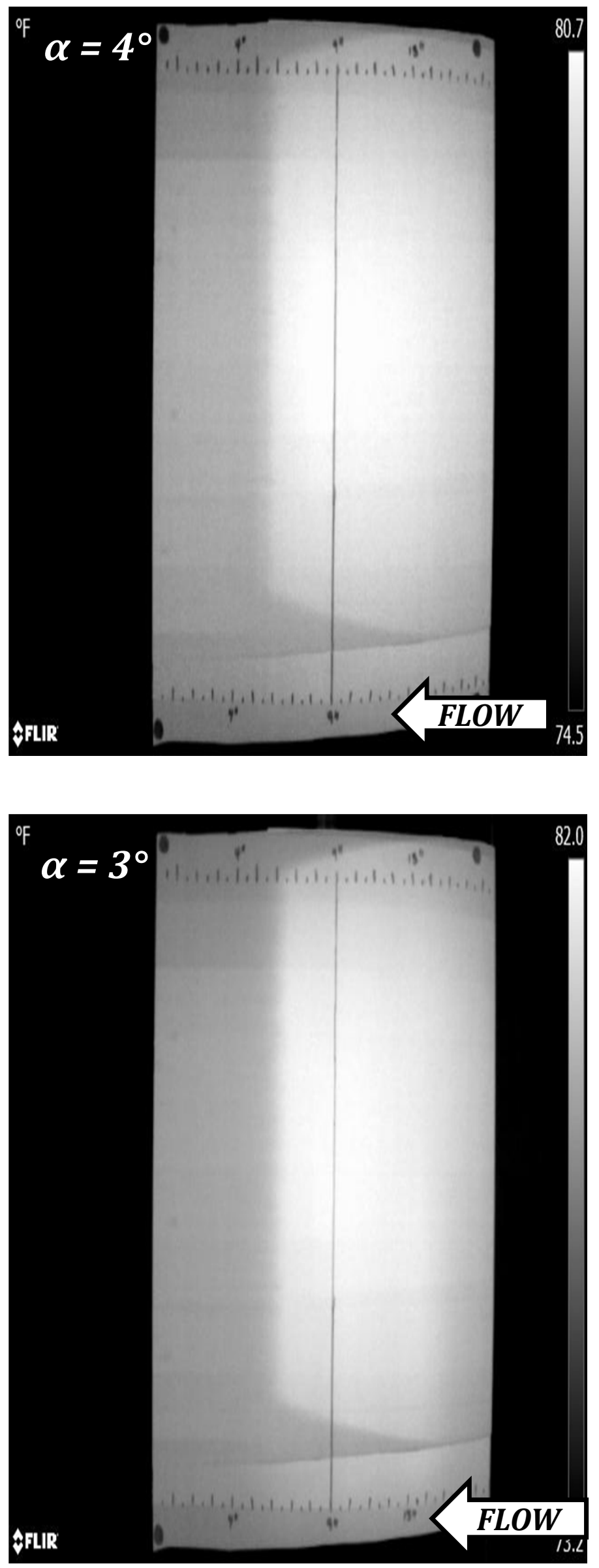

SUCTION SIDE
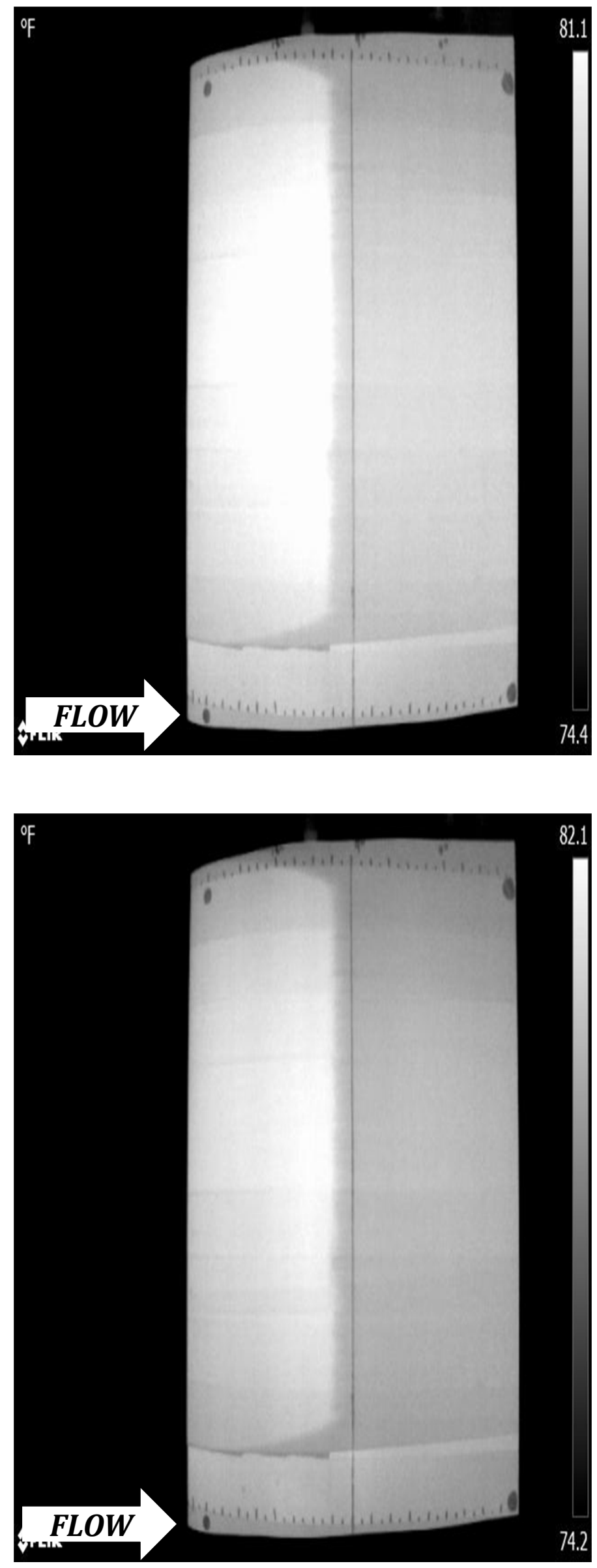


\subsection{6-m DU96-W-180 at Re $=2 \times 10^{5}$}

\section{PRESSURE SIDE}
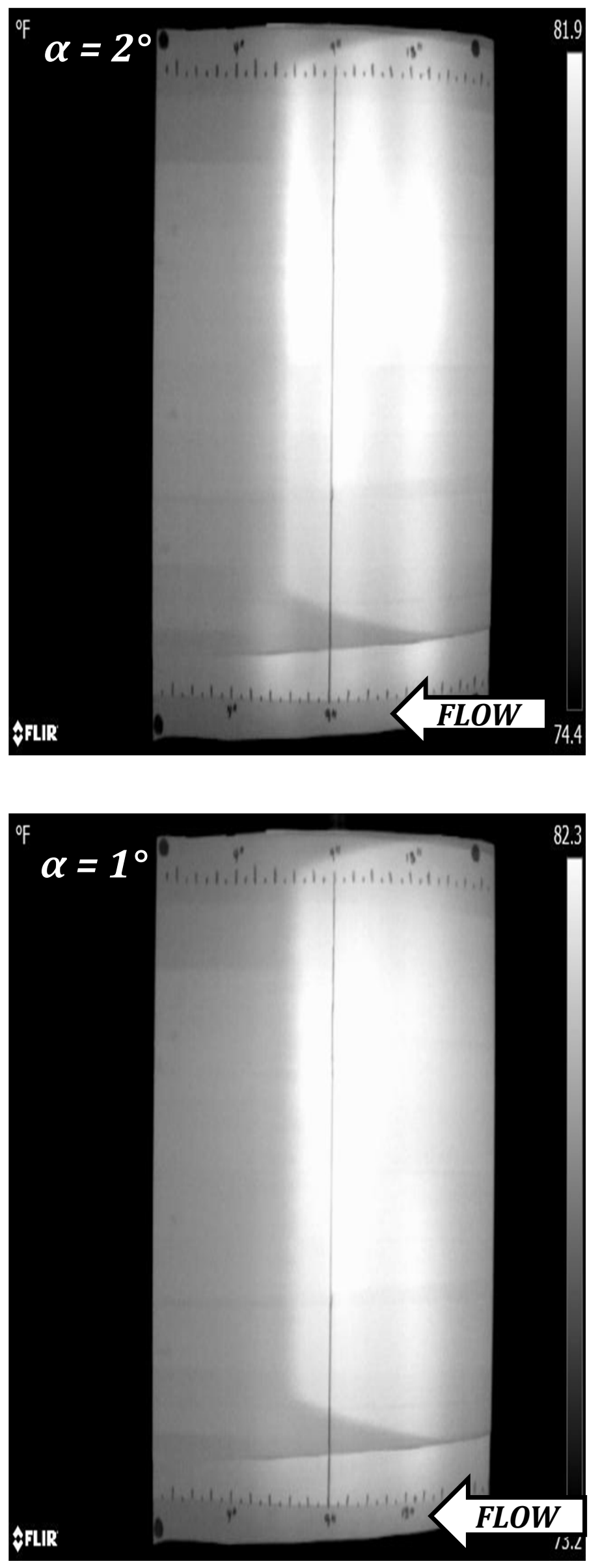

SUCTION SIDE
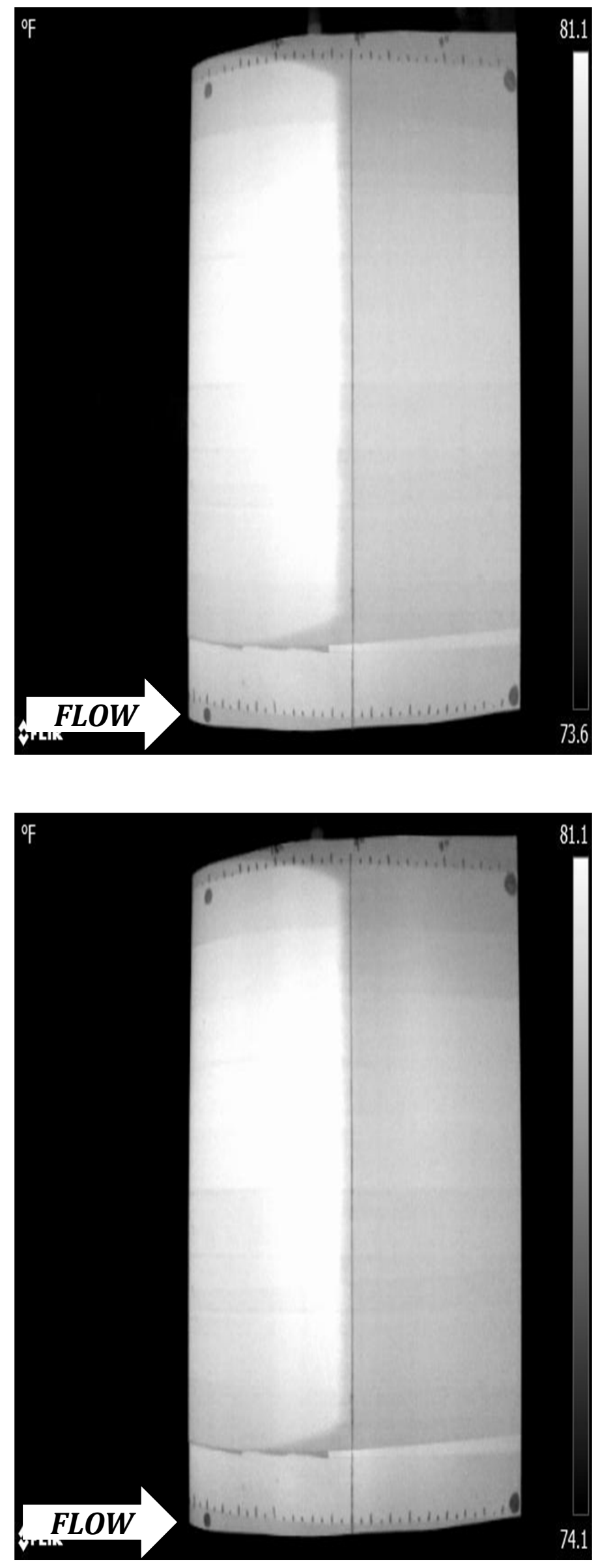


\subsection{6- $m$ DU96-W-180 at Re $=2 \times 10^{5}$}

\section{PRESSURE SIDE}
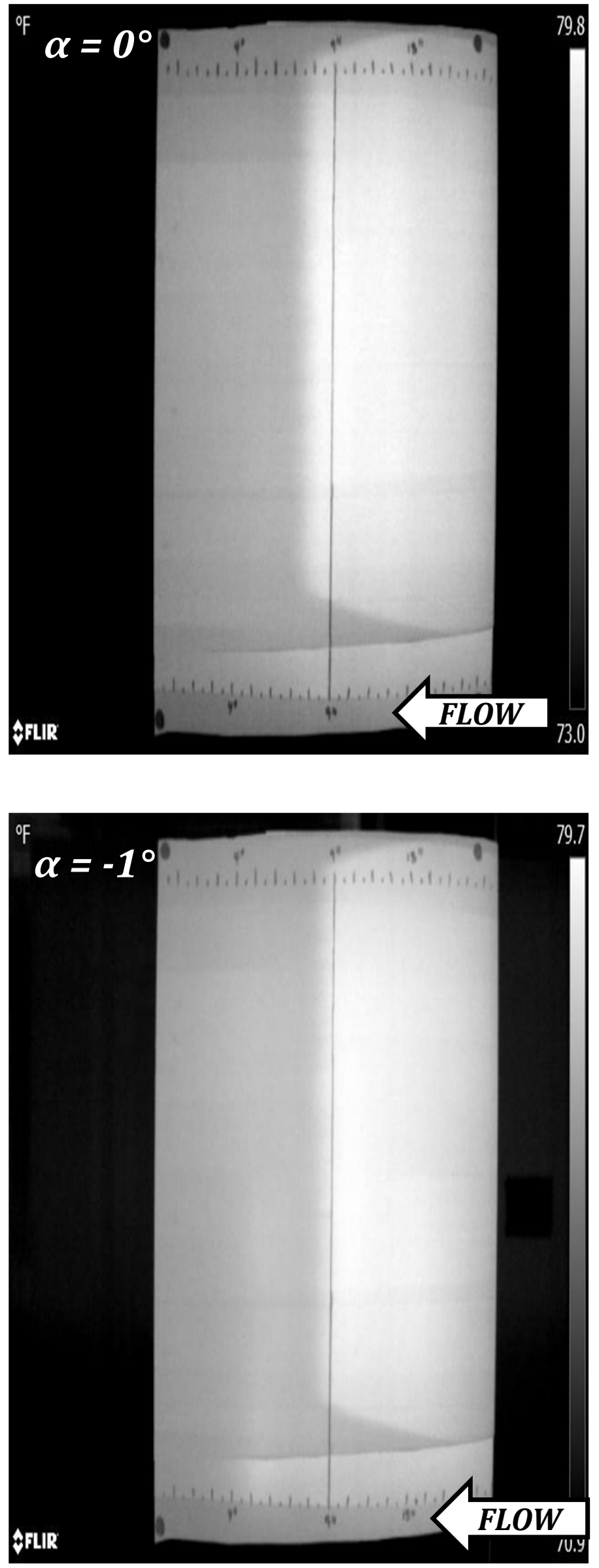

SUCTION SIDE
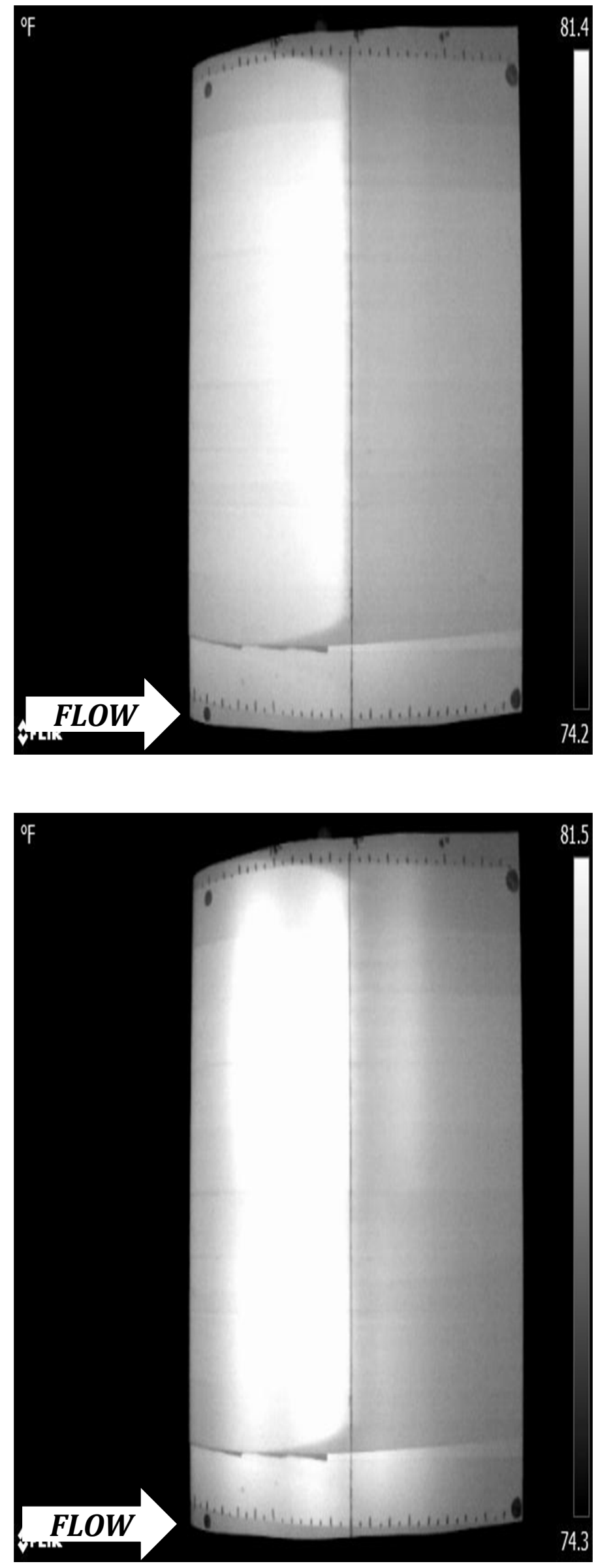


\subsection{6-m DU96-W-180 at $R e=2 \times 10^{5}$}

\section{PRESSURE SIDE}
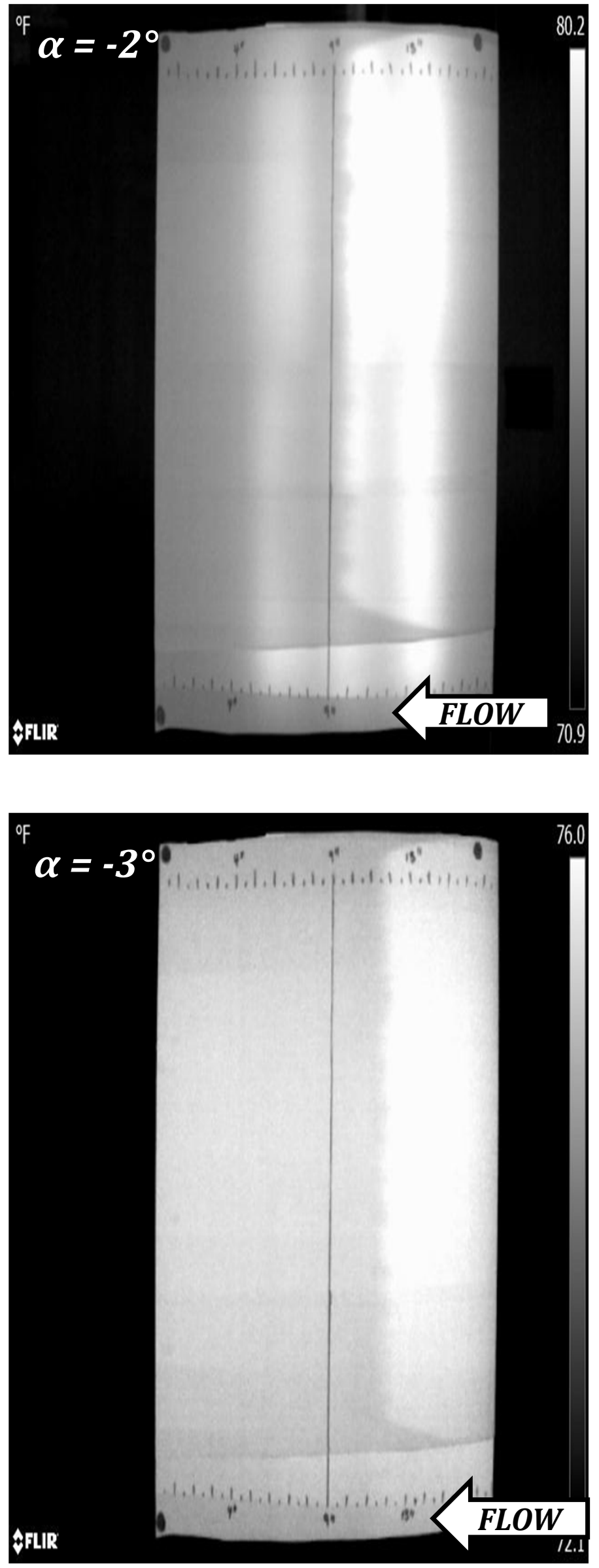

SUCTION SIDE
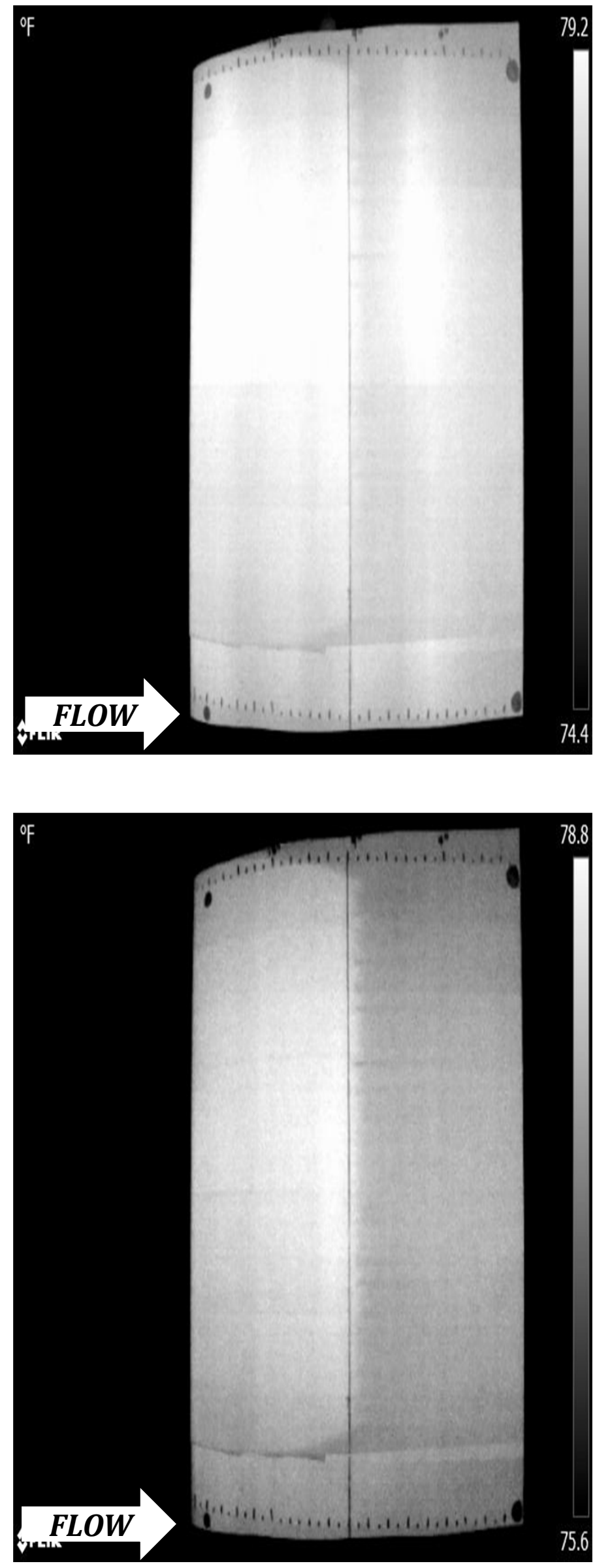


\subsection{6-m DU96-W-180 at $R e=2 \times 10^{5}$}

\section{PRESSURE SIDE}
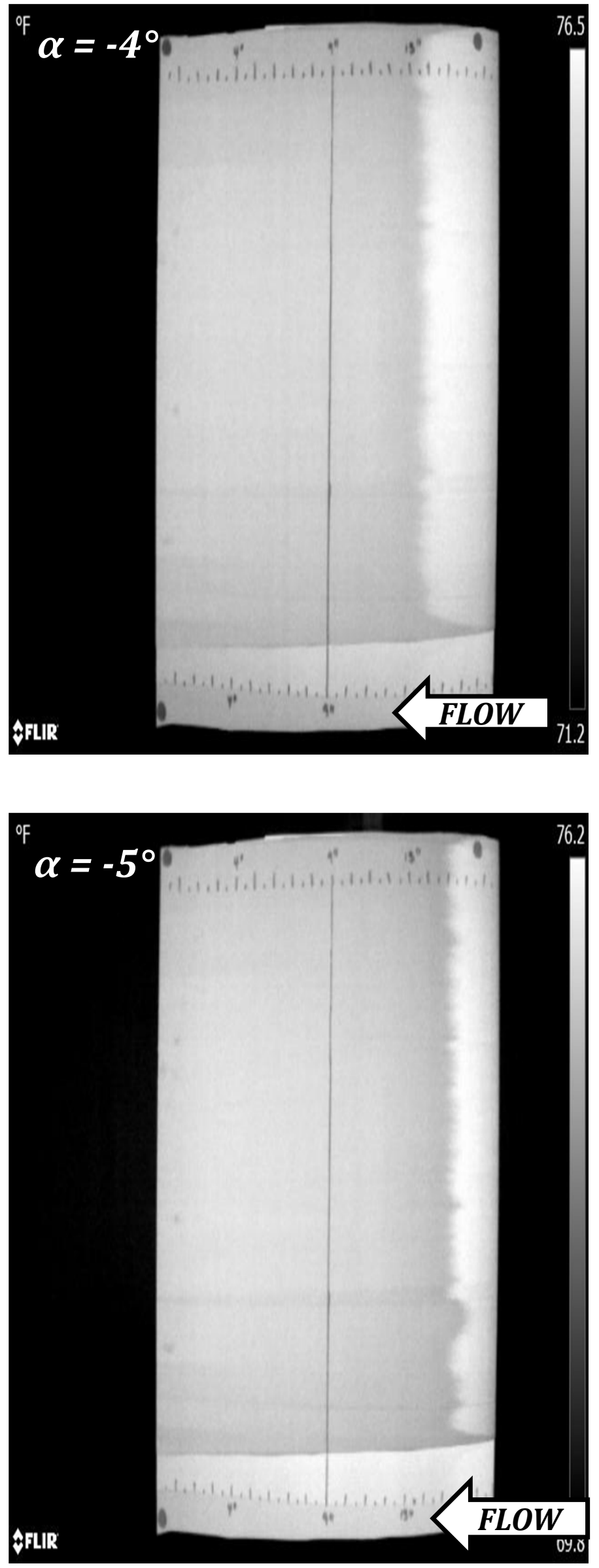

\section{SUCTION SIDE}
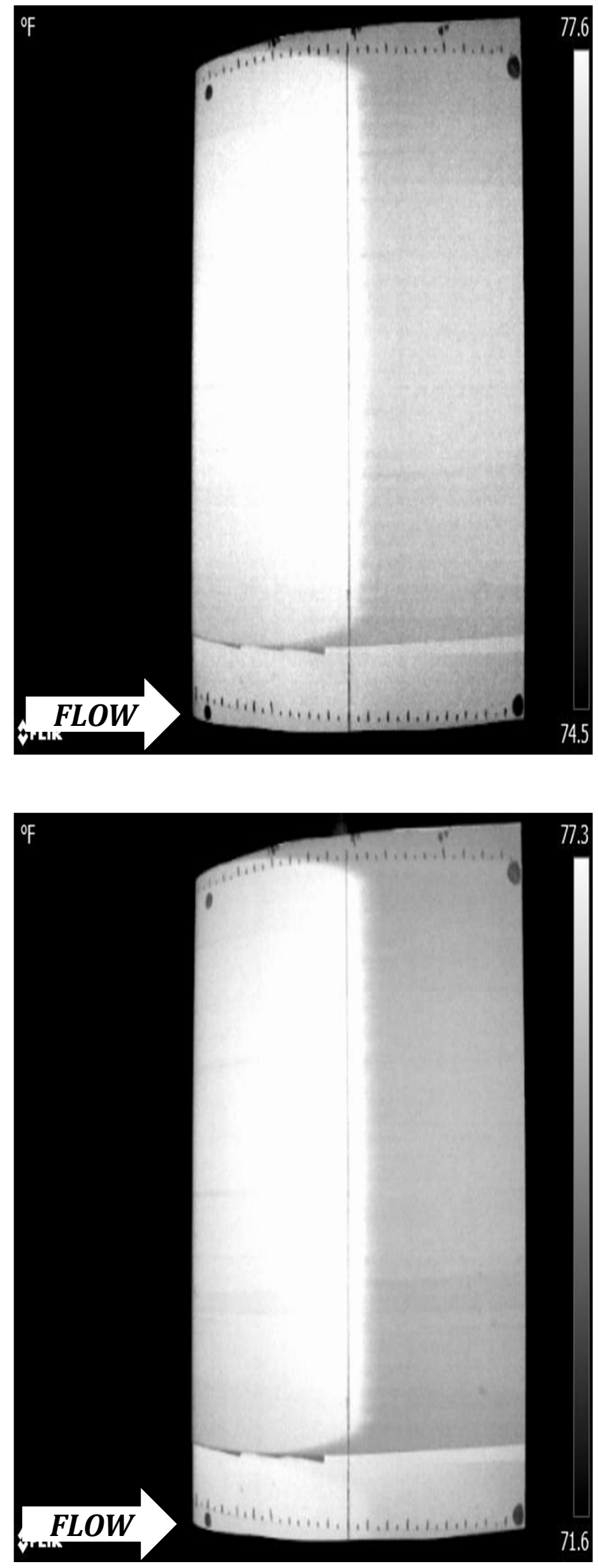


\subsection{6-m DU96-W-180 at Re $=2 \times 10^{5}$}

\section{PRESSURE SIDE}
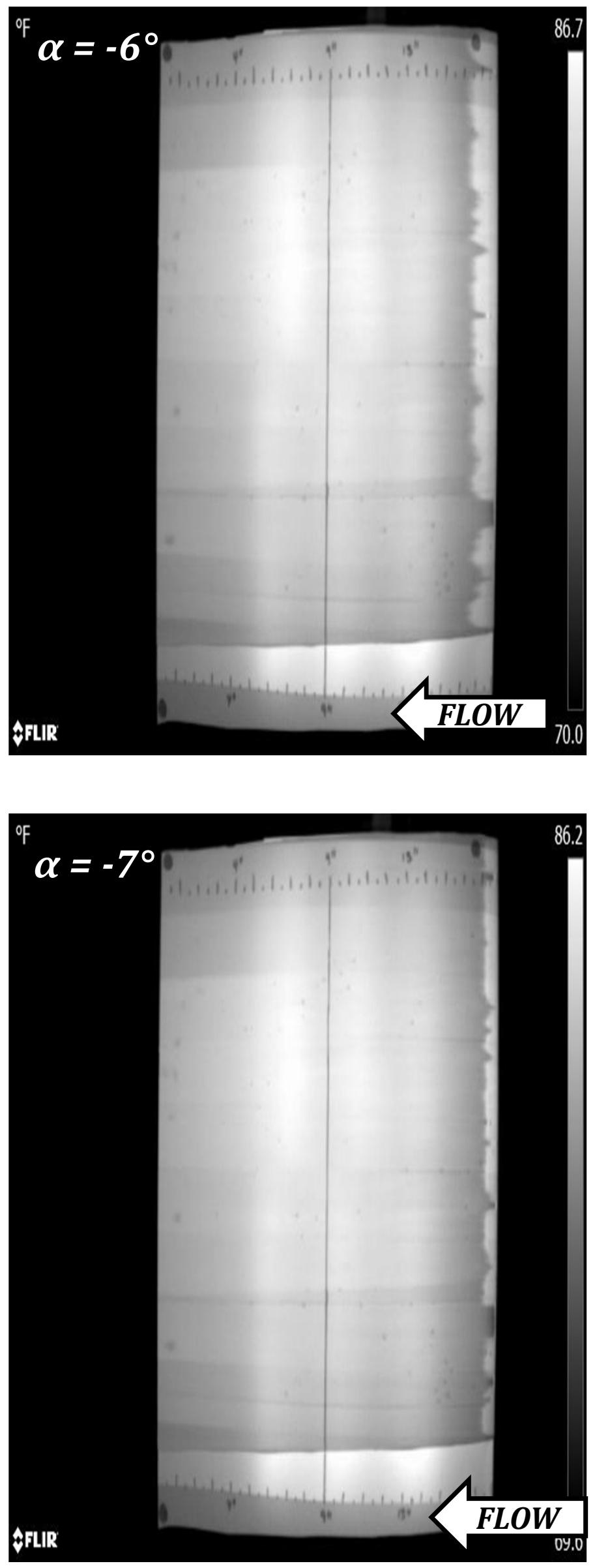

SUCTION SIDE
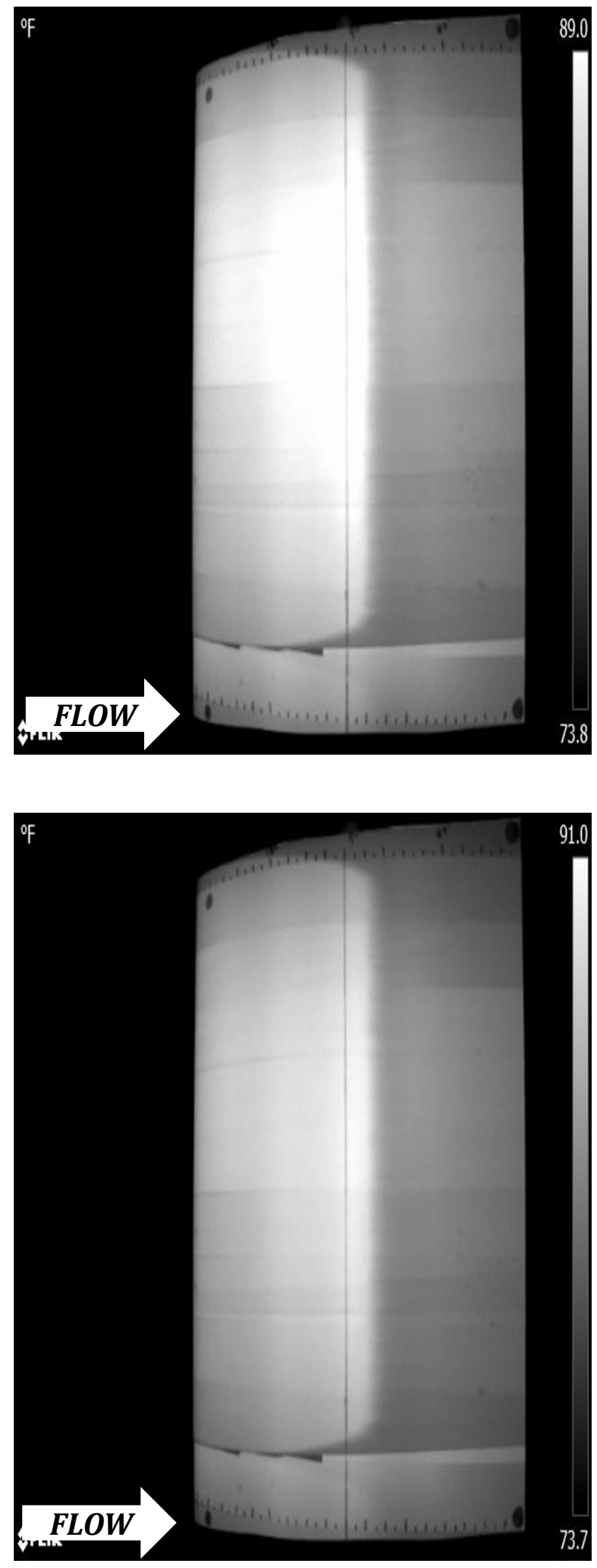


\subsection{6-m DU96-W-180 at Re $=2 \times 10^{5}$}

\section{PRESSURE SIDE}
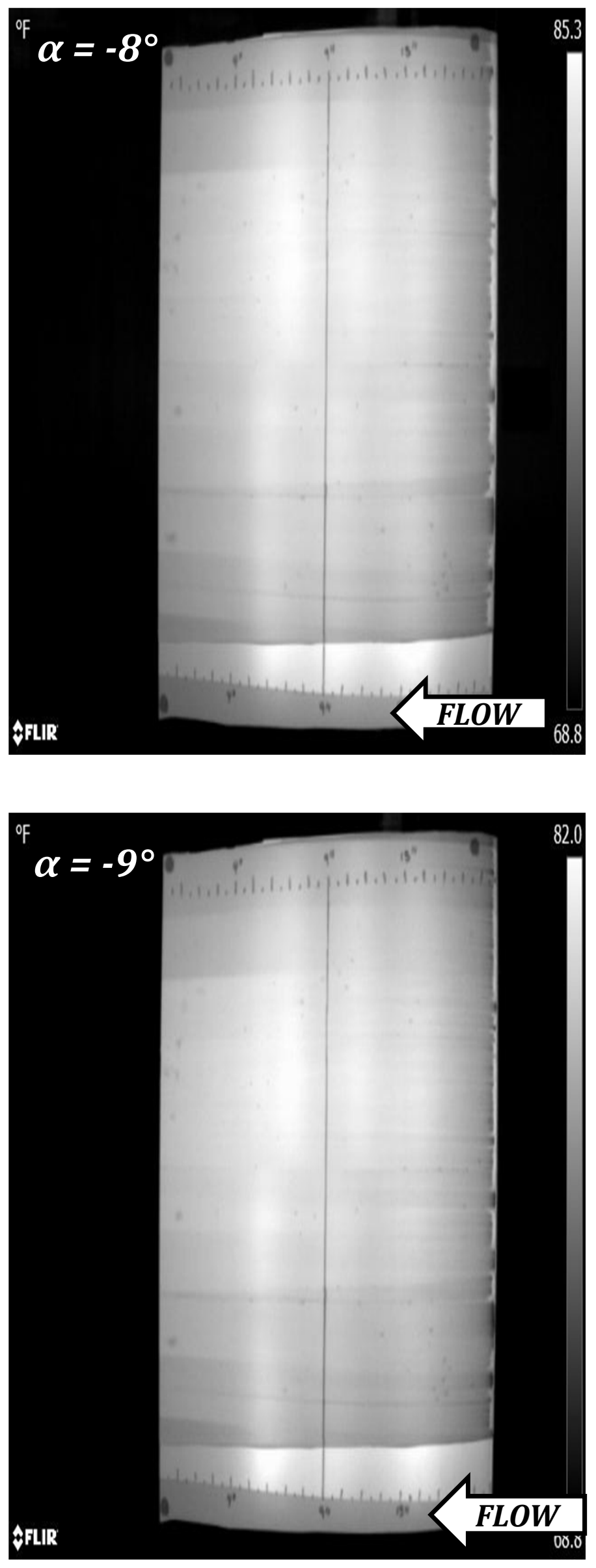

SUCTION SIDE
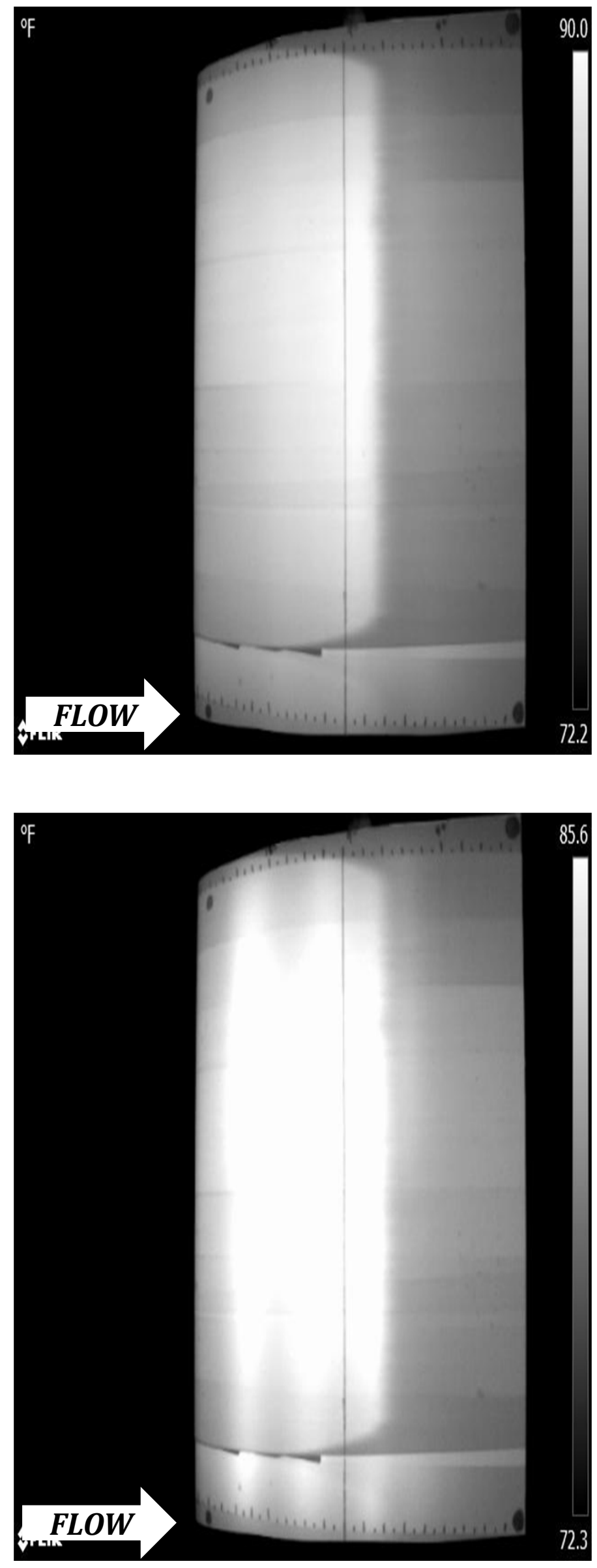


\subsection{6- $\mathrm{m}$ DU96-W-180 at $R e=2 \times 10^{5}$}

\section{PRESSURE SIDE}
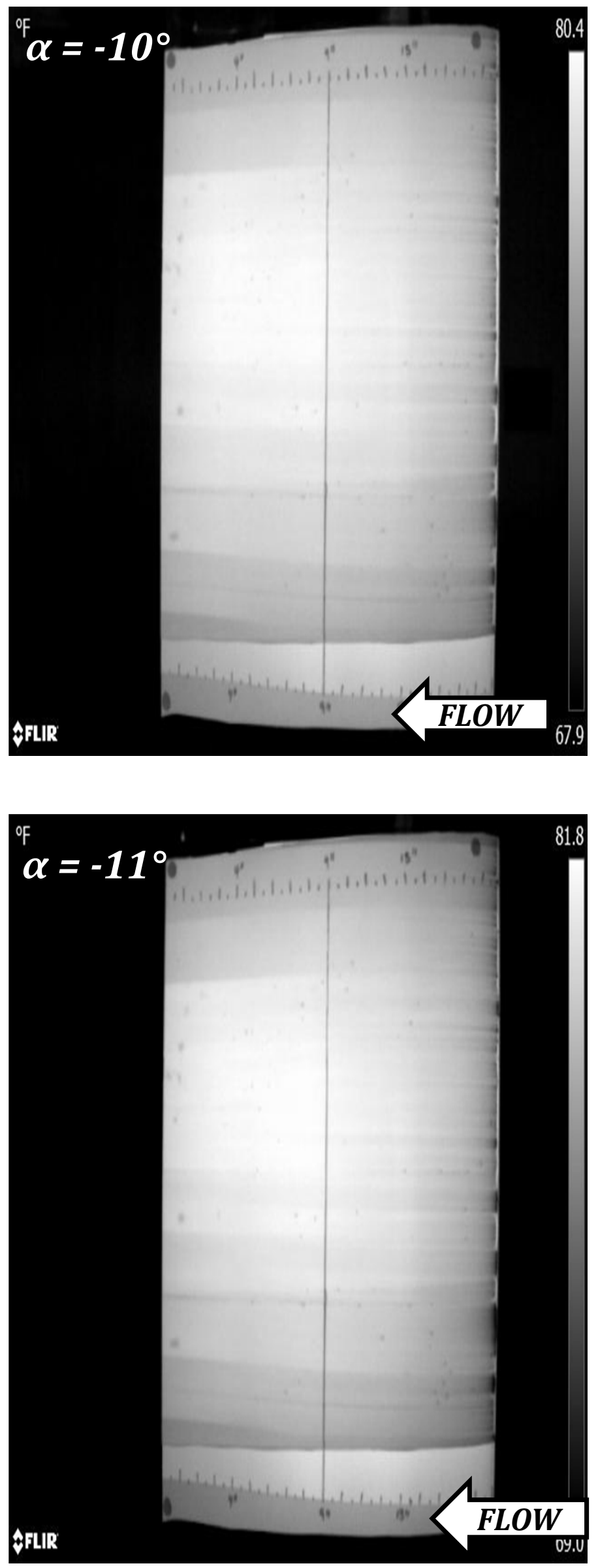

SUCTION SIDE
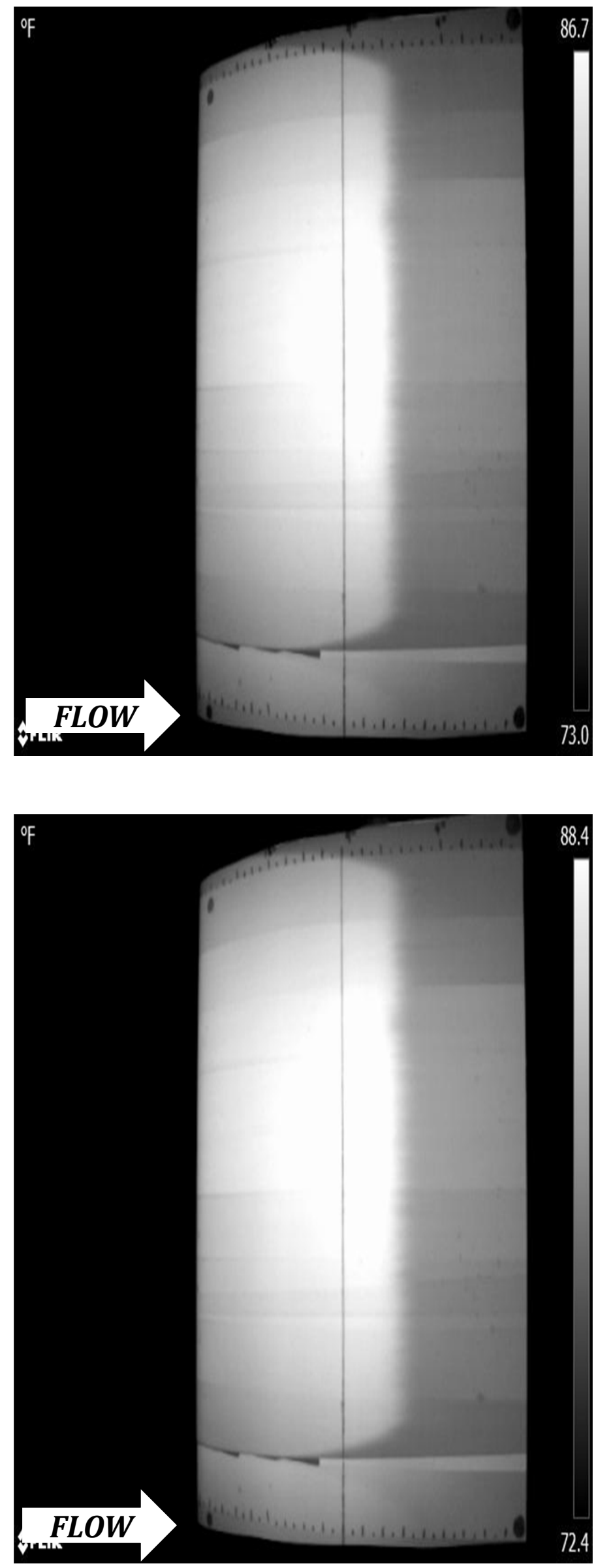


\subsection{6- $\mathrm{m}$ DU96-W-180 at $R e=2 \times 10^{5}$}

\section{PRESSURE SIDE}
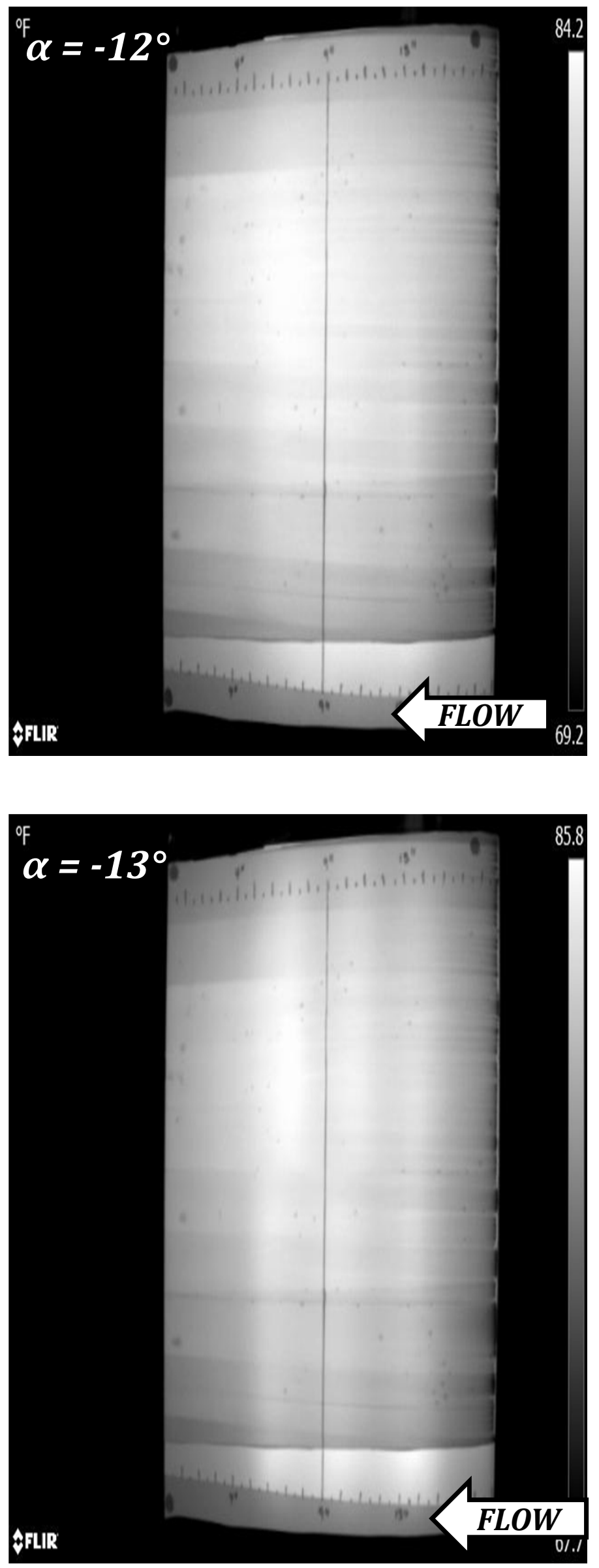

SUCTION SIDE
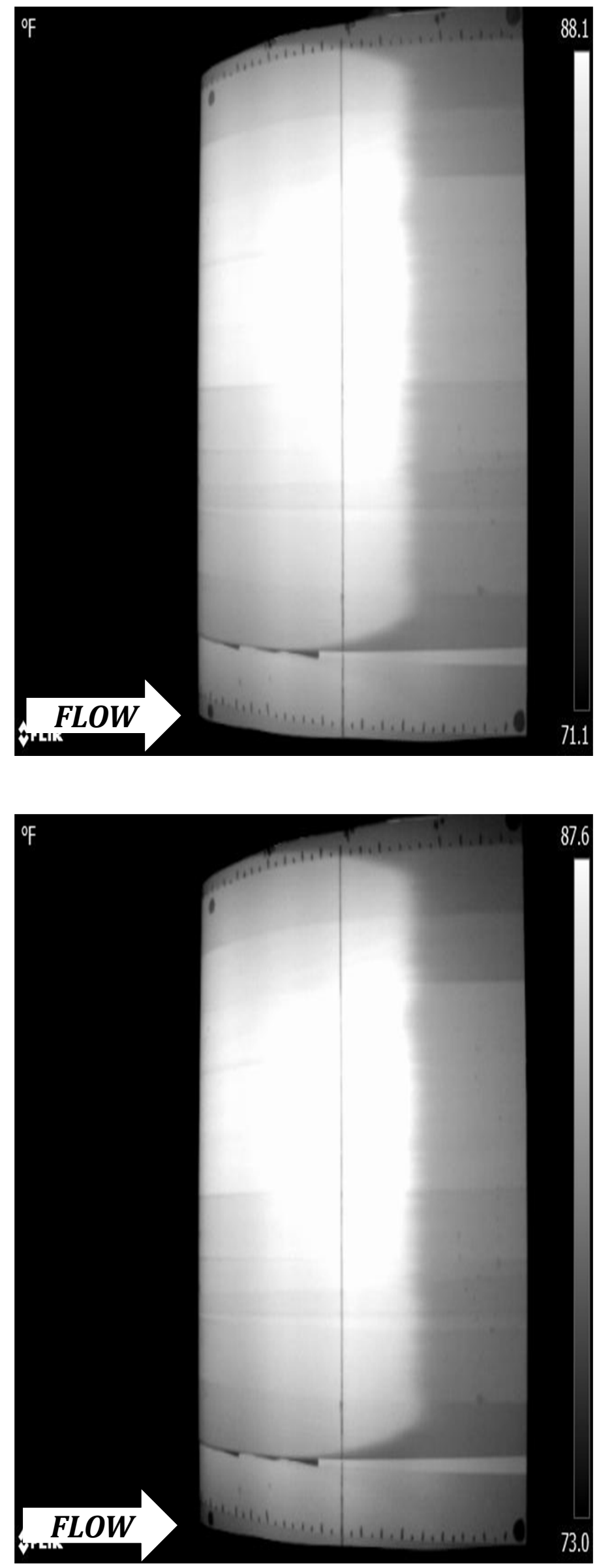


\subsection{6-m DU96-W-180 at $R e=2 \times 10^{5}$}

\section{PRESSURE SIDE}
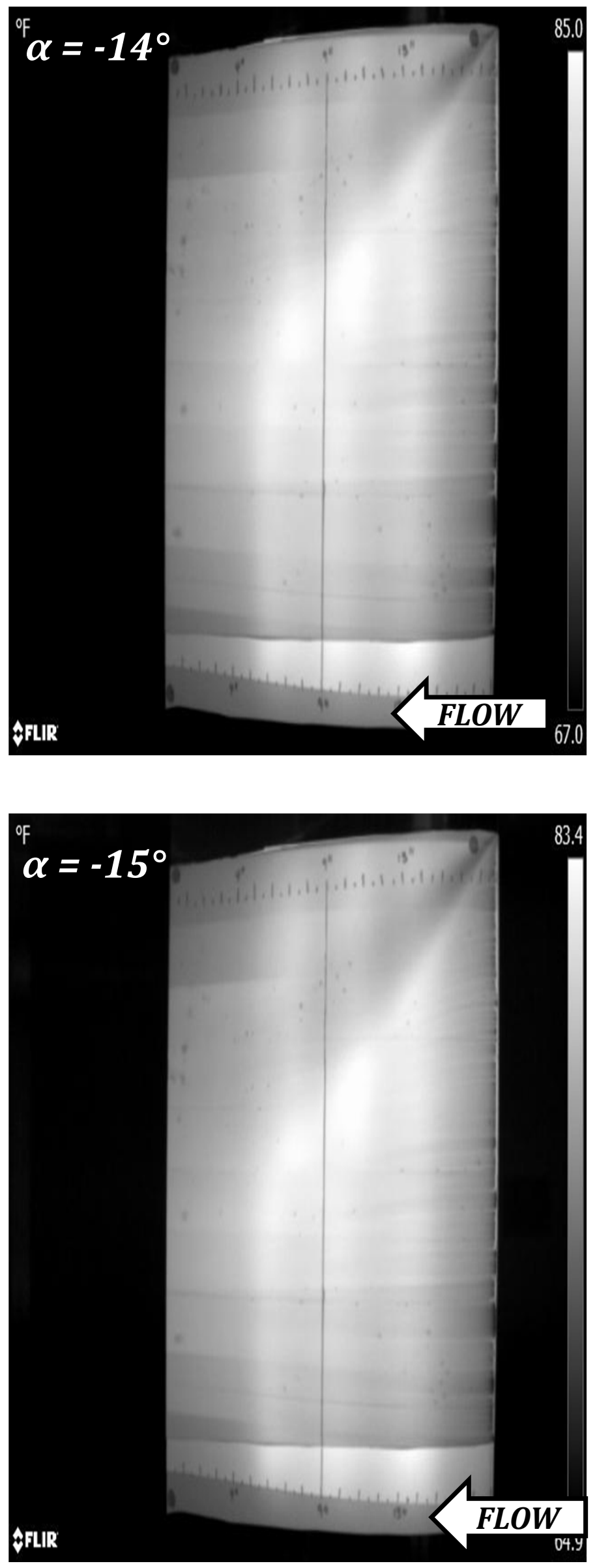

\section{SUCTION SIDE}
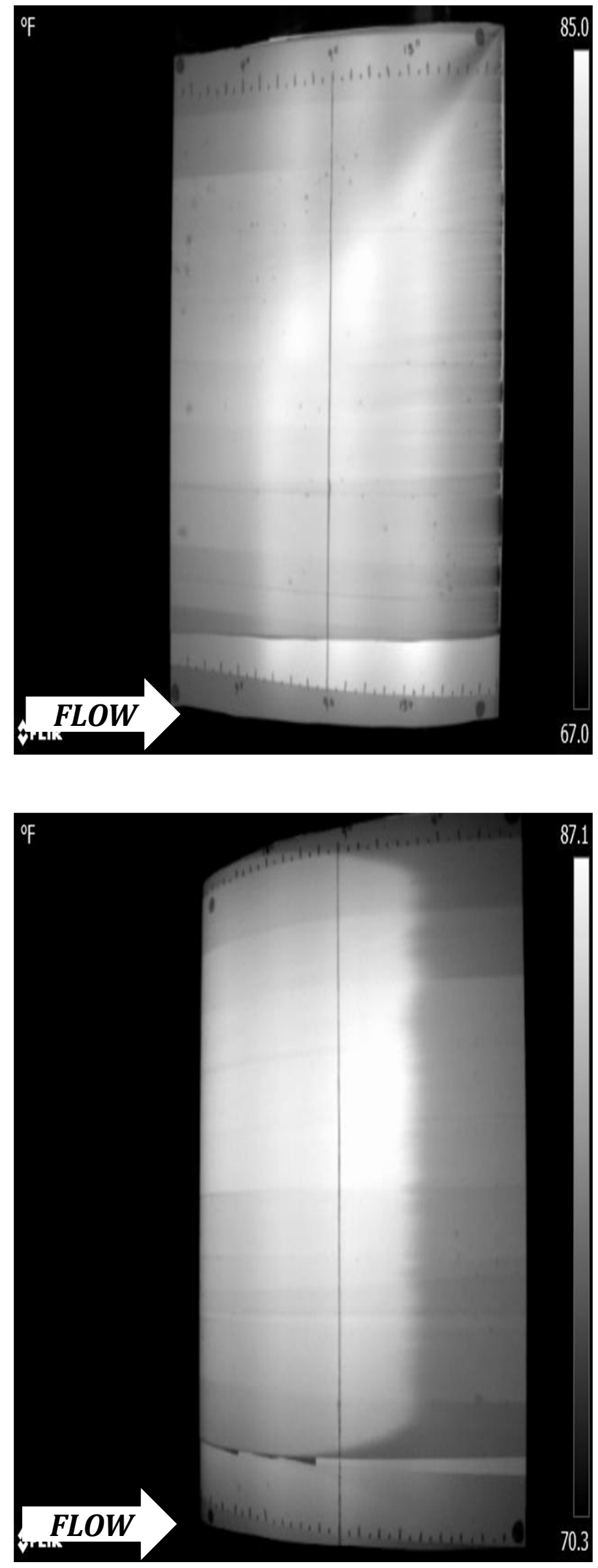


\subsection{6-m DU96-W-180 at Re $=2 \times 10^{5}$}

\section{PRESSURE SIDE}
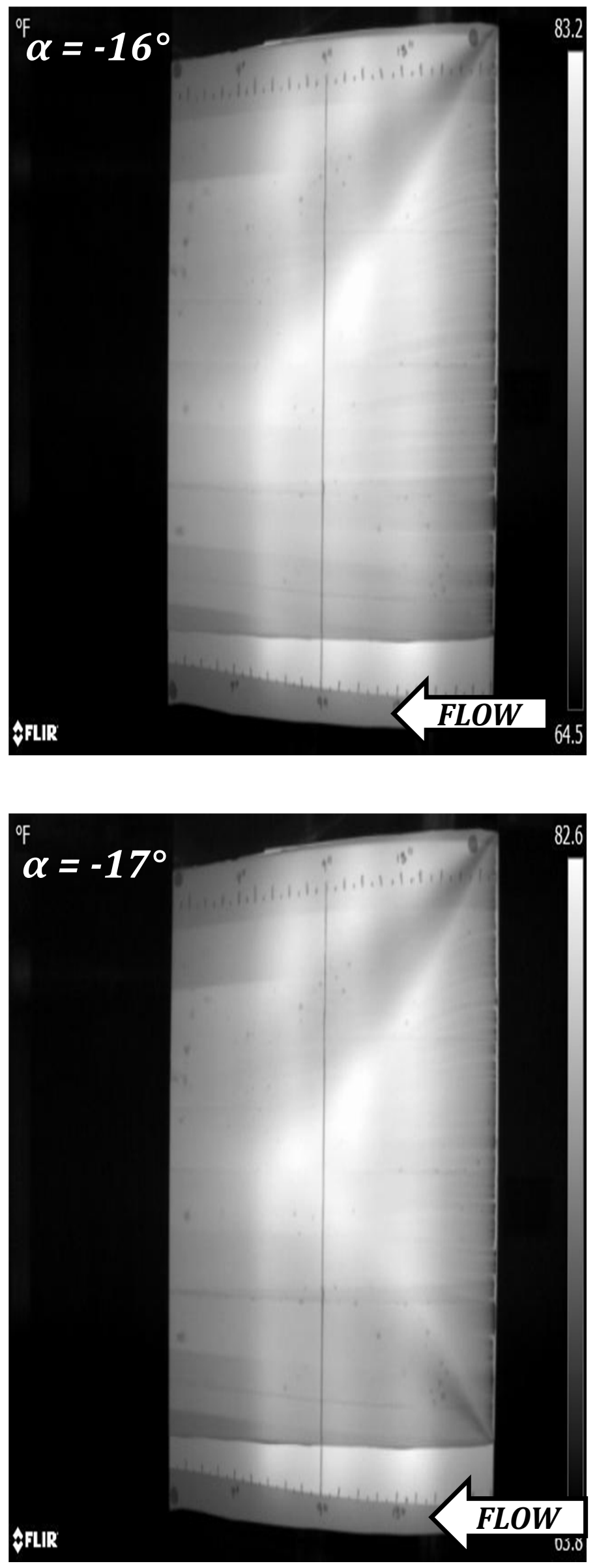

SUCTION SIDE
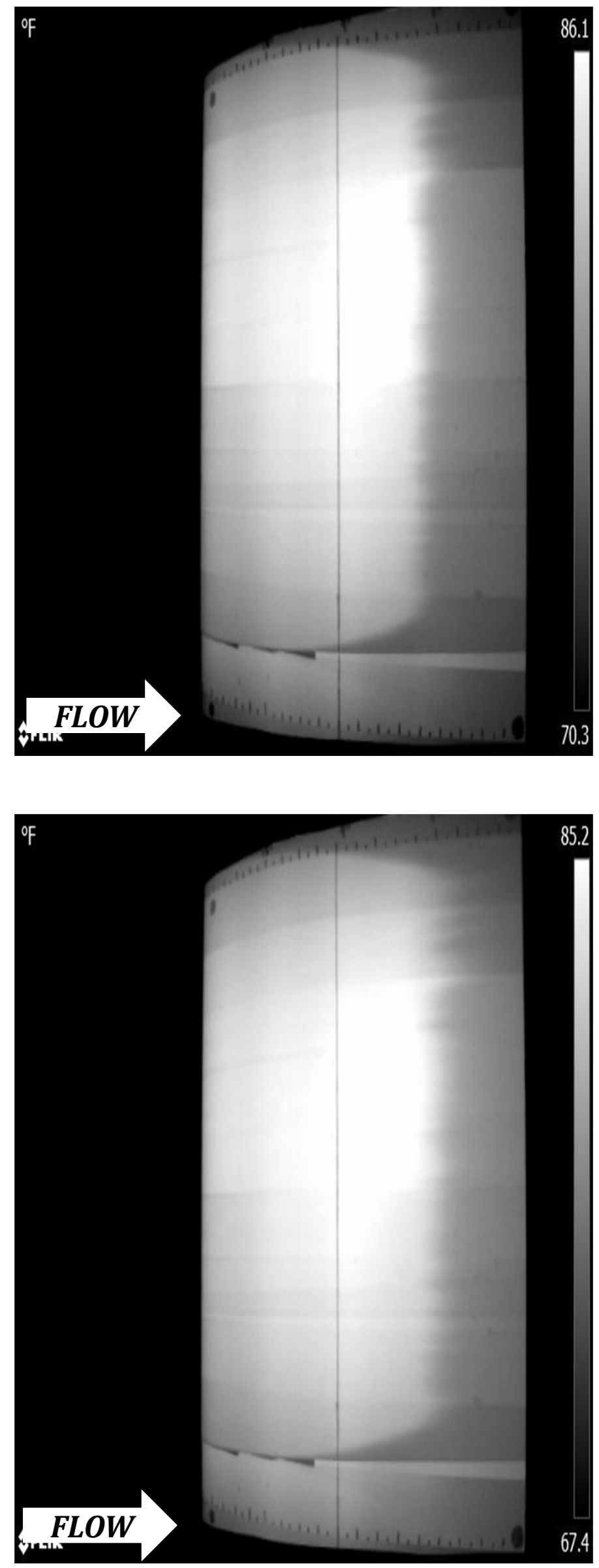


\subsection{6-m DU96-W-180 at Re $=2 \times 10^{5}$}

\section{PRESSURE SIDE}
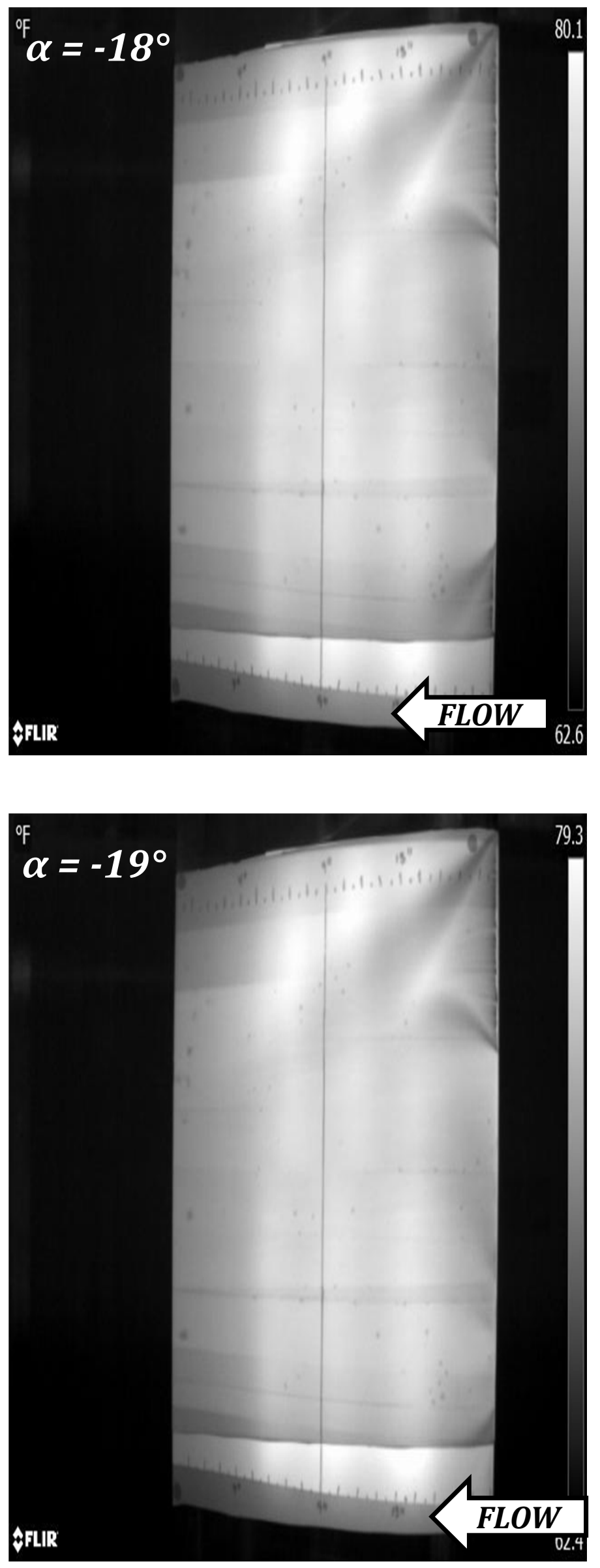

SUCTION SIDE
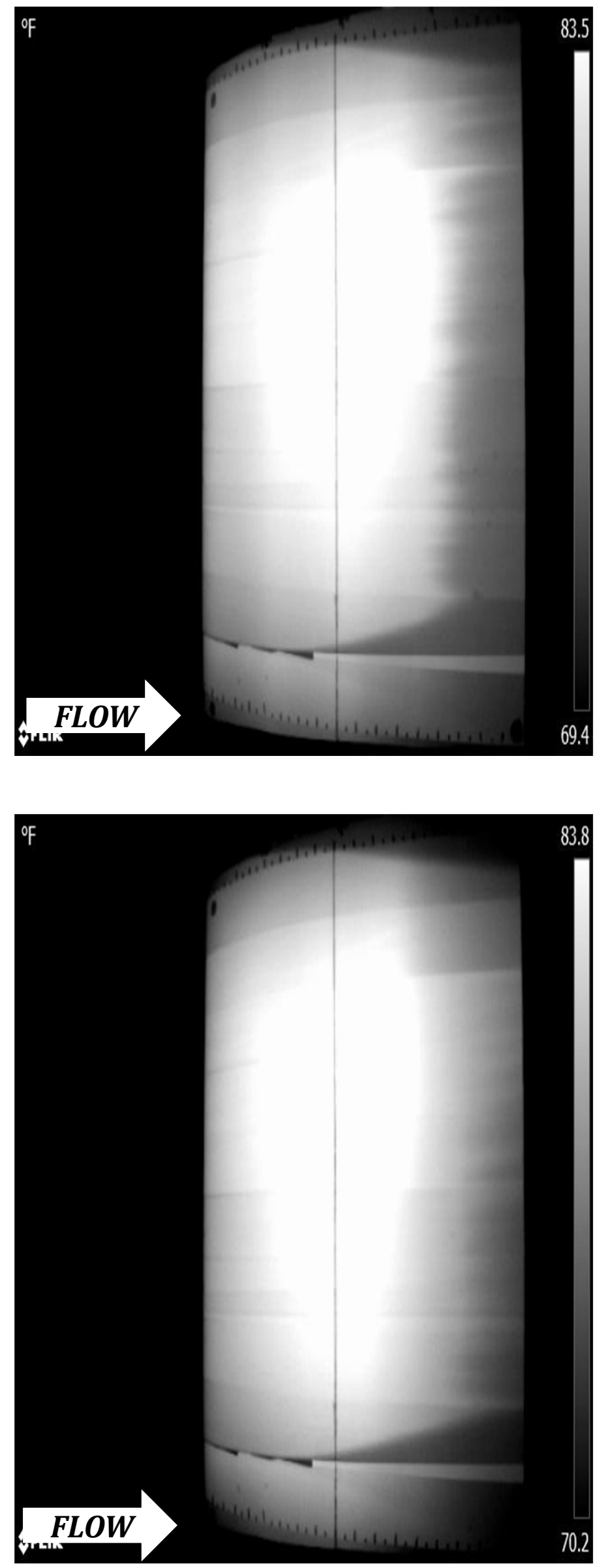


\subsection{6- $\mathrm{m}$ DU96-W-180 at $R e=2 \times 10^{5}$}

\section{PRESSURE SIDE}

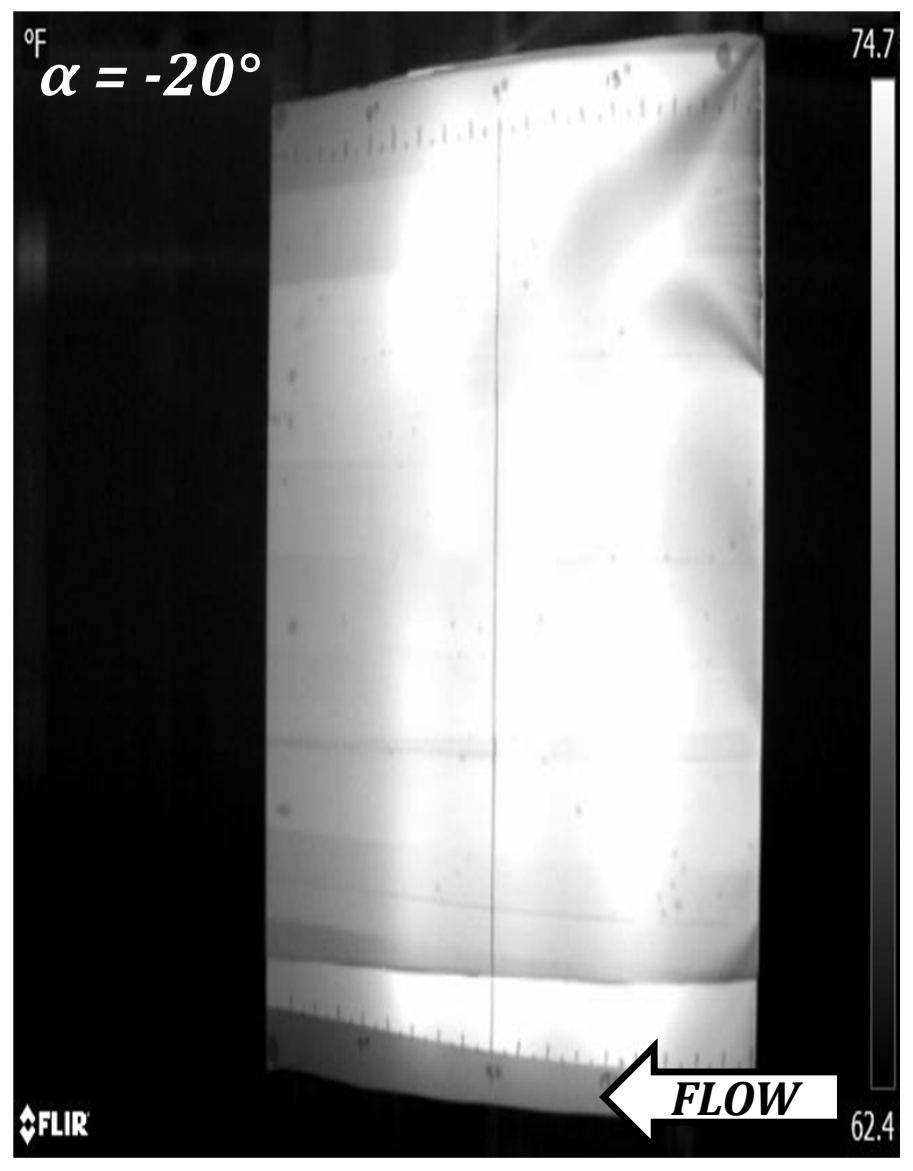

SUCTION SIDE

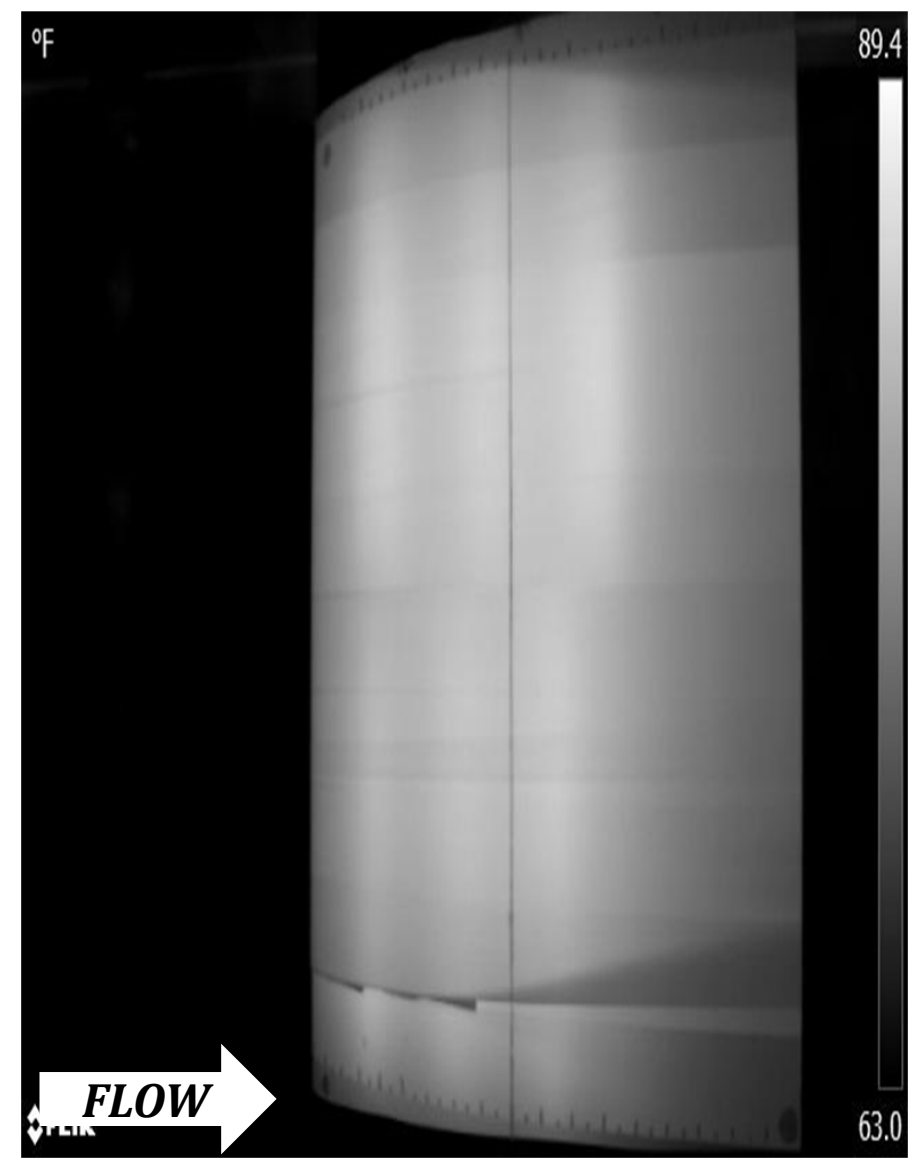

